\title{
9th World Congress on Developmental Origins of Health and Disease
}

4096

Location and preventive measures for pressure ulcers in paediatric age

lurdes lomba ${ }^{1}$, Rita Bessa ${ }^{2}$, Silvia Santos ${ }^{3}$

${ }^{1}$ Coimbra Nursing School; ${ }^{2}$ Dudley Group NHS Foundation

Trust, England; ${ }^{3}$ Unidadde de Cuidados Continuados de

Cantanhede. Portugal

Research question: what are the most frequent anatomical locations of pressure ulcers in pediatrics and the more effective preventive measures used by nurses?

Methods: An integrative review of literature was conducted using online scientific database and selecting studies that answered the research question. A research from the references of the selected studies was added. From the evaluation of the results, 5 studies were selected: 3 address the most common anatomical locations and 2 focus on effective preventive measures.

Results: The most mentioned anatomical sites are occipital and sacrococcygeal, existing some discrepancy between authors. Despite the lack of studies about the efficacy of the materials and measures focusing mainly in adults, the most effective preventive measures selected are the nutritional appointments, alternance of decubitus, use of cushions to relieve the pressure, having suitable surfaces and applying gel plates.

Conclusions: Studies reveal a disagreement about the most frequent sites of pressure ulcers which is justified by the anatomical differences among children and the changes in the pressure locations according to their growth. There is also a dearth of empirical evidence about the effectiveness of the preventive measures. Being vital the recognition of this issue in pediatrics, further investigation is suggested.

\section{3}

Health practices and microbiological evaluation associated with the handling of urethral and peripheral intravenous catheters

Joao Manuel Garcia Graveto ${ }^{1}$, Santos Daniela ${ }^{2}$, Taborda Joana $^{3}$, Manuel Chaves ${ }^{4}$, Anabela Oliveira ${ }^{5}$, Nadia Osório ${ }^{6}$, Precisosa Sousa ${ }^{7}$

${ }^{1}$ Nursing School of Coimbra, Portugal; ${ }^{2}$ Central Hospital of Santarem, Portugal; ${ }^{3}$ Nurse in Central University Hospital of Coimbra, Portugal; ${ }^{4}$ Nursing School of Coimbra, Portugal;
${ }^{5}$ Nursing School of Coimbra, Portugal; ${ }^{6}$ Health School of Coimbra, Portugal; ${ }^{7}$ Health School of Coimbra, Portugal

Introduction: Microbial biofilms are one of the major reasons for nosocomial infections (NIs) and persist in various sites in the human body, especially in association with medical devices (ex.Urethral Catheters-UC and Peripheral Intravenous Catheter-PIC).

Research question: What are the health practices and microbiological results associated with the handling of urethral and peripheral intravenous catheters?

Methods: Cross-sectional, multicenter, analytical-correlational research. After a content analysis of the protocols in force on UC and PIC handling, the teams were training to standardize practices, control study variables and collection data procedures.

Results/Conclusions: Results shows that there are some gaps between the real practice of nursing professionals and the standards implemented by the health units. The microbiological results of UC and PICs suggest suggests the review of some practices and protocols.

\section{5}

Impact of Peritoneal Dialysis On The Family Life Of Children With Chronic Kidney Disease

lurdes lomba ${ }^{1}$, alexandra lameirinhas ${ }^{2}$, Ana Margarida Silva ${ }^{3}$, Juliana Brito $^{4}$

${ }^{1}$ Coimbra Nursing School; ${ }^{2}$ Frimley Park Hospital; ${ }^{3}$ Coimbra nursing school; ${ }^{4}$ Coimbra nursing school

Research question: What are the difficulties experienced by the family in caring for the child on Peritoneal Dialysis and what are the strategies used by nurses to minimize these difficulties?

Methods: A Systematic literature review without meta-analysis was conducted using different search engines and databases with predefined inclusion criteria and descriptors. After critical appraisal of the results, 7 articles were obtained: 5 from MEDLINE and 2 from CINHAL.

Results: Changes in family daily lives are consequence of the disease. The results showed that parents, particularly the mothers, are the main caregivers of these children. They mentioned difficulties related to maintaining a steady job, financial constraints and increased medical costs. None of the studied articles focus on results from the implementation of specific nurse interventions, they only make suggestions about the nurseâs role. 
Conclusion: The changes imposed by the Chronic Kidney Disease adversely affect the family dynamics. Thus, the nursesâ interventions should focus on identifying the difficulties experienced during the adaptation process, sharing care and promoting the familyâs well-being. Future studies on the nursesâ interventions with these families of children on Peritoneal Dialysis are recommended.

\section{6}

Using Telomere Length as Predictive Marker for Susceptibility and Resiliency to Poor Outcomes Following Pediatric Concussion

Richelle Mychasiuk ${ }^{1}$, Harleen $\mathrm{Hehar}^{2}$, Irene $\mathrm{Ma}^{3}$, Michael Esser ${ }^{4}$

${ }^{1}$ University of Calgary; ${ }^{2}$ University of Calgary; ${ }^{3}$ University of Calgary; ${ }^{4}$ University of Calgary

As there is often no detectable damage to the brain following a concussion, diagnosis is often subjective and based on selfreport. What's more, there are no scientifically validated methods for prognosis of injury severity. Although $\sim 15 \%$ of children develop lasting impairments there are no reliable measures to distinguish between those that improve without incident and those requiring intervention. Telomeres are regions of repetitive DNA at the end of chromosomes that have been used to predict lifespan, cancer risk, and susceptibility to the common cold. This study examined the predictive utility of telomeres as markers for prognosis of concussion outcomes in a rodent model, given a variety of perinatal experiences; a high-fat, high-sugar diet; a calorically restricted diet; exercise exposure; and controls. Rats were exposed to one of the aforementioned conditions during pregnancy, weaning, and the remaining experimental time-course. Rats experienced a pediatric concussion or sham injury, were subjected to a behavioural test battery, and sacrificed. This study found that perinatal experience significantly altered telomere length, and that telomere length was strongly associated with the rodent's behavioural response to the concussion. Although further investigation is required, telomeres may be a valuable tool for the prediction of injury resiliency and susceptibility.

\section{8}

Escitalopram effectivity and tolerability in hemodialiysis patients

Pervin Erdem MD ${ }^{1}$, Aylın Yazıcı MD², Abdullah Erdem $\mathrm{MD}^{3}$

${ }^{1}$ Mersin State Hospital, Psychratry Department, Turkey; ${ }^{2}$ Mersin University, Psychiatry Department, Turkey; ${ }^{3}$ Mersin Ortadogu

Private Hospital, Nephrology Department, Turkey

Major depressive Disorder (MDD) is one of the most common psychiatric disorders.
End Stage Renal Disease (ESRD) incidance is increasing and social and economical aspects of the disease becomes more important over the world. The lifetime of ESRD patients becomes longer according to the new developments of medical care.

Numerous studies have shown that; the incidance of MDD is higher at ESRD patients than the general population. Depression not only affects the life quality but the immunity, nutritional status and survival of the patients. According to these factors, depression treatment becomes more important for ESRD patients.

In this study we evaluated 145 hemodialysis patients and determined major depressive disorder at 67 patients. 62 patients included into the study. Patients were divided into two groups and escitalopram and placebo were given to the groups. 58 patients completed the study. 30 patients were from escitalopram group and 28 were from placebo group.

Hamilton depression rating scale was used to determine depressive symptoms. Treatment with escitalopram compared to placebo was associated with significantly greater improvements on the depressive symptoms. The side effect profile and severity of escitalopram compared to placebo was found tolerable.

As a result we found escitalopram effective and tolerable in the treatment of depression at the ESRD patients.

KeyWords: Escitalopram, major depressive MDD, ESRD

4423

A Nationwide register study on maternal and foetal factors in bladder exstrophy in sweden

Ängla Mantel ${ }^{1}$, Magdalena Fossum ${ }^{2}$, Agneta Nordenskjöld ${ }^{3}$

${ }^{1}$ Karolinska Institute; ${ }^{2}$ Karolinska Institute; ${ }^{3}$ Karolinska Institute

Bladder exstrophy is a rare congenital malformation where the underlying cause is largely unknown with both environmental and genetic mechanisms involved.

We conducted a nationwide epidemiological study related to maternal and foetal risk factors of bladder exstrophy in Sweden 1973-2011.

120 patients were identified in the Medical Birth Register with ICD codes; 753.50, 753F and Q64.1, 1973-2011. Cases were matched with 5 controls/patient for calendar year of delivery and sex, and a case-control study was performed by linkage between several national registers.

The study showed a total live-birth prevalence of 2.98:100 000 with a 1.14:1 male-to-female ratio, demonstrating a higher risk among mothers with Nordic origin of birth, age, BMI and smoking. Parity and assisted conception were not identified as risk factors. Delivery mode, birth weight, gestational week, Apgar and survival did not differ from controls. Major associated malformations were present in $7.5 \%$ of the cases. 
$41 \%$ had surgery for inguinal hernia and $11 \%$ of the boys for retentio testis.

This national register study on bladder exstrophy demonstrated a prevalence of 2.98:100 000 live-births with an almost equal sex ratio. Majority of cases were isolated without major associated malformations. Nordic maternal origin of birth, age, $\mathrm{BMI}$ and smoking were associated with higher risk.

\section{8}

Management through Starving and distinguishing the cancer cells as strategy to augment the conventional chemo, immune and radiotherapy, surgery, and marrow transplantation

Prof. Asenath Sigot ${ }^{1}$, Dr. John Okoth ${ }^{2}$, Bridget Musuke ${ }^{3}$

${ }^{1}$ Masinde Muliro University of Science and Technology; ${ }^{2}$ Masinde Muliro University of Science and Technology; ${ }^{3}$ Masinde Muliro University of Science and Technology

Cancer cells are the body cells that grow and divide at an unregulated and quickened pace. In its etiology, cancer goes through a multi stage process described in three progressive phases. Its prevalence, incidences and mortality are on the rise, being listed the fourth leading cause of death with $70 \%$ of which occurring in Low and Middle income countries. Multidisciplinary approach is key in the management of cancer especially when it has to be strategically applied to correct nutritional deficiencies, minimize weight loss but without feeding the tumor cells that is 'staving the cancer cells' and for chemotherapy apply innovative therapeutic dietetics to afford the amplification and enhance affinity of antineoplasts directed to the tumor cells and not the normal cells. This paper has explored the therapeutic foods with scientifically analyzed nutritional and phytochemical values to inform the practitioners on beneficial nutrient, phytochemical, drug and excipient interactions that potentiates medical management of cancer.

\section{3}

Maternal risk factors and perinatal characteristics of hirschsprung's disease

Anna Svenningsson ${ }^{1}$, Eva Hagel ${ }^{2}$, Agneta Nordenskjöld ${ }^{3}$, Tomas Wester ${ }^{4}$

\section{${ }^{1}$ Karolinska Institutet; ${ }^{2}$ Karolinska Institutet; ${ }^{3}$ Karolinska Institutet; ${ }^{4}$ Karolinska Institutet}

Research question: Hirschsprung's disease (HSCR) is a multifactorial disease caused by both genetic and environmental factors. The aim of this study was to examine maternal risk factors and perinatal characteristics for HSCR and to study the Swedish perinatal incidence of the disease.

Method: A Swedish nationwide, population-based, casecontrol cohort containing all children born in Sweden between 1/1 1982 till 31/12 2013 was used for this study. Five age- and sex-matched controls were randomly selected among children without HSCR in the cohort. The association between studied risk factors and HSCR were analyzed using conditional logistic regression to calculate odds ratio (ORs) and 95\% confidence intervals (CIs).

Results: The study included 600 cases of HSCR (466 males) and 3000 controls (2330 males). The incidence of HSCR was $1,88 / 10000$ live newborns. The only maternal factor associated with increased risk for HSCR was high maternal BMI (Body Mass Index) 30,0-34,9 OR 1,89 (1,31-2,74). The perinatal characteristics showed that children with HSCR were born at a lower gestational age.

Conclusion: The study shows a Swedish incidence of HSCR of 1,88/10000 live newborns. Children with Hirschsprung's disease were born at a lower gestational age than controls. Maternal obesity may increase the risk for HSCR.

\section{8}

A secular trend for pubertal timing in Swedish men born 1946-1991 - the BEST cohort

Maria Bygdell $^{1}$, Jenny Kindblom ${ }^{2}$, Claes Ohlsson ${ }^{3}$

${ }^{1}$ Institute of medicine; ${ }^{2}$ Institute of medicine; ${ }^{3}$ Institute of medicine

Studies of male pubertal timing are scarce. We collected growth data from Child Health Care centers in Gothenburg, Sweden, for children born 1946 and later and established a unique population-based cohort, the BMI Epidemiology study (BEST; $\mathrm{n} \approx 400000$ ). The overall aim is to determine the role of childhood obesity and pubertal timing for diseases later in life. The aim with this BEST sub-study was to study male pubertal timing. Men born every five years from 1946 to 1991 were evaluated $(n=300$ for each birth year, $n=3,000$ for the entire sub-cohort). Age at Peak Height Velocity (PHV) was calculated from growth data and used as an objective measurement of pubertal timing.

The mean age at PHV was $13.9 \pm 1.1$ years. Linear regression analyses revealed that age at PHV was 1.4 months earlier for every 10 -year increase in birth year $\left(\mathrm{p}=2.6^{*} 10-17\right)$. Similar results were seen after adjustment for changes in ethnic composition between birth cohorts. When childhood BMI was included as a covariate, the effect of birth year on PHV was maintained but attenuated.

In conclusion, we provide compelling evidence of a secular trend for pubertal timing in Swedish men. The effect is partly but not completely explained by increased childhood BMI.

\section{3}

Mode of delivery is associated with plasma adiponectin changes from late pregnancy to postpartum: a prospective cohort in Rio de Janeiro, Brazil

Fernanda Rebelo ${ }^{1}$, Claudio Struchiner ${ }^{2}$, Gilberto Kac $^{3}$

${ }^{1}$ National School of Public Health/ Fiocruz; ${ }^{2}$ National School of Public Health/ Fiocruz; ${ }^{3}$ Rio de Janeiro Federal University 
Research question: Considering the potential role of adiponectin in determining important outcomes of maternal and child health, this study aims to evaluate the association between mode of delivery and changes on maternal plasma adiponectin from late pregnancy to postpartum.

Methods: Prospective cohort with follow-up at 30-36th gestational weeks and 30-45 days postpartum. Subjects were between 20-40 years old, free of chronic and infectious diseases and present singleton pregnancy. Mode of delivery was classified as vaginal (VD) or cesarean section (CS). Plasma adiponectin $(\mu \mathrm{g} / \mathrm{mL})$ was measured using commercial ELISA kits. Statistical analyses included Wilcoxon rank-sum test and adjusted linear mixed effects model.

Results: Postpartum adiponectin were higher for the VD group ( $\mathrm{n}=99$; median: 8.25, IQR: 5.85-11.90), compared to the CS group ( $\mathrm{n}=60$; median: 7.34, IQR: 4.36-9.76; $\mathrm{p}=0.037$ ). This difference was not observed for third trimester samples. Women who had a CS presented a lower rate of increase in adiponectin from third trimester to postpartum, compared to those who had VD $(\beta=-0.15,95 \%$ CI: $-0.28,-0.02$, $\mathrm{p}=0.026)$.

Conclusion: Women who delivered by CS had lower rates of increase in plasma adiponectin after delivery, compared to those who had a VD. This difference resulted in lower absolute concentrations of this adipokine 30-45 days postpartum.

\section{6}

The Literacy in Professional Nursing in Peripheral Intravenous CatheterizationProcedures - An Integrative Review

Graveto Joao Manuel, Oliveira Ana Catarina,

Martins Ana Rita

Nursing School of Coimbra - Portugal

Introduction: The peripheral intravenous catheterization (PIC) is a common practice in the hospital setting with risk of nosocomial infections. Few empirical studies have evaluated the relationship between nurses' knowledge of national/international recommendations on this procedure.

Objective: To know which scientific evidence on the literacy of nurses in this procedure.

Methods: Integrative review of scientific studies published between 2009 and 2014, following the Cochrane (PICOD) model with inclusion/exclusion criteria.

Results: Mostly nurses have knowledge about the guidelines of PIC procedure, however not always mobilize for the provision of nursing care. $89.7 \%$ of nurses said they needed to wear gloves in the salinization of PIC, yet only $30.5 \%$ used gloves (Moncaico \& Figueiredo, 2009). Also the educational activities allowed reflective and update the knowledge of professionals about this procedure times, conceiving objective and desired changes in behavior and increase adherence to recommendations, with consequences in the pratice. Ahlqvist et al.(2006) capud Oliveira \& Parreira, 2010, p.142) mentions that after the implementation of the guidelines, the number of satisfactory nursing care increased by $12 \%$.

Conclusions: Nursing care related to PVC procedure should follow the recommendations based on scientific evidence, in order to reduce complications and costs associated with the care and increase the safety and welfare of the patient. We recognize the contribution as required for further research in this area.

\section{7}

Young obese pre-diabetic rats are resistance to wound healing

Jean Besson $^{1}$, Luzmarina Hernandes ${ }^{2}$, Claudinéia Franco ${ }^{3}$, Rosiane Miranda $^{4}$, Carina Previate ${ }^{5}$, Isabela Martins ${ }^{6}$, Karina Morikawa ${ }^{7}$, Débora Luz ${ }^{8}$, Rodrigo Gomes ${ }^{9}$, Graciette Matioli ${ }^{10}$, Paulo Mathias ${ }^{11}$

${ }^{1}$ Laboratory of Animal Histology, Department of Morfological Sciences, State University of Maringá; ${ }^{2}$ Laboratory of Animal Histology, Department of Morfological Sciences, State University of Maringá; ${ }^{3}$ State University of Maringá Laboratory of Secretion Cell Biology, Department of Biotechnology, Genetics and Cell Biology; ${ }^{4}$ State University of Maringá Laboratory of Secretion Cell Biology, Department of Biotechnology, Genetics and Cell Biology; ${ }^{5}$ State University of Maringá Laboratory of Secretion Cell Biology, Department of Biotechnology, Genetics and Cell Biology; ${ }^{6}$ State University of Maringá - Laboratory of Secretion Cell Biology, Department of Biotechnology, Genetics and Cell Biology; ${ }^{7}$ Laboratory of Animal Histology, Department of Morfological Sciences, State University of Maringá; ${ }^{8}$ Laboratory of Animal Histology, Department of Morphological Sciences, State University of

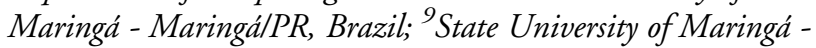
Department of Physiological Sciences; ${ }^{10}$ Laboratory of Enzimatic Biotechnology, Department of Pharmacie, State University of Maringá; " ${ }^{1}$ State University of Maringá - Laboratory of Secretion Cell Biology, Department of Biotechnology, Genetics and Cell Biology

Background: Impaired cutaneous wound healing is a major health concern. Recent evidence indicates that aberrant wound site inflammation may be an underlying cause for delayed healing. Type 2 diabetes and obesity are common metabolic disorders characterized by resistance in the actions of insulin resulting in a wound healing low-level. Whether or not topic insulin treatment improved wound healing in pre-diabetic rats, was tested.

Methods: Pre-diabetic-rats were obtained by neonatal treatment with monosodium L-glutamate (MSG) and controls saline. Three experimental groups were designed: MSGanimals treated or untreated with topical exogenous insulin 
and control-rats treated with topical exogenous insulin. Treatment was initiated at 60-days-old. After a period of $4,7,10$ and 14 days, of treatment the animals were sacrificed to inflammation (myeloperoxidase), cell migration (keratinocytes) and neovascularization evaluation.

Results: After 7 days, MSG-insulin-treated or -untreated rats showed high levels of myeloperoxidase compared control animals $(\mathrm{p}<0.05)$. After all treatment periods $(4,7,10$ and 14 days) was observed retarded keratinocyte migration and neovascularization with insulin topical treatment for wound healing.

Conclusion: MSG-rats presented severe resistance to exogenous insulin for wound healing, revealing alterations in inflammatory conditions and failures in process as cell proliferation and angiogenesis.

\section{4}

Prenatal exposure to maternal diabetes mellitus and risk of childhood mortality among children with birth defects in TEXAS, USA

Wendy Nembhard ${ }^{1}$, Shams Rahman ${ }^{2}$, David Fixler ${ }^{3}$, Mary Ethen ${ }^{4}$, Alfred Mbah ${ }^{5}$, Mark Canfield ${ }^{6}$

${ }^{1}$ University of Arkansas for Medical Sciences \& Arkansas Children's Hospital Research Institute; ${ }^{2}$ University of South Florida, College of Public Health; ${ }^{3}$ Division of Cardiology, Department of Pediatrics, University of Texas Southwestern Medical Center; ${ }^{4}$ Birth Defects Epidemiology and Surveillance Branch, Texas Department of State Health Services; ${ }^{5}$ University of South Florida, College of Public Health; ${ }^{6}$ Birth Defects Epidemiology and Surveillance Branch, Texas Department of State Health Services

Research question: What is the association between prenatal maternal diabetes mellitus (DM) and childhood mortality risk among children with birth defects and does the association vary by maternal race/ethnicity?

Methods: We conducted a retrospective cohort study of children with major structural birth defects born January 1999 - December 2008 in the Texas Birth Defect Registry. Prenatal maternal DM status was determined by medical and birth record review. Hazard ratios (HR) and 95\% confidence intervals (CIs) were calculated from multivariable Coxproportional hazards regression models.

Results: After adjusting for covariates, maternal Type 2 and gestational DM did not increase risk of childhood mortality. Type 1 DM was associated with decreased risk of childhood mortality (HR $=0.69$; 95\% CI: 0.47-1.01). Overall, maternal Type 1, Type 2 and gestational DM were not associated with increased risk of childhood mortality by race/ ethnicity.

However, children born to non-Hispanic (NH)-black mothers with pre-pregnancy DM or DM not-specified had increased risk of childhood mortality $(\mathrm{HR}=1.74 ; 95 \%$
CI: $1.00-3.03$ and $\mathrm{HR}=1.67$; 95\% CI: 0.94-2.97, respectively) compared to children of NH-black mothers without DM.

Conclusions: Prenatal maternal DM exposure did not increase overall childhood mortality but may increase childhood mortality for NH-black children with birth defects.

\section{5}

Has Neonatal Resuscitation Program (NRP) made a difference on the Apgar score at 5 minutes (Apg5) in the last 30 years?

Jagjit Singh Teji

Unversity of Chicago

Background: NRP was instituted in 1987 and revised in 2011. Apg5, has been inversely associated with neonatal morbidity and mortality, NMM, and infant mortality, IMR.

Objective: How has the Apg5 changed in the last 30 years in the USA?

Design/Methods: The data from NCHS vital statistics USA for the years 1983 thru 2012. Yearly variables used for analysis consisted of Apg5, year of birth, gestational age, birthweight, plurality, race, ethnicity, maternal age and education, maternal risk factors and mortality. STATA 13.0 and Excel 2010 were used for analysis.

Results: Data from 120 million newborns were used from 1983 thru 2012. The most significant decline occurred for Apg5 of 10 and 0 that declined from $24.96 \%$ to $3.32 \%$ and $0.07 \%$ to $0.03 \%$, from 1983 to 2012 , respectively. While Apg 5 of 9 increased from $62.16 \%$ to $83.14 \%$. Infant mortality demonstrated OR 150.93 (CI 148-152), P > 0.0005 for Apg5 from 0-3; and 25.23 (CI 24.8-25.2) P > 0.000 for Apg5 from 4-6;

Conclusions: 1.This is the largest assessment of Apg5 for newborns to date. 2. NRP may have provided for better assessment and care of baby's condition at birth and thereby impact on later health and disease.

\section{6}

Delivery Pattern of Multiple births in the last 30 years in the USA and its impact on DOHaD

Jagjit Singh Teji

Unversity of Chicago

Background: The delivery timings of singleton and multiple births have demonstrated a variation in the literature. In the USA multiracial, multi-ethnic, and intermix of these groups create new and diverse group potentially with unpredictable perinatal outcomes.

Objective: Determine the delivery pattern of multiple births in the USA over the last 30 years. 
Design/Methods: The NCHS data was used for 1983 thru 2012. Variables consisted of yr, ethnicity, plurality, gestational age, birthweight and maternal risk factors were used for analysis with STATA 13 and Excel 2010.

Results: Over 120 million births were analyzed from 1983 thru 2012. Singleton and multiple births for every week of gestation are occurring relatively earlier in the latter part of the study period. Earlier births were more common in the non-Hispanic blacks, unmarried, teenage or advanced maternal age of mother, and in maternal risk factors.

Conclusions: 1.This is the largest study of delivery pattern of multiple births to date. 2. Delivery of all levels of plurality occurred earlier in the latter part of the study period. 3. Delivery of singleton or multiples occurring earlier than prior years if unabated can have detrimental impact on the burden of disease particularly the metabolic and cardiovascular diseases in future.

\section{8}

Population based nationwide study of hypospadias in Sweden, 1973 to 2009: incidence and risk factors

Anna Skarin Nordenvall ${ }^{1}$, Louise Frisén ${ }^{2}$,

Anna Nordenström ${ }^{3}$, Paul Lichtenstein ${ }^{4}$,

Agneta Nordenskjöld ${ }^{5}$

${ }^{I}$ Department of Women's and Children's health, Karolinska

Institute; ${ }^{2}$ Department of Clinical Neuroscience, Karolinska

Institute; ${ }^{3}$ Department of Women's and Children's Health,

Karolinska Institute; ${ }^{4}$ Department of Medical Epidemiology and

Biostatistics, Karolinska Institute; ${ }^{5}$ Department of Women's and Children's Health, Karolinska Institute

We studied the incidence of hypospadias in Sweden during a 40-year period to determine if changes were associated with known risk factors.

Prospectively collected data from nationwide health care and demographic registers was analyzed and collected for all males born in Sweden between 1973 and 2009. The incidence of hypospadias per 1,000 live-born boys was calculated as number of cases divided by total number of births yearly. The association between hypospadias and risk factors was estimated using logistic regression, expressed as OR's.

The nationwide incidence of boys diagnosed with hypospadias was approximately 4.5 per 1,000 live-born boys until 1990 , increasing to 8 per 1,000 boys during the following decade. Mild and severe phenotypes comprised the increase. Boys born small for gestational age (OR 4.34), as a twin (OR 1.8 ) or as a result of in vitro fertilization (OR 1.15) were at increased risk for hypospadias. Multivariate analyses revealed that changes in risk factors did not explain the increased incidence.

This study demonstrates an increased incidence of hypospadias diagnoses in Sweden from 1990 to 1999 that is not attributable to previously known risk factors. The increase includes mild and severe phenotypes, suggesting that shifts in the diagnostic criteria are not the underlying cause.

\section{4}

Early developmental considerations of chronic pelvic pain

John Jarrell

University of Calgary Canada

Introduction: The relationship of pain and pain sensitization has not been evaluated in terms of fetal loss. Pain sensitization occurs when a subject becomes more sensitive and has more pain with less provocation. When the pain is severe and of long duration, pain sensitization emerges. The specific object was to observe fetal loss rates per woman among those with chronic pelvic pain and pain sensitization.

Methods: 184 women with chronic pain for more than 6 months agreed to provide clinical history and physical findings. Data from multiple sources were collected to estimate the pattern of increasing menstrual function from hunter gatherers to present. Information regarding fetal loss was estimated by subtracting parity from gravidity. Women were compared for differences in fetal loss on the basis of both chronic pain and pain sensitization. Sensitization was determined by the presence or absence of viscerally related cutaneous allodynia.

Results: Women now have an estimated 400 lifetime menstrual cycles - an evolved increase. Overall severe dysmenorrhea is estimated at $2-29 \%$ while women with chronic pelvic pain had a higher rate of $78 \%$. Severe dysmenorrhea $(88.2 \%$ vs. $59.6 \%, \mathrm{P}<0.001)$ and continuous pain $(72.0 \%$ vs. $36.7 \%$ $\mathrm{P}<0.001)$. are greater with sensitization. Pregnancy loss prior rate per woman was $65 \%$ among all women with chronic pelvic pain but there was no difference among those with and without sensitization ( $69 \%$ vs. $56 \%)$.

Comment: The shift to contraception and increasing menstrual function increases the risks of dysmenorrhea, chronic pelvic pain and pain sensitization - a maladaptive state due to trade-offs. The increased fetal loss per woman with chronic pelvic pain may suggest an extreme form of developmental origin of disease independent of pain sensitization. These findings may have implications for early treatment of dysmenorrhea and prenatal counselling of women with chronic pelvic pain.

\section{4}

Childhood hypertension and family history of hypertension in primary school children in Port Harcourt, Nigeria

Boma Okoh ${ }^{1}$, Edward Alikor ${ }^{2}$

${ }^{1}$ University of Port Harcourt; ${ }^{2}$ University of Port Harcourt 
Research question: Is there a relationship between childhood hypertension and positive family history?

Methods: Pupils were recruited using a stratified multi-staged sampling technique. Data was collected using a pretested questionnaire. The average of three blood pressure measurements, weight and height were taken for each pupil. Hypertension was defined as average systolic and/or diastolic blood pressure $\hat{a} \%$ o 95 thpercentile for age, gender and height using standard blood pressure charts. Family history of hypertension was defined as either parent indicating that they were hypertensive (diagnosed by a physician and/ or on antihypertensive drugs) or had a family history of hypertension.

Results: A total of 1302pupils: 717(55.1\%) females and 585 $(44.9 \%)$ males were studied. The mean age of pupils was $8.82 \hat{A} \pm 1.91$ years. Sixty one $(4.7 \%)$ of the pupils had hypertension. A total of $316(24.3 \%)$ had a family history of hypertension. The mean systolic (p-value).

\section{9}

\section{Abdominal circumference and weight at birth are both associated with metabolic risk factors in early childhood}

Mette Tanvig ${ }^{1}$, Christina Anne Vinter ${ }^{2}$, Jan Stener Jørgensen ${ }^{3}$, Sonja Wehberg ${ }^{4}$, Per Glud Ovesen ${ }^{5}$, Henrik Thybo Christesen ${ }^{6}$, Dorte Møller Jensen ${ }^{7}$

${ }^{1}$ Department of Gynecology and Obstetrics, Odense University Hospital; ${ }^{2}$ Department of Gynecology and Obstetrics, Odense University Hospital; ${ }^{3}$ Department of Gynecology and Obstetrics, Odense University Hospital; ${ }^{4}$ Centre for Clinical Epidemiology, Odense University Hospital, Odense, Denmark; ${ }^{5}$ Department of Gynecology and Obstetrics, Aarhus University Hospital, Skejby, Denmark; ${ }^{6}$ Hans Christian Andersen Children's Hospital, Odense University Hospital, Odense, Denmark; ${ }^{7}$ Department of Endocrinology, Odense University Hospital

Research question: Birth weight (BW) is a well-established predictor for later risk of adverse metabolic outcome, whereas less is known about the predictive value of neonatal abdominal fat deposition. We aimed to investigate the associations between birth abdominal circumference (BAC, an indicator of neonatal abdominal fat deposition), and BW and metabolic profile in early childhood.

Methods: In a secondary analysis of data from a follow-up of a randomized controlled trial (the Lifestyle in Pregnancy and Offspring study), we investigated metabolic risk factors in 150 offspring of obese mothers and 97 offspring of normal weight mothers. At 2.8 years, abdominal circumference (AC), blood pressure and fasting plasma glucose, insulin, high density lipoprotein (HDL) and triglycerides were measured. Regression models were used to analyze associations between BAC and $\mathrm{BW}$ and outcomes.
Results: BAC and BW were associated (all $\mathrm{p}<0.02$ ) with (regression coefficients per increase in $\mathrm{BAC}$ and $\mathrm{BW}$ of 1 standard deviation score, respectively): AC (0.57; $0.70)$, plasma glucose $(0.11 ; 0.09)$, insulin $(4.33 ; 3.13)$ and triglycerides $(0.07 ; 0.07)$, but not with blood pressure or HDL.

Conclusion: BAC and BW were both associated with later metabolic risk factors, indicating that $\mathrm{BAC}$ in addition to $\mathrm{BW}$ is predictive of later metabolic profile.

\section{7}

Long term effects of the undernutrition upon the hypothalamus-pituitary-adrenal and -gonadal axes in adolescent rats

Júlio Cezar de Oliveira ${ }^{1}$, Egberto Gaspar de Moura ${ }^{2}$, Rosiane Aparecida Miranda ${ }^{3}$, Luiz Felipe Barella ${ }^{4}$, Audrei Pavanello ${ }^{5}$, Ananda Malta ${ }^{6}$, Claudinéia Conationi da Silva Franco 7 , Isabela Peixoto Martins ${ }^{8}$, Rosana Torrezan ${ }^{9}$, Paulo Cezar de Freitas Mathias ${ }^{10}$, Patrícia Cristina Lisboa ${ }^{11}$

${ }^{1}$ Laboratory of Secretion Cell Biology, Department of Biotechnology, Cell Biology and Genetics, State University of Maringa, Maringá, PR, Brazil; Department of Physiological Sciences; ${ }^{2}$ Department of Physiological Sciences, Roberto Alcântara Gomes Biology Institute, State University of Rio de Janeiro, Rio de Janeiro, RJ, Brazil; ${ }^{3}$ Laboratory of Secretion Cell Biology, Department of Biotechnology, Cell Biology and Genetics, State University of Maringa, Maringá, PR, Brazil; ${ }^{4}$ Laboratory of Secretion Cell Biology, Department of Biotechnology, Cell Biology and Genetics, State University of Maringa, Maringá, PR, Brazil and Molecular Signaling Section, Labo; ${ }^{5}$ Laboratory of Secretion Cell Biology, Department of Biotechnology, Cell Biology and Genetics, State University of Maringa, Maringá, PR, Brazil; ${ }^{6}$ Laboratory of Secretion Cell Biology, Department of Biotechnology, Cell Biology and Genetics, State University of Maringa, Maringá, PR, Brazil; ${ }^{7}$ Laboratory of Secretion Cell Biology, Department of Biotechnology, Cell Biology and Genetics, State University of Maringa, Maringá, PR, Brazil; ${ }^{8}$ Laboratory of Secretion Cell Biology, Department of Biotechnology, Cell Biology and Genetics, State University of Maringa, Maringá, PR, Brazil; ${ }^{9}$ Department of Physiological Sciences, State University of Maringa, Maringá, PR, Brazil; ${ }^{10}$ Laboratory of Secretion Cell Biology, Department of Biotechnology, Cell Biology and Genetics, State University of Maringa, Maringá, PR, Brazil; ${ }^{11}$ Department of Physiological Sciences, Roberto Alcântara Gomes Biology Institute, State University of Rio de Janeiro, Rio de Janeiro, RJ, Brazil

Research question: Glucocorticoid dyshomeostasis disrupts body metabolism. The effects of low-protein diet on hypothalamus-pituitary-adrenal (HPA) and -gonadal (HPG) axes in adolescent rats and their roles on metabolism as a longterm consequence were studied. 
Methods: Thirty days-old Wistar male rats were fed a lowprotein diet $(4 \%$, LP) until 60-day-old; while their counterparties were fed a normal-protein diet (23\%, NP). Body weight and food intake were assessed throughout the experimental period. Both rats at 60 - and 120 -days of age were sacrificed and studied. Pancreatic islets and testicles were isolated to study insulin secretion and testosterone production, respectively. Fat depots as well as fasting glucose, insulin, leptin, corticosterone, adrenocorticotropic hormone (ACTH) and testosterone were evaluated. Data were analyzed with Student's t-test.

Results: At 60-day-old, LP rats displayed a lean phenotype, hypoglycemia, hypoinsulinemia, hypoleptinemia, hypercorticosteronemia, and high ACTH and low testosterone levels $(p<0.05)$. In addition, weak insulin secretion and testosterone production $(p<0.05)$ were found. On the contrary, 120-days-old LP rats were hyperglycemic, hyperinsulinemic and hyperleptinemic $(\mathrm{p}<0.01)$. Moreover, low $\mathrm{ACTH}$ and testosterone levels were found, and increased insulin secretion ability associated with an obese phenotype $(\mathrm{p}<0.05)$.

Conclusion: Pubertal undernutrition disrupts HPA and HPG axes crosstalk inducing insulin secretion impairment and obesity-correlated complications later in life.

\section{1}

Prenatal food and micronutrient supplementation and associations with metabolic status in the offspring at 4.5 years. The randomized MINIMat-trial in rural Bangladesh

Eva-Charlotte Ekström ${ }^{1}$, Rubhana Raqib ${ }^{2}$, Shams El Arifeen ${ }^{3}$, Samar Basu ${ }^{4}$, Kerstin Brismar ${ }^{5}$, Lars-Åke Persson ${ }^{6}$

${ }^{1}$ International Maternal and Child Health, Women's and Children's Health, Uppsala University, Sweden;

${ }^{2}$ International Centre for Diarrhoeal Disease Research, Bangladesh (ICDDR, B), Dhaka, Bangladesh; ${ }^{3}$ International Centre for Diarrhoeal Disease Research, Bangladesh (ICDDR,B), Dhaka, Bangladesh; ${ }^{4}$ Oxidative Stress and Inflammation, Department of Public Health and Caring Sciences, Uppsala University, Uppsala, Sweden, and Chaire d' Excellence Program, Department of Biochemistry; ${ }^{5}$ Department of Molecular Medicine and Surgery, Karolinska Institutet, Karolinska University Hospital; ' International Maternal and Child Health, Women's and Children's Health, Uppsala University, Sweden

Research question: Effects of prenatal food- and micronutrient supplements on metabolic markers in the offspring at 4.5 years.

Methods: Women were recruited Jan 1-Dec 21, 2002 (the MINIMat-trial, Bangladesh, ISRCTN16581394). Supplementation with capsules of iron $30 \mathrm{mg} /$ folic acid $400 \mu \mathrm{g}$, iron $60 \mathrm{mg} /$ folic acid $400 \mu \mathrm{g}$, or multiple micronutrients was combined with food supplementation $(608 \mathrm{kcal}, 6$ days $/$ week) randomized to either early $(\mathrm{GW}$ 9) or usual invitation $(\mathrm{GW} \sim 20)$. Biomarkers were assessed at 4.5 years, $\mathrm{n}=1354$ (lipids, glucose/insulin, CRP, growth factors, oxidative stress).

Results: Children of mothers randomized to early food had lower cholesterol $(-0.079 \mathrm{mmol} / \mathrm{L}, 95 \%$ CI $-0.156 ;-0.003)$, LDL $(-0.068 \mathrm{mmol} / \mathrm{L}, 95 \%$ CI $-0.126 ;-0.011)$ and apoB $(-0.017 \mathrm{~g} / \mathrm{L}, 95 \% \mathrm{CI}-0.033 ;-0.001)$. Multiple micronutrients resulted in lower HDL $(-0.028 \mathrm{mmol} / \mathrm{L}, 95 \%$ CI -0.053 ; $-0.002)$, lower glucose $(-0.099 \mathrm{mmol} / \mathrm{L}, 95 \% \mathrm{CI}-0.179$; -0.019 ) and lower IGF-1 (difference $\log$ scale $-0.141 \mu \mathrm{g} / \mathrm{L}$, $95 \%$ CI $-0.254 ;-0.028$, corresponding to $14 \%$ difference in median). There were no combined supplementation effects, or effects on CRP or oxidative stress.

Conclusion: Early invitation to prenatal food supplementation was associated with favorable blood lipids in children 4.5 years. Multiple micronutrients were associated with lower HDL, glucose and IGF-1. This suggests chronic disease risk indicators in childhood may be modified by early prenatal interventions.

\section{1}

Maternal protein intake in pregnancy and offspring metabolic health at age 9-16 - results from a high-risk Danish cohort

Susanne Hansen ${ }^{1}$, Louise Groth Grunnet ${ }^{2}$, Marin Strøm ${ }^{3}$, Anne Ahrendt Bjerregaard ${ }^{4}$, Line Hjort ${ }^{5}$,

Camilla Møller Madsen ${ }^{6}$, Anne Cathrine Baun Thuesen ${ }^{7}$, Bodil Hammer Bech ${ }^{8}$, Thorhallur Ingi Halldorsson', Charlotta Granström ${ }^{10}$, Allan Vaag ${ }^{11}$, Sjurdur F Olsen ${ }^{12}$

${ }^{1}$ Centre for Fetal Programming, Department of Epidemiology Research, Statens Serum Institut, Copenhagen, Denmark; ${ }^{2}$ Department of Endocrinology, Diabetes and Metabolism, Rigshospitalet, Copenhagen Denmark; ${ }^{3}$ Centre for Fetal Programming, Department of Epidemiology Research, Statens Serum Institut, Copenhagen, Denmark; ${ }^{4}$ Centre for Fetal Programming, Department of Epidemiology Research, Statens Serum Institut, Copenhagen, Denmark; ${ }^{5}$ Department of Endocrinology, Diabetes and Metabolism, Rigshospitalet, Copenhagen Denmark; ${ }^{6}$ Department of Endocrinology, Diabetes and Metabolism, Rigshospitalet, Copenhagen Denmark; ${ }^{7}$ Department of Endocrinology, Diabetes and Metabolism, Rigshospitalet, Copenhagen Denmark; ${ }^{8}$ Department of Public Health, University of Aarhus, Aarhus, Denmark; ${ }^{9}$ Faculty of Food Science and Nutrition, University of Iceland, Reykjavik, Iceland; ${ }^{10}$ Centre for Fetal Programming, Department of Epidemiology Research, Statens Serum Institut, Copenhagen, Denmark; ${ }^{11}$ Department of Endocrinology, Diabetes and Metabolism, Rigshospitalet, Copenhagen Denmark; ${ }^{12}$ Centre for Fetal Programming, Department of Epidemiology Research, Statens Serum Institut, Copenhagen, Denmark 
Research question: Is protein intake in pregnancy associated with offspring metabolic health at age 9-16 in a cohort that over-sampled mothers with gestational diabetes mellitus (GDM).

Methods: 609 women with a GDM index pregnancy and 626 control women enrolled in the Diabetes and Women's Health Study were used for the analysis. Protein intake was assessed using a food frequency questionnaire in gestational week 25 . The offspring underwent a clinical examination including fasting blood samples and DXA scan (subset of 666) from which metabolic outcomes were derived. Analyses were conducted applying a 1:1 substitution of carbohydrates for protein.

Results: Mean (SD) protein intake in pregnancy was $92(27) \mathrm{g} /$ day $(16(3) \% \mathrm{E})$. There were suggestive trends for maternal protein intake with an unfavourable offspring body composition (lower lean mass \% and higher total fat mass \%). These associations appeared strongest among offspring of control mothers. Low protein intake $(<=12 \% \mathrm{E})$ was inversely associated with HOMA-IR (ratio of geometric means: 0.84, $95 \%$ CI: $0.72,0.98)$ and fasting insulin $(0.83,95 \%$ CI: 0.73 , $0.95)$, but not body composition. Results were similar across GDM and control offspring.

Conclusion: Low maternal protein intake in pregnancy was related to improved measures of glucose homeostasis in the offspring; only minor effects were seen on body composition.

\section{7}

Hypertension induced by low protein nutrition during adolescence in rats depends on autonomic nervous system

Laize Peron Tófolo ${ }^{1}$, Leticia Diniz Crepaldi ${ }^{2}$, Ananda Malta ${ }^{3}$, Tatiane Aparecida da Silva Ribeiro ${ }^{4}$, Flavio Andrade Francisco 5 , Edmara Baroni ${ }^{6}$, Paulo Cezar de Freitas Mathias ${ }^{7}$, Paulo Cezar de Freitas Mathias ${ }^{8}$

${ }^{1}$ Laboratory of Secretion Cell Biology, Department of Biotechnology, Genetics and Cell Biology, Universidade Estadual de Maringá - MaringálPR, Brazil; ${ }^{2}$ Department of Physiologic Science, Universidade Estadual de Maringá MaringálPR, Brazil; ${ }^{3}$ Laboratory of Secretion Cell Biology, Department of Biotechnology, Genetics and Cell Biology, Universidade Estadual de Maringá - MaringálPR, Brazil; ${ }^{4}$ Laboratory of Secretion Cell Biology, Department of

Biotechnology, Genetics and Cell Biology, Universidade Estadual de Maringá - MaringálPR, Brazil; ${ }^{5}$ Laboratory of Secretion Cell Biology, Department of Biotechnology, Genetics and Cell Biology, Universidade Estadual de Maringá - MaringálPR, Brazil; ${ }^{6}$ Department of Physiologic Science, Universidade Estadual de Maringá - MaringálPR, Brazil; ${ }^{7}$ Laboratory of Secretion Cell Biology, Department of Biotechnology, Genetics and Cell Biology, Universidade Estadual de Maringá - MaringálPR, Brazil;

${ }^{8}$ Laboratory of Secretion Cell Biology, Department of
Biotechnology, Genetics and Cell Biology, Universidade Estadual de Maringá - MaringálPR, Brazil

Protein restriction during critical phases of life may lead to hypertension. Recently, we have shown that adolescence is, as well, a susceptible phase for programming to metabolic syndrome. In this context we hypothesised that low protein diet during adolescence may lead to hypertension dependent on hyper activation of sympathetic nervous system.

Adolescent Wistar rats (30 to 60 day-old) were exposed to a low protein (LP) diet (4\% of protein). Control animals had access to normal commercial (NP) chow (23\% of protein). Blood pressure and pulse interval were recorded in 120-day-old rats. Vascular and cardiac sympathetic and cardiac parasympathetic activity was estimated via spectral analyses. Renal function was evaluated through glomerular filtration rate, proteinuria and 24 hours urine volume. Student t-test was used to compare groups.

LP animals showed $12 \%$ greater blood pressure levels compared NP animals $(\mathrm{p}<0.01)$. Pulse interval was similar between groups. Vascular and cardiac sympathetic activity was, respectively, $42 \%$ and $86 \%$ greater in LP animals ( $p<0.05$ ). Cardiac parasympathetic activity was $38 \%$ reduced in LP compared with NP rats $(\mathrm{p}<0.05)$. Renal function was not different between groups.

LP diet exposition during adolescence programs to hypertension later in life, which may depend on autonomic dysfunction, but not the kidney.

\section{4}

Is percentage body-fat at birth related to age-dependant cardiovascular dysfunction?

Fiona Tout ${ }^{1}$, Kyra Sim ${ }^{2}$, Girvan Malcolm ${ }^{3}$, Garry Cohen ${ }^{4}$

${ }^{1}$ Charles Perkins Centre, The University of Sydney, Australia;

${ }^{2}$ Charles Perkins Centre, The University of Sydney, Australia;

${ }^{3}$ Royal Prince Alfred Hospital Women and Babies, Camperdown, Australia; ${ }^{4}$ Karolinska Institutet, Sweden

Research question: Is there a relationship between percentage body-fat measured by air displacement plethysmography (ADP) and cardiovascular dysfunction?

Vascular function is altered early in newborns who are small and preterm, however percentage body-fat measured by ADP has been shown to be a better measure than birthweight for assessing neonatal morbidity. Its association with cardiovascular stress reactivity is unknown.

Methods: Prospective cohort of 30 term singleton newborns delivered at a tertiary hospital with a PEAPOD® assessment performed within 24-hours of birth. Three prespecified groups of ten newborns were included: low body-fat $(<10$ th percentile), normal body-fat (10-90th percentile) and high body-fat (> 90th percentile). Blood pressure and heart rate were recorded using a wrist cuff (Finometer) at the level of the heart in 
quiet sleep. Cardiovascular stress reactivity was assessed by blood pressure response to $60^{\circ}$ head up tilt using a purpose built tilt table.

Outcomes: Multivariate analysis will be used to determine contribution of body composition to cardiovascular stress reactivity accounting for confounders such as age, gender and gestation.

Conclusion: If an association between body-fat and cardiovascular dysfunction exists it may offer opportunities to develop a simple test at birth for later life cardiometabolic disease.

\section{6}

Muscle fitness and the cardio-metabolic risk factors from childhood through to late adolescence: The Raine Study

Lawrence Beilin ${ }^{1}$, Beth Hands ${ }^{2}$, Sally Burrows ${ }^{3}$, Kay Cox ${ }^{4}$, Trevor Mori ${ }^{5}$

${ }^{1}$ University of Western Australia; ${ }^{2}$ Notre Dame University;

${ }^{3}$ University of Western Australia; ${ }^{4}$ University of Western

Australia; ${ }^{5}$ University of Western Australia

Background: Muscle strength and endurance are important aspects of physical fitness. There is a lack of data examining the evolution of relationships between muscle function and cardiometabolic risk factors in childhood and adolescence.

Methods: 1916 participants from the Western Australian Pregnancy Cohort (Raine) Study at ages 10, 14 and 17 years. Longitudinal linear mixed model analyses were performed.

Results: Hand grip strength was significantly associated with a higher systolic BP over the 10-17 year age span in both males and females $(\mathrm{P}=<0.001)$ in models adjusted for gender and BMI, but the strength of association was attenuated over time. Back endurance was significantly associated with a higher systolic BP at 14 years and 17 years $(\mathrm{P}<0.05)$. Hand grip strength and back endurance were significantly inversely associated with HOMA at 14 and 17 years of age in both sexes (coefficient $-0.01, \mathrm{P}<0.001$. and coefficient $-0.003, \mathrm{P}<0.001)$.

Conclusion: The positive association seen with muscle fitness and systolic BP is contrary to that seen in some adult populations. The inverse associations seen with both muscle strength and back endurance and HOMA are consistent with adult findings. Further studies as the population moves into adult life will be of interest.

\section{0}

Maternal stress during pregnancy and anemia in the second year of an infant's life

Antonio Augusto Moura da Silva ${ }^{1}$, Rosangela Fernandes Lucena Batista $^{2}$, Vanda Maria Ferreira Simoes ${ }^{3}$, Marcelo Zubaran Goldani ${ }^{4}$, Viviane Cunha Cardoso ${ }^{5}$, Heloisa Bettiol ${ }^{6}$, Marco Antonio Barbieri ${ }^{7}$

${ }^{1}$ Department of Public Health, Federal University of Maranhao, Brazil; ${ }^{2}$ Department of Public Health, Federal University of Maranhao, Brazil; ${ }^{3}$ Department of Public Health, Federal
University of Maranhao, Brazil; ${ }^{4}$ Department of Pediatrics, Faculty of Medicine, Federal University of Rio Grande do Sul, Brazil; ${ }^{5}$ Department of Pediatrics, Ribeirao Preto Medical School, University of Sao Paulo, Brazil; ' Department of Pediatrics, Ribeirao Preto Medical School, University of Sao Paulo, Brazil; ${ }^{7}$ Department of Pediatrics, Ribeirao Preto Medical School, University of Sao Paulo, Brazil

Research question: The prevalence of iron-deficiency anemia among children $<5$ years is high, estimated at $47.4 \%$. Among monkeys, maternal stress during pregnancy has been associated with iron deficiency in the pups and with impairment of innate immunity. To verify if stress during pregnancy is associated with anemia in infants aged 13 to 23 months a convenience cohort started during the prenatal period in Sao Luis, Brazil, was used.

Methods: The level of stress during pregnancy was determined in a prenatal visit from the $22 \mathrm{nd}$ and 25 th week of pregnancy using the Perceived Stress Scale. The women were interviewed during the postpartum period and their babies were evaluated in the second year of life. Anemia was considered to be present when hemoglobin was $<11 \mathrm{~g} / \mathrm{dL}$. A total of 761 infants were evaluated. The risk ratio (RR) was estimated by Poisson regression.

Results: The prevalence of anemia was $27.7 \%$ among infants whose mothers suffered stress during pregnancy and $12.7 \%$ among the others $(p<0.001)$, with a RR of $2.18(95 \% \mathrm{CI}$ 1.59-3.01). After adjustment, stress during pregnancy $(\mathrm{RR}=$ $2.20,1.58-3.06)$ continued to be associated with anemia.

Conclusion: Stress during pregnancy was an important predictor of anemia in the second year of an infant's life.

\section{2}

Maternal vomiting during early pregnancy and cardiovascular risk factors at school-age. The Generation R Study Sunayna Bahadoer ${ }^{1}$, Vincent W.V. Jaddoe ${ }^{2}$, Oscar H. Franco ${ }^{2}$, Albert Hofman $^{2}$, Eric A.P. Steegers ${ }^{2}$, Romy Gaillard ${ }^{2}$

${ }^{1}$ Erasmus $M C ;{ }^{2}$ Erasmus $M C$

Research question: To examine the associations of maternal daily vomiting during early pregnancy, as measure of hyperemesis gravidarum, with childhood cardiovascular outcomes.

Methods: In a population-based prospective cohort study among 4769 mothers and their children, we related maternal vomiting during early pregnancy, assessed by questionnaire with cardiovascular outcomes at 6 years (body mass index, total fat mass percentage, android/gynoid fat ratio, preperitoneal fat mass, blood pressure, lipids and insulin levels).

Results: As compared to children from mothers without daily vomiting during early pregnancy, those from mothers with daily vomiting during early pregnancy had a higher childhood total body fat mass (difference 0.12 Standard Deviation Score (SDS) (95\% Confidence Interval (CI):0.03 to 0.20), android/ gynoid fat mass ratio (difference 0.13 SDS (95\% CI: 0.04 to 0.23 ), and preperitoneal fat mass area (difference 0.10 SDS (95\% CI: 0 to 0.20 ). These associations were not explained by 
birth characteristics but partly explained by higher infant weight gain. Maternal daily vomiting during early pregnancy was not associated with other childhood cardiovascular risk factors.

Conclusion: Maternal daily vomiting during early pregnancy was associated with higher childhood total fat, abdominal and preperitoneal fat mass, but not with other cardiovascular risk factors.

\section{9}

Maternal and paternal family history of diabetes and metabolic outcomes at age 5-6 years: results of the ABCD study

Adriëtte Oostvogels ${ }^{1}$, Tessa Roseboom ${ }^{2}$, Karien Stronks ${ }^{3}$, Tanja Vrijkotte ${ }^{4}$

${ }^{1}$ Academic Medical Centre, Amsterdam; ${ }^{2}$ Academic Medical Centre, Amsterdam; ${ }^{3}$ Academic Medical Centre, Amsterdam;

${ }^{4}$ Academic Medical Centre, Amsterdam

Research question: Do maternal and paternal family history of diabetes contribute equally to adverse metabolic health outcomes in their children?

Methods: We compared, at age 5-6 years, body mass index (BMI), waist-height-ratio (WHtR), fat percentage (fat\%), fasting glucose, C-peptide and HOMA-IR in children without a family history of diabetes (controls; $n=2242$ ), with children with maternal $(n=353)$, paternal $(n=318)$ or both maternal and paternal (parental; $\mathrm{n}=187$ ) family history of diabetes. Children of women with diabetes during pregnancy were excluded.

Results: Children with maternal family history of diabetes had increased fat $\%(\beta=1.04 ; 95 \% \mathrm{CI}: 0.34,1.73)$ and children with parental family history of diabetes had increased BMI $(\beta=0.41 ; 95 \%$ CI:0.19,0.63), WHtR $(\beta=0.005 ; 95 \%$ CI:0.000, 0.009), fat $\%(\beta=2.08 ; 95 \% \mathrm{CI}: 1.17,3.00)$ and decreased C-peptide $(\beta=-0.26 ; 95 \%$ CI:-0.40, -0.11) and HOMA-IR ( $\beta=-0.13$; 95\% CI:0.-021, -0.06). Adjustments for maternal, paternal and childhood characteristics rendered the results on BMI, WHtR and fat $\%$ non-significant, but C-peptide $(\beta=-0.21 ; 95 \%$ CI:-0.36, -0.07) and HOMA-IR $(\beta=-0.11 ; 95 \%$ CI:-0.18, -0.04) remained lower.

Conclusion: We found evidence for some maternal influences on the body composition of the child, however both maternal and paternal family history influenced glucose metabolism. These findings do therefore not support the fetal overnutrition hypothesis.

\section{3}

Delivery by Cesarean Section and Blood Pressure from Age 7 to 15 Years

Teresa Attina $^{1}$, Jan Blustein ${ }^{2}$, Jianmeng Liu ${ }^{3}$, Trasande Leonardo ${ }^{4}$

${ }^{1}$ Department of Pediatrics, New York University School of

Medicine, New York, NY, US; ${ }^{2}$ Department of Health Policy,
Medicine, and Population Health, NYU School of Medicine, NYU/Wagner School of Public Service, New York, NY, US; ${ }^{3}$ Institute of Reproductive and Child Health/Ministry of Health Key Laboratory of Reproductive Health and Department of Epidemiology and Biostatistics, School of Public Health, Pekin; ${ }^{4}$ Departments of Pediatrics, Environmental Medicine, and Population Health, New York University School of Medicine, New York, NY, US; New York University Wagner School of Public Service

Research question: Prevalence of cesarean section has been increasing worldwide, with concerns about the long-term implications for child health. Our aim was to investigate the association between mode of delivery and childhood blood pressure in the Avon Longitudinal Study of Parents and Children (ALSPAC).

Methods: We analyzed data from 7,170 mother-child pairs. Our primary outcome was the age- and gender-standardised systolic and diastolic BP z-score at 7, 9, 11 and 15 years of age. Results: Of the 7,170 children, 648 (9.04\%) were delivered by cesarean section. In our multivariable model, after adjusting for several key clinical confounders, we detected a significant association between cesarean delivery and age- and genderstandardised systolic blood pressure outcome at age 15, which was present with or without the inclusion of child BMI in the model [SBP z-score increments +0.09 SD units, 95\% CI 0.005 to $0.18 ; \mathrm{p}=0.039$ (adjusting for BMI); +0.11 SD units, $95 \%$ CI 0.02 to $0.20 ; \mathrm{p}=0.022$ (without adjusting for BMI)]. Evidence of the association was significant and qualitatively greater among children born of overweight/obese mothers.

Conclusion: In this cohort, cesarean delivery was associated with increased blood pressure at 15 years of age. Further research is needed to understand the mechanisms underlying this association.

\section{1}

Early childhood growth patterns and airway resistance, fractional exhaled nitric oxide and asthma at school age - The Generation R Study

Maribel Casas ${ }^{1}$, Herman T. den Dekker ${ }^{2}$, Claudia J. Kruithof ${ }^{3}$, Johan C. de Jongste ${ }^{4}$, Martine Vrijheid ${ }^{5}$,

Vincent W.V. Jaddoe ${ }^{6}$, Liesbeth Duijts ${ }^{7}$

${ }^{1}$ The Generation R Study Group, Department of PediatricsDivision of Respiratory Medicine, Erasmus MC, University Medical Centre, Rotterdam, the Netherlands. Centre for Research in E; ${ }^{2}$ The Generation R Study Group, Department of PediatricsDivision of Respiratory Medicine, Department of Epidemiology, Erasmus MC, University Medical Centre, Rotterdam, the Netherland; ${ }^{3}$ The Generation R Study Group, Department of Epidemiology, Erasmus MC, University Medical Centre, Rotterdam, the Netherlands; ${ }^{4}$ Department of Pediatrics, Division of Respiratory Medicine, Erasmus MC, University Medical Centre, Rotterdam, the Netherlands; ${ }^{5}$ Centre for Research in 
Environmental Epidemiology, Barcelona, Spain; ${ }^{6}$ The Generation $R$ Study Group, Department of Epidemiology, Department of Pediatrics-Division of Neonatology, Erasmus MC, University Medical Centre, Rotterdam, the Netherlands; ${ }^{7}$ Department of Pediatrics-Division of Respiratory Medicine, Department of Pediatrics-Division of Neonatology, Erasmus MC, University Medical Centre, Rotterdam, the Netherlands

Research question: To assess the associations of early childhood growth patterns with respiratory outcomes at school-age. Methods: The study included 5,370 children participating in the Generation R Study, a prospective population-based cohort. Repeated growth measurements between 0 and 3 years of age were used to derive peak height velocity (PHV), peak weight velocity (PWV), body mass at adiposity peak (BMIAP), and age at adiposity peak (AGEAP). Airway resistance (Rint) and fractional exhaled nitric oxide (FeNO) were measured at age 6 years. Asthma and wheezing patterns (never, early, late, persistent) were prospectively assessed by questionnaires. All findings were expressed as standard deviation score (SDS).

Results: An increase of one SDS PHV was associated with a slightly lower Rint [SDS-score (95\% CI): -0.03 (-0.04, -0.01)] and higher risk of asthma [RR (95\% CI): $1.01(1.00,1.03)]$. A higher PWV and BMIAP were associated with increased risks of early wheezing [RRs (95\% CI): 1.11 (1.06, 1.16), 1.26 (1.11, 1.42), respectively] and persistent wheezing [RRs (95\% CI): 1.09 (1.03, 1.16), 1.37 (1.17, 1.60), respectively]. No other associations were observed.

Conclusion: Higher PHV, PWV and BMIAP in early childhood are associated with lower Rint or increased risks of wheezing patterns and asthma at school-age.

\section{4}

Relation of growth in early infancy with cardio-metabolic risk factors during childhood in term low birth weight Indian subjects

Manpreet Kaur ${ }^{1}$, Sikha Sinha ${ }^{2}$, Geeta Trilok Kumar ${ }^{3}$, Suzanne Filteau ${ }^{4}$, Anura V Kurpad ${ }^{5}$, Caroline HD Fall ${ }^{6}$, Clive Osmond ${ }^{7}$, Harshpal Singh Sachdev ${ }^{8}$

${ }^{1}$ Institute of Home Economics, University of Delhi, Delhi, India; ${ }^{2}$ Sitaram Bhartia Institute of Science and Research, New Delhi, India; ${ }^{3}$ Institute of Home Economics, University of Delhi, Delhi, India; ${ }^{4}$ London School of Hygiene and Tropical Medicine, London, UK; ${ }^{5}$ St. John's Research Institute, Banglore, India; ${ }^{6} M R C$ Lifecourse Epidemiology Unit, University of Southampton, UK; ${ }^{7}$ MRC Lifecourse Epidemiology Unit, University of Southampton, UK; ${ }^{8}$ Sitaram Bhartia Institute of Science and Research, New Delhi, India

Research question: Is early infant growth related to blood pressure, motor development and body composition at childhood in term low birth weight (LBW) subjects?
Methods: 2079 term LBW newborns, enrolled in a vitamin D supplementation trial from urban poor setting, were measured serially from 0-26 weeks. Childhood (3-7 years, $n=912)$ evaluations included blood pressure (BP), motor development (grip strength, running, squats and ASQ-2), bone ultrasound and body composition (equations derived from deuteriumdilution in 229). Conditional SD scores from 0-6 weeks, 6-26 weeks and 26 weeks-childhood, computed from WHO BMI-for-age z-scores, were related to these outcomes.

Results: At 26 weeks, $62 \%$ were predominantly/exclusively breastfed and $96 \%$ immunized. Higher BMI gain (26 weeks-childhood) was significantly associated with systolic $(2.4 \mathrm{mmHg} / \mathrm{SD})$ and diastolic $(0.6 \mathrm{mmHg} / \mathrm{SD}) \mathrm{BP}$. Motor development and bone ultrasound were unrelated to BMI gain. Birth BMI and BMI gain (all intervals) predicted $(\mathrm{P}<0.001)$ fat- and lean-mass; the former ( 0.11 to $0.61 \mathrm{~kg} / \mathrm{m} 2 / \mathrm{SD})$ more strongly than latter $(0.10$ to 0.45 $\mathrm{kg} / \mathrm{m} 2 / \mathrm{SD}$ ). However, on restricting to deuterium-dilution estimates, these BMI gain associations were stronger for lean-mass till 6 weeks and for fat-mass thereafter.

Conclusion: In term LBW infants, faster BMI gain predicts higher $\mathrm{BP}$ and adiposity more strongly than lean-mass at 3-7 years.

\section{9}

Fetal and postnatal growth patterns related to cardiometabolic risk factors in preschool children

Damaskini Valvi ${ }^{1}$, David Martinez ${ }^{2}$, Ferran Ballester ${ }^{3}$, Jesus Ibarluzea $^{4}$, Adonina Tardon ${ }^{5}$, Jordi Sunyer ${ }^{6}$, Martine Vrijheid ${ }^{7}$

${ }^{1}$ Centre for Research in Environmental Epidemiology, Barcelona, Spain and Harvard T.H. Chan School of Public Health, Boston, United States; ${ }^{2}$ Centre for Research in Environmental Epidemiology, Barcelona, Spain; ${ }^{3}$ Center for Public Health Research (CSISP-

FISABIO), Valencia, Spain; ${ }^{4}$ Biodonostia Health Research Institute, San Sebastian, Basque Country, Spain; ${ }^{5}$ Faculty of Medicine, University of Oviedo, Oviedo, Asturias; ${ }^{6}$ Centre for Research in Environmental Epidemiology, Barcelona, Spain; ${ }^{7}$ Centre for Research in Environmental Epidemiology, Barcelona, Spain

Research question: Do fetal and postnatal growth patterns predict cardiometabolic risk in preschool children?

Methods: We analysed 734 children from the Spanish INMA birth cohort study. Longitudinal patterns of fetal growth parameters were defined as the SD-score difference between gestational weeks 12-20 and 20-34. Latent class growth curve models defined five body mass index (BMI) SD-score trajectories from birth to age 4 years. A continuous score of child cardiometabolic risk at 4 years (mean; range: $0 ;-6,9)$ was generated as the sum of age- and sex-specific SD-scores for BMI, mean arterial pressure, high-density lipoprotein cholesterol and triglyceride serum concentrations.

Results: Fetal weight and abdominal circumference SD score differences between 20 and 34 weeks were positively associated to the cardiometabolic risk score ( $\beta ; 95 \%$ CI: $0.21 ; 0.02,0.42$ and $0.14 ;-0.01,0.32$, respectively). Children that presented 
lower or higher sizes at birth followed by a rapid BMI gain had on average higher cardiometabolic risk scores than children with average sizes at birth and slower BMI gains at later ages. Postnatal BMI trajectories mediated the associations between fetal growth and cardiometabolic risk score.

Conclusions: Findings suggest that fetal growth patterns in late pregnancy and postnatal BMI trajectories may predict cardiometabolic risk in preschool children.

\section{2}

Longitudinal growth during fetal life and infancy and cardiovascular outcomes at school-age. The Generation R Study

Liza Toemen, Layla de Jonge, Olta Gishti, Lennie van Osch-Gevers, Rob Taal, Eric Steegers, Albert Hofman, Willem Helbing, Vincent Jaddoe

${ }^{1}$ Erasmus MC, University Medical Center, Rotterdam, the Netherlands

Research question: Low birth weight is associated with cardiovascular disease in later life. We studied longitudinal fetal and infant growth patterns associated with cardiovascular outcomes in children.

Methods: In a population-based prospective cohort study among 6,239 children, we measured fetal femur length and estimated weight by 20 and 30 weeks ultrasounds, child length and weight at birth, 0.5, 1, 2 and 6 years and blood pressure, carotid-femoral pulse wave velocity, aortic root diameter, left ventricular mass and fractional shortening at 6 years. Longitudinal regression analyses were used to associate growth patterns with cardiovascular outcomes.

Results: Lower gestational age, birth weight and gestational age adjusted birth weight were associated with higher blood pressure and smaller aortic root diameter. Fetal growth restricted children with accelerated infant growth had the highest blood pressure. Longitudinal analyses showed that children with increased blood pressure tend to be smaller during late fetal life followed by higher growth rates during infancy. Children with increased aortic root diameter or left ventricular mass at 6 years, had higher growth rates during fetal life and infancy.

Conclusions: Specific fetal and infant growth patterns are associated with different cardiovascular outcomes in children. Further studies are needed to identify the long-term clinical cardiovascular consequences.

\section{3}

Effect of tempol on blood pressure, renal inflamation and function in gestational high fat diet offspring

Noemi Angélica Vieira Roza ${ }^{1}$, Patrícia Aline Boer ${ }^{2}$, José Antônio Rocha Gontijo ${ }^{3}$

${ }^{1}$ Department of Internal Medicine School of Medicine, State University of Campinas; ${ }^{2}$ Department of Internal Medicine School of Medicine, State University of Campinas; ${ }^{3}$ Department of Internal Medicine School of Medicine, State University of Campinas

Obesity and arterial hypertension have been frequently associated with high circulating levels of oxidative metabolites. Increased oxidative stress in obese and hypertensive patients appears to contribute to the prevalence of chronic kidney disease leading to progression of renal vascular dysfunction and inflammation. The use of antioxidant Tempol ${ }^{R}$ (SOD analogue) by dams who consume a high fat diet (HFD) can attenuate the effects of increased blood pressure and prevent changes in kidney structure and function. Female Sprague-Dawley rats were divided into four groups: Control (standard diet), DHL (60\% HFD), CTempol ${ }^{R}$ (standard diet+Tempol $-1 \mathrm{mmol} / \mathrm{L}$ in drinking water) and $D T e m p o l^{R}(\mathrm{HFD}+\mathrm{Tempol}-1 \mathrm{mmol} / \mathrm{L}$ in drinking water), treated for six weeks and before and during the pregnancy and weaning, as a chronic treatment. The offspring were sacrificed with 12 days, 8 and 16 -weeks of life. DTempol ${ }^{R}$ group presented high blood pressure levels $(\mathrm{p}<0.001)$ beyond 12 weeks of age associated with reduction of glomerular filtration rate $(\mathrm{p}=0.0029)$ and increased FENa $(\mathrm{p}=0.0350)$ and FEK $(\mathrm{p}<0.0001)$ at 16 -weeks old. Increased renal CD $11 \mathrm{~b} / \mathrm{c}^{+}$ cells was observed in DHL group $(\mathrm{p}=0.0316)$ by Flow Cytometry. We conclude that enhanced blood pressure was not reversed by Tempol; otherwise, the kidney function is preserved by SOD analogue use.

\section{3}

Associations of linear growth and relative weight gain during early life with childhood fasting glucose and insulin resistance

Marieke de Beer ${ }^{1}$, Tanja G.M. Vrijkotte ${ }^{2}$, Caroline H.D. Fall ${ }^{3}$, Clive Osmond $^{4}$, Reinoud J.B.J. Gemke

${ }^{1}$ University Medical Center, Amsterdam, the Netherlands;

${ }^{2}$ University Medical Center, Amsterdam, the Netherlands;

${ }^{3}$ University Medical Center, Amsterdam, the Netherlands;

${ }^{4}$ University Medical Center, Amsterdam, the Netherlands;

${ }^{5}$ University Medical Center, Amsterdam, the Netherlands

Research question: Growth during early life has been associated with later life insulin resistance. The timing of this effect and the relative importance of linear growth and relative weight gain need to be further established.

Methods: Of 5551 children with collected growth data in a prospective cohort study (Amsterdam Born Children and their Development), fasting glucose and homeostasis model assessment of insulin resistance (HOMA-IR) at age of 5-6 years were available for 1604 and 1252 children respectively. We assessed how statistically independent measures representing linear growth and relative weight gain in 5 periods from infancy to childhood were associated with these outcomes.

Results: Both linear growth after age 1 year and relative weight gain after age 1 year were positively associated with childhood 
fasting glucose and insulin resistance. The associations with insulin resistance were stronger for relative weight gain than for linear growth (HOMA-IR z score: $\beta$ coefficient 0.19 (95\% CI 0.13 to 0.24$), \mathrm{P}<0.001$ and 0.08 (0.02 to 0.13 ), $\mathrm{P}=0.005$ per SD change in relative weight gain and linear growth between $1-5$ years respectively).

Conclusion: Faster childhood linear growth and faster childhood relative weight gain are associated with higher fasting glucose and insulin resistance at age 5-6 years.

\section{6}

Air pollution in infancy and chronic rheumatic heart disease: evidence for a link

David Phillips

MRC Lifecourse Epidemiology Unit

Chronic rheumatic heart disease (RHD) is the long-term consequence of a systemic, inflammatory, autoimmune response initiated by infection with $\beta$-haemolytic streptococci. It is the main cause of heart disease in many resource poor countries and has been consistently and strongly linked to poor living conditions. Using historical data from the UK, we have shown a strong geographical correlation between exposure to domestic smoke pollution in infancy and adult mortality from RHD. These analyses were based on current adult mortality between 1993 and 2012 in men and women aged 35 to 74 living in 78 local authority areas, which accounted for approximately a third of the population of England and Wales. Smoke production was obtained from published records of regional coal consumption in 1952. The correlations were present in both genders and were independent of potential confounding factors such as social class, overcrowding, population density and educational level. Burning relatively low-grade, sulphurous domestic coal, as was common in the post war years, would have produced lots of smoke rich in a wide variety of potentially toxic compounds which could increase the susceptibility to $\beta$-haemolytic streptococcal infection by means of a wide variety of mechanisms.

\section{8}

Exposition to ambient air pollution during perinatal life programs metabolic disruption in adult rat offspring

Rosiane Aparecida Miranda ${ }^{1}$, Stéphane Firmin ${ }^{2}$, Anthony Verdin $^{3}$, Gabriel Sergio Fabrício ${ }^{4}$, Flávio Andrade Francisco ${ }^{5}$, Claudinéia Conationi da Silva Franco ${ }^{6}$, Carina Previate ${ }^{7}$, Rodrigo Mello Gomes ${ }^{8}$, Marlon E. Cerf ${ }^{9}$, Latifa AbdennebiNajar $^{10}$, Paulo Cezar de Freitas Mathias ${ }^{11}$

${ }^{1}$ Laboratory of Secretion Cell Biology, Department of Biotechnology, Genetics and Cell Biology, State University of Maringá, 87020-900 Maringá, PR, Brazil; ${ }^{2}$ Institut
Polytechnique LaSalle Beauvais, EGEAL-UP 2012.10.101., 19 rue Pierre Waguet, 60026 Beauvais Cedex, France; ${ }^{3}$ Université du Littoral Côte d' Opale, Unité de Chimie Environnementale et Interactions sur le Vivant (UCEIV), EA4492, Maison de la Recherche en Environnement Industriel de Dunkerque; ${ }^{4}$ Institut Polytechnique LaSalle Beauvais, EGEAL-UP 2012.10.101., 19 rue Pierre Waguet, 60026 Beauvais Cedex, France; ${ }^{5}$ Laboratory of Secretion Cell Biology, Department of Biotechnology, Genetics and Cell Biology, State University of Maringá, 87020-900 Maringá, PR, Brazil; ' Laboratory of Secretion Cell Biology, Department of Biotechnology, Genetics and Cell Biology, State University of Maringá, 87020-900 Maringá, PR, Brazil; ' Laboratory of Secretion Cell Biology, Department of Biotechnology, Genetics and Cell Biology, State University of Maringá, 87020-900 Maringá, PR, Brazil; ${ }^{8}$ Laboratory of Secretion Cell Biology, Department of Biotechnology, Genetics and Cell Biology, State University of Maringá, 87020-900 Maringá, PR, Brazil; ${ }^{9}$ Diabetes Discovery Platform, South African Medical Research Council, Tygerberg, South Africa; ${ }^{10}$ Institut Polytechnique LaSalle Beauvais, EGEALUP 2012.10.101., 19 rue Pierre Waguet, 60026 Beauvais Cedex, France; " ${ }^{11}$ Laboratory of Secretion Cell Biology, Department of Biotechnology, Genetics and Cell Biology, State University of Maringá, 87020-900 Maringá, PR, Brazil

Background: Urbanization, with its associated air pollution, contributes to respiratory disorders and affects metabolism. Studies on the effects of air contaminant exposition during perinatal life on adult offspring metabolism are limited.

Methods: Samples of $>10 \mu \mathrm{m}$ particulate matter $(\mathrm{PM}>10)$ were collected in an urban area of Cotonou, Benin. The PM > 10 solution was prepared with corn oil. Throughout pregnancy and lactation, dams were gavaged with $200 \mu \mathrm{g}$ PM > $10 / \mathrm{kg}$ of body weight/day. After birth, the metabolism of dams and their 21 and 90 day old male offspring were assessed.

Results: Gestational and lactational air contaminant administration did not alter maternal glycemic homeostasis; however, in exposed progeny, normoglycemic weaned and adult offspring presented hyperinsulinemia, insulin resistance, dyslipidemia and overweight $(\mathrm{p}<0.05)$.

Conclusion: Exposition to ambient particulate matter $(\mathrm{PM}>10)$ during perinatal life alters metabolism in infancy and adulthood demonstrating a programming effect.

\section{6}

An attempt of development of PCBs exposure assessment using Food Frequency Questionnaire in Japanese national birth cohort

Norimichi Suzuki ${ }^{1}$, Akifumi Eguchi ${ }^{2}$, Masae Otake ${ }^{3}$, Masamichi Hanazato ${ }^{4}$, Yoshiharu Matsuno ${ }^{5}$, Hiroko Nakaoka ${ }^{6}$, Emiko Todaka ${ }^{7}$, Chisato Mori ${ }^{8}$

${ }^{1}$ Center for Preventive Medical Science, Chiba University;

${ }^{2}$ Clinical Cell Biology and Medicine, Graduate School of Medicine, Chiba University; ${ }^{3}$ Center for Preventive Medical 
Science, Chiba University; ${ }^{4}$ Center for Preventive Medical Science, Chiba University; ${ }^{5}$ Center for Preventive Medical Science, Chiba University; ${ }^{6}$ Center for Preventive Medical Science, Chiba University; ${ }^{7}$ Center for Preventive Medical Science, Chiba University; ${ }^{8}$ Center for Preventive Medical Science, Chiba University

Health effects caused by fetal exposures to PCBs have been concerned. If highly contaminated group can be identified from Food Frequency Questionnaire (FFQ), it will become possible to save cost and time to detect high risk group. In this study, the correlation between FFQ and blood PCB levels of the participants of Japan Environment and Children's Study (JECS) will be reported.

Blood PCB levels in 1332 mothers were analyzed and calculated by GC/ECD. PCB concentrations, FFQ, and questionnaire were summarized and removal of outliers using $95 \%$ Hotelling'sT2 statistics $(n=1278)$. The answers of FFQ and blood PCB level was analyzed with Partial Least Squares (PLS) analysis.

PCB level expected values are gained from the answers to FFQ (Consumptions of fish, soybeans, snacks and potato), age, delivery times in each models and the blood PCB level correlated $(\mathrm{r} 2=0.262)$ by PLS analysis.

This study was conducted as an adjunct study of JECS. This study was conducted as an Adjunct Study of JECS. This study was partly supported by the JECS funding from the Ministry of the Environment, Japan. The findings and conclusions of this article are solely the responsibility of the authors and do not represent the official views of the above government.

\section{2}

Relationship between maternal blood PCB level and distance towards the nearest fishing port in Japanese national birth cohort

Masae Otake ${ }^{1}$, Akifumi Eguchi ${ }^{2}$, Norimichi Suzuki ${ }^{3}$, Chie Koga ${ }^{4}$, Yoshiharu Matsuno ${ }^{5}$, Hiroko Nakaoka ${ }^{6}$, Emiko Todaka ${ }^{7}$, Chisato Mori ${ }^{8}$

${ }^{1}$ Center for Preventive Medical Sciences, Chiba University;

${ }^{2}$ Clinical Cell Biology and Medicine, Graduate School of

Medicine, Chiba University; ${ }^{3}$ Center for Preventive Medical

Sciences, Chiba University; ${ }^{4}$ Department of Bioenvironmental

Medicine, Graduate School of Medicine, Chiba University;

${ }^{5}$ Center for Preventive Medical Sciences, Chiba University;

${ }^{6}$ Center for Preventive Medical Sciences, Chiba University;

${ }^{7}$ Center for Preventive Medical Sciences, Chiba University;

${ }^{8}$ Department of Bioenvironmental Medicine, Graduate School of Medicine, Chiba University

Health effects caused by fetal exposures to PCBs have been concerned. Moreover, seafood is the main source of the PCBs in Japan. In this study, the relationship between the proximity of the subjects' residences to fishing ports and their blood PCB levels was examined. The study subjects were selected from the participants of the Japan Environment and Children's Study (JECS). Blood PCB levels in 1228 mothers who lives in Chiba Study Location were determined by GC/ECD. We measured the distance between the subjects' residence and the nearby fishing ports. The subjects were divided into the short and long-distance group. The relationship between the distance-groups and blood PCB levels were analyzed with ANOVA. The mean concentration of PCBs was $0.32 \mathrm{ng} / \mathrm{g}$-wet in nulliparous. Moreover, the PCB levels were significantly higher in short-distance group. The result indicates that the distance towards fishing port may relate a high risk of exposure to PCB. This study was conducted as an Adjunct Study of JECS. This study was partly supported by the JECS funding from the Ministry of the Environment, Japan. The findings and conclusions of this article are solely the responsibility of the authors and do not represent the official views of the government.

\section{6}

Perinatal Pesticide exposure results in metabolic dysfunction in adult rat offspring

Tatiane Ribeiro ${ }^{1}$, Audrei Pavanello ${ }^{2}$, Ananda Malta ${ }^{3}$, Laize Tófolo ${ }^{4}$, Isabela Martins ${ }^{5}$, Deborah Sloboda ${ }^{6}$, Gabriel Fabrício ${ }^{7}$, Flávio Francisco ${ }^{8}$, Stéphane Firmin ${ }^{9}$, Latifa Najar ${ }^{10}$, Paulo Mathias ${ }^{11}$

${ }^{1}$ Universidade Estadual de Maringa; ${ }^{2}$ Universidade Estadual de Maringa; ${ }^{3}$ Universidade Estadual de Maringa; ${ }^{4}$ Universidade Estadual de Maringa; ${ }^{5}$ Universidade Estadual de Maringa; ${ }^{6}$ MacMaster University; ${ }^{7}$ UPSP-EGEAL Institut Polytechnique LaSalle de Beauvais; ${ }^{8}$ Universidade Estadual de Maringa; ${ }^{9}$ UPSP-EGEAL Institut Polytechnique LaSalle de Beauvais; ${ }^{10}$ UPSP-EGEAL Institut Polytechnique LaSalle de Beauvais;

${ }^{11}$ Universidade Estadual de Maringa

Background/Aims: Orgaphosphorous pesticides inhibit acetylcholinesterase activity and can cause serious health problems in adults. Pesticides are used ubiquitously in agriculture and, pregnant women will be exposed to these chemicals through alimentation. Whether or not low doses of orgaphosphorous chemicals during perinatal life may impact metabolic development is unknown.

Methods: Pregnant Wistar rats were given oral acephate daily $(2.5 \mathrm{mg} / \mathrm{kg} \mathrm{BW} /$ day (ORG) by gavage) throughout pregnancy and lactation or corn oil (control, OIL). After birth, offspring were divided into 2 groups, normal litters (NL) of 9 pups from both OIL and ORG dams and small litters of 3 pups (SL), from OIL or ORG dams. Adult offspring 90th days old had their metabolic outcomes investigated.

Results Perinatal pesticide exposure resulted in low birth weight. We observed an interaction between perinatal pesticide and lactational nutrition: where pernatal pesticide exposure reduced body weight compared to untreated animals, but only in the presence of nutritional excess (SL). CON+SL, but not ORG+SL offspring were obese, but despite the lack of increased adiposity, 
$\mathrm{ORG}+\mathrm{SL}$ offspring were hyperinsulinic, and glucose intolerant compared to CON+SL.

Conclusion: Perinatal pesticide exposure results in impaired glucose homeostasis in adult offspring.

\section{7}

The influence of pre- and postnatal exposure to dioxins and PCBs on ADHD symptoms and cognitive functions in Norwegian preschool children

Helle K. Knutsen ${ }^{1}$, Anne Lise Brantsæter ${ }^{2}$, Margaretha Haugen $^{3}$, Helen Engelstad Kvalem ${ }^{4}$, Jan Alexander ${ }^{5}$, Helle Margrete Meltzer ${ }^{6}$, Pål Zeiner ${ }^{7}$, Heidi Aase ${ }^{8}$

${ }^{1}$ Norwegian Institute of Public Health; ${ }^{2}$ Norwegian Institute of Public Health; ${ }^{3}$ Norwegian Institute of Public Health; ${ }^{4}$ Norwegian Institute of Public Health; ${ }^{5}$ Norwegian Institute of Public Health; ${ }^{6}$ Norwegian Institute of Public Health; ${ }^{7}$ Norwegian Institute of Public Health; ${ }^{8}$ Norwegian Institute of Public Health

Research question: Does low level exposure to dioxins and polychlorinated biphenyls (PCBs), during pregnancy and breastfeeding affect neurodevelopment in children and result in impaired cognitive functions and ADHD?

Methods: We used the Norwegian Mother and Child Cohort Study (MoBa), with the ADHD sub-study ( $\mathrm{n}=1195)$, to examine early-life exposure to dioxins and PCBs and associations with early $\mathrm{ADHD}$ symptoms, IQ, working memory, and language skills in children at 3.5 years. The influence of maternal dietary exposure to dioxins/PCB during pregnancy was investigated using linear and negative binomial regression. Data from MoBa also included breastfeeding duration and relevant covariates.

Results: The estimated maternal exposure to dioxins and PCBs in MoBa was generally low and median intake of dioxins and dioxin-like PCB was 3.6 times lower than the tolerable weekly intake of $14 \mathrm{pg} \mathrm{TEQ} / \mathrm{kg}$ bw/week. An interquartile range increase in PCB-153 exposure was associated with 1.1\% (95\% CI: -1.5 , 3.8) higher ADHD symptom scores and -0.2 IQ points $(95 \%$ CI: $-0.4,0.04)$. The associations were not statistically significant. Conclusion: Maternal dietary exposure to dioxins and PCBs in MoBa was generally low, and not significantly associated with early ADHD symptoms and IQ in preschool children.

\section{5}

Biomonitoring for Lead, Cadmium, Total Mercury and Methylmercury Concentrations in Maternal Blood at 2nd Trimester and Umbilical Cord Blood at Birth

Yu-Mi Kim ${ }^{1}$, Young-Seoub Hong ${ }^{2}$, Jin-Young Chung ${ }^{3}$, Byoung-Gwon Kim ${ }^{4}$

${ }^{1}$ Department of Preventive Medicine, Dong-A University College of Medicine, Busan, Republic of Korea; ${ }^{2}$ Department of Preventive Medicine, Dong-A University College of Medicine, Busan, Republic of Korea; ${ }^{3}$ Dong-A University Heavy Metal Exposure
Environmental Health Center, Dong-A University College of Medicine, Busan, Republic of Korea; ${ }^{4}$ Department of Preventive Medicine, Dong-A University College of Medicine, Busan, Republic of Korea

Background: Considering the increased concerns about heavy metal exposures in pregnancy and early childhood period, the importance of a biomonitoring has been emerging recently. This study was conducted to summarize the distributions of lead, cadmium and mercury blood concentrations on pregnancy and early childhood period.

Methods: The analytic population was 104 mother and child pairs who completed baseline maternal blood sampling and the umbilical cord sampling at birth. The lead and cadmium concentrations were analyzed by ICP-MS (Inductively coupled plasma-mass) spectrometer (Agilent Technologies 7700 series, Santa Clara, USA). Total blood mercury was analyzed using the gold amalgamation method by an automatic mercury analyzer (SP-3DS, Nippon Instrument Corp., Tokyo, Japan). For methylmercury analysis, an automated methylmercury analytic systems (MERX, Brooks Rand Co., USA) was applied with the cold vapor atomic fluorescence spectrophotometry (CVAFS).

Results: The geometric means of maternal lead, cadmium, total mercury and methylmercury concentration at $1 \mathrm{st}$ trimester were respectively $1.02 \pm 1.39 \mathrm{ug} / \mathrm{dl}, 0.61 \pm 1.51 \mathrm{ug} / \mathrm{l}$, $2.97 \pm 1.45 \mathrm{ug} / \mathrm{l}$ and $2.39 \pm 1.45 \mathrm{ug} / \mathrm{l}$. The values for newborns at the birth were $0.71 \pm 1.42 \mathrm{ug} / \mathrm{dl}, \quad 0.01 \pm 5.31 \mathrm{ug} / \mathrm{l}$, $4.44 \pm 1.49 \mathrm{ug} / \mathrm{l}$ and $3.67 \pm 1.51 \mathrm{ug} / \mathrm{l}$. Mean ratios of umbilical cord to mother were calculated to 0.72 for lead, 1.76 for total mercury and 1.81 for methylmercury.

Conclusions: The levels of most measures in pregnant women and infants were higher than industrialized western countries. Though placenta seemed to be protective to cadmium, accumulated patterns were shown in total mercury and methylmercury.

\section{9}

Air pollution - a potential early life programming agent?

David Phillips ${ }^{1}$, Clive Osmond ${ }^{2}$

${ }^{1} M R C$ Lifecourse Epidemiology Unit; ${ }^{2} M R C$ Lifecourse Epidemiology Unit

The short term effects of air pollution are well-documented but it is not known whether early life exposure to pollutants has long term effects. We have related estimates of air pollution and social conditions developed by Daly in 1951-2 for 83 UK cities to their mortality rates at ages 35-74 in men and women during 19932012 .There were strong associations between domestic air pollution and all-cause mortality (relative risk per SD 1.102, 95\% CI 1.100-1.104), ischaemic heart disease (1.126, 1.122-1.131), cerebrovascular disease $(1.113,1.105-1.122)$ and chronic bronchitis $(1.184,1.172-1.196)$. There were no separate associations with industrial air pollution. Social class, education, crowding and population density were further independent predictors of 
mortality rates but adjustment for these variables suggested that air pollution was an independent and additive risk factor.

The pollution estimates were from coal consumption data when fuel rationing was still in operation and the combustion of available low-grade, sulphurous domestic fuel would have produced a wide variety of toxic compounds. Industrial pollution seems to have little additional effect possibly because of higher chimneys. Our data raise the possibility that early life exposure to these sources of pollution may have important long-term effects on the health of the population.

\section{7}

Methodological challenges for DOHaD studies: Disentangling pre- and postnatal exposure to persistent organic pollutants in a large pregnancy cohort

Anne Lise Brantsæter ${ }^{1}$, Margaretha Haugen ${ }^{2}$, Helle Margrete Meltzer $^{3}$, Merete Eggesb $\varnothing^{4}$, Helle K Knutsen ${ }^{5}$

${ }^{1}$ Norwegian Institute of Public Health; ${ }^{2}$ Norwegian Institute of Public Health; ${ }^{3}$ Norwegian Institute of Public Health; ${ }^{4}$ Norwegian Institute of Public Health; ${ }^{5}$ Norwegian Institute of Public Health

Research question: What are the methodological challenges in epidemiological studies attempting to disentangle prenatal and postnatal exposure to persistent organic pollutants (POPs) and their effects on child health?

Methods: The Norwegian Mother and Child Cohort Study $(\mathrm{MoBa})$ is a population-based pregnancy cohort study comprising >100000 mothers. Pregnant women were enrolled in gestational week 17-18 from all over Norway between 1999 and 2008 and follow-up is ongoing. We aim to quantify both pre- and postnatal exposure to POPs based on questionnaire data on maternal and child diet and concentrations in measured in plasma from subsamples of mothers and children.

Results: Plasma concentrations of POPs were higher in children at 3 years than in pregnant women (1.2-1.8-fold higher), and breastfeeding duration was a major determinant of concentrations in children. Although breastfeeding increases children's POPs during postnatal life, deleterious effects of POPs on child development are often attributed to prenatal exposure. The concentrations of most POPs are correlated in maternal serum, cord serum and breast milk, due to their high persistency in fat tissue.

Conclusion: There is a need for more knowledge on how to model pre- and postnatal exposure to POPs in pregnancy cohorts.

\section{9}

Gestational exposure to bisphenol-a: a new window of susceptibility for the development of diabetes in mothers later in life

Paloma Alonso-Magdalena ${ }^{1}$, Marta García-Arévalo ${ }^{2}$, Ivan Quesada ${ }^{3}$, Angel Nadal ${ }^{4}$

${ }^{1}$ Departamento de Biología Aplicada. Universidad Miguel Hernández; ${ }^{2}$ Instituto de Bioingeniería. Universidad Miguel
Hernández; ${ }^{3}$ Instituto de Bioingenieria. Universidad Miguel Hernandez; ${ }^{4}$ Instituto de Bioingenieria. Universidad Miguel Hernandez

Research question: We evaluate the metabolic disorders that BPA exposure during pregnancy may cause in the mother and whether it may predispose to type 2 diabetes and obesity later in life.

Methods: Pregnant mice were treated with BPA at a dose of 10 or $100 \mathrm{ug} / \mathrm{kg} /$ day during days 9 to 16 of gestation.

Results: Pregnant mice treated with BPA developed glucose intolerance, hyperinsulinemia and altered insulin sensitivity. This altered metabolic situation was resolved after parturition but was triggered again several months later. Six months after delivery BPA mice showed marked insulin resistance and profound glucose intolerance, as well as increased body weight. In addition, in vivo and in vitro insulin secretion was decreased in these mice, which resembled a decline of pancreatic b-cell function. Reduced b-cell mass was also observed which was correlated with decreased proliferation capacity and an increase of apoptosis in b-cells. In addition, peripheral tissues, including adipose tissue and liver, showed cellular aging signals that might influence in the impaired insulin sensitivity and accelerate the development of metabolic disorders.

Conclusions: BPA exposure during pregnancy could be considered a new risk factor for the deterioration of maternal glucose metabolism and the increased occurrence of diabetes.

\section{4}

Estimating burden and disease costs of exposure to endocrine disrupting chemicals

Leonardo Trasande ${ }^{1}$, R. Thomas Zoeller ${ }^{2}$, Ulla Hass ${ }^{3}$, Andreas Kortenkamp ${ }^{4}$, Philippe Grandjean ${ }^{5}$, John Peterson Myers ${ }^{6}$, Russ Hauser ${ }^{7}$, Martine Bellanger ${ }^{8}$, Pat Hunt ${ }^{9}$, Paul Fowler ${ }^{10}$, Sheela Sathyanarayana ${ }^{11}$, Ruthann Rudel ${ }^{12}$, Juliette Legler ${ }^{13}$, Niels E. Skakkebaek ${ }^{14}$, Jerrold J Heindel ${ }^{15}$

${ }^{1}$ NYU School of Medicine; ${ }^{2}$ University of Massachusetts;

${ }^{3}$ Technical University of Denmark; ${ }^{4}$ Brunel University;

${ }^{5}$ University of Southern Denmark \& Harvard School of Public Health; ${ }^{6}$ Environmental Health Sciences; ${ }^{7}$ Harvard School of

Public Health; ${ }^{8}$ EHESP School of Public Health; ${ }^{9}$ Washington

State University; ${ }^{10}$ University of Aberdeen; ${ }^{11}$ Seattle Children's

Research Institute; ${ }^{12}$ Silent Spring Institute; ${ }^{13} \mathrm{VU}$-Amsterdam;

${ }^{14}$ Rigshospitalet \& University of Copenhagen; ${ }^{15}$ NIEHS

Rapidly increasing evidence has documented that endocrine disrupting chemicals (EDCs) contribute substantially to disease and disability, yet little data exist on health and economic costs that can be reasonably attributed to EDCs. We recently adapted the Intergovernmental Panel on Climate Change weight-of-evidence characterization for probability of causation based upon available epidemiologic and toxicologic evidence for contributing to disease by an endocrine disruptor mechanism. The WHO Grading of Recommendations Assessment, Development and Evaluation 
criteria were adapted to evaluate epidemiologic evidence. Expert panels achieved consensus for probable $(>20 \%)$ causation for IQ loss; intellectual disability; autism; attention deficit hyperactivity disorder; childhood obesity; endometriosis; fibroids; breast cancer; adult obesity; adult diabetes; cryptorchidism; male infertility and mortality associated with reduced testosterone. Monte Carlo simulations produced a median cost of $€ 157$ billion in Europe (1.23\% of EU Gross Domestic Product). EDC exposures are likely to contribute substantially to disease and dysfunction across the life course with costs in the hundreds of billions/year globally. These estimates represent only those EDCs with highest probability of causation; a broader analysis would have produced greater estimates of burden of disease and costs. Costs are likely on the same order of magnitude in other regions insofar as EDC exposures are similar.

\section{1}

Environmental contaminant exposures in $\mathrm{DOHaD}$ - building the Human Early-Life Exposome (HELIX)

Martine Vrijheid ${ }^{1}$, Oliver Robinson ${ }^{2}$, Xavier Basagaña ${ }^{3}$, Leda Chatzi $^{4}$, Muireann Coen ${ }^{5}$, Regina Grazuleviciene ${ }^{6}$, Peter van den $\mathrm{Hazel}^{7}$, Mark Nieuwenhuijsen ${ }^{8}$, Remy Slama ${ }^{9}$, Catherine Thomsen $^{10}$, John Wright ${ }^{11}$

${ }^{1}$ CREAL, Barcelona; ${ }^{2}$ CREAL; ${ }^{3}$ CREAL; ${ }^{4}$ University of Crete, Greece; ${ }^{5}$ Imperial College London, UK; ${ }^{6}$ Vytauto Didziojo Universitetas, Lithuania; ${ }^{7}$ VGGM, the Netherlands; ${ }^{8}$ CREAL; ${ }^{9}$ INSERM, France; ${ }^{10}$ NIPH, Norway; ${ }^{11}$ Bradford Teaching Hospitals NHS Foundation Trust (BTHFT), UK

Research question: Environmental hazards form a heterogeneous, interacting, multi-level part of disease causation, but epidemiology has almost uniquely focused on single exposureoutcome relationships. The "exposome" concept was proposed to encompass the totality of exposures from conception onwards, complementing the genome. New analytical frameworks are required to interpret multi-dimensional exposome data.

Methods: HELIX is a EC-FP7 collaborative research project commissioned in the European Comission's exposome programme. HELIX uses six existing, prospective birth cohort studies to measure a range of chemical and physical environmental hazards in food, consumer products, water, air, noise, and the built environment, in pre and postnatal periods, and link these with molecular omics profiles (of metabolites, proteins, RNA transcripts and DNA methylation) and child health outcomes.

Results: We will present first results of the HELIX project, including a description of the correlation structure of multiple exposure data as a first step in developing analytical tools appropriate to exposome data.

Conclusion: The exposome concept provides an important new framework to improve knowledge on the environmental component of disease aetiology, with early life as a crucial developmental period. Challenges in the measurement, analysis, and interpretation of complex longitudinal exposomehealth associations require efficient collaboration between environmental health, life-course epidemiology and other fields.
5008

Maternal occupational exposure to extremely low frequency magnetic fields and electrical shocks and birth outcomes across Europe

Monica Guxens ${ }^{1}$, Maribel Casas ${ }^{2}$, Albert Dalmau-Bueno ${ }^{3}$, Martine Vrijheid ${ }^{4}$, Roel Vermeulen ${ }^{5}$, Anke Huss ${ }^{6}$

${ }^{1}$ Centre for Research in Environmental Epidemiology (CREAL), Barcelona, Spain; ${ }^{2}$ Centre for Research in Environmental Epidemiology (CREAL), Barcelona, Spain; ${ }^{3}$ Centre for Research in Environmental Epidemiology (CREAL), Barcelona, Spain;

${ }^{4}$ Centre for Research in Environmental Epidemiology (CREAL), Barcelona, Spain; ${ }^{5}$ Institute for Risk Assessment Sciences (IRAS), Utrecht University, the Netherlands; ${ }^{6}$ Institute for Risk Assessment Sciences (IRAS), Utrecht University, the Netherlands

Research question: To assess the association between maternal occupational exposure to extremely low frequency magnetic fields (ELF-MF) and electrical shocks during pregnancy and birth outcomes across Europe.

Methods: We included 186,433 mother-child pairs from 12 European birth cohorts. Individual data on maternal occupation during pregnancy was linked with job exposure matrices of ELF-MF and electrical shocks. Outcomes assessed included birthweight, gestational age, preterm birth, term low birthweight, small for gestational age, and infant sex ratio. Analyses were adjusted for several potentially confounding variables. We performed meta-analyses of cohort-specific estimates.

Results: Exposure to high levels of ELF-MF during pregnancy was associated with a higher odds of term low birthweight $(\mathrm{OR}=1.99 ; 95 \% \mathrm{CI}=1.00,3.99)$ compared to low levels of ELF-MF. Exposure to high risk of electrical shocks during pregnancy was associated with a longer gestational length of 0.91 days $(95 \% \mathrm{CI}=0.07,1.68)$ compared to low risk of electrical shocks. Associations were consistent across cohorts with little heterogeneity. No associations were found with the other outcomes.

Conclusion: This large study suggests that exposure to high levels of ELF-MF during pregnancy might be associated with impaired fetal growth at birth, whereas exposure to high risk of electrical shocks during pregnancy seems to be associated with longer gestational age.

\section{1}

Multigenerational effects of paternal exposure to environmental contaminants

Janice Bailey ${ }^{1}$, Clotilde Maurice ${ }^{2}$, Serge McGraw ${ }^{3}$, Romain Lambrot $^{4}$, Nancy Côte ${ }^{5}$, Arnaud Droit ${ }^{6}$, Donovan Chan ${ }^{7}$, Jacquetta Trasler $^{8}$, Sarah Kimmins ${ }^{9}$

${ }^{1}$ Université Laval; ${ }^{2}$ Université Laval; ${ }^{3}$ McGill University;

${ }^{4}$ McGill University; ${ }^{5}$ Université Laval; ${ }^{6}$ Université Laval;

${ }^{7} \mathrm{Mc}$ Gill University; ${ }^{8} \mathrm{Mc}$ Gill University; ${ }^{9} \mathrm{Mc}$ Gill University

Research question: How does early exposure to environmentally-relevant organochlorines (OC) affect male 
reproductive development and his ability to produce healthy future generations?

Methods: Sprague Dawley female rats were gavaged with corn oil (Controls) or an OC mixture designed to mimic that which contaminates the Arctic food chain and at levels to represent human exposure. Females were mated with untreated males to produce the prenatally-exposed $\mathrm{F} 1$ generation. The prenatally-exposed F1 sires were mated with untreated females to produce F2, F3 and F4 generations. The phenotypes of these males and the sperm methylome of the F1 sires were assessed in comparison with the Control paternal lineage.

Results: Prenatal exposure to OC induced subfertility in the F1 males. Numerous health defects, including male subfertility were observed throughout the F2, F3 and F3 generations. In the sperm from the F1 prenatally-exposed males, $>200$ genes were differentially methylated, including those in regions involved in embryo development. Pathologies linked to the altered genes include metabolic, neurological, psychological and cardiovascular disorders, cancers and reproductive dysfunction.

Conclusion: Early paternal exposure to OC induces reproductive dysfunction and developmental pathologies in his offspring and future generations, possibly due to epimutations in his sperm DNA.

\section{7}

Preventive intervention to reduce human exposure of PCBs/Dioxins

Chisato Mori ${ }^{1}$, Akifumi Eguchi ${ }^{2}$, Masae Otake ${ }^{3}$,

Masamichi Hanazato ${ }^{4}$, Norimichi Suzuki ${ }^{5}$,

Masahiro Watanabe ${ }^{6}$, Yoshiharu Matsuno ${ }^{7}$, Hiroko Nakaoka ${ }^{8}$, Emiko Todaka ${ }^{9}$

${ }^{1}$ Center for Preventive Medical Sciences Chiba University;

${ }^{2}$ Center for Preventive Medical Sciences Chiba University;

${ }^{3}$ Center for Preventive Medical Sciences Chiba University;

${ }^{4}$ Center for Preventive Medical Sciences Chiba University;

${ }^{5}$ Center for Preventive Medical Sciences Chiba University;

${ }^{6}$ Center for Preventive Medical Sciences Chiba University;

${ }^{7}$ Center for Preventive Medical Sciences Chiba University;

${ }^{8}$ Center for Preventive Medical Sciences Chiba University;

${ }^{9}$ Center for Preventive Medical Sciences Chiba University

Adverse health effects by fetal and children's exposures to PCBs have been concerned. Our previous studies revealed that colestimide (fiber; a medication for hyperlipidemia) was effective to reduce PCB level in human. In this study, a method to reduce $\mathrm{PCB}$ exposure was proposed from the data of Japan Environment and Children's Study (JECS). JECS is a long term cohort study of children. Blood PCB levels of 197 fathers who were recruited in Chiba Regional Center of JECS were analyzed and calculated by Packed Column Gas Chromatography Electron Capture Detector (GC/ECD). The relationships between Body Mass Index, ages, FFQ answers and PCB concentration were analyzed by Partial least squares and Bayesian ridge regression with variable selection by widely applicable information criterion. The results showed that consumption of fruits and vegetables had significantly negative correlation with $\mathrm{PCB}$ concentration. It indicated that fibers contained in fruits and vegetables might reduce PCBs. This study was conducted as an adjunct study of JECS, and partly supported by a grant for JECS from the Ministry of the Environment, Japan. The findings and conclusions of this article are solely the responsibility of the authors and do not represent the official views of the above government.

\section{5}

Bisphenol-a exposure in utero disrupts pancreatic beta-cell function and mass in mice one month after delivery

Marta Garcia-Arévalo ${ }^{1}$, Paloma Alonso-Magdalena ${ }^{2}$, Esther Fuentes $^{3}$, Ivan Quesada ${ }^{4}$, Joan M. Servitja ${ }^{5}$, Anna Novials ${ }^{6}$

${ }^{1}$ Instituto de Bioingeniería, Universidad Miguel Hernández;

${ }^{2}$ Departamento de Biología Aplicada, Universidad Miguel Hernández; ${ }^{3}$ Instituto de Bioingeniería, Universidad Miguel Hernández; ${ }^{4}$ Instituto de Bioingeniería, Universidad Miguel Hernández; ${ }^{5}$ Diabetes \& Obesity Laboratory, IDIBAPS, Barcelona, Spain; ${ }^{6}$ Diabetes \& Obesity Laboratory, IDIBAPS, Barcelona, Spain

Research questions: Bisphenol-A (BPA) is a widespread endocrine disrupting chemical. The aim of this work was to study BPA actions on metabolic parameters as well as in betacell mass and function in BPA-exposed animals.

Methods: OF1 pregnant mice were treated with either vehicle or BPA (10 or $100 \mathrm{microg} / \mathrm{kg} /$ day) during days 9 to 16 of gestation. One month after delivery, glucose homeostasis and pancreatic beta cell function and mass were studied. Gene expression was analyzed.

Results: Male mice showed lower body weight at birth compared to control animals. No differences in glucose tolerance or insulin sensitivity were found in at one month of age compared to controls. In vivo glucose-stimulated insulin secretion was increased in both BPA groups. In addition, higher serum insulin, leptin and c-peptide levels were increased. We observed increased pancreatic -cell mass in BPA animals compared to control group. This increment was correlated with increased proliferation capacity and decreased apoptosis rate in beta-cells. Global gene expression analysis using microarrays revealed that genes related to cell cycle and mitosis were significantly enriched.

Conclusion: Early exposure to BPA during embryonic development altered pancreatic beta-cell function and mass and global gene expression.

Funding: MINECO BFU2011-28358 and SAF2014-58335-P 
5352

Bisphenol A (BPA) increases neural progenitor cell proliferation and enhances differentiation of appetite versus satiety neurons

Michael G. Ross ${ }^{1}$, Mina Desai ${ }^{2}$, Guang $\operatorname{Han}^{3}$, Marie H Beall ${ }^{4}$

${ }^{1}$ Geffen School of Medicine at UCLA; ${ }^{2}$ LABioMed at HarborUCLA Med. Ctr, Torrance, CA, United States; ${ }^{3}$ LABioMed at Harbor-UCLA Med. Ctr, Torrance, CA, United States; ${ }^{4} L A$ Perinatal Assoc, LA, CA, United States

Abstract: Increases in childhood and adult obesity have been attributed, in part to altered development of the hypothalamic appetite center (arcuate nucleus; ARC) resulting from programming effects of developmental exposures. The ARC contains two populations of neurons with opposing actions on food intake.

ARC appetite (AgRP, agouti-related protein and NPY, neuropeptide Y) and ARC satiety (POMC, pro-opiomelanocortin). Bisphenol A (BPA) is a ubiquitous chemical widely used in plastics (e.g. water bottles). BPA is an endocrine disruptor (EDC) chemical which has estrogen receptor effects, and significant levels are consistently observed in pregnant women and fetal plasma and amniotic fluid. We hypothesized that BPA exposure impacts fetal hypothalamic ARC development, resulting in an increase in appetite (AgRP/NPY) versus satiety (POMC) neurons.

Methods: Newborn rats born from normal dams were sacrificed and brains dissected. Hypothalamic neuroprogenitor cells (NPCs) from control newborns were cultured in both complete and differentiation medium and treated with BPA $(10 \mathrm{mM})$ or DMSO (control) for 5 days. NPC proliferation (MTT assay) was assessed, and protein expression (Western Blot) of NPC markers (Nestin), NPC proliferative and neurogenic factors (Hes1, Ngn3), and peptide markers of differentiated appetite (AgRP/NPY) and satiety (POMC) neurons were analyzed.

Results: In response to BPA exposure, NPC proliferation markedly increased, as measured by MTT assay as well as Nestin (1.3-fold) and Hes1 (1.4-fold). In differentiation media, BPA increased NPC expression of Ngn3 (1.5-fold). When NPCs were differentiated, there was marked increased expression of AgRP (1.8-fold) and NPY (1.6-fold) and reduced expression of POMC (0.6-fold).

Conclusions: Exposure to BPA causes increased NPC proliferation and differentiation, in part via upregulated Hes1 and Ngn3. The preferential upregulation of AgRP/NPY and downregulation of POMC suggests that BPA enhancement of appetite drive is a putative factor contributing to BPA-induced offspring obesity.

\section{0}

Development of a nutritional wellness program for staff at a further education and training college

Siveshnee Devar

University of Mpumalanga
Background: The rate of absenteeism and prevalence of NCDs in South Africa is extremely high. This is consistent with other educational institutions and workplaces around the globe. In most cases the absence of health and the presence of one or more non communicable diseases coupled with the lack of physical exercise is a major factor in absenteeism. Absenteeism at the workplace comes at a huge cost to the employer and the country as a whole.

Aim: Findings from this study was to develop a suitable nutritional wellness program for the workplace.

Methodology: A needs analysis in the form of 24-hour recall, food frequency, health and socio demographic questionnaires was undertaken to determine the need for a wellness program for the institution. Anthropometric indices such as BMI, waist circumference and blood pressure were also undertaken to determine the state of health of the staff.

Results: This study has found that obesity, central obesity, hypertension as well as deficiencies in nutrients and minerals were prevalent in this group. Fruit and vegetable consumption was also below the WHO recommendation. This study showed a link between diet, physical activity and diseases of lifestyle. There were positive correlations between age and systolic blood pressure, waist circumference and systolic blood pressure, waist circumference and diastolic blood pressure and waist-to-height ratio and BMI.

Conclusion: The results indicated the need for immediate intervention in the form of a wellness program. Nutrition education is important for both the workplace and out. Education and knowledge are important factors for lifestyle changes. The proposed intervention is aimed at improving presenteeism and decreasing the incidence of non- communicable diseases. Presenteeism and good health are important factors for quality education at all educational institutions.

\section{2}

Child protection actions after preterm, early term and post term birth - a follow up register-linkage study

Suvi Alenius ${ }^{1}$, Eero Kajantie ${ }^{2}$, Pieta Näsänen-Gilmore ${ }^{3}$, Marja Vääräsmäki ${ }^{4}$, Mika Gissler ${ }^{5}$, Petteri Hovi ${ }^{6}$

${ }^{1}$ National Institute for Health and Welfare, Helsinki, Finland;

${ }^{2}$ National Institute for Health and Welfare, Helsinki, Finland; Children's Hospital, Helsinki University Hospital and University of Helsinki, Helsinki, Finland, Department of Obstet; ${ }^{3}$ National Institute for Health and Welfare, Helsinki, Finland; ${ }^{4}$ National Institute for Health and Welfare, Oulu, Finland; Department of Obstetrics and Gynecology, MRC, Oulu, Oulu University Hospital and University of Oulu, Oulu, Finland; ${ }^{5}$ National Institute for Health and Welfare, Helsinki, Finland; ${ }^{6}$ National Institute for Health and Welfare, Helsinki, Finland; Children's Hospital, Helsinki University Hospital and University of Helsinki, Helsinki, Finland

Research question: The aim of this nationwide register-linkage study was to assess how gestational age (GA) at birth predicts the rate of children ever placed outside home. 
Method: We used data from the Finnish Medical Birth Register to identify singleton born infants $(n=224751)$ of five GA-categories born between Jan 1st 1987 and Sep 30th 1990. Register of Child Welfare provided follow-up data (7757 first placements outside home, 3.5\%) until 18th birthday. We analyzed the effect of GA by logistic regression.

Results: We compared odds for outside home placement to those born at full term (39-41 gestational weeks) first with a model adjusted for index child's sex and maternal age. ORs for placement were $2.24(1.86,2.70)$ for early preterm (<34weeks), $1.63(1.46,1.81)$ for late preterm $(34-<37)$, $1.26(1.19,1.33)$ for early term $(37-<38)$ and 1.19 (1.07, 1.32) for post term $(>42)$. When adjusted for birth year, maternal smoking during pregnancy and her highest attained education, the $<34$-wk OR attenuated to $1.78(1.47,2.16)$ with other ORs unchanged.

Conclusion: Early environmental conditions may have life-long consequences for individual, family and society: As compared to full term children, preterm, early term and post term children are predisposed to placement outside home.

\section{2}

Mother's accurate perception is insufficient in maintaining normal-weight in a Singaporean cohort of pre-school children

Tuck Seng Cheng ${ }^{1}$, See Ling Loy ${ }^{2}$, Yin Bun Cheung ${ }^{3}$, Jerry Kok Yen Chan ${ }^{4}$, Mya Thway Tint ${ }^{5}$, Keith Godfrey ${ }^{6}$, Peter Gluckman ${ }^{7}$, Kenneth Kwek ${ }^{8}$, Seang Mei Saw ${ }^{9}$, Yap-Seng Chong $^{10}$, Lynette Shek ${ }^{11}$, Yung Seng Lee ${ }^{12}$, Fabian Yap ${ }^{13}$, Ngee Lek ${ }^{14}$

${ }^{1} K K$ Women's and Children's Hospital, Singapore; ${ }^{2} K K$ Women's and Children's Hospital, Singapore; ${ }^{3}$ Duke-NUS Graduate Medical School, Singapore; 'KK Women's and Children's Hospital, Singapore; ${ }^{5}$ National University of Singapore, Singapore; ${ }^{6}$ University of Southampton, Southampton, UK; ${ }^{7}$ Agency for Science, Technology and Research, Singapore; ${ }^{8} \mathrm{KK}$ Women's and Children's Hospital, Singapore; ${ }^{9}$ National University of Singapore, Singapore; ${ }^{10}$ National University of Singapore, Singapore; ${ }^{11}$ National University of Singapore, Singapore; ${ }^{12}$ National University of Singapore, Singapore; ${ }^{13} \mathrm{KK}$ Women's and Children's Hospital, Singapore; ${ }^{14} K K$ Women's and Children's Hospital, Singapore

Background: We hypothesized that mother's accurate perception is important in maintaining child's normalweight status, and investigated the association between maternal perception and child's actual weight status 12 months later.

Methods: Mother-child pairs $(\mathrm{n}=575)$ were drawn from a Singapore birth cohort. At 36 and 48 months, respectively, the children were classified as underweight, normal-weight and overweight based on body mass index using the International Obesity Task Force criteria. Maternal perception of child's weight status at 36 months was evaluated using verbal questions and visual silhouettes, and compared with child's weight status at 48 months.

Results: At 36 months, 413 (72\%) children were normalweight. Among these normal-weight children, 313 mothers (76\%) accurately perceived their child's weight status both verbally and visually. At 48 months, 352 children remained normal-weight, of whom 271 mothers (77\%) had accurate perception at 36 months; significantly, 24 children became overweight despite accurate perception in 20 mothers (83\%), and 37 children became underweight despite accurate perception in 22 mothers (59\%); $\mathrm{p}=0.03$.

Conclusion: Children who were accurately perceived by their mothers as having normal-weight at 36 months could still become overweight or underweight at 48 months. This suggests that mother's accurate perception is insufficient in maintaining normal-weight in pre-schoolers.

\section{3}

\section{Why do pregnant women participate in trials?}

Stephen Jeffares ${ }^{1}$, Farah Sadrudin ${ }^{2}$, Nicole Huisman ${ }^{3}$, Hema Venkataraman ${ }^{4}$, Ponnusamy Saravanan ${ }^{5}$

${ }^{1}$ Institute of Local Government Studies, University of Birmingham; ${ }^{2}$ Imperial NHS Trust; ${ }^{3}$ South West Deanary NHS Foundation Trust; ${ }^{4}$ George Eliot Hospital, University of Warwick; ${ }^{5}$ Division of Metabolic and Vascular Health University of Warwick \& George Eliot Hospital

Introduction: Patient participation in clinical trials is a growing area of interest. Knowing why participants agree to take part allows trial designs to be attractive to patients. However, our knowledge on pregnant women with regards to this is limited. This qualitative study aims to explore reasons why pregnant women agree to take part in trials.

Methodology: We chose a novel methodology; Q-methodology, to investigate opinions of women within the PRiDE study. Q-methodology provides holistic view points on subject matters. 40 reasons for participation were derived from a literature review and informal interviewing. 30 participants were asked to rank these statements in order of agreement, and objective analysis correlated these against each other to find commonalities and distinctions.

Results: Correlating the data identified three major view points; helping the future of medicine, personal connection, and no inconvenience. Participants were made aware during consenting that taking part would not affect their general care.

Conclusions: A genuine interest in helping medicine progress, feeling a personal connection to the disease, and taking part without interference to their lives are main factors for pregnant women. Investigations need to be conducted with women who declined to take part, and those from different socioeconomic backgrounds or ethnicities. 
4562

A questionnaire survey to determine understanding of $\mathrm{DOHaD}$ concepts in populations of future registered dietitian nutritionists, and present/future midwives in Japan

Aya Endo ${ }^{1}$, Kanae Sato ${ }^{2}$, Manabu Musashi ${ }^{3}$, Masahito Oyamada ${ }^{4}$

${ }^{1}$ Tenshi College, Nikko Memorial Hospital, and Fuji Women's University; ${ }^{2}$ Tenshi College; ${ }^{3}$ Tenshi College; ${ }^{4}$ Fuji Women's University

Research question: To determine understanding of $\mathrm{DOHaD}$ concepts in populations of future registered dietitian nutritionists (RDNs), and present/future midwives.

Methods: A questionnaire survey was performed using a questionnaire adapted from Gage et al. (2011) with a question added about the influence of a woman's diet during pregnancy on adult health. Undergraduate nutrition students and postgraduate midwifery students in a Japanese college, and midwives in Hokkaido of Japan were invited to complete the questionnaire.

Results and Conclusion: A total of 186 present/future health professionals including 84 undergraduate nutrition students, 44 postgraduate midwifery students, and 58 midwives completed the questionnaire. Overall, diets during pregnancy and infancy were rated an extremely/very important influence on adult health by $61 \%$ and $63 \%$, respectively, but were deemed less significant than diet and physical activity in childhood/adolescence $(86 \%$, $73 \%)$ and in adulthood $(84 \%, 71 \%)$, air pollution $(87 \%)$, exposure to cigarette smoke (96\%), and genetics/inheritance $(72 \%)$. A cluster analysis demonstrated that answers to the questions about influences of diets during pregnancy and infancy constitute a separate group from those to the other questions. These results of Japanese future RDNs and present/future midwives showed the relatively low levels of recognition of the effect of early nutrition on lifelong health.

\section{5}

Characterising the potential of undergraduate courses to facilitate understanding of $\mathrm{DOHaD}$ concepts in populations of future health professionals

Masahito Oyamada ${ }^{1}$, Sarah Morgan ${ }^{2}$, Robyn Dixon ${ }^{3}$, Clare Wall $^{4}$, Anecita Lim ${ }^{5}$, Jacquie Bay ${ }^{6}$

${ }^{1}$ Fuji Women's University; ${ }^{2}$ Liggins Institute, University of Auckland, and Gravida: National Centre for Growth and Development; ${ }^{3}$ University of Auckland; ${ }^{4}$ University of Auckland;

${ }^{5}$ University of Auckland; ${ }^{6}$ Liggins Institute, University of Auckland, and Gravida: National Centre for Growth and Development

Research question: To evaluate the relationship between $\mathrm{DOHaD}$ related course content and change in awareness of $\mathrm{DOHaD}$ concepts during undergraduate courses for future registered dietitian nutritionists.

Methods: A standardised questionnaire developed by researchers in New Zealand and Japan (http://urx.nu/dQJO) was used to ascertain knowledge of $\mathrm{DOHaD}$ concepts in undergraduate nutrition students at the beginning of year 1 and the end of year 3 in two universities in New Zealand and Japan. Results: At the beginning of year 1, undergraduate nutrition students demonstrated fairly low levels of awareness of $\mathrm{DOHaD}$ concept. Although knowledge of this concept was significantly increased at the end of year 3 until which lectures containing $\mathrm{DOHaD}$ evidence had been presented, the knowledge level was still relatively low. The Japanese students showed that exposure to $\mathrm{DOHaD}$ concepts in the course achieved a high level of awareness of the term $\mathrm{DOHaD}$, but it did not create the understanding of $\mathrm{DOHaD}$ concept in a large proportion.

Conclusion: These results suggest that inclusion of $\mathrm{DOHaD}$ related content in undergraduate nutrition education could enhance application of $\mathrm{DOHaD}$ concepts by future healthcare professionals, but we need to review the $\mathrm{DOHaD}$ aspects of the learning programme and design a revised programme to translate the concepts effectively.

4569

Social network as a health promotion tool for pregnant women: the Welcome Baby Project

Ana Maria Escobar ${ }^{1}$, Murilo Wadt ${ }^{2}$, André Covic ${ }^{3}$, Carolina Hofmeister Mansu ${ }^{4}$, Filumena Gomes ${ }^{5}$, Maria Helena Valente ${ }^{6}$, Grisi Sandra ${ }^{7}$, Joana Abucham Ribeiro ${ }^{8}$

${ }^{1}$ University of Sao Paulo Medical School; ${ }^{2}$ University of São Paulo, School of Economics, Business Administration and Accounting; ${ }^{3}$ State University of Campinas, Computer Science Institute and Institute of Philosophy and Human Sciences; ${ }^{4}$ Universtity of Sao Paulo School of Medicine; ${ }^{5}$ São Paulo c; ${ }^{6}$ São Paulo University Medical School; ${ }^{7}$ São Paulo University Medical School; ${ }^{8}$ AJR Heath Education

Research question: can a social network be used as a tool for health promotion?

Methods: we are following pregnant women since the first trimester of pregnancy until 30 days after childbirth, in a closed group within Facebook, the social network used. We called it the Welcome Baby Project. Participation is on a voluntary basis. All pregnant women who were interested, accepted the terms and conditions, and filled out an online form. Five structured questionnaires are used with the pregnant women's habits and health information.

Results: the sample included 649 pregnant women who are daily receiving health information.

Educational level: $6.9 \%$ of pregnant women have until first grade, $31.1 \%$ have high school; $41.5 \%$ have university degree and $20.48 \%$ have post graduation. Regarding family income, $23.2 \%$ received up to 2 minimum wages, $63.8 \% 2-10$ wages and $12.7 \%$ more than 10 minimum wages per month.

Conclusion: this is an innovative project in its form and content. The social network can be used as a tool to promote health, clarify important issues and encourage the need of prenatal consultation, since it covers all education extracts and family income. 
4570

Pregnant women, educational level and most used health information search sites

Ana Maria Escobar ${ }^{1}$, Murilo Wadt ${ }^{2}$, André Covic ${ }^{3}$, Carolina Hofmeister Mansu ${ }^{4}$, Filumena Gomes ${ }^{5}$, Maria Helena Valente $^{6}$, Grisi Sandra ${ }^{7}$, Joana Abucham Ribeiro ${ }^{8}$

${ }^{1}$ University of Sao Paulo Medical School; ${ }^{2}$ University of São Paulo, School of Economics, Business Administration and Accounting; ${ }^{3}$ State University of Campinas, Computer Science Institute and Institute of Philosophy and Human Sciences; ${ }^{4}$ Universtity of Sao Paulo School of Medicine; ${ }^{5}$ University of Sao Paulo Medical School; ${ }^{6}$ University of Sao Paulo Medical School;

${ }^{7}$ University of Sao Paulo Medical School; ${ }^{8}$ AJR Heath Education

Research question: where pregnant women seek health information?

Methods: 649 pregnant women are followed since the first trimester of pregnancy, in a closed group within Facebook, a social network. They accepted the terms and conditions. We asked what media they used to obtain health information. Results: magazines, 40.2\%; newspapers, 20.7\%; TV, 48.9\%; internet (except social network), $87.0 \%$; their mothers, $34.6 \%$; other members of the family, $18.1 \%$; friends, $21.3 \%$; doctors, $72.9 \%$; teachers, $7.4 \%$. The group with less than 8 years of education had less access to the media. The average sources for the more educated group is 3.73 and the less educated group 1.50. Using Pearson $99 \%$ reliability: magazines, $p=0.002$; newspapers, $\mathrm{p}=0.004$; TV, $\mathrm{p}<0.001$; internet, $\mathrm{p}<0.001$; their mothers, $\mathrm{p}=0.395$; family, $\mathrm{p}=0.154$; friends, $\mathrm{p}=0.079$; doctors, $\mathrm{p}<0.001$ and teachers $\mathrm{p}=0.708$.

Conclusions: online social network mobilize people of diverse educational levels. People with less education level have access to fewer sources of information, particularly doctors, television, other sources than social network in the internet, magazines and newspapers. The social network can be used as a tool to promote health in all education and income levels.

\section{2}

Is there a relationship between maternal body mass index and pregnancy intention?

Kate Cheney ${ }^{1}$, Marilena Pelosi ${ }^{2}$, Joanne Ludlow ${ }^{3}$, Alexandra Barrett ${ }^{4}$, Kirsten Black ${ }^{5}$

${ }^{1}$ Royal Prince Alfred Hospital Women and Babies, Camperdown, Australia; ${ }^{2}$ Royal Prince Alfred Hospital Women and Babies, Camperdown, Australia; ${ }^{3}$ Royal Prince Alfred Hospital Women and Babies, Camperdown, Australia; ${ }^{4}$ School of Public Health, The University of Sydney, Australia; ${ }^{5}$ Sydney Medical School, The University of Sydney, Australia

Research question: Is there a relationship between body mass index (BMI) and pregnancy intention?

Obesity increases the risk of obstetric complications which can have long-term consequences for both the mother and child.
Pregnancy planning therefore has important public health implications given the rising obesity rates.

Methods: A cross-sectional study was conducted from November 2013-February 2015, at an early pregnancy assessment clinic within a tertiary teaching hospital in Sydney, Australia. The clinic reviews pregnant women $>16$ years of age with concerns or with pain and/or vaginal bleeding, at $<20$ weeks gestation. Pregnancy intention was assessed using a self-completed, validated, pregnancy intention questionnaire. Anthropometry was measured.

Results: Forty-three percent (219) had clearly intended their pregnancy, 39\% (194) were ambivalent and 18\% (88) had not planned this pregnancy. There was no relationship found between BMI categorisation and pregnancy intention (overweight/obese [95\% CI: $0.60-1.26 ; \mathrm{P}=0.16]$ ); or obesity alone (95\% CI: 0.55-1.56; $\mathrm{P}=0.78)$.

Conclusion: Pregnancy intention was not found to be related to maternal weight, contrary to previous research. Given the increasing proportion of overweight and obese women presenting in obstetric practice, and the known associated weight-related complications, this study demonstrates tailoring preconception care recruitment by BMI status is not required.

\section{4}

Ensuring empowerment of women and girls is at the center of $\mathrm{DOHaD}$ translation programs

Mark Vickers ${ }^{1}$, Susan Morton ${ }^{2}$

${ }^{1}$ Liggins Institute, University of Auckland; ${ }^{2}$ University of Auckland

Research question: To examine the impact of gender on participation by adolescents in school-based $\mathrm{DOHaD}$ communication and translation programs.

Methods: Learning modules presenting $\mathrm{DOHaD}$ evidence were used by teachers within science programs in Year 7-10 classes in 10 schools. Evaluation was via matched pre/post questionnaires and interviews, to 12-months post-intervention. Results: Participation positively impacted understanding of the association between nutritional environment during pregnancy and later-life NCD risk. Understanding moved from $50 \%$ pre- to $78 \%$ post-intervention, sustained to 12 -months at $69 \%$, with no gender-based difference. However, males showed a lower initial understanding of the importance of nutrition for health. This lifted to the same level as females post-intervention, sustained to 12months. Positive dietary action was sustained across both genders. Conclusion: The program design was effective irrespective of gender. For adolescent girls to become change agents, they must understand the potential that they hold, via nutritional choices, to break the intergenerational cycle of obesity-related ill-health in their families. However, gender equity issues potentially challenge this. Therefore boys must also understand the importance of nutrition for women, and be supported to potentially challenge cultural norms and prioritize the nutrition of women and children through through evidence-based actions. 


\section{Neonatal outcomes of unplanned pregnancy in the GUSTO birth cohort study}

See Ling Loy ${ }^{1}$, Tuck Seng Cheng ${ }^{2}$, Yin Bun Cheung ${ }^{3}$, Keith Godfrey $^{4}$, Peter Gluckman ${ }^{5}$, Kenneth Kwek ${ }^{6}$, Seang Mei Saw ${ }^{7}$, Yap-Seng Chong ${ }^{8}$, Fabian Yap ${ }^{9}$, Ngee Lek $^{10}$, Jerry Kok Yen Chan ${ }^{11}$

${ }^{1}$ KK Women's and Children's Hospital, Singapore; 'KK Women's and Children's Hospital, Singapore; ${ }^{3}$ Duke-NUS Graduate Medical School, Singapore; ${ }^{4}$ University of Southampton, Southampton, UK; ${ }^{5}$ Agency for Science, Technology and Research, Singapore; 'KK Women's and Children's Hospital, Singapore;

${ }^{7}$ National University of Singapore, Singapore; ${ }^{8}$ National University of Singapore, Singapore; ${ }^{9} \mathrm{KK}$ Women's and Children's Hospital, Singapore; ${ }^{10}$ KK Women's and Children's Hospital, Singapore; ${ }^{11} \mathrm{KK}$ Women's and Children's Hospital, Singapore

Research question: Pregnancy intention may play an important role in health consequences of offspring. We investigated neonatal outcomes of unplanned pregnancy.

Methods: This was a prospective cohort study which included 861 mother-infant pairs in Singapore. Data on pregnancy intention and socio-demography were collected at 26-28 weeks' gestation, while neonatal outcomes were retrieved after delivery. Multivariate regression analyses were performed to examine the relationships between pregnancy intention and associated outcomes.

Results: There were 481 (56\%) planned pregnancies, 334 (39\%) mistimed (unplanned and enthusiastic) and 46 (5\%) unintended (unplanned and unenthusiastic) pregnancies. Babies born to mothers of mistimed pregnancy were less likely to require special care after birth $(\mathrm{OR}=0.59,95 \% \mathrm{CI}=0.42,0.83)$ and were associated with lower length-for-age $Z$ score $(\beta=-0.17,95 \%$ $\mathrm{CI}=-0.33,-0.01)$ at birth. The significant association between mistimed pregnancy and higher weight-for-length $\mathrm{Z}$ score disappeared after adjusting for potential confounders $(\beta=0.17$, $95 \% \mathrm{CI}=-0.02,0.36)$. No differences in neonatal outcomes were found between unintended and planned pregnancies.

Conclusion: Mother's intention to conceive was not significantly associated with adverse neonatal outcomes in this cohort. Further investigation is needed to study the offsprings' long term health consequences.

\section{1}

The Healthy Start to Life Education for Adolescents Project: Indicators of early success in adaptation for use in Small Island Developing States

Delaney Yaqona ${ }^{1}$, Karen Tairea ${ }^{2}$, Sarah Morgan ${ }^{3}$, Tania Morgan $^{4}$, Mark Vickers ${ }^{5}$, Celeste Barrett-Watson ${ }^{6}$, Jackson Martin $^{7}$, Upokoina Herrmann ${ }^{8}$

${ }^{1}$ Nukutere College, Cook Islands; ${ }^{2}$ Te Marae Ora, Cook Islands Ministry of Health; ${ }^{3}$ Liggins Institute, University of Auckland;
${ }^{4}$ Tereora College, National College of the Cook Islands; ${ }^{5}$ Liggins Institute, University of Auckland; ${ }^{6}$ Tereora College, National College of the Cook Islands; ${ }^{7}$ Tereora College, National College of the Cook Islands; ${ }^{8}$ Maraurau o te Pai Api' i, Ministry of Education, Cook Islands

Research question: To explore the potential for adaptation of the Healthy Start to Life Education for Adolescents project (HSLEAP) to support reduction in NCD risk for adolescents and their future offspring in Small Island Developing States (SIDS). Methods: HSLEAP, an adolescent DOHaD communication and translation program, proven effective in New Zealand, was adapted for use in Pacific SIDS. Evaluation via matched pre/ post questionnaires and interviews with participants, teachers and families is planned in two contrasting SIDS, to 24-months post-intervention.

Results: In the Cook Islands, adolescents in all schools on the island of Rarotonga (population 8,000, adult obesity 65\%, adult diabetes $25 \%$ ) will be exposed to the program over two years. Validation phase evidence shows changes in understanding of the importance of nutrition before and during pregnancy for the infant (strongly agree (SA) pre 36\% to post $70 \%)$ and later-life health (pre $13 \%$ to post $36 \%)(n=69)$. In post-intervention interviews $(n=30) \quad 80 \%$ of adolescents offered examples of family communication and positive behavior change arising from participation.

Conclusion: SIDS are burdened with poverty, isolation, and high rates of obesity and related disease. Utilizing WHO STEPS, Global-School-Health-Survey, and DOHAD evidence, we have demonstrated that HSLEAP is adaptable to support $\mathrm{DOHaD}$ translation in developing nations. Early impact data is positive.

\section{4}

\section{The Political Ecology of Perinatal Nutrition in Khayelitsha, South Africa}

Michelle Pentecost

University of Oxford

Research question: South African nutrition policy has adopted the global health focus on 'the first thousand days' as the window of opportunity for nutrition interventions to target undernutrition, childhood communicable diseases, and a potential future burden of adult non-communicable disease. This study examines the local uptake of this new perinatal nutrition policy in Khayelitsha to assess policy responsiveness to this local context.

Methods: This research spanned 14 months and employed qualitative and ethnographic methodologies. 60 participants were recruited at 2 clinics in Khayelitsha at antenatal visits, and participated in a baseline survey, and a standardised telephonic interview after delivery. A smaller group of 15 participants were followed up closely over 12 months with initial life history interviews, fortnightly follow up visits, and close participant observation by the researcher. 
Results: Participants' health knowledge from the clinics did not align with practice in the majority of observed cases due to financial pressures, family expectation and personal preference, among other reasons.

Conclusion: This paper offers a rich ethnographic illustration of the complex set of factors that structure the nutritional profile of pregnant women and infants in Khayelitsha, only some of which are taken into account in the formulation of nutrition policy.

\section{5}

\section{Shapes of nourishment: how ingestion edits life in the Cape Winelands}

Carina Truyts

University of Cape Town

Question: Considering that nourishment in physical, emotional and psychological forms of care is essential to health and lifelong development starting from the 'first 1000 days' this paper explores strategies for nourishment - particularly as it pertains to pregnant women and infants - in the context of precarious livelihoods.

In terms of food systems and public health discourse, the paper uses epigenetics and the first thousand days 'window' to examine the discourses around care relating to environmental factors such as kinship, belonging, and contextual history.

Methods: Qualitative anthropological fieldwork was conducted for 5 months where inter-generational and broader care networks were observed through participant observation, interviews, focus groups and network analysis. Food intake and larger systems of food distribution were explored through interviews, 24 hour recall, and participant observation.

Results: Nourishment took multiple forms and played out in complicated ways that required language use that deviates from that offered by biomedical and food security discourses. Precariousness due to seasonal variability, familial and broader relations, substance use, insider/outsider status and other factors impacted nutrition and food access.

Conclusion: The data demonstrated that attention to everyday lived experiences can reveal important information about the causes and ways in which people deal with uncertainty.

\section{3}

Perceptions of reproductive medicine clinical staff involved in a long-term, longitudinal study in pregnancy

Gail Pounder ${ }^{1}$, Hema Venkataraman ${ }^{2}$, Sammy Case ${ }^{3}$, Chin Wei Wong ${ }^{4}$, Ponnusamy Saravanan ${ }^{5}$

${ }^{1}$ George Eliot Hospital NHS Trust; ${ }^{2}$ University of Warwick;

${ }^{3}$ University of Warwick; ${ }^{4}$ University of Warwick; ${ }^{5}$ George Eliot Hospital NHS Trust and University of Warwick

Research question: Participation of maternity staff is poor and is critical for improving research in $\mathrm{DOHaD}$. Our question was, 'Why do clinical staff participate in research on pregnant women and what are their perceived barriers?'

Methods: An anonymous questionnaire-based pilot study was carried out in a research-naïve district general hospital contributing to a large longitudinal pregnancy study (PRiDE study). Two focus group interviews $(n=6)$ were conducted to identify the perceived barriers.

Results: The response rate was $65 \%(26 / 40)$ from a range of clinicians (hospital midwives-16, community midwives-2, healthcare assistants-1, phlebotomists-3, doctors-4, unspecified-4). Majority said their main reasons were contribution to evidence-based practice (92\%) and improving maternal-child health $(81 \%)$. To maintain their interest, $40 \%$ felt more regular updates from the research team would be helpful (newsletters, posters, face-face interactions). $25 \%$ admitted that research participation was additional workload. The focus group interviews revealed that poor instructions from senior management and a lack of incentives were the main barriers.

Conclusion: Contribution to evidence and improving maternal and child health were the main reasons for clinicians to be involved in research. Engagement of senior management, incentives and regular updates from the research team may improve further contribution. Funding bodies should encourage research teams to focus on such activities.

\section{9}

Maternal preferences for infant body size are associated with feeding practices and infant obesity among American Samoans

Nicola L Hawley ${ }^{1}$, Renu Nadkarni ${ }^{2}$, Jeanette SternbergLamb $^{3}$, Matauitafa Faiai ${ }^{4}$, Bethel T Muasau-Howard ${ }^{5}$, Aileen Solaita ${ }^{6}$, Stephen T McGarvey ${ }^{7}$

${ }^{1}$ Yale University; ${ }^{2}$ Yale University; ${ }^{3}$ Brown University; ${ }^{4}$ Chaminade University; ${ }^{5}$ Lyndon B Johnson Tropical Medical Center, American Samoa; ${ }^{6}$ Department of Health, American Samoa; ${ }^{7}$ Brown University

Research question: Parental preferences and beliefs about healthy weight and diet in infants have been associated with infant obesity among US minority populations. We aimed to examine preferences for infant body size among American Samoans, a population who have historically venerated large body size.

Methods: Mothers of infants 0-12 months $(n=149)$ were surveyed in 2014. The Infant Body Habitus Scale was used to determine preferences for infant body size. Associations among infant body size preferences, maternal characteristics, feeding practices, and measured infant body size were explored.

Results: Thirty-two percent of mothers preferred an infant body size larger than what they themselves thought was healthiest. This preference was not associated with maternal demographic characteristics, BMI, or infant gender. It was, however, associated with earlier cessation of breastfeeding and 
larger infant body size. Interestingly, those who preferred a smaller baby were most likely to think formula alone was the best way to feed an infant. Fifty-seven percent of mothers thought their older family members would prefer a baby larger than what she thought was healthy.

Conclusion: The observed associations between infant body size preferences, feeding behaviors and measured infant body size warrant further exploration and consideration in obesity intervention design.

\section{6}

Epigenetic programming, respect for autonomy and intergenerational justice: a new ethical battleground in connection with the fight against obesity

Lyne Létourneau ${ }^{1}$, Louis-Étienne Pigeon ${ }^{2}$

${ }^{1}$ Université Laval; ${ }^{2}$ Université Laval

In recent years, scientific knowledge in epigenetics has demonstrated in several animal species, including humans, that a propensity to obesity is transmitted to the next generation as a susceptibility to accumulate excess weight despite a normal diet. This new knowledge comes at a time when calls for the fight against obesity are increasing and the urgency to act is underscored emphatically. Based on the view that reducing obesity will produce significant health benefits and decrease the economic burden of rising health care costs, the fight against obesity finds additional support in epigenetic programing. It is now possible to include "benefit to others" (i.e. immediate descendants and future generations) as a credible justification for action. Such a rationalization however raises a number of questions in terms of liberal ethical theory. Indeed, the possibility of invoking benefit to others in order to prescribe the adoption of healthy eating habits is associated with two important ethical issues: respect for autonomy and intergenerational justice. It is imperative to clarify the relation between these two complex issues and the normative consequences of their enactment.

\section{5}

OPERA: the Optimal Pregnancy Environment Risk Assessment program, an initiative of the Preterm Birth International Collaborative (PREBIC)

David Olson

\section{University of Alberta, Edmonton, Canada}

OPERA is an international, interdisciplinary program of women's health researchers, care providers, foundations and agencies dedicated to discovering and disseminating inexpensive and accessible tools to diagnose women at risk for preterm birth (PTB) or other adverse pregnancy outcomes as early in pregnancy. OPERA's initial efforts are focusing on risk diagnosis in populations of vulnerable women.
OPERA intends to build a sustainable international organization that will provide guidance and support for studies of cohorts of pregnant women in order to develop evidence to 1) determine how to assess risk for PTB simply, inexpensively and as early as possible in pregnancy, 2) to identify the women in the highest (20\%) at-risk group (with $80 \%$ precision), and 3) to share this information with interested parties.

During its first formal meeting in San Francisco, March 22-23, 2015, thirty-eight invited participants discussed various vulnerable populations (victims of natural disasters, pollution and megacities, low income countries, refugees, marginalized women in replete societies, and excesses of modern societies), mechanisms leading to PTB, current approaches to risk assessment, existing studies predicting adverse pregnancy outcomes, and interventions to mitigate adverse pregnancy outcomes. See www.operamtg.org for presentations.

OPERA is sponsored by PREBIC, Worldwide Universities Network and March of Dimes.

\section{1}

Mothers, Daughters and Pregnancy Outcome: Narratives Across Time from Sana'a, Yemen

Annica Kempe

Karolinska Institutet

4304

Patterns of Body Mass Trajectory and Impact of Lifestyle Factors among Japanese Children

Chiyori Haga $^{1}$, Yoko Aihara ${ }^{2}$

${ }^{1}$ The University of Okayama; ${ }^{2}$ Kobe City College of Nursing

We aimed to conduct an explorative analysis for identifying variations in the developmental patterns of body size and the impact of lifestyle factors in Japanese children.

The data included details of all 155 children born from April 2, 2005 to March 31, 2006 in Japan. We used a discrete mixture model to explore the patterns of body mass index (BMI) trajectories. It was measured at birth and then annually until the age of 9 years. We also used a multinomial logistic regression to identify factors associated with overweight in children.

Five patterns of BMI trajectory were identified in both boys and girls. The three patterns associated with obesity or overweight in boys were being obese since the age of 5 years $(4.0 \%)$, being overweight since the age of 5 years $(20.0 \%)$, and gradually being overweight since the age of 7 years. The only pattern associated with overweight in girls was being overweight since the age of 4 years.

The results show that preschool age is a crucial period for becoming overweight in childhood. We also found that maternal working and childrenâs extracurricular activities were 
associated with overweight or obesity. However, the factors may differ by sex.

\section{5}

\section{Age at menarche in relation to digit ratio among high school girls in Kuwait}

Abdullah Al-Taiar

Faculty of Medicine, Kuwait University.

Research question: The link between age of menarche and early-life exposure to androgen as marked by digit ratio remains under intense debate. This study aimed to estimate the age of menarche among high school girls in Kuwait and to explore the association between age of menarche and both digit ratio and breastfeeding.

Methods: A cross-sectional study was conducted on 810 randomly selected female high school students in Kuwait. Data on age of menarche was collected by self-administered questionnaire; while the length of the second and fourth finger was measured using a digital caliper.

Results: Out of the 810 students selected, 50 (6.2\%) were absent or refused to participate. The mean (SD) age at menarche was 12.32 (1.21) years (95\% CI:12.23-12.41). There was no significant association between age of menarche and digit ratio in either hand before and after adjusting for potential confounders. No association was found also between age of menarche and breastfeeding in the first four months of life.

Conclusion: The estimated age of menarche among contemporary girls in Kuwait is similar to that in industrialized countries. Trends in age at menarche should be monitored because of their public health implications; and cohort studies are recommended to investigate if breastfeeding has a spinoff benefit in

\section{8}

Ethnic differences and determinants of infant BMI peak in a multi-ethnic Asian population

Izzuddin Aris ${ }^{1}$, Jonathan Bernard ${ }^{2}$, Mya Thway Tint ${ }^{3}$, Ling Wei Chen ${ }^{4}$, Wei Wei Pang ${ }^{5}$, Shu E Soh ${ }^{6}$, Seang Mei Saw ${ }^{7}$, Kenneth Kwek ${ }^{8}$, Keith Godfrey ${ }^{9}$, Peter Gluckman ${ }^{10}$, Yap Seng Chong $^{11}$, Fabian Yap ${ }^{12}$, Yung Seng Lee ${ }^{13}$

${ }^{1}$ Singapore Institute for Clinical Sciences, Agency for Science Technology and Research; ${ }^{2}$ Singapore Institute for Clinical Sciences, Agency for Science Technology and Research; ${ }^{3}$ Yong Loo Lin School of Medicine, National University of Singapore, Department of Obstetrics and Gynaecology; ${ }^{4}$ Yong Loo Lin School of Medicine, National University of Singapore, Department of Paediatrics; ${ }^{5}$ Yong Loo Lin School of Medicine, National University of Singapore, Department of Obstetrics and Gynaecology; ${ }^{6}$ Singapore Institute for Clinical Sciences, Agency for Science Technology and Research; ${ }^{7}$ Yong Loo Lin School of Medicine, National University of Singapore; ${ }^{8} \mathrm{KK}$ Womn's and
Children's Hospital; ${ }^{9}$ University of Southampton; ${ }^{10}$ University of Auckland, Liggins Institute; ${ }^{11}$ Yong Loo Lin School of Medicine, National University of Singapore, Department of Obstetrics and Gynaecology; ${ }^{12}$ KK Women's and Children's Hospital; ${ }^{13}$ Yong Loo Lin School of Medicine, National University of Singapore, Department of Paediatrics

Research question: To examine ethnic differences and determinants of infant body mass index (BMI) peak in an Asian population.

Methods: Among 1086 infants with seven measures of BMI from 3rd week to 18-months of age, AGEpeak and BMIpeak at infancy was estimated from individually-fitted BMI trajectories using mixed effect models with restricted cubic spline functions. Multivariable linear regression was used to examine the effects of ethnicity and other maternal and infant factors on AGEpeak and BMIpeak.

Results: The mean (SD) AGEpeak and BMIpeak were 6.58 $(0.12)$ months and $17.4(0.9) \mathrm{kg} / \mathrm{m} 2$ respectively. Compared to Chinese infants, Malay and Indian infants had a later AGEpeak (by 0.04 (95\% CI: 0.02, 0.07) and $0.07(0.05,0.10)$ months, respectively) $(\mathrm{p}<0.01$ for both). Compared to Chinese infants, Malay infants had a higher BMIpeak $[+0.17 \mathrm{~kg} / \mathrm{m} 2(0.03$, $0.32)]$, while Indian infants had lower BMIpeak $[-0.30 \mathrm{~kg} / \mathrm{m} 2$ $(-0.46,-0.13)](\mathrm{p}<0.05$ for both). Other determinants of AGEpeak and BMIpeak included maternal pre-pregnancy BMI, gestational weight gain, parity and infant gender. Independent of potential confounders, higher birthweight and longer breastfeeding duration were associated with a younger AGEpeak, while birthweight and length were positively associated with BMIpeak.

Conclusion: Both AGEpeak and BMIpeak are strongly associated with ethnicity and other maternal.

\section{9}

Comparing the body composition of preterm infants born Margaret Sheridan-Pereira ${ }^{1}$, Edna Roche ${ }^{2}$

${ }^{1}$ Trinity College Dublin/Coombe Women and Infants University Hospital; ${ }^{2}$ Trinity College Dublin/AMNCH, Tallaght,

Dublin 24

Research question: Preterm birth has been shown to have an adverse effect on infant body composition. Our hypothesis was that infants born less than 32 weeks would have a higher body fat percentage at term corrected age and six weeks corrected gestational age when compared with infants born between 32 and 37 weeks gestation.

Methods: Preterm infants born less than 37 weeks gestation were recruited to have body composition measurements performed by air displacement plethysmography at term and six weeks corrected gestational age.

Results: There was no difference in weight at term corrected age in infants born $<32$ weeks and those born 32-37 weeks, however infants born $<32$ weeks had higher body fat percentage $(3.12+/-0.49 \mathrm{~kg}, 17.4+/-4.7 \%$ and $3.16+/-0.64 \mathrm{~kg}$, 
$13.8+/-4.2 \%$ respectively, $\mathrm{p}<0.001, \mathrm{n}=130)$. By six weeks corrected gestational age weight and body fat percentage were similar between both groups $(4.16+/-0.70 \mathrm{~kg}, 20.8+/-4.3 \%$ and $4.68+/-0.80 \mathrm{~kg}, 21.2+/-5.2 \%, \mathrm{n}=100)$.

Conclusion: While very preterm infants had higher body fat percentage at term corrected age than late preterm infants, this was not the case at six weeks corrected gestational age. It is uncertain whether this early higher fat accretion which is reversed by six weeks corrected age has life-long effects on the very preterm infant.

\section{0}

The effect of gender on body composition in preterm infants

Margaret Sheridan-Pereira ${ }^{1}$, Edna Roche ${ }^{2}$

${ }^{1}$ Trinity College Dublin/Coombe Women and Infants University Hospital; ${ }^{2}$ Trinity College Dublin/AMNCH, Tallaght,

Dublin 24

Research question: Female infants born at term (37-42 weeks gestation) have lower birth weight and higher body fat percentage than male infants at birth and early infancy. The purpose of this study was to observe the effect of gender on the body fat percentage of preterm infants at term and six weeks corrected gestational age.

Methods: Preterm infants born less than 37 weeks gestation were recruited to have body composition measurements performed by air displacement plethysmography at term and six weeks corrected gestational age.

Results: There was no difference in weight or body fat percentage between male and female preterm infants at term corrected age $(3.19+/-0.62 \mathrm{~kg}, 14.9+/-4.7 \%$ and $3.10+/-0.54 \mathrm{~kg}$, $15.7+/-4.8 \%$ respectively, $\mathrm{n}=130)$. At six weeks corrected gestational age preterm male infants were heavier but had similar body fat percentage compared with preterm female infants $(4.80+/-0.73 \mathrm{~kg}, 21.0+/-4.6 \%$ and $4.51+/-0.76,21.1$ $+/-5.1$ respectively, $\mathrm{n}=100$ ).

Conclusion: Unlike term infants, preterm males and females have similar body fat percentage at term and six weeks corrected gestaitonal age. This supports the hypothesis that preterm birth has more of a negative impact on males compared with females.

\section{7}

Low cost body composition measurement for nutrition assessment using Near Infrared (NIR) light reflection

Angela Carberry ${ }^{1}$, Emily Bek ${ }^{2}$, Fatin Hamim Mustafa ${ }^{3}$, Peter Jones ${ }^{4}$, Alistair McEwan ${ }^{5}$, Heather Jeffery ${ }^{6}$

${ }^{1}$ University of Sydney; ${ }^{2}$ University of Sydney; ${ }^{3}$ University of Sydney; ${ }^{4}$ University of Sydney; ${ }^{5}$ University of Sydney; ${ }^{6}$ University of Sydney
Research question: How well does NIR light reflection predict body fat percentage $(\mathrm{BF} \%)$ with reference to a gold standard in term neonates.

Methods: Eligibility included well term ( $\geqslant 37$ weeks) infants born within Royal Prince Alfred (RPA) Hospital, Sydney, Australia in 2014. NIR measurements were undertaken on the skin surface on the anterior and medial thigh. BF\% measurements were undertaken by air displacement plethysmography (ADP) (gold standard).

Results: Out of the total cohort, $n=20$ neonates had valid measurements using a cosine corrector. The demographics of these neonates were; 65\% Male, Length $(\mathrm{cm})$ 50.57 2.96 , Birth weight (kg) $3.30 \pm 0.53$, Gestational age (weeks) $39.29 \pm 1.87$.

The $\mathrm{R}$ squared, Root Mean Squared Error (RMSE) and $\mathrm{p}$ values for the anterior and medial thigh measurements were: 0.884 (R square), 0.457 (RMSE) (<0.001) (p-value) (anterior thigh) and 0.892 (R square), 0.44 (RMSE) $(<0.001)$ (p-value) (medial thigh).

Conclusion: NIR light reflection anterior and medial R squared values explain 88 and $89 \%$ of variance respectively. These preliminary findings show a strong correlation between NIR in comparison with a gold standard technique (ADP) in term neonates. Further research is required in varied income settings, ethnicity and age groups (birth up 2 years).

\section{1}

Maternal determinants of differences in size and adiposity between Pakistani origin and White British origin children age $4 / 5$

Lesley Fairley ${ }^{1}$, Emily Petherick ${ }^{2}$, John Wright ${ }^{3}$, Debbie A Lawlor ${ }^{4}$

${ }^{1}$ University of Leeds; ${ }^{2}$ Bradford Institute for Health Research;

${ }^{3}$ Bradford Institute for Health Research; ${ }^{4}$ University of Bristol

Research question: Maternal pregnancy exposures could contribute to ethnic differences in childhood adiposity. Our research question was whether BMI and glucose intolerance during pregnancy are associated with size at age 4/5 in Pakistani and White British children.

Methods: Data from 938 Pakistani and 566 White British mothers and offspring from the Born in Bradford study were used. All mothers completed an oral glucose tolerance test in pregnancy. Associations of BMI, gestational diabetes (GD) and fasting/postload glucose with offspring height, weight, subscapular (SSF) and triceps skinfolds (TSF) were examined.

Results: With the exception of postload glucose, all of the maternal exposures were positively associated with height, weight and SSF, but had null or weak associations with TSF. Where there was evidence of ethnic differences, associations tended to be stronger in White British participants. For example, each $\mathrm{mmol} / \mathrm{l}$ greater fasting glucose was associated with $0.50 \mathrm{~mm}(0.07,0.94)$ and $0.12 \mathrm{~mm}(-0.20,0.45)$ greater 
SSF in White British and Pakistani respectively. Equivalent results for TSF were $0.29 \mathrm{~mm}(-0.41,0.98)$ and $0.13 \mathrm{~mm}$ $(-0.29,0.55)$.

Conclusion: Maternal adiposity and glucose influence central, more than peripheral adiposity in childhood and these associations appear stronger in White British than Pakistani children.

\section{3}

Supraclavicular Brown Adipose Tissue Composition at Birth Predicts Adiposity Gain Over the First Year of Life

Sonja Entringer ${ }^{1}$, Jerod Rasmussen ${ }^{2}$, Pathik Wadhwa ${ }^{3}$, Claudia Buss ${ }^{4}$

${ }^{1}$ Department of Medical Psychology, Charité University Medicine Berlin; ${ }^{2}$ Department of Pediatrics, University of California, Irvine; ${ }^{3}$ Department of Psychiatry and Human Behavior, University of California, Irvine; ${ }^{4}$ Department of Medical Psychology, Charité University Medicine Berlin

Objective: Brown Adipose Tissue (BAT) appears to play a beneficial role in reduced obesity/adiposity risk and improved metabolic function. Very little is known about the relationship of BAT characteristics in early life and implications for obesity/ adiposity in subsequent periods of life. We investigated if brown fat composition at birth is associated with changes in adiposity over the first year of life.

Methods: Using a Dixon MRI protocol, fat- and water-based images of $\mathrm{N}=26$ neonates were acquired within the first month of life, BAT depots identified and mean fat fraction (FF) within BAT depots was computed. A higher FF is associated with a more white-fat-like composition. Dual-energy x-ray absorptiometry (DXA) was performed at birth and one year age to quantify total body fat $(\% \mathrm{BF})$.

Results: After adjusting for infant sex and gestational age at birth, a more brown-like composition of the supraclavicular BAT depot (smaller FF) was associated with a lower increase in $\% \mathrm{BF}$ over the first year of life $(\mathrm{p}<.005, \mathrm{R}=0.60, \mathrm{CI}$ $(\mathrm{R}, 95 \%)=0.24-0.82)$.

Conclusion: These findings suggest that a more brown-like (metabolically active) BAT composition at birth is protective of obesity/adiposity risk in early life.

\section{2}

Tracking of abdominal fat mass during childhood. The Generation R Study

Suzanne Vogelezang ${ }^{1}$, Olta Gishti ${ }^{2}$, Romy Gaillard ${ }^{3}$, Eline van der Beek ${ }^{4}$, Marieke Abrahamse ${ }^{5}$, Albert Hofman ${ }^{6}$, Janine Felix ${ }^{7}$, Vincent Jaddoe ${ }^{8}$

${ }^{1}$ The Generation R Study Group, Erasmus Medical Center, Rotterdam, The Netherlands; Department of Epidemiology, Erasmus Medical Center, Rotterdam, The Netherlands;
Department of Pedi; ${ }^{2}$ The Generation R Study Group, Erasmus Medical Center, Rotterdam, The Netherlands; Department of Epidemiology, Erasmus Medical Center, Rotterdam, The Netherlands; Department of Pedi; ${ }^{3}$ The Generation R Study Group, Erasmus Medical Center, Rotterdam, The Netherlands; Department of Epidemiology, Erasmus Medical Center, Rotterdam, The Netherlands; Department of Pedi; ${ }^{4}$ Nutricia Research, Danone Nutricia Early Life Nutrition, Utrecht, the Netherlands; ${ }^{5}$ Nutricia Research, Danone Nutricia Early Life Nutrition, Utrecht, the Netherlands; ${ }^{6}$ Department of Epidemiology, Erasmus Medical Center, Rotterdam, the Netherlands; ${ }^{7}$ The Generation R Study Group, Erasmus Medical Center, Rotterdam, The Netherlands; Department of Epidemiology, Erasmus Medical Center, Rotterdam, The Netherlands; Department of Pedi; ${ }^{8}$ The Generation R Study Group, Erasmus Medical Center, Rotterdam, The Netherlands; Department of Epidemiology, Erasmus Medical Center, Rotterdam, The Netherlands; Department of Pedi

Research question: We examined the extent of tracking of abdominal fat mass during the first 6 years of life.

Methods: We performed a prospective cohort study among 393 Dutch children followed from the age of 2 years $(90 \%$ range $1.9 ; 2.3)$ to 6 years $(90 \%$ range $5.7 ; 6.2)$. At both ages, we performed abdominal ultrasound to measure subcutaneous and preperitoneal fat distances and areas.

Results: Subcutaneous abdominal fat distance and area and preperitoneal fat area at 2 years were correlated with their corresponding measures at 6 years ( $p$-values $<0.01$ ), with the strongest coefficients for subcutaneous fat measures. Children with high subcutaneous abdominal fat measures, but not high preperitoneal fat measures, at 2 years had increased risks of remaining in the upper $15 \%$ at 6 years (Odds Ratios 5.1 (95\% Confidence Interval (CI) 2.4 to 10.8$)$ and 12.8 (95\% CI 5.6 to 29.1) for subcutaneous fat distance and area, respectively).

Conclusions: Our findings suggest that abdominal fat mass tracks during childhood. Tracking between 2 and 6 years of age seems to be stronger for subcutaneous abdominal fat than for preperitoneal fat. This implies that, depending on the location of adipose tissue, there might be a differential pattern for fat depot development.

4597

Pattern of undernutrition among $\mathrm{u}-5$ and primary school children in Nigeria using anthropometic indices

Rachael Akano ${ }^{1}$, Rasaki Sanusi ${ }^{2}$

${ }^{1}$ TAi Solarin University of Education, Nigeria; ${ }^{2}$ University of Ibadan, Nigeria

Research question: Is there a difference in the prevalence of undernutrition among U-5 and Primary School Children (PSC) in Nigeria? 
Methods: A descriptive cross-sectional study was carried out among U-5 and PSC in Ijebu-Ode Local Government Area (ILGA). Validated, semi-structured and pretested intervieweradministered questionnaire was used to obtain information on demographic data of the children. Indices of malnutrition were derived from the children's height and weight measurements. U-5 children (400) and PSC (2019) were randomly selected from 4 immunization centers and 13 public primary schools respectively. Nutritional status of respondents was determined using WHO Anthro version 3.2. and WHO Anthro plus electronic softwares. Data were analysed using descriptive statistics, Chi square test and ANOVA at $\mathrm{p}=0.05$.

Results: Age of respondents was 3.4 \pm 1.2 years and $9.4 \pm 1.3 y$ years for U-5 and PSC respectively. Prevalence of wasting and underweight were higher $(8.6 \%$ and 19.0$)$ among PSC than the U-5 (6.0\% and 7.8\%) respectively, however, for stunting, the prevalence was higher among U-5 (18.5\%) than PSC (16.3\%). There was a statistically significant difference between prevalence undernutrition among U-5 and PSC $(\mathrm{p}<0.05)$.

Conclusions: In this study, undernutrition is prevalent among U-5 and primary school children. Nutrition interventions are urgently required to reduce its prevalence among Nigerian children.

\section{5}

More evidence for 'Thin-fat' Asian babies from a high risk UK population: Preliminary results from the PRiDE pregnancy cohort

Hema Venkataraman ${ }^{1}$, Amina Dawoodji ${ }^{2}$, Samina Zaheer ${ }^{3}$, Nithya Sukumar ${ }^{4}$

${ }^{1}$ University of Warwick; ${ }^{2}$ University of Warwick; ${ }^{3}$ University of Warwick; ${ }^{4}$ University of Warwick

Research question: What are the ethnic differences in neonatal anthropometry in women with high risk of GDM?

Methods: Maternal and offspring data were collected from women recruited in the ongoing, multicentre, prospective PRiDE study. The participants were women at high risk of GDM based on selective screening criteria. Offspring anthropometry (measured within 72-hours of delivery) was compared between South Asians (SA) and White Caucasians (WC). Ethnic specific BMI thresholds were used to define obesity.

Results: Of 855 deliveries from May 2013 to Mar 2015, detailed anthropometric data was available in 567 women. $14.2 \%$ were SA and $71.1 \%$ were WC. $18.6 \% \& 61.4 \%$ of SA and $65.4 \% \& 77.0 \%$ of WC were obese and overweight respectively $(\mathrm{p}<0.007)$. SA had overall lower birth weights [median(IQR): 3072.5(2710-3380)g vs. 3497.5(3088-3830) g], abdominal [32,(29.5-33) vs 33.5, IQR $(31.5-34.9) \mathrm{cm}$, mid-arm $[10.5(9.8-11.5)$ vs $11.05(10.2-12) \mathrm{cm}]$, chest [33(31-34) vs $34.2(32.9-35.6) \mathrm{cm}]$ and head circumferences [24(34-25) vs $35(33.5-36) \mathrm{cm}$ ] but higher HC/AC ratio (1.09 (1.04-1.13) vs 1.04(1.0-1.1)], ( $<<0.02$ for all). However, the length, triceps and subscapular skin-fold thickness was similar to WC women. AC and $\mathrm{HC}$ remained lower in SA despite adjustments for gestational age, offspring sex, maternal age, height, BMI and smoking status.

Conclusion: SA offspring had lower overall body size but similar subcutaneous fat measures when compared to offspring of more obese/overweight WC mothers. This "thin-fat" SA body composition with higher $\mathrm{HC} / \mathrm{AC}$ ratios and higher subcutaneous adiposity may signify an intra-uterine insult.

\section{0}

Late prematurity and adiposity in adolescents: evidence from "Children of 1997" Birth Cohort

Connie Hui ${ }^{1}$, H. Simon Lam ${ }^{2}$, Gabriel M. Leung ${ }^{3}$,

C. Mary Schooling ${ }^{4}$

${ }^{1}$ University of Hong Kong; ${ }^{2}$ The Chinese University of Hong Kong; ${ }^{3}$ University of Hong Kong; ${ }^{4}$ University of Hong Kong

Research question: To assess the association of late premature birth with adiposity at 14 years and to test whether any associations were mediated by infant weight growth.

Method: In the Chinese birth cohort "Children of 1997", we used linear regression to assess the association of late premature (34-36 gestational weeks, $n=295,85 \%$ follow-up) compared to term (37 to 42 gestational weeks, $\mathrm{n}=6874,88 \%$ follow-up) birth with BMI, waist-hip ratio (WHR) and waist-height ratio (WHtR) at 14 years adjusted for sex, socio-economic position, mother's place of birth, maternal smoking during pregnancy. We also assessed the mediation by accelerated infant growth (change in weight $z$-score of $\geqslant 0.67$ at $0-12$ months).

Findings: Late premature birth had little association with early growth faltering or socio-economic position. Late premature births had greater z-score in BMI $(0.21,95 \%$ CI $0.07,0.35)$, WHR $(0.16,95 \%$ CI $0.03,0.29)$ and WHtR $(0.27,95 \%$ CI $0.14,0.40)$ in adolescence. Only the association of late prematurity with higher BMI was mediated by accelerated infant growth.

Conclusions: Late premature birth was associated with greater adolescent adiposity, but only the association with BMI was mediated by infant growth, suggesting vulnerability to metabolic risk in late premature births may arise through multiple pathways.

\section{3}

Associations of infant subcutaneous fat mass with total and abdominal fat mass at school-age. The Generation R Study

Susana Santos ${ }^{1}$, Romy Gaillard ${ }^{2}$, Andreia Oliveira ${ }^{3}$, Henrique Barros $^{4}$, Marieke Abrahamse-Berkeveld ${ }^{5}$, Eline $M$ van der Beek $^{6}$, Albert Hofman ${ }^{7}$, Vincent WV Jaddoe ${ }^{8}$

${ }^{1}$ EPIUnit, Institute of Public Health, University of Porto, Porto, Portugal; The Generation R Study Group, Erasmus MC, 
University Medical Center, Rotterdam, the Netherlands; ${ }^{2}$ The Generation R Study Group, Erasmus MC, University Medical Center, Rotterdam, The Netherlands; Department of Pediatrics, Erasmus MC, University Medical Center, Rotterdam, The Net; ${ }^{3}$ EPIUnit, Institute of Public Health, University of Porto, Porto, Portugal; Department of Clinical Epidemiology, Predictive Medicine and Public Health, University of Porto Medical S; ${ }^{4}$ EPIUnit, Institute of Public Health, University of Porto, Porto, Portugal; Department of Clinical Epidemiology, Predictive Medicine and Public Health, University of Porto Medical S; ${ }^{5}$ Nutricia Research, Danone Nutricia Early Life Nutrition, Utrecht, the Netherlands; ${ }^{6}$ Nutricia Research, Danone Nutricia Early Life Nutrition, Utrecht, the Netherlands: ${ }^{7}$ Department of Epidemiology, Erasmus MC, University Medical Center, Rotterdam, the Netherlands; ${ }^{8}$ The Generation R Study Group, Erasmus MC, University Medical Center, Rotterdam, The Netherlands; Department of Pediatrics, Erasmus MC, University Medical Center, Rotterdam, The Netherlands

Research question: To examine the associations of infant subcutaneous fat with total and abdominal fat at school-age. Methods: In a population-based cohort study among 821 children, we measured biceps, triceps, suprailiacal and subscapular skinfold thicknesses and estimated total subcutaneous fat and central-to-total subcutaneous fat ratio at 1.5 and 24 months. At 6 years, we measured fat mass index, central-to-total fat ratio and android-to-gynoid fat ratio by dual-energy X-ray absorptiometry and preperitoneal fat area by abdominal ultrasound.

Results: Central-to-total subcutaneous fat ratio at 1.5 months was positively associated with fat mass index and central-tototal fat ratio at 6 years, whereas both total and central-to-total subcutaneous fat ratio at 24 months were positively associated with all childhood adiposity measures $(\mathrm{p}<0.05)$. Increased risk of childhood overweight was observed for 1-standard-deviation scores (SDS) higher body mass index (BMI) at 1.5 months and for 1-SDS higher BMI (Odds Ratio (OR) 2.76, 95\% Confidence Interval (CI) 2.07,3.69) and total subcutaneous fat at 24 months (OR 1.70, 95\% CI 1.36,2.12). Stronger associations were observed among girls than boys.

Conclusion: Infant subcutaneous fat is associated with total and abdominal fat at school-age. Further studies are needed to assess the long-term cardiovascular consequences of infant body fat.

\section{3}

Early childhood growth in term low birth weight Indian newborns: Follow-up of Delhi Infant Vitamin D Supplementation Study, India

Manpreet Kaur ${ }^{1}$, Geeta Trilok Kumar ${ }^{2}$, Sikha Sinha ${ }^{3}$, Suzanne Filteau ${ }^{4}$, Clive Osmond ${ }^{5}$, Harshpal Singh Sachdev ${ }^{6}$

${ }^{1}$ Institute of Home Economics, University of Delhi, Delhi, India;

${ }^{2}$ Institute of Home Economics, University of Delhi, Delhi, India;

${ }^{3}$ Sitaram Bhartia Institute of Science and Research, New Delhi,

India; ${ }^{4}$ London School of Hygiene and Tropical Medicine,
London, UK; ${ }^{5}$ MRC Lifecourse Epidemiology Unit, University of Southampton, UK; ${ }^{6}$ Sitaram Bhartia Institute of Science and Research, New Delhi, India

Research question: What is the subsequent growth trajectory of term low birth weight (LBW) Indian newborns adhering to prescribed nutrition and health care recommendations?

Methods: 2079 term LBW newborns from an urban poor setting were followed-up for growth from 0 to 26 weeks $(n=1282)$ and at 3-7 years $(n=912)$. Using Cole's LMS approach, age and sex specific internal Z-scores were computed and subsequently adjusted for the effect of a vitamin $\mathrm{D}$ intervention and potential bias due to attrition. Back-transformed measurements were then used to compute WHO Z-scores for height-for-age (HAZ), weight-for-age (WAZ), and BMI-for-age (BMIZ).

Results: HAZ remained virtually stable; mean change from birth till 6 weeks, 26 weeks and 3-7 years was 0.07, 0.04 and $0.2 \mathrm{SD}$, respectively. BMIZ and WAZ showed considerable catch-up; 0.69, 1.84 and 1.38 SD for BMIZ and 0.25, 0.89 and $0.60 \mathrm{SD}$ for WAZ, respectively. Maximal catch-up occurred at 26 weeks with some growth faltering thereafter.

Conclusion: Term LBW infants reared under adverse socio-economic conditions in childhood show substantial catch-up growth in BMIZ and WAZ but not in HAZ. The potential impact of this excess weight over length gain in later life, especially for cardio-metabolic risk factors and human capital development, needs urgent evaluation.

\section{0}

The long-term effects of childhood overweight and obesity on bone health in later life

Hongbo Dong ${ }^{1}$, Dongqing $\mathrm{Hou}^{2}$, Yinkun Yan ${ }^{3}$, Junting Liu ${ }^{4}$, Hong Cheng, Jie $\mathrm{Mi}^{6}$

${ }^{1}$ Peking Union Medical College; Capital Institute of Pediatrics;

${ }^{2}$ Capital Institute of Pediatrics; ${ }^{3}$ Peking Union Medical College; Capital Institute of Pediatrics; ${ }^{4}$ Capital Institute of Pediatrics;

${ }^{5}$ Capital Institute of Pediatrics; ${ }^{6}$ Capital Institute of Pediatrics

Background/Objectives: To investigate the long-term association between childhood obesity and adulthood bone mineral density (BMD).

Methods: We used the "Beijing BP Cohort Study", where 1259 subjects aged 6-18 years old were followed over 24 years from childhood (1987) to early or middle adulthood (2011). Anthropometric measures were obtained at baseline and follow-up examinations. Childhood overweight and obesity was defined using area under the curve (AUC) of body mass index (BMI) computed from longitudinal growth curve models. Bone mineral density (BMD, $\mathrm{g} / \mathrm{cm} 2$ ) of the whole body, hip and lumbar spine were measured by dual-energy $\mathrm{X}$-ray absorptiometry to assess bone health in early or middle adulthood.

Results: There was a statistically positive association between AUC of childhood BMI and adult BMD of whole body, hip 
and lumbar spine (coefficient after adult age and sex adjustment, 0.16 to 0.29 , all $\mathrm{p}$ values $<0.01$ ). Compared to those with normal BMI, overweight children had higher adult BMD in both sexes. In additional regression models, childhood overweight other than childhood obesity predicted independently later-life all three BMD parameters after controlling for adult risk factors.

Conclusions: Our findings suggest that childhood overweight may exert positive effects on BMD in the later adulthood.

\section{8}

\section{Nutritional Status and Growth of Infants in a South African Birth Cohort Study}

Shrish Budree ${ }^{1}$, Elizabeth Goddard ${ }^{2}$, Kirsty Brittain ${ }^{3}$, Whitney Barnett ${ }^{4}$, Landon Myer ${ }^{5}$, Heather Zar ${ }^{6}$

${ }^{1}$ Department of Paediatrics and Child Health, Red Cross War Memorial Children's Hospital and MRC Unit on Child and Adolescent Health, University of Cape Town, South Africa; ${ }^{2}$ Department of Paediatrics and Child Health, Red Cross War Memorial Children's Hospital and MRC Unit on Child and Adolescent Health, University of Cape Town, South Africa; ${ }^{3}$ Department of Paediatrics and Child Health, Red Cross War Memorial Children's Hospital and MRC Unit on Child and Adolescent Health, University of Cape Town, South Africa; ${ }^{4}$ Department of Paediatrics and Child Health, Red Cross War Memorial Children's Hospital and MRC Unit on Child and Adolescent Health, University of Cape Town, South Africa; ${ }^{5}$ Division of Epidemiology and Biostatistics, School of Public Health and Family Medicine, University of Cape Town, Cape Town, South Africa; ${ }^{6}$ Department of Paediatrics and Child Health, Red Cross War Memorial Children's Hospital and MRC Unit on Child and Adolescent Health, University of Cape Town, South Africa

Early infant growth is associated with later development of non-communicable diseases. Few longitudinal studies have investigated patterns of infant growth in low/middle-income countries. We aimed to investigate infant growth in the Drakenstein Child Health Study, a South African birth cohort. Methods: Peri-urban mothers were enrolled antenatally and mother-infant dyads followed until 12 months of age. Growth was analysed as z-scores for weight and height. Linear mixed effects models were used to investigate predictors of birth weight and infant growth.

Results: Anthropometric data of 812 infants, 50\% Black African $(\mathrm{n}=406)$ and $49 \%$ mixed race $(\mathrm{n}=395)$ were included. Black African infants had higher birth weight vs. mixed race infants (Weight-for-age $z$-score: -0.4 vs. -0.7 , $\mathrm{p}<0.001)$ and remained heavier and taller throughout infancy. Stunting was prevalent $[18 \%$ at 2 months and $12 \%$ at 12 months]. Maternal height, smoking, ethnicity and socioeconomic status (SES) were significant independent predictors of birth weight. Ethnicity, SES and birth weight were significant independent predictors of growth during the first year.
Conclusion: Birth weight is an important predictor of growth trajectory during infancy. Birth weight and infant growth are influenced by several modifiable factors.

Acknowledgements: Harry-Crossley Foundation; SA Thoracic Society; Bill and Melinda Gates Foundation (OPP1017641);MRC/NRF.

\section{8}

Long-term effects of internal displacement during early life on adolescent growth and final height in post-conflict northern Uganda

Stine Schramm ${ }^{1}$, Henrik Ravn ${ }^{2}$, Marianne Skytte Jakobsen ${ }^{3}$, Morten Sodemann ${ }^{4}$

${ }^{1}$ Centre for Global Health, University of Southern Denmark;

${ }^{2}$ Research Centre for Vitamins and Vitamins, Statens Serum

Institute, Denmark; ${ }^{3}$ Department of Paediatrics, Centre

Lillebaelt, Denmark; ${ }^{4}$ University of Southern Denmark, Centre for Global Health, Denmark

Research question: What are the long-term, including sexdifferential-, effects of being internally displaced during early life on adolescent growth and final height?

Method: A longitudinal survey within the Gulu Health and Demographic Surveillance System (HDSS) is currently being carried out (December 2014 - April 2017). At baseline, all residents within selected areas of Gulu HDSS aged 15-24 years were eligible to participate. Trained assistants conducted anthropometric measurements (height and knee-to-heel length) and administered questionnaires with information on frequency, timing and duration of having been displaced, socioeconomic and -demographic information, and pubertal stage. Three follow-up visits every six months will be conducted.

Preliminary Results: At baseline, 1,217 participants (54.7\% male) were interviewed and measured. The prevalence of short stature was $9.3 \%$ male and $1.1 \%$ for female.

Displacement characteristics: $87 \%$ had been displaced, ranging from 0 - 5 times, $14.5 \%$ born in displacement. Median years of displacement was 7.3 [4.9-9.4], and median age at first displacement was 3.8 years [1.2-6.1]. Further analysis is in process.

Conclusion: The study will provide unique growth monitoring data beyond childhood, and build a foundation to study early life exposures, growth, height, nutrition and chronic diseases in later life in low-income settings.

\section{6}

Prevalence of overweight and obesity among primary school children in a developing country: NW-CHILD longitudinal data of 6-9-yr-old children in South Africa

Anita Pienaar

${ }^{1}$ School for Biokinetics, Recreation and Sport Sciences Northwest University, Potchefstroom Campus 
Research question: Is changes in overweight and obesity in pre pubertal children living in the NW Province of South Africa similar to the widespread trends of increasing child obesity that are reported for developing countries?

Methods: International age adjusted BMI cut-off points were used to determine overweight and obesity, 3-years apart in children of 20 schools, across 5 school SES levels (quintiles), in 4 educational districts ( $\mathrm{N}=574,282$ boys, 292 girls; 407 black, 143 white). Mixed models were used to analyse the effects of sex, ethnicity and socio-economic status (SES) of the school.

Results: A 4\% increase was found with significant increases in whites, blacks, boys and girls. SES effects were significant where children in schools associated with higher SES, had the highest rate of increase and the highest prevalence of obesity.

Conclusions: Obesity prevalence rose significantly from 6-9-years while obesity, compared to overweight, increased more. The rate of increase differed markedly in different sexes, ethnic groups and SES, masking the extent of the problem. Shifting towards an unhealthy BMI was more common than obtaining a healthier BMI over the 3-year period. Early prevention strategies are needed based on the trends that were found, with special attention to white children living in high SES regions, and black children in economic transition.

\section{0}

Maternal Serum Adiponectin During Pregnancy is Associated with Infant's Adiposity in The Universiti Sains Malaysia Pregnancy Cohort Study

Hamid Jan Jan Mohamed ${ }^{1}$, Marhazlina Mohamed ${ }^{2}$, See Ling Loy ${ }^{3}$, Yu Wang ${ }^{4}$

${ }^{1}$ Universiti Sains Malaysia; ${ }^{2}$ Universiti Sains Malaysia; ${ }^{3} \mathrm{KK}$ Women's and Children's Hospital; ${ }^{4}$ University of Hong Kong

This study aimed to investigate the association of maternal total serum adiponectin with infant adiposity. A total of 155 Malay women aged 19 to 40 years old were randomly recruited into the Universiti Sains Malaysia Pregnancy Cohort Study from April 2010 to December 2012. Fasting blood samples were drawn at second and third trimester of pregnancy. Total serum adiponectin was determined using enzyme-linked immunoassay (ELISA) method. Infant adiposity indicators such as body weight, body mass index-for-age (BAZ), abdominal circumference and triceps skinfold were measured at birth and at 12 months of age. Results showed median total serum adiponectin at second and third trimester were $7.06 \mu \mathrm{mol} / \mathrm{L}$ $(\mathrm{IQR}=4.35-10.91)$ and $6.98 \mu \mathrm{mol} / \mathrm{L}(\mathrm{IQR}=4.78-9.12)$, respectively. Multivariate regression analyses showed maternal total serum adiponectin at second trimester were negatively associated with infant BAZ at birth $(\beta=-0.87, p=0.006)$. There were significant negative associations between maternal total serum adiponectin at third trimester with infant body weight $(\beta=-0.47, p=0.004)$ and abdominal circumference $(\beta=-2.52, p=0.009)$ at birth, and infant body weight $(\beta=-1.02, p=0.009)$ and $\operatorname{BAZ}(\beta=-1.00, p=0.018)$ at 12 months of age. In conclusion, these findings indicate the possible role of maternal total serum adiponectin in regulating adiposity in the first year of life of infants.

\section{3}

Preliminary study of infant growth in 'SARAS KIDs', follow up of Project SARAS, a food based intervention before and during pregnancy

Caroline Fall ${ }^{1}$, Sirazul Sahariah ${ }^{2}$, Meera Gandhi ${ }^{3}$, Harsha Chopra ${ }^{4}$, Harshad Sane ${ }^{5}$, Monika Dayama ${ }^{6}$, Nick Brown ${ }^{7}$, Barrie Margetts ${ }^{8}$

${ }^{1}$ MRC Lifecourse Epidemiology Unit, University of Southampton, $U K ;{ }^{2}$ Centre for the Study of Social Change; ${ }^{3}$ Centre for the Study of Social Change; ${ }^{4}$ Centre for the Study of Social Change; ${ }^{5}$ Centre for the Study of Social Change; ${ }^{6}$ Centre for the Study of Social Change; ${ }^{7}$ Salisbury District Hospital, UK; ${ }^{8}$ Public Health Nutrition, University of Southampton, $U K$

Aim: To study infant anthropometry in "SARAS KIDS $(n=1024)$, follow up study of Project Saras [a food based intervention trial in slum women, 3 months before and during pregnancy (Jan 2006 to June 2012) for infant weight gain of $100 \mathrm{gm}$ ]. Methodology: Anthropometric data collected during "SARAS KIDS" Project was analysed. Internal Z-scores were calculated at 1, 3, 6 and 12 months accounting for the wide age range.

Results: 1 . At one month, the treatment group children have larger weight, chest, abdomen and mid upper arm circumference than the control group children by approximately 0.2 Z-scores.

2. By three months, they are still bigger in weight, head, chest, abdomen and mid upper arm circumference in the per protocol analysis.

3. By 6 and 12 months the differences in size disappears between treatment and controls.

Conclusion: Project SARAS, food based intervention has an effect on infant growth up to 3 months post-partum. This means that infants in the treatment group are at an advantage during critical periods of brain, heart, and visceral development.

Follow-up of the children at 6 years which is under way will determine the functional benefits of this advantage on body composition, cognition and cardio metabolic parameters.

\section{4}

Maternal dietary practices and anthropometric status of infants aged 0-6 weeks in Makuya village, Thulamela municipality, Vhembe district of Limpopo province

Zwidofhelangani Matsea ${ }^{1}$, Tjale Mahopo ${ }^{2}$

${ }^{1}$ University of venda; ${ }^{2}$ University of Venda 
Research question: What are the maternal dietary practices and anthropometric status of infants aged 0-6 weeks in Makuya village, Thulamela municipality, Vhembe district of Limpopo province.

Methods: Descriptive study design and quantitative methods were used. The study was conducted at Makuya clinic in Vhembe district. Sixty (60) participants participated in the study. Statistical Package of Social Science (SPSS), version 22, was used to analyse the data.

Results: The age of the mothers ranged from 15 to 41 years. Nearly two thirds of mothers (63.3\%) were aged 20 to 30 years, and only $15 \%$ were 19 years and under. About $13.3 \%$ of participants during pregnancy were underweight, $30 \%$ were overweight and $6.7 \%$ were obese. More than third quarter of infants $(80 \%)$ at birth had normal WAZ $(-1 S D$ to $\leqslant+2 S D)$, while $6.7 \%$ of infants were underweight $(<-3$ SD to $<-2 S D)$. About $8.3 \%$ of infants at birth were stunted $(<-3$ SD to $<-2 \mathrm{SD})$. The majority of participants $(91.7 \%)$ received folate and iron supplements, while $8.3 \%$ received iron, folate and calcium supplements during pregnancy.

Conclusion: Underweight, wasting and stunting were found prevalent in Makuya immediately after birth. However, Stunting was more prevalent than wasting and underweight.

\section{4}

A maternal 'junk food' diet alters mu-opioid receptor mRNA expression in late but not early postnatal development in female offspring

Jessica Gugusheff ${ }^{1}$, Iain Clarke ${ }^{2}$, Zhi Yi Ong ${ }^{3}$

${ }^{1}$ The University of Adelaide; ${ }^{2}$ Monash University; ${ }^{3}$ University of Pennsylvania

Background: Perinatal junk food exposure reduces the sensitivity of opioid signalling and increases the preference for palatable diets in the offspring. This study aimed to further explore the mechanisms underlying this desensitisation of the opioid pathway, by determining when in development changes in mu-opioid receptor $(\mathrm{MuRc})$ expression occur.

Methods: 10 Albino Wistar rats were provided with either a junk food $(n=5)$, or standard diet $(n=5)$, during pregnancy and lactation. Brain tissue was collected from offspring at birth and postnatal weeks 1,3 and 4 and in situ hybridisation used to determine MuRc expression in the nucleus accumbens (NAc) and ventral tegmental area (VTA).

Results: The number of cells expressing MuRc mRNA in the VTA of female JF offspring was 32\% (week 3) and 57\% (week 4) lower than Controls $(\mathrm{P}<0.05)$. There were no differences at earlier time points or in males. The number of MuRc-positive cells in the NAc was lower $(\mathrm{P}<0.05)$ during weeks 1-2 than in weeks 3-4, independent of maternal diet.

Conclusions: These results suggest that programming of the reward pathway by maternal junk food occurs later in development, and provides critical insights into the potential window of opportunity during which to intervene to prevent or reverse these effects.
4403

Effect of maternal high-fat diet on cardiac function of male and female juvenile offspring

Suelen Guedes Zeca ${ }^{1}$, Taina Moraes de Oliveira ${ }^{2}$, Natalia D’Assupção Lima Rangel ${ }^{3}$, Isalira Peroba Ramos ${ }^{4}$, Carmem Cabanelas Pazos-Moura ${ }^{5}$, Isis Hara Trevenzoli ${ }^{6}$, Norma Aparecida dos Santos Almeida ${ }^{6}$

${ }^{1}$ Federal Rural University of Rio de Janeiro; ${ }^{2}$ Federal Rural University of Rio de Janeiro; ${ }^{3}$ Federal Rural University of Rio de Janeiro; ${ }^{4}$ Federal University of Rio de Janeiro; ${ }^{5}$ Federal University of Rio de Janeiro; ${ }^{6}$ Federal University of Rio de Janeiro; ${ }^{7}$ Federal Rural University of Rio de Janeiro

Research question: Maternal high-fat diet (HFD) induces male offspring obesity. At weaning, the offspring had excess thyroid hormone $(\mathrm{TH})$ and higher adrenal catecholamine content, catecholamines and TH may impact cardiovascular function. This study investigated if HFD alters the cardiac expression of $\beta 1-A R$ and $T R \alpha 1$ receptors and the cardiac function in juvenile offspring in a sex dependent manner.

Methods: Female Wistar rats received a standard diet (C; $9 \%$ of fat) or a high-fat diet (HF; $28 \%$ of fat) during perinatal period. At weaning, the cardiac expression of $\beta 1-\mathrm{AR}$ and TR $\alpha 1$ receptors were measured by Western blot. The cardiac function was analyzed by echocardiography at 30th day, being $\mathrm{p}<0.05$ statistically different. Results: At weaning, the cardiac expression of the $\beta 1$-AR was higher in HF female offspring (+93\%) while TR $\alpha 1$ expression was increased in HF male ( +1.85 fold $)$ vs control offspring. At 30 days, the HF female offspring showed impaired systolic function $(-9 \%$ ejection fraction and $+33 \%$ end-systolic volume), and HF male offspring had lower ejection fraction $(-5 \%)$ and higher ventricle mass $(+10 \%)$.

Conclusion: Maternal HFD induced early changes in cardiac function independent of the sex of the offspring, but male and female seems to have different mechanisms for the establishment of such dysfunction.

\section{6}

Low-protein diet during last third of gestation malprograms HPA-axis leading to impaired insulin secretion in adult rat offspring

Júlio Cezar de Oliveira ${ }^{1}$, Patrícia Cristina Lisboa ${ }^{2}$,

Rodrigo Mello Gomes ${ }^{3}$, Rosiane Aparecida Miranda ${ }^{4}$, Luiz

Felipe Barella ${ }^{5}$, Isabela Peixoto Martins ${ }^{6}$, Vander Alves Silva ${ }^{7}$, Flavio Andrade Francisco ${ }^{8}$, Egberto Gaspar de Moura ${ }^{9}$,

Paulo Cezar de Freitas Mathias ${ }^{10}$

${ }^{1}$ Health Sciences Institute, Federal University of Mato Grosso, Sinop, MT, Brazil; Laboratory of Secretion Cell Biology, Department of Biotechnology, Cell Biology and Genetics, State;

${ }^{2}$ Department of Physiological Sciences, Roberto Alcântara Gomes Biology Institute, State University of Rio de Janeiro, Rio de Janeiro, RJ, Brazil; ${ }^{3}$ Department of Physiological Sciences, State 
University of Maringa, Maringá, PR, Brazil; ${ }^{4}$ Laboratory of Secretion Cell Biology, Department of Biotechnology, Cell Biology and Genetics, State University of Maringa, Maringá, PR, Brazil; ${ }^{5}$ Laboratory of Secretion Cell Biology, Department of Biotechnology, Cell Biology and Genetics, State University of Maringa, Maringá, PR, Brazil and Molecular Signaling Section, Labo; ${ }^{6}$ Laboratory of Secretion Cell Biology, Department of Biotechnology, Cell Biology and Genetics, State University of Maringa, Maringá, PR, Brazil; ${ }^{7}$ Laboratory of Secretion Cell Biology, Department of Biotechnology, Cell Biology and Genetics, State University of Maringa, Maringá, PR, Brazil; ${ }^{8}$ Laboratory of Secretion Cell Biology, Department of Biotechnology, Cell Biology and Genetics, State University of Maringa, Maringá, PR, Brazil; ${ }^{9}$ Department of Physiological Sciences, Roberto Alcântara Gomes Biology Institute, State University of Rio de Janeiro, Rio de Janeiro, RJ, Brazil; ${ }^{10}$ Laboratory of Secretion Cell Biology, Department of Biotechnology, Cell Biology and Genetics, State University of Maringa, Maringá, PR, Brazil

Research question: Low birthweight has been implicated in glucocorticoid dyshomeostasis inducing metabolic syndrome. We aimed to evaluate the effect of low-protein diet offered to pregnant dams during the last third of gestation on offspring metabolism and pancreatic islet function.

Methods: Virgin female Wistar rats aged 70-days-old were mated and the vaginal smear used to assess conception by the presence of spermatozoa. At the last third of pregnancy, dams were fed a protein-restricted diet (4\%, IUPR) until delivery. Control dams were fed a normal-protein diet (23\%, Control). The offspring was weighed at birth and throughout experimental period. At 90-day-old, the offspring was sacrificed and pancreatic islets isolated to study insulin secretion under different glucose and acetylcholine concentrations. Fasting glucose, insulin, adiponectin, leptin, corticosterone and adrenocorticotropic hormone (ACTH) were evaluated as well as the lipid profile. Data were analyzed by Student's t-test.

Results: At 90-day-old, IUPR rats were heavier, dyslipdemic and displayed hyperglycemia, hyperinsulinemia, hyperleptinemia and hypercorticosteronemia $(\mathrm{p}<0.01)$. In addition, adiponectin and $\mathrm{ACTH}$ were reduced and the insulinotropic action of glucose and acetylcholine was impaired in IUPR rats $(\mathrm{p}<0.05)$.

Conclusion: Maternal undernutrition held only during the last third of gestation disrupts pancreatic islets insulinotropic response and induces obesity-correlated complications as longterm consequences.

\section{0}

Maternal Supplementation with a Soluble Fibre (Polydextrose) Prevents the Adverse Effects of Maternal Obesity on Offspring Energy Expenditure in Mice

Xanthi Maragkoudaki ${ }^{1}$, Matthew Naylor ${ }^{2}$, Joaquim Pombo ${ }^{3}$, Lucilla Poston ${ }^{4}$, Paul Taylor ${ }^{5}$

${ }^{1}$ Division of Women's Health \& Women's Health Academic Centre, Faculty of Life Sciences \& Medicine, King's College London;

${ }^{2}$ Division of Women's Health \& Women's Health Academic Centre,
Faculty of Life Sciences \& Medicine, King's College London; ${ }^{3}$ Division of Women's Health \& Women's Health Academic Centre, Faculty of Life Sciences \& Medicine, King's College London; ${ }^{4}$ Division of Women's Health \& Women's Health Academic Centre, Faculty of Life Sciences \& Medicine, King's College London;

${ }^{5}$ Division of Women's Health \& Women's Health Academic Centre, Faculty of Life Sciences \& Medicine, King's College London

Research question: We hypothesised that maternal dietinduced-obesity has adverse consequences for offspring energy balance and susceptibility to obesity in adulthood, which is prevented by maternal intervention with polydextrose (PDX). Methods: Female mice were fed a control (Con), or obesogenic diet $(\mathrm{Ob})$ or Ob supplemented with 5\% PDX (ObPDX) 6 weeks before mating and throughout pregnancy and lactation. Offspring were weaned onto control diet. At 3 and 6 months energy intake (EI) and energy expenditure (EE) were measured by indirect calorimetry, and glucose-tolerance-tests performed. Offspring of control (OffCon) obese (OffOb) and supplemented (OffObPDX) dams were also challenged for 3-weeks with obesogenic diet.

Results: At 6 months, OffOb were obese, with increased white adipose tissue mass $(\mathrm{P}<0.001)$, had decreased brown adipose tissue (BAT, $\mathrm{P}<0.01)$ and lower $\mathrm{EE}(\mathrm{P}<0.001)$ and impaired glucose metabolism $(\mathrm{P}<0.05)$ versus OffCon. The obesogenic dietary challenge resulted in greater $\mathrm{EI}$, reduced $\mathrm{EE}$ and accelerated weight gain in $\mathrm{OffOb}$ versus controls $(\mathrm{P}<0.05)$. These were prevented by Maternal supplementation with PDX. Conclusions: Maternal obesity adversely influences energy balance and risk of obesity in offspring obesity, which is prevented by maternal dietary intervention with soluble fibre. Associated changes in gut microbiota composition and mitochondrial function are implicated.

\section{4}

Coconut Water improves Reproductive Indices in Adult Offspring of High Fat Diet Fed Dams

Opeyemi Akindele ${ }^{1}$, Yinusa Raji ${ }^{2}$

${ }^{1}$ University of Ibadan; ${ }^{2}$ University of Ibadan

Research Question: Coconut water (CW) is hypolipidemic in the presence of a high fat diet (HFD). HFD consumption during pregnancy adversely affects reproductive indices of offspring. This study explored the effects of CW and HFD during gestation on some reproductive indices in offspring of Wistar rats.

Methods: Twenty-four pregnant rats were assigned into four groups namely; control, CW ( $1 \mathrm{ml} / 100 \mathrm{~g} /$ day CW), HFD (30\% butter in feed ad libitum) and CW+HFD. Treatments were administered from gestation day (GD) 1-21 after which dams were allowed to litter naturally. The anogenital distance index (AGDi) of the pups was calculated within 24 hours after parturition and at 4 months postnatal life. Onset of puberty was monitored. Sperm characteristics were analysed at 4 months postnatal life. Data were expressed as mean \pm SEM 
and compared using ANOVA followed by post hoc analysis. $p \leqslant 0.05$ was considered statistically significant.

Results: AGDi was increased in CW+HFD pups at 4 months. Testis descent was delayed in HFD compared to CW+HFD. $\mathrm{CW}$ reduced the HFD-induced delay in preputial separation and vaginal opening. Sperm count was reduced in HFD.

Conclusion: The results suggest that coconut water administration during pregnancy can protect reproductive functions of offspring from the adverse programming effect of a high fat diet.

\section{6}

Later pregnancy appetite, dietary intake, and small-forgestational-age

Fuying Tian ${ }^{1}$, Xiaozhong $\mathrm{Wen}^{2}$, Chuanbo Xie ${ }^{3}$, Jianmiao $\mathrm{Lin}^{4}$, Shixin Yuan ${ }^{5}$, Xiaoling Guo ${ }^{6}$, Weiqing $\mathrm{Chen}^{7}$

${ }^{1}$ Sun Yat-Sen University; ${ }^{2}$ State University of New York at Buffalo; ${ }^{3}$ Sun Yat-Sen University; ${ }^{4}$ Shenzhen Women and Children's Hospital; ${ }^{5}$ Shenzhen Women and Children's Hospital; ${ }^{6}$ Foshan Women and Children's Hospital; ${ }^{7}$ Sun Yat-Sen University

Research question: To investigate the effect of appetite after 20 gestational weeks and diet on risk of small-for-gestationalage (SGA).

Methods: We investigated 3,525 pregnant women in Guangdong, China, 2009-2010. The women retrospectively self-reported appetite (bad, average, and good) after 20 gestational weeks, and diet during pregnancy. We defined SGA referring to the standard of Asian newborns. We fitted Logistic regression model for the associations of appetite and diet with SGA, adjusting for demographic characteristics, pre-pregnancy body mass index, and physical activity during pregnancy.

Results: Pregnant women with average (odds ratio, 0.71 [95\% confidence interval, 0.51 to 0.98$]$ ) or good appetite (0.42 [0.30 to 0.60$]$ ) after 20 gestational weeks had lower risk for SGA than those with bad appetite. Pregnant women who consumed $100 \mathrm{~g}-200 \mathrm{~g}(0.48$ [0.28 to 0.82$])$ or $>=200 \mathrm{~g}(0.51$ [0.30 to $0.87]$ ) vegetable per day had lower risk of having SGA newborns than those who consumed $<100 \mathrm{~g}$, and who often consumed fish ( 0.57 [ 0.38 to 0.85 ] for $>=200 \mathrm{~g}$ per day, 0.72 [0.53 to 0.99 ] for $<100 \mathrm{~g}$ ) were less likely to have SGA newborns than those did not consume fish.

Conclusion: Good appetite during later pregnancy, higher intake of vegetable and fish might reduce risk of SGA.

\section{8}

Maternal dietary mineral intake during gestation and the association with infant crown-heel length

Sharon Brennan-Olsen ${ }^{1}$, John Wark ${ }^{2}$, Kathy Bennett ${ }^{3}$, Julie Pasco ${ }^{4}$

${ }^{1}$ Deakin University \& University of Melbourne; ${ }^{2}$ University of Melbourne; ${ }^{3}$ Deakin University; ${ }^{4}$ Deakin University \& University of Melbourne
Research Question: Is gestational dietary consumption of Magnesium $(\mathrm{Mg})$, phosphorus $(\mathrm{P})$, zinc $(\mathrm{Zn})$, calcium $(\mathrm{Ca})$ and potassium $(\mathrm{K})$ associated with infant crown-heel length?

Methods: Pregnant women were recruited from Geelong Hospital, Australia (2002-04) $(\mathrm{n}=475) .372 / 475$ (78.3\%) recorded dietary intake using a validated food frequency questionnaire. At birth trained personnel measured infant crown-heel length. Linear regression models were used to determine associations between maternal mineral intake and crown-heel length and adjust for potential confounders including maternal height, energy intake and gestation length.

Results: Weak correlations were observed between intake of $\mathrm{Mg}, \mathrm{P}, \mathrm{Zn}, \mathrm{Ca}$ and $\mathrm{K}$ and crown-heel length $(\mathrm{r}=0.14$, $\mathrm{p}=0.01 ; \mathrm{r}=0.14, \mathrm{p}=0.01 ; \mathrm{r}=0.11, \mathrm{p}=0.04 ; \mathrm{r}=0.09$, $p=0.08, r=0.08 p=0.13$, respectively). The associations with $\mathrm{Mg}, \mathrm{P}$ and $\mathrm{Zn}$ were not attenuated after adjustment for maternal height $(\beta 1.10, \mathrm{SE} \pm 0.44 ; \mathrm{p}=0.01, \beta 1.18, \mathrm{SE} \pm 0.45$, $\mathrm{p}=0.01 ; \beta 0.86, \mathrm{SE} \pm 0.40, \mathrm{p}=0.03$, respectively); these relationships persisted after additional adjustment for gestation length and total energy intake ( 2.38, SE \pm 0.75 ; $\mathrm{p}=0.002, \quad 3.02 \mathrm{SE} \pm 0.80, \mathrm{p}>0.001 ; 2.31, \mathrm{SE} \pm 0.68, \mathrm{p}=$ 0.001 , respectively).

Conclusion: A greater consumption of $\mathrm{Mg}, \mathrm{P}$ and $\mathrm{Zn}$ during gestation was associated with a longer crown-heel length in infants. This knowledge may inform future disease prevention strategies and thereby reduce the predisposition of offspring to non-communicable disease, such as osteoporosis, in adulthood.

\section{5}

Micronutrients in Pregnancy as a Risk Factor for gestational Diabetes and Effects on mother and baby - PRiDE study rationale and protocol

Amitha Gopinath ${ }^{1}$, Nithya Sukumar ${ }^{2}$, Hema Venkataraman ${ }^{3}$, Caroline Fall ${ }^{4}$, Jad Siblini ${ }^{5}$, Philesha Walters ${ }^{6}$

${ }^{1}$ George Eliot Hospital NHS Trust and University of Warwick;

${ }^{2}$ University of Warwick; ${ }^{3}$ University of Warwick; ${ }^{4}$ University of southampton; ${ }^{5}$ University of Warwick; ${ }^{6}$ University of Warwick

Research question: Several maternal factors during early development, foetal growth restriction and exposure to gestational diabetes(GDM) increases offspring's diabetes and obesity risk. Maternal vitamin B12 insufficiency and hyperhomocysteinaemia, is associated with an increased risk of GDM in Indian mothers and insulin resistance in the children. Given the rising prevalence of metabolic syndrome globally, our research question is, "what is the role of B12, folate and homocysteine on the risk of GDM and the offspring?".

Methods: A prospective longitudinal cohort in early pregnancy $(n=4500)$ in two ethnic groups who are at high metabolic risk will be established. A nested case-control study will assess the differences in B12, folate and homocysteine with/ 
without GDM. All women undergo glucose-tolerance-test (24-28weeks). Detailed pheno- and genotyping of mothers (detailed history, anthropometry, blood and urine) and neonates (anthropometry, cord blood, cord and placenta) will be conducted.

Results: More than 1500 women are recruited from 3 centres. GDM rates are as expected and the study is expected to complete in Dec 2016.

Discussion: When fully formed, PRiDE will be the largest cohort in high-risk women of two ethnic groups from early pregnancy. It has the potential to address several gaps in evidence in GDM, including the long-term effects in offspring.

\section{2}

The Effect of Prenatal n-3 Fatty Acid Supplementation in Infants on Cardiometabolic Risk Factors at Birth and at the 12-year Follow-up

Valene HL See ${ }^{1}$, Rae-Chi Huang ${ }^{2}$, Trevor A Mori ${ }^{3}$, Susan L Prescott ${ }^{4}$, Lawrence J Beilin ${ }^{5}$, Sally Burrows ${ }^{6}$

${ }^{1}$ School of Medicine and Pharmacology; School of Paediatrics and Child Health, University of Western Australia; ${ }^{2}$ Telethon Kids Institute, University of Western Australia; ${ }^{3}$ School of Medicine and Pharmacology, University of Western Australia; ${ }^{4}$ School of Paediatrics and Child Health; Telethon Kids Institute, University of Western Australia; ${ }^{5}$ School of Medicine and Pharmacology, University of Western Australia; ${ }^{6}$ School of Medicine and Pharmacology, University of Western Australia

Does n-3 fatty acid (FA) supplementation in pregnancy program improvements in cardiometabolic risk factors postnatally? Pregnant allergic women $(n=98)$ were randomised to $4 \mathrm{~g} /$ day of n-3 FA or olive oil [OO] from 20 weeks of gestation until delivery. Measurements in the offspring included anthropometry, adiponectin and leptin at birth and at 12 years (12Y) and skinfold thickness, fasting blood biochemistry, blood pressure and heart rate at $12 \mathrm{Y}$.

At birth, 83 mothers and children completed the study; 55 children completed the $12 \mathrm{Y}$ follow up. n-3 FA significantly increased adiponectin in the offspring at birth but not at $12 \mathrm{Y}$ compared with the $\mathrm{OO}$ group. Adiponectin significantly decreased in n-3 FA and OO groups from birth to $12 \mathrm{Y}$. At $12 \mathrm{Y}$, between-group comparisons revealed lowered cholesterol levels in the OO group and marginally lowered heart rate in the n-3 FA group. There were no significant differences between groups for other $12 \mathrm{Y}$ clinical and biochemical measurements.

Maternal n-3 FA supplementation from 20 weeks of gestation until delivery significantly increased offspring adiponectin levels at birth, with the effect attenuating at 12Y. Adiponectin plays a role in normalizing insulin resistance and adiposity, thus increasing adiponectin by dietary n-3 FA may play an essential role in the reduction of cardiometabolic risk.
4647

Effects of Prenatal n-3 Fatty Acid Supplementation on Offspring Resolvins at Birth and at 12 Years of Age

Valene HL See ${ }^{1}$, Emilie Mas ${ }^{2}$, Trevor A Mori ${ }^{3}$, Rae-Chi Huang ${ }^{4}$, Susan L Prescott ${ }^{5}$, Lawrence J Beilin ${ }^{6}$, Sally Burrows ${ }^{7}$

${ }^{1}$ School of Medicine and Pharmacology; School of Paediatrics and Child Health, University of Western Australia; ${ }^{2}$ School of Medicine and Pharmacology, University of Western Australia; ${ }^{3}$ School of Medicine and Pharmacology, University of Western Australia; ${ }^{4}$ Telethon Kids Institute, University of Western Australia; ${ }^{5}$ School of Paediatrics and Child Health; Telethon Kids Institute, University of Western Australia; ${ }^{6}$ School of Medicine and Pharmacology, University of Western Australia; ${ }^{7}$ School of Medicine and Pharmacology, University of Western Australia

Does n-3 fatty acid (FA) supplementation in pregnancy program improvements in specialized proresolving mediators (SPM) of inflammation postnatally?

Pregnant allergic women $(n=98)$ were randomised to $4 \mathrm{~g} /$ day of n-3 FA or olive oil (OO) from 20 weeks of gestation until delivery. SPM were measured in the offspring at birth and at 12 years of age (12Y) by liquid chromatography-tandem mass spectrometry.

At birth, n-3 FA supplementation significantly increased cord blood RvE2, RvE3 and the precursor 18R/S-HEPE (derived from eicosapentaenoic acid), and 17R/S-HDHA (derived from docosahexaenoic acid), relative to the OO group. Other SPM including RvE1, 18R-RvE3, RvD1, 17R-RvD1, RvD2, and 14R/S-HDHA were not significantly different between groups. There were no significant differences between groups for any of the plasma SPM at $12 \mathrm{Y}$.

Maternal n-3 FA supplementation from 20 weeks of gestation until delivery significantly increased SPM in the offspring at birth, but the effects were not sustained to $12 \mathrm{Y}$. The concentrations of SPM in blood were comparable to those shown to be biologically active in healthy humans. These findings suggest that patients with impaired immune responses that are likely present during pregnancy and at birth, may benefit from n-3 FA supplementation.

\section{6}

Maternal high-fat diet programs endocannabinoid metabolism in male and female rat offspring at weaning

Camilla Dias-Rocha ${ }^{1}$, Mariana Almeida ${ }^{2}$, Andre Silva ${ }^{3}$, Mariana Muros ${ }^{4}$, Carmen Pazos-Moura ${ }^{5}$, Isis Trevenzoli ${ }^{6}$, Juliana Franco ${ }^{7}$

${ }^{1}$ Federal University of Rio de Janeiro; ${ }^{2}$ Federal University of Rio de Janeiro; ${ }^{3}$ Federal University of Rio de Janeiro; ${ }^{4}$ Federal University of Rio de Janeiro; ${ }^{5}$ Federal University of Rio de Janeiro; ${ }^{6}$ Federal University of Rio de Janeiro; ${ }^{7}$ Federal University of Rio de Janeiro 
Research question: Obesity has been associated with over activation of the endocannabinoid system (ECS). It is unknown, however, whether ECS participates in the developmental origins of obesity. We hypothesized that maternal highfat diet (HFD) would alter the endocannabinoid metabolizing enzymes Monoacylglycerol Lipase (MAGL) and Fatty Acid Amide Hydrolase (FAAH) in adipose tissue of offspring rats.

Methods: Female rats received a standard diet (C; 9\% fat) or a HFD (28\% fat) during perinatal period. At weaning, offspring from both $\mathrm{C}$ and HFD dams were killed and we measured FAAH and MAGL protein and mRNA in visceral (VAT) and subcutaneous (SAT) adipose tissue.

Results: Maternal HFD increased body weight and adiposity in both male and female offspring. In VAT, maternal HFD increased FAAH protein and mRNA, and MAGL protein in male without changes in female offspring. In SAT, FAAH protein was increased in both male and female while MAGL mRNA was increased only in female offspring.

Conclusion: Maternal HFD induced early obesity in the offspring with changes in the adipose tissue ECS. In the VAT, these changes were gender-dependent. We speculate that ECS is over activated in HFD offspring and an increased degradation of EC could be a possible adaptive defense mechanism.

\section{1}

Maternal high-fat diet programs obesity and sex-specific differences in cannabinoid receptor expression in adipose tissue of weanling rats

Mariana Macedo de Almeida ${ }^{1}$, Camilla Pereira Dias-Rocha ${ }^{2}$, André de Souza Silva ${ }^{3}$, Juliana Gastão Franco ${ }^{4}$,

Carmen Cabanelas Pazos de Moura ${ }^{5}$, Norma Aparecida dos Santos Almeida ${ }^{6}$, Aline Cordeiro ${ }^{7}$, Isis Hara Trevenzoli ${ }^{8}$

${ }^{1}$ Federal University of Rio de Janeiro; ${ }^{2}$ Federal University of Rio de Janeiro; ${ }^{3}$ Federal University of Rio de Janeiro; ${ }^{4}$ Federal University of Rio de Janeiro; ${ }^{5}$ Federal University of Rio de Janeiro; ${ }^{6}$ Federal Rural University of Rio de Janeiro; ${ }^{7}$ Federal University of Rio de Janeiro; ${ }^{8}$ Federal University of Rio de Janeiro

Research question: Obesity is associated with over activation of the endocannabinoid system (ECS). However, the effect of maternal obesity on offspring ECS is unknown. We hypothesized that maternal obesity would regulate type 1 and type 2 cannabinoid receptors (CB1/Cnr1 and CB2/Cnr2) in a sex and adipose depot dependent manner. Methods: Female rats received a standard diet (C; 9\% fat) or a high-fat diet (HFD; $28 \%$ fat) during perinatal period. At weaning, we analyzed leptinemia and cannabinoid receptor expression in visceral (VAT) and subcutaneous (SAT) adipose tissue of male and female offspring.

Results: Maternal HFD induced offspring obesity and hyperleptinaemia. Maternal HFD increased CB1 in VAT of female offspring while decreased CB1 in SAT of male and female offspring, without changes in Cnr1 mRNA levels. Maternal HFD decreased CB2 in VAT of female offspring while decreased and increased CB2 in SAT of male and female offspring, respectively. Maternal HFD increased Cnr2 mRNA levels in VAT of male offspring while decreased $\mathrm{Cnr} 2$ in SAT of female offspring.

Conclusion: Maternal obesity programmed cannabinoid receptors in a sex and adipose depot dependent manner. We speculate that $\mathrm{CB} 1$ changes are involved with lipogenesis while $\mathrm{CB} 2$ changes can be associated with proinflammatory profile in the offspring.

\section{8}

Formula milk derived CML induces RAGE activation, long term inflammation and oxidative stress in IUGR piglets

Ghada Elmhiri ${ }^{1}$, Dler F Mahmood ${ }^{2}$, Celine Niquet-Leridon ${ }^{3}$, Philippe Jacolot ${ }^{4}$, Stephane Firmin ${ }^{5}$, Lydie Guigand ${ }^{6}$, Frederic J. Tessier ${ }^{7}$, Thibaut Larcher $^{8}$, Latifa Abdennebi-Najar ${ }^{9}$

${ }^{1}$ UP-EGEAL 2012.10.101 Institut Polytechnique LaSalle Beauvais; ${ }^{2}$ UMR 8256 UPMC Université Paris 6 -CNRS INSERM Dept of 8 Adaptation and Ageing Biology (UMR 8256 । ERL-1164); ${ }^{3} U P$-EGEAL 2012.10.101 Institut Polytechnique LaSalle Beauvais; ${ }^{4}$ UP-EGEAL 2012.10.101 Institut Polytechnique LaSalle Beauvais; ${ }^{5}$ UP-EGEAL 2012.10.101 Institut Polytechnique LaSalle Beauvais; ${ }^{6} I N R A$, UMR 703 APEX, Ecole Nationale 10 Vétérinaire Agroalimentaire et de l'Alimentation Nantes-Atlantique (Oniris), Nantes, France.; ${ }^{7} U P-E G E A L$

2012.10.101 Institut Polytechnique LaSalle Beauvais; ${ }^{8}$ INRA, UMR 703 APEX, Ecole Nationale 10 Vétérinaire Agroalimentaire et de l'Alimentation Nantes-Atlantique (Oniris), Nantes, France.;

${ }^{9}$ UP-EGEAL 2012.10.101 Institut Polytechnique LaSalle Beawvais

Research question: Formula-derived dietary-advancedglycation end products (AGEs) may promote programming of inflammation and oxidative stress in the kidney of intrauterine growth retardation (IUGR) piglets.

Methods: IUGR piglets received either a low heated formula (LHF, $n=8$ ), a high heated formula (HHF: $n=8$ ) or suckled naturally for $3 \mathrm{wk}$ postnatally. Then they were fed with normal ad libitum regular diet. $\mathrm{N}(\varepsilon)$-carboxymethyllysine (CML) was measured in plasma, feces, and formula by HPLC/MS-MS.

Results: CML was detected by immunofluorescence in kidney cells. Target renin-angiotensin-apoptotic, pro-inflammatory genes p62-NF- $\mathrm{kB}$, and soluble receptor of AGE (sRAGE) levels were quantified. Compared with that in controls, free $\mathrm{CML}$ and plasma urea increased significantly in the HHF-fed group at PND36 $(\mathrm{p}<0.05)$. CML was detected in the nuclei of renal tubular cells of formula-fed piglets but not in suckled ones. Furthermore, the activation of sRAGE was increased in HHF group $(\mathrm{p}<0.01)$. AT1, AT2, caspase 3 and 8, p62-NF$\kappa \mathrm{B}$, and total protein oxidation in kidney were higher in HHFfed group as compared to LHF-fed group $(\mathrm{p}<0.05)$.

Conclusion: Food processes aimed at reducing the concentration of AGEs in infant formula are urgently needed and may be therapeutically relevant for premature and/or IUGR. 
4693

A prenatal/lactation diet rich in omega-3 PUFAs may provide protection against diabetes in adulthood: a rat model of low diabetes prevalence among Yup'ik Alaskan Natives

Daniel Benyshek ${ }^{1}$, Julie Kachinski ${ }^{2}$, Hongbin Jin ${ }^{3}$

${ }^{1}$ University of Nevada, Las Vegas; ${ }^{2}$ University of Nevada, Las Vegas; ${ }^{3}$ University of Nevada, Las Vegas

Research question: Yup'ik Alaskans continue to have a relatively low prevalence of diabetes (3.3\%) despite a diet consisting of less locally harvested, and more store bought foods, and increasing obesity. The traditional Yup'ik diet, high in omega3-polyunsaturated fatty acids (omega-3-PUFAs), may provide protection from diabetes in adulthood. We asked: i) What effect does a maternal prenatal/lactation diet high in omega-3-PUFAs have on the insulin sensitivity of adult Sprague Dawley rat offspring weaned to a western diet?; and ii) What effect does a postweaning offspring diet high in omega-3 PUFAs have on adult insulin sensitivity under varying maternal prenatal/lactation dietary conditions?

Methods: Utilizing an experimental animal design, pregnant/ lactating rat dams were fed one of three diets: high omega-3PUFA; control; or high saturated-fat 'western'. Postweaning offspring diets were varied similarly. The primary outcome measure was offspring insulin sensitivity (HOMA-IR), at $120 \mathrm{~d}$.

Results and Conclusions: Our results suggest that a maternal prenatal/lactation diet high in omega-3 PUFAs may provide some protection from insulin resistance among adult offspring even when weaned to a high saturated-fat western diet, and that the protective metabolic effects of a postweaning diet high in omega-3-PUFAs is insufficient to overcome the deleterious effects of a prenatal/lactation.

\section{5}

Zinc supplementation during mice gestation modifies breast cancer susceptibility of the female offspring in adulthood

Raquel Santana ${ }^{1}$, Fábia De Oliveira Andrade ${ }^{2}$, Sônia De Assis ${ }^{3}$, Mariana Papaléo Rosim ${ }^{4}$, Mayara Lilian P. Miranda ${ }^{5}$, Mari Cleide Sogayar $^{6}$, Pedro Vitoriano Oliveira ${ }^{7}$, Inar Alves de Castro $^{8}$, Luís Fernando Barbisan ${ }^{9}$, Thomas Prates Ong ${ }^{10}$

${ }^{1}$ University of Sao Paulo, Sao Paulo, Brazil; ${ }^{2}$ University of Sao Paulo, Sao Paulo, Brazil; ${ }^{3}$ Georgetown Lombardi Compreensive Cancer Center, Washington D.C, USA $;{ }^{4}$ University of Sao Paulo, Sao Paulo, Brazil; ${ }^{5}$ University of Sao Paulo, Sao Paulo, Brazil; ${ }^{6}$ University of Sao Paulo, Sao Paulo, Brazil; ${ }^{7}$ University of Sao Paulo, Sao Paulo, Brazil; ${ }^{8}$ University of Sao Paulo, Sao Paulo, Brazil; ${ }^{9}$ Sao Paulo State University, Botucatu, Brazil;

${ }^{10}$ University of Sao Paulo, Sao Paulo, Brazil
Zinc is an essential micronutrient required for several process including cell proliferation, differentiation and gene expression regulation. The aim of this study was evaluate if zinc deficiency or supplementation during gestation influence female offspring susceptibility to breast cancer in adulthood. Black C57BL/6 female mice consumed during gestation control diet (AIN-93G; CO group); zinc-deficient diet (8 ppm; ZnD group) and zinc-supplemented diet (45 ppm; $\mathrm{ZnS}$ group). Mammary tumors were induced by subcutaneous administration of $15 \mathrm{mg}$ of medroxyprogesterone to 6-week-old female offspring, followed by oral administration of $1 \mathrm{mg} 7,12$-dimethylbenz[a] anthracene once-a-week for 4 weeks. Non-initiated mammary glands of 7 -week-old female offspring from all groups were used to evaluate gene expression by q-PCR. Compared to $\mathrm{CO}$ group offspring, $\mathrm{ZnS}$ presented increased $(\mathrm{P}<0.05)$ mammary tumor incidence, number of terminal end buds and cell proliferation (Ki67). There were no differences $(P \geqslant 0.05)$ concerning these variables between $\mathrm{CO}$ and $\mathrm{ZnD}$ group. Compared to $\mathrm{CO}$ group offspring, $\mathrm{ZnS}$ also showed higher $(\mathrm{P}<0.05)$ expression of RASSF1 and ZPF382 genes, with no differences $(\mathrm{P} \geqslant 0.05)$ between $\mathrm{CO}$ and $\mathrm{ZnD}$. $\mathrm{ZnS}$ showed marginal increased expression of STAT3 compared to $\mathrm{CO}(\mathrm{P}=0.07)$ and $\mathrm{ZnD}(\mathrm{P}=0.057)$. In conclusion, zinc supplementation in early-life may be associated with an increased susceptibility to breast cancer development in adulthood.

\section{8}

Maternal dietary patterns and infant birth outcomes in a multi-ethnic Asian population

Airu Chia ${ }^{1}$, Jamie de Seymour ${ }^{2}$, Marjorelee Colega ${ }^{3}$, Yiong-Huat Chan ${ }^{4}$, Izzuddin Aris ${ }^{5}$, Mya-Thway Tint ${ }^{6}$, Phaik-Ling Quah ${ }^{7}$, Keith Godfrey ${ }^{8}$, Kenneth Kwek ${ }^{9}$, Seang-Mei Saw ${ }^{10}$, Peter Gluckman ${ }^{11}$, Yap-Seng Chong ${ }^{12}$, Philip Baker ${ }^{13}$, Yung Seng Lee ${ }^{14}$, Mary Chong ${ }^{15}$

${ }^{1}$ Singapore Institute for Clinical Sciences, $A^{*} S T A R$, Singapore; Department of Obstetrics \& Gynaecology, Yong Loo Lin School of Medicine, National University of Singapore, Singapore; ${ }^{2}$ Liggins Institute, University of Auckland, New Zealand; ${ }^{3}$ Singapore Institute for Clinical Sciences, $A^{*} S T A R$, Singapore; ${ }^{4}$ Biostatistics Unit, Yong Loo Lin School of Medicine, National University of Singapore, Singapore; ${ }^{5}$ Department of Paediatrics, Yong Loo Lin School of Medicine, National University of Singapore, Singapore; ${ }^{6}$ Department of Obstetrics \& Gynaecology, Yong Loo Lin School of Medicine, National University of Singapore, Singapore;

${ }^{7}$ Singapore Institute for Clinical Sciences, $A^{*} S T A R$, Singapore;

${ }^{8}$ Medical Research Council Lifecourse Epidemiology Unit and NIHR Southampton Biomedical Research Centre, University of Southampton and University Hospital Southampton NHS Foundation; ${ }^{9}$ Department of Maternal Fetal Medicine, KK Women's and Children's Hospital, Singapore; ${ }^{10}$ Saw Swee Hock School of Public Health, National University of Singapore and National University Health System, Singapore; " ${ }^{11}$ Singapore 
Institute for Clinical Sciences, $A^{*}$ STAR; Liggins Institute, University of Auckland, New Zealand; ${ }^{12}$ Singapore Institute for Clinical Sciences, $A^{*} S T A R$, Singapore; Department of Obstetrics \& Gynaecology, Yong Loo Lin School of Medicine, National University of Singapore, Singapore; ${ }^{13}$ Liggins Institute, University of Auckland, New Zealand; ${ }^{14}$ Singapore Institute for Clinical Sciences, $A^{*}$ STAR, Singapore; Department of Paediatrics, Yong Loo Lin School of Medicine, National University of Singapore, Singapore; Division of $P ;{ }^{15}$ Singapore Institute for Clinical Sciences, $A^{*} S T A R$, Singapore; Department of Paediatrics, Yong Loo Lin School of Medicine, National University of Singapore, Singapore; Clinical Nutr

Research question: There is limited evidence examining associations between maternal dietary patterns and birth outcomes, particularly in Asian populations. We examined for associations among women in the Singapore GUSTO motheroffspring cohort.

Methods: Dietary patterns were identified by factor analysis using 24-h recalls obtained during 26-28th gestational weeks. Birth measurements and gestational age data were obtained from hospital records. Associations between dietary patterns and birth outcomes were assessed by multivariate logistic (preterm risk) and linear regression (birth anthropometry).

Results: Of 820 infants, $7.6 \%$ were born preterm. Mean \pm SD birth weight was $3093 \pm 430 \mathrm{~g}$. Three maternal dietary patterns were identified: vegetable, fruit and rice-based (VFR); seafood and noodle-based (SfN); pasta and processed meat (PPM). VFR diet was positively associated with gestational age-adjusted birthweight (28.5g per SD score increment; 95\% CI:2.3, 54.7) and ponderal index $(0.33 \mathrm{~kg} / \mathrm{m} 3$ per $\mathrm{SD} ; 0.12,0.54)$ in offspring and inversely associated with risk of preterm birth (OR per $S D=0.71 ; 0.52,0.99)$. VFR diet corresponded with higher protein (highest versus lowest tertile of VFR score: $16.5 \%$ vs $14.9 \%$ of energy) and lower fat intakes (31.5\% vs $34.4 \%$; $<<0.05$ for all). No associations were seen between $\mathrm{SfN}$ and PPM diets and birth outcomes.

Conclusion: A vegetable, fruit and rice-based diet during pregnancy was associated with larger birth size and lower incidence of preterm birth.

\section{3}

Impact of maternal and/or paternal blackberry (Rubus spp.) extract consumption on female offspring susceptibility to chemically-induced breast carcinogenesis

Vanessa Cardoso Pires ${ }^{1}$, Sara Lima Anacleto ${ }^{2}$, Gabriela Rezende Costa ${ }^{3}$, Laila Abicair Sangaletti Santos ${ }^{4}$, Neuza Mariko Aymoto Hassimotto ${ }^{5}$, Franco Maria Lajolo ${ }^{6}$, Odair Aguiar $\mathrm{Jr}^{7}$, Thomas Prates $\mathrm{Ong}^{8}$

${ }^{1}$ University of Sao Paulo; ${ }^{2}$ University of Sao Paulo; ${ }^{3}$ University of Sao Paulo; ${ }^{4}$ University of Sao Paulo; ${ }^{5}$ University of Sao Paulo;

${ }^{6}$ University of Sao Paulo; ${ }^{7}$ Federal University of Sao Paulo;

${ }^{8}$ University of Sao Paulo
Breast cancer is the most frequent type of cancer among women. Maternal and paternal food consumption can influence the risk of breast cancer in the offspring. Blackberry contains bioactive compounds that display antioxidant and anticancer effects. Our aim was to evaluate the effects of maternal and/or paternal consumption of a blackberry methanolic extract on female offspring susceptibility to chemicallyinduced breast carcinogenesis. C57BL/6 mice were divided into four groups: father blackberry (FB), mother blackberry $(\mathrm{MB})$, father and mother blackberry (FMB) and control (CTRL). The blackberry extract (BE) was given through drinking water to fathers from prepuberty onwards and to mothers during gestation and lactation. Blackberry consumption by the fathers increased their plasma antioxidant capacity (PAC) $(\mathrm{p}<0.001)$, reduced testicular superoxide dismutase (SOD) and catalase (CAT) activity $(\mathrm{p}<0.05$ and $\mathrm{p}<0.001$, respectively) and improved sperm production and morphology $(\mathrm{p}<0.001)$. PAC was decreased in FB and MB groups female offspring ( $p<0.001$ and $p<0.01$, respectively) and increased in FMB group offspring $(\mathrm{p}<0.001)$ compared to controls. FMB group offspring showed lower incidence of mammary tumors, that presented however higher tumor weight $(\mathrm{p}<0.01)$ and volume $(\mathrm{p}<0.05)$. Maternal and/or paternal $\mathrm{BE}$ consumption was able to modulate the antioxidant capacity and breast carcinogenesis susceptibility in the female offspring.

\section{8}

A proposal for determining the associations of iron and fatty acid status with birth outcomes in urban South African pregnant women

Elize Symington ${ }^{1}$, Marius Smuts ${ }^{2}$, Jeannine Baumgartner ${ }^{3}$

${ }^{1}$ Unisa; ${ }^{2}$ North West University; ${ }^{3}$ North West University

Research question: Little is known about iron and long-chain polyunsaturated fatty acid (LCPUFA) intake and status of pregnant South African women. Low maternal levels of both iron and LCPUFA have been associated with shorter gestation and low birth weight. Furthermore, iron is a co-factor of enzymes involved in LCPUFA metabolism. We will assess iron and LCPUFA intake and status throughout pregnancy, and determine associations with birth outcomes.

Methods: The proposed research will have an observational, longitudinal design. Pregnant women ( $<16$ weeks gestation) $(n=250)$ will be recruited from primary healthcare clinics in Johannesburg and followed up at a provincial hospital. Intake and status of iron and LCPUFA will be assessed at $<16,24$ and 36 weeks gestation. At birth, cord blood will be collected and gestational age and birth weight determined.

Results: We expect poor intakes and status of iron and LCPUFA throughout pregnancy, and that iron deficiency during pregnancy compromises LCPUFA status. We hypothesize that low maternal levels of both iron and LCPUFA are associated with shorter gestation and low birth weight. 
Conclusion: It is important to understand associations between maternal nutrition and birth outcomes to provide a basis for nutritional interventions which can improve birth outcomes and long-term health.

\section{7}

Effects of a maternal high-fat, high-sucrose diet and folate supplements on insulin-signaling and lipid metabolism inheritance in male rat offspring

Dan Ramdath ${ }^{1}$, Candace Cuthbert ${ }^{2}$, Aileen Hawke ${ }^{3}$, Jerome Foster $^{4}$

${ }^{1}$ Guelph Food Research Centre, Ontario N1G 5C9, Canada;

${ }^{2}$ Faculty of Medical Sciences, The University of the West Indies, Trinidad \& Tobago; ${ }^{3}$ Guelph Food Research Centre, Ontario

N1G 5C9, Canada; ${ }^{4}$ Faculty of Medical Sciences, The University of the West Indies, Trinidad \& Tobago

Research question: Does a maternal high-fat, high-sucrose (HFS) diet alter insulin-signaling and lipid metabolism gene and protein expression in male, Sprague-Dawley rat offspring and is this effect modulated by folic acid-supplementation (HFS/F)?

Methods: Pregnant Sprague-Dawley rats were randomly fed a control, HFS or HFS/F diet. Offspring were fed an AIN control diet for 10 weeks then sacrificed: fasting plasma insulin and glucose were analyzed and HOMA-IR derived. Liver and skeletal muscle gene and protein expression in offspring tissue were assayed by RT-PCR and western blotting. Mean differences were assessed by one-way ANOVA with Bonferroni's post-hoc test.

Results: HFS/F offspring had significantly lower fasting plasma glucose and higher insulin concentrations vs. HFS and control, respectively ( $\mathrm{p} \leqslant 0.05)$. HOMA-IR was high in both HFS groups. Hepatic acetyl-CoA carboxylase gene expression was significantly down-regulated and protein expression significantly lower in HFS vs. control $(\mathrm{p} \leqslant 0.05)$. Similarly, skeletal muscle v-raf-1 murine leukemia viral oncogene homolog 1 gene expression was significantly down-regulated and phosphorylated protein expression significantly lower in HFS vs. control offspring ( $\mathrm{p} \leqslant 0.05)$.

Conclusion: Maternal HFS diet alters HOMA-IR, insulinsignaling and lipid metabolism gene and protein expression in offspring liver and skeletal muscle: these are not modulated by HFS/F.

\section{3}

Prenatal nutrient restriction induces reproductive dysfunction and an early loss of ovarian follicles

Kaitlyn Chan ${ }^{1}$, Dr. Deborah Sloboda ${ }^{2}$, Nicole Yokubynas ${ }^{3}$

${ }^{1}$ McMaster University, Department of Biochemistry and Biomedical Sciences; ${ }^{2}$ McMaster University, Department of Biochemistry and Biomedical Sciences; ${ }^{3}$ McMaster University
Research question: We have shown that offspring born to nutrient restricted mothers are born small and display early ovarian aging. We investigated the mechanisms regulating follicle loss.

Methods: Pregnant rats were fed either a control diet during pregnancy and lactation (CON), or mothers fed $50 \%$ of control intake during pregnancy (UN) and then fed a control diet during lactation. Offspring ovaries were collected to assess follicle numbers. Follicles were isolated using laser microdissection and key factors were analyzed using IHC and qPCR. Serum levels of gonadotropins, and sex steroids were measured using ELISA.

Results: As neonates (P4) and juveniles (P27), UN offspring had increased follicular recruitment proteins pAkt and pFOXO3A and at P27 had lower estradiol levels compared to CON offspring As young adults (P60) UN offspring had irregular estrous cycles, decreased antral follicles and corpora lutea number, increased atretic follicles, and reduced serum $\mathrm{AMH}$ levels. These changes were not associated with altered gene expression patterns.

Conclusion: Prenatal UN impaired offspring reproductive cyclicity, impaired ovulation and resulted in a loss of antral follicles very early in life. It appears that pAkt mediated follicle recruitment could be accelerated already during neonatal life that would, contribute to follicle loss by young adulthood.

\section{9}

Associations of maternal folate and vitamin B12 status with offspring's linear growth in a multi-ethnic Asian motheroffspring cohort study

Ling-Wei Chen ${ }^{1}$, Ai Lin $\mathrm{Lim}^{2}$, Marjorelee Colega ${ }^{3}$, Mya-Thway Tint ${ }^{4}$, Izzuddin M. Aris ${ }^{5}$, Jonathan Y. Bernard ${ }^{6}$, Peter D. Gluckman ${ }^{7}$, Keith M. Godfrey ${ }^{8}$, Kenneth Kwek ${ }^{9}$, Seang-Mei Saw ${ }^{10}$, Yap-Seng Chong ${ }^{11}$, Fabian Yap ${ }^{12}$, Rob M. van Dam ${ }^{13}$, Mary Foong-Fong Chong ${ }^{14}$, Yung Seng Lee ${ }^{15}$

${ }^{1}$ Department of Paediatrics, Yong Loo Lin School of Medicine, National University of Singapore and National University Health System, Singapore; Khoo Teck Puat-National University C;

${ }^{2}$ Singapore Institute for Clinical Sciences, $A^{*} S T A R$, Singapore;

${ }^{3}$ Singapore Institute for Clinical Sciences, $A^{*} S T A R$, Singapore;

${ }^{4}$ Department of Obstetrics \& Gynaecology, Yong Loo Lin School of Medicine, National University of Singapore and National University Health System, Singapore; ${ }^{5}$ Singapore Institute for Clinical Sciences, $A^{*} S T A R$, Singapore; ${ }^{6}$ Singapore Institute for Clinical Sciences, $A^{*} S T A R$, Singapore; ${ }^{7}$ Singapore Institute for Clinical Sciences, $A^{*} S T A R$, Singapore; Liggins Institute, University of Auckland, New Zealand; ${ }^{8}$ Medical Research Council Lifecourse Epidemiology Unit and NIHR Southampton Biomedical Research Centre, University of Southampton and University Hospital Southampton NHS Foundation; ${ }^{9}$ Department of Maternal Fetal Medicine, KK Women's and Children's Hospital, Singapore; ${ }^{10}$ Saw Swee Hock School of Public Health, National University of Singapore and National 
University Health System, Singapore; ${ }^{11}$ Singapore Institute for Clinical Sciences, $A^{*} S T A R$, Singapore; Department of Obstetrics \& Gynaecology, Yong Loo Lin School of Medicine, National University of Singapore and National; ${ }^{12}$ Department of Paediatric Endocrinology, KK Women's and Children's Hospital, Singapore; Duke-NUS Graduate Medical School, Lee Kong Chian School of Medicine, Singapore; ${ }^{13}$ Saw Swee Hock School of Public Health, National University of Singapore and National University Health System, Singapore; Department of Medicine, Yong Loo Lin School of Medicine, $N ;{ }^{14}$ Department of Paediatrics, Yong Loo Lin School of Medicine, National University of Singapore and National University Health System, Singapore; Khoo Teck Puat-National University C; ${ }^{15}$ Department of Paediatrics, Yong Loo Lin School of Medicine, National University of Singapore and National University Health System, Singapore; Khoo Teck Puat-National University $C$

Research question: Does maternal folate and vitamin B12 status influence offspring's linear growth?

Methods: Maternal blood samples $(n=999)$ collected during 26th-28th week gestation were assayed for plasma folate and vitamin B12 concentrations. Infants' lengths were measured at birth, week 3, and month 3, 6, 9, 12, 15, 18, 24. Associations of folate and vitamin B12 with linear growth were assessed using multivariable linear regressions.

Results: Higher maternal folate level was associated with a higher birth length $(0.08 \mathrm{~cm}$ per SD increase of folate, $P=0.19 ; \beta=0.16 \mathrm{~cm}, P=0.02$ after mutual adjustment with vitamin $\mathrm{B} 12$ ), while higher vitamin $\mathrm{B} 12$ was associated with a lower birth length $(-0.19 \mathrm{~cm}, \mathrm{P}<0.01)$. After birth, higher folate was associated with lower length velocity $(\mathrm{cm} / \mathrm{month})$ (indicative of catch-down growth), while higher vitamin B12 was associated with higher length velocity (indicative of catchup growth). Independent of birth length, higher folate was significantly associated with lower conditional length change (SD/SD) from birth until week $3(\beta=-0.07, \mathrm{P}=0.02)$ and month $9(\beta=-0.08, P=0.02)$. These relationships appeared clearer in Chinese, the biggest ethnic group in this study.

Conclusion: Maternal folate and vitamin B12 status appeared to have different influences on offspring's linear growth.

\section{4}

Dietary patterns and the development of the PCOS phenotype: the chance of ongoing pregnancy

Nicole Huijgen ${ }^{1}$, Joop Laven ${ }^{2}$, Yvonne Louwers ${ }^{3}$, Sten Willemsen $^{4}$, Jeanne de Vries ${ }^{5}$, Régine Steegers-Theunissen ${ }^{6}$

${ }^{1}$ Erasmus $M C$, University Medical Centre; ${ }^{2}$ Erasmus $M C$, University Medical Centre; ${ }^{3}$ Erasmus MC, University Medical Centre; ${ }^{4}$ Erasmus MC, University Medical Centre; ${ }^{5}$ Wageningen University; ${ }^{6}$ Erasmus MC, University Medical Centre

Research question: Healthy nutrition is essential for ovarian follicle development. Recently, we observed an impaired diet quality in PCOS patients. Here we investigate the dietary patterns of PCOS patients and the chance of ongoing pregnancy.

Methods: In a periconception cohort, we included patients visiting the preconception outpatient clinic. Food frequency and general questionnaires were completed in 266 patients of which 55 patients were diagnosed PCOS. Anthropometrics and bloods samples were obtained. Principal component factor analysis was used to summarize dietary patterns and multivariable logistic regression analysis was applied.

Results: High adherence to the healthy dietary pattern (comprising fruits, vegetables, fish and whole grains) was negatively associated with the severe hyperandrogenic (HA) PCOS phenotype (OR0.27; p<0.05; 95\% CI 0.07-0.98), after adjustment for energy intake, BMI and age. PCOS patients with high adherence to the healthy dietary pattern also showed a 3-fold higher chance of ongoing pregnancy (OR3.38; $\mathrm{p}<0.05 ; 95 \%$ CI 1.01-11.36). After additional adjustment for age and BMI this chance attenuated to 3.09 (p0.083; 95\% CI 0.86-11.05).

Conclusion: A healthy dietary pattern is associated with PCOS severity and an increased chance of ongoing pregnancy. Therefore, healthy nutritional support should be incorporated into reproductive care and treatment plan PCOS.

\section{3}

Low vitamin B12 in pregnancy is associated with maternal obesity and gestational diabetes

Nithya Sukumar ${ }^{1}$, Sean Wilson ${ }^{2}$, Hema Venkataraman $^{3}$, Ponnusamy Saravanan ${ }^{4}$

${ }^{1}$ Warwick Medical School, University of Warwick, UK; ${ }^{2}$ George Eliot Hospital, Nuneaton, UK; ${ }^{3}$ Warwick Medical School, University of Warwick; ${ }^{4}$ Warwick Medical School, University of Warwick

Research question: A study from India showed that B12 insufficiency in pregnancy was associated with higher risk of gestational diabetes (GDM), mediated by BMI. It is not known whether the same association exists among pregnant women in the UK.

Methods: A retrospective study was done on women attending our antenatal clinic. Information including 1st trimester BMI, B12, folate and glucose were collected and multivariate regression models applied.

Results: 247 women (114 GDM, 133 controls) who had B12 levels checked in early 3rd trimester were included. $26.7 \%$ had B12 values $<150$ pmol.1-1. 1st trimester BMI was the only significant predictor of 3rd trimester B12(r=-0.203, $p=$ $0.001 ; \beta=-0.21, p=0.002$ after adjusting for age, parity, ethnicity and folate). GDM mothers were older, more obese and had significantly lower mean B12 (186 vs. 225 pmol.1-1, 
$\mathrm{p}=0.01)$ but similar folate levels. Women in the lowest B12 tertile had significantly higher odds of GDM than the highest tertile (AOR: 2.27, 95\% CI: 1.07, 4.78), after adjusting for $\mathrm{BMI}$ and other variables as above.

Conclusion: Obese pregnant women in UK are at risk of B12 insufficiency, which in turn is independently associated with GDM. Whether early pregnancy B12 insufficiency predicts the onset of GDM require longitudinal studies.

\section{7}

Vitamin B12 deficiency impairs insulin signalling in human adipocytes by down regulating Akt via PTEN and Trb3

Antonysunil Adaikalakoteswari ${ }^{1}$, Manu Vatish ${ }^{2}$, Sudhesh Kumar $^{3}$, Philip McTernan ${ }^{4}$, Ponnusamy Saravanan ${ }^{5}$, Gyanendra Tripathi ${ }^{6}$

${ }^{1}$ Division of Metabolic and Vascular Health, University of Warwick, Warwick, United Kingdom; ${ }^{2}$ Nuffeld Department of Obstetrics \& Gynaecology, University of Oxford, UK; ${ }^{3}$ Division of Metabolic and Vascular Health, University of Warwick, Warwick, United Kingdom; ${ }^{4}$ Division of Metabolic and Vascular Health, University of Warwick, Warwick, United Kingdom;

${ }^{5}$ Division of Metabolic and Vascular Health, University of Warwick, Warwick, United Kingdom; Department of Pathology \& Academic Department of Diabetes and Endocrinology, George Eliot; ${ }^{6}$ Division of Metabolic and Vascular Health, University of Warwick, Warwick, United Kingdom

Research question: Longitudinal studies and animal models showed that low maternal vitaminB12 deficiency resulted in the development of insulin-resistance and metabolic syndrome phenotype in the offspring. This provided impetus to investigate the molecular mechanisms of insulin signalling in adipocyte dysfunction due to vitamin B12 deficiency. We chose adipocytes as an in-vitro model system as they undergo several cycles of differentiation and maturation, mimicking in-vivo intergenerational effect (pregnancy-offspring).

Methods and Results: In three independent clinical studies, we consistently observed that women at childbearing age, at early pregnancy and at delivery with low B12 status had higher BMI. Adipocytes cultured in B12 deficient conditions showed reduced basal and insulin stimulated glucose uptake. Protein expression of p-Akt, $\mathrm{p}$-Akt activity and its downstream targets (p-GSK3- $\beta$, p-FOXO1 and p-S6K) were altered. The negative regulators of Akt, (PTEN and TRB3) were upregulated. Adipocytes treated with PTEN inhibitor or transfected with Trb3 siRNA alleviated Akt inhibition and restored insulin stimulated glucose uptake. Similarly ex vivo study using adipose tissue from mothers with low B12 showed reduced protein expression of p-Akt and p-Akt activity.

Conclusions: Our study provides evidence that adipocytes with low B12 develop insulin resistance due to impaired Akt signalling cascade, which appears partly through activation of PTEN/Trb3.

\section{5}

Resveratrol supplementation during pregnancy in an animal model of maternal obesity

EA Domfeh, E Richardson, N Maicas Blasco,

AM Samuelsson, K Macé, L Poston, E Castañeda-Gutiérrez, PD Taylor

${ }^{1}$ Division of Women's Health \& Women's Health Academic Centre, Faculty of Life Sciences \& Medicine, King's College

London. ${ }^{*}$ Nestlé Research Centre. Department of Nutrition and Health. Switzerland

Research question: The objective of the study was to investigate the benefit of resveratrol supplementation during pregnancy and lactation on metabolic outcomes in obese rat dams and their offspring.

Methods: Rats were fed either a balanced control or a highly palatable obesogenic diet (high fat \& high sugar) from $6 \mathrm{wk}$ before mating until the end of lactation (21d postpartum). At mating, dams were further assigned to either resveratrol (50 $\mathrm{mg} / \mathrm{kg} / \mathrm{d}$ ) or placebo, presented daily in a gelatine pellet throughout gestation and lactation. Dams were euthanized at $16 \mathrm{~d}$ of gestation; at 4, 9 and $18 \mathrm{~d}$ of lactation. A subgroup gave birth and their offspring were analysed for glucose tolerance (GTT) at $90 \mathrm{~d}$ of age.

Results: Supplementation with resveratrol had no apparent effect on energy intake, glucose homeostasis or weight gain during pregnancy. Resveratrol supplementation in obese dams significantly reduced maternal weight loss during lactation $(\mathrm{P}<0.05)$, and hepatic mRNA expression of PPAR $\gamma$ $(\mathrm{P}<0.001)$ and SIRT-1 $(\mathrm{P}<0.01)$. Male offspring of resveratrol-treated control dams had impaired glucose homeostasis at $90 \mathrm{~d}$ of age, as shown by greater peak and AUC after GTT $(\mathrm{P}<0.05)$.

Conclusions: Supplementation with resveratrol did not improve metabolic outcomes in obese dams or their offspring. [Nestlé, Tommy's].

\section{6}

\section{Association between sweetened beverages during pregnancy and gestational weight gain}

Shu E Soh ${ }^{1}$, Wen Fang Seoh ${ }^{2}$, Joren Colega ${ }^{3}$,

Peter D. Gluckman ${ }^{4}$, Keith M. Godfrey ${ }^{5}$, Seang Mei

Saw $^{6}$, Kenneth Kwek ${ }^{7}$, Yap Seng Chong ${ }^{8}$, Mary Foong-Fong Chong ${ }^{9}$

${ }^{1}$ Singapore Institute for Clinical Sciences; ${ }^{2}$ Singapore Institute for Clinical Sciences; ${ }^{3}$ Singapore Institute for Clinical Sciences;

${ }^{4}$ Singapore Institute for Clinical Sciences; ${ }^{5}$ Singapore Institute for Clinical Sciences; ${ }^{6}$ Saw Swee Hock School of Public Health; ${ }^{7}$ KK Women's and Children's Hospital; ${ }^{8}$ Singapore Institute for Clinical Sciences, National University of Singapore; ${ }^{9}$ Singapore Institute for Clinical Sciences 
Research question: Diet is one of the crucial modifiable factors for excessive gestational weight gain (GWG) which can adversely affect maternal and child outcomes. We aim to study the association of food groups consumed during pregnancy and GWG.

Methods: Dietary intakes of 900 mothers enrolled in the Singapore GUSTO birth cohort study were ascertained through 24-hour recalls at 26-28th weeks' gestation and food items categorized into food groups. Total GWG was calculated as the difference between booking weight and the last measured weight before delivery. Linear regression was performed to adjust for maternal age, ethnicity, alcohol consumption, smoking, exercise, gestational diabetes, parity, child's gender, marital status, education, pre-pregnancy BMI, total energy and weeks at last antenatal visit.

Results: Among key food groups examined, consumption of sweet beverages excluding tea and coffee was significantly associated with greater GWG $(\beta=200 \mathrm{~g}$ increase in GWG per $100 \mathrm{ml}$, 95\% CI: $0.001-0.003$ ) after adjustments for confounders. Meat, fish, seafood, eggs, dessert and snacks, fast food and total dairy intake were not found to be independently associated with GWG.

Conclusion: Consumption of sweetened beverages is positively associated with GWG. Their intakes should be cautioned during pregnancy to mitigate risk of excessive weight gain.

\section{4}

GLP-1 response to a glucose load and its associated outcomes in gestational diabetes (GDM) pregnancies and controls: Rationale and Protocol

Nithya Sukumar ${ }^{1}$, Gail Pounder ${ }^{2}$, Ilona Goljan ${ }^{3}$, Selvin Selvamoni ${ }^{4}$, Hema Venkataraman ${ }^{5}$, Ponnusamy Saravanan ${ }^{6}$

${ }^{1}$ Warwick Medical School, University of Warwick, UK; ${ }^{2}$ George Eliot Hospital, Nuneaton, UK; ${ }^{3}$ George Eliot Hospital, Nuneaton, UK; ${ }^{4}$ George Eliot Hospital, Nuneaton, UK; ${ }^{5}$ Warwick Medical School, University of Warwick, UK; ${ }^{6}$ Warwick Medical School, University of Warwick, UK

Research Question: Glucagon-like peptide-1 (GLP-1) is a hormone which potentiates post-prandial insulin secretion. Levels are reduced by $20-50 \%$ in type 2 diabetes and pre-diabetes but its role in GDM is inconclusive. Our primary aim is to assess whether such reduced response is present in women at risk of GDM. Methods: This is a sub-study within the longitudinal PRiDE cohort. To demonstrate a $20 \%$ lower mean AUC for GLP-1 in GDM with $90 \%$ power at $5 \%$ significance, the estimated sample size is 26 cases (recruitment target: 30 GDM, 60 controls). Blood will be drawn at $30 \mathrm{~min}$ intervals during $75 \mathrm{~g}$ 2-hour glucose tolerance test. $40 \mathrm{uL} / \mathrm{ml}$ of a DPP- 4 inhibitor added to prevent degradation of GLP-1.

Results: 45 women have been recruited into the sub-study at a mean gestation of $25+6$ weeks. Their baseline characteristics are $($ mean $\pm S D)$ : age $29.4 \pm 4.3$ years; prepregnancy BMI $30.9 \pm 7.2 \mathrm{~kg} / \mathrm{m} 2 ; \quad 1$ st trimester waist circumference
$97.4 \pm 17.4 \mathrm{~cm} .11 \%$ of women have been diagnosed with GDM using the modified WHO criteria.

Conclusion: Recruitment will be completed by end-2015 followed by analysis of GLP-1 and related biochemical parameters. This study will enable further characterization of women at high-risk of developing GDM as well as the effects of GLP-1 on adverse outcomes due to GDM.

\section{7}

Diabetic pregnancies have augmented umbilical venous supply to the right fetal liver lobe linearly related to accelerated growth

Agnethe Lund ${ }^{1}$, Ebbing Cathrine ${ }^{2}$, Torvid Kiserud ${ }^{3}$, Jørg Kessler ${ }^{4}$

${ }^{1}$ Dept. of obstetrics and gynecology, Haukeland University Hospital, Norway; ${ }^{2}$ Dept. of obstetrics and gynecology, Haukeland University Hospital; ${ }^{3}$ University of Bergen and Haukeland University Hospital, Dept Ob/Gyn; ${ }^{4}$ Dept. of obstetrics and gynecology, Haukeland University Hospital

Research question: Umbilical venous flow to the fetal liver determines growth. This blood perfuses the entire left lobe and to a varying degree the right lobe via the left portal vein (LPV) reflected in the magnitude of blood velocity in the LPV.

Research question: Is umbilical blood distribution to the fetal liver altered in diabetic pregnancies, and is it related to growth? Method: In accordance with ethical approval this longitudinal study of women with pre-gestational diabetes included ultrasound examination at gestational week 20, 24, 28, 32 and 36. Time-averaged maximum blood velocity in the LPV was measured and birthweight noted and compared with a reference population using $\mathrm{z}$-scores.

Results: For 22 completed diabetic pregnancies the median gestational age at birth was $38+2$ weeks (range $30+5-40+4$ ), and birthweight was $3570 \mathrm{~g}$ (range 1900-5020g). LPV blood velocity and birthweight were increased (z-score $0.91,95 \%$ CI $0.61-1.18$ and $1.03,95 \%$ CI $0.76-1.30$, respectively) compared with the reference population. There was a linear relation (Beta $0.31, p=0.08$ ) between LPV velocity beyond 30 weeks of gestation and birthweight z-scores.

Conclusion: Diabetic pregnancies are associated with augmented umbilical venous supply to the right liver lobe, which is linearly correlated to accelerated growth in the last trimester.

\section{1}

Low breast-feeding rates in women with high risk of gestational diabetes mellitus: Differences between South-Asians and Caucasians

Rajni Vekaria ${ }^{1}$, Ree'Thee Bhatt ${ }^{2}$, Angeliki Xiarchou ${ }^{3}$, Karen Shorthose ${ }^{4}$, Hema Venkataraman ${ }^{5}$, Nithya Sukumar ${ }^{6}$, Ponnusamy Saravanan ${ }^{7}$

${ }^{1}$ University of Warwick; ${ }^{2}$ University of Warwick; ${ }^{3}$ George Eliot Hospital NHS Trust; ${ }^{4}$ George Eliot Hospital NHS Trust; ${ }^{5}$ George 
Eliot Hospital NHS Trust, University of Warwick; ${ }^{6}$ University of Warwick; ${ }^{7}$ George Eliot Hospital NHS Trust, University of Warwick

Research question: Breastfeeding reduces the metabolic risk of mothers and offspring. Investigating breastfeeding rates, predictors and ethnic differences in women at risk of gestational diabetes mellitus (GDM).

Methods: Self-reported breastfeeding rates were collected retrospectively from a sub-set of (230/573) women at least 6-months postpartum. Logistic regression and Chi2test were performed to study ethnic differences.

Results: $65 \%$ initiated breastfeeding (national average $73.9 \%$; $\mathrm{p}=0.002$ ) and at 6-weeks, $30 \%$ continued (national average $47.2 \% ; \mathrm{p}<0.001)$. South Asians (SA) were more likely to initiate breastfeeding (OR:3.9, $\mathrm{p}=0.015)$ and exclusively breastfed for longer compared to Caucasians (mean $\pm \mathrm{SD}$ : $237.3 \pm 93.7$ vs $122.0 \pm 68.3$ days, $\mathrm{p}<0.001)$.

After adjusting for BMI and ethnicity, household income above $£ 20$, 800 was an independent predictor of initiating breastfeeding (OR: 2.246, $\mathrm{p}=0.014$ ), which also predicted continuation at 6-weeks (OR:2.750, $\mathrm{p}=0.044)$ along with higher occupational class (OR:2.577, $\mathrm{p}=0.021$ ).

Mothers with higher qualifications were more likely to breastfeed after adjusting for age, BMI and ethnicity (OR:2.754, $\mathrm{p}=0.005)$.

Conclusions: Breastfeeding rates in this population were lower than the national average with SAs more likely to breastfeed than Caucasians and continue exclusively for longer. Lower household income, qualifications and employment class predicted low breastfeeding initiation rates. Educating this subpopulation is vital to reduce future metabolic risk to both mother and offspring.

\section{7}

Developing Computational Intelligence-based Practical Predictive Analytics and Decisioning Systems Frameworks for Gestational Diabetes Management

Zacchaeus Oni Omogbadegun ${ }^{1}$, Sena Rita Okuboyejo ${ }^{2}$

${ }^{1}$ Covenant University, Ota, Nigeria; ${ }^{2}$ Covenant University, Ota, Nigeria

Research question: Gestational Diabetes Mellitus (GDM), a syndrome characterized by glucose intolerance with onset or first recognition during pregnancy, is one of pregnancy's most common medical complications. How could we predict and diagnose GDM early in pregnancy to reduce global fetal and infant loss, perinatal morbidity, neonatal mortality, and malformations rates as there are no tools for an early diagnosis of GDM, or, even more importantly, a protocol that allows prevention of GDM?

Methods: Comprehensive literature review on institutional protocol for GDM diagnostic criteria carried out. Distributed questionnaires and interviewed Obstetricians/Gynaecologists and Dieticians in Reproductive Health (OGDRH) selected from public and private healthcare facilities in Nigeria. Qualitative analytical approach, a hybridization of computational intelligence (CI) technologies, and ninety-seven contingently combined possible GDM application rules stored in a built knowledge base used to develop practical predictive analytics and decisioning systems frameworks empowering OGDRH detect and manage GDM with precision.

Results: CI-based frameworks where healthcare system leverages on the predictive power of artificial intelligence tools to predict high and accurate GDM probabilities with significant effects on prevention of ambiguity, vagueness, and imprecision inherent in medical diagnosis.

Conclusion: The increasing comorbid epidemics of GDM conditions mandates a thorough examination of best therapies and prevention strategies.

\section{9}

Maternal dietary patterns and Gestational Diabetes Mellitus in a multi-ethnic Asian cohort

Jamie V de Seymour ${ }^{1}$, Airu Chia ${ }^{2}$, Marjorelee Colega ${ }^{3}$, Beatrix Jones ${ }^{4}$, Cai Shirong ${ }^{5}$, Keith Godfrey ${ }^{6}$, Kenneth Kwek ${ }^{7}$, Saw Seang Mei ${ }^{8}$, Peter Gluckman ${ }^{9}$, Cath Conlon ${ }^{10}$, Yap Seng Chong ${ }^{11}$, Philip Baker ${ }^{12}$, Mary FF Chong ${ }^{13}$

${ }^{1}$ Liggins Institute, The University of Auckland, New Zealand;

${ }^{2}$ Singapore Institute for Clinical Sciences, $A^{*} S T A R$, Singapore;

${ }^{3}$ Singapore Institute for Clinical Sciences, $A^{*} S T A R$, Singapore;

${ }^{4}$ Massey University, New Zealand; ${ }^{5}$ Department of Obstetrics \& Gynaecology, Yong Loo Lin School of Medicine, National University of Singapore, Singapore; ${ }^{6}$ Medical Research Council Lifecourse Epidemiology Unit and NIHR Southampton Biomedical Research Centre, University of Southampton and University Hospital Southampton NHS Foundation;

${ }^{7}$ Department of Maternal Fetal Medicine, KK Women's and Children's Hospital, Singapore; ${ }^{8}$ Saw Swee Hock School of Public Health, National University of Singapore and National University Health System, Singapore; ${ }^{9}$ Singapore Institute for Clinical Sciences, $A^{*} S T A R$, Singapore \& Liggins Institute, The University of Auckland, New Zealand; ${ }^{10}$ Massey University, New Zealand; ${ }^{11}$ Singapore Institute for Clinical Sciences, $A^{*} S T A R$, Singapore; ${ }^{12}$ Liggins Institute, The University of Auckland, New Zealand; ${ }^{13}$ Singapore Institute for Clinical Sciences, $A^{*} S T A R$, Singapore \& Department of Paediatrics, Yong Loo Lin School of Medicine, National University of Singapore, Singapore

Background: Evidence examining the association between maternal dietary patterns and gestational diabetes mellitus (GDM) risk is limited, particularly in Asian populations. We examined this in the Singapore GUSTO mother-offspring cohort.

Methods: At 26-28 weeks gestation, maternal dietary intakes from participants of the GUSTO study were ascertained using 
24hour dietary recalls, and $75 \mathrm{~g}$ oral glucose tolerance tests were conducted with GDM diagnosis according to 1999 WHO guidelines. Dietary patterns were identified using factor analysis, and multivariate logistic regression analyses performed.

Results: In 822 mothers, $18 \%$ were diagnosed with GDM. Three dietary patterns were identified: a vegetable, fruit and rice-based diet (VFR), a seafood and noodle-based diet ( $\mathrm{SfN}$ ), and a pasta, cheese and processed meat diet (PCP). After adjusting for confounding variables, the SfN pattern was significantly associated with lower likelihood of GDM (OR per $\mathrm{SD}=0.77 ; 0.62,0.95)$. Women with $\mathrm{SfN}$ scores in the highest tertile had higher total calorie, protein, and fat but lower carbohydrate intakes than those in the lowest tertile (2058 vs 1830 $\mathrm{kcal} /$ day; 16.2 vs $15.2 \%$; 33.7 vs $31.2 \%$; 50.1 vs $53.6 \%$ of energy respectively; $\mathrm{p}<0.05$ for all). No associations were seen between VFR and PCP diets with GDM risk.

Conclusion: A seafood and noodle-based dietary pattern was associated with lower risk of GDM.

\section{9}

Epigenome-wide and transcriptome-wide analyses reveal gestational diabetes is associated with alterations in the Human Leukocyte Antigen complex

Alexandra M. Binder ${ }^{1}$, Jessica LaRocca ${ }^{2}$, Corina Lesseur ${ }^{3}$, Carmen Marsit ${ }^{4}$

${ }^{1}$ Obstetrics and Gynecology Epidemiology Center, Department of Obstetrics, Gynecology and Reproductive Biology, Brigham and Women's Hospital, Harvard Medical School, Boston, MA, USA; ${ }^{2}$ Harvard University Center for the Environment, Harvard University, Cambridge, MA, USA; ${ }^{3}$ Department of Pharmacology and Toxicology, and Section of Biostatistics and Epidemiology, Department of Community and Family Medicine, Geisel School of Medicine at Dartmouth, Hanov; ${ }^{4}$ Department of Pharmacology and Toxicology, and Section of Biostatistics and Epidemiology, Department of Community and Family Medicine, Geisel School of Medicine at Dartmouth, Hanov

Research question: Gestational diabetes mellitus (GDM) affects approximately $18 \%$ of pregnancies in the United States and increases the risk of adverse health outcomes in the offspring. Since adult disease propensities may be set by anatomical and molecular alterations in the placenta associated with GDM, we aimed to assess epigenetic changes in the placenta resulting from GDM that may affect fetal programming. Methods: We measured genome-wide methylation (Infinium HumanMethylation450 Beadchips) and expression (Affymetrix Transcriptome Microarrays) in placental tissue of 41 GDM cases and 41 matched pregnancies without maternal complications from the Harvard Epigenetic Birth Cohort.

Results: Specific transcriptional and epigenetic perturbations associated with GDM status included alterations in the major histocompatibility complex (MHC) region, which were validated in an independent cohort, the Rhode Island Child
Health Study. Gene ontology enrichment among gene regulation influenced by GDM revealed an over-representation of immune response pathways among differential expression, reflecting these coordinated changes in the MHC region.

Conclusions: Our study represents the largest investigation of transcriptomic and methylomic differences associated with GDM, providing comprehensive insight into the molecular basis of GDM induced fetal (re)programming.

\section{2}

The double burden of disease in the rural and urban Free State

Corinna Walsh ${ }^{1}$, Reinette Tydeman-Edwards ${ }^{2}$, Cornel Van Rooyen $^{3}$

${ }^{1}$ University of the Free State; ${ }^{2}$ University of the Free State;

${ }^{3}$ University of the Free State

Research question: This cross-sectional study formed part of the Assuring Health for All in the Free State (AHA FS) study and aimed to determine whether a double burden of undernutrition in children and overnutrition in adults was present. Methods: Diet and anthropometric status of adults (25-64 years) in the rural and urban Free State were compared. Associations between anthropometric status of adults and preschool children $(<7$ years old $)$ from the same households were determined. 553 adults from the rural Southern Free State and 419 adults from urban Mangaung participated. A qualitative food frequency questionnaire was used to assess dietary patterns. Body mass index and waist circumference were determined in adults and height-for-age, weight-for-age and weight-for-height in 176 children.

Results: High sugar, salt and fat intakes and low fruit and vegetable intakes were common in both rural and urban participants. About two-thirds of both rural (65.6\%) and urban (66.2\%) women were overweight or obese. Fewer men (23.3\% rural and $16 \%$ urban) were overweight or obese. More than two thirds of stunted, underweight and wasted children lived with an overweight or obese caregiver.

Conclusion: Unhealthy eating patterns, accompanied by a double burden of disease were identified in both rural and urban communities.

\section{4}

Cohort profile: The Across-generation Study in Chinese Females

Wanghong $\mathrm{Xu}^{1}$, Hong Fang ${ }^{2}$, Guoyou Qin ${ }^{3}$

${ }^{1}$ School of Public Health Fudan University; ${ }^{2}$ Minhang CDC of Shanghai, China; ${ }^{3}$ School of Public Health Fudan University

Research question: Malnutrition in early life has been an important risk factor for subsequent chronic non-communicable 
diseases (NCDs). However, its effect on the risk of NCDs in offspring and biological mechanisms remain unclear.

Methods: We recruited 10,324 females from 3,888 families in Shanghai, China, between 2012 and 2013 to evaluate the cross-generational impact of birth weight on the risk of NCDs in Chinese females.

Results: All participants completed a baseline survey and anthropometrics. Noteworthy characteristic of this cohort is that about 10\% (380) families included F1, F2 and F3 generations and were with the F1 generation exposing to the 1959-61 nation-wide famine in early life. Currently, the cohort of females is being followed via triennial in-person recontact to collect blood samples and information on incident NCDs.

Conclusion: The resources from the cohort will be valuable to better understand the main drive of the rapidly increasing trend of NCDs in China, and to develop an effective intervention approach to curb the upward trend in the country.

\section{7}

Dental biomarkers for evaluating chronic disease risk later in life: longitudinal evidence from the Bolivian Amazon

Philippe P Hujoel $^{1}$, Dan T.A. Eisenberg ${ }^{2}$, Daniel A. Enquobahrie $^{3}$, Paula S. Nurius ${ }^{4}$, Annette L. Fitzpatrick ${ }^{5}$

${ }^{1}$ Departments of Oral Health Sciences and Epidemiology, University of Washington; ${ }^{2}$ Department of Anthropology and the Center for Studies in Demography and Ecology, University of Washington; ${ }^{3}$ Department of Epidemiology, University of Washington; ${ }^{4}$ Department of Social Work, University of Washington; ${ }^{5}$ Departments of Family Medicine, Epidemiology and Global Health, University of Washington

Research question: Are dental markers (influenced by childhood malnutrition) associated with adolescent anthropometrics and health indicators?

Methods: This study extends a prospective cohort from the Bolivian Amazon. The study sample was comprised of approximately 600 adolescents, aged 11-17 years, who were included in a 9-year longitudinal study as young children. The presence of any enamel hypoplasia $(\mathrm{EH})$ in the permanent dentition and dental occlusion, quantified using digital photography, was evaluated in relation to measures of adolescent growth and health including blood pressure, HbAlc, hemoglobin, and white blood cell count.

Results: The dual burden of under-nutrition in childhood (45\%) and overweight in adulthood (23\%) afflict the study population. Regression analyses, adjusted for age and gender, will indicate whether dental markers are associated with outcomes and estimate the prevalence ratio for each. Additional analyses will aim to better understand the role of anthropometrics in the dental marker-health outcomes relationship. [Data collection concludes in August 2015 and specific results will be presented.]
Conclusion: This study has the potential to demonstrate associations between permanent dental markers, reflecting early childhood exposures, and adolescent health. This measure may ultimately be used as a biomarker for evaluating chronic disease risk later in life in a low-resource settings.

\section{5}

Risk factors for development of future chronic disease from an Indigenous Australian cohort

Loretta Weatherall ${ }^{1}$, Roger Smith ${ }^{2}$, Clare Collins ${ }^{3}, \mathrm{Kym} \mathrm{Rae}^{4}$, Eugenie Lumbers 5

${ }^{1}$ University of Newcastle; ${ }^{2}$ University of Newcastle; ${ }^{3}$ University of Newcastle; ${ }^{4}$ University of Newcastle; ${ }^{5}$ University of Newcastle

Research question: The life expectancy of Indigenous Australians is amongst the poorest and burden of chronic diseases is partly to blame. Pregnancy cohort studies in Indigenous populations are unfortunately scarce as they may be of great value in determining the causes and trajectories of poor health in these populations.

Methods: Prospective longitudinal pregnancy cohort design comprising 224 Indigenous women (mean age 26yrs, range: 13.8-40.9yrs) to date (Ethics Approval No: 08/05/21/4.01).

Results: Median birth weight was 3180g (910-5430g) with $28.4 \%$ of the infants born small for gestational age (SGA; 90th centile). Although the median gestational age at delivery was 39.1 weeks (31.5-43 weeks), $13.6 \%$ of babies were born preterm (<37 wks). $39.3 \%$ of infants were admitted to neonatal intensive care nurseries. $47.5 \%$ of women reported smoking during their pregnancy. The mean BMI was $30.7 \mathrm{~kg} / \mathrm{m} 2$ (15-52). 61.5\% of the women in the study were classified as being overweight/obese. The mean \% body fat was $41.7 \%$ (1763\%). 6\% of the participants report GDM diagnosis and $2.6 \%$ report Type II diabetes.

Conclusion: Analysis of the birth outcomes from the first 110 women in the cohort demonstrates that infants born from this cohort are at significantly greater risk of chronic disease development later in life.

\section{4}

GLP-1 response to a glucose load and its associated outcomes in gestational diabetes (GDM) pregnancies and controls: Rationale and Protocol

Nithya Sukumar ${ }^{1}$, Gail Pounder ${ }^{2}$, Ilona Goljan ${ }^{3}$, Selvin Selvamoni ${ }^{4}$, Hema Venkataraman ${ }^{5}$, Ponnusamy Saravanan ${ }^{6}$

${ }^{1}$ Warwick Medical School, University of Warwick, UK; ${ }^{2}$ George Eliot Hospital, Nuneaton, UK; ${ }^{3}$ George Eliot Hospital, Nuneaton, $U K ;{ }^{4}$ George Eliot Hospital, Nuneaton, UK; ${ }^{5}$ Warwick Medical School, University of Warwick, UK; ${ }^{6}$ Warwick Medical School, University of Warwick, UK 
Research Question: Glucagon-like peptide-1 (GLP-1) is a hormone which potentiates post-prandial insulin secretion. Levels are reduced by $20-50 \%$ in type 2 diabetes and pre-diabetes but its role in GDM is inconclusive. Our primary aim is to assess whether such reduced response is present in women at risk of GDM.

Methods: This is a sub-study within the longitudinal PRiDE cohort. To demonstrate a 20\% lower mean AUC for GLP-1 in GDM with $90 \%$ power at $5 \%$ significance, the estimated sample size is 26 cases (recruitment target: 30 GDM, 60 controls). Blood will be drawn at $30 \mathrm{~min}$ intervals during $75 \mathrm{~g} \mathrm{2-}$ hour glucose tolerance test. $40 \mathrm{uL} / \mathrm{ml}$ of a DPP-4 inhibitor added to prevent degradation of GLP-1.

Results: 45 women have been recruited into the sub-study at a mean gestation of $25+6$ weeks. Their baseline characteristics are $($ mean $\pm \mathrm{SD})$ : age $29.4 \pm 4.3$ years; prepregnancy BMI $30.9 \pm 7.2 \mathrm{~kg} / \mathrm{m} 2$; $1 \mathrm{st}$ trimester waist circumference $97.4 \pm 17.4 \mathrm{~cm} .11 \%$ of women have been diagnosed with GDM using the modified WHO criteria.

Conclusion: Recruitment will be completed by end-2015 followed by analysis of GLP-1 and related biochemical parameters. This study will enable further characterization of women at high-risk of developing GDM as well as the effects of GLP-1 on adverse outcomes due to GDM.

\section{1}

Low breast-feeding rates in women with high risk of gestational diabetes mellitus: Differences between South-Asians and Caucasians

Rajni Vekaria ${ }^{1}$, Ree'Thee Bhatt ${ }^{2}$, Angeliki Xiarchou ${ }^{3}$, Karen Shorthose ${ }^{4}$, Hema Venkataraman ${ }^{5}$, Nithya Sukumar ${ }^{6}$, Ponnusamy Saravanan ${ }^{7}$

${ }^{1}$ University of Warwick; ${ }^{2}$ University of Warwick; ${ }^{3}$ George Eliot Hospital NHS Trust; ${ }^{4}$ George Eliot Hospital NHS Trust; ${ }^{5}$ George Eliot Hospital NHS Trust, University of Warwick; ${ }^{6}$ University of Warwick; ' George Eliot Hospital NHS Trust, University of Warwick

Research question: Breastfeeding reduces the metabolic risk of mothers and offspring. Investigating breastfeeding rates, predictors and ethnic differences in women at risk of gestational diabetes mellitus (GDM).

Methods: Self-reported breastfeeding rates were collected retrospectively from a sub-set of (230/573) women at least 6-months postpartum. Logistic regression and Chi2test were performed to study ethnic differences.

Results: $65 \%$ initiated breastfeeding (national average $73.9 \%$; $\mathrm{p}=0.002$ ) and at 6-weeks, 30\% continued (national average $47.2 \% ; \mathrm{p}<0.001)$. South Asians (SA) were more likely to initiate breastfeeding (OR:3.9, $\mathrm{p}=0.015)$ and exclusively breastfed for longer compared to Caucasians (mean $\pm S D$ : $237.3 \pm 93.7$ vs $122.0 \pm 68.3$ days, $\mathrm{p}<0.001)$.

After adjusting for BMI and ethnicity, household income above $£ 20,800$ was an independent predictor of initiating breastfeeding (OR:2.246, $\mathrm{p}=0.014)$, which also predicted continuation at 6-weeks (OR:2.750, $\mathrm{p}=0.044$ ) along with higher occupational class (OR:2.577, $\mathrm{p}=0.021$ ).

Mothers with higher qualifications were more likely to breastfeed after adjusting for age, BMI and ethnicity (OR:2.754, p = 0.005).

Conclusions: Breastfeeding rates in this population were lower than the national average with SAs more likely to breastfeed than Caucasians and continue exclusively for longer. Lower household income, qualifications and employment class predicted low breastfeeding initiation rates. Educating this subpopulation is vital to reduce future metabolic risk to both mother and offspring.

\section{9}

Maternal dietary patterns and Gestational Diabetes Mellitus in a multi-ethnic Asian cohort

Jamie V de Seymour ${ }^{1}$, Airu Chia ${ }^{2}$, Marjorelee Colega ${ }^{3}$, Beatrix Jones ${ }^{4}$, Cai Shirong ${ }^{5}$, Keith Godfrey ${ }^{6}$, Kenneth Kwek ${ }^{7}$, Saw Seang $\mathrm{Mei}^{8}$, Peter Gluckman ${ }^{9}$, Cath Conlon ${ }^{10}$, Yap Seng Chong ${ }^{11}$, Philip Baker ${ }^{12}$, Mary FF Chong ${ }^{13}$

${ }^{1}$ Liggins Institute, The University of Auckland, New Zealand;

${ }^{2}$ Singapore Institute for Clinical Sciences, $A^{*} S T A R$, Singapore;

${ }^{3}$ Singapore Institute for Clinical Sciences, $A^{*} S T A R$, Singapore;

${ }^{4}$ Massey University, New Zealand; ${ }^{5}$ Department of Obstetrics \& Gynaecology, Yong Loo Lin School of Medicine, National University of Singapore, Singapore; ${ }^{6}$ Medical Research Council Lifecourse Epidemiology Unit and NIHR Southampton Biomedical Research Centre, University of Southampton and University Hospital Southampton NHS Foundation; ${ }^{7}$ Department of Maternal Fetal Medicine, KK Women's and Children's Hospital, Singapore; ${ }^{8}$ Saw Swee Hock School of Public Health, National University of Singapore and National University Health System, Singapore; ${ }^{9}$ Singapore Institute for Clinical Sciences, $A^{*} S T A R$, Singapore \& Liggins Institute, The University of Auckland, New Zealand; ${ }^{10}$ Massey University, New Zealand; ${ }^{11}$ Singapore Institute for Clinical Sciences, $A^{*} S T A R$, Singapore; ${ }^{12}$ Liggins Institute, The University of Auckland, New Zealand; ${ }^{13}$ Singapore Institute for Clinical Sciences, $A^{*} S T A R$, Singapore \& Department of Paediatrics, Yong Loo Lin School of Medicine, National University of Singapore, Singapore

Background: Evidence examining the association between maternal dietary patterns and gestational diabetes mellitus (GDM) risk is limited, particularly in Asian populations. We examined this in the Singapore GUSTO mother-offspring cohort.

Methods: At 26-28 weeks gestation, maternal dietary intakes from participants of the GUSTO study were ascertained using 24 hour dietary recalls, and 75 g oral glucose tolerance tests were conducted with GDM diagnosis according to 1999 WHO guidelines. Dietary patterns were identified using factor analysis, and multivariate logistic regression analyses performed.

Results: In 822 mothers, 18\% were diagnosed with GDM. Three dietary patterns were identified: a vegetable, fruit and 
rice-based diet (VFR), a seafood and noodle-based diet ( $\mathrm{SfN}$ ), and a pasta, cheese and processed meat diet (PCP). After adjusting for confounding variables, the SfN pattern was significantly associated with lower likelihood of GDM (OR per $\mathrm{SD}=0.77 ; 0.62,0.95)$. Women with $\mathrm{SfN}$ scores in the highest tertile had higher total calorie, protein, and fat but lower carbohydrate intakes than those in the lowest tertile (2058 vs 1830 $\mathrm{kcal} /$ day; 16.2 vs $15.2 \%$; 33.7 vs $31.2 \%$; 50.1 vs $53.6 \%$ of energy respectively; $\mathrm{p}<0.05$ for all). No associations were seen between VFR and PCP diets with GDM risk.

Conclusion: A seafood and noodle-based dietary pattern was associated with lower risk of GDM.

\section{1}

Moderate exercise with low frequency training improves thermogenic activity in early overfeeding rats without changes in VO2max capacity

Audrei Pavanello ${ }^{1}$, Ananda Malta ${ }^{2}$, Tatiane Aparecida Ribeiro $^{3}$, Laíze Perón Tófolo ${ }^{4}$, Thauany Fabbri ${ }^{5}$, Adriane Barreto Gôngora ${ }^{6}$, Lucas Eduardo Cardoso ${ }^{7}$, Kesia Palma-Rigo ${ }^{8}$, Paulo Cezar de Freitas Mathias ${ }^{9}$

${ }^{1}$ Laboratory of Secretion Cell Biology, Department of Biotechnology, Genetics and Cell Biology, State University of Maringá, MaringálPR - Brazil; ${ }^{2}$ Laboratory of Secretion Cell Biology, Department of Biotechnology, Genetics and Cell Biology, State University of Maringá, MaringálPR - Brazil; ${ }^{3}$ Laboratory of Secretion Cell Biology, Department of Biotechnology, Genetics and Cell Biology, State University of Maringá, MaringálPR - Brazil;

${ }^{4}$ Laboratory of Secretion Cell Biology, Department of Biotechnology, Genetics and Cell Biology, State University of Maringá, MaringálPR - Brazil; ${ }^{5}$ Laboratory of Secretion Cell Biology, Department of Biotechnology, Genetics and Cell Biology, State University of Maringá, MaringálPR - Brazil; ${ }^{6}$ Laboratory of Secretion Cell Biology, Department of Biotechnology, Genetics and Cell Biology, State University of Maringá, MaringálPR - Brazil; ${ }^{7}$ Laboratory of Secretion Cell Biology, Department of Biotechnology, Genetics and Cell Biology, State University of Maringá, MaringálPR - Brazil; ${ }^{8}$ Laboratory of Secretion Cell Biology, Department of Biotechnology, Genetics and Cell Biology, State University of Maringá, MaringálPR - Brazil, ${ }^{9}$ Laboratory of Secretion Cell Biology, Department of Biotechnology, Genetics and Cell Biology, State University of Maringá, MaringálPR - Brazil

Hypothesis: Is moderate intensity and low frequency exercise training enough to change physical aerobic capacity and interscapular brown adipose tissue (iBAT) activity in early-overfeedinduced obesity (SL)?

Methods: Rat litters were adjusted to 9 pups for normal litters (NL) and 3 pups for SL group, weaned at 21 days-old. Youngest were separated in sedentary and exercised in treadmill (NL SED, SL SED, and NL SED,SL EXE). Performance tests at 30, 45, 60 and 80 days-old were applied to measured VO2max capacity. Training were made from 30- to 80-days- old, 3 times/week, at $60 \%$ of VO2max. Temperature transponders were implanted beneath iBAT. At 100 days-old animals were euthanize. IBAT fat pad was dissected, weighted and frozen to further analysis.

Results: Exercise reduce iBAT fat in SL rats (SL SED vs SL EXE, $p<0,001)$. iBAT activity was improved in both groups during lights-on period (NL SED vs NL EXE, $\mathrm{p}<0,05$; SL SED vs SL EXE, $\mathrm{p}<0,01)$. Exercise improved workload (NL SED vs NL EXE, $\mathrm{p}<0,001$; SL SED vs SL EXE, $\mathrm{p}<0,001$ ). VO2max increased in exercised NL (NL SED vs NL EXE $p<0,05)$; although, no changes were observed in SL. Conclusion: Improving thermogenesis by moderate training might be crucial to balance metabolism, attenuating obesity.

\section{9}

Pancreatic sympathectomy causes metabolic disorders and impaired insulin secretory response by pancreatic beta cells kelly Valério Prates ${ }^{1}$, Ananda Malta ${ }^{2}$, Rosiane Aparecida Miranda ${ }^{2}$, Flavio Andrade Francisco ${ }^{3}$, Claudinéia Conationi da Silva Franco ${ }^{4}$, Elaine Vieira ${ }^{5}$, Kesia Palma Rigo ${ }^{6}$, Paulo Cezar de Freitas Mathias ${ }^{7}$

${ }^{1}$ State University of Maringá - MaringálPR, Brazil; ${ }^{2}$ State University of Maringá - MaringálPR, Brazil; ${ }^{3}$ State University of Maringá - MaringálPR, Brazil; ${ }^{4}$ State University of Maringá MaringálPR, Brazil; ${ }^{5}$ State University of Maringá - MaringálPR, Brazil;; ${ }^{6}$ State University of Maringá - MaringálPR, Brazil; ${ }^{7}$ State University of Maringá - MaringálPR, Brazil

Background: Impairment of autonomic nervous system (ANS) is one of hallmarks of obesity. It has been shown that sympathetic (SNS) activity is decreased in obesity. The aim of the present work was to investigate whether suppression of SNS activity to the pancreas could induce obesity and could lead to alterations in pancreatic beta cell function.

Methods: At 60-day-old, rats underwent surgery for ablation of neural sympathetic termination of the pancreas (Sym group). Another group of rats was used as control (Sham group). At 120-day-old, blood glucose and insulin were measured. Protein expression was detected by western blotting technique. Data were analysed using one-way ANOVA or Student t-test by the GraphPad Prism version 6.01.

Results: Sym rats exhibited increase in body weight, food intake and fat pad as compared to Sham rats $(\mathrm{p}<0.01)$. Sympathectomy caused glucose intolerance and hypoinsulinemia. Isolated islets from Sym rats had decrease in glucose-stimulated insulin secretion as compared to Sham rats $(\mathrm{p}<0.01)$. This effect was in parallel with upregulation of the $\alpha-2$ adrenergic receptors expression in islets isolated from Sym rats.

Conclusion: Pancreatic sympathectomy alters energy metabolism and causes increased expression of the $\alpha 2$-adrenergic receptors that could explain altered pancreatic beta-cell response in this animal model. 
4481

\section{Enalapril Restores Endothelium-Derived Hyperpolarizing Factor-Mediated Relaxation in Mesenteric Artery of Prenatal Testosterone Exposed Adult Rats}

K Sathishkumar ${ }^{1}$, Amar More ${ }^{2}$, Jay Mishra ${ }^{3}$, Gary Hankins ${ }^{4}$, Chandra Yallampalli ${ }^{5}$

${ }^{1}$ University of Texas Medical Branch; ${ }^{2}$ University of Texas Medical Branch; ${ }^{3}$ University of Texas Medical Branch;

${ }^{4}$ University of Texas Medical Branch; ${ }^{5}$ Baylor

We tested whether the angiotensin-converting enzyme inhibitor enalapril restores endothelium-derived hyperpolarizing factor (EDHF) function through regulating the activities of small (SK3) and intermediate (IK1) conductance calciumactivated potassium channels in the mesenteric arteries of adult rats exposed to elevated testosterone during fetal life. Pregnant Sprague-Dawley rats were injected with vehicle or testosterone propionate $(0.5 \mathrm{mg} / \mathrm{kg} /$ day from gestation day 15-19, subcutaneously), and their 6-month-old male offspring were examined. A subset of rats in these 2 groups was administered enalapril ( $40 \mathrm{mg} / \mathrm{kg} /$ day) for 2 weeks through drinking water. Blood pressures were significantly higher in testosterone offspring compared to controls, and treatment with enalapril significantly attenuated blood pressure in testosterone offspring. EDHF relaxation in mesenteric arteries of testosterone offspring was reduced compared to controls, and it was significantly restored by enalapril treatment. IK1 channel expression and function were similar between control and testosterone rats, and it was not affected by enalapril treatment. The relaxations mediated by the SK3 were impaired in testosterone offspring, and it was normalized by enalapril treatment. Furthermore, enalapril treatment restored expression levels of SK3 channels. These results indicate that renin angiotensin signaling may be the underlying mechanism that links prenatal androgen exposure and adult life endothelial dysfunction.

\section{3}

Multigenerational sex-specific effects of maternal BPA exposure on glucose and KIC stimulated insulin secretion and islet gene expression in mice

Changhong $\mathrm{Li}^{1}$, Martha Susiarjo ${ }^{2}$, Tom van der Meer ${ }^{3}$, Cetewayo Rashid ${ }^{4}$, Amita Bansal ${ }^{5}$, Marisa Bartolomei ${ }^{6}$, Rebecca Simmons ${ }^{7}$

${ }^{1}$ Division of Endocrinology and Metabolism, The Children's Hospital of Philadelphia, 802B Abramson Research Center, Philadelphia, PA; ${ }^{2}$ Center of Excellence in Environmental Toxicology, University of Pennsylvania Perelman School of Medicine, Philadelphia, PA; Department of Cell and Developmental Biology, Perelman S; ${ }^{3}$ Center for Research on Reproduction and Women's Health, Perelman School of Medicine,
University of Pennsylvania, Philadelphia, PA; Department of Pediatrics, University of Groningen; ${ }^{4}$ Center for Research on Reproduction and Women's Health, Perelman School of Medicine, University of Pennsylvania, Philadelphia, PA; Center of Excellence in Environmental Toxicology; ${ }^{5}$ Center for Research on

Reproduction and Women's Health, Perelman School of Medicine, University of Pennsylvania, Philadelphia, PA; Center of Excellence in Environmental Toxicology; ${ }^{6}$ Center of Excellence in Environmental Toxicology, University of Pennsylvania Perelman School of Medicine, Philadelphia, PA; Department of Cell and Developmental Biology, Perelman S; ${ }^{7}$ Center for Research on Reproduction and Women's Health, Perelman School of Medicine, University of Pennsylvania, Philadelphia, PA; Center of Excellence in Environmental Toxicology

Background: Bisphenol A (BPA), an endocrine disruptor, maternal exposure leads to high body fat and impaired glucose tolerance in F1 and F2 male, but not female mice; the underlying mechanisms remain unknown. We determined multigenerational effects of maternal BPA exposure on glucose (GSIS) and $\alpha$-ketoisocaproate stimulated insulin secretion (KICSIS), and islet gene expression in mice. Methods: Islets were isolated from adult F1 and F2 offspring ( $\mathrm{n}=4-6$ per sex per group) of F0 mothers exposed to $10 \mu \mathrm{g} / \mathrm{kg} /$ day (LowerB), $10 \mu \mathrm{mg} / \mathrm{kg} /$ day (UpperB) BPA and 7\% corn oil (Control) diets. GSIS and KICSIS were determined by perifusion ramp studies; data were analysed by 2-way ANOVA. mRNA levels were determined by qPCR. P $<0.05$ was considered significant. Results: LowerB F1 and F2 males, but not females, had reduced GSIS and increased igf2 and ucp2 mRNA expression than Controls; F2 males also had increased ogdh mRNA expression. UpperB F1 and F2 males had reduced KICSIS and increased igf1 mRNA expression than Controls; F1 males also had increased basal insulin secretion, pdx1, igf2 and hnf1a mRNA expression. Conclusion: Early life lower and upper dose BPA exposure at representative human exposure levels altered insulin secretion, mitochondrial function and islet gene expression across two generations in male mice.

\section{2}

Early treatment with antidiabetic glibenclamide inhibits rat growth tumor independent of metabolism improvement

Carina Previate $^{1}$, Claudinéia Franco ${ }^{2}$, Rosiane Miranda ${ }^{3}$, Ananda Malta ${ }^{4}$, Tatiane Ribeiro ${ }^{5}$, Juliane Sant'Anna ${ }^{6}$, Marialba Prado ${ }^{7}$, Hely Morais ${ }^{8}$, Helenir Souza ${ }^{9}$, Rodrigo Gomes $^{10}$, Flávio Francisco ${ }^{11}$, Paulo Mathias ${ }^{12}$

${ }^{1}$ State University of Maringá - Laboratory of Secretion Cell Biology, Department of Biotechnology, Genetics and Cell Biology;

${ }^{2}$ State University of Maringá - Laboratory of Secretion Cell Biology, Department of Biotechnology, Genetics and Cell Biology; ${ }^{3}$ State University of Maringá - Laboratory of Secretion Cell Biology, Department of Biotechnology, Genetics and Cell Biology; ${ }^{4}$ State University of Maringá - Laboratory of Secretion Cell Biology, Department of Biotechnology, Genetics and Cell Biology; 
${ }^{5}$ State University of Maringá - Fellowship of CNPq-Brazilian Research Agency; ${ }^{6}$ State University of Maringá - Laboratory of Mutagenesis \& Genetics, Department of Cell Biology and Genetics; ${ }^{7}$ State University of Maringá - Laboratory of Mutagenesis \& Genetics, Department of Cell Biology and Genetics; ${ }^{8}$ Laboratory of Physiology, Department Physiological Sciences, State University of Londrina - Londrina/PR, Brazil; ${ }^{9}$ Laboratory of Physiology, Department Physiological Sciences, State University of Londrina - Londrina/PR, Brazil; ${ }^{10}$ Department of Physiological Sciences, State University of Maringa, Maringa, PR, Brazil; ${ }^{11}$ State University of Maringá Laboratory of Secretion Cell Biology, Department of Biotechnology, Genetics and Cell Biology; ${ }^{12}$ State University of Maringá Laboratory of Secretion Cell Biology, Department of Biotechnology, Genetics and Cell Biology

Background/Aims: Antidiabetic metformin reduces insulin resistance and equilibrates metabolic discords of diabetics; also, anticancer effects have been observed. Antitumoral metformin mechanisms are not yet disclosed; however, among other hypothesis, regarding proliferative action of insulin, metformin could be decrease tumor growth by reducing blood insulin concentration. Other antidiabetics, using different pathways to improve metabolism, such as glibenclamide, that stimulate the increase of insulin secretion, can be use as antitumoral drug testing insulin-metabolic hypothesis.

Methods: Weaned, 21-days-old, male rats by gavage received glibenclamide $2 \mathrm{mg} / \mathrm{kg}$ bw/day until they completed 100-daysold, controls received water. Pre-diabetic rats that were neonatal treated with glutamate monosodium (MSG) were also used. All adult 100-day-old rats $24 \mathrm{~h}$ after the treatment received rat breast carcinoma cells, Walker 256, to observe growth tumor.

Results: Glibenclamide improved metabolism of MSG-rats, reducing obesity, hyperinsulinemia and glucose intolerance $(\mathrm{p}<0.05)$. Walker 256 tumor was growing with same magnitude in lean and MSG-obese rats $(\mathrm{p}<0.05)$. Glibenclamide treatment reduced in $27 \%$ the tumor growth to both rat groups $(\mathrm{p}<0.05)$. Conclusion: Glibenclamide early treatment has antitumoral effects, which might not depend on metabolic changes.

\section{3}

Early treatment with metformin inhibits obesity onset and muscarinic receptor dysfunction in pancreatic islets of rats

Adriane Barreto ${ }^{1}$, Rosiane Miranda ${ }^{2}$, Luiz Barella ${ }^{3}$, Kelly Prates $^{4}$, Veridiana Moreira ${ }^{5}$, Lucas Cardoso ${ }^{6}$, Lucas Saavedra ${ }^{7}$, Flávio Francisco ${ }^{8}$, Júlio de Oliveira ${ }^{9}$, Paulo Mathias ${ }^{10}$, Claudinéia Franco ${ }^{11}$

${ }^{1}$ State University of Maringá - Laboratory of Secretion Cell Biology, Department of Biotechnology, Genetics and Cell Biology; ${ }^{2}$ State University of Maringá - Laboratory of Secretion Cell Biology, Department of Biotechnology, Genetics and Cell Biology; ${ }^{3}$ State University of Maringá - Laboratory of Secretion Cell Biology,
Department of Biotechnology, Genetics and Cell Biology; ${ }^{4}$ State University of Maringá - Laboratory of Secretion Cell Biology, Department of Biotechnology, Genetics and Cell Biology; ${ }^{5}$ State University of Maringá - Laboratory of Secretion Cell Biology, Department of Biotechnology, Genetics and Cell Biology; 'State University of Maringá - Laboratory of Secretion Cell Biology, Department of Biotechnology, Genetics and Cell Biology; ${ }^{7}$ State University of Maringá - Laboratory of Secretion Cell Biology, Department of Biotechnology, Genetics and Cell Biology; ${ }^{8}$ State University of Maringá - Laboratory of Secretion Cell Biology, Department of Biotechnology, Genetics and Cell Biology; ${ }^{9}$ Health Sciences Institute, Federal University of Mato Grosso, Sinop, MT, Brazil; ${ }^{10}$ State University of Maringá - Laboratory of Secretion Cell Biology, Department of Biotechnology, Genetics and Cell Biology; ${ }^{11}$ State University of Maringá - Laboratory of Secretion Cell Biology, Department of Biotechnology, Genetics and Cell Biology

Background/Aims: Metformin is an antidiabetic used actually for metabolic syndrome treatment. Autonomic nervous system (ANS) imbalance is associated with metabolic diseases. The goal of study was to test whether metformin could improve ANS activity in pre-diabetic rats.

Methods: Pre-diabetic rats were obtained by neonatal treatment with monosodium L-glutamate (MSG), which were treated, from weaning to 100-day-old, with metformin 250 $\mathrm{mg} / \mathrm{kg}$ body weight/day, or saline. After the end of metformin treatment, the animals were sacrificed to biometric and biochemical evaluation. ANS electrical activity was recorded. Isolated pancreatic islets were used to $\mathrm{mAChR}$ quantification by western blotting. Anti-muscarinic drugs were used to study the $\mathrm{mAChR}$ function, in vitro.

Results: Metformin treatment normalized hyper vagal response in MSG rats. Insulin secretion stimulated by glucose in isolated islets was increased in MSG rats; while, acetylcholine (Ach) induced lower insulin release. Metformin treatment did not change insulin secretion. Insulinostatic effect of $\mathrm{M} 3 \mathrm{mAChR}$ selective antagonist was significantly high in both groups. $\mathrm{M} 2 \mathrm{mAChR}$ selective antagonist increased cholinergic response in untreated rats and decreased in treated animals. The $\mathrm{M} 3 \mathrm{mAChR}$ expression was decreased in MSG tread by $38 \%$.

Conclusion: Chronic metformin treatment was effective to alleviate obesity, leading to a normalization of ANS activity and muscarinic receptors protein expression.

\section{7}

Kidney Function and Pathology in Adult Rats Following Hyperoxia Exposure during Nephrogenesis

Mariane Bertagnolli ${ }^{1}$, Anik Cloutier ${ }^{2}$, Chanel Béland ${ }^{3}$, Marie-Amélie Lukaszewski ${ }^{4}$, Megan R. Sutherland ${ }^{5}$, Anne Monique Nuyt ${ }^{6}$

${ }^{1}$ CHU Sainte-Justine Research Center and the University of Montreal; ${ }^{2} \mathrm{CHU}$ Sainte-Justine Research Center and the University of Montreal; ${ }^{3} \mathrm{CHU}$ Sainte-Justine Research Center and the 
University of Montreal; ${ }^{4} \mathrm{CHU}$ Sainte-Justine Research Center and the University of Montreal; ${ }^{5} \mathrm{CHU}$ Sainte-Justine Research Center and the University of Montreal; ${ }^{6} \mathrm{CHU}$ Sainte-Justine Research Center and the University of Montreal

Research question: Preterm neonates, born at a time when nephrogenesis (development of nephrons in the kidneys) is ongoing, are prematurely exposed to high oxygen levels at birth which may adversely impact renal development. The aim of this study was to determine the long-term effects of neonatal hyperoxia exposure on renal function and pathology.

Methods: Sprague-Dawley rat pups were raised in a hyperoxic environment (80\% oxygen) from P3-P10, during ongoing postnatal nephrogenesis. Control litters were kept in room air $(n=8$ litters/group; 1 male, 1 female /litter/age). Kidney function (urine and plasma creatinine, sodium, and protein) and morphology (renal corpuscle size, glomerulosclerosis, fibrosis, and glomerular crescents) were assessed at 1,5 and 11 months of age.

Results: Neonatal hyperoxia exposure had no impact on body or kidney weights. Creatinine clearance was significantly reduced following hyperoxia exposure at $5 \mathrm{mo}$; there was no significant effect on renal function at $1 \mathrm{mo}$ or $11 \mathrm{mo}$. The percentage of crescentic glomeruli (indicative of glomerular injury) was markedly increased in $11 \mathrm{mo}$ hyperoxia-exposed males. Renal corpuscle size, glomerulosclerosis index, and renal fibrosis were not affected.

Conclusion: Findings suggest that exposure to high oxygen levels during development may impact renal functional capacity and increase susceptibility to renal disease in adulthood.

\section{2}

Postnatal overnutrition programs thyroid hormone metabolism and function in adult rat progeny

Patricia Cristina Lisboa ${ }^{1}$, Elaine de Oliveira ${ }^{2}$, Ellen P. S. Conceição ${ }^{3}$, Egberto Gaspar Moura ${ }^{4}$

${ }^{1}$ State University of Rio de Janeiro; ${ }^{2}$ State University of Rio de Janeiro; ${ }^{3}$ State University of Rio de Janeiro; ${ }^{4}$ State University of Rio de Janeiro

Research Question: Early overnutrition (EO) during lactation leads to obesity and lower serum thyroid hormones (TH) at adulthood. Here, we elucidate the mechanism of thyroid dysfunction in this programming model.

Methods: EO was induced by litter size reduction (3 pups/ litter, SL group) on PN3, while control group had 10 pups/ litter. Rats were euthanized at PN180.

Results: SL group presented lower hypothalamic TRH, intrapituitary TSH and basal TSH release, D1 activity in thyroid and white adipose tissue (WAT), but higher D2 activity (hypothalamus, pituitary, brown adipose tissue and WAT), in accordance to its hypothyroid status. Thyroid, heart and testis D2 activities were unchanged, suggesting that other factors, which were programmed by postnatal EO, are more important regulators of these enzymes than $\mathrm{TH}$, at least in these tissues.
Liver D1, mGPD and TR $\beta 1$ were unchanged in adult SL rats, suggesting that the $\mathrm{TH}$ conversion and action were preserved in liver, even with the presence of lower TH. In hypothalamus and pituitary, TR $\beta 2$ was unchanged; while TR $\beta 1$ in WAT was lower, which can contribute to the lower catabolic WAT status. Conclusion: EO change thyroid function in adult life in a tissue-specific way, helping the understanding obesogenesis in this model.

\section{0}

Secondary signalling responses shared between skin fibroblasts of patients with clinically severe and marginally affected 3-methylcrotonyl-CoA carboxylase deficiency

Lizelle Zandberg ${ }^{1}$, Alberdina Aike van Dijk ${ }^{2}$

${ }^{1}$ North-West University; ${ }^{2}$ North-West University

Isolated 3-methylcrotonyl-CoA carboxylase (MCC) deficiency is an autosomal recessive inherited metabolic disease of leucine catabolism that has a highly variable phenotype. The lack of understanding of the pathomechanism and poor characterisation of the underlying molecular interactions involved with the disease makes it difficult to estimate the long term impact on health and clinical outcome. Here we present the first comparative transcriptome and functional networks for clinically severe and marginally affected MCC deficient patients using immortalized human skin fibroblasts. Whole genome expression profiles of immortalized human skin fibroblast cell cultures from two clinically severe MCC deficient patients, two asymptomatic marginally affected MCC deficient and two healthy control individuals were generated using Affymetrix ${ }^{\circledR H u E x S T} 1.0$ arrays. Comparative analyses identified 686 well-defined significantly differentially expressed transcript IDs that overlap between the clinically severe and marginally affected MCC deficient transcriptomes. The functional relationships and networks showed association with cellular assembly and organization, nervous system-, digestive system- and cardiovascular system development and function. Similarities were clear in lipid signalling and inflammatory responses, whereas differences between the clinically severe and marginally affected MCC deficient transcriptomes were detected in networks related to small molecule biochemistry, drug metabolism, molecular transport and cholesterol biosynthesis.

\section{7}

Melanocortin- 4 receptor mediates leptin associated renal dysfunction secondary to neonatal hyperleptinaemia

Sarah Wylie ${ }^{1}$, Nuria Macais Blasco ${ }^{2}$, Nynke R. Oosterhuis ${ }^{3}$, Joles A. Joles ${ }^{4}$, Joaquim M. Pombo ${ }^{5}$, Nina Balthasar ${ }^{6}$, Paul D. Taylor ${ }^{7}$, Lucilla Poston ${ }^{8}$

${ }^{1}$ Division of Women's Health \& Women's Health Academic

Centre, Faculty of Life Sciences \& Medicine, King's College 
London; ${ }^{2}$ Division of Women's Health \& Women's Health Academic Centre, Faculty of Life Sciences \& Medicine, King's College London; ${ }^{3}$ Department of Nephrology and Hypertension, University Medical Center, Utrecht, the Netherlands;

${ }^{4}$ Department of Nephrology and Hypertension, University

Medical Center, Utrecht, the Netherlands; ${ }^{5}$ Division of Women's Health \& Women's Health Academic Centre, Faculty of Life Sciences \& Medicine, King's College London; 'School of Physiology and Pharmacology, University of Bristol, Bristol, UK; ${ }^{7}$ Division of Women's Health \& Women's Health Academic Centre, Faculty of Life Sciences \& Medicine, King's College London; ${ }^{8}$ Division of Women's Health \& Women's Health Academic Centre, Faculty of Life Sciences \& Medicine, King's College London

Research question: Maternal obesity in rodents leads to juvenile sympathetic mediated hypertension. This is associated with an exaggerated leptin surge in early postnatal life. The aim of this study was to determine the impact of an exaggerated neonatal leptin surge on the long-term structure and function of the kidney, independent of the influence of hypertension.

Methods: We used melanocortin- 4 receptor deficient (MC4R-/-) mice to investigate the impact of neonatal hyperleptinaemia on kidney pathology. Neonatal MC4R-/- and wildtype (WT) mice were treated with leptin (L-Tx,3mg/kgIP) or saline (S-Tx), postnatal day 9-14. At 6 months of age arterial pressure and glomerular filtration rate were measured using radiotelemetry and creatinine clearance respectively and renal injury were evaluated by immunohistochemistry and QPCR.

Results: Hypertensive L-TxWT mice showed decreased creatinine clearance [GFR, $\mathrm{ml} / \mathrm{min}, \mathrm{L}-\mathrm{Tx}, 0.28 \pm 0.02$ vs. S-Tx, $0.46 \pm 0.01, n=6, p<0.01]$ and increased urinary albumin excretion. There was no added detrimental effect of $\mathrm{L}-\mathrm{Tx}$ in the obese normotensive MC4R-/-. L-TxMc4r-/- appeared protected against inflammatory induced renal injury with reduced oxidative stress, macrophage infiltration and cyclooxygenase- 2 (COX-2) mRNA expression.

Conclusions: Neonatal hyperleptinaemia causes renal dysfunction. The melanocortin- 4 receptor plays a dominant role in the regulation of neonatal leptin induced inflammation in the kidney (British Heart Foundation \& Tommy's).

\section{1}

Renal denervation reverses the development of juvenile hypertension, renal dysfunction and renal injury secondary to neonatal hyperleptinaemia

Ramon Fernandes ${ }^{1}$, Nynke R. Oosterhuis ${ }^{2}$, Nuria Macais Blasco $^{3}$, Jaap A. Joles ${ }^{4}$, Joaquim M. Pombo ${ }^{5}$, Paul D. Taylor ${ }^{6}$, Lucilla Poston ${ }^{7}$

${ }^{1}$ Division of Women's Health \& Women's Health Academic Centre, Faculty of Life Sciences \& Medicine, King's College London, London, UK; ${ }^{2}$ Department of Nephrology and Hypertension, University Medical Center, Utrecht, the Netherlands; 'Division of Women's Health \& Women's Health
Academic Centre, Faculty of Life Sciences \& Medicine, King's College London, London, UK; ${ }^{4}$ Department of Nephrology and Hypertension, University Medical Center, Utrecht, the Netherlands; ${ }^{5}$ Division of Women's Health \& Women's Health Academic Centre, Faculty of Life Sciences \& Medicine, King's College London; ${ }^{6}$ Division of Women's Health \& Women's Health Academic Centre, Faculty of Life Sciences \& Medicine, King's College London; ${ }^{7}$ Division of Women's Health \& Women's Health Academic Centre, Faculty of Life Sciences \& Medicine, King's College London

Research question: Maternal obesity in rodents leads to juvenile sympathetic mediated hypertension. This is associated with an exaggerated leptin surge in early postnatal life. The aim of this study was to examine the effect of renal denervation on early hypertension and renal pathology arising from maternal obesity.

Methods: Neonatal rats were treated with leptin (L-Tx; 3mg/ $\mathrm{kg}$ IP, postnatal day 9-14). Bilateral renal denervation (DNX) or sham-operation (SHAM) was performed at day 22. At 30 days of age, rats were allocated to examine renal structure and functions were examined. A further group underwent unilateral renal denervation (UNX) and kidneys were perfusion-fixed for stereological estimates of glomerular dimensions and glomerulosclerosis, or frozen for immunohistochemistry.

Results: DNX reversed the juvenile hypertension in the L-Tx rats. UNX had no effect, but resulted in decreased renal injury (NGAL) and oxidative stress (Nox-4, nitrotyrosine). Glomerular filtration rate (GFR) was rescued in L-Tx-DNX (male, $1.4 \pm 0.1$, female, $0.9 \pm 0.1 \mathrm{ml} / \mathrm{min}$ ) compared with $\mathrm{L}-\mathrm{Tx}$ SHAM (male, $1.0 \pm 0.1$, female, $0.5 \pm 0.1 \mathrm{ml} / \mathrm{min} ; \mathrm{P}<0.05$ ). L-Tx rats showed increased sodium retention, ENAC (epithelial sodium channel) activity, and urinary renin and angiotensinogen concentrations which were all normalised after DNX.

Conclusions: Renal denervation is a potential therapy against early sympathetic-mediated renal dysfunction (British Heart Foundation, Tommy's).

\section{1}

Vitamin B12 deficiency alters adipogenesis and associated microRNA's in human adipocytes

Antonysunil Adaikalakoteswari ${ }^{1}$, Hema Venkataraman ${ }^{2}$, Manu Vatish ${ }^{3}$, Philip McTernan ${ }^{4}$, Gyanendra Tripathi ${ }^{5}$, Ponnusamy Saravanan ${ }^{6}$

${ }^{1}$ Division of metabolic and vascular health, University of Warwick, Warwick, UK; ${ }^{2}$ Division of metabolic and vascular health, University of Warwick, Warwick, UK; ${ }^{3}$ Nuffield Department of Obstetrics \& Gynaecology, University of Oxford, UK; ${ }^{4}$ Division of metabolic and vascular health, University of Warwick, Warwick, UK; ${ }^{5}$ Division of metabolic and vascular health, University of Warwick, Warwick, UK; ${ }^{6}$ Division of 
metabolic and vascular health, University of Warwick, Warwick, UK; Department of Pathology \& Academic Department of Diabetes and Endocrinology, George Eliot Hospital, $N$

Research question: Human cohorts and animal models provide compelling evidence suggesting the role of the B12 in modulating the risk of diabetes and adiposity via developmental programming. We recently demonstrated that neonates born to mothers with low B12 levels in pregnancy have adverse cord blood lipid profile. We hypothesized that maternal vitamin B12 deficiency enhances adipogenic differentiation. We chose adipocytes as an in-vitro model system as they undergo several cycles of differentiation and maturation, mimicking in-vivo intergenerational effect (pregnancy-offspring) and investigated the role of vitamin B12 deficiency on adipogenesis.

Methods: Primary human pre-adipocytes were differentiated in various B12 concentrations (1) Control: (B12-500 nM); (2) LowB12 (0.15 nM) (3) NoB12: (B12-0 nM). Maternal venous blood samples $(n=91)$ and adipose tissue $(n=42)$ were collected at delivery.

Results: Adipocytes cultured in B12 deficient conditions showed increased lipid accumulation, lipolysis and reduced basal and insulin-stimulated glucose uptake. In our clinical study, mothers with low B12 status had higher BMI and adverse lipid profile. Gene expression of adipogenic regulators (PPAR $\gamma, \mathrm{CEBP} \alpha, \mathrm{RXR} \alpha$ ), lipid coating protein (perilipin), lipogenesis (FASN, ACC1) and development-related genes (Zfp423, EZH2, WISP2) were altered in adipocytes cultured in B12 deficient conditions and in adipose tissue from mothers with low B12. Expression of anti-adipogenic microRNAs (miR-27b and miR-195a targeting PPAR $\gamma$ and Zfp423, respectively) were down-regulated in adipocytes cultured in B12 deficient conditions.

Conclusion: Our study highlights that low B12 enhances adipogenesis and triggers specific microRNAs. Thus suggesting B12 deficiency alters adipocyte commitment and differentiation via epigenetic alterations leading to the development of obesity and insulin resistance in later life.

\section{0}

Experimental hyperleptinaemia in neonatal rats mimics the effects of maternal obesity on POMC expression in the arcuate nucleus

Sung Eun Bae ${ }^{1}$, Timothy South ${ }^{2}$, Paul Taylor ${ }^{3}$, Lucilla Poston ${ }^{4}$, Clive Coen ${ }^{5}$

'Division of Women's Health and Women's Health Academic Centre, Faculty of Life Sciences and Medicine, King's College London; 'Division of Women's Health and Women's Health Academic Centre, Faculty of Life Sciences and Medicine, King's College London; 'Division of Women's Health and Women's Health Academic Centre, Faculty of Life Sciences and Medicine, King's College London; 'Division of Women's Health and Women's Health Academic Centre, Faculty of Life Sciences and Medicine, King's College London; ${ }^{5}$ Division of Women's Health and Women's Health Academic Centre, Faculty of Life Sciences and Medicine, King's College London

Research question: Previously, we have shown that offspring of obese rat dams that were fed a highly palatable obesogenic diet throughout pregnancy and lactation (OffOb) display a prolonged postnatal leptin surge, associated with adult leptin resistance, hyperphagia and obesity compared to controls (OffCon). Moreover, neonatal experimental hyperleptinaemia affects sucrose preference and obesity development. We, therefore, compared appetite-regulatory transcript expression in the arcuate nucleus (ARC) in OffOb and in rats exposed to experimental hyperleptinaemia.

Methods: Four groups of male Sprague-Dawley rats were studied: (1) OffOb; (2) OffCon; (3) neonatal rats treated twice daily on postnatal days $9-15$ with either leptin $(0.3 \mathrm{mg} / \mathrm{kg}$, ip, LTx) or (4) saline treated controls. Offspring were weaned onto standard chow. Rats were euthanized (day 30) and brains removed for examination of NPY, AgRP, POMC and CART-mRNA levels in the ARC by in situ hybridization.

Results: Both OffOb and LTx juveniles showed a significant increase in POMC expression in the ARC. There were no differences in NPY, AGRP or CART expression.

Conclusions: These findings demonstrate comparability between OffOb and LTx groups in regard to POMC expression. Exposure to elevated levels of leptin in the second postnatal week permanently affects this component of the appetite-regulatory circuitry.

\section{6}

Sex differences in early life programming of the Hypothalamic-Pituitary-Adrenal axis in humans suggest increased vulnerability in females

Thomas Carpenter ${ }^{1}$, Sheila Grecian ${ }^{2}$, Rebecca M Reynolds ${ }^{3}$

${ }^{1}$ Endocrinology Unit, University/BHF Centre for Cardiovascular Science, Queen's Medical Research Institute, Edinburgh UK;

${ }^{2}$ Endocrinology Unit, University/BHF Centre for Cardiovascular Science, Queen's Medical Research Institute, Edinburgh UK;

${ }^{3}$ Endocrinology Unit, University/BHF Centre for Cardiovascular Science, Queen's Medical Research Institute, Edinburgh UK

Research question: Fetal glucocorticoid overexposure is a key mechanism linking early development with later life disease. In humans low birthweight associates with increased fasting cortisol, hypothalamic-pituitary-adrenal (HPA) axis reactivity and with cardiovascular risk and cognitive decline. Animal models suggest the HPA axis of female offspring is more susceptible to programming insults. We hypothesised that there would be similar sex differences in humans.

Methods: We conducted a systematic review, searching databases to June 2014. Two independent researchers screened articles for eligibility. The 29 included studies investigated the 
consequences of maternal stressors of asthma, psychosocial stress and glucocorticoid medications on HPA axis outcomes of placental glucocorticoid biology, and HPA axis function in early- and later-life.

Results: Female offspring had lower birthweight, increased therapeutic responsiveness to glucocorticoids, increased diurnal cortisol secretion and HPA axis reactivity, compared to males. Further, the female placenta increased its permeability to maternal glucocorticoids following maternal stress with downregulation of the enzyme 11-beta-hydroxysteroid dehydrogenase (which converts active cortisol to inactive cortisone) in response to maternal glucocorticoid exposure or maternal asthma.

Conclusion: The female HPA axis is more vulnerable to programming; this suggests a mechanism underlying sex differences in later life diseases including depression and cardiometabolic disease.

\section{6}

From embryos to adults: transcriptional signatures throughout development following preimplantation embryo manipulation

Sky Feuer ${ }^{1}$, Xiaowei Liu ${ }^{2}$, Rhodel Simbulan ${ }^{3}$, Annemarie Donjacour ${ }^{4}$, Paolo Rinaudo ${ }^{5}$

${ }^{1}$ University of California, San Francisco; ${ }^{2}$ University of California, San Francisco; ${ }^{3}$ University of California, San

Francisco; ${ }^{4}$ University of California, San Francisco; ${ }^{5}$ University of California, San Francisco

Research question: Investigate if transcriptional changes occurring during the preimplantation period that result from embryo manipulation persist throughout development and are maintained in adult tissues.

Methods: Microarray profiling was performed on mouse blastocysts: 1) cultured in vitro from the zygotic stage in 4 different culture conditions; 2) generated by in vitro fertilization (IVF); or 3) derived after natural mating (control). Microarray analysis was additionally performed on pancreatic islets, gonadal fat, skeletal muscle and liver tissue isolated from IVF and control offspring.

Results: In embryos, the effects of IVF and culture were condition-specific: each culture condition uniquely altered blastocyst transcriptional signature, with only 5 genes commonly misexpressed in all 5 in vitro conditions. Adult IVF mice also displayed tissue-specific molecular signatures with only 4 genes concordantly misexpressed. Pathway analysis revealed that more stressful conditions were associated with more significant alteration to the transcriptome. Common pathways affected by preimplantation stress included systemic oxidative stress, mitochondrial dysfunction, impaired insulin and adipogenic signaling.

Conclusion: Acute changes induced by embryo manipulation are differentially affected by subsequent development. While a common gene signature of preimplantation embryo stress is not present in blastocysts or adult tissues, there is evidence of oxidative stress and mitochondrial dysfunction.
4870

Modulation of SIRT1 expression by resveratrol: a protective role against accelerated senescence of endothelial progenitor cells in preterm infants

Yzydorczyk Catherine ${ }^{1}$, Simoncini Stéphanie ${ }^{2}$, Boubred Farid ${ }^{3}$, Sabatier Florence ${ }^{4}$, Dignat-George françoise ${ }^{5}$, Simeoni Umberto ${ }^{6}$

${ }^{1}$ CHUV-Department of Pediatrics-Lausanne-Switzerland;

${ }^{2}$ Aix-Marseille University, Inserm, VRCM, UMR_S 1076,

Marseille, France; ${ }^{3}$ Aix-Marseille University, Inserm, VRCM,

UMR_S 1076, Marseille, Francel APHM, CHU de la

Conception, Department of Neonatology, France; ${ }^{4}$ Aix-Marseille

University, Inserm, VRCM, UMR_S 1076, Marseille, Francel

APHM, CHU de la Conception, Laboratory of cell therapy,

Marseille, France; ${ }^{5}$ aix-Marseille University, Inserm, VRCM,

UMR_S 1076, Marseille, France/APHM, CHU de la

Conception, Laboratory of hematology, Marseille, France;

${ }^{6}$ CHUV-Department of Pediatrics-Lausanne-Switzerland

Research question: Preterm (PT) birth is a risk factor for hypertension at adulthood. A putative mechanism of such long-term effect is an altered vascular tree development. We recently showed that endothelial colony forming cells (ECFCs), considered as biomarkers of endothelial dysfunction, from PT neonates display impaired angiogenesis and accelerated senescence via decreased SIRT1 expression that can be modulated by resveratrol (R). Oxidative stress (OS) plays a key role in vascular complications of PT. We thus hypothesized that OS is involved in the accelerated senescence observed in PT.

Methods: ECFCs were isolated from venous umbilical cord blood of 25 PT and term neonates (CTRL). On ECFCs from PT vs. CTRL, we measured +/- pretreatment with R: expressions (western blot) of superoxide dismutase (SOD1), catalase (CAT), endothelial nitric oxide synthase (eNOS); superoxide anion (SA) and NO productions (fluorescent dye).

Results: In ECFCs from PT vs. CTRL: expressions of CAT, SOD1 and eNOS were decreased $(p<0.01)$. SA and NO productions were increased $(p<0.001)$ and decreased ( $\mathrm{p}<0.05)$, respectively. Pretreatment with $\mathrm{R}$ restored CAT, SOD1 and eNOS expressions, normalized SA levels and improved NO production.

Conclusion: Manipulating SIRT1 expression using resveratrol is protective against $\mathrm{OS}$, and may therefore improve angiogenic capacity in PT infants.

\section{9}

Early maternal DEX administration alters fetal ovine hepatic and placental glucose regulation in a sex-specific manner

Braun Thorsten $^{1}$, Huaisheng $\mathrm{Xu}^{2}$, Hongkai Shang ${ }^{3}, \mathrm{Li}_{\text {Shaofu }}{ }^{4}$, Ehrlich Loreen ${ }^{5}$, Henrich Wolfgang ${ }^{6}$, Newnham John ${ }^{7}$, Challis John ${ }^{8}$, Plagemann Andreas ${ }^{9}$, Sloboda Deborah ${ }^{10}$

${ }^{1}$ Departments of Obstetrics and Division of Experimental Obstetrics, Charité, Berlin, Germany; ${ }^{2}$ Departments of Obstetrics 
and Gynecology, Linyi people's hospital, China; ${ }^{3}$ Department of Obstetrics and Gynecology, Hangzhou first people's hospital, Zhejiang, China; 'School of Women's and Infants' Health, King Edward Memorial Hospital, The University of Western Australia, Australia; ${ }^{5}$ Departments of Obstetrics and Division of Experimental Obstetrics, Charité, Berlin, Germany;

${ }^{6}$ Departments of Obstetrics and Division of Experimental Obstetrics, Charité, Berlin, Germany; 'School of Women's and Infants' Health, King Edward Memorial Hospital, The University of Western Australia, Australia; 'School of Women's and Infants' Health, King Edward Memorial Hospital, The University of Western Australia, Australia and Department of Physiology, Obstetrics and Gynecology, Univer; ${ }^{9}$ Departments of Obstetrics and Division of Experimental Obstetrics, Study Group in Perinatal Programming, Charité Campus Virchow, Berlin, Germany; ${ }^{10}$ Departments of Biochemistry and Biomedical Sciences, Obstetrics \& Gynecology and Pediatrics, McMaster University, Canada

Research question: We have shown an increase in male fetal glucose levels after maternal Dexamethasone (DEX) administration early in pregnancy. We set out to determine whether early DEX treatment altered fetal hepatic glucose homeostasis and placental glucose transporter expression.

Methods: Pregnant ewes carrying singleton fetuses $(n=119)$ were treated with saline ( $2 \mathrm{ml}$ saline/ewe; control) or DEX (i.m. injections $0.14 \mathrm{mg} / \mathrm{kg}$ ewe weight/ $12 \mathrm{~h}$ ) at 40-41 days of gestation (dG). At 50, 100, 125, $140 \mathrm{dG}$, hepatic IGF1/2, IGFR1/2, IGFBP1/3, G6Pase, G6PD and PECK and placental GLUT1/3 mRNA levels were analyzed.

Results: Maternal glucose was similar between groups. In DEX males, higher fetal glucose levels were associated with increased hepatic G6Pase at $140 \mathrm{dG}$. IGF-1 was increased at $100 \mathrm{dG}$, but IGF2 and IGFR1/2 were unaltered by DEX treatment. Placental GLUT3 levels were increased at 125 dG. In females, DEX decreased PEPCK and increased IGFBP-1 m RNA levels at $100 \mathrm{dG}$. At $140 \mathrm{dG}$, DEX increased IGF-1, which was inversely correlated with placental GLUT3 levels.

Conclusions: In male fetuses, DEX induced increases in glucose levels were accompanied by increased hepatic G6Pase [rate limiting enzyme in the hepatic gluconeogenic pathway] and placental GLUT3 levels. In females, DEX treatment reduced hepatic gluconeogenesis at $100 \mathrm{dG}$, independent of placental glucose transporter expression.

\section{4}

\section{Neonatal Adiposity Increases with Rising Cord Blood Insulin-like Growth Factor 1 Levels}

Rachel Kadakia ${ }^{1}$, Jami Josefson ${ }^{2}$

${ }^{1}$ Northwestern University/Ann and Robert H. Lurie Children's Hospital of Chicago; ${ }^{2}$ Northwestern University/Ann and Robert H. Lurie Children's Hospital of Chicago
Background: Neonatal adiposity may be an important predictor of childhood obesity and adverse metabolic health. Identifying early life markers of obesity can inform earlier prevention efforts. Insulin-like growth factor 1 (IGF-1) is important for fetal growth; however, its effects on fetal adipose tissue are incompletely understood.

Hypothesis: IGF-1 levels in cord blood are associated with neonatal adiposity.

Methods: We utilized a cohort of healthy maternal-neonatal pairs. Neonatal body composition was measured by air displacement plethysmography within 72 hours of life. Cord blood IGF-1 and leptin levels were assayed. Associations between IGF-1 and birth weight, \%body fat, and leptin were assessed using generalized linear models and adjusted for sex, race, gestational age, maternal age, and maternal pre-pregnancy BMI.

Results: Cord blood IGF-1 levels were positively correlated with birth weight $(r=0.62, p=0.0007)$, percent body fat $(r=0.51, p=0.001)$, and cord blood leptin levels $(r=0.32$, $\mathrm{p}=0.037)$ when adjusted for neonatal sex, race, gestational age, maternal age at delivery, and maternal pre-pregnancy BMI. For every $10 \mathrm{ng} / \mathrm{ml}$ increase in IGF-1, birth weight and \%body fat increased by $60 \mathrm{~g}$ and $0.5 \%$ respectively.

Conclusion: Cord blood IGF-1 levels are directly related to neonatal adiposity and may be a useful marker of obesity risk.

\section{6}

Assessment of the effect of low doses of food contaminants on Min6 and rat isolated pancreatic cells stress response

Firmin Stéphane ${ }^{1}$, Malta Ananda ${ }^{2}$, Elmhiri Ghada ${ }^{3}$, Bahi-Jaber Narges ${ }^{4}$, Abdennebi-Najar Latifa ${ }^{5}$

${ }^{1}$ UP 2012.10.101. EGEAL-Institut Polytechnique LaSalle Beauvais 60000-Beauvais France; ${ }^{2}$ Laboratory of Secretion Cell Biology, Department of Biotechnology, Genetics and Cell Biology, State University of Maringá, 87020-900 Maringá, PR, Brazil; ${ }^{3}$ UP 2012.10.101. EGEAL-Institut Polytechnique LaSalle Beauvais 60000-Beauvais France; ${ }^{4} U P$ 2012.10.101. EGEALInstitut Polytechnique LaSalle Beauvais 60000-Beauvais France; ${ }^{5}$ UP 2012.10.101. EGEAL-Institut Polytechnique LaSalle Beauvais 60000-Beauvais France

Research question: Fetus/infants are exposed to many chemicals food contaminants that are suspected to be involved in long term risk of diabetes. In this study, we aim to investigate the pancreatic cellular stress responses to chemical mixture commonly present in maternal food Methods: Min 6 cell line and Langerhans islets isolated from adult rats were subsequently co-exposed for 24,48 and $72 \mathrm{~h}$ to increasing concentrations $(0,001 \mu \mathrm{M}$ to $1 \mu \mathrm{M})$ of cadmium, deoxynivalenol, aroclor 1254, malathion and malaoxon mixture alone or in combination with methylglyoxal $(10 \mu \mathrm{M})$. A cytotoxicity assay was performed using mitochondrial deshydrogenase activity $(\mathrm{MDH})$, as an indicator of chemical stress response

Results: A significant ( $p$ 
5077

Neurokinins expression in dorsal root ganglia, renal sodium handling and hypertension in maternal proteinrestricted rats: role of renal nerves

Augusto Henrique Custódio ${ }^{1}$, Marcelo Cardoso de Lima ${ }^{2}$, Patrícia Aline Boer ${ }^{3}$, José Antônio Rocha Gontijo ${ }^{4}$

${ }^{1}$ Department of Internal Medicine School of Medicine, State University of Campinas; ${ }^{2}$ Department of Internal Medicine School of Medicine, State University of Campinas; ${ }^{3}$ Department of Internal Medicine School of Medicine, State University of Campinas; ${ }^{4}$ Department of Internal Medicine School of Medicine, State University of Campinas

Neurokinins expression in dorsal root ganglia, renal sodium handling and hypertension in maternal protein-restricted rats: role of renal nerves.

Augusto Henrique Custdio, Marcelo Cardoso de Lima, Patrcia Aline Boer and Jos Antnio Rocha Gontijo.

Department of Internal Medicine School of Medicine, State University of Campinas, 13083-887 Campinas, SP, Brazil

Many evidences have supporting the idea which events in critical periods of fetal development may cause functional and structural changes of many organs. The renal neural activity play a role to maintain the water and salt balance and, the hemodynamic disorders are often associated with arterial hypertension. Here, up to 10 -wk old offspring from gestational protein-restricted dams (LP) was observed an enhanced systolic pressure (LP: $14314.6 \mathrm{mmHg}$ ) compared to normoprotein intake controls (NP: $1277.5 \mathrm{mmHg}$ ); additionally, the higher blood pressure was significantly mitigated by bilateral renal denervation (LP DNx: 129.83.7 vs. LP Sham: $149.99 .3 \mathrm{mmHg}$ ). Renal function test showed that renal denervation also reverse the huge antinatriuresis in 10-wk old LP compared to sham-operated offspring. (LPDNx $0.250 .02 \%$ : vs. LP-Sham: $0.02 \% 0.15$ ), returning to the basal level 16th week (LP-DNx: 0.040.01\% vs. LP-Sham: $0.040 .008 \%$ ), after kidney reinnervation. Dorsal root ganglia study, showed significant reduction of immunoreactivity to neurotransmitters substance P, CGRP in addition to increasing the NK1 receptor in the LP group. This study demonstrated that reduced renal afferent neural activity may contribute to the hypertension development in adulthood in rats from gestational protein-restricted dams, hypothetically, by elevation of sympathetic nerve activity.

\section{8}

Single cell transcriptional analysis: delineating cell lineage and pluripotency during human blastocyst formation

Sophie Petropoulos ${ }^{1}$, Edsgärd Daniel ${ }^{2}$, Deng Qiaolin ${ }^{3}$, Sandberg Rickard ${ }^{4}$, Lanner Fredrik ${ }^{5}$

${ }^{1}$ Karolinska Institute, Stockholm; ${ }^{2}$ Karolinska Institute, Stockholm; ${ }^{3}$ Karolinska Institute, Stockholm; ${ }^{4}$ Karolinska Institute, Stockholm; ${ }^{5}$ Karolinska Institute, Stockholm
Characterizing the first week of human development is of great importance. During this window, the embryo divides to form the trophectoderm (TE), primitive endoderm (PE) and epiblast (EPI). Insults during this period may therefore affect the developmental trajectory of these three lineages, ultimately resulting in profound downstream implications. To date, limited information exists pertaining to the transcriptional landscape and what drives lineage segregation in humans. Using Smart-seq2 single-cell RNA-sequencing, we have now established a transcriptional road map from embryonic (E) day 3-7 (71 embryos, $\sim 1600$ cells). Our data describe the temporal progression of lineage segregation where TE vs inner cell mass (ICM) segregation occurs from E4 to E5 and at E5 a priming of ICM cells towards EPI and PE is observed. During this progression, numerous genes (e.g. GATA2/3, PTGES, DAB2, FN1, PDGFRA, SOX2 and FGF4) and key signaling pathways (Nodal, Notch and Wnt) have been identified. The fundamental knowledge elucidated from this study is crucial in identifying mechanistic pathways underlying lineage segregation and the establishment of pluripotency, thus being of great importance for understanding human development and regenerative medicine. Further, we have now established a platform by which the effects of environmental perturbations on embryo development can be elucidated.

\section{6}

Glibenclamide early treatment improves rat autonomic nervous system activity in MSG-induced obesity

Elaine Vieira ${ }^{1}$, Rosiane Miranda ${ }^{2}$, Laize Tófolo ${ }^{3}$, Flávio Francisco ${ }^{4}$, Vander Alves ${ }^{5}$, Rosana Torrezan ${ }^{6}$, Júlio de Oliveira $^{7}$, Jean Besson ${ }^{8}$, Carina Previate ${ }^{9}$, Isabela Martins $^{10}$, Paulo Mathias ${ }^{11}$, Claudinéia Franco ${ }^{12}$

${ }^{1}$ State University of Maringá - Laboratory of Secretion Cell Biology, Department of Biotechnology, Genetics and Cell Biology; ${ }^{2}$ State University of Maringá - Laboratory of Secretion Cell Biology, Department of Biotechnology, Genetics and Cell Biology; ${ }^{3}$ State University of Maringá - Laboratory of Secretion Cell Biology, Department of Biotechnology, Genetics and Cell Biology; ${ }^{4}$ State University of Maringá - Laboratory of Secretion Cell Biology, Department of Biotechnology, Genetics and Cell Biology; ${ }^{5}$ State University of Maringá - Laboratory of Secretion Cell Biology, Department of Biotechnology, Genetics and Cell Biology; ${ }^{6}$ State University of Maringá - Laboratory of Secretion Cell Biology, Department of Biotechnology, Genetics and Cell Biology; ${ }^{7}$ Health Sciences Institute, Federal University of Mato Grosso, Sinop, MT, Brazil; ${ }^{8}$ Laboratory of Animal Histology, Department of Morphological Sciences, State University of Maringá - Maringál PR, Brazil; ${ }^{9}$ State University of Maringá - Laboratory of Secretion Cell Biology, Department of Biotechnology, Genetics and Cell Biology; ${ }^{10}$ State University of Maringá - Laboratory of Secretion Cell Biology, Department of Biotechnology, Genetics and Cell Biology; ${ }^{1}$ State University of Maringá - Laboratory of Secretion Cell Biology, Department of Biotechnology, Genetics and Cell 
Biology; ${ }^{12}$ State University of Maringá - Laboratory of Secretion Cell Biology, Department of Biotechnology, Genetics and Cell Biology

Research question: Autonomic nervous system (ANS) imbalance is associated with metabolic diseases. Glibenclamide is an antidiabetic used in the treatment of type 2 diabetes. This sulphonylurea stimulates insulin secretion from pancreatic beta-cells. It is scarce data concerning ANS activity in diabetic patients, treated with glibenclamide. The aim this work was test whether glibenclamide can improve ANS activity in pre-diabetic-rats.

Methods: Pre-diabetes was induced by treatment with monosodium L-glutamate (MSG) in neonatal rats. MSG-rats were treated with saline (Control group) or glibenclamide $2 \mathrm{mg} / \mathrm{kg}$ body weight (bw)/day (Glib group) by gavage. Bw, food intake, fasting glucose, insulin levels and fat tissue accumulation were measured. The vagus and sympathetic nerve activity were measure by electrical records. Insulin secretion was measured in isolated islets challenged with glucose and acetylcholine by radioimmunoassay technique.

Results: MSG-rats presented glucose intolerant, hyperinsulinemia, increased in fat tissue and low sympathetic and high parasympathetic activity. Glib group had increased food consumption but no changes in bw $(p<0.05)$. Glibenclamide treatment prevented the onset of obesity $(p<0.05)$, normalized the sympathetic and parasympathetic activity, increased the insulinotropic effect and unaltered cholinergic actions in islets from both groups.

Conclusion: Early glibenclamide treatment might prevent MSG-obesity onset, including balanced ANS activity.

\section{3}

Body Mass Index (BMI), its determinants and association with adult chronic disease at 10-year follow-up in the Lifeways Cross-Generation Cohort Study

Mirjam Heinen ${ }^{1}$, Pauline O'Reilly ${ }^{2}$, Sally McKey ${ }^{3}$, Rebecca Somerville ${ }^{4}$, Karien Viljoen ${ }^{5}$, Ricardo Segurado ${ }^{6}$, Celine Murrin ${ }^{7}$, Cecily Kelleher ${ }^{8}$

${ }^{1}$ UCD School of Public Health, Physiotherapy and Population Science; ${ }^{2}$ UCD School of Public Health, Physiotherapy and Population Science; ${ }^{3}$ UCD School of Public Health, Physiotherapy and Population Science; ${ }^{4}$ UCD School of Public Health, Physiotherapy and Population Science; ${ }^{5}$ UCD School of Public Health, Physiotherapy and Population Science; ${ }^{6} U C D$ School of Public Health, Physiotherapy and Population Science; ${ }^{7}$ UCD School of Public Health, Physiotherapy and Population Science; ${ }^{8}$ UCD School of Public Health, Physiotherapy and Population Science

Research question: To evaluate risk factors, including own and infant birth-weight (BW) for raised body mass index, BMI, in adult parents and grandparents and for grandparents only, associations of BMI with Diabetes Mellitus (DM) and cardiovascular disease (CVD) at 10 year follow-up.
Methods: The sample comprised 1803 adults in 822 families with an index child or grandchild recruited during pregnancy who had questionnaire or examination data for BMI, sociodemographic and health status data and grandparents $(65 \%$ of this sample) also had general practice morbidity follow-up records. Adjusted multivariate analyses were undertaken for outcomes of BMI, DM or CVD, employing family identity as a random effects parameter.

Results: In model one, comprising 807 adults with selfreported BW at baseline, age $(p<0.001)$, lineage group $(p=0.013)$, education level $(p=0.001)$ and index grandchild's BW $(p=0.002)$ but not own BW $(p=0.679)$ were significantly associated with BMI. In model 2 of 1132 grandparents only, BMI was strongly associated with both DM $(\mathrm{p}<0.001)$ and CVD $(\mathrm{p}<0.001)$ adjusted for demographic factors and index grandchild's BW.

Conclusion: These findings in a rare three-generation study confirm an association of BMI with adult chronic disease and also a cross-generation association for infant BW and adults' BMI.

\section{0}

Longitudinal changes in glucose and lipid metabolism in relation to maternal bmi: results from the stork study

Marie Cecilie Paasche Roland ${ }^{1}$, Camilla M Friis ${ }^{2}$, Kristin Godang ${ }^{3}$, Jens Bollerslev ${ }^{4}$, Tore Henriksen ${ }^{5}$

${ }^{1}$ Department of Obstetrics and Gynecology, Drammen Hospital; ${ }^{2}$ Department of Obstetrics, Oslo University Hospital; ${ }^{3}$ Department of Endocrinology; ${ }^{4}$ Department of Endocrinology and University of Oslo; ${ }^{5}$ Department of Obstetrics and University of Oslo

Research question: Increased BMI is associated with disturbances in glucose and lipid metabolism, which undergo profound changes during normal pregnancy. We hypothesized that longitudinal changes in glucose and lipid metabolism differ between women categorized according to BMI.

Methods: 1031 healthy women were followed prospectively and categorized according to BMI; BMI < 25 ( $n=628)$, BMI 25-30 $(\mathrm{n}=286)$ and BMI $>30(\mathrm{n}=89)$. Fasting plasma glucose $(\mathrm{FPG})$, insulin and lipids were measured at 15 weeks/v1 and 31 weeks/v3 or 36 weeks/v4. Analyses:paired t-tests and oneway ANOVA.

Results: mean (SD) mmol/l for each category At v1 all concentrations (except HDL) increased significantly with increasing BMI-category; FPG 3.92(0.36), 4.07(0.41), 4.24(0.42), insulin(median) 23.0, 33.0, 49.5, total cholesterol 4.85(0.85), 4.99(0.81), 5.14(0.96), LDL 2.49(0.75), 2.66(0.69), 2.88 (0.84) and triglycerides $1.10(0.36), 1.28(0.46), 1.57(0.47)$. HDL decreased; 1.86(0.4), 1.75(0.37), 1.55(0.39).

All concentrations (except HDL) increased significantly from v1 to v3/v4; FPG 0.09(0.42), 0.17(0.46), 0.23(0.47), insulin $10.0,19.0,20.5$, total cholesterol 1.95(0.97), 1.66(0.96), 1.28 (1.03), LDL 1.44(0.87), 1.13(0.87), 0.73(0.84), triglycerides $1.29(0.64), 1.35(0.70), 1.23(0.84)$. HDL decreased in normal $\mathrm{BMI}$ and overweight, but not in obese. 
The longitudinal changes differed between BMI-categories for glucose and insulin (larger with increasing BMI-category, $\mathrm{p}<0.05$ ), total cholesterol and LDL (smaller with increasing BMI-category, $\mathrm{p}<0.001$ ) but not for HDL and triglycerides (ns). Conclusion: BMI influences glucose, insulin and lipid values in early pregnancy and magnitude of longitudinal changes. Overweight/obese women have larger changes in glucose metabolism, but less pronounced changes in lipids than normal weight women.

\section{8}

Digit ratio (2D:4D), parameters of reproductive history and sex hormones in a rural population

Magdalena Klimek ${ }^{1}$, Andrzej Galbarczyk ${ }^{2}$, Ilona Nenko ${ }^{3}$, Grazyna Jasienska ${ }^{4}$

${ }^{1}$ Institute of Public Health Jagiellonian University Medical College; ${ }^{2}$ Institute of Public Health Jagiellonian University Medical College; ${ }^{3}$ Institute of Public Health Jagiellonian University Medical College; ${ }^{4}$ Institute of Public Health Jagiellonian University Medical College

Digit ratio has been suggested to be an indicator of sex hormone exposure during prenatal development. It is hypothesized that women with more feminine digit ratio were exposed to higher prenatal estrogen levels, and men with more masculine 2D:4D to higher testosterone levels, what may permanently influence functioning of reproductive system. Therefore, it might be expected that $2 \mathrm{D}: 4 \mathrm{D}$ will be related to reproductive characteristics and adult sex hormone levels.

Methods: The study participants were 319 women and 688 men from Mogielica Human Ecology Study Site located in southern Poland, in a village with high fertility.

Results: Women with more feminine 2D:4D had higher number of children $(P=0.002)$, higher number of daughters $(P=0.02)$, gave birth to the last child at later age $(P=0.02)$ and had a longer reproductive period $(P=0.04)$, but did not differ in sex hormone levels from women with more masculine 2D:4D. Men with more masculine 2D:4D had higher number of children $(\mathrm{P}=0.04)$ and higher testosterone concentration $(\mathrm{P}=0.04)$ than men with more feminine $2 \mathrm{D}: 4 \mathrm{D}$.

Conclusion: Our results indicate that early developmental hormonal environment may influence future reproductive parameters, but not adult sex hormone levels among women.

\section{9}

Maternal metabolic outcomes in women with a history of hypertensive pregnancy disorders

Laura Benschop ${ }^{1}$, Jeanine E Roeters-van Lennep ${ }^{2}$, Sarah Schalekamp-Timmermans ${ }^{3}$, Vincent WV Jaddoe ${ }^{4}$, Nienke E Bergen $^{5}$, Eric AP Steegers ${ }^{6}$

${ }^{1}$ Department of Obstetrics and Gynecology, Erasmus Medical Center, Rotterdam, the Netherlands; ${ }^{2}$ Department of Internal
Medicine, Erasmus Medical Center, Rotterdam, the Netherlands; ${ }^{3}$ Department of Obstetrics and Gynecology, Erasmus Medical Center, Rotterdam, the Netherlands; ${ }^{4}$ Department of Epidemiology, Erasmus Medical Center, Rotterdam, the Netherlands; ${ }^{5}$ Department of Obstetrics and Gynecology, Erasmus Medical Center, Rotterdam, the Netherlands; 'Department of Obstetrics and Gynecology, Erasmus Medical Center, Rotterdam, the Netherlands

Research question: We assessed whether women with preeclampsia or pregnancy-induced hypertension (PIH) have more unfavorable metabolic outcomes six years after pregnancy as compared to normotensive women.

Methods: This study was embedded in the Generation R study. Information on pregnancy and metabolic outcomes six years after pregnancy was available in 4933 women. We measured total body and abdominal fat distribution, weight and plasma lipid concentrations (total cholesterol, LDL-c, HDL-c, triglycerides, Apolipoprotein-B (Apo-B), lipoprotein (a) $(\mathrm{lp}-\mathrm{a}))$.

Results: Compared with normotensive women, women with PIH had higher Apo-B (0.06 g/l; 95\% CI 0.03, 0.09), LDL-c $(0.14 \mathrm{mmol} / \mathrm{l} ; 95 \% \mathrm{CI} 0.05,0.24)$ and triglyceride concentrations $(0.11 \mathrm{mmol} / \mathrm{l} ; 95 \% \mathrm{CI} 0.001,0.21)$ and lower HDL-c concentrations (-0.06 mmol/1; 95\% CI -0.11, -0.001). Women with PIH or preeclampsia both had increased risks of clustering of metabolic risk factors (OR 2.4; 95\% CI 1.5, 3.7 and OR 2.7; 95\% CI 1.4, 5.1, respectively) compared with normotensive women. These associations attenuated after adjustment for maternal weight gain after pregnancy. No differences were observed in lipid concentrations between normotensive and preeclamptic women.

Conclusion: Hypertensive pregnancy disorders were associated with adverse metabolic outcomes and an increased risk of clustering of metabolic risk factors six years after pregnancy as compared to normotensive women.

4520

Adipose tissue adiponectin expression and its relation to metabolic and endocrine parameters in women with gestational diabetes and their offspring

Rebecca C. Rancourt ${ }^{1}$, Jens H. Stupin ${ }^{2}$, Kerstin Melchior ${ }^{3}$, Thomas Ziska ${ }^{4}$, Wolfgang Henrich ${ }^{5}$, Andreas Plagemann ${ }^{6}$

${ }^{1}$ Clinic of Obstetrics, Division of 'Experimental Obstetrics', Charité - University Medicine Berlin, Campus VirchowKlinikum, Berlin, Germany; ${ }^{2}$ Clinic of Obstetrics, Division of 'Experimental Obstetrics', Charité - University Medicine Berlin, Campus Virchow-Klinikum, Berlin, Germany; ${ }^{3}$ Clinic of Obstetrics, Division of 'Experimental Obstetrics', Charité University Medicine Berlin, Campus Virchow-Klinikum, Berlin, Germany; ${ }^{4}$ Clinic of Obstetrics, Division of 'Experimental Obstetrics', Charité - University Medicine Berlin, Campus Virchow-Klinikum, Berlin, Germany; ${ }^{5}$ Clinic of Obstetrics, 
Division of 'Experimental Obstetrics', Charité - University

Medicine Berlin, Campus Virchow-Klinikum, Berlin, Germany;

'Clinic of Obstetrics, Division of 'Experimental Obstetrics', Charité - University Medicine Berlin, Campus Virchow-

Klinikum, Berlin, Germany

Research question: Identification of relationships between adiponectin gene expression in maternal adipose tissues and metabolic/endocrine parameters in women with gestational diabetes (GDM) and/or gestational overweight and their offspring.

Methods: Subcutaneous (SAT) and visceral adipose tissue (VAT) samples were collected from women with GDM $(n=29)$ or normal glucose tolerance (NGT; $n=35)$, accompanied by anthropometric, metabolic and hormonal characterization of mothers and newborns.

Results: Adiponectin gene expression was significantly decreased in SAT $(\mathrm{p}<0.05)$ and VAT $(\mathrm{p}<0.001)$ in GDM vs. NGT. Across all subjects, adiponectin expression correlated negatively with maternal BMI and fasting glucose at delivery (SAT: $r=-0.36 ; r=-0.26 ;$ VAT: $r=-0.27 ; r=-0.28$; $\mathrm{p}<0.05$ for all). SAT adiponectin expression correlated positively with maternal plasma adiponectin $(\mathrm{r}=0.53$; $\mathrm{p}<0.0001)$ and inversely with cord-blood leptin $(\mathrm{r}=-0.32 ; \mathrm{p}<0.01)$. Maternal plasma adiponectin showed a negative relationship with maternal BMI at delivery, AUC-oGTT, prepartum fasting glucose and birthweight $(r=-0.34 ; r=-0.28 ; r=-0.44$; $\mathrm{r}=-0.28$; all $\mathrm{p}<0.05$ ). Cord-blood adiponectin, however, neither differed between groups nor correlated with birthweight, ponderal index, neonatal leptin and C-peptide.

Conclusion: Adipose tissue adiponectin expression is altered in GDM/gestational overweight, adversely affecting materno-fetal metabolism. In contrast, no associations were observed between cord-blood adiponectin and adverse neonatal outcome. Data so far does not support 'malprogramming' of adiponectin in GDM offspring.

\section{3}

PRE-PLAN: A randomised controlled trial assessing the impact of preconception weight loss for overweight or obese women

Kyra Sim ${ }^{1}$, Rachel O'Connell ${ }^{2}$, Michael Peek ${ }^{3}$, Jon Hyett ${ }^{4}$, Ralph Nanan ${ }^{5}$, Kirsten Black ${ }^{6}$, Margaret Allman-Farinelli ${ }^{7}$, Jennie Brand-Miller ${ }^{8}$, Adrian Bauman ${ }^{9}$, Ian Caterson ${ }^{10}$

${ }^{1}$ Charles Perkins Centre, The University of Sydney, Australia; ${ }^{2}$ NHMRC Clinical Trials Centre, The University of Sydney, Australia; ${ }^{3}$ Charles Perkins Centre, The University of Sydney, Australia; ${ }^{4}$ Sydney Medical School, The University of Sydney, Australia; ${ }^{5}$ Charles Perkins Centre, The University of Sydney, Australia; ${ }^{6}$ Sydney Medical School, The University of Sydney, Australia; ${ }^{7}$ Charles Perkins Centre, The University of Sydney, Australia; ${ }^{8}$ Charles Perkins Centre, The University of Sydney, Australia; ${ }^{9}$ Charles Perkins Centre, The University of Sydney,

\section{Australia; ${ }^{10}$ Charles Perkins Centre, The University of Sydney, Australia}

Research question: Can a multidisciplinary preconception weight-loss program, for overweight and obese women improve pregnancy outcomes, preconception health and early childhood markers of later life obesity and cardiovascular disease?

Methods: A phase II randomised controlled trial will recruit 225 women $\geqslant 18-40$ years, who are overweight $\left(\mathrm{BMI} \geqslant 27 \mathrm{~kg} / \mathrm{m}^{2}\right)$ or obese $\left(\mathrm{BMI} \geqslant 30 \mathrm{~kg} / \mathrm{m}^{2}\right)$ and intending pregnancy within 6-12 months.

Participants will be randomised centrally using minimisation in a 2:1 ratio to a 12-week multidisciplinary intervention (including a very-low-energy diet, physical activity and behavioural support) versus standard care based on current guidelines. All participants will receive preconception health appointments at baseline and 3-months and follow up visits at 15 months. Children of participants will be seen at 12 months of age.

Primary outcome: Composite adverse outcome (at least one of: gestational diabetes, pregnancy-induced hypertension, preeclampsia, caesarean section, premature birth, perinatal mortality, nursery admission, large-for-gestational age).

Secondary outcomes: Maternal, infant and child adiposity; time-to-pregnancy; metabolic and cardiovascular biomarkers; placental pathology; physical activity; dietary intake; psychological and acceptance measures; preconception knowledge, child arterial intima media-thickness and cost effectiveness.

Conclusion: This intervention has the potential to improve significant short-term morbidities for mothers and babies as well as obesity and cardiovascular outcomes for the next generation.

\section{9}

Psychosocial exposures during childhood and development of adult obesity: a co-twin control study

Marianne Vamosi ${ }^{1}$, Kirsten Ohm Kyvik ${ }^{2}$, Berit L Heitmann ${ }^{3}$

${ }^{1}$ Institut Public Health, Aarhus University, Denmark; ${ }^{2}$ Department Head, Professor, Department of Clinical Research, University of

Southern denmark; ${ }^{3}$ Professor, Ph. Institute of Preventive Medicine, Bispebjerg and Frederiksberg Hospitals, The Capital Region

The purpose of the study was to examine if parental antipathy and neglect during childhood were associated with obesity in adulthood. From the Danish Twin Registry 146 adult samesexed twin pairs discordant for Body Mass Index (BMI) were identified. Criteria for being discordant were that one of the twins should have a BMI between 20 and $25 \mathrm{~kg} / \mathrm{m}^{2}$ (normal weight) and the co-twin a $\mathrm{BMI} \geqslant 30 \mathrm{~kg} / \mathrm{m}^{2}$ (obesity). In total 236 out of $289(81.7 \%)$ eligible twin individuals participated in an interview and a physical examination.

A part of the Childhood Experience of Care and Abuse, the parental care and neglect questionnaire, by Bifulco et al, was used to assess perceived parental antipathy and neglect. Data were analyzed by means of intra-pair comparisons. 
Our results showed that recalled maternal antipathy $(p=0.04)$ and maternal neglect $(p=0.01)$ were both associated with adult obesity. Paternal neglect and antipathy were not related with adult obesity.

The study demonstrates that experience in childhood maternal antipathy and neglect may contribute to the development of obesity at age 20 and later in adulthood.

\section{3}

Proton pump inhibitors detrimentally affect motile sperm count in men of reproductive age

Nicole Huijgen ${ }^{1}$, Maria de Ridder ${ }^{2}$, Ann Vanrolleghem ${ }^{3}$, Katia Verhamme ${ }^{4}$, Miriam Sturkenboom ${ }^{5}$, Joop Laven ${ }^{6}$, Régine Steegers-Theunissen ${ }^{7}$

${ }^{1}$ Erasmus MC, University Medical Centre; ${ }^{2}$ Erasmus $M C$, University Medical Centre; ${ }^{3}$ Erasmus MC, University Medical Centre; ${ }^{4}$ Erasmus MC, University Medical Centre; ${ }^{5}$ Erasmus $M C$, University Medical Centre; ${ }^{6}$ Erasmus MC, University Medical Centre; ${ }^{7}$ Erasmus MC, University Medical Centre

Research question: The use of prescribed medication is high (45-63\%) in men of reproductive age. Does the use of proton pump inhibitors (PPIs) detrimentally affect semen quality?

Methods: A nested case-control study was conducted in a population-based cohort of men visiting the general practitioner. We selected 2,045 men contemplating pregnancy and with data on semen quality in the Dutch Integrated Primary Care Information database.

Controls were matched to cases (total motile sperm count $(\mathrm{TMSC}) \leqslant 1$ ) on practice.

The effect of PPI use in two consecutive periods of 6 months preceding semen analysis was analysed using conditional logistic regression with adjustment for age.

Results: After exclusion of 786 men using other medication then PPIs and 505 men with incomplete medication history (<year) and no PPI use, 129 PPI users and 625 controls not using any prescribed medication were available for analyses. The use of PPIs 12-6 months before semen analysis was associated with a 5-fold higher risk of low TMSC (OR5.359; p0.017). PPI use 6 months before semen analysis was not associated with low TMSC.

Conclusion: Long term PPI use is associated with a more than 5-fold higher risk of a low motile sperm count in men contemplating pregnancy.

\section{9}

Maternal waist circumference in early pregnancy and truncal adiposity during late pregnancy are associated with glucose intolerance

Judith Plester $^{1}$, Hema Venkataraman ${ }^{2}$, Karen Rouault ${ }^{3}$, Pareena Patel $^{4}$, Natalie Maltseva ${ }^{5}$, Ponnusamy Saravanan ${ }^{6}$

${ }^{1}$ George Eliot Hospital NHS Trust; ${ }^{2}$ George Eliot Hospital NHS Trust and University of Warwick; ${ }^{3}$ George Eliot Hospital
NHS Trust; ${ }^{4}$ University of Warwick; ${ }^{5}$ University of Warwick; ${ }^{6}$ George Eliot Hospital NHS Trust and University of Warwick

Research question: What are the associations between various maternal anthropometric measures during pregnancy and insulin resistance?

Methods: Maternal anthropometry [body mass index (BMI), waist circumference (WC), triceps and subscapular skinfold thickness] was measured at two time points in the multi-centre PRiDE study. 75 g glucose tolerance tests (GTT) carried out at 24-28weeks.

Results: Anthropometry was obtained in early pregnancy (mean \pm SD $12.4 \pm 9.6$ weeks, $\mathrm{n}=1284$ ) and at GTT $(26.5 \pm 2.4$ weeks, $n=996)$. Skinfold thickness was done on a sub-sample of 224 and 281 women during the two visits, respectively. The baseline results (mean $\pm \mathrm{SD}$ ) were: BMI: $31.0 \pm 7.8 \mathrm{~kg} / \mathrm{m}^{2}$, WC: $98.8 \pm 16.9 \mathrm{~cm}$, triceps: $25.1 \pm 7.5$ $\mathrm{mm}$, and subscapular skinfold thickness: $26.4 \pm 8.5 \mathrm{~mm}$. Gestational weight gain (GWG) was $5.6 \pm 4.8 \mathrm{~kg}$ between the two visits. In addition to BMI, early pregnancy WC correlated with glucose at 0 and $120 \mathrm{mins}(\mathrm{r}=0.23, \mathrm{p}<0.01$ and $\mathrm{r}=0.10, \mathrm{p}<0.01)$. Apart from fasting glucose, truncal obesity (2nd trimester subscapular thickness) was the strongest positive predictor of glucose at $120 \mathrm{mins}(\beta=0.36$, $\mathrm{p}=0.03$ ) after adjusting for baseline BMI, anthropometry and GWG. None of these factors significantly predicted fasting glucose.

Conclusion: In addition to BMI, early pregnancy WC and late pregnancy truncal adiposity are associated with glucose intolerance. Waist circumference at booking could be used to target women to reduce risk of glucose intolerance.

\section{6}

Gestational weight gain in obese pregnant women is associated with higher central adiposity

Jacqueline Farmer ${ }^{1}$, Hema Venkataraman ${ }^{2}$, Ellie Wright ${ }^{3}$, Hannah Brown ${ }^{4}$, Ponnusamy Saravanan ${ }^{5}$

${ }^{1}$ George Eliot Hospital NHS Trust; ${ }^{2}$ University of Warwick;

${ }^{3}$ University of Warwick; ${ }^{4}$ University of Warwick; ${ }^{5}$ University of Warwick and George Eliot Hospital NHS Trust

Research Question: The Institute of Medicine (IOM) published guidelines in 2009 about recommended gestational weight gain (GWG) according to BMI categories. Our aim was to investigate the rate of GWG and its association with insulin resistance.

Methods: Maternal anthropometry (height, weight, waist circumference, triceps and subscapular skinfold thickness) was measured as part of the longitudinal PRiDE study.

Results: Anthropometric data was obtained $(n=966)$ in early 2nd (mean \pm SD gestation $12.4 \pm 9.6$ weeks) and 3rd trimesters $(26.5 \pm 2.4$ weeks). According to IOM-2009, the GWG 
between these visits in underweight, normal, overweight and obese women should be $7.0,6.3,3.9$ and $3.1 \mathrm{~kg}$, respectively. The mean \pm SD and percentage increase above recommended weight gain (in brackets) in the 4 groups were: 6.6 2.9 $(-5.4 \%), 6.8 \pm 3.5(+7.4 \%), 7.0 \pm 4.0(+80.5 \%)$ and $4.6 \pm 5.4$ $(+49.7 \%)$, respectively. In obese women, subscapular skinfold thickness at the 2 nd visit (but not triceps) was positively associated with GWG $(r=0.21, \mathrm{p}<0.01)$ and higher mean readings in those with greater than recommended GWG (29.5 vs, $26.9 \mathrm{~mm}, \mathrm{p}<0.05)$ suggests that these women acquired more central adiposity. There was no relationship between GWG and glucose intolerance.

Conclusion: Women who are overweight or obese gained more than $50 \%$ of the recommended weight between 12 and 26 weeks, with proportionately higher central (truncal) adiposity.

\section{4}

Perinatal prebiotic supplementation has later consequences on adult metabolism and immunity in pigs used as a human model

Cindy Le Bourgot ${ }^{1}$, Sophie Blat ${ }^{2}$, Stéphanie Ferret-Bernard ${ }^{3}$, Emmanuelle Apper ${ }^{4}$, Frédérique Respondek ${ }^{5}$, Isabelle Le Huërou-Luron ${ }^{6}$

${ }^{1}$ National Institute for Agricultural Research; ${ }^{2}$ National Institute for Agricultural Research; ${ }^{3}$ National Institute for Agricultural Research; ${ }^{4}$ Tereos; ${ }^{5}$ Tereos; ${ }^{6}$ National Institute for Agricultural Research

Perinatal nutrition, a key factor controlling gut bacterial colonization, may have lasting consequences on health. We investigated whether early short-chain fructooligosaccharide (scFOS) supplementation, known to well-balance microbiota, impacted adult metabolic and immunologic responses to a high-fat (HF) diet.

Sows received a supplementation with scFOS or not for the last 4 weeks of gestation and the lactation. The scFOS supplementation was maintained in piglets weaned from scFOS sows till postnatal day (PND)56. Twenty four piglets were then fed a standard diet until PND190, then a HF diet for 12 weeks.

Early scFOS supplementation increased faecal short-chain fatty acid production after 3 weeks of HF diet but this effect was not observed later. Ongoing metagenomics analysis will give more insights on microbiota composition changes. Insulin response to a glucose challenge tended to increase in scFOS group, but no other metabolic parameter was modified (lipid metabolism, basal glycaemia and insulinemia, insulin pancreatic content). Preliminary results showed a modulation of the pro-inflammatory cytokine secretion (IFN $\gamma$, TNF $\alpha$, IL-8) by ex vivo stimulated ileal explants and MLN cells, revealing a modification of local immunity orientation with perinatal scFOS supplementation.
Our results underline the key role of early microbiota modulation on adult responses to a nutritional challenge.

\section{7}

The associations of leptin and other maternal variables with gestational weight gain differ according to pre-gestational body mass index categories

Ana Beatriz Franco-Sena ${ }^{1}$, Fernanda Rebelo ${ }^{2}$, Thatiana Pinto ${ }^{3}$, Dayana Farias ${ }^{4}$, Gabrielle Esteves ${ }^{5}$, Gilberto $\mathrm{Kac}^{6}$

${ }^{1}$ Nutritional Epidemiology Observatory, Department of Social and Applied Nutrition, Institute of Nutrition Josué de Castro, Rio de Janeiro Federal University, Brazil; ${ }^{2}$ Nutritional Epidemiology Observatory, Department of Social and Applied Nutrition, Institute of Nutrition Josué de Castro, Rio de Janeiro Federal University, Brazil; ${ }^{3}$ Nutritional Epidemiology Observatory, Department of Social and Applied Nutrition, Institute of Nutrition Josué de Castro, Rio de Janeiro Federal University, Brazil; ${ }^{4}$ Nutritional Epidemiology Observatory, Department of Social and Applied Nutrition, Institute of Nutrition Josué de Castro, Rio de Janeiro Federal University, Brazil; ${ }^{5}$ Nutritional Epidemiology Observatory, Department of Social and Applied Nutrition, Institute of Nutrition Josué de Castro, Rio de Janeiro Federal University, Brazil; ${ }^{6}$ Nutritional Epidemiology Observatory, Department of Social and Applied Nutrition, Institute of Nutrition Josué de Castro, Rio de Janeiro Federal University, Brazil

Research question: Are the associations of leptin and other selected maternal variables with gestational weight gain (GWG) the same in normal weight (NW), overweight (OW) and obese $(\mathrm{OB})$ women?

Methods: rospective cohort of 228 women followed at the 5-13th, 20-26th and 30-36th gestational weeks. The associations of the independent variables with GWG in pregestational NW $\left(\mathrm{BMI}=18.5-24.9 \mathrm{~kg} / \mathrm{m}^{2}\right)$, OW $(\mathrm{BMI}=$ $\left.25.0-29.9 \mathrm{~kg} / \mathrm{m}^{2}\right)$ and $\mathrm{OB}\left(\mathrm{BMI} \geqslant 30.0 \mathrm{~kg} / \mathrm{m}^{2}\right)$ women were assessed using longitudinal linear mixed-effects models.

Results: In the multiple model for NW women, the following covariates remained associated with GWG: leptin and HDL-c concentrations, QUICKI values, maternal height and sleep duration. For OW women, the associated variables were leptin concentrations, maternal height and QUICKI. For pregestational $\mathrm{OB}$ women, HDL-c concentrations, maternal height, sleep duration, QUICKI, marital status and smoking habit were the variables associated to GWG. A negative interaction between leptin concentrations and sleep duration was found only in OW women.

Conclusions: Leptin concentrations were positively associated with GWG in NW and OW women, but not in $\mathrm{OB}$ ones. However, in OW women, sleep duration decreased the effect of leptin on GWG. Other factors associated with GWG throughout pregnancy present effects specifically in some pre-gestational BMI categories. 
4706

Sweet dreams in pregnancy: Influence of antenatal sleep quality and sleep duration on maternal blood glucose in women with risk factors for gestational diabetes

Shirong $\mathrm{Cai}^{1}{ }^{1}$, Sara Tan ${ }^{2}$, Peter D. Gluckman ${ }^{3}$, Keith M. Godfrey ${ }^{4}$, Seang Mei Saw ${ }^{5}$, Kok-Hian Tan ${ }^{6}$, George SH Yeo ${ }^{7}$, Kenneth Kwek ${ }^{8}$, Michael J. Meaney ${ }^{9}$, Yap-Seng Chong ${ }^{10}$, Joshua J. Gooley ${ }^{11}$

${ }^{1}$ Department of Obstetrics and Gynaecology, Yong Loo Lin School of Medicine, National University of Singapore; ${ }^{2}$ Duke-NUS Graduate Medical School; ${ }^{3}$ Liggins Institute, University of Auckland; ${ }^{4}$ MRC Life course Epidemiology Unit, University of Southampton; ${ }^{5}$ Saw Swee Hock School of Public Health, National University of Singapore; ${ }^{6}$ Department of Obstetrics and Gynaecology, KK Women's and Children's Hospital; ${ }^{7}$ Department of Maternal Fetal Medicine, KK Women's and Children's Hospital; ${ }^{8}$ Department of Maternal Fetal Medicine, KK Women's and Children's Hospital; ${ }^{9}$ Department of Psychiatry and Neurology and Neurosurgery, McGill University;

${ }^{10}$ Department of Obstetrics and Gynaecology, Yong Loo Lin School of Medicine, National University of Singapore; ${ }^{I}{ }^{I} D u k e-N U S$ Graduate Medical School

Research Question: To examine the influence of maternal sleep quality and sleep duration on maternal antenatal glucose levels in a multi-ethnic Asian population of women, with and without risk factors for gestational diabetes mellitus (GDM)

Methods: 726 pregnant women completed the Pittsburgh Sleep Quality Index (PSQI) and a 75-g oral glucose tolerance test (OGTT) during 26-28 weeks gestation. Risk factors for GDM were based on the guidelines recommended by UK National Institute for Health and Clinical Excellence (NICE). Results: In women with at least 1 risk factor for GDM, fewer hours of night sleep was associated with a higher maternal fasting glucose $(\beta=0.05 \mathrm{mmol} / \mathrm{L}, \mathrm{p}=0.021)$. Amongst them, poor sleepers (PSQI global score $>5$ ) were found to have higher fasting blood glucose [ $+0.17 \mathrm{mmol} / \mathrm{L}$ ( $95 \%$ CI: 0.05 to 0.29 ). Sleep duration and sleep quality did not significantly affect maternal blood glucose in women without any risk factors. All results were adjusted for maternal BMI at 26-28 weeks, age and ethnicity.

Conclusion: Poor sleep quality and shorter sleep duration were associated with elevated fasting maternal blood glucose concentrations in women with at least 1 risk factor for GDM.

\section{3}

Severe early childhood infection, low socioeconomic status and adverse cardiometabolic risk phenotypes in adulthood: The Cardiovascular Risk in Young Finns Study

Richard S. Liu ${ }^{1}$, David P. Burgner ${ }^{2}$, Matthew A. Sabin ${ }^{3}$, Costan G. Magnussen ${ }^{3}$, Michael Cheung ${ }^{3}$, Mika Kähönen ${ }^{3}$,
Terho Lehtimäki ${ }^{3}$, Nina Hutri-Kähönen ${ }^{3}$, Eero Jokinen ${ }^{3}$, Tomi Laitinen $^{3}$, Leena Taittonen ${ }^{3}$, Päivi Tossavainen ${ }^{3}$, Terence Dwyer ${ }^{3}$, Jorma S.A. Viikari ${ }^{3}$, Olli T. Raitakari ${ }^{3}$

${ }^{1}$ Murdoch Childrens Research Institute, Parkville, Victoria, Australia; ${ }^{2}$ Murdoch Childrens Research Institute, Parkville, Victoria, Australia; ${ }^{3}$ Murdoch Childrens Research Institute

Research question: Early life socioeconomic disadvantage is associated with increased adult cardiovascular and metabolic disease. We hypothesised that interactions between serious childhood infection and low socioeconomic status may contribute to adverse cardiometabolic parameters in adulthood.

Methods: We analysed prospective longitudinal data from the Cardiovascular Risk in Young Finns Study. Using fully adjusted models, we investigated relationships between childhood infection-related hospitalisation and socioeconomic status (family income), and adult cardiometabolic parameters.

Results: Of 1015 individuals (3-18 y.o at baseline; $30-45$ y.o at follow-up), 133 (12.6\%) were hospitalised with infection $<5$ years, with no differences by socioeconomic status. Childhood infection-related hospitalisation was significantly associated with increased adult body mass index $\left(\beta \pm S E 2.4 \pm 0.8 \mathrm{~kg} / \mathrm{m}^{2}\right.$, $\mathrm{P}=0.008)$, waist circumference $(7.4 \pm 2.3 \mathrm{~cm}, \mathrm{P}=0.004)$, and reduced brachial flow mediated dilatation $(-2.7 \pm 0.9 \%$, $\mathrm{P}=0.002)$ in those from families with below median income compared with those from higher income families. No associations between childhood infection and adult cardiometabolic parameters were observed in those from higher income families.

Conclusion: Infection was associated with increased cardiometabolic risk only in those from lower socioeconomic backgrounds. Childhood infection may widen the social gradients observed in cardiometabolic disease risk in adults.

\section{1}

Metabolic syndrome, objectively measured physical activity and early growth - the helsinki birth cohort sudy

Minna Salonen ${ }^{1}$, Niko Wasenius ${ }^{2}$, Mikaela Von Bonsdorff ${ }^{3}$, Eero Kajantie ${ }^{4}$, Johan Eriksson ${ }^{5}$

${ }^{1}$ Folkhälsan Research Center, Helsinki, Finland; Department of Health, National Institute for Health and Welfare, Helsinki, Finland; ${ }^{2}$ Department of General Practice and Primary Health Care, University of Helsinki, Finland; ${ }^{3}$ Gerontology Research Center and Department of Health Sciences, University of Jyväskylä, Jyväskylä, Finland; ${ }^{4}$ Children's Hospital, Helsinki University Central Hospital, University of Helsinki, Finland; Department of Health, National Institute for Health and Welfare, Helsinki, Finland; Depa; ${ }^{5}$ Department of General Practice and Primary Health Care, University of Helsinki, FinlandFolkhälsan Research Center, Helsinki, Finland; Department of Health, National Institute for Health and Welfare, Helsinki, Finland 
Research question: We examined associations between intensity and volume of physical activity (PA) and the metabolic syndrome (MS). Furthermore we examined whether birth size and childhood growth predict PA and MS or explain their relationship.

Methods: We studied 712 subjects born 1934-44, who have birth and growth data available from several medical records. MS and PA were assessed in 2011-2013. PA was measured objectively (SenseWear Armband $囚, 5$ days) and expressed in metabolic equivalents of task (MET).

Results: The prevalence of MS was 29\% (for men 31\% and women 28\%). Increasing intensity (MET) and volume (MET-hours) of PA was associated with a decreased likelihood of having MS, odds ratios (ORs) being 0.23 (95\% CI: 0.15-0.37) for those in the highest third of PA volume category and 0.57 (95\% Ci: $0.38-0.85)$ for the middle third compared to those within the lowest. Birth size or childhood growth did not modify these findings. In men, gain in weight and BMI between 2 and 11 years, was inversely associated with PA.

Conclusion: Higher total mean intensity and volume rates of PA had a beneficial impact on the likelihood of having MS. Higher weight gain in mid childhood predicts decreased volume of PA in elderly men.

\section{2}

Current Status of gestational weight gain in China: A systematic review and meta-analysis

Hongbo Dong ${ }^{1}$, Jie Dong ${ }^{2}$, Fangfang Chen ${ }^{3}$, Jie $\mathrm{Mi}^{4}$

${ }^{1}$ Peking Union Medical College; Capital Institute of Pediatrics; ${ }^{2}$ Peking Union Medical College; Capital Institute of Pediatrics; ${ }^{3}$ Capital Institute of Pediatrics; ${ }^{4}$ Capital Institute of Pediatrics

Background: Gestational weight gain (GWG) is considered one of the risk factors of future obesity in the offspring. However, information on the epidemiology of GWG is limited in China. This study aimed to present the most recent status and geographic variability of GWG adequacy according to the 2009 Institute of Medicine (IOM) recommendations in China. Methods: Hospital-based, cross-sectional surveys were systematically reviewed using the keywords "gestational weight gain", "Institute of Medicine ", "China" in electronic databases. Only singleton pregnancies described by the authors were included. Quality of the literature was evaluated through the Newcastle-Ottawa scale. The pooled proportion was estimated using the Stata 11.0 software.

Results: In total, 10 studies were selected out of 112 hits. The overall prevalence of adequate GWG was 30.33\% (95\% CI, $30.03 \%-30.64 \%$ ) according to 2009 IOM recommendation. The prevalence of inadequate and excessive weight gain was $17.36 \%$ (95\% CI, 17.10\%-17.63\%) and 52.31\% (95\% CI, 51.98\%-52.65\%), respectively. Compared with southern regions, there was a higher prevalence of excessive GWG (57.21\% vs $41.57 \%)$ but lower adequate GWG $(31.98 \%$ vs $38.53 \%)$ and inadequate GWG $(0.21 \%$ vs $19.90 \%)$ in northern regions.

Conclusions: Prevalence of excessive GWG was high based on IOM guidelines in China. Early screening and prevention programs on should be emphasized and promoted.

\section{2}

Childhood obesity affects adult metabolic syndrome and diabetes

Hong Cheng ${ }^{1}$, Dongqing $\mathrm{Hou}^{2}$, Xiaoyuan $\mathrm{Zhao}^{3}$, Junting Liu ${ }^{4}$, Yinkun Yan ${ }^{5}$, Jie $\mathrm{Mi}^{6}$

${ }^{1}$ Capital Institute of Pediatrics; ${ }^{2}$ Capital Institute of Pediatrics;

${ }^{3}$ Capital Institute of Pediatrics; ${ }^{4}$ Capital Institute

of Pediatrics; ${ }^{5}$ Capital Institute of Pediatrics; ${ }^{6}$ Capital Institute of Pediatrics

Objectives: We seek to observe the association between childhood obesity by different measures and adult obesity, metabolic syndrome (MetS), and diabetes.

Methods: 1209 subjects from "Beijing Blood Pressure Cohort Study" were followed $22.9 \pm 0.5$ years in average from childhood to adulthood. We defined childhood obesity using body mass index (BMI) or left subscapular skinfold (LSSF), and adult obesity as BMI $\geqslant 28 \mathrm{~kg} / \mathrm{m}^{2}$. MetS was defined according to the joint statement of International Diabetes Federation and American Heart Association with modified waist circumference ( $\geqslant 90 / 85 \mathrm{~cm}$ for men/women). Diabetes was defined as fasting plasma glucose $\geqslant 7.0 \mathrm{mmol} / \mathrm{L}$ or blood glucose $2 \mathrm{~h}$ after oral glucose tolerance test $\geqslant 11.1 \mathrm{mmol} / \mathrm{L}$ or currently using blood glucose-lowering agents.

Results: Compared to children without obesity, children obese by LSSF only or by both had higher risk of diabetes. After controlling for adult obesity, childhood obesity predicted independently long-term risks of diabetes (odds ratio 2.8, $95 \%$ confidence interval 1.2-6.3) or abdominal obesity $(2.7,1.6-4.7)$ other than MetS as a whole $(1.2,0.6-2.4)$.

Conclusions: Childhood obesity predicts long-term risk of adult diabetes, and the effect is independent of adult obesity. LSSF is better than BMI in predicting adult diabetes.

\section{1}

Associations of Socio-demographic factors with Body Mass Index among women of Reproductive age living in slums of Mumbai, India

Sirazul Sahariah ${ }^{1}$, Caroline Fall ${ }^{2}$, Meera Gandhi ${ }^{3}$, Harshad Sane $^{4}$, Harsha Chopra ${ }^{5}$, Swati Jha ${ }^{6}$, Patsy Coakley ${ }^{7}$, Nick Brown $^{8}$, Sarah Kehoe ${ }^{9}$, Ramesh Potdar ${ }^{10}$

${ }^{1}$ Centre for the Study of Social Change, Mumbai, India; ${ }^{2} M R C$ Lifecourse Epidemiology Unit, Unversity of Southampton, UK; 
${ }^{3}$ Centre for the Study of Social Change, Mumbai, India;

${ }^{4}$ Centre for the Study of Social Change, Mumbai, India;

${ }^{5}$ Centre for the Study of Social Change, Mumbai, India;

${ }^{6}$ Centre for the Study of Social Change, Mumbai, India;

${ }^{7}$ MRC Lifecourse Epidemiology Unit, Unversity of

Southampton, UK; ${ }^{8}$ Children's Unit, Salisbury General

Hospital, Wiltshire, UK; ${ }^{9} M R C$ Lifecourse Epidemiology Unit,

Unversity of Southampton, UK; ${ }^{10}$ Centre for the Study of Social

Change, Mumbai, India

Introduction: Body Mass Index (BMI) is important indicator of nutritional status, and strong predictor of reproductive outcome. Low maternal BMI means increased risk of low birth weight and subsequently with increased risk of noncommunicable diseases in adult life.

Methods: Socio-demographic, dietary and food security data from a randomized controlled trial of a pre-conceptional nutritional supplement for women of reproductive age were collected between 2006 and 2012.

Result: 6513 women participated. The mean (SD) age and BMI were $25.0( \pm 3.99)$ years and $20.8( \pm 6.13) \mathrm{kg} / \mathrm{m}^{2}$ respectively. Prevalence of underweight, overweight and obesity was $32 \%, 12 \%$ and $3 \%$ respectively. The median daily calorie intake was higher with increasing BMI. Proportion of food insecure women were highest in underweight (3.7\%) compared to overweight and obese (1.5\%).

Higher education and socio-economic score were associated with lower risk of underweight. Risk of underweight increased among women in unskilled occupations (OR:1.21), using tobacco (OR:1.26), currently breast feeding (OR:1.27), and living in joint family (OR:1.24). Overweight and obesity were associated with Muslim religion and Hindi as mother tongue. Higher educational attainment was associated with overweight, but not to obesity.

Conclusion: One third of women were underweight which is likely to increase non-communicable diseases in next generation.

\section{0}

Maternal fatty acid profiles, hemodynamic adaptations and gestational hypertensive disorders. The Generation $\mathbf{R}$ Study

Romy Gaillard ${ }^{1}$, Aleksandra Jelena Vidakovic ${ }^{2}$, Henning Tiemeier ${ }^{3}$, Albert Hofman ${ }^{4}$, Eric Steegers ${ }^{5}$, Vincent Jaddoe ${ }^{6}$

${ }^{1}$ Generation R Study Group, Erasmus University Medical Center; ${ }^{2}$ Generation R Study Group, Erasmus University Medical Center; ${ }^{3}$ Child and Adolescent Psychiatry, Erasmus University Medical Center; ${ }^{4}$ Department of Epidemiology, Erasmus University Medical Center; ${ }^{5}$ Deparment of Obstetrics and Gynaecology; ${ }^{6}$ Generation R Study Group, Erasmus University Medical Center
Research question: Adverse fatty acids profiles are associated with adult cardiovascular disease. We examined whether maternal fatty acids during pregnancy affect maternal blood pressure development, umbilical and uterine artery resistance indices and the risk of gestational hypertensive disorders.

Methods: This study was embedded in a population-based prospective cohort study among 6,823 pregnant women. Maternal fatty acid levels (saturated (SFA), monounsaturated (MUFA), n-3 polyunsaturated (n-3 PUFA) and n-6 polyunsaturated (n-6 PUFA)) were measured in midpregnancy. We measured maternal blood pressure in each trimester of pregnancy, umbilical artery and uterine artery vascular resistance in second and third trimester, and obtained information about gestational hypertensive disorders from medical records.

Results: Higher maternal SFAs, but not MUFAs, were associated with a higher maternal blood pressure from first trimester onwards (p-values).

\section{9}

Non-alcoholic Fatty Liver Disease among adult survivors of Severe Acute Malnutrition

Debbie Thompson ${ }^{1}$, Ingrid Tennant ${ }^{2}$,

Deanne Soares ${ }^{3}$, Tamika Royal-Thomas ${ }^{4}$,

Christopher Byrne ${ }^{5}$, Terrence Forrester ${ }^{6}$,

Michael Boyne

${ }^{1}$ University of the West Indies; ${ }^{2}$ University of the

West Indies; ${ }^{3}$ University of the West Indies; ${ }^{4}$ University of the West Indies; ${ }^{5}$ University of Southampton; ${ }^{6}$ University of the West Indies; ${ }^{7}$ University of the West Indies

Research question: We investigated whether malnutrition survivors had more liver fat than controls and whether marasmus survivors (Ms) had more liver fat than kwashiorkor survivors (Ks).

Methods: We used birth weight (BW) from hospital records and measured anthropometry and body composition (DEXA). Liver fat was assessed as a continuous outcome, using single, cross-sectional CT scanning at T12/ L1. Subjects were age, sex and BMI-matched. Data were analyzed using multivariate linear regression.

Results: We studied $45 \mathrm{Ms}, 43 \mathrm{Ks}$ and 84 controls (age $29.0 \pm 8.4$ years and BMI $23.5 \pm 5.0 \mathrm{~kg} / \mathrm{m}^{2}$ ). Malnutrition survivors had similar liver fat to controls $(64.5 \pm 4.4$ $\mathrm{HU}$ vs. $64.0 \pm 4.6 \mathrm{HU} ; \mathrm{P}=0.49)$. Ms had lower BW than Ks $(-0.51 \mathrm{~kg} ; \mathrm{p}=0.02)$, smaller waists $(\mathrm{P}=0.03)$ and were thinner $(P=0.01)$, with less body fat $(P=0.05)$. Ms had more liver fat than Ks after adjusting for age, sex and BW $(\beta=$ 2.62, $95 \%$ CIs; -4.95 to -0.29 ; $\mathrm{P}=0.03)$. Lower $\mathrm{BW}$ infants had less liver fat after adjusting for diagnosis $(\beta=-1.51$, $\mathrm{P}=0.04)$. 
Conclusion: Fatty liver occurs at lower BMI and body fat in Ms compared with Ks and is not mediated by birth weight.

\section{2}

Pre-conception Anaemia and Body Mass Index among participants of a study evaluating foetal programming for Non-communicable Diseases (FOETALforNCD) in Tanzania

Christentze Schmiegelow ${ }^{1}$, Omari Abdul ${ }^{2}$, Daniel Minja ${ }^{3}$, Sofie Lykke ${ }^{4}$, Ib C Bygbjerg ${ }^{5}$, John Lusingu ${ }^{6}$, Vaag Allan ${ }^{7}$, Birgitte Bruun Nielsen $4^{8}$, Reginald Kavishe ${ }^{9}$

${ }^{1}$ Centre for Medical Parasitology, Department of International Health, Microbiology and Immunology, University of Copenhagen, Denmark; ${ }^{2}$ National Institute for Medical Research, Tanga Center, Tanga, Tanzania; ${ }^{3}$ National Institute for Medical Research, Tanga Center, Tanga, Tanzania; ${ }^{4}$ Global Health, Department of Public Health, University of Copenhagen, Copenhagen, Denmark; ${ }^{5}$ Global Health, Department of Public Health, University of Copenhagen, Copenhagen, Denmark; ${ }^{6}$ National Institute for Medical Research, Tanga Center, Tanga, Tanzania; ${ }^{7}$ Diabetes and Metabolism, Copenhagen University Hospital), Copenhagen, Denmark; ${ }^{8}$ Department of Obstetrics and Gynecology, Aarhus University Hospital, Aarhus, Denmark; ${ }^{9}$ Kilimanjaro Christian Medical University College, Tanzania

Research question: Over nutrition has dramatically increased with the nutrition transition while under nutrition and micronutrients deficiencies persist. Poor nutritional status early in pregnancy may partially influence foetal programming for non communicable disease but little is known on its magnitude in the preconception period. Clustering of opposite ends of malnutrition spectrum in developing countries can present a unique difficulty for public health interventions.

Methods: We assessed the magnitude of the underweight, overweight, obesity and anaemia among rural 684 non pregnant Tanzanian women aged 18-40 and explored the sociodemographic patterns of individual double burden from a baseline survey women participating in cohort study (FOETALforNCD) in north eastern Tanzania.

Results: Overall, $51 \%$ of the women had anaemia before conception out of which $21(3 \%)$ had mild to moderate anaemia $(\mathrm{Hb} \leqslant 8 \mathrm{~g} / \mathrm{dL})$. The prevalence of obesity, overweight and underweight was $13 \% 20 \%$ and $11 \%$, respectively. Microcytic hypo chromic anaemia was prevalent (40\%). Marital status, short stature and having no toilet were associated with anaemia and underweight while parity and woman occupation were significantly associated with overweight/ obesity.
Conclusion: A substantial proportion of women have poor nutritional status in the preconception period that could have short and long-term adverse effects on newborn's health as well as women's.

\section{2}

Associations of early stunting with cardiometabolic risk factors in adulthood

Emanuella De Lucia Rolfe, Giovanny V A França, Denise Gigante, Jaime Mirand, John Yudkin, Ken Ong

Introduction: Early stunting may have long-term effects on body composition, resulting in obesity related co-morbidities. We tested the hypothesis that individuals stunted in early childhood may be at higher cardiometabolic risk later in adulthood.

Methods: 1753 men and 1781 women participating in the 1982 Pelotas (Brazil) birth cohort study had measurements of body composition and cardiometabolic traits at age 30y. Early stunting was defined as height-for-age Z-score below -2 at age $2 \mathrm{y}$ using the $\mathrm{WHO}$ criteria.

LinBernardo Horta ear regression models were performed stratified by sex, controlling for maternal and child's skin colour and family income.

Results: Stunted individuals were shorter (Men: $\beta=-1.15$ $\mathrm{z}$-score; $\mathrm{p}<0.001 /$ Women: $\beta=-1.08$ z-score $; \mathrm{p}<0.001)$, had substantially lower lean mass (Men: $\beta=-0.84 ; \mathrm{p}<0.001 /$ Women: $\beta=-0.76 ; p<0.001$ ), fat mass (Men: $\beta=-0.30$; $\mathrm{p}<0.001 /$ Women: $\beta=-0.44 ; \mathrm{p}<0.001)$, and subcutaneous fat (Men: $\beta=-0.34 ; p<0.001 /$ Women: $\beta=-0.27 ; p=0.001$ ), but no difference in visceral fat (Men: $\beta=-0.03 ; p=0.7$ / women: $\beta=-0.02 ; p=0.8)$. Men who were stunted had higher glucose levels $(\beta=0.16 ; \mathrm{p}=0.04)$.

Conclusions: Stunted individuals appear to have the tendency to accumulate less lean mass and subcutaneous fat, and this body composition profile is unlikely to be beneficial in terms of metabolic risk, particularly in men.

\section{5}

Prenatal maternal stress increases the risk of asthma?

Heinrich Joachim

Institute and Outpatient Clinic for Occupational, Social, and environmetal Medicine, Ludwig Maximilians University Munich

Maternal stress adversely affects a mother's wellbeing and health, and also negatively impacts her offspring. That such a relationship also holds true for maternal stress during the pregnancy period is intuitive. However, whether maternal stress increases the risk of asthma development in her offspring is less clear and will be evaluated in this review. 
This is a narrative review with a focus on past five years papers. There is some evidence from murine models to suggest that maternal stress during pregnancy increases the risk of asthma in the offspring. Whilst results from several observational studies in human populations are mostly consistent with these experimental findings, the epidemiological evidence is weaker. Almost all existing observational studies suffer from severe methodological limitations.

Nevertheless, the results from experimental work on maternal stress during pregnancy and asthma risk in the offspring, in concert with obvious other adverse health effects in the offspring, are sufficient to justify a recommendation to reduce maternal stressors, particularly during pregnancy.

\section{0}

Does comorbid Attention deficit/hyperactivity disorder effects cognition in Bipolar Disorder?

Cem Gündüz ${ }^{1}$, Mehmet Çevik ${ }^{2}$

${ }^{1} \ddot{U}$ nye State Hospital; ${ }^{2}$ Samsun Psychiatry Hospital

Research question: Cognitive impairment is frequently observed among individuals with bipolar disorder during euthymic phases of the illness. Adult attention deficit/ hyperactivity disorder (ADHD) occurs $9-35 \%$ in the $\mathrm{BD}$ patients. The aim of this study is to determine the frequency of adult ADHD comorbidity with lifetime bipolar disorder, and the influence of this comorbidity on cognitive impairment. Methods: Between 18 and 65 year old patients who had already received a diagnosis of $\mathrm{BD}$ according to DSM-IV and were in remission for at least 1 months at the time of inclusion in the study were recruited. All patients were also assessed for the presence of ADHD according to DSM-IV criterions. After that Wisconsin Card Sorting Test (WCST) was applied to all patients by specialist psycholog to establish cognitive impairment.

Results: Sixty nine consecutive bipolar patients were evaluated and 8 (\%11.6) patients had comorbid adult ADHD. There is no significant difference in WCST between Bipolar and Bipolar comorbid adult ADHD groups.

Conclusions: This study concluded that Bipolar disorder comorbid adult ADHD didn't cause further more cognitive impairment but this data has to be verified with further studies with increasing number of cases.

\section{8}

Brighter Children: The Association between Child Season of Birth and IQ

Steegers-Theunissen R.P.M. ${ }^{1}$, Tiemeier H. ${ }^{2}$

${ }^{1}$ Erasmus MC, University Medical Center, Rotterdam, the Netherlands; ${ }^{2}$ Erasmus MC, University Medical Center, Rotterdam, the Netherlands
Research question: Season of birth has repeatedly been found to be a risk indicator for adverse neurodevelopment outcomes. Here, we tested seven explanations that has been put forward to explain this association.

Methods: In the Generation $\mathrm{R}$ birth cohort (Rotterdam, The Netherlands, born in 2002-2006) we examined the association between season of birth and non-verbal intelligence quotient (IQ) at age 6 years among 6,034 children. We explored maternal exposure to infections during pregnancy, maternal nutritional intake, maternal socio-demographic factors and IQ, birth outcomes, cord blood vitamin D concentrations, and child age relative to peers in class as explanatory mechanisms.

Results: We found spring birth to be associated with a more than 1 point lower non-verbal IQ ( $\beta-1.24,95 \%$ CI -2.31 ; -0.17, $P=0.02)$. Adjustment for the different possible mechanisms led to a substantial reduction $(63.7 \%)$ of this association, to which maternal socio-demographic factors and IQ were prominent contributing factors.

Conclusions: Season of birth is an indicator of many underlying processes related to IQ. Parental socio-demographic factors related to planning of pregnancy, and not biological confounders, are of particular importance in the general population.

\section{7}

Early nutritional intervention prevents the early-life adversity induced cognitive impairments

Eva F.G. Naninck ${ }^{1}$, Kit-Yi Yam ${ }^{2}$, Lidewij Schipper ${ }^{3}$, Torsten Plosch ${ }^{4}$, Hans J.B. van Goudoever ${ }^{5}$, Annemarie Oosting ${ }^{6}$, Eline M. van der Beek ${ }^{7}$, Paul J. Lucassen ${ }^{8}$

${ }^{1}$ University of Amsterdam, SILS/CNS, Amsterdam, the Netherlands; ${ }^{2}$ University of Amsterdam, SILS/CNS, Amsterdam, the Netherlands; ${ }^{3}$ Nutricia Research - Danone Nutricia Early Life Nutrition, Utrecht, the Netherlands; ${ }^{4}$ Department of Obstetrics \& gynecology, Universitair Medisch Centrum Groningen, Groningen, the Netherlands; ${ }^{5}$ Department of Mother \& Child, Emma Children's Hospital, Academic Medical Center, Amsterdam, the Netherlands;

${ }^{6}$ Nutricia Research - Danone Nutricia Early Life Nutrition, Utrecht, the Netherlands; ${ }^{7}$ Nutricia Research - Danone Nutricia Early Life Nutrition, Singapore; ${ }^{8}$ University of Amsterdam, SILS/ CNS, Amsterdam, the Netherlands

Early-life stress (ES) is associated with cognitive decline in adulthood. The mechanisms underlying this programming are unknown and the possible role of nutrition has been largely ignored. Essential nutrients as methyl donors (MD; B-vitamins, methionine) and polyunsaturated fatty acids (PUFAs) are key for brain development and epigenetic machinery, known to be involved in programming by ES. We study 1) how chronic ES affects level of these nutrients, 
2) if early dietary intervention (DI) with MD or PUFAs prevents the ES-induced cognitive impairments and 3) the mechanisms mediating the effects of these DIs at hormonal, structural, and epigenetic level.

We used a chronic ES mouse model consisting of exposing dams to limited nesting/bedding material from postnatal day (P) P2-P9, resulting in cognitive decline in adulthood. We show that i) ES leads to alterations in levels of MDs and PUFAs in the P9 offspring's plasma and brain and ii) that DI with MD during P2-P9 can prevent (at least partly) the ES-induced cognitive impairments. Effects of DI with PUFAs, and possible mechanisms mediating these beneficial effects of DIs are currently under investigation.

These results highlight early DI as an entirely new non-invasive target to prevent the ES-induced deficiencies.

\section{9}

Childhood parental income, anthropometrics at age 24 months and cognitive abilities at age 20 years - The Helsinki Birth Cohort Study 1934-1944

H. Maiju Mikkonen ${ }^{1}$, Katri Räikkönen ${ }^{2}$, Markus Henriksson ${ }^{3}$, Johan G. Eriksson ${ }^{4}$, Eero Kajantie ${ }^{5}$

${ }^{1}$ National Institute for Health and Welfare, Helsinki, Finland o Department of Public Health, University of Helsinki, Helsinki, Finland; ${ }^{2}$ Institute of Behavioral Sciences, University of Helsinki, Helsinki, Finland; ${ }^{3}$ Centre for Military Medicine, Finnish Defence Forces, Helsinki, Finland; ${ }^{4}$ National Institute for Health and Welfare, Helsinki, Finland \& Folkhälsan Research Centre, Helsinki, Finland \& Institute of Clinical Medicine, University of Helsinki, Helsinki, Fin; ${ }^{5}$ National Institute for Health and Welfare, Helsinki, Finland \& Children's Hospital, Helsinki University Central Hospital and University of Helsinki, Helsinki, Finland \& Department

We examined how early-life parental income is related to cognitive abilities in 474 men born at Helsinki University Central Hospital 1934-1939, who performed the Finnish Defense Forces Basic Ability Test at the average age of 20.3 years and whose parents' taxable income was available for at least one year 1934-1939 from Helsinki municipal tax records. Anthropometrics from child welfare clinic records and education from Finnish census data were examined as mediators with linear regression.

Education was the strongest predictor of cognitive abilities (0.41 SD increase in test scores for upper secondary vs. less), followed by the direct association of parental income $(0.15$ SD increase for 1 SD higher income), both $\mathrm{p}<0.01$, controlling for age at testing, being firstborn or evacuated abroad during World War 2. When anthropometrics at 24 months were individually added to the model, test scores increased by 0.11 SD $(p=0.01), 0.05$ SD $(p=0.21)$ and 0.08 SD $(p=0.06)$ per 1 SD higher weight, height and BMI, respectively. Without mediators, income predicted $0.23 \mathrm{SD}$ higher test scores per $1 \mathrm{SD}$ higher income. These results suggest that childhood parental income is associated with adult cognitive abilities, mediated by education and childhood anthropometrics.

\section{7}

Cadmium-affected cognitive and behavioral development to 10 years of age: A prospective cohort study in Bangladesh

Fahmida Tofail ${ }^{1}$, Annachiara Malin Igra ${ }^{2}$, Jena Hamadani ${ }^{3}$, Syed Moshfiqur Rahman ${ }^{4}$, Marie Vahter ${ }^{5}$

${ }^{1}$ International Centre for Diarrhoeal Disease Research, Bangladesh (icddr,b), Dhaka, Bangladesh; ${ }^{2}$ Institute of Environmental Medicine, Karolinska Institutet, Stockholm, Sweden; ${ }^{3}$ International Centre for Diarrhoeal Disease Research, Bangladesh (icddr,b), Dhaka, Bangladesh; ${ }^{4}$ Institute of Environmental Medicine, Karolinska Institutet, Stockholm, Sweden and International Centre for Diarrhoeal Disease Research, Bangladesh (icddr,b), Dhaka, Bangladesh; ${ }^{5}$ Institute of Environmental Medicine, Karolinska Institutet, Stockholm, Sweden

In our longitudinal mother-child cohort originating from a multimicronutrient trial (MINIMat) in pregnancy in Bangladesh, we have found that children's pre- and postnatal exposure to cadmium, a common food pollutant, affected blood DNA methylation in the newborns and IQ at pre-school age. We now aim to explore whether the effects on child development persist at 10 years of age and if they are potentiated by the continued exposure. Children born from October 2002 to December 2003 were selected for follow-up of development at 5 and 10 years $(n=1530)$. Cadmium exposure was assessed by concentrations in urine (marker of long-term exposure) of the mothers' during pregnancy and the children's at 5 and 10 years, measured using ICP-MS. Cognitive function (IQ) was assessed using Wechsler Intelligence Scales (WPPSI-III and WISC-IV) and behavior using the Strengths and Difficulties Questionnaire. Preliminary, multivariable-adjusted regression analyses indicate inverse associations between children's urinary cadmium (median $0.24 \mu \mathrm{g} / \mathrm{L}$ ) and full scale IQ (B - $0.80 ; 95 \%$ CI - 1.3 to -0.31 ), and the other four IQ scores at 10 years. Additional longitudinal analyses, using linear mixedeffects model fit by maximum likelihood estimation in combination with quantile regression analyses, will provide information on critical periods of exposure and potential susceptible subgroups.

\section{6}

The Relationship Between Perfectionism and Depression in Panic Disorder

Emrah Yigit

Darica Farabi State Hospital 
Research between August 2009 and January 2010, Bakirköy Prof. Dr. Mazhar Osman Mental Health and Neurological Diseases Training and Research Hospital and admitted to the Outpatient Department, DSM-IV - TR criteria had been diagnosed with panic disorder were compared to 115 patients.

Structured interview after the personal information form, Multidimensional Perfectionism Scale, Liebowitz Social Anxiety Symptoms Scale, Panic and Agoraphobia Scale, Beck Depression Inventory, Beck Anxiety Inventory was used.

Total MPS group with depression scores were significantly higher than the group without depression $(\mathrm{p}=0.0001)$. MPS yourself for the group with depression scores were significantly higher than the group without depression $(p=0.003)$. MPS scores of others towards the depressed group was significantly higher in the group without depression $(p=0.0001)$. MPS scores for the social order of the depressed group was significantly higher in group Like being in depression $(\mathrm{p}=0.0001)$.

Beck Depression Inventory and the Beck Anxiety Inventory scores and the total MPS, there was a significant relationship between the MPS and MPS scores for social order against others. Depression in patients with deeper levels of guilt and feelings of worthlessness and decreases towards self confidence.

\section{2}

Effects of inadequate iodine status in pregnancy on infant development. Results from a Norwegian longitudinal observational study

Maria Wik Markhus ${ }^{1}$, Ingvild Eide Graff ${ }^{2}$, Camilla Fløysvik Seldal ${ }^{3}$, Lisbeth Dahl ${ }^{4}$, Livar Frøyland ${ }^{5}$, Jannike Øyen ${ }^{6}$, Siv Skotheim ${ }^{7}$, Hanne Cecilie Braarud ${ }^{8}$, kjell Morten Stormark ${ }^{9}$, Marian Kjellevold Malde ${ }^{10}$

${ }^{1}$ NIFES $;{ }^{2}$ NIFES; ${ }^{3}$ NIFES; ${ }^{4}$ NIFES; ${ }^{5}$ NIFES $;{ }^{6}$ NIFES $;{ }^{7}$ RKBU; ${ }^{8} \mathrm{RKBU} ;{ }^{9} \mathrm{RKBU} ;{ }^{10} \mathrm{NIFES}$

Research question: Women have an increased need for iodine during pregnancy and infants are vulnerable to iodine deficiency. The research question in this study was: do infants of mothers with an inadequate urinary iodine concentration (UIC) during pregnancy have different developmental status than infants of mothers with an adequate UIC during pregnancy?

Method: Urine samples were collected in gestational week 28 $(\mathrm{n}=64)$ and three months postpartum $(\mathrm{n}=85)$ and infant developmental status were assessed using The Ages and Stages Questionnaire at six $(n=89)$ and 12 months of age $(\mathrm{n}=84)$.

Results: Only $42 \%$ of the mothers had an adequate iodine status in pregnancy (UIC $>150 \mu \mathrm{g} / \mathrm{L}$ ). Infants of mothers with inadequate iodine status in pregnancy had borderline significant lower communication skills $($ median $=35(15,55))$ at 12 months og age than infants of mothers with an adequate iodine status in pregnancy $($ median $=43(5,60)(\mathrm{U}=205$, $\mathrm{n}=35, \mathrm{p}=0,05)$.

Conclusions: The iodine status was suboptimal for $58 \%$ of the mothers in pregnancy. Infants of mothers with inadequate iodine status in pregnancy had poorer communication skills than infants of mothers with adequate iodine status.

\section{4}

The Association between Autism Spectrum Traits and Visual Processing in Young Adults with Very Low Birth Weight: Helsinki Study of Very Low Birth Weight Adults

Elina Seppä ${ }^{1}$, Anu-Katriina Pesonen ${ }^{2}$, Kati Heinonen ${ }^{3}$, Marius Lahti ${ }^{4}$, Riikka Pyhälä ${ }^{5}$, Eero Kajantie ${ }^{6}$, Petteri Hovi ${ }^{7}$, Sture Andersson ${ }^{8}$, Katri Räikkönen ${ }^{9}$

${ }^{1}$ Institute of Behavioral Sciences, University of Helsinki, Finland; ${ }^{2}$ Institute of Behavioral Sciences, University of Helsinki, Finland;

${ }^{3}$ Institute of Behavioral Sciences, University of Helsinki, Finland;

${ }^{4}$ Institute of Behavioral Sciences, University of Helsinki, Finland;

5 Institute of Behavioral Sciences, University of Helsinki, Finland;

${ }^{6}$ Department of Health, Chronic Disease Prevention Unit, National Institute for Health and Welfare, Helsinki, Finland;

${ }^{7}$ Department of Health, Chronic Disease Prevention Unit, National Institute for Health and Welfare, Helsinki, Finland;

${ }^{8}$ Children's Hospital, University of Helsinki and Helsinki

University Hospital, Finland; ${ }^{9}$ Institute of Behavioral Sciences,

University of Helsinki, Finland

Research question: We examined if Autism Spectrum Disorder (ASD) -related traits and performance in visual processing tasks are related in a group of young adults born preterm at very low birth weight (VLBW; <1500 g).

Methods: Our sample comprised 101 VLBW young adults of the Helsinki Study of Very Low Birth Weight Adults. They completed the Autism-Spectrum Quotient (AQ) yielding two sum scores on autism spectrum traits, and the Rey-Osterrieth Complex Figure Test (ROCF) yielding three time and three raw scores on visual processing. The effect of AQ scores on ROCF test scores was examined using linear regression analysis and necessary adjustments were made.

Results: The more autistic traits the VLBW group reported on the AQ Total sum score and Social interaction sum score, the slower they performed on all ROCF tasks (P-values < 0.05 ). There were no significant associations between the AQ sum scores and ROCF raw scores.

Conclusions: Our results imply that in adults born preterm at VLBW a higher level of ASD-related traits is associated with a slower global visual processing style. This is a unique study showing that individuals born preterm at VLBW are at an increased risk for a cognitive visual processing style related to ASD. 
4624

Pregnant women of South Asian origin have higher prevalence of symptoms suggestive of anxiety and depression in early pregnancy

Selvin Selvamoni ${ }^{1}$, Hema Venkatraman ${ }^{2}$, Nithya Sukumar ${ }^{3}$, Judith Plester ${ }^{4}$, Jessica Laidlaw ${ }^{5}$, James Hesford ${ }^{6}$, Ponnusamy Saravanan ${ }^{7}$

${ }^{1}$ Warwick University; ${ }^{2}$ Warwick University; ${ }^{3}$ Warwick Unversity; ${ }^{4}$ George Eliot Hospital; ${ }^{5}$ Warwick University;

${ }^{6}$ Warwick University; ${ }^{7}$ Warwick University

Research question: Maternal anxiety and depression are known to adversely affect offspring's health. We studied the ethnic specific prevalence of anxiety and depression.

Methods: Prospective data $(n=1386)$ from an ongoing longitudinal study (PRiDE) of women of high metabolic risk in the UK. Anxiety (GAD7), depression (PhQ9) and wellbeing (WMSWBS) were analysed by questionnaires in early pregnancy.

Results: Study population: $14.2 \%$ South Asians (SA), 71.1\% White Caucasians (WC) and $14.7 \%$ others. Mean age: 29.8 ( \pm 5.3 years); mean BMI:30.8 $\left( \pm 7.2 \mathrm{~kg} / \mathrm{m}^{2}\right)$ and mean gestational age:88 ( \pm 8.3 days).

Both anxiety [median (IQR) 3.0(0.0-0.7) vs. 2.0(0.0-0.6); $\mathrm{p}=0.02]$ and depression $[5.0(2.0,9.0)$ vs. 4.0(2.0,7.0); $\mathrm{p}=0.002]$ scores were higher in SA compared to WC. SA ethnicity $(p=0.02)$ and lower household income $(p=0.017)$ were independent predictors of anxiety (adjusted for age, BMI, smoking, marital status, educational attainment and employment status). Similarly, SA ethnicity $(\mathrm{p}=0.002)$ and lower income $(\mathrm{p}=0.002)$ were independent predictors of depression after adjustment for the same covariates. However, wellbeing was similar in SA and WC [54.0(46.0, 60.0) vs 53.0(46.0, $58.0) ; \mathrm{p}=0.381]$, which is probably contributed by marital status $(p=0.011)$ and higher household income $(p=0.014)$ after adjustment for the same covariates.

Conclusion: SA ethnicity and lower household income are associated with higher levels of anxiety and depression in highrisk pregnant women. Further studies are needed to evaluate the impact of this on offspring's health.

\section{6}

Multigenerational Programming of Anxiety Related Gene Expression in the Prefrontal Cortex after Antenatal Glucocorticoid: Paternal Transmission

Andrea Constantinof ${ }^{1}$, Vasilis Moisiadis ${ }^{2}$, Alisa Kostaki ${ }^{3}$, Stephen Matthews ${ }^{4}$

${ }^{1}$ University of Toronto; ${ }^{2}$ University of Toronto; ${ }^{3}$ University of Toronto; ${ }^{4}$ University of Toronto

Research question: Prenatal exposure to excess glucocorticoids is implicated in the development of psychiatric disorders through dysregulation of genes in the prefrontal cortex (PFC). We investigated whether prenatal synthetic glucocorticoids (sGC) program transcription of anxiety- and stress- related genes in the PFC across two generations following paternal transmission.

Methods: Pregnant guinea pigs received 3 courses of betamethasone (Beta; $1 \mathrm{mg} / \mathrm{kg} ; \mathrm{n}=12)$ or saline $(\mathrm{C} ; \mathrm{n}=11)$ at $75 \%$ of gestation. Male offspring were mated with naïve females to generate F2 animals. Gnb1, Fkbp5, Gr and $\mathrm{Mr}$ mRNA were measured by $\mathrm{q}-\mathrm{RTPCR}$ in PFC from $\mathrm{F} 1$ (Females: $\mathrm{C} n=6$, Beta $\mathrm{n}=4$; Males: $\mathrm{C} n=8$, Beta $\mathrm{n}=8$ ) and F2 (Females: $C n=7$, Beta $n=5$; Males: $C n=8$, Beta $n=8$ ) offspring at PND 40.

Results: $\mathrm{Mr}$ expression was reduced in F2 Beta females $(\mathrm{p}<0.05)$ compared to $\mathrm{C}$, with a trend towards reduced $\mathrm{Mr}$ in F1 Beta females $(p=0.057)$. Gnb1 expression was reduced in F1 Beta females $(\mathrm{p}<0.05)$. Fkbp5 and Gr expression were not altered in females. Gene expression was not altered in males.

Conclusion: Prenatal exposure to sGC resulted in sex-specific reduction in expression of genes associated with anxiety (Gnb1) and stress response $(\mathrm{Mr})$ in the PFC in two generations of juvenile female offspring.

\section{0}

Does Prenatal exposure to arsenic affect offspring cognition and educational outcomes?

James Jungius ${ }^{1}$, Sarah Lewis ${ }^{2}$, Carolina Bonilla ${ }^{3}$, Caroline Relton ${ }^{4}$, George Davey Smith ${ }^{5}$

${ }^{1}$ University of Bristol; ${ }^{2}$ University of Bristol; ${ }^{3}$ University of Bristol; ${ }^{4}$ University of Bristol; ${ }^{5}$ University of Bristol

Arsenic exposure during pregnancy impairs neural development. Arsenic is metabolized by methyltransferases including N6AMT1 and AS3MT. In this study we tested associations between 10 maternal AS3MT and 10 N6AMT1 SNPs on offspring cognition and educational outcomes in the ALSPAC cohort. We investigated offspring IQ at age 8(WISC score), standardised Key stage 2 (KS2) and 3 (KS3) results, and GCSE results with adjustment for offspring genotype and principal components. We found no association of any AS3MT SNPs investigated with any educational outcome. Seven of the N6AMT1 SNPs were negatively associated with offspring IQ at age 8 that endured adjustments (maximum decrease -2.11 (95\% C.I $-0.95,-3.25)$ IQ points per effect allele $(\mathrm{p}<0.05)$ ). Of these SNPs 4 were associated with standardised KS2 results $(p<0.05)$. None of these SNPs were associated with KS3 or categorical GCSE results when unadjusted. Adjustment for offspring genotype showed weak negative association with the same four SNPs. N6AMT1 and AS3MT are involved with different stages of arsenic metabolism. The association of N6AMT1 SNPs with offspring IQ at age 8 but lack of N6AMT1 association suggests that arsenic exposure in utero can have detrimental effects on offspring's cognitive 
development however this may not carry forward to educational outcomes.

\section{2}

Cognitive function can be affected by the physical properties of dietary lipids in early life

Lidewij Schipper ${ }^{1}$, Laus M. Broersen ${ }^{2}$, Maarten Loos ${ }^{3}$, Anton J.W. Scheurink ${ }^{4}$, Gertjan van Dijk ${ }^{5}$, Nana Bartke ${ }^{6}$

${ }^{1}$ Nutricia Research, Danone Nutricia Early Life Nutrition, Utrecht, the Netherlands; ${ }^{2}$ Nutricia Research, Advanced Medical Nutrition, Utrecht, the Netherlands; ${ }^{3}$ Sylics (Synaptologics BV), Amsterdam, the Netherlands; ${ }^{4}$ Dept Neuroendocrinology, Center for Behavior and Neurosciences, University of Groningen, the Netherlands; ${ }^{5}$ Dept Neuroendocrinology, Center for Behavior and Neurosciences, University of Groningen, the Netherlands;

${ }^{6}$ Nutricia Research, Danone Nutricia Early Life Nutrition, Utrecht, the Netherlands

Infant cognitive development can be positively influenced by prolonged breastfeeding, and differences in dietary lipid quality between Human Milk (HM) and Infant Milk Formula (IMF) may contribute. Indeed, alterations in the dietary supply of omega-3 fatty acids can affect cognitive function. We studied whether another aspect of lipid quality, i.e. physical properties of lipid globules, may also contribute to cognitive development. Between 16 and 42 days of life, male C57Bl/6j mice were subjected either to control diet or to diet containing complex lipid matrix (CLM), which more closely resembles physical properties of HM lipid globules (i.e. larger lipid droplets coated by phospholipids, Nuturis $\left.{ }^{\circledR}\right)$. Thereafter, regular rodent chow was fed until animals were sacrificed at day 101. During adolescence and young adulthood all mice were subjected to a test battery targeting various cognitive domains.

In adolescence, CLM fed mice showed improved attention and working memory. Some of these effects were sustained into adulthood. Spatial reference memory however remained unaffected during adolescence and in adulthood by the early diet. In conclusion, early life dietary exposure directly following lactation to a diet containing a lipid structure closer to BM lipid globules, can improve specific cognitive functions.

\section{2}

Periconceptional folic acid supplementation and child risk of autism spectrum disorder. Analysis from 89,293 motherchild pairs from the Danish Naitonal Birth Cohort

Marin Strøm ${ }^{1}$, Alberto Ascherio ${ }^{2}$, Kristen Lyall ${ }^{3}$, Sjúrdur Fródi Olsen ${ }^{4}$

${ }^{1}$ Centre for Fetal Programming, Statens Serum Institut; ${ }^{2}$ Dept Nutrition and Dept Epidemiology, Harvard T.H.Chan School of Public Health; ${ }^{3}$ Dept Nutrition, Harvard T.H.Chan School of Public Health; ${ }^{4}$ Centre for Fetal Programming, Statens Serum
Institut AND Dept Nutrition Harvard T.H. Chan School of Public Health

Research question: Folate is necessary for normal fetal spine, brain and skull development, due to its role in nucleotide synthesis, methylation processes, DNA integrity, and transcription. Recently, results from the large Norwegian $\mathrm{MoBa}$ Cohort showed periconceptional folic acid supplementation was associated with child autism spectrum disorder (ASD). Our aim was to test this hypothesis in the large prospective Danish National Birth Cohort.

Methods: We used data from 89,293 mother-children pairs from the Danish National Birth Cohort. Data on folic acid supplementation from 4 weeks prior to pregnancy until gestational week (gw) 12 was collected by a form completed at recruitment (typically between gw 6 to 9). ICD-10 diagnosis codes on ASD (F840, F841, F845, F848 and F849) were obtained from the mandatory Danish Central Psychiatric Research Registry.

Results: There were 1269 children with a diagnosis of ASD. We could detect no association between maternal folic acid supplementation and ASD; adjusted HR for gw -4 to 12 was 0.98 (95\% CI 0.85; 1.13). Similar conclusion was obtained when we looked at 4 week intervals separately.

Conclusion: In this large prospective cohort study we were not able to substantiate an association between maternal folic acid supplementation during the periconceptional period and child risk of ASD.

\section{4}

Birth weight and school performance using twin status as an instrumental variable: evidence from Hong Kong's 'Children of 1997' birth cohort

Shilin Lin ${ }^{1}$, GM Leung ${ }^{2}$, CM Schooling ${ }^{3}$

${ }^{1}$ The University of Hong Kong; ${ }^{2}$ The University of Hong Kong;

${ }^{3}$ The University of Hong Kong

Research question: What is the association of birth weight with school performance in late childhood? - evidence from a Chinese birth cohort.

Methods: In 3987 children from Hong Kong's "Children of 1997" birth cohort, we used ordinary least squares (OLS) regression to examine the adjusted association of sex- and gestational age-specific birth weight $\mathrm{z}$-score with school performance (on a Likert scale 1-6) at around 14 years. We also used twin status as an instrumental variable to obtain a less-confounded association.

Results: In OLS, birth weight z-score was positively associated with school performance $(0.06,95 \%$ confidence interval (CI) 0.02 to 0.10 ), adjusting for parental education, maternal age and migrant status. In instrumental variable analysis (IVA), there was no such association $(-0.02,95 \% \mathrm{CI}-0.27$ to 0.31 , F statistic $=11.7)$. 
Conclusions: Observationally, birth weight was positively associated with school performance at 14 years. However, as suggested by IVA, the positive association, usually observed, could be due to residual confounding by socio-economic position or reflect a biological mechanism which leads to low birth weight and impaired brain development, rather than reflecting the effect of low birth weight.

\section{4}

Age at onset of puberty and adolescent depressive symptoms: Evidence from Hong Kong's 'Children of 1997' birth cohort

Hui Wang ${ }^{1}$, Mary Catherine Schooling ${ }^{2}$, Shi Lin Lin ${ }^{3}$, Grbriel M Leung ${ }^{4}$

${ }^{1}$ The University of Hong Kong; ${ }^{2}$ The University of Hong Kong;

${ }^{3}$ The University of Hong Kong; ${ }^{4}$ The University of Hong Kong

Purpose: Timing of onset of puberty has fallen steeply with profound consequences for health and wellbeing. Whether timing of onset of puberty relates to depression is unclear.

Method: The adjusted associations of age at onset of puberty based on clinically assessed breast/genitalia and pubic hair development with depressive symptoms assessed from Patient Health Quesionnaire-9 z-score at $\sim 13$ years ( $\mathrm{n}=5795,73 \%$ ) were examined using multivariable linear regression in a population representative Hong Kong Chinese birth cohort 'Children of 1997'. We also assessed whether associations varied by sex.

Results: Associations between onset of puberty varied by sex ( $\mathrm{p}$ value $<0.05)$. Earlier breast development $(-0.17$, 95\% confidence interval $(-0.28,-0.05))$ and pubic hair development in girls rather than in boys $(-0.15,95 \%$ confidence interval $(-0.28,-0.03))$ were associated with depressive symptoms adjusted for age, socio-economic position, birth order, secondhand smoke exposure, parents' age at birth, survey mode and sex-specific birth weight z-score in girls. Similarly adjusted, earlier age of menarche was associated with more depressive symptoms $(-0.26,95 \%$ confidence interval $(-0.39,-0.12))$.

Conclusions: Early onset of puberty in girls was associated with depressive symptoms. Whether these findings are indicators of the effects of the hormones or of social pressures remain to be determined.

\section{0}

Is early inflammation associated with Attention Deficit/ Hyperactivity Disorder?

Desiree Silva ${ }^{1}$, Lyn Colvin ${ }^{2}$, Carol Bower ${ }^{3}$

${ }^{1}$ Telethon Kids Institute, School of Paediatrics and Child Health, Joondalup Health Campus; ${ }^{2}$ Telethon Kids Institute; ${ }^{3}$ Telethon Kids Institute
Research question: Do conditions causing inflammation in early life increase the risk of developing Attention Deficit/ Hyperactivity Disorder (ADHD).

Method: 16,883 children and adolescents (cases) aged 4-25 years had been prescribed stimulant medication in WA for $\mathrm{ADHD}$ and were recorded on the Monitoring of Drugs Dependency System (MODDS). A stratified random sample of birth records with no linkage to MODDS formed a comparison group $(32,728)$. Case and comparison records were linked to the Midwives Notification System and the Hospital Morbidity Database.

Results: Mothers of non- Aboriginal and Aboriginal children with ADHD were significantly more likely to smoke in pregnancy, have pre-eclampsia and threatened preterm labour. Children under 4 years who were subsequently diagnosed with ADHD, adjusted for maternal, perinatal and socioeconomic factors had significantly more early hospital admissions for infections and inflammatory conditions irrespective of race. Having an early surgical procedure involving a general anaesthetic also increased this risk.

Discussion: Early exposure to infections and conditions causing inflammation increased the risk of subsequently being diagnosed with ADHD irrespective of gender and race. Further studies need to understand the potential effects of inflammation on early brain development.

\section{3}

Cognitive performance in Indian children: Relationship to maternal vitamin $\mathrm{D}$ status during pregnancy

Sargoor Veena ${ }^{1}$, Ghattu Krishnaveni ${ }^{2}$, Krishnamachari Srinivasan ${ }^{3}$, Kotrangada Thajna ${ }^{4}$, Caroline Fall ${ }^{5}$

${ }^{1}$ Epidemiology Research Unit, CSI Holdsworth Memorial Hospital, Mysore, India; ${ }^{2}$ Epidemiology Research Unit, CSI Holdsworth Memorial Hospital, Mysore, India; ${ }^{3}$ St. John's Medical College, St. John's National Academy of Health Sciences, Bangalore, India; ${ }^{4}$ Epidemiology Research Unit, CSI Holdsworth Memorial Hospital, Mysore, India; ${ }^{5}$ MRC Lifecourse Epidemiology Unit, University of Southampton, Southampton, UK

Research question: To examine whether maternal 25-hydroxyvitamin D concentration during pregnancy is associated to offspring cognitive performance in an Indian population.

Methods: Cognitive performance was assessed in children from the Mysore Parthenon birth cohort during childhood (age 9-10 years; $n=468$ ) and adolescence (age 13-14 years; $n=472$ ) using 3 core tests from the Kaufman Assessment Battery for children and additional tests measuring learning, long-term retrieval/ storage, short-term memory, reasoning, visuo-spatial ability, verbal fluency, and attention and concentration. Maternal plasma 25-hydroxyvitamin D concentration was measured at $30 \pm 2$ weeks of gestation. 
Results: During pregnancy 320 (68\%) women had 'vitamin D deficiency' (plasma 25-hydroxyvitamin D concentration $<50$ $\mathrm{nmol} / \mathrm{l})$. Girls scored better than boys in short-term memory, reasoning, verbal fluency, and attention ( $p<0.05$ for all). Maternal vitamin D status (low as well as across the entire range) was unrelated to offspring cognitive performance at both ages, either unadjusted or after adjustment for the child's current age, sex, maternal age, parity, season at the time of blood sampling, socio-economic status, parents' education, maternal intelligence and home environment.

Conclusions: In this population, despite a high prevalence of maternal vitamin $\mathrm{D}$ deficiency, there was no evidence of an association between maternal vitamin $\mathrm{D}$ status during pregnancy and offspring cognitive performance.

\section{1}

Risk, resiliency, and early child development: a community based study

Dr. Suzanne Tough ${ }^{1}$, Dr. Sheila McDonald ${ }^{2}$, Heather Kehler ${ }^{2}$, Hamideh Bayrampour ${ }^{2}$, Nonie Fraser-Lee ${ }^{2}$

${ }^{1}$ University of Calgary; ${ }^{2}$ University of Calgary

Research question: Understanding risk and protective factors for early child development can inform prevention and early intervention. This study examines risk factors for development delay, as well as the role of resiliency within the context of poor maternal mental health or socioeconomic adversity.

Methods: Women participating in a prospective cohort study $(\mathrm{n}=3200)$ completed 3 questions in the perinatal period up to 1 year post birth. Maternal depression, anxiety, stress, social support, and parenting efficacy were measured using standardized tools. The child's development status at one year was measured using the Ages and Stages Questionnaire. The relationship between risk factors, resiliency, and early child development was examined using multivariable logistic regression and chi square tests with statistical significance set at p-value.

\section{0}

Maternal pre-pregnancy obesity and child neuropsychological development at 4 years

Maribel Casas ${ }^{1}$, Joan Forns ${ }^{2}$, David Martínez ${ }^{3}$, Martine Vrijheid ${ }^{4}$

${ }^{1}$ Centre for Research in Environmental epidemiology (CREAL); ${ }^{2}$ Centre for Research in Environmental epidemiology (CREAL); Department of Genes and Environment, Division of Epidemiology, Norwegian Institute of Public Health, Oslo, Norway; ${ }^{3}$ Centre for Research in Environmental epidemiology (CREAL); ${ }^{4}$ Centre for Research in Environmental epidemiology (CREAL)

Research question: To assess the association of maternal prepregnancy obesity with child neuropsychological development at 4 years of age.
Methods: The study included 1,746 children participating in the INMA Project, a prospective population-based cohort. Cognitive and psychomotor development was assessed using McCarthy Scales of Children's Abilities. Attention-deficit hyperactivity disorder (ADHD) symptoms were assessed using the Criteria of Diagnostic and Statistical Manual of Mental Disorders, Fourth Edition (ADHD-DSM-IV) form and autism spectrum disorder (ASD) using the Childhood Asperger Syndrome Test (CAST). Paternal BMI was used as negative control exposure.

Results: The percentage of obese mothers and fathers was $8 \%$ and $12 \%$, respectively. Children of obese mothers had lower cognitive and psychomotor development scores than those of normal weight mothers in the unadjusted model. After full adjustment for socioeconomic covariates and paternal BMI only the verbal subscale remained statistically significantly reduced $(\beta=-3.1 ; 95 \% \mathrm{CI}=-5.7,-0.5)$. Both maternal and paternal overweight and obesity were associated with an increased risk of ADHD and ASD symptoms.

Conclusion: Maternal pre-pregnancy obesity is associated with reduced verbal development scores at 4 years. The reported associations with ADHD and ASD could be attributed to unmeasured familial confounding.

\section{3}

Chronic Maternal Stress (CMS) during Early Pregnancy in Sheep Increases Fetal Hormonal Stress Response at 0.7 Gestation

Michelle Haase ${ }^{1}$, Michelle Haase ${ }^{2}$, Sven Rupprecht ${ }^{3}$, Rene Schiffner ${ }^{4}$, Schubert Harald ${ }^{5}$, Bischoff Sabine ${ }^{6}$, Nathanielsz Peter ${ }^{7}$, Schwab Matthias ${ }^{8}$

${ }^{1}$ Department of Neurology, Jena University Hospital, Jena, Germany; ${ }^{2}$ Department of Neurology, Jena University Hospital, Jena, Germany; ${ }^{3}$ Department of Neurology, Jena University Hospital, Jena, Germany; ${ }^{4}$ Department of Neurology, Jena University Hospital, Jena, Germany; ${ }^{5}$ Institute of Lab Animal Sciences, Jena University Hospital, Jena, Germany; ${ }^{6}$ Institute of Lab Animal Sciences, Jena University Hospital, Jena, Germany;

${ }^{7}$ Department of Animal Science, University of Wyoming, USA;

${ }^{8}$ Department of Neurology, Jena University Hospital, Jena, Germany

Research question: Does CMS during early pregnancy accentuate fetal stress response?

Methods: Six pregnant sheep underwent repeated isolation stress between 0.2 and 0.66 gestation (CMS group). Six pregnant ewes functioned as controls. At 0.7 gestation, fetuses were chronically instrumented and underwent acute isolation stress for $2 \mathrm{~h}$.

Results: Control and CMS ewes responded to isolation stress with an increase in maternal blood pressure, heart rate, cortisol and norepinephrine and a decrease in uterine blood flow (UBF, $\mathrm{P}<0.05)$. UBF decrease was more prolonged and accentuated following CMS (22\% for $36 \mathrm{~min}$ vs. $27 \%$ for $58 \mathrm{~min}, \mathrm{P}<0.05$ ). 
After CMS, fetal baseline norepinephrine concentration was elevated (0.70.1nmol.L-1 vs. $2.80 .7 \mathrm{nmol} . \mathrm{L}-1, \mathrm{P}<0.05)$ and cortisol baseline tended to be elevated. All fetuses showed no rise in cortisol but a prolonged norepinephrine increase over 60 to $120 \mathrm{~min}$ which was higher following CMS (1.40.4nmol.L-1 vs. 6.31.6nmol.L-1, P $<0.05)$.

Conclusion: CMS during early pregnancy accentuated fetal stress response by an increase of uterine vasculature sensitivity to maternal catecholamines.

\section{6}

Oxytocin receptor gene and psychosocial measures associated with mother-infant relationship

João Maroco $^{1}$, Jorge Sinval ${ }^{2}$, Adriana Saur ${ }^{3}$, Heloisa Bettiol ${ }^{4}$

${ }^{1}$ ISPA - Instituto Universitário de Ciências Psicológicas, Sociais e da Vida; ${ }^{2}$ Universidade do Porto and Universidade de São Paulo; ${ }^{3}$ Universidade de São Paulo and Centro Universitário Barão de Mauá; ${ }^{4}$ Universidade de São Paulo

Recent studies have suggested that maternal genetic susceptibility may be associated with different patterns of establishment of emotional bonds between mother and baby. We investigated which variables are predictors for the mother-infant relationship, including the oxytocin receptor gene (OXTR) and psychosocial measures. Brazilian mothers $(n=1057)$ completed assessments of mother-infant relationship (Postpartum Bonding Questionnaire), stress, depression and psychiatric symptoms using standardized instruments and were genotyped for OXTR rs53576 (AA/AG × $\mathrm{GG})$. Multiple linear regression was used to test the association of mother-infant relation with the above variables. Results indicated that the model achieves an acceptable amount of the total variance explained for the mother-infant relationship results ( $F$ $(4,1016)=70.36 ; \mathrm{p}<0.001, \mathrm{Ra} 2=0.214)$. The significant predictors were psychiatric symptoms $(\beta=0.176$; $\mathrm{t}(1016)=4.672$; $\mathrm{p}<0.001)$ and stress $(\beta=0.297 ; \mathrm{t}(1016)=8.242 ; \mathrm{p}<0.001)$. The variables genotype and depression were not significant. We concluded that psychosocial measures seem to be a more important predictor of emotional bonds between the mother and her child than genetic factors.

\section{4}

Feeding practices of mothers and/or caregivers of infants below the age of 6 months in four provinces in South Africa

Johann Jerling ${ }^{1}$, Sussana M Hanekom ${ }^{2}$, Edelweiss Wentzel-Viljoen $^{3}$

${ }^{1}$ North-West University; ${ }^{2}$ North-West University; ${ }^{3}$ North-West University

Breastfeeding is one of the primary strategies of enhancing infant nutrition as well as improving child survival worldwide. The International Conference on Nutrition (ICN) Rome
Declaration on Nutrition (2014) seeks to increase the rate of EBF in the first 6 months up to at least $50 \%$ globally. At national level, little data on infant and young child feeding is available.

Method: Fixed structured interviews were conducted for this cross-sectional study. A total of 580 mothers/caregivers were interviewed.

Results: The exclusive breastfeeding (EBF) rate at 6 months was $12 \%$. Most women that delivered full term babies had 6.2 higher odds of initiating breastfeeding within the first hour after delivery. Breastfeeding cessation (28\%) and introduction of complementary food $(17 \%)$ were done at one month. Only one $(0.2 \%)$ infant met the minimum standards of dietary diversity. Returning to work $(29 \%)$ or school $(12 \%)$, the mother's health status (25\%) and perceived "insufficient" milk supply (12.5\%) influenced the mothers' feeding practices. Conclusion: New strategies should address the gaps in key breastfeeding awareness messages with special focus on community involvement and participation. There is also a need for the whole nutrition fraternity, including the government, academia and development industries to interject and set out more innovative approaches.

\section{0}

Assessment of selected labelling practices observed on infant formula (IF) and follow-up formula (FUF) in four developing countries

Lara Sweet ${ }^{1}$, Alison Feeley ${ }^{2}$, Rosalyn Ford ${ }^{3}$, Jane Badham ${ }^{4}$, Elizabeth Zehner ${ }^{5}$

${ }^{1} J B$ Consultancy; ${ }^{2} J B$ Consultancy; ${ }^{3} J B$ Consultancy;

${ }^{4} J B$ Consultancy; ${ }^{5}$ Helen Keller International

Research question: What are the characteristics of the labels of FUF sold in four countries including descriptive name, colour scheme, design, age of introduction and use of images, that are the same/similar to IF made by the same manufacturer?

Methods: A cross-sectional survey was conducted in which available IF and FUF for sale in Cambodia, Nepal, Senegal and Tanzania were purchased and selected labelling practices assessed to determine similarities.

Results: The number of products purchased was 113, 15, 36 and 22 respectively. FUF and IF included similar or the same names/colour scheme/layout/design and/or similar/same slogans/mascots/symbols, as well as pack-shots and/or textual reference to other FUF or IF from the same manufacturer.

Conclusion: Similar labelling resulting in cross-promotion of FUF and IF manufactured by the same company is widespread and as a result may mislead the consumer, circumvent provisions of the Code and undermine global breastfeeding recommendations. FUF should either fall under the Code, or should be labelled in a different manner and not make reference to IF in order to promote optimal infant feeding. 
4471

Realities and challenges concerning claims on foods consumed by infants and young children: Assessment and Research in Child Feeding (ARCH)

Lara Sweet ${ }^{1}$, Alison Feeley ${ }^{2}$, Rosalyn Ford ${ }^{3}$, Jane Badham ${ }^{4}$, Elizabeth Zehner ${ }^{5}$

${ }^{1} J B$ Consultancy; ${ }^{2} J B$ Consultancy; ${ }^{3} J B$ Consultancy;

${ }^{4} J B$ Consultancy; ${ }^{5}$ Helen Keller International

Research question: What claims are being made on foods fed to IYC in 2 African countries?

Methods: Cross-sectional study in Senegal and Tanzania whereby labels of commercially produced foods marketed to (CPCF) and commonly fed to (CPF) children $<2$ years were assessed against international guidance and national legislation. Results: Claims were prevalent. Eighty nine percent of CPCF in Senegal made claims - $44 \%$ nutrient content and $42 \%$ nonnutrition. In Tanzania, where $92 \%$ of products included a claim, nutrient function/ other function/ implied health (38\%) and non-nutrition claims $(31 \%)$ were most common. In Senegal half of the $59 \%$ of CPF claims were non-nutrition and $37 \%$ nutrient content. In Tanzania, where $77 \%$ of CPF products made a claim, $43 \%$ were non-nutrition, $30 \%$ nutrient content and $27 \%$ nutrient function/ other function/ implied health claims.

Conclusions: As claims commonly occur on foods for IYC, global guidance on the appropriateness of all claims is necessary to support optimal IYCF. Guidance should ensure that restrictions on claims for nutritionally appropriate CPCF foods do not result in the unintended negative consequence of caregivers selecting other less nutritious foods which have fewer claim and promotion restrictions.

\section{6}

Iron and a mixture of docosahexaenoic and eicosapentaenoic acid supplementation, alone and in combination, affect bioactive lipid signalling and morbidity of iron deficient South African school childr

Jeannine Baumgartner ${ }^{1}$, Lizelle Zandberg ${ }^{2}$, Philip Calder ${ }^{3}$, Cornelius (Marius) Smuts ${ }^{4}$

${ }^{1}$ Centre of Excellence for Nutrition, North-West University; ${ }^{2}$ Centre of Excellence for Nutrition, North-West University; ${ }^{3}$ The Human Development and Health Academic Unit, Faculty of Medicine, University of Southampton, Southampton, United Kingdom and the NIHR Southampton Biomedical Research Centre in $N ;{ }^{4}$ Centre of Excellence for Nutrition, North-West University

Both iron and n-3 polyunsaturated fatty acids (PUFA) act through multiple mechanisms to influence immune cell responses. We recently reported that iron supplementation increased respiratory morbidity in iron deficient South African children. This increase, however, was attenuated when iron was provided in combination with a mixture of docosahexaenoic and eicosapentaenoic acid (DHA/EPA).
To explore potential underlying mechanisms, we examined the effects of iron and DHA/EPA, alone and in combination, on plasma lipid-derived immune modulator concentrations and related gene expression in peripheral blood mononuclear cells (PBMC). Lipid-derived immune modulators were analysed with liquid chromatography tandem mass spectrometry and gene expression with quantitative real time PCR.

DHA/EPA decreased inflammatory 12-hydroxyeicosatetraenoic acid and tended to increase anti-inflammatory and pro-resolving 17-hydroxydocosahexaenoic acid (17-HDHA), while iron decreased 17-HDHA. However, in combination with iron, the anti-inflammatory effect of DHA/EPA was maintained. These results were supported by directionally altered targeted PBMC gene expression results and predicted functional pathways. Thus, the biochemical findings presented here may explain the prevention of iron-induced respiratory morbidity which we observed when iron was supplemented in combination with DHA/EPA during the 8.5 mo randomised controlled trial. Also, this novel finding might lead to a safer approach of delivering iron supplementation.

\section{8}

Nutritional characteristics of soy malt beverage developed from blends of sorghum (sorghum bicolor) and soybean (glycine max)

Gloria Elemo $^{1}$, Babtunde Kosoko², Samuel Owolabi ${ }^{3}$, Bamke Adeyoju $^{4}$

${ }^{1}$ Federal Institute of Industrial Research, Oshodi, Lagos, Nigeria; ${ }^{2}$ Federal Institute of Industrial Research, Oshodi, Lagos, Nigeria;

${ }^{3}$ Federal Institute of Industrial Research, Oshodi, Lagos, Nigeria;

${ }^{4}$ Federal Institute of Industrial Research, Oshodi, Lagos, Nigeria

Research Question: Development of high nutrient-dense beverage from specially processed blends of sorghum as sorghum malt and soybeans as soymilk could improve nutritional status of school age children (age 5-13 years).

Methods used: A simple process technology was developed involving malting of sorghum, kilning, drying of the malted sorghum, wet milled into paste of less than 250 micron and sifted. Soymilk was produced from clean wholesome soybeans. The suspension thus obtained was combined with the soymilk with predetermined levels of preservatives (food grade), sweetener and flavourants followed by pasteurization at 90 degrees for 20-25 minutes.

Nutritional and safety evaluation studies using 60 experimental rats (95.4g average weight) for a period of six weeks were determined.

Results: The developed drink had a biological value of $100 \%$, 99.2\% Net protein utilization and $99.5 \%$ percent digestibility. Haematological profile of the rats showed that there was increased production of white blood cells (6070-6240) when rats were given their normal diet and the developed drink, the packed cell volume also increased ( 42.65 to $44.12 \%)$ as well as 
red blood cells (5840 to 5960) and blood platelets (2.76 to $2.91)$ in experimental rats. There was also gradual weight gain over time by the rats, the level of liver enzymes were safe as well as creatinine $(42.98$ to $50.93 \mathrm{mmmol} / \mathrm{L})$.

Conclusion: The developed Soymalt drink could be suitable for adoption as part of a school lunch program for school age children.

\section{3}

\section{Predominantly night-time feeding predicts infants gaining more weight}

Tuck Seng Cheng ${ }^{1}$, See Ling Loy ${ }^{2}$, Jia Ying Toh $^{3}$, Yin Bun Cheung ${ }^{4}$, Jerry Kok Yen Chan ${ }^{5}$, Keith Godfrey ${ }^{6}$, Peter Gluckman $^{7}$, Seang Mei Saw ${ }^{8}$, Yap-Seng Chong ${ }^{9}$, Yung Seng Lee $^{10}$, Ngee Lek ${ }^{11}$, Mary Foong-Fong Chong ${ }^{2}$, Fabian Yap ${ }^{13}$

${ }^{1} K K$ Women's and Children's Hospital, Singapore; ${ }^{2} K K$ Women's and Children's Hospital, Singapore; ${ }^{3}$ Agency for Science,

Technology and Research, (A*STAR), Singapore; ${ }^{4} D u k e-N U S$

Graduate Medical School, Singapore; ${ }^{5}$ KK Women's and Children's Hospital, Singapore; ${ }^{6}$ University of Southampton, Southampton, UK; ${ }^{7}$ Agency for Science, Technology and Research, ( $\left.A^{*} S T A R\right)$, Singapore; ${ }^{8}$ National University of Singapore, Singapore; ${ }^{9}$ National University of Singapore, Singapore; ${ }^{10}$ National University of Singapore, Singapore; ${ }^{11}$ KK Women's and Children's Hospital, Singapore; ${ }^{12}$ Agency for Science, Technology and Research, (A*STAR), Singapore; ${ }^{13} \mathrm{KK}$ Women's and Children's Hospital, Singapore

Background: It is unknown whether predominantly day-time or night-time feeding influences infant growth. We examined the association of infant meal timing with growth changes and body size at 24 months.

Methods: Mothers from a Singapore birth cohort reported food intake and meal timings of their children at 12 months of age, obtained by 24 -hour recall or a 72 -hour diary. Day-time (0700-1879h) and night-time (1900-0659h) feeders were defined by their respective periods of greater energy intake. Body Mass Index $Z$ scores (BAZ) were calculated using WHO Child Growth Standards 2006 to determine changes in BAZ at 12-24 months and weight status at 24 months. Associations between meal timing and growth were tested using multivariate regression.

Results: There were 587 day-time and 126 night-time feeders, with similar total daily caloric intakes [759.50 (SD 205.29) vs 782.65 (SD 213.41) kcal, $\mathrm{p}=0.254$ ]. Compared to day-time feeders, higher BAZ gain at 12-24 months (adjusted $\beta=0.27$, $95 \% \mathrm{CI}=0.077,0.462 ; \mathrm{p}=0.006)$ and an increased risk of becoming overweight (adjusted OR: 3.18, 95\% CI $=1.04$, 9.72; $\mathrm{p}=0.042)$ but not underweight were observed in nighttime feeders.

Conclusion: Night-time feeding may predispose infants to being overweight and is a modifiable risk factor against childhood obesity.
4602

Dietary supplementation with essential micronutrients during early postnatal life partly prevents chronic earlylife stress-induced cognitive impairments

Eva F. G. Naninck ${ }^{1}$, Kit-Yi Yam² ${ }^{2}$ Johannes B. van Goudoever $^{3}$, Torsten Plosch ${ }^{4}$, Paul J Lucassen ${ }^{5}$, Aniko Korosi ${ }^{6}$

${ }^{1}$ Swammerdam Institute for Life Sciences, Center for Neuroscience, University of Amsterdam; ${ }^{2}$ Swammerdam Institute for Life Sciences, Center for Neuroscience, University of Amsterdam; ${ }^{3}$ Department of Mother \& Child, Academic Medical Center, Amsterdam, the Netherlands; ${ }^{4}$ Department of Obstetrics o gynecology, Universitair Medisch Centrum Groningen, the Netherlands; ${ }^{5}$ Swammerdam Institute for Life Sciences, Center for Neuroscience, University of Amsterdam; ${ }^{6}$ Swammerdam Institute for Life Sciences, Center for Neuroscience, University of Amsterdam

Early-life stress (ES) lastingly impairs brain structure and function. We investigate a possible role of nutrition in this programming and focus on methyldonors (MD) (e.g. methionine, vitamin B's) important for development and epigenetic modifications, implicated in programming. We study: i) if ES alters micronutrient availability; ii) the epigenetic, structural and behavioral effects of ES and, iii) if early MD-supplementation can reverse ES-induced changes in the offspring.

ES was induced in C57Bl/6 mice from postnatal day (P) 2-9, while dams received either control or MD-enriched diet. Nutrient content was measured at $\mathrm{P} 9$ in stomach milk, plasma and brain of the offspring. Next, we studied effects of MD-diet on ES-induced alterations in maternal behavior, the offspring's stress axis activity, neurogenesis, DNA methylation levels (global and $\mathrm{Nr} 3 \mathrm{C} 1$ specific) and DNA methyltransferase expression, in the hippocampus, a brain region important for cognition.

We show that ES reduces methionine levels in offspring's plasma and brain. Importantly, MD-diet ameliorates ES-induced cognitive impairments in adulthood, abolishing deficits in spatial learning and memory. MD-diet does not only restore methionine levels in the offspring, but also appears to prevent ES-induced stress axis hyperactivity. Our findings show that a short, early nutritional intervention can prevent lasting ES-effects on hippocampal functions.

\section{3}

Developmental changes in brain LCP accumulation: effect of lowering $n 6$ LA or increasing n3 LCP's in the maternal diet during lactation

Annemarie Oosting ${ }^{1}$, Lidewij Schipper ${ }^{2}$

${ }^{1}$ Nutricia Research, Danone Nutricia Early Life Nutrition, Utrecht, the Netherlands; ${ }^{2}$ Nutricia Research, Danone Nutricia Early Life Nutrition, Utrecht, the Netherlands 
$\mathrm{N} 3$ long-chain polyunsaturated fatty acids (LCPs) are important for brain development. Brain LCP accretion depends on dietary supply of LCPs and their C18 precursors. The contemporary dietary increase in linoleic acid (LA; C18:2n6) and decrease in n3LCP intake is also reflected in human milk fatty acid composition. As human milk is the sole source of nutrition for newborn infants this could also impact brain development. We investigated whether increased maternal n3LCP or reduced LA intake during lactation could enhance n3LCP incorporation in offspring brain.

C57Bl/6j dams with litter were subjected to control diet, reduced LA diet (low-LA), or increased n3LCP diet (High-n3) during lactation. At several times milk was collected and pups sacrificed to collect brains.

Maternal High-n3 resulted in increased n3LCP and reduced n6LCP levels in milk and offspring brain. The low-LA reduced LA and n6LCP without affecting n3LCP in milk, whereas in offspring brain n3LCP was increased and n6LCP reduced.

In conclusion, both reduced maternal dietary LA and increased n3LCPs improve offspring brain n3LCP status. However, because LA inhibits n3LCP biosynthesis and incorporation in the developing brain, lowering LA intake during lactation may be a more effective way to increase infant brain n3LCPs accretion than supplementing with n3LCPs.

\section{4}

Long-term impact of early feeding with hydrolyzed compared to regular cow's milk formula: the German Infant Nutritional Intervention Study GINI

Sibylle Koletzko ${ }^{1}$, Andrea v. Berg, Birgit Filipiak-Pittroff, Holger Schulz, Ute Hoffmann, Elke Link, Maike Sußmann, Markus Schnappinger, Irene Brüske, Marie Standl, Ursula Krämer, Barbara Hoffmann, Joachim Heinrich, Carl-Peter Bauer, Dietrich Berdel

${ }^{1}$ Dr. von Hauner Children's Hospital, University of Munich

Research question: What is the long-term impact of feeding infants hydrolyzed compared to regular cow's milk formula (CMF)?

Methods: Between 1995 -1998 2252 newborns at allergic risk were randomly allocated to receive double blind partial whey hydrolyzate (pHF-W), extensive whey hydrolyzate (eHF-W), extensive casein hydrolyzate (eHF-C) or CMF during the first four months of life as breast milk substitute if necessary. Children were followed at 1, 2, 3, 4, 6, 10 and 15 years. Outcomes included cumulative incidence and period prevalence of physician-diagnosed allergies and sensitization to common allergens. Logistic and Log-binomial regression models using generalized estimation equation for repeated measures were performed.

Results: Of 2252 infants (ITT population), 637 were exclusively breastfed for 6 months, of the remaining 1615 (modified
ITT), 988 consumed study formula adhering to the protocol (PP). Of the PP (ITT) population $67.3 \%(64.4 \%)$ and $66.0 \%$ (61.1\%) provided data at 10 and 15 years, respectively. Risk reduction for eczema occurred during the first year for eHF-C and $\mathrm{pHF}-\mathrm{W}$ and persisted without rebound. A reduced period prevalence for eHF-C (PP) at age 10 years point to a long-term preventive effect.

Conclusion: Early feeding with certain hydrolyzed formulas compared with CMF has long-term health

\section{8}

Determinants of breastfeeding practices and success in a multi-ethnic Asian population

Wei Wei Pang ${ }^{1}$, Izzuddin M. Aris ${ }^{2}$, Doris Fok ${ }^{3}$, Shu-E Soh ${ }^{4}$, Mei Chien Chua ${ }^{5}$, Sok Bee Lim $^{6}$, Seang-Mei Saw ${ }^{7}$, Kenneth

Kwek $^{8}$, Peter D. Gluckman ${ }^{9}$, Keith M. Godfrey ${ }^{10}$, Rob M. van $\mathrm{Dam}^{11}$, Michael S. Kramer ${ }^{12}$, Yap-Seng Chong ${ }^{13}$

${ }^{1}$ National University of Singapore; ${ }^{2}$ Singapore Institute for Clinical Sciences (SICS), Agency for Science, Technology and Research $\left(A^{*} S T A R\right) ;{ }^{3}$ National University of Singapore;

${ }^{4}$ Singapore Institute for Clinical Sciences (SICS), Agency for Science, Technology and Research (A*STAR); ${ }^{5} K K$ Women's and Children's Hospital (KKH); 'KK Women's and Children's Hospital (KKH); ${ }^{7}$ National University of Singapore; ${ }^{8} K K$ Women's and Children's Hospital (KKH); ${ }^{9}$ Singapore Institute for Clinical Sciences (SICS), Agency for Science, Technology and Research ( $\left.A^{*} S T A R\right) ;{ }^{10}$ University of Southampton and University Hospital Southampton NHS Foundation Trust; ${ }^{11}$ National University of Singapore; ${ }^{12} \mathrm{Mc}$ Gill University; ${ }^{13}$ Singapore Institute for Clinical Sciences (SICS), Agency for Science, Technology and Research ( $\left.A^{*} S T A R\right)$

Research question: To assess the prevalence, duration and mode of breastfeeding (direct or expressed) among mothers of three Asian ethnic groups in Singapore.

Methods: Participants were 1030 women from the GUSTO (Growing Up in Singapore Toward healthy Outcomes) cohort study. Data collected included early breastfeeding experiences, breastfeeding initiation and duration and mode of breastfeeding. Cox regression models identified factors associated with discontinuation of any breastfeeding and exclusive breastfeeding. Logistic regression analyses assessed the association of ethnicity with mode of breastfeeding.

Results: At 6 months postpartum, the prevalence of any breastfeeding was $46 \%$ for Chinese mothers, $22 \%$ for Malay mothers and $41 \%$ for Indian mothers; prevalences of exclusive breastfeeding were $11 \%, 2 \%$ and $5 \%$, respectively. A higher proportion of Chinese mothers fed their infants expressed breastmilk, as opposed to directly breastfeeding them, compared with the other two ethnic groups. Adjusting for maternal education, breastfeeding duration was similar in the 3 ethnic groups, but ethnicity remained a significant predictor of mode of breastfeeding. 
Conclusions: Breastfeeding rates and duration need improvement in this multi-ethnic Asian population. Further work is needed to understand the cultural differences in mode of breastfeeding and its implications for maternal and infant health.

\section{9}

The Role of Early Nutrition in Programming of Adult Body Composition - the Helsinki Study of Very Low Birth Weight Adults

Juho Kuula Kuula ${ }^{1}$, Hanna-Maria Matinolli Matinolli ${ }^{2}$, Petteri Hovi Hovi ${ }^{3}$, Satu Männistö Männistö ${ }^{4}$, Marika SipolaLeppänen Sipola-Leppänen ${ }^{5}$, Johan G Eriksson Eriksson ${ }^{6}$, Outi Mäkitie Mäkitie ${ }^{7}$, Anna-Liisa Järvenpää Järvenpää ${ }^{8}$, Sture Andersson Andersson ${ }^{9}$, Eero Kajantie Kajantie ${ }^{10}$

${ }^{1}$ National Institute for Health and Welfare; ${ }^{2}$ National Institute for Health and Welfare; ${ }^{3}$ National Institute for Health and Welfare, ildren's Hospital, University of Helsinki and Helsinki University Central Hospital; ${ }^{4}$ National Institute for Health and Welfare; ${ }^{5}$ National Institute for Health and Welfare; ${ }^{6}$ National Institute for Health and Welfare, Unit of General Practice, Helsinki University Central Hospital, Folkhälsan Research Centre, Vasa Central Hospital; ${ }^{7}$ Children's Hospital, University of Helsinki and Helsinki University Central Hospital, Vasa Central Hospital; ${ }^{8}$ Children's Hospital, University of Helsinki and Helsinki University Central Hospital; ${ }^{9}$ Children's Hospital, University of Helsinki and Helsinki University Central Hospital; ${ }^{10}$ National Institute for Health and Welfare, Children's Hospital, University of Helsinki and Helsinki University Central HospitalDepartment of Obstetrics and Gynaecology, Oulu Univer

Background: Suboptimal nutrition during fetal life and early childhood has been proposed to play a crucial role in increasing the risk of adverse health effects in later life.

Objective: We studied the associations between early postnatal nutrition and adult body size and composition among subjects born preterm at very low birth weight (VLBW; <1500 g).

Design/Methods: We collected comprehensive information on daily nutritional intake of 127 young adults born with VLBW in 1978-1985 from the hospital records of their initial hospital stay. We calculated mean daily intakes of protein, fat and carbohydrate for 3-week periods between birth and 9 weeks. At the mean age of 22.5 (SD 2.1) years, body composition was measured by DXA.

Results: Protein intake during the first 3 weeks, which was below the current recommendations, predicted adult body composition. Similar associations were seen with early fat but not with carbohydrate intake. The results were little changed when adjusted for pre- and postnatal medical complications. Conclusions: Among preterm infants with relatively low protein intakes, a higher protein intake predicts a higher lean body mass over 20 years later. Our results emphasize the importance of variation in nutrition during early postnatal life as an important contributor in programming later metabolic health.
4719

Associations between toddlers' food neophobia and dietary habits. A cross-sectional study

Nina Cecilie $\emptyset_{\text {verby }}{ }^{1}$, Sissel Heidi Helland ${ }^{2}$, Elling Bere ${ }^{3}$

${ }^{1}$ Department of Public Health, Sports and Nutrition, University of Agder, Kristiansand, Norway; ${ }^{2}$ Department of Public Health, Sports and Nutrition, University of Agder, Kristiansand, Norway; ${ }^{3}$ Department of Public Health, Sports and Nutrition, University of Agder, Kristiansand, Norway

Research question: Is there an association between food neophobia and dietary habits in Norwegian toddlers?

Methods: We recruited parents of 2-year-olds from 309 kindergartens in Norway. In total 605 parents agreed to participate, and 496 filled in a food frequency questionnaire (FFQ) regarding child diet and child food neophobia (CFN). CFN was measured using Child Food Neophobia Scale. Of the original 10 items, four items were excluded for not being ageappropriate. Higher score indicated greater degree of CFN. From the FFQ we made sum scores of consumption of fruits and vegetables, snacks and high processed foods, respectively.

Results: Mean score on Child Food Neophobia Scale was 18.2 (SD: 9.4) among 2-year olds (53\% boys). Mean consumption of fruits and vegetables was 25 times/week (SD: 9.9), snacks was $11.5 \mathrm{t} /$ week (SD:2.8) and high processed foods was $16.2 \mathrm{t} /$ week (SD: 3.3) In a multiple regression analysis, total intake of fruits and vegetables was significantly associated with CFNSscores, both crude (B:-0.317, SE:0.046, p <0,001) and when adjusting for parental education (B:-0.316 (SE: 0.047), $\mathrm{p}<0.001)$. There were no significant associations between other food groups and food neophobia.

Conclusion: Our study indicates a lower intake of fruit and vegetables when with higher scores on food neophobia.

\section{2}

Nutritional and Safety Characteristics of Soymalt Beverage for School Age Children in Low Income Countries

Oluwatoyin Oluwole ${ }^{1}$, Gloria Elemo ${ }^{2}$, Babatunde Kosoko ${ }^{3}$, Samuel Owolabi ${ }^{4}$, Banke Adeyoju ${ }^{5}$

${ }^{1}$ Federal Institute of Industrial Research, Oshodi, Lagos, Nigeria; ${ }^{2}$ Federal Institute of Industrial Research, Oshodi, Lagos, Nigeria; ${ }^{3}$ Federal Institute of Industrial Research, Oshodi, Lagos, Nigeria; ${ }^{4}$ Federal Institute of Industrial Research, Oshodi, Lagos, Nigeria; ${ }^{5}$ Federal Institute of Industrial Research, Oshodi, Lagos, Nigeria

Research question: Development of high nutrient dense beverage from Sorghum malt and Soymilk could improve nutritional status of school age children (age 5 to 13 years).

Methods used: A process technology was developed involving malting of Sorghum, kilning, drying followed by wet milling into paste and sifting. Soymilk was produced from clean wholesome soybeans. The suspension thus obtained was 
combined with the soymilk with predetermined levels of sweetener and flavourants followed by pasteurization at 90degrees for 20-25 minutes.

Nutritional and safety evaluation studies using 60 experimental rats (95.4g mean weight) for a period of six weeks were determined.

Results: Biological value of $100 \%, 99.2 \%$ Net protein utilization and $99.5 \%$ percent digestibility was recorded in the developed beverage. White blood cells, packed cell volume, red blood cells, blood platelets and creatinine levels were (60706240), (42.65 to $44.12 \%)$, (5840 to 5960$)$, (2.76 to 2.91$)$ and 42.98 to $50.93 \mathrm{~mm} \mathrm{~mol} / \mathrm{L}$ for white blood cells, packed cell volume, red blood cells, blood platelets and creatinine levels respectively. The level of the liver enzymes were safe.

Conclusion: The developed Soy malt beverage is suitable for clinical trials using human subjects.

\section{3}

Nutritional and Anaemic Status associated with Asymptomatic Malaria in school aged children in Nkassomo, Centre region-Cameroon: Pilot study

Clovis Hugues Seumen Tiogang ${ }^{1}$, Clovis Hugues Seumen Tiogang ${ }^{2}$, Francis Zeukeng ${ }^{3}$

${ }^{1}$ The Biotechnology Cente/University of Yaounde $i{ }^{2}$ The Biotechnology Cente/University of Yaounde i; ${ }^{3}$ The Biotechnology Center/University of Yaounde I

Research question: Undernutrition and anaemia among children remain a challenging public health problem in developing countries where malaria is endemic. Is the any association between nutritional status, anaemia and malaria among children in Nkassomo (a village in the center region-Cameroon).

Methodology: A cross- sectional survey was carried out in November 2014. Overall, 68 children aged 2-16 years were enrolled in the study. Anthropometric parameters were measured, Weight for age (WA) and Height for age (HA) z-scores were calculated to assess underweight and stunting respectively. Finger-prick blood samples were collected for haematocrit, malaria parasite determination and Plasmodial genomic DNA extraction from filter paper.

Results: 38 children (55.88\%) harbored malaria parasites. Plasmodium falciparum was the only species involved. 44 (64.7\%) were anaemic; 24 (35.29\%) had moderate anaemia and $20(29.41 \%)$ mild anemia. A significant association was found between malaria status and anaemia. However, there was no association between malaria status and the severity of anemia. Globally, 27 (39.7\%) and 36 (52.9\%) were underweight and stunted respectively. However, there was no statistically significant association between stunting and malaria status or underweight and malaria status.

Conclusion: Malaria is highly prevalent in children in Nkassomo village, it is associated with anaemia but not with malnutrition.

\section{5}

Classification of mixed milk-fed infants by infancy dried blood spot lipidomic profiles

Philippa Prentice ${ }^{1}$, Animesh Acharjee ${ }^{2}$, Ken $\mathrm{K} \mathrm{Ong}^{3}$, James Smith ${ }^{4}$, Carlo L Acerini ${ }^{5}$, Ieuan A Hughes ${ }^{6}$, David B Dunger ${ }^{7}$, Albert Koulman ${ }^{8}$

${ }^{1}$ Department of Paediatrics, University of Cambridge, $U K ;{ }^{2} M R C$ Human Nutrition Research, Cambridge; ${ }^{3}$ MRC Epidemiology Unit, University of Cambridge, UK; ${ }^{4} M R C$ Human Nutrition Research, Cambridge; ${ }^{5}$ Department of Paediatrics, University of Cambridge, UK; ${ }^{6}$ Department of Paediatrics, University of Cambridge, UK; ${ }^{7}$ Department of Paediatrics, University of Cambridge, UK; ${ }^{8}$ MRC Human Nutrition Research, Cambridge

Research question: We previously demonstrated substantial differences between 3-month lipidomic profiles of exclusively breast-fed and formula-fed infants, using a novel method with dried blood spots (DBS). These early changes may contribute to metabolic programming. We now investigate whether formula milk intake affects lipidomic profiles in mixed (breast +formula milk)-fed infants.

Methods: 40 new DBS samples were analysed at age 3-months (16 breast-fed, 11 formula-fed, 13 mixed-fed); 39 at 6-months, from a representative birth cohort. High-resolution massspectrometry identified 100 lipids: sphingomyelins (SM), phosphatidylcholines (PC), cholesterol-esters and triglycerides. Lipid species and ratios previously predictive of breast-feeding, e.g. lower $\mathrm{PC}(34: 2): \operatorname{SM}(36: 2)$, were related to feeding type and formula milk volume, assessed by questionnaire.

Results: Breast-fed vs. formula-fed infants were successfully classified by lipidomic profile ( $3 \%$ error), validating previous work. Infants with limited formula intake $(<200 \mathrm{mls}=1-2$ feeds/day) had lipid profiles mirroring exclusively breast-fed infants e.g. low $\mathrm{PC}(34: 2): \operatorname{SM}(36: 2)$ ratio; those consuming large formula volumes representing profiles of exclusively formula-fed infants, others showing intermediary profiles. Formula milk intake also affected lipidomic profile at age 6months (after 86\% had commenced weaning).

Conclusions: Lipidomic profiles classify mixed-fed infants according to formula milk intake. Preliminary results suggest that consuming 1-2 formula feeds/day may not significantly alter lipidomic profiles from those of exclusively breast-fed infants.

\section{4}

Iodine status and thyroid function of breastfed infants aged 2-4 months and their mothers residing in a South African township

Jennifer Osei ${ }^{1}$, Olivia van der Reijden ${ }^{2}$, Susanne Dold ${ }^{3}$, Maria Andersson ${ }^{4}$, Cornelius M Smuts ${ }^{5}$, Jeannine Baumgartner ${ }^{6}$

${ }^{1}$ Centre of Excellence for Nutrition, North-West University, Potchefstroom, South Africa; ${ }^{2}$ Human Nutrition Laboratory, Institute of Food, Nutrition, and Health, ETH Zurich; ${ }^{3}$ Human 
Nutrition Laboratory, Institute of Food, Nutrition, and Health, ETH Zurich; ${ }^{4}$ Human Nutrition Laboratory, Institute of Food, Nutrition, and Health, ETH Zurich; ${ }^{5}$ Centre of Excellence for Nutrition, North-West University, Potchefstroom, South Africa; ${ }^{6}$ Centre of Excellence for Nutrition, North-West University, Potchefstroom, South Africa

Research Question: We assessed iodine nutrition in breastfed infants and lactating women and the relative contribution of iodine in salt (SIC) and maternal salt intake.

Methods: Using a cross-sectional design, we measured SIC, breast-milk iodine concentrations (BMIC), urinary iodine concentrations (UIC) and thyroid function of 100 mothers and their 2-4 month-old breastfed infants.

Results: Median (m) UIC of infants $(373 \mu \mathrm{g} / \mathrm{L})$ and mothers $(118 \mu \mathrm{g} / \mathrm{L})$ were sufficient. However, $49 \%$ of mothers had UIC $<100 \mu \mathrm{g} / \mathrm{L}$. In contrast, $53 \%$ of infants had $\mathrm{UIC}>300 \mu \mathrm{g} / \mathrm{L}$. mSIC was $44.1 \mu \mathrm{g} / \mathrm{g}$. SIC and maternal urinary sodium excretion predicted UIC of mothers. mBMIC was $179 \mu \mathrm{g} / \mathrm{L}$. Age of infants, SIC, maternal UIC and urinary sodium excretion predicted BMIC. BMIC and infant age predicted UIC of infants. Infants from households with SIC above the recommended upper level of 40 ppm (59\%) had higher odds for having UIC $>300 \mu \mathrm{g} / \mathrm{L}(\mathrm{OR}=2.8,95 \% \mathrm{CI}$ : $1.1,7.2)$. Infants with UIC $>300 \mu \mathrm{g} / \mathrm{L}$ had higher odds for having elevated thyroglobulin concentrations $(\mathrm{OR}=1.69,95 \% \mathrm{CI}: 1.37$, 2.08) compared to infants with a UIC within the normal range $(100 \geqslant \mathrm{UIC} \leqslant 300 \mu \mathrm{g} / \mathrm{L})$.

Conclusion: Our results suggest that the salt iodization program in South Africa supplies sufficient iodine to infants and lactating mothers. However, better monitoring of salt iodization is needed to avoid "over-iodization", which may cause iodine overload in breastfed infants.

\section{0}

\section{Effects of Maternal PUFA Status and Breastfeeding on Early Childhood Infection Risks}

Germaine Jia Min Yong ${ }^{1}$, Wei Wei Pang ${ }^{2}$, Philip C Calder ${ }^{3}$, Keith M Godfrey ${ }^{4}$, Peter D Gluckman ${ }^{5}$, Seang-Mei Saw ${ }^{6}$, Kenneth Kwek ${ }^{7}$, Yap Seng Chong ${ }^{8}$, Mary Foong Fong Chong ${ }^{9}$, Lynette Pei-Chi Shek ${ }^{10}$, Shu E Soh ${ }^{11}$

${ }^{1}$ Singapore Institute of Clinical Sciences, Agency for Science, Technology and Research; ${ }^{2}$ Department of Obstetrics and Gynaecology, Yong Loo Lin School of Medicine, National University of Singapore, National University Health System; ${ }^{3}$ Human Development and Health Academic Unit, Faculty of Medicine, University of Southampton; NIHR Southampton Biomedical Research Centre, University of Southampton; Department of Bi; ${ }^{4}$ Human Development and Health Academic Unit, Faculty of Medicine, University of Southampton; NIHR Southampton Biomedical Research Centre, University of Southampton; Medical Research; ${ }^{5}$ Singapore Institute of Clinical Sciences, Agency for Science, Technology and Research; Liggins
Institute, University of Auckland; ' ${ }^{6}$ Saw Swee Hock School of Public Health, National University of Singapore, National University Health System; ${ }^{7}$ Department of Maternal Fetal Medicine, KK Women's and Children's Hospital; 'Singapore Institute of Clinical Sciences, Agency for Science, Technology and Research; Department of Obstetrics and Gynaecology, Yong Loo Lin School of Medicine, National Universit; ${ }^{9}$ Clinical Nutrition Research Centre, Singapore Institute of Clinical Sciences, Agency for Science, Technology and Research; Department of Pediatrics, Yong Loo Lin School of Medicine; ${ }^{10}$ Department of Pediatrics, Yong Loo Lin School of Medicine, National University of Singapore, National University Health System; Singapore Institute of Clinical Sciences, Agency for; ${ }^{11}$ Singapore Institute of Clinical Sciences, Agency for Science, Technology and Research

Research question: Infant breastfeeding and polyunsaturated fatty acid (PUFA) intake have been linked with early childhood infections; less is known about the possible role of maternal PUFA status during pregnancy. We examined how maternal PUFA status during pregnancy and infant breastfeeding collectively relate to early childhood infection.

Methods: We analyzed plasma phosphatidylcholine n-3, n-6, and total PUFA levels (26-28th weeks' gestation) in 951 Singapore GUSTO cohort mothers. Offspring breastfeeding status and infection symptoms (bronchiolitis/bronchitis, vomiting, diarrhoea, and fever) were ascertained using repeated questionnaires from age $0-18 \mathrm{mo}$. Offspring breastfeeding status was grouped as high (exclusive/predominantly breastfed until $4 \mathrm{mo}$, then at least partially breastfed until $\geqslant 6 \mathrm{mo}$ ), low (exclusively formula-fed by $3 \mathrm{mo}$ ), or intermediate (all others). Logistic regression analyses were adjusted for childcare, household income, education and number of siblings.

Results: Independent of breastfeeding status, higher maternal n-3 PUFA status was associated with lower risks of infant bronchiolitis/bronchitis, diarrhoea, and vomiting, while a higher maternal n-6:n-3 PUFA ratio correlated with infant diarrhoea and vomiting. Interestingly, high and intermediate breastfeeding groups were associated with higher fever risk, a novel observation amongst similar studies. Bronchiolitis/bronchitis and vomiting were not associated with breastfeeding status.

Conclusion: Maternal PUFA status may have in utero effects on offspring infection risk of infection during infancy.

\section{4}

One Carbon metabolism in young Indian girls: use of methionine load test

Prachi Katre ${ }^{1}$, Suyog Joshi ${ }^{2}$, Dattaray Bhat Bhat ${ }^{3}$, Neha Gurav $^{4}$, Swati Pandit ${ }^{5}$, Himangi Lubree ${ }^{6}$, Sadanand Naik ${ }^{7}$, Anura Kurpad $^{8}$, Satish Kalhan ${ }^{9}$, Chittaranjan Yajnik ${ }^{10}$

${ }^{1}$ Diabetes Unit, King Edward Memorial Hospital Research Centre, Pune, India; ${ }^{2}$ Diabetes Unit, King Edward Memorial Hospital Research Centre, Pune, India; ${ }^{3}$ Diabetes Unit, King 
Edward Memorial Hospital Research Centre, Pune, India; ${ }^{4}$ Diabetes Unit, King Edward Memorial Hospital Research Centre, Pune, India; 5 Diabetes Unit, King Edward Memorial Hospital Research Centre, Pune, India; ${ }^{6}$ Diabetes Unit, King Edward Memorial Hospital Research Centre, Pune, India; ${ }^{7}$ Diabetes Unit, King Edward Memorial Hospital Research Centre, Pune, India; ${ }^{8}$ Department of Physiology, Division of Nutrition, St John's Medical College, Bangalore, India; ${ }^{9}$ Department of Pathobiology, Lerner Research Institute. The Cleveland Clinic Foundation, Cleveland, Ohio, United States;

${ }^{10}$ Diabetes Unit, King Edward Memorial Hospital Research Centre, Pune, India

Research question: To investigate 1-C metabolism in severely B12 deficient girls before and after supplementation with B12 and multimicronutrients (MMN).

Methods: Thirty nine B12 deficient adolescent girls were randomized to receive $2.0 \mu \mathrm{g} \mathrm{B} 12(\mathrm{n}=19)$ or $\mathrm{B} 12+\mathrm{MMN}$ (including $2.0 \mu \mathrm{g}$ of $\mathrm{B} 12,1 \mathrm{mg}$ of $\mathrm{B} 6$ and $1.8 \mathrm{mg}$ of $\mathrm{B} 2+$ other $)+$ milk powder $(5 \mathrm{gm}$ protein). A methionine load test $(50 \mathrm{mg} / \mathrm{kg}$ of body weight) was performed before and 6 months after supplementation. Biomarkers of one carbon metabolism were measured in the plasma.

Results: Girls were 17 years old with a BMI of $19.0 \mathrm{~kg} / \mathrm{m}^{2}$. Their median plasma B12 concentration was $92 \mathrm{pmol} / \mathrm{L}$ and homocysteine was 41.5 micromoles/L. Supplementation resulted in a significant and similar rise in plasma levels of B12 (+68 pmol/L) and a fall in t-Hcy (-26 micromoles/L), hyperhomocysteinimia reduced by $60 \%$. Rise in plasma homocysteine concentration during methionine load test was attenuated by supplementation in both groups by $87 \%$.

Conclusion: Supplementation of B12 deficient young girls with $2 \mathrm{mcg}$ of B12/day for 6 months reduced hyperhomocysteinimia significantly, but did not normalize the levels. Multimicronutrients and protein had no additional effect. Dose-response and isotopic investigations will help study contribution of different metabolic cycles to deranged 1-C metabolism.

\section{3}

\section{Breast milk cholesterol concentration is independent of} LDL-receptor expression in mice

Mirjam AM Lohuis ${ }^{1}$, Lidiya G Dimova ${ }^{2}$, Uwe JF Tietge ${ }^{3}$, Henkjan J Verkade ${ }^{4}$

${ }^{1}$ Department of Pediatrics, University of Groningen, University Medical Center Groningen, Hanzeplein 1, 9713 GZ Groningen, the Netherlands; ${ }^{2}$ Department of Pediatrics, University of Groningen, University Medical Center Groningen, Hanzeplein 1, 9713 GZ Groningen, the Netherlands; ${ }^{3}$ Department of Pediatrics, University of Groningen, University Medical Center Groningen, Hanzeplein 1, 9713 GZ Groningen, the Netherlands; ${ }^{4}$ Department of Pediatrics, University of Groningen, University Medical Center Groningen, Hanzeplein 1, 9713 GZ Groningen, the Netherlands
Research question: Breast milk contains high cholesterol concentrations, which conceivably impact long-term cholesterol homeostasis in the offspring. However, origin and regulation of milk cholesterol concentrations are largely unknown. Here, we studied whether low-density lipoproteins (LDL) contribute to milk cholesterol concentrations using a dietary intervention and a genetic (LDL receptor knock-out) approach.

Methods: Blood and milk were sampled on day 7 of lactation from C57BL/6J(WT) and LDLR-KO dams on control or high cholesterol (HCh; 0.5\%, 2 weeks) diets after 3-4h separation from their pups.

Results: HCh diet slightly increased plasma cholesterol in WT, mainly within the (V)LDL fraction, but did not affect milk cholesterol concentration $(1.4 \mathrm{mmol} / \mathrm{L})$. LDLR-KO mice had a 3.6-fold higher plasma (V)LDL-cholesterol on control diet compared to WT (p-value).

\section{0}

Effect of infant formula with protein content of $1.6 \mathrm{~g} /$ 100kcal fed between 3-12 months on growth at 3 and 5 years of age

Ekhard E. Ziegler ${ }^{1}$, David A. Fields ${ }^{2}$, Steven D. Chernausek ${ }^{3}$, Philippe Steenhout ${ }^{4}$, Dominik Grathwohl ${ }^{5}$, Janice M. Jeter ${ }^{6}$, Steven E. Nelson ${ }^{7}$, Nicholas P. Hays ${ }^{8}$, Ferdinand Haschke ${ }^{9}$

${ }^{1}$ Department of Pediatrics, University of Iowa, Iowa City, IA, USA; ${ }^{2}$ Department of Pediatrics, University of Oklahoma Health Sciences Center, Oklahoma City, OK, USA; ${ }^{3}$ Department of Pediatrics, University of Oklahoma Health Sciences Center, Oklahoma City, OK, USA; ${ }^{4}$ Nestlé Clinical Development Unit, Lausanne, Switzerland; ${ }^{5}$ Nestlé Clinical Development Unit, Lausanne, Switzerland; ${ }^{6}$ Department of Pediatrics, University of Iowa, Iowa City, IA, USA; ${ }^{7}$ Department of Pediatrics, University of Iowa, Iowa City, IA, USA; ${ }^{8}$ Nestlé Nutrition ROD, King of Prussia, PA, USA; ${ }^{9}$ Department of Pediatrics, PMU Salzburg, Austria

Research question: Do children fed formula with unconventionally low protein content during infancy have normal growth at age 3 and 5 years?

Methods: Children randomized as healthy infants to receive experimental (EXPL; $1.61 \mathrm{~g}$ protein $/ 100 \mathrm{kcal}, 67.2 \mathrm{kcal} / \mathrm{dL}$ ) or control (CTRL; $2.15 \mathrm{~g}$ protein $/ 100 \mathrm{kcal}, 64.6 \mathrm{kcal} / \mathrm{dL}$ ) formula from age $3-12 \mathrm{mo}(\mathrm{n}=97 /$ group $)$, or enrolled as a nonrandomized breastfed group $(\mathrm{BF} ; \mathrm{n}=112)$, were contacted for follow-up visits at 3 and 5y. Follow-up measures included anthropometry and body composition (reported separately). Treatment differences $(\mathrm{n}=192$ at $3 \mathrm{y} ; \mathrm{n}=173$ at $5 \mathrm{y})$ were estimated by ANCOVA correcting for baseline (3-mo value), sex, and maternal pre-pregnancy BMI.

Results: There were no significant differences between EXPL and CTRL in any anthropometric measure at 3 or $5 y$. Weight, BMI, and BMI-for-age Z-scores at $5 y$ were significantly higher 
(all $\mathrm{p}<0.05$ ) in CTRL vs. BF, with no significant differences between EXPL and BF. Although height-for-age Z-scores were significantly higher in both CTRL and EXPL vs. BF at $3 y$ $(\mathrm{p}<0.01)$, differences at $5 \mathrm{y}$ were not significant.

Conclusion: Children fed formula with a protein content of $1.61 \mathrm{~g} / 100 \mathrm{kcal}$ have normal growth at 3 and 5 years of age. Higher BMI values at age 5 years among children fed higherprotein formula vs. breast milk during infancy suggest a possible programming effect.

\section{4}

Effect of formula derived Advanced Glycation End products on liver antioxidant systems of IUGR piglets

Firmin Stéphane ${ }^{1}$, Elmhiri Ghada ${ }^{2}$, Bahi-Jaber Narges ${ }^{3}$, Abdennebi-Najar Latifa ${ }^{4}$

${ }^{1}$ UP 2012.10.101. EGEAL-Institut Polytechnique LaSalle Beauvais 60000-Beauvais France; ${ }^{2}$ UP 2012.10.101. EGEALInstitut Polytechnique LaSalle Beauvais 60000-Beauvais France; ${ }^{3}$ UP 2012.10.101. EGEAL-Institut Polytechnique LaSalle Beauvais 60000-Beauvais France; ${ }^{4} U P$ 2012.10.101. EGEALInstitut Polytechnique LaSalle Beauvais 60000-Beauvais France

Research question: We recently reported lipid peroxidation products in liver of intrauterine growth retarded (IUGR) piglets fed Advanced Glycation End products derived formula (AGEs-F) in their early postnatal life. Since the mechanisms underlying these negative effects remain to be elucidated, we aimed to investigate whether antioxidant enzymes involved in the control of redox homeostasis were altered in tissues of these IUGR piglets.

Methods: Superoxide dismutase (SOD) and glutathione peroxidase (GPx) activities were measured in liver of 54 day old pigs fed low temperature heated formula (LHF: $n=8$ ) or a high temperature heated formula (HHF: $n=8$ ) during suckling period.

Results: AGEs induced oxidative damages were accompanied with a significant increase of SOD activity in liver of HHF compared to LHF animals (p-value).

\section{9}

Effect of Vitamin B12 and MMN intervention on one carbon (1C) indices in rural Indian children

Shradha Gangulikar ${ }^{1}$, Nilam Memane ${ }^{2}$, Deepa Raut ${ }^{3}$, Pallavi Hardikar ${ }^{4}$, Charudatta Joglekar ${ }^{5}$, Pallavi Yajnik ${ }^{6}$, Caroline $\mathrm{Fall}^{7}$, Chittaranjan Yajink ${ }^{8}$

${ }^{1}$ Diabetes Unit, Kem Hospital Research Center, Pune, India; ${ }^{2}$ Diabetes Unit, Kem Hospital Research Center, Pune, India;

${ }^{3}$ Diabetes Unit, Kem Hospital Research Center, Pune, India;

${ }^{4}$ Diabetes Unit, Kem Hospital Research Center, Pune, India;

${ }^{5}$ Diabetes Unit, Kem Hospital Research Center, Pune, India;

${ }^{6}$ Diabetes Unit, Kem Hospital Research Center, Pune, India;
${ }^{7}$ MRC Lifecourse Epidemiology Unit, UK; ${ }^{8}$ Diabetes Unit, Kem Hospital Research Center, Pune, India

Research question: We aimed to study the efficacy of vitamin B12 intervention on $1 \mathrm{C}$ metabolites in Pune Intervention trial. Methods: The children $(\mathrm{n}=557)$ were randomized into three groups: Vitamin B12 $(2 \mu \mathrm{g})$, vitamin B12 $(2 \mu \mathrm{g})+\mathrm{MMN}+$ milk powder and placebo. We measured total homocysteine (tHcy), cysteine, glutathione, folate and vitamin B12 at screening and follow up (8-15months after start of intervention). Iron and Folic acid were given to all. Group identity and vitamin B12 are not shown due to blinding.

Results: At screening, median hemoglobin, B12, folate concentration were $13.4 \mathrm{~g} / \mathrm{dl}, 175 \mathrm{pM}$ and $18 \mathrm{nM} / \mathrm{L}$ respectively. $50 \%$ were B12 deficient and 3\% were folate deficient. Median tHcy in the three groups were 25.6, 22.6 and $23.4 \mu \mathrm{M} / \mathrm{L}$ respectively. The prevalence of hyperhomocysteinemia was 89 , 87 and $84 \%$ respectively. At follow up, median tHcy were $12.0,24.0$ and $12.2 \mu \mathrm{M} / \mathrm{L}$ respectively and the prevalence of hyperhomocysteinemia was 36,75 and $35 \%$ respectively. The intervention caused a significant fall in the tHcy in two groups. There were no changes in hemoglobin, folate, cysteine and glutathione concentrations.

Conclusions: Our intervention seems to be efficacious in reducing plasma tHcy. We expect this will have favorable effect on fetal growth and epigenetic programming.

\section{1}

Long term modification of microbiota and antioxidant defenses by high formula derived ages in iugr individuals

Ghada Elmhiri ${ }^{1}$, Samir Dou ${ }^{2}$, Thibaut Larcher ${ }^{3}$, Narges BahiJaber $^{4}$, Stephane Firmin ${ }^{5}$, Latifa Abdenebbi-Najar ${ }^{6}$

${ }^{1}$ UP-EGEAL 2012.10.101 Institut Polytechnique LaSalle Beauvais-France; ${ }^{2}$ UP-EGEAL 2012.10.101 Institut Polytechnique LaSalle Beauvais-France; ${ }^{3}$ INRA, UMR 703 APEX, Ecole Nationale Vétérinaire Agroalimentaire et de ' Alimentation Nantes-Atlantique (Oniris), Nantes, France; ${ }^{4}$ UP-EGEAL 2012.10.101 Institut Polytechnique LaSalle Beauvais; ${ }^{5}$ UP-EGEAL 2012.10.101 Institut Polytechnique LaSalle Beauvais; ${ }^{6} U P-E G E A L$ 2012.10.101 Institut Polytechnique LaSalle Beauvais

Research question: We have shown that Formula-derived dietary advanced glycation end products (F-AGEs) promote programming of inflammation and oxidative stress in kidney of intrauterine growth retardation (IUGR) individuals. In this study we aim to determine whether F-AGEs modify microbial set-up and disturb the antioxidant defenses in colon at adult age.

Methods: IUGR piglets received either a low heated formula (LHF, $\mathrm{n}=8$ ) or a high heated formula (HHF, $\mathrm{n}=8$ ) for 3 weeks. Piglets were fed ad libitum regular diet until postnatal day (PND) 54. Feces and Ceco-colonic microbiota were 
characterized at PND36 and 54 by CE-SSCP and qPCR analysis. At PND54, colon histological analysis, GPX enzyme activity and oxidative stress status were determined.

Results: Fecal Bacterial diversity and abundance were modified at PND36 in HHF as compared to LHF animals (p-value).

\section{0}

Impact of infant feeding on growth trajectory patterns from birth to six years: a four cohorts analysis in the Early Nutrition project

Berthold Koletzko ${ }^{1}$, Peter Rzehak ${ }^{2}$, Wendy H. Oddy ${ }^{3}$, Maria Luisa Mearin ${ }^{4}$, Merete Eggesbø $\varnothing^{5}$, Veit Grote ${ }^{6}$, Trevor A. Mori $^{7}$, Martina Weber ${ }^{8}$, Lawrence J. Beilin ${ }^{9}$, Rae-Chi Huang ${ }^{10}$

${ }^{1}$ Ludwig-Maximilians-University of Munich Medical Centre, Munich, Germany; ${ }^{2}$ Ludwig-Maximilians-University of Munich Medical Centre, Munich, Germany; ${ }^{3}$ Ludwig-MaximiliansUniversity of Munich Medical Centre, Munich, Germany; ${ }^{4}$ Ludwig-Maximilians-University of Munich Medical Centre, Munich, Germany; ${ }^{5}$ Ludwig-Maximilians-University of Munich Medical Centre, Munich, Germany; ${ }^{6}$ Ludwig-MaximiliansUniversity of Munich Medical Centre, Munich, Germany; ${ }^{7}$ Ludwig-Maximilians-University of Munich Medical Centre, Munich, Germany; ${ }^{8}$ Ludwig-Maximilians-University of Munich Medical Centre, Munich, Germany; ${ }^{9}$ Ludwig-MaximiliansUniversity of Munich Medical Centre, Munich, Germany;

${ }^{10}$ Ludwig-Maximilians-University of Munich Medical Centre, Munich, Germany

Research question: We aimed to test the hypothesis that early infant feeding is associated with programming of growth and obesity in childhood. Our objective was to define growth trajectories of children up to age 6 years across four cohorts.

Methods: We used data on four cohort studies participating in the European Union funded Early Nutrition research project were included (West Australian Pregnancy Cohort (Raine) Study, European Childhood Obesity Trial (CHOP Study), HUMIS Norway, Prevent CD Netherlands) on anthropometry, infant feeding and maternal characteristics. Data from the cohorts were combined into one model using latent growth mixture modelling. Predictors of the identified body mass index (BMI) (standard deviation score) trajectories were identified and tested (breastfeeding duration, maternal prepregnancy BMI, maternal age and education).

Results: In multivariate analysis, breastfeeding duration of less than 3 months compared to breastfeeding duration of 3 months or longer, was significantly associated with either a 4.5 fold increased odds for children to develop a persistent, accelerating rapid growth pattern or a 1.78-fold odds to develop early, non-persistent rapid growth rather than a normative growth pattern.

Discussion: A shorter duration of breastfeeding predicts rapid, accelerated growth in infancy and until at least 6 years of age.
4402

Epigenome-wide association study identifies novel CpG loci associated with perimembranous ventricular septal defects in very young children

K.P.J. Wijnands ${ }^{1}$, J. Chen ${ }^{2}$, L. Liang ${ }^{3}$, X. Lin ${ }^{4}$, W.A. Helbing $^{5}$, P.J. van der Spek ${ }^{6}$, A.G. Uitterlinden ${ }^{7}$, R.P.M. Steegers-Theunissen ${ }^{8}$

${ }^{1}$ Department of Obstetrics and Gynaecology, Erasmus MC, University Medical Centre, Rotterdam, The Netherlands; ${ }^{2}$ Department of Biostatistics, Harvard School of Public Health, Boston, MA, United States of America. Department of Biomedical Statistics and Informatics, Mayo Clinic, Rochester, MN;

${ }^{3}$ Department of Biostatistics and Department of Epidemiology, Harvard School of Public Health, Boston, MA, United States of America; ${ }^{4}$ Department of Biostatistics, Harvard School of Public Health, Boston, MA, United States of America; ${ }^{5}$ Department of Paediatrics, Division of Paediatric Cardiology, Erasmus MC, University Medical Centre, Rotterdam, The Netherlands; ${ }^{6}$ Department of Bioinformatics, Erasmus $M C$, University Medical Centre, Rotterdam, The Netherlands; ${ }^{7}$ Genetic Laboratory of the Department of Internal Medicine and Department of Epidemiology, Erasmus MC, University Medical Centre, Rotterdam, the Netherlands; ${ }^{8}$ Department of Obstetrics and Gynaecology and Department of Clinical Genetics, Erasmus MC, University Medical Centre, Rotterdam, The Netherlands

Research question: We performed an epigenome-wide association study in DNA derived from peripheral white blood cells of very young children to identify novel 5'-cytosine-phosphoguanosine $(\mathrm{CpG})$ loci associated with perimembranous ventricular septal defects (pVSD).

Methods: We investigated 455,741 CpG loci in 84 case children with pVSD and 196 control children at 17 months of age using the Illumina Infinium ${ }^{\circledR}$ HumanMethylation450 BeadChip.

Results: Using conservative Bonferroni correction, we identified one $\mathrm{CpG}$ locus on chromosome 1 within the gene body of PRDM16 that was higher methylated in pVSD children $(+4.8 \%$ in cases, P-value $=9.17 \mathrm{E}-08)$. At $15 \%$ false discovery rate, we identified seven additional CpG loci mapped to eight genes on five chromosomes. Absolute methylation differences were observed in CpG loci mapped to ESAM $(-2.2 \%$ in cases, P-value $8.09 \mathrm{E}-07)$, PPFIA4 $(+2.4 \%$ in cases, $\mathrm{P}$-value $=8.46 \mathrm{E}-07)$, NFIA $(-0.8 \%$ in cases, $\mathrm{P}$-value $=9.13 \mathrm{E}-$ $-07)$, HNRNPU $(-0.7 \%$ in cases, $\mathrm{P}$-value $=1.87 \mathrm{E}-06)$, HIF3A $(-2.8 \%$ in cases, $\mathrm{P}$-value $=2.08 \mathrm{E}-06), \mathrm{CDK} 4(-0.4 \%$ in cases, $\mathrm{P}$-value $=2.11 \mathrm{E}-06)$ and MOAP1 and C14orf109 $(-0.4 \%$ in cases, P-value $=2.42 \mathrm{E}-06$ ).

Conclusion: We demonstrate an association between one higher methylated CPG locus within PRDM16 and increased risk of $\mathrm{pVSD}$. Moreover, differential methylation of CpG loci mapped to eight genes involved in embryogenesis, cardiogenesis or related to severe heart defects was shown. 
4435

Pregnancy induces selective changes in the maternal hepatic transcriptome, which may be regulated by DNA methylation

Samuel Hoile ${ }^{1}$, Mark Hanson ${ }^{2}$, Karen Lillycrop ${ }^{3}$, Graham Burdge $^{4}$

${ }^{1}$ University of Southampton; ${ }^{2}$ University of Southampton;

${ }^{3}$ University of Southampton; ${ }^{4}$ University of Southampton

Research question: The mechanisms underlying pregnancyassociated structural adaptations to the liver are poorly understood. We hypothesised that these adaptations involve alterations to the transcriptome and to epigenetic processes. To address this, we measured the expression of the liver transcriptome and methylome during pregnancy.

Methods: C57/Blk6 mice (90 day old, $n \geqslant 6$ ) were culled, or mated and culled on $\mathrm{d} 7, \mathrm{~d} 14$ or $\mathrm{d} 18$ gestation. Cell size was determined by histology. Expression of the liver transcriptome was measured by microarray ( $>2$-fold signal threshold). Global methylation and hydroxymethylation were measured by ELISA.

Results: Cell size increased from d7 onwards. 115 genes were altered at $\mathrm{d} 14$, and 123 at $\mathrm{d} 18$ compared to non-pregnant animals. These were associated primarily with cell proliferation and apoptosis, and predicted increased cell proliferation at $\mathrm{d} 14$ and $\mathrm{d} 18$, and lower apoptosis at $\mathrm{d} 18$. Global methylation and hydroxymethylation was decreased significantly in pregnant compared to non-pregnant liver. This was accompanied by lower DNA methyltransferase-3 and higher Tet2 expression.

Conclusion: Together these findings show liver growth in early pregnancy involved hypertrophy and hyperplasia, while late pregnancy involved cell proliferation and decreased apoptosis. These results also support the suggestion that altered epigenetic processes are involved.

\section{7}

Impact of maternal endotoxin challenge and omega-3 fatty acid supplementation on ovine placental and fetal hippocampal gene expression

Rebecca Fisher-Heffernan ${ }^{1}$, Margaret Quinton ${ }^{2}$, Niel Karrow ${ }^{3}$

${ }^{1}$ University of Guelph; ${ }^{2}$ University of Guelph; ${ }^{3}$ University of Guelph

Research Question: Increased occurrence of inflammatory diseases in adulthood may result from stressors encountered in utero. Therefore, it is proposed that maternal supplementation with anti-inflammatory omega-3 polyunsaturated fatty acids (n-3 PUFA) such as fishmeal (FM) may protect the fetus from stress-induced changes in gene expression pertaining to the hypothalamic-pituitary-adrenal axis (HPAA).

Methods: In late gestation, ewes were supplemented with FM or soybean meal (SM). Half the ewes in each treatment received a lipopolysaccharide (LPS) endotoxin or saline (CON) bolus, and subsequently fetal tissues were collected. Gene expression was assessed in fetal tissues by real-time qPCR and included genes involved in HPAA regulation including 11- $\beta$-hydroxysteroid dehydrogenase types 1 and 2 (11- $\beta$-HSD $1 / 2)$, cyclooxygenases (COX-1/2) and interleukin-1 in the placenta, mineralocorticoid receptor (MR) in the fetal hippocampus and melanocortin-2 receptor in the fetal adrenals.

Results and conclusion: It was found that placental 11- $\beta$ HSD2 expression is significantly lower in FM compared to SM treatments and trends in COX-2 expression indicate reduced expression in $\mathrm{FM}+\mathrm{CON}$ compared to $\mathrm{SM}+\mathrm{CON}$. A trend in hippocampal MR expression indicates reduced expression in the $\mathrm{SM}+\mathrm{LPS}$ group compared to the $\mathrm{SM}+\mathrm{CON}$ and $\mathrm{FM}+\mathrm{LPS}$ groups. This study demonstrates that fetal HPAA gene expression is influenced by LPS exposure and n-3 PUFA supplementation.

\section{5}

Epigenome of circadian clock related genes in pre-eclampsia Caroline van den Berg ${ }^{1}$, Ines Chaves ${ }^{2}$, Emilie Herzog ${ }^{3}$, Sten Willemsen ${ }^{4}$, Bert van der Horst ${ }^{5}$, Régine Steegers-Theunissen ${ }^{6}$ ${ }^{1}$ Erasmus MC University Medical Centre Rotterdam; ${ }^{2}$ Erasmus MC University Medical Centre Rotterdam; ${ }^{3}$ Erasmus MC University Medical Centre Rotterdam; ${ }^{4}$ Erasmus MC University Medical Centre Rotterdam; ${ }^{5}$ Erasmus MC University Medical Centre Rotterdam; ${ }^{6}$ Erasmus MC University Medical Centre Rotterdam

Research question: The placenta is important in providing a healthy environment for the fetus and plays a central role in the pathophysiology of pre-eclampsia (PE). There is some evidence that pre-eclampsia is related to an altered circadian homeostasis. Our objective was to compare DNA methylation at circadian clock genes between PE and control pregnancies.

Methods: In a nested case-control study pregnancies complicated with PE and controls with comparable gestational age at delivery were enrolled. Umbilical cord white blood cells, endothelial cells, and placental biopsies of $29 \mathrm{PE}$ and 20 control pregnancies (gestational age median range) were investigated. Genome-wide methylation analysis (Illumina 450K DNA methylation arrays) was performed. ANCOVA of the methylation of CpGs at circadian clock genes was used, adjusted for gestational age at sampling and for multiple comparisons.

Results: DNA methylation differed significantly between early-onset PE and controls at 6 circadian clock gene related CpGs in placenta and $22 \mathrm{CpGs}$ in umbilical cord white blood cells $(\mathrm{P}<0.05)$.

Conclusion: Variations in DNA methylation at circadian clock genes in early-onset compared to late-onset PE suggests differences in origins and severity of this placental-related disease. In the future DNA methylation markers may be used as early predictors of health of the offspring. 
4493

Patterns of epigenome-wide differential DNA methylation in maternal, placental and fetal bio-samples between term low and normal birth weight newborns

Fuying Tian ${ }^{1}$, Chuanbo Xie ${ }^{2}$, Xiaozhong Wen ${ }^{3}$, Zhongzheng $\mathrm{Niu}^{4}$, Peng Ding ${ }^{5}$, Yanhui $\mathrm{He}^{6}$, Jianmiao Lin $^{7}$, Shixin Yuan ${ }^{8}$, Xiaoling Guo ${ }^{9}$, Deqin Jia ${ }^{10}$, Weiqing Chen ${ }^{11}$

${ }^{1}$ Sun Yat-Sen University; ${ }^{2}$ Sun Yat-Sen University; ${ }^{3}$ State University of New York at Buffalo; ${ }^{4}$ Sun Yat-Sen University; ${ }^{5}$ Sun Yat-Sen University; ${ }^{6}$ Guangdong Provincial Center for Disease Control and Prevention; ${ }^{7}$ Shenzhen Women and Children's Hospital; ${ }^{8}$ Shenzhen Women and Children's Hospital; ${ }^{9}$ Foshan Women and Children's Hospital; ${ }^{10}$ Foshan Women and Children's Hospital; ${ }^{11}$ Sun Yat-Sen University

Research question: Development Origins of Health and Disease postulates that early environmental perturbations contribute to low birth weight (LBW) and the long-term health through epigenetic. This study will explore epigenetic links among mother-placenta-fetus as a whole unit in the term LBW. Methods: Maternal and cord blood and placenta of six term LBW cases and six controls matched for age, gestational week, pre-pregnant BMI, parity and newborn gender were sampled from mother-child pairs at birth. DNA methylation was measured by Infinium Human Methylation 450K BeadChip and a series of bioinformatics analysis were conducted.

Results: 153 differentially methylated regions (DMRs) were identified in LBW for maternal blood, 684 for placenta and 184 for cord blood. Enrichment analysis showed that dominant methylation changes were metabolism in maternal and cord blood and placenta, and the magnitude of changes was the greatest in cord blood followed by placenta and maternal blood. Maternal DNA aberrant methylation is mainly embodied in the detoxification process, and the placental DNA methylation modifications were enriched in biological adhesion, cell communication, and development process.

Conclusion: Results implicated that DNA methylation modification patterns were distinct in three tissues and these changes had functional associations with each other.

\section{9}

Epigenome-wide differentially methylated regions in tissues of placental and fetal origin

Emilie M. Herzog ${ }^{1}$, Alex J. Eggink ${ }^{2}$, Sten P. Willemsen ${ }^{3}$, Jun Chen ${ }^{4}$, Anton J.M. Roks ${ }^{5}$, Eric A.P. Steegers ${ }^{6}$, Regine P.M. Steegers-Theunissen ${ }^{7}$

${ }^{1}$ Department of Obstetrics and Gynaecology, Erasmus MC, University Medical Centre Rotterdam, the Netherlands; ${ }^{2}$ Department of Obstetrics and Gynaecology, Erasmus MC, University Medical Centre Rotterdam, the Netherlands;

${ }^{3}$ Department of Biostatistics, Erasmus MC, University Medical

Centre Rotterdam, the Netherlands; ${ }^{4}$ Department of Biostatistics,
Harvard School of Public Health, Boston, MA, United States of America; ${ }^{5}$ Department of Internal Medicine, Section of Vascular Medicine and Pharmacology, Erasmus MC, University Medical Centre Rotterdam, the Netherlands; ${ }^{6}$ Department of Obstetrics and Gynaecology, Erasmus MC, University Medical Centre Rotterdam, the Netherlands; ${ }^{7}$ Department of Obstetrics and Gynaecology, Erasmus MC, University Medical Centre Rotterdam, the Netherlands

Research question: Periconception epigenetic programming is essential for lineage differentiation and embryonic development. DNA methylation is an epigenetic mechanism of programming mostly studied in DNA derived from easily accessible white blood cells. We aimed to identify epigenomewide differentially methylated regions in tissues of placental and fetal origin.

Methods: We conducted a nested case-control study, embedded in the Rotterdam periconceptional birth cohort (Predict Study). In 36 uncomplicated term pregnancies, we collected UCWBC $(n=25)$ and placental tissue $(n=25)$. Endothelial cells (HUVEC) were obtained by collagenase perfusion of the umbilical vein $(n=25)$. DNA methylation of cytosine-guanine dinucleotides (CpGs) was measured by the Illumina HumanMethylation $450 \mathrm{~K}$ BeadChip. Statistical analysis was performed using a linear mixed model with gestational age adjustment and Bonferroni correction ( $\alpha 1.099 \times 10-7)$.

Results: Epigenome-wide analysis revealed differentially methylated CpGs between UCWBC and placenta (335.236 CpGs, 74\%), between placenta and HUVEC (334.375 CpGs, 74\%), and between UCWBC and HUVEC (199.207 CpGs, 44\%). Further identifications and functional assessments of the tissue-specific differentially methylated regions are in progress. Conclusion: Placental and fetal tissues demonstrate epigenome-wide tissue-specific differentially methylated regions. To improve the validity of future epigenetic association studies in birth cohorts, tissue specificity should be addressed.

\section{8}

Effects of non-steroid vs steroid-induced preterm birth in sheep on expression of genes involved in insulin secretion and hepatic insulin sensitivity

Amita Bansal ${ }^{1}$, Jane Alsweiler ${ }^{2}$, Hui Hui Phua ${ }^{3}$, Kristin Connor ${ }^{4}$, Mike Dragunow ${ }^{5}$, Rob De Matteo ${ }^{6}$, Jane Harding ${ }^{7}$, Frank Bloomfield ${ }^{8}$

${ }^{1}$ Liggins Institute, University of Auckland, Auckland, New Zealand; Gravida: National Centre for Growth \& Development, New Zealand; Current address: Center for Research on Reproducti; ${ }^{2}$ Liggins Institute, University of Auckland, Auckland, New Zealand; Department of Paediatrics: Child \& Youth Health, University of Auckland, Auckland, New Zealand; Gravida: National; ${ }^{3}$ Liggins Institute, University of Auckland, Auckland, New Zealand; ${ }^{4}$ Liggins Institute, University of Auckland, Auckland, New Zealand; Samuel Lunenfeld Research Institute, 
Mount Sinai Hospital, Toronto, Canada; Gravida: National Centre for Growth o Development, New Zealand; ${ }^{5}$ Centre of Brain Research, University of Auckland, Auckland, New Zealand; Gravida: National Centre for Growth \& Development, New Zealand; ${ }^{6}$ Ritchie Centre, MIMR-PHI Institute of Medical Research, Melbourne, Australia; ${ }^{7}$ Liggins Institute, University of Auckland, Auckland, New Zealand; ${ }^{8}$ Liggins Institute, University of Auckland, Auckland, New Zealand; Department of Paediatrics: Child \& Youth Health, University of Auckland, Auckland, New Zealand; Gravida: National

Background: Antenatal steroids (ANS), gold standard therapy for women at risk of preterm birth, may contribute to the increased risk of impaired glucose tolerance following preterm birth. We aimed to investigate mRNA expression of genes involved in insulin secretion and hepatic insulin sensitivity in lambs born preterm (137 d gestation) following labour induced with (S-Prem), or without (NS-Prem), steroids compared with term-born lambs (Term; $148 \mathrm{~d}$ ). Methods: Pancreata and liver were collected at 4 weeks ( $n=6$ per group) and 12 months of age ( $n=12-13$ per group). mRNA levels were determined by qPCR normalised to 3 housekeeping genes. Results: At 4 weeks there were minimal differences amongst groups. At 12 months, pancreatic glucokinase, pdx1, igf1, igf 2 and insulin levels and hepatic ppar $\alpha$ were reduced by $21-63 \%$ in NS-Prem compared with S-Prem, but hepatic glut2 and pepck levels were 1.8-2.2 fold higher (all $\mathrm{P}<0.01$ ). mRNA levels in S-Prem were not different from those of Term for most genes; where there were differences, these were small $(<15 \%)$. Conclusion: In sheep, preterm delivery without ANS results in greater adverse effects on the pancreas and liver than preterm delivery preceded by ANS, contrary to the prevailing dogma regarding potential long-term adverse metabolic effects of ANS.

\section{0}

\section{Establishing a reference DNA methylome for umbilical cord blood}

Jane YL Tan ${ }^{1}$, Xinyi Lin ${ }^{2}$, Yonghui $\mathrm{Wu}^{3}$, Samantha J Liew ${ }^{4}$, Bhuvaneshwari Shunmuganathan ${ }^{5}$, Kai-Lyn $\mathrm{Ng}^{6}$, Yap-Seng Chong $^{7}$, Walter Stunkel ${ }^{8}$, Peter Gluckman ${ }^{9}$, Neerja Karnani ${ }^{10}$, Clara Y Cheong ${ }^{11}$

${ }^{1}$ Singapore Institute for Clinical Sciences; ${ }^{2}$ Singapore Institute for Clinical Sciences; ${ }^{3}$ Singapore Institute for Clinical Sciences; ${ }^{4}$ Singapore Institute for Clinical Sciences; ${ }^{5}$ Singapore Institute for Clinical Sciences; ${ }^{6}$ Yong Loo Lin School of Medicine, National University Health System; ${ }^{7}$ Singapore Institute for Clinical Sciences; ${ }^{8}$ Singapore Institute for Clinical Sciences; ${ }^{9}$ Singapore Institute for Clinical Sciences; ${ }^{10}$ Singapore Institute for Clinical Sciences; ${ }^{11}$ Singapore Institute for Clinical Sciences

Research question: Epigenetic variation that results from exposure differences during the gestational phase is thought to underpin both acute and long-term risks for metabolic health. Umbilical cord blood is often used as a tissue for epigenetic biomarker discovery. However, less consideration has been given to the heterogeneity of blood and methylation variation that results from this. We aim to isolate and establish a methylome profile for each major blood cell type.

Methods: Cord blood was collected at delivery from women with normal uncomplicated pregnancies. Specific leukocyte cell types were enriched using magnetic bead-bound antibodies against cell surface markers of interest. Cell purity and abundance were determined by flow cytometry. The DNA methylation profiles for each cell type will be assayed with the HumanMethylation 450 Bead chip.

Results: We have successfully isolated multiple cell types at $>90 \%$ purity, representing over $90 \%$ of leukocytes. Comparisons of our isolated cell type profiles weighted by cell abundance will be made against whole cord blood methylation profiles.

Conclusion: The utility of a cell-type specific methylation profile for a complex heterogenous tissue is particularly important to refine the epigenetic signature of whole cord blood. This allows for greater accuracy in identifying true epigenetic markers for phenotype.

\section{3}

Early digestive microbiota characteristics are associated to immune-related gene expression and susceptibility to postweaning diarrhea

Dou Samir ${ }^{1}$, Gadonna-Widehem Pascale ${ }^{2}$, Hamoudi Dounia ${ }^{3}$, Rome Véronique ${ }^{4}$, Rhazi Larbi ${ }^{5}$, Lakhal Lyes ${ }^{6}$, Bahi-Jaber Narges ${ }^{7}$, Pinon-Quintana Arturo ${ }^{8}$, Guyonvarch Alain ${ }^{9}$, Abdennebi-Najar Latifa ${ }^{10}$, Le Huërou-Luron Isabelle ${ }^{11}$

${ }^{1}$ Institut Polytechnique LaSalle Beauvais; ${ }^{2}$ Institut Polytechnique LaSalle Beauvais; ${ }^{3}$ Institut polytechnique LaSalle; ${ }^{4}$ Institut Polytechnique LaSalle; ${ }^{5}$ Institut Polytechnique LaSalle; ${ }^{6}$ Institut Polytechnique LaSalle; ${ }^{7}$ Institut Polytechnique LaSalle; ${ }^{8}$ INVIVO-NSA; ${ }^{9}$ INVIVO-NSA; ${ }^{10}$ Institut Polytechnique LaSalle; ${ }^{11} I N R A$ (ADNC)

Bacterial colonization impacts intestinal immune development, in a sustainable way, with consequences on susceptibility to developing immune responses in adulthood. Our hypothesis was that distinct gut microbiota composition and structure in infancy might confer gene expression alterations, predisposing to postweaning diarrhea. Using a porcine model, 40 neonates from two husbandry conditions were followed up upon onset diarrhoea in a challenging postweaning environment. Faecal bacterial community was characterized with cultivation-based microbiological analyses and 16S rRNA gene approaches coupled with capillary electrophoresis single-strandedconformation-polymorphism (CE-SSCP) and quantitative polymerase chain reaction (qPCR) at postnatal day (PND) 7 , 
14, 21, 30 and 38. At PND50, colon transcriptome profiling was carried out using porcine microarrays enriched with immunity-related genes. From PND38 to 47, thirteen pigs had diarrhea (D) while the others were resistant (R). At PND7, the faecal bacterial community of $\mathrm{R}$ pigs displayed a higher bacterial diversity but a lower evenness than that of $\mathrm{D}$ ones, regardless of husbandry conditions. Differentially expressed genes in $\mathrm{D}$ pigs stressed an up-regulation of cellular oxidative stress and an up-regulation of proflammatory pathways. Interestingly, interleukin-17 gene expression was negatively correlated to bacterial diversity at PND7 $(r=-0.61)$. Ongoing analysis of epigenetic markers would give more insights on mechanisms involved.

\section{0}

Whole-transcript expression analysis identifies new candidate genes involved in renal tubular programming after uteroplacental insufficiency in rats

Eva Nüsken ${ }^{1}$, Lisa Sprenger ${ }^{2}$, Maria Wohlfarth ${ }^{3}$, Gregor Lippach ${ }^{4}$, Felix Lechner ${ }^{5}$, Jörg Dötsch ${ }^{6}$

${ }^{1}$ University of Cologne, Department of Pediatrics; ${ }^{2}$ University of Cologne, Department of Pediatrics; ${ }^{3}$ University of Cologne, Department of Pediatrics; ${ }^{4}$ University of Cologne, Department of Pediatrics; ${ }^{5}$ University of Cologne, Department of Pediatrics;

${ }^{6}$ University of Cologne, Department of Pediatrics

Background: During pathologic intrauterine conditions, alteration of gene expression may cause altered organ development resulting in long-term organ dysfunction. Our aim was to identify candidate genes involved in renal programming after experimental uteroplacental insufficiency in rats.

Methods: Kidneys from neonatal rats after bilateral uterine vessel ligation (LIG, $\mathrm{n}=5$ ) at day $\mathrm{E} 19$ of gestation (term is E22.5) were compared to kidneys from offspring of un-operated control dams $(\mathrm{C}, \mathrm{n}=5)$. RNA was isolated and a gene microarray was performed (Affymetrix Rat Gene 2.0 ST Array).

Results: Seventeen of $\sim 27.000$ protein-coding transcripts showed significant and more than \pm 1.5 -fold changes in LIG. In these genes, we performed a Pubmed research which identified 5 genes to be relevant in kidney development or injury. Interestingly, all are especially relevant in renal tubules: Glutathione S-transferase alpha 2 (-2.8fold), kidney androgen regulated protein ( -2.2 fold), chemokine (C-C motif) ligand 20 (+1.9fold), hydroxyprostaglandin dehydrogenase 15 $(-1.6$ fold $)$ and 4-hydroxyphenylpyruvate dioxygenase (-1.6fold).

Discussion: In line with a tubular phenotype of our animals (increased salt loss and tubular proliferation in later life), transcript expression analysis reveals altered expression of genes relevant for tubular development and function. Further projects should clarify the role of these genes for functional and morphological nephrogenesis.
4751

Dietary fatty acids as a means of targeted modification of DNA methylation in adults: a proof-of-concept study

Graham Burdge ${ }^{1}$, Samuel Hoile ${ }^{2}$, Rebecca Clarke-Harris ${ }^{3}$, Trevor Mori ${ }^{4}$, Lawrence Beilin ${ }^{5}$, Rae-Chi Huang ${ }^{6}$, Karen Lillycrop ${ }^{7}$

${ }^{1}$ University of Southampton; ${ }^{2}$ University of Southampton;

${ }^{3}$ University of Southampton; ${ }^{4}$ University of Western Australia;

${ }^{5}$ University of Western Australia; ${ }^{6}$ University of Western Australia; ${ }^{7}$ University of Southampton

Research question: Altered epigenetic processes can mediate the effects of the early life environment on disease risk. Targeting interventions to modify such effects represents a major challenge. We investigated the specificity of the effect of dietary supplementation with fish oil (FO) or olive oil (OO) on the DNA methylation of genes involved in polyunsaturated fatty acid biosynthesis in humans.

Methods: Subjects $(56 \pm 1 \mathrm{y})$ received either FO $(\mathrm{M} 8, \mathrm{~F} 7)$ or OO (M8,F6) (4g/d) for 8 weeks. Leukocyte DNA was collected at baseline and end. FADS1, FADS2, ELOVL2 and ELOVL5 DNA methylation was measured by pyrosequencing. Transcripts were measured by qRTPCR.

Results: FADS1 and ELOVL2 methylation was unaltered. In men, $\mathrm{OO}$ and FO altered ( $\geqslant 10 \%$ ) 2/19 FADS2 CpG loci, one locus in opposite directions. OO altered 1/12 ELOVL5 CpG loci. In women, $\mathrm{OO}$ altered $(\geqslant 10 \%) 8 / 19$ and FO $4 / 19$ FADS2 CpG loci. OO altered 3/12 and FO 2/12 ELOVL 5 CpG loci. These changes were associated negatively with mRNA levels.

Conclusions: Dietary oils induce differential changes in DNA methylation of $\mathrm{CpG}$ loci. Thus modest modifications to dietary fat may facilitate targeted changes in DNA methylation. This could form a basis of interventions to modify the effect.

\section{4}

Life course-adjusted associations between intrauterine environment and DNA methylation in young adult women of a Jerusalem Perinatal Study sub-cohort

Jonathan Huang ${ }^{1}$, David Siscovick ${ }^{2}$, Hagit Hochner ${ }^{3}$, Yechiel Friedlander ${ }^{4}$, Daniel Enquobahrie ${ }^{5}$

${ }^{1}$ Institute for Health and Social Policy, McGill University; ${ }^{2} \mathrm{New}$ York Academy of Medicine; ${ }^{3}$ Braun School of Public Health, Hebrew University-Hadassah Medical Center; ${ }^{4}$ Braun School of Public Health, Hebrew University-Hadassah Medical Center;

${ }^{5}$ Department of Epidemiology, University of Washington

Question: Intrauterine environment (IUE), including maternal gestational weight gain (GWG) and pre-pregnancy BMI (ppBMI), have been associated with offspring cardiometabolic phenotype. We investigated whether offspring adult DNA methylation may play a role. 
Methods: Among 589 adult women (mean = 32 years) of the Jerusalem Perinatal Study Family Follow-Up, we quantified peripheral blood DNA methylation in five candidate genes drawn from literature (ABCA1, INS-IGF2, LEP, HSD11B2, and NR3C1) using MassARRAY EpiTYPER. We used multivariable linear regression and marginal structural models to estimate GWG - offspring methylation associations, controlling for life course factors.

Results: Higher GWG was inversely related to ABCA1 methylation $(\beta=-1.1 \%$ per quartile, [95\% CI: $-2.0,-0.3])$, after adjustment for ancestry, parental confounders, own education, parity, and smoking, and multiple testing. This association was larger in women with ppBMI $<25$, however the interaction $p$-value was $>0.05$. In exploratory analyses, ABCA1 methylation appeared to mediate the pathway between maternal GWG and beta-cell function (indirect effect on log $($ HOMA- $\beta$ ) $=-0.6 \%$, [95\% CI: $-1.0,-1.1], \mathrm{p}=0.014)$.

Conclusions: We found some evidence for inverse associations between maternal GWG and offspring ABCA1 methylation, supporting previous fetal cord blood associations. In future work, we consider cell composition contributions and potential mechanisms.

\section{3}

Genome wide methylation analysis identifies differentially methylated CpG loci associated with severe obesity in childhood

Rae-Chi Huang ${ }^{1}$, Emma S Garratt ${ }^{2}$, Hong Pan $^{3}$, Yong Hui $\mathrm{Wu}^{4}$, Elizabeth A Davis ${ }^{5}$, Sheila J Barton ${ }^{6}$, Graham C Burdge ${ }^{7}$, Keith M Godfrey ${ }^{8}$, Joanna D Holbrook ${ }^{9}$, Karen A Lillycrop ${ }^{10}$

${ }^{1}$ Telethon Kids Institute, The University of Western Australia;

${ }^{2}$ 2Academic Unit of Human Development and Health, Faculty of

Medicine, University of Southampton, Southampton, UK; ${ }^{3}$ Singapore Institute for Clinical Sciences (SICS), A*STAR, Brenner Centre for Molecular Medicine, Singapore, 117609 and School of Computer Engineering, Nanyang Technological Univer; ${ }^{4}$ Singapore Institute for Clinical Sciences (SICS), $A^{*} S T A R$, Brenner Centre for Molecular Medicine, Singapore, 117609; ${ }^{5}$ Telethon Kids Institute, The University of Western Australia; ${ }^{6} M R C$ Lifecourse Epidemiology Unit, University of Southampton, Southampton, UK; ${ }^{7}$ Academic Unit of Human Development and Health, Faculty of Medicine, University of Southampton, Southampton, UK; ${ }^{8} M R C$ Lifecourse Epidemiology Unit, University of Southampton, Southampton, UK. and NIHR Southampton Biomedical Research Centre, University of Southampton and University Hospital Sou; ${ }^{9}$ Singapore Institute for Clinical Sciences (SICS), A*STAR, Brenner Centre for Molecular Medicine, Singapore, 117609 and Yong Loo Lin School of Medicine, National University of Singa; ${ }^{10}$ Academic Unit of Human Development and Health, Faculty of Medicine, University of Southampton, Southampton, UK and Faculty of Natural and Environmental Sciences, University of Sout

Research question: Is differential DNA methylation associated with childhood obesity?

Methods: DNA methylation profiles in whole blood from 78 obese (mean BMI z-score 2.6) and 71 age/sex matched controls (mean BMI z-score 0.1) were analysed (Human Methylation $450 \mathrm{~K}$ BeadChip array). Samples from the obese were pooled separately form the controls. The associations between the methylation of selected differentially methylated $\mathrm{CpG}$ (DMCpG) loci with childhood obesity were validated in individual subjects using sodium bisulfite pyrosequencing across loci within the FYN, PIWIL4 and TAOK3 genes.

Results: Comparison between obese and controls revealed 129 DMCpG loci associated with 80 unique genes with greater than $10 \%$ difference in methylation ( $\mathrm{p}$-value).

\section{0}

Epigenetic marks at birth in dopamine beta-hydroxylase associated with childhood cognitive outcomes in the Southampton Women's Survey Cohort (SWS)

Paula Costello ${ }^{1}$, Philip Titcombe ${ }^{2}$, Shelia Barton ${ }^{3}$, Robert Murray ${ }^{4}$, Emma Garratt ${ }^{5}$, Rebecca Clarke-Harris ${ }^{6}$, Graham Burdge $^{7}$, Cyrus Cooper ${ }^{8}$, Hazel Inskip ${ }^{9}$, Catherine Gale ${ }^{10}$, Yap Seng Chong ${ }^{11}$, Karen Lillycrop ${ }^{12}$, Keith Godfrey ${ }^{13}$

${ }^{1}$ University of Southampton; ${ }^{2}$ University of Southampton;

${ }^{3}$ University of Southampton; ${ }^{4}$ University of Southampton;

${ }^{5}$ University of Southampton; ${ }^{6}$ University of Southampton;

${ }^{7}$ University of Southampton; ${ }^{8}$ University of Southampton;

${ }^{9}$ University of Southampton; ${ }^{10}$ University of Southampton;

${ }^{11}$ Singapore Institute of Clinical Sciences; ${ }^{12}$ University of

Southampton; ${ }^{13}$ University of Southampton

Early life environment has been linked to later neurocognitive function. To investigate the contribution of epigenetic marks induced during development to later neurocognitive function, we determined the relationship between the perinatal methylation status of dopamine beta-hydroxylase (DBH), which is important for cognitive development, and the child's later cognition.

Methods: Methylation of $\mathrm{CpG}$ loci within the $\mathrm{DBH}$ gene promoter region in umbilical cord (UC) tissue from infants in the SWS was measured by pyrosequencing. Neurocognition was assessed in separate groups of 4 and 7 year-olds.

Results: At 4 years, in 127 SWS children, higher UC DBH -926 CpG methylation was associated with lower four-year Wechsler Pre-School and Primary Scale of Intelligence performance $(\beta=-35.926)$ and full IQ $(\beta=-33.594)$ (both $p$

\section{6}

Maternal obesity (MO) programs down regulation of expression of hepatic cell cycle genes in the adult male rat offspring liver at postnatal day 110

Luis Reyes-Castro ${ }^{1}$, Guadelupe Rodriguez ${ }^{2}$, Claudia Bautista ${ }^{3}$, Claudia Vega ${ }^{4}$, Carlos Ibáñez ${ }^{5}$, Consuelo Lomas ${ }^{6}$, Jeremy Glenn ${ }^{7}$, Laura A Cox ${ }^{8}$, Elena Zambrano ${ }^{9}$

${ }^{1}$ Reproductive Biology, Instituto Nacional de Ciencias Médicas y Nutrición Salvador Zubirán, Mexico City, Mexico; ${ }^{2}$ Reproductive Biology, Instituto Nacional de Ciencias Médicas y Nutrición 
Salvador Zubirán, Mexico City, Mexico; ${ }^{3}$ Reproductive Biology, Instituto Nacional de Ciencias Médicas y Nutrición Salvador Zubirán, Mexico City, Mexico; ${ }^{4}$ Reproductive Biology, Instituto Nacional de Ciencias Médicas y Nutrición Salvador Zubirán, Mexico City, Mexico; ${ }^{5}$ Reproductive Biology, Instituto Nacional de Ciencias Médicas y Nutrición Salvador Zubirán, Mexico City, Mexico; ${ }^{6}$ Reproductive Biology, Instituto Nacional de Ciencias Médicas y Nutrición Salvador Zubirán, Mexico City, Mexico; ${ }^{7}$ Deparartment of Genetics and Southwest National Primate Research Center, Texas Biomedical Research Institute, San Antonio, TX; ${ }^{8}$ Deparartment of Genetics and Southwest National Primate Research Center, Texas Biomedical Research Institute, San Antonio, TX; ${ }^{9}$ Reproductive Biology, Instituto Nacional de Ciencias Médicas y Nutrición Salvador Zubirán, Mexico City, Mexico

Research question: Most programming studies on gene expression changes following sub-optimal maternal diets have been conducted in undernutrition models. Few data are available on offspring transcriptome changes programmed by $\mathrm{MO}$ and over nutrition. We hypothesized that changes in cell cycle signaling pathways are a mechanism contributing to dysfunctional postnatal growth outcomes resulting from exposure to MO during development.

Methods: We evaluated effects of $\mathrm{MO}$ induced in female rats placed at their weaning on a high fat diet maintained through pregnancy and lactation as well as control mothers raised on normal laboratory chow. All offspring ate normal chow from weaning. We evaluated effects on 110 day male offspring liver transcriptome with RNA Seq and pathway analysis.

Results: 25 cell cycle signaling pathway genes were differentially expressed in $\mathrm{MO}$ offspring with $92 \%$ down regulated including Tgf $\beta 1$, Tp53, Cdk4, Cdk6, Orc4L, Orc5L, Tp53, Wee, Bub3.

Conclusions: We conclude that exposure to MO during fetal and early neonatal life programs down regulation of key cell cycle genes. While MO offspring generally weigh more than those of lean mothers due to fat mass, cell number may not be increased as in sarcopenia and organ dysfunction resulting in premature death from decreased cell division and repair.

\section{1}

Maternal obesity (MO) programs key genes associated with epigenetic and transcriptional processes in livers of adult 110 day old male rat offspring

Guadalupe Rodriguez ${ }^{1}$, Claudia J Bautista ${ }^{2}$, Claudia Vega ${ }^{3}$, Luis Reyes-Castro ${ }^{4}$, Carlos Ibáñez ${ }^{5}$, Consuelo Lomas ${ }^{6}$, Jeremy Glenn ${ }^{7}$, Laura A Cox $^{8}$, Elena Zambrano ${ }^{9}$

${ }^{1}$ Reproductive Biology, Instituto Nacional de Ciencias Médicas y Nutrición Salvador Zubirán, Mexico City, Mexico; ${ }^{2}$ Reproductive Biology, Instituto Nacional de Ciencias Médicas y Nutrición Salvador Zubirán, Mexico City, Mexico; ${ }^{3}$ Reproductive Biology, Instituto Nacional de Ciencias Médicas y Nutrición Salvador Zubirán, Mexico
City, Mexico; ${ }^{4}$ Reproductive Biology, Instituto Nacional de Ciencias Médicas y Nutrición Salvador Zubirán, Mexico City, Mexico; ${ }^{5}$ Reproductive Biology, Instituto Nacional de Ciencias Médicas y Nutrición Salvador Zubirán, Mexico City, Mexico; ${ }^{6}$ Reproductive Biology, Instituto Nacional de Ciencias Médicas y Nutrición Salvador Zubirán, Mexico City, Mexico; ${ }^{7}$ Deparartment of Genetics and Southwest National Primate Research Center, Texas Biomedical Research Institute, San Antonio, TX, USA; ${ }^{8}$ Deparartment of Genetics and Southwest National Primate Research Center, Texas Biomedical Research Institute, San Antonio, TX, USA; ${ }^{9}$ Reproductive Biology, Instituto Nacional de Ciencias Médicas y Nutrición Salvador Zubirán, Mexico City, Mexico

Research question: We asked the question "To what extent does developmental programming by $\mathrm{MO}$ alter factors that regulate the transcriptional machinery across the transcriptome?"

Methods: We evaluated effects of MO induced in female rats placed at their weaning on a high fat diet maintained through pregnancy and lactation as well as control mothers raised on normal laboratory chow. All offspring were fed normal chow from weaning. We determined changes in genes that affect methylation, histone function and ribosomal proteins in their adult 110 day old male offspring liver transcriptome with RNA Seq.

Results: Of the genes differentially expressed between control and MO livers forty-one genes (16\%) were up regulated and 2454 (84\%) genes were down regulated. Genes known to regulate methylation and other epigenetic process that were down regulated in the MO offspring included Mthfd1, Dmap1, Ehmt2 and Gnmt as were histone deacetylases 3, 8, 20 and 11 and multiple genes encoding ribosomal and mitochondrial ribosomal subunits.

Conclusions: The adult liver of MO adult offspring shows global down regulation of genes that are required for normal function of the transcription process. Further studies are needed to determine the extent to which epigenetic marks are laid down and removed.

\section{2}

Trans-generational epigenetic effects of a maternal high-fat high-sucrose diet on insulin-signaling, lipid and carbohydrate metabolism genes in rat

Dan Ramdath ${ }^{1}$, Candace Cuthbert ${ }^{2}$, Aileen Hawke ${ }^{3}$, Jerome Foster ${ }^{4}$

${ }^{1}$ Guelph Food Research Centre, Ontario N1G 5C9, Canada;

${ }^{2}$ Faculty of Medical Sciences, The University of the West Indies, St. Augustine, Trinidad \& Tobago; ${ }^{3}$ Guelph Food Research Centre, Ontario N1G 5C9, Canada; ${ }^{4}$ Faculty of Medical Sciences, The University of the West Indies, St. Augustine, Trinidad \& Tobago

Research question: Does maternal high-fat, high-sucrose (HF/ HS) diet alter the DNA methylation (\%Me) of insulin-signaling, carbohydrate and lipid metabolism genes in male offspring, and what is the role of prenatal folic acid supplementation? 
Design and Methods: Pregnant Sprague Dawley rats were randomly assigned ( $n=5 /$ group) to Control, HF/HS or folic acid-supplemented (HF/HS/FA) diet. Male litter was fed an AIN control diet for $10 \mathrm{wk}$ before sacrifice. Skeletal and liver \% Me were measured using focused RT-PCR. Dam livers were also assessed for \%Me. Mean differences were assessed by one-way ANOVA with Bonferroni's post-hoc test.

Results: HF/HS offspring had significantly higher weights than control or HF/HS/FA from postnatal weeks 7 to 10 $(\mathrm{p} \leqslant 0.05)$. Overall, carbohydrate and lipid gene \%Me were significantly lower in $\mathrm{HF} / \mathrm{HS} / \mathrm{FA}$ vs $\mathrm{HF} / \mathrm{HS}$ offspring $(\mathrm{p} \leqslant 0.05)$. In both groups hepatic genes were generally hypermethylated while skeletal muscle genes were hypomethylated. Significant differences existed between Control and HF/HS/FA dams in \%Me for the insulin receptor substrate 1 and lipoprotein lipase genes; 95\% CI (95\% CI: 0.133, 9.183 and $0.241,1.90$, respectively).

Conclusions: Maternal diet induced methylation of offspring liver and skeletal muscle DNA can be modulated by maternal folic acid-supplementation.

\section{4}

Differential expression of miR138-1 in preeclampsia and limited epigenetic deregulation at the maternal-fetal interface of the placenta

Milena N. Leseva ${ }^{1}$, Alexandra M. Binder ${ }^{2}$

${ }^{1}$ Institute for Prevention and Cancer Epidemiology, University Medical Center Freiburg, Freiburg, Germany; Obstetrics and Gynecology Epidemiology Center, Department of Obstetrics, Gy; ${ }^{2}$ Obstetrics and Gynecology Epidemiology Center, Department of Obstetrics, Gynecology and Reproductive Biology, Brigham and Women's Hospital, Harvard Medical School, Boston MA, USA

Research question: Preeclampsia is a frequent cause of maternal morbidity and unfavorable pregnancy outcomes. The aim of this study was to identify changes in the epigenetic signature at the maternal-fetal interface associated with this condition.

Methods: A total of $\mathrm{n}=28$ preeclamptic placentas from participants in the Harvard Epigenetic Birth Cohort were matched with healthy controls. DNA methylation was assessed using the $450 \mathrm{~K}$ DNA methylation microarray. Pyrosequencing was used to validate any differentially methylated regions. Gene expression profiles were assessed in a subset of cases $(n=19)$ and matched controls using the Affymetrix HTA 2.0 gene expression microarray.

Results: Using a bump hunting approach we identified DMRs within the promoters of ZNF300, MYG1, NAPRT1 and succinate dehydrogenase complex genes but none of these remained statistically significant after controlling for the FWER at $\alpha=0.05$. We found no correlation between DNA methylation differences in cases versus controls and expression of genes in close proximity. However, we observed a statistically significant up-regulation of the miR138-1 miRNA in preeclamptic placentas when compared to healthy controls.
Conclusion: In contrast to prior reports, we did not observe extensive epigenetic deregulation in preeclamptic placentas. None of our top DMRs reached genome-wide significance, perhaps due to the small sample size.

\section{2}

Epigenetics in gestational protein restriction intake: miRNA profile expression in offspring heart

Heloisa Balan Assalin ${ }^{1}$, José Antonio Rocha Gontijo ${ }^{2}$, Patricia Aline Boer ${ }^{3}$

${ }^{1}$ Department of Internal Medicine School of Medicine, State University of Campinas; ${ }^{2}$ Department of Internal Medicine School of Medicine, State University of Campinas; ${ }^{3}$ Department of Internal Medicine School of Medicine, State University of Campinas

Epigenetic mechanisms are involved in altered offspring gene expression determined by gestational nutrient restriction and miRNAs could play important role. Our prior results, using gestational low-protein intake model have identified adult heart disorders in programmed rats. Then, we evaluated cardiac left ventricle miRNA expression profile of gestational protein restricted rats. Pregnant Wistar rats were allocate in two groups, according to protein supply during pregnancy: NP (17\%) or $\mathrm{LP}(6 \%)$. We evaluated left ventricle miRNAs expression in offspring male rats at 12-days (12d) and 16-weeks (16w) after birth. LP-12d had significantly increased mir-184/192/376c/ $380-3 p / 380-5 p / 451 / 582-3 p$ and significantly decreased mir-547/743a versus NP-12d. LP-16w had significantly increased let-7b/125a-3p/142-3p/182/188-5p and significantly decreased let-7g/107/127/181a/181c/184/324-5p/ 383/423-5p/484 versus NP-16w. For exclude possible hypertension effects in adult ventricle, we did the mRNA target prediction for all altered miRNA's expression and elected those that alteration is present in $12 \mathrm{~d}$ and $16 \mathrm{w}$. LP-12d had lower expression of mRNAs Bbs 1 and $\mathrm{Calml} 3$ and higher Dnmt3a/Oxct1/Rictor and Trps1 than NP-12d. LP-16w had higher Adrkb1/ Bbs1/ Dnmt3a/Gpr22/Inppl1 and Oxct1 expression than NP-16w. In conclusion, the gestational protein restriction leads to early heart miRNA expression changes that are perpetuate into adulthood and are involved in heart congenital disease, DNA methylation and ketone body catabolism.

\section{1}

Regulatory landscape of the human neonatal epigenome Mei-lyn Ong ${ }^{1}, \mathrm{AL} \mathrm{Teh}^{2}, \mathrm{C} \mathrm{Li}^{3}$, P Hong ${ }^{4}, \mathrm{~L} \mathrm{Xinyi}^{5}, \mathrm{Y} \mathrm{Wu}{ }^{6}, \mathrm{CY}$ Cheong $^{7}$, SM Saw ${ }^{8}$, K Kwek ${ }^{9}$, MS Kobor ${ }^{10}$, KM Godfrey ${ }^{11}$, MJ Meaney ${ }^{12}$, PD Gluckman ${ }^{13}$, YS Chong ${ }^{14}$, JD Holbrook ${ }^{15}$, $\mathrm{N}$ Karnani ${ }^{16}$

${ }^{1}$ Singapore Institute for Clinical Sciences; ${ }^{2}$ Singapore Institute for Clinical Sciences; ${ }^{3}$ Singapore Institute for Clinical Sciences; 
${ }^{4}$ Singapore Institute for Clinical Sciences; ${ }^{5}$ Singapore Institute for Clinical Sciences; ${ }^{6}$ Singapore Institute for Clinical Sciences;

${ }^{7}$ Singapore Institute for Clinical Sciences; ${ }^{8}$ Singapore Institute for Clinical Sciences; ${ }^{9}$ Singapore Institute for Clinical Sciences;

${ }^{10}$ Singapore Institute for Clinical Sciences; ${ }^{11}$ Singapore Institute for Clinical Sciences; ${ }^{12}$ Singapore Institute for Clinical Sciences;

${ }^{13}$ Singapore Institute for Clinical Sciences; ${ }^{14}$ Singapore Institute for Clinical Sciences; ${ }^{15}$ Singapore Institute for Clinical Sciences;

${ }^{16}$ Singapore Institute for Clinical Sciences

Research question: The human neonatal epigenome is influenced by both the inherited genotype and quality of the in utero environment, and can strongly influence one's health over the lifecourse. However, there is a limited understanding of the genomic regulation of these methylation variations across the genome.

Methods: Briefly, 1,148 umbilical cord samples from healthy babies, which are part of the GUSTO birth cohort study, were selected (Soh et al., 2013). Genotyping was performed on the Illumina Omniexpress + exome array and processed in a standard fashion. DNA methylation profiling was performed on the Infinium Mehthylation 450 Bead Chip. Data was processed as described previously (Pan et al., 2012).

Variably and differentially methylated regions (VMR \& DMR) were detected as previously described (Ong \& Holbrook, 2013). Results: We first characterized genomic regions by their degree of inter-individual methylation variation across the neonatal population. We then segregated these regions into either SNPdriven or complex interactions-driven. We further mapped out the hyper and hypo-methylated regions in the umbilical cord epigenome. Finally, we overlay these distinctively driven methylation regions with maps of gene regulatory elements and biological pathways to decipher their regulatory roles.

Conclusion: We generated, for the umbilical cord epigenome, a comprehensive methylation map characterized by interindividual variability, hyper- or hypo-methylated status, and SNP driven or complex interactions driven effect. We find that distinct classes of methylation regions exhibit different regulatory functions, and may reflect specific in utero environmental influences.

\section{4}

Endothelial heterogeneity in umbilical arteries of IUGR \& LGA fetuses: the role of epigenetic programming of vascular function

Bernado Krause $^{1}$, A Caniuguir ${ }^{2}$, Carrasco-Wong I ${ }^{3}$, C Hernandez ${ }^{4}$, E Muñoz ${ }^{5}$, R Uauy ${ }^{6}$, P Casanello $^{7}$

${ }^{1}$ Division of Obstetrics and Gynecology, 2Division of Pediatrics, School of Medicine, Pontificia Universidad Católica de Chile, Santiago, Chile; ${ }^{2}$ Division of Obstetrics and Gynecology, 2Division of Pediatrics, School of Medicine, Pontificia Universidad Católica de Chile, Santiago, Chile; ${ }^{3}$ Division of Obstetrics and Gynecology, 2Division of Pediatrics, School of
Medicine, Pontificia Universidad Católica de Chile, Santiago, Chile; ${ }^{4}$ Division of Obstetrics and Gynecology, 2Division of Pediatrics, School of Medicine, Pontificia Universidad Católica de Chile, Santiago, Chile; ${ }^{5}$ Division of Obstetrics and Gynecology, 2Division of Pediatrics, School of Medicine, Pontificia Universidad Católica de Chile, Santiago, Chile; ${ }^{\sigma}$ Division of Obstetrics and Gynecology, 2Division of Pediatrics, School of Medicine, Pontificia Universidad Católica de Chile, Santiago, Chile; ${ }^{7}$ Division of Obstetrics and Gynecology, 2Division of Pediatrics, School of Medicine, Pontificia Universidad Católica de Chile, Santiago, Chile

The association of low and excessive fetal weight with cardiovascular risk are in the central interest of programming.

Aim: Due to the key role of endothelium in the umbilical vascular function we have studied markers of endothelial epigenetic heterogeneity in HUAEC from IUGR, AGA and LGA fetuses.

Methods: In Human Umbilical Artery Endothelium (HUAEC) from IUGR-AGA and LGA fetuses the expression of eNOS, Arg2, NOX4, GPX1, SOD1, Nrf2 and HO1 was determined under basal, hypoxia and oxidative stress conditions. The methylation status (pyrosecuencing) of the promoter of these genes as well as histone modifications ( $\mathrm{H} 3 \mathrm{~K} 4$ Me2/H3K4 Me3/H3K9 Me2/H3K9 Ac/H4K12 Ac) by ChIP were performed.

Results: In IUGR and LGA-derived HUAEC there is an increase in the basal expression of eNOS and NOX4. The mRNA of Arg2 and eNOS are induced by hipoxia in AGAHUAEC but no change is observed in IUGR or LGAHUAEC. Both eNOS and NOX1 showed significant changes in the methylation status at their promoter región. ChIP showed an important amount of opened chromatin markers (H3K9 Ac, H4K12 Ac) mainly in the eNOS promoter.

Conclusion: In summary both extreme phenotypes (IUGR and LGA) show changes in some key endotelial genes as well as genes involved in the response to oxidative stress. These changes are associated to epigenetic marks of vascular programming.

\section{4}

\section{In silico Construction of Personalized DNA Methylation Biomarkers}

Evan Gatev

University of British Columbia, Canada

DNA Methylation variability across individuals can be exploited to construct personalized biomarkers for disease and risk. This requires the integration of genetic and DNA methylation data. The typical scenario of a limited number of observation poses several challenges, including over-fitting, perormance assessment and interpretability of the results. We offer a novel approach to the construction of personalized DNA 
methylation biomarkers, that addresses these challenges. Our approach is based on constructions of informative reduced representations of the multiple big "Omics" data, and their integration in a novel way. We use a three stage pipeline with genomic feature construction and selection, relevant DNAmethylation probe selection and data integration to demonstrate a proof-of-concept cortisol personalized biomarker in a small cohort of 187 individuals.

\section{9}

Epigenome-wide and transcriptome-wide analyses reveal gestational diabetes is associated with alterations in the Human Leukocyte Antigen complex

Alexandra M. Binder ${ }^{1}$, Jessica LaRocca ${ }^{2}$, Corina Lesseur ${ }^{3}$, Carmen Marsit ${ }^{4}$

${ }^{1}$ Obstetrics and Gynecology Epidemiology Center, Department of Obstetrics, Gynecology and Reproductive Biology, Brigham and Women's Hospital, Harvard Medical School, Boston, MA, USA;

${ }^{2}$ Harvard University Center for the Environment, Harvard University, Cambridge, MA, USA; ${ }^{3}$ Department of Pharmacology and Toxicology, and Section of Biostatistics and Epidemiology, Department of Community and Family Medicine, Geisel School of Medicine at Dartmouth, Hanov; ${ }^{4}$ Department of Pharmacology and Toxicology, and Section of Biostatistics and Epidemiology, Department of Community and Family Medicine, Geisel School of Medicine at Dartmouth, Hanov

Research question: Gestational diabetes mellitus (GDM) affects approximately $18 \%$ of pregnancies in the United States and increases the risk of adverse health outcomes in the offspring. Since adult disease propensities may be set by anatomical and molecular alterations in the placenta associated with GDM, we aimed to assess epigenetic changes in the placenta resulting from GDM that may affect fetal programming.

Methods: We measured genome-wide methylation (Infinium HumanMethylation450 Beadchips) and expression (Affymetrix Transcriptome Microarrays) in placental tissue of 41 GDM cases and 41 matched pregnancies without maternal complications from the Harvard Epigenetic Birth Cohort.

Results: Specific transcriptional and epigenetic perturbations associated with GDM status included alterations in the major histocompatibility complex (MHC) region, which were validated in an independent cohort, the Rhode Island Child Health Study. Gene ontology enrichment among gene regulation influenced by GDM revealed an overrepresentation of immune response pathways among differential expression, reflecting these coordinated changes in the MHC region.

Conclusions: Our study represents the largest investigation of transcriptomic and methylomic differences associated with GDM, providing comprehensive insight into the molecular basis of GDM induced fetal (re)programming.
Impact of pregnancy overweight and depression on the newborn gut microbiome

Anita Kozyrskyj ${ }^{1}$, Petya Koleva ${ }^{2}$, Ji-Sun Kim ${ }^{3}$, Sarah Bridgman ${ }^{4}$, Angela Chow ${ }^{5}$, David Guttman ${ }^{6}$, Malcolm Sears ${ }^{7}$, Allan Becker ${ }^{8}$, Piush Mandhane9, Padmaja Subbarao $^{10}$, Stuart Turvey ${ }^{11}$, James Scott ${ }^{12}$, CHILD Study Investigators

${ }^{1}$ University of Alberta; ${ }^{2}$ University of Alberta; ${ }^{3}$ University of Toronto; ${ }^{4}$ University of Alberta; ${ }^{5}$ University of Alberta;

${ }^{6}$ University of Toronto; ${ }^{7}$ McMaster University; ${ }^{8}$ University of Manitoba; ${ }^{9}$ University of Alberta; ${ }^{10}$ University of Toronto;

${ }^{11}$ University of British Columbia; ${ }^{12}$ University of Toronto

Research question: The fetal gut is not sterile and can be shaped by in utero exposures. The aim of our study was to assess the impact of maternal conditions that affect infant growth on the microbial composition of newborn first stool.

Methods: This study comprised a sub-set of 57 mothers and their full-term infants from the Winnipeg site of the Canadian Healthy Infant Longitudinal Development (CHILD) cohort. Microbiota of the meconium (first stool) were characterized by Illumina sequencing of the hypervariable V4 region of the 16S rRNA gene. Measures of mother's prenatal health (overweight status, history of depression) were obtained from standardized questionnaires and hospital records.

Results: Microbiota were detected in 13 of 57 newborn meconium samples. Pre-pregnancy overweight was recorded in 8 of these 13 mothers (61\%), while $5(38 \%)$ had a history of depression or were taking anti-depressants while pregnant. Significantly higher ratios of Lactobacillales to Bacteroidaceae ( $\mathrm{p}$

\section{1}

Maternal health conditions related to the ratio of placental weight and birth weight.

Filumena Maria da Silva Gomes ${ }^{1}$, Maria Helena Valente ${ }^{2}$, Ana Carolina Novo ${ }^{3}$, Ana Maria de Ulhôa Escobar ${ }^{4}$, Leide Irislayne Macena da Costa Silva ${ }^{5}$, Sandra J. F. E. Grisi ${ }^{6}$

${ }^{1}$ Faculdade de Medicina da Universidade de São Paulo;

${ }^{2}$ Faculdade de Medicina da Universidade de São Paulo;

${ }^{3}$ Faculdade de Medicina da Universidade de São Paulo;

${ }^{4}$ Faculdade de Medicina da Universidade de São Paulo;

${ }^{5}$ Faculdade de Medicina da Universidade de São Paulo;

${ }^{6}$ Faculdade de Medicina da Universidade de São Paulo

Research question: The present research aims to study obstetric conditions associated with placental weight ratios in newborn $\geqslant 37$ weeks gestation, in hospital's records from the University of São Paulo, Brazil. 
Methods: Identified 281 newborn. Group studies were constituted: placental weight ratios (PWR) was assessed as quartile $<25$ and $>75$ percentile.

Results: Mean birth weight was 3,225 (1,725-4,390) grams (g); Average placental weight was $624 \mathrm{~g}$ (255-985); and average PWR was 0.1936 (0.087 to 0.296). Prepregnancy maternal $\quad B M I>25.0 \quad \mathrm{~kg} / \mathrm{m}^{2} \quad(\mathrm{Chi} 2$ sig 0.017$)$ and maternal anemia during pregnancy (Chi2 sig 0.015 ) was associated with PWR $<25$ percentile. Maternal anemia during pregnancy (Chi2 sig 0.006) was also associated with PWR $>75$ percentile.

Conclusions: pre-gestational and gestational maternal conditions are related PWR atypical.

\section{4}

Placental malaria exposure does not affect clinical profile or fitness level in young tanzanian men and women

Dirk Lund Christensen ${ }^{1}$, Fannie LaJeunesse-Trempe ${ }^{2}$, Kaushik Ramaiya $^{3}$, Theonest Kahangwa Mutabingwa ${ }^{4}$, Daniel Faurholt-Jepsen ${ }^{5}$, Jannie Nielsen ${ }^{6}$, Allan Vaag ${ }^{7}$, Ib Christian Bygbjerg ${ }^{8}$, Lars Sander Koch ${ }^{9}$

${ }^{1}$ University of Copenhagen; ${ }^{2}$ University of Copenhagen; ${ }^{3}$ Shree Hindu Mandal Hospital; ${ }^{4}$ Hubert Kairuki Memorial Hospital; ${ }^{5}$ Copenhagen University Hospital; ${ }^{6}$ University of Copenhagen; ${ }^{7}$ Copenhagen University Hospital; ${ }^{8}$ University of Copenhagen;

${ }^{9}$ Steno Diabetes Center

Research question: This study aimed at measuring if exposure to placental malaria in the foetal state affected clinical and fitness outcomes in young adults in Tanzania.

Methods: We traced 45 men and women aged 19.6 (1.1) years from Tanzania, and their birth files were used to determine the status of their placental malaria exposure (PM groups by placental blood smear). We measured anthropometrics, blood pressure (BP), standard oral glucose tolerance test, and aerobic fitness (watt-max test on stationary bicycle). Between-group differences of placental malaria exposure were assessed by ANOVA analyses for outcome variables.

Results: Mean fasting plasma glucose was 4.6 (0.6) and 2-h glucose $6.4(1.5) \mathrm{mmol} / \mathrm{L}$, with between PM-group differences of $p=0.24$ and $p=0.49$, respectively. Systolic BP was 126 (13), and diastolic BP was 76 (7) $\mathrm{mmHg}$ with between PM-group differences of $p=0.70$ and $p=0.52$, respectively. Mean waist circumference was $76.3(4.5) \mathrm{cm}$ with between PM-group difference of $p=0.15$. Mean aerobic fitness was $27.5(9.3) \mathrm{mlO} 2 / \mathrm{kg} / \mathrm{min}$ with between PM-group difference of $\mathrm{p}=0.70$.

Conclusion: PM exposure does not affect blood glucose, blood pressure, anthropometric measurements, or aerobic fitness level in young Tanzanian adults. PM exposure may affect physiological and epigenetic disease mechanisms underlying metabolic diseases, but not clinical outcomes in early adulthood.

\section{5}

Measures of Placental Growth in Relation to Birth Weight: a Latin America study

Grandi Carlos ${ }^{1}$, Ligia Nascente ${ }^{2}$, Ricardo Cavalli ${ }^{3}$, Viviane Cunha Cardoso ${ }^{4}$

${ }^{1}$ Department of Pediatrics, Sarda Maternity Hospital, University of Buenos Aires, Argentina; ${ }^{2}$ Department of Pediatrics, Ribeirão Preto Medical School, University of São Paulo, Brazil; ${ }^{3}$ Department of Gynecoly and Obstetric. Ribeirão Preto Medical School, University of São Paulo, Brazil; ${ }^{4}$ Department of Pediatrics, Ribeirão Preto

Medical School, University of São Paulo, Brazil

Research question: To explore the relationship between measures of placental growth measures (PGM) and birth weight (BW) in a public maternity hospital.

Methods: Observational study of 875 singleton liveborn children of the Sarda Maternity Hospital, University of Buenos Aires, Argentina, between January 2011 and August 2012 with complete data of PGM. The following manual measurements of the placenta according to standard methods were performed: weight $(\mathrm{PW}, \mathrm{g})$, larger and smaller diameters $(\mathrm{cm})$, ellipsivility, thickness $(\mathrm{cm})$, shape, area $\left(\mathrm{cm}^{2}\right), \mathrm{BW} / \mathrm{PW}$ and PW/BW ratios, and efficiency. Associations between BW and PGM were examined using multiple linear regression.

Results: Placental weight alone accounted for $49 \%$ of birthweight variation $(\mathrm{p}<0,001)$, whereas all PGM accounted for $53.6(\mathrm{p}<0,001)$. Combined, PGM, maternal characteristics (parity, pre-eclampsia, tobacco), gestational age and gender explained $77.8 \%$ of birth weight variation $(\mathrm{p}<0,001)$. Among preterm births, 59 percent of BW variance was accounted for by PGM, compared with 44 percent at term.

Conclusion: Reliable measures of placental growth estimate $53.6 \%$ of birth weight variance, and project this outcome to a greater degree in preterm births than at term. These findings would contribute to the understanding of the maternal-placental programming of chronic disease.

\section{9}

Late pregnancy maternal dietary methyl donor supplementation partially reverses effects of placental restriction on cutaneous hypersensitivity

Amy Wooldridge ${ }^{1}$, Robert Bischof ${ }^{2}$, Hong Liu ${ }^{3}$,

Damien Hunter ${ }^{4}$, Lynne Giles ${ }^{5}$, Karen Kind ${ }^{6}$, Julie Owens ${ }^{7}$, Vicki Clifton ${ }^{8}$, Kathryn Gatford ${ }^{9}$

${ }^{1}$ University of Adelaide; ${ }^{2}$ Monash University; ${ }^{3}$ University of Adelaide; ${ }^{4}$ University of Adelaide; ${ }^{5}$ University of Adelaide;

${ }^{6}$ University of Adelaide; ${ }^{7}$ University of Adelaide; ${ }^{8}$ University of Adelaide; ${ }^{9}$ University of Adelaide

Research question: Fetal growth restriction reduces fetal methyl donor abundance and decreases allergy rates or susceptibility, including in the placentally-restricted (PR) 
adolescent sheep. Maternal dietary methyl donor supplementation in late pregnancy increases risk of allergy in offspring. We therefore investigated the role of methyl donor supply in fetal programming of allergic susceptibility.

Methods: Outcomes were measured in lambs from control (49, CON), PR (28) or PR ewes fed methyl donors from d120 of pregnancy until term (25, PR+METHYL). We measured serum antibody responses to sensitisation with house dust mite (HDM) and ovalbumin, reactions to intradermal antigen at 28 weeks, and skin mast cell density at 52 weeks.

Results: Birth weight was greater in $\mathrm{CON}$ than $\mathrm{PR}$ or $\mathrm{PR}$ + METHYL lambs. HDM-specific IgE increases were greater in PR and PR+METHYL than CON. Fewer PR than CON sheep responded positively to intradermal ovalbumin, with intermediate responses in PR+METHYL. In preliminary analyses, mast cell density in the upper dermis did not differ between groups.

Conclusion: PR increases antibody responses to sensitisation without increasing cutaneous hypersensitivity, suggesting that PR suppresses inflammatory responses to allergens downstream of antibody responses. Responses to maternal methyl supplementation, suggest that epigenetic mechanisms may contribute to fetal programming of inflammatory responses.

\section{2}

Placental weight is not altered by early pregnancy maternal BMI or late pregnancy hyperglycaemia

Ilona Goljan ${ }^{1}$, Hema Venkataraman ${ }^{2}$, Nithya Sukumar ${ }^{3}$, Adaikala Antonysunil ${ }^{4}$, Phoebe Brobbey ${ }^{5}$, Harvinder Mann ${ }^{6}$, Ponnusamy Saravanan ${ }^{7}$

${ }^{1}$ George Eliot Hospital NHS Trust, The University of Warwick, UK; ${ }^{2}$ George Eliot Hospital NHS Trust, The University of Warwick, UK; ${ }^{3}$ George Eliot Hospital NHS Trust, The University of Warwick, UK;

${ }^{4}$ The University of Warwick, UK; ${ }^{5}$ The University of Warwick, UK;

${ }^{6}$ The University of Warwick, UK; ${ }^{7}$ George Eliot Hospital NHS

Trust, The University of Warwick, UK

Research question: What are the predictors of placental weight and its relationship between maternal and fetal characteristics?

Methods: A sub-study of all women at high-risk of GDM from the multicentre, prospective PRiDE study (delivered between Sep2014-Mar2015). Maternal and offspring characteristics were recorded, including detailed anthropometry and placental weight. Placenta weight was obtained within 24-hours of delivery.

Results: $122 / 140$ deliveries during this period, had complete data including placental weight. At booking ( $<12$ weeks): mean age-28.4 \pm 5.22 years, BMI-32.3 $\pm 8.2 \mathrm{Kg} / \mathrm{cm}^{2}$, height$164.5 \pm 7.5 \mathrm{~cm}$ and waist circumference (WC) $-104.0 \pm 18.9 \mathrm{~cm}$. $12.7 \%$ were current smokers. Mean birth weight was $3439.3 \pm 632.8 \mathrm{~g}$. Mean fasting and 2-hour post-load glucose were $4.54 \pm 0.4(\mathrm{FPG}) \& 5.7 \pm 1.2(2 \mathrm{hPG})$ respectively. Mean placenta weight $-655.1 \pm 172.7 \mathrm{~g}$.
Placental weight was an independent predictor of birth weight after adjustment for height, BMI, gestational age, smoking status and maternal glucose- $(\mathrm{p}<0.001)$.

Height- $(\mathrm{p}<0.001)$, gestational age- $(\mathrm{p}=0.044)$ and sex$(\mathrm{p}=0.005)$ of baby independently predicted placental weight after adjustment for all above factors and explained 26.5\% variation in placental weight.

Maternal adiposity (BMI, WC or skin folds), smoking status or glucose did not impact on placental weight.

Conclusion: Placental weight was an independent predictor of offspring weight irrespective of maternal characteristics. Placental weight is preserved across different BMI and glucose categories. Majority of factors affecting placental weight are unknown.

\section{0}

Fetal growth under restricted food conditions in livebearing animals with and without placentas: Implications for the evolution of the placenta

Bart Pollux ${ }^{1}$, Bart Pollux ${ }^{2}$, David Reznick ${ }^{3}$

${ }^{1}$ Wageningen University; ${ }^{2}$ Wageningen University (the

Netherlands); ${ }^{3}$ University of California Riverside (USA)

In highly variable environments females may plastically adjust offspring phenotype in a way that enhances fitness. Here, we hypothesize that (1) in live-bearing organisms the ability of females to adjust offspring phenotype during their pregnancy arises during the evolution of the placenta and (2) having a placenta is disadvantageous when living in an unpredictable resource environment.

To test these hypotheses, we performed a food-manipulation experiment with live-bearing fish species that either lack a placenta (Poeciliopsis gracilis) or have a well-developed placenta (Poeciliopsis turneri). We studied changes in neonate traits (birth weight and fat reserve) that were induced by changes in maternal food availability during the pregnancy. First, we found that only live-bearing fish with a placenta can adjust offspring phenotype during their pregnancy, suggesting that this ability evolved in close association with the placenta. Second, we found that in placental fish restrictions in maternal food availability during pregnancy lead to severe reductions in birth weight (up to 50\%) and neonate fat reserves (up to $75 \%$ ). This suggests that having a placenta is a maladaptive strategy under fluctuating food conditions and, hence, that the placenta most likely evolved in stable high-resource environments.

\section{4}

Altered placental transport and immune status following maternal malnutrition has implications for fetoplacental development

KL Connor $^{1}$, E Bloise ${ }^{2}$, M Kibschull ${ }^{3}$, E Matysiak ${ }^{4}$, M Lee $^{5}$, R Maganga $^{6}$, SG Matthews ${ }^{7}$, SJ Lye ${ }^{8}$

${ }^{1}$ Lunenfeld-Tanenbaum Research Institute, Mount Sinai Hospital; ${ }^{2}$ Physiology, University of Toronto and Laboratory of 
Translational Endocrinology, Federal University of Rio de Janeiro;

${ }^{3}$ Lunenfeld-Tanenbaum Research Institute, Mount Sinai

Hospital; ${ }^{4}$ Lunenfeld-Tanenbaum Research Institute, Mount

Sinai Hospital; ${ }^{5}$ Lunenfeld-Tanenbaum Research Institute,

Mount Sinai Hospital; ${ }^{6}$ Lunenfeld-Tanenbaum Research

Institute, Mount Sinai Hospital; ${ }^{7}$ Physiology, University of

Toronto and Lunenfeld-Tanenbaum Research Institute, Mount

Sinai Hospital; ${ }^{8}$ Lunenfeld-Tanenbaum Research Institute,

Mount Sinai Hospital

Background: The placenta regulates nutrient supply and acts as a protective barrier against infection and harmful compounds present in the maternal circulation. We hypothesise that maternal malnutrition impacts placental immune status and fatty acid (FA)/drug transporters, altering developmental trajectories.

Methods: Mice were fed a control diet during pregnancy $(\mathrm{CON})$, a $30 \%$ calorie reduced diet from d5.5-18.5 (CR) or a $60 \%$ high fat diet before and during pregnancy (HF). At d 18.5 hormones were measured in blood, mRNA and protein expression of placental transporters, inflammatory and differentiation markers were measured ( $\mathrm{p} 0.05$ ).

Results: CR reduced maternal glucose, insulin, leptin, triglycerides and weight. HF increased maternal leptin and reduced adiponectin, triglycerides and free FA. CR fetuses/placentae were lighter with increased inflammatory biomarkers. CR and HF placentae had decreased labyrinth and junctional zone areas. HF had reduced trophoblast glycogen cell markers (Pcdh12/Cx31.1). FA transporter FAT/CD36 expression was lower with CR, whereas FABPpm mRNA and FATP4 protein were higher with CR vs. HF. Drug transporter Abcbla mRNA was lower, whereas Abcg2 was higher, in HF vs. CR.

Conclusions: Placental transport and inflammatory markers are altered with maternal malnutrition, which may have implications for fetoplacental development, particularly in pregnancies where malnutrition, inflammation and/or medication use coexist.

\section{3} Regulators of Placental neovascularization in
hemangiomagenesis

Peace Zwane ${ }^{1}$, Sanah Nkadimeng ${ }^{2}$

${ }^{1}$ University of Pretoria; ${ }^{2}$ University of Pretoria

The insulin/Insulin growth factor (IGF) system is important in the regulation of placental development. Insulin growth factor 2 (IGF-2) in particular is a placental marker associated with vascularization. Other factors identified as important in the regulation of vessel growth in the placenta are vascular endothelial growth factor (VEGF) and basic fibroblast growth factor (bFGF). The most common tumors of infancy, infantile hemangiomas (IH), appear to have a placental origin. The aim of this study was to investigate angiogenesis, as well as the expression of these regulators of neovascularization in hemangioma. Tissue biopsies from patients with
IH were cultured to study angiogenesis. We further studied the expression of IGF-2, and the downstream regulators of IGF/IGF2R signaling in a mouse model of hemangioma. The expression of VEGF and bFGF were studied using immunobotting. Our results revealed excessive angiogenesis in IH biopsies. Mouse studies further revealed that tumor growth was associated with increased Akt activity. In vitro, the inhibition of PI3k using Wortmannin lead to the inhibition of endothelial cell migration and invasion, as well as a reduction in angiogenesis. It is possible that IGF signaling promotes angiogenesis via PI3k/Akt, and that targeting of this pathway may have therapeutic potential in these lesions.

\section{5}

Placental size relative to birth weight in term deliveries in Japanese population

Keiji Suzuki ${ }^{1}$, Erie Imai ${ }^{2}$, Hitoshi Ishimoto ${ }^{3}$, Goki Yahata ${ }^{4}$

${ }^{1}$ Tokai University School of Medicine; ${ }^{2}$ Tokai University School of Medicine; ${ }^{3}$ Tokai University School of Medicine; ${ }^{4}$ Yahata

Women's Clinic

Background: Size of the placenta at birth is indicated to be associated with later health and disease issues.

Objective: To investigate weights of placenta in term deliveries in two hospitals of different levels (level1 and level3) in Japan. Methods: Delivery records were reviewed and most recent data (2014-2015) of term deliveries collected from an obstetric clinic (Yahata Women's Clinic; representing level1) and from Perinatal Center, Tokai University Hospital (representing level3). Data of gestational age, birth weight, sex, mode of delivery and placenta weight were collected. Placenta to birth weight ratio was also calculated and compared between groups. Results: Data from 77 deliveries (38 males and 39 females) were collected from the level1 clinic, and 36 (17 males and 19 females) from the level3 hospital. Birth weights were 3028 $+/-305 \mathrm{~g}$ and $2932+/-453 \mathrm{~g}$, placenta weights were $541+/-85 \mathrm{~g}$ and $618+/-120 \mathrm{~g}$; placenta/birth weight ratios were 0.18 $+/-0.02$ and $0.21+/-0.03$, respectively.

Conclusion: Placenta/birth weight ratio from the level1 clinic was comparable to those of previous reports from different countries (0.14-0.19). However, it was significantly higher in the level3 hospital, which might be related with difference in the level of intra-uterine fetal well being between these populations.

\section{5}

Placental expression of cubilin, megalin and VDR is associated with neonatal size and body composition

Claire Simner ${ }^{1}$, Sheila Barton ${ }^{2}$, Hazel Inskip ${ }^{3}$, Keith Godfrey ${ }^{4}$, Mark Hanson ${ }^{5}$, Cyrus Cooper ${ }^{6}$, Nicholas Harvey ${ }^{7}$, Rohan Lewis ${ }^{8}$

${ }^{1}$ Institute of Developmental Sciences, University of Southampton;

${ }^{2}$ MRC Lifecourse Epidemiology Unit, Southampton; ${ }^{3}$ MRC Lifecourse 
Epidemiology Unit, Southampton; ${ }^{4}$ Institute of Developmental Sciences, MRC Lifecourse Epidemiology Unit, NIHR Southampton Biomedical Research Centre, Southampton UK; ${ }^{5}$ Institute of Developmental Sciences, NIHR Southampton Biomedical Research Centre, Southampton UK; ${ }^{6} M R C$ Lifecourse Epidemiology Unit, NIHR Southampton Biomedical Research Centre, Southampton UK, NIHR Musculoskeletal Biomedical Research Unit, Oxford UK.; ${ }^{7}$ MRC Lifecourse Epidemiology Unit, NIHR Southampton Biomedical Research Centre, Southampton UK; ${ }^{8}$ Institute of Developmental Sciences, University of Southampton

Research question: Placental vitamin $\mathrm{D}$ transfer to the fetus is important for normal development. However, vitamin D transport across the placenta and actions within the placenta are not fully understood. The placenta expresses genes required for vitamin $\mathrm{D}$ transport (megalin and cubilin) and signalling (VDR and $\operatorname{RXR} \alpha$ ). We investigated whether these genes are associated with neonatal outcomes.

Methods: Placental samples were collected at delivery $(\mathrm{n}=102)$ from the Southampton Women's Survey cohort (3,158 pregnancies), with ethics approval and informed consent. Megalin, cubilin, RXR $\alpha$ and VDR mRNA expression were measured by quantitative-PCR and related to measures of neonatal size using Pearson's partial-correlation.

Results: VDR expression associated negatively with neonatal birth weight $(r=-0.19, \quad p=0.06)$, crown-heel length $(\mathrm{r}=-0.22, \mathrm{p}=0.03)$ lean mass, $(\mathrm{r}=-0.24, \mathrm{p}=0.02)$ fat mass $(\mathrm{r}=-0.22, \mathrm{p}=0.03)$ and placental weight $(\mathrm{r}=-0.22, \mathrm{p}=0.03)$. Cubilin expression associated positively with neonatal head circumference $(r=0.22, p=0.03)$ and crown-heel length $(\mathrm{r}=0.21, \mathrm{p}=0.04)$. Megalin expression associated positively with birth weight $(\mathrm{r}=0.2, \mathrm{p}=0.05)$.

Conclusion: Relationships between placental genes involved in vitamin $\mathrm{D}$ handling and neonatal size suggests the placenta mediates the amount of vitamin D transferred to the fetus. Vitamin D signalling via VDR and $\mathrm{RXR} \alpha$ may also influence expression of genes important for placental function and affect fetal growth.

This work is supported by the Medical Research Council (MC_U147585827, MC_ST_U12055), British Heart Foundation (RG/07/009), NIHR Southampton Biomedical Research Centre, University of Southampton and University Hospital Southampton NHS Foundation Trust, and the Gerald Kerkut Charitable Trust.

\section{1}

\section{Association between placental weight and birth weight in a Brazilian birth cohort}

Ligia Nascente ${ }^{1}$, Davi Aragon ${ }^{2}$, Ricardo Cavalli ${ }^{3}$, Carlos Grandi ${ }^{4}$, Viviane Cardoso 5

${ }^{1}$ Department of Pediatrics, Ribeirão Preto Medical School, University of São Paulo; ${ }^{2}$ Department of Pediatrics, Ribeirão Preto Medical School, University of São Paulo; ${ }^{3}$ Department of Obstetrics and Gynecology, Ribeirão Preto Medical School,
University of São Paulo, Brazil; ${ }^{4}$ Department of Pediatrics, Sarda Maternity Hospital, University of Buenos Aires, Argentina; ${ }^{5}$ Department of Pediatrics, Ribeirão Preto Medical School, University of São Paulo

Research question: To investigate the association between placental weight and birth weight in a Brazilian birth cohort. Methods: Observational study of 958 trios of mothers, placentas and singleton newborns delivered at a tertiary hospital at Ribeirão Preto, São Paulo, Brazil, during 2010 and 2011. Mothers were interviewed after delivery in order to answer a standardized questionnaire about their demographic, social and health characteristics. Gestational age and birth weight were collected from medical files, while placental measures were available from pathology reports. Simple and multiple nonlinear regression models were fitted to evaluate the effect of placental weight on birth weight, adjusting for maternal characteristics (age, parity, body mass index before pregnancy, hypertension, diabetes, anemia and smoking), gestational age and neonate sex.

Results: Placental weight alone explained 53\% (R2) of birth weight total variability whereas placental weight and gestational age together explained $72 \%$ of it. Combined, placental weight, gestational age, neonate sex and maternal characteristics explained $74 \%$ of birth weight.

Conclusion: $53 \%$ of birth weight variance was explained only by placental weight in this Brazilian birth cohort.

\section{5}

Characterization of gut microbiota among healthy Chinese infants

Mingyang Yuan ${ }^{1}$, Shenghui $\mathrm{Li}^{2}$, Yong Guo ${ }^{3}$, Yashu Kuang ${ }^{4}$, Hongwen Deng ${ }^{5}$, Huimin Xia ${ }^{6}, \mathrm{Xiu}_{\mathrm{Qiu}}{ }^{7}$

${ }^{1}$ Guangzhou Women and Children's Medical Center;

${ }^{2}$ Guangzhou Women and Children's Medical Center;

${ }^{3}$ Guangzhou Women and Children's Medical Center;

${ }^{4}$ Guangzhou Women and Children's Medical Center; 5 Tulane University School of Public Health and Tropical Medicine;

${ }^{6}$ Guangzhou Women and Children's Medical Center;

${ }^{7}$ Guangzhou Women and Children's Medical Center

Background: Acquisition and development of the gut microbial community have a long-term effect on health. Here we characterized the gut microbiota of healthy Chinese neonates and infants by analyzing their fresh feces, and studied the potential factors that can affect the bacterial colonization.

Methods: Twenty-nine babies were recruited at the Guangzhou Women and Children's Medical Center. Feces were collected in sterile flask container by perianal stimulation. Total bacterial DNA was extracted, and high-throughput sequencing for the $16 S$ rDNA genes was performed.

Results: The fecal microbiota of Chinese infants were dominated by Proteobacteria, Firmicutes, Bacteroidetes and Actinobacteria. At genus level, the neonate's fecal microbiota were mainly composed of Escherichia/Shigella, Enterococcus, Klebsiella and 
Streptococcus, while the 1-3 month-old infants had a wide variety of genera. These observes were different from the findings in European and American infants, due to the significantly higher of Enterobacteriaceae in Chinese infants. We also observed delivery mode was a determinant for fecal microbiota.

Conclusions: Our study adds to understanding of the gut microbiota during early life and provides new evidence for the effects of delivery mode, which could help to improve the infant healthy.

\section{6}

Early free access to inulin prebiotic alleviates pesticide induced metabolic disorders in adult rat offspring

Julie Reygner ${ }^{1}$, Lydia Lichtenberger ${ }^{2}$, Ghada Elmhiri ${ }^{3}$, Narges Bahi-Jaber $^{4}$, Larbi Rhazi ${ }^{5}$, Flore Depeint ${ }^{6}$, Véronique Bach ${ }^{7}$, Hafida Khorsi-Cauet ${ }^{8}$, Latifa Abdennibi-Najar ${ }^{9}$

${ }^{1}$ PERITOX UMR-IO1 INERIS Amiens- UP EGEAL 201210 101 Beauvais France; ${ }^{2}$ CHU Amiens, Amiens France; ${ }^{3} U P$ EGEAL-2012-10-101 Institut Polytechnique LaSalle Beauvais, Beauvais France; ${ }^{4}$ UP EGEAL-2012-10-101 Institut Polytechnique LaSalle Beauvais, Beauvais France; ${ }^{5} U P$ EGEAL-2012-10-101 Institut Polytechnique LaSalle Beauvais, Beauvais France; ${ }^{6}$ UP EGEAL-2012-10-101 Institut Polytechnique LaSalle Beauvais, Beauvais France; ${ }^{7}$ PERITOX UMR-I01 INERIS UPJV Amiens France; ${ }^{8}$ PERITOX UMR-IO1 INERIS UPJV Amiens France; ${ }^{9}$ UP EGEAL-2012-10-101 Institut Polytechnique LaSalle Beauvais, Beauvais France

Research question: We investigated whether perinatal supplementation with prebiotics alleviates offspring pesticide induced metabolic disorders.

Methods: 5 groups ( $\mathrm{n}=7$ to 11 ) of Wistar rats received during gestation and lactation Chlorpyrifos (CPF) at the dose of $1 \mathrm{mg}$ (CPF1) or $3.5 \mathrm{mg} / \mathrm{kg} /$ day $(\mathrm{CPF} 3.5)$ with free access to inulin $(10 \mathrm{~g} /$ liter in drinking water). Metabolic profile, leptin sensitivity and gut microbiota composition were analyzed at postnatal day 59 (PND59).

Results: At birth, CPF offspring presented a higher body weight $(\mathrm{BW})$ compared to control animals ( $\mathrm{p}$

\section{8}

A prebiotic treatment can prevent pesticide-induced dysbiosis in an artificial human gut model

Julie Reygner ${ }^{1}$, Claire Joly Condette ${ }^{2}$, Camille Mayeur ${ }^{3}$, Flore Depeint ${ }^{4}$, Stéphane Delanaud ${ }^{5}$, Véronique Bach ${ }^{6}$, Latifa Abdennebi-Najar ${ }^{7}$, Hafida Khorsi-Cauet ${ }^{8}$

${ }^{1}$ PERITOX UMR-IO1 INERIS UPJV Amiens-UP EGEAL 2012 10101 Beauvais France; ${ }^{2}$ EA 4666 Lymphocyte NormalPathologique et cancers; ${ }^{3}$ Commensal and Probiotics-Host Interactions Laboratory, INRA, UMR 1319 MICALIS, F-78350 Jouy-en-Josas; ${ }^{4}$ UP EGEAL-2012-10-101 Institut Polytechnique
LaSalle Beauvais, Beauvais France; ${ }^{5}$ PERITOX UMR-IOI INERIS UPJV, Amiens France; ${ }^{6}$ PERITOX UMR-IO1 INERIS UPJV, Amiens France; ${ }^{7}$ UP EGEAL-2012-10-101 Institut Polytechnique LaSalle Beauvais, Beauvais France; ${ }^{8}$ PERITOX UMR-I01 INERIS UPJV, Amiens France

Research question: Daily exposure to food pesticides have serious effects on the maturation and development of organs such as intestine, leading to public health concerns. Our previous study on an artificial intestine model (SHIME®) showed that chronic exposure to a low dose of Chlorpyrifos (CPF) caused intestinal dysbiosis resulting in a decrease of Lactobacilli and Bifidobacteria. The aim of this study was to investigate the association of CPF (1 mg) together with inulin on microflora stability.

Method: The SHIME® consists of 6 reactors representing the different parts of the gastrointestinal tract. The last 3 reactors representing colon were inoculated with a mix of human feces. Samples are collected at D0, D15, D30.

Results: With CPF alone, we observed an increase in Clostridii and Enterobacteriaceae (D0 vs D15 p < 0.001) whereas no increase was observed when combined with inulin. We also noticed a significant increase in Bifidobacreria ( $p$-value).

\section{6}

Energy and hormonal composition of breast milk program obese or lean adult rat offspring

Vander Silva Alves ${ }^{1}$, Júlio Cezar de Oliveira ${ }^{2}$, Claudinéia Conationi da Silva Franco ${ }^{3}$, Luiz Felipe Barella ${ }^{4}$, Kelly Valério Prates ${ }^{5}$, Tatiane Aparecida Ribeiro ${ }^{6}$, Laize Peron Tófolo ${ }^{7}$, Audrei Pavanello ${ }^{8}$, Rosana Torrezan ${ }^{9}$, Paulo Cezar de Freitas Mathias ${ }^{10}$, Ellen Paula Santos da Conceição ${ }^{11}$, Patrícia Cristina Lisboa $^{12}$, Rosiane Aparecida Miranda ${ }^{13}$

${ }^{1}$ Laboratory of Secretion Cell Biology, Department of Biotechnology, Genetics and Cell Biology, State University of Maringá, 87020-900 Maringá, PR, Brazil; ${ }^{2}$ Health Sciences Institute, Federal University of Mato Grosso, Sinop/MT, Brazil; ${ }^{3}$ Laboratory of Secretion Cell Biology, Department of Biotechnology, Genetics and Cell Biology, State University of Maringá, 87020-900 Maringá, PR, Brazil; ${ }^{4}$ National Institute of Health (HIN), Washington/DC, USA; ${ }^{5}$ Laboratory of Secretion Cell Biology, Department of Biotechnology, Genetics and Cell Biology, State University of Maringá, 87020-900 Maringá, PR, Brazil; ${ }^{6}$ Laboratory of Secretion Cell Biology, Department of Biotechnology, Genetics and Cell Biology, State University of Maringá, 87020-900 Maringá, PR, Brazil; ${ }^{7}$ Laboratory of Secretion Cell Biology, Department of Biotechnology, Genetics and Cell Biology, State University of Maringá, 87020-900 Maringá, PR, Brazil; ${ }^{8}$ Laboratory of Secretion Cell Biology, Department of Biotechnology, Genetics and Cell Biology, State University of Maringá, 87020-900 Maringá, PR, Brazil; ${ }^{9}$ Department of Physiological Sciences, State University of Maringá-MaringálPR, Brazil; ${ }^{10}$ Laboratory of Secretion Cell Biology, Department of 
Biotechnology, Genetics and Cell Biology, State University of Maringá, 87020-900 Maringá, PR, Brazil; ${ }^{11}$ Department of Physiology Sciences, Institute of Biology, State University of Rio de Janeiro - Rio de Janeiro/RJ, Brazil; ${ }^{12}$ Department of Physiology Sciences, Institute of Biology, State University of Rio de JaneiroRio de Janeiro/RJ, Brazil; ${ }^{13}$ Laboratory of Secretion Cell Biology, Department of Biotechnology, Genetics and Cell Biology, State University of Maringá, Maringá, Paraná, Brazil

Background/Aims: Maternal obesity has been associated with metabolic disturbances in adulthood. Nevertheless, changes in lactation period can modify the offspring development. We tested whether breast milk from obese mothers can programming metabolic dysfunction later in life.

Methods: Cross-fostering during lactation was used to test the hypothesis. Neonate female rats were treated during the first 5 days of life with monosodium L-glutamate (MSG), $4 \mathrm{mg} / \mathrm{g}$ body mass. Controls received saline. At adult age, control and obese female rats were mated. Pups were identify as from control mothers, CONF2; from obese mothers MSGF2; crossfostered obese with from control mothers CR-MSGF2 and cross-fostered control pups with from obese mothers CRCONF2. Milk composition was evaluated. Offspring with 120-day-old were assessed to evaluated metabolism and leptin pathway signalling in hypothalamus.

Results: Breast milk composition in obese mothers exhibits higher triglycerides, glucose, insulin and lower leptin content $(\mathrm{p}<0.05)$. As expected, MSGF2 rats displayed higher body weight, fat pad storage, insulin and leptin levels regarding CONF2 $(p<0.05)$. Overall leptin pathway was reduced in MSGF2 and CR-CONF2 animals $(p<0.05)$. Adoption by control mothers restores hormone levels and central leptin signal transduction.

Conclusions: Breast milk composition can program metabolism of adult offspring associate to central changes in leptin sensitivity.

\section{9}

Soya bean isoflavones attenuates early-overnutritioninduced rat obesity

Rosana Torrezan ${ }^{1}$, Audrei Pavanello ${ }^{2}$, Ananda Malta ${ }^{3}$, Laize Peron Tófolo ${ }^{4}$, Isabela Peixoto Martins ${ }^{5}$, Claudinéia Conationi da Silva Franco ${ }^{6}$, Rosiane Aparecida Miranda ${ }^{7}$, Júlio Cezar de Oliveira $^{8}$, Kesia Palma Rigo ${ }^{9}$, Paulo Cezar de Freitas Mathias $^{10}$, Tatiane Aparecida Ribeiro ${ }^{11}$

${ }^{1}$ Laboratory of Secretion Cell Biology, Department of Cell Biology and Genetics, State University of Maringá - (MaringálPR, Brazil); ${ }^{2}$ Laboratory of Secretion Cell Biology, Department of Cell Biology and Genetics, State University of Maringá - (MaringálPR, Brazil); ${ }^{3}$ Laboratory of Secretion Cell Biology, Department of Cell Biology and Genetics, State University of Maringá - (Maringá/PR, Brazil); ${ }^{4}$ Laboratory of Secretion Cell Biology, Department of Cell Biology and Genetics, State University of Maringá - (MaringálPR, Brazil);
${ }^{5}$ Laboratory of Secretion Cell Biology, Department of Cell Biology and Genetics, State University of Maringá - (MaringálPR, Brazil); ${ }^{6}$ Laboratory of Secretion Cell Biology, Department of Cell Biology and Genetics, State University of Maringá - (MaringálPR, Brazil); ${ }^{7}$ Laboratory of Secretion Cell Biology, Department of Cell Biology and Genetics, State University of Maringá - (MaringálPR, Brazil); ${ }^{8}$ Health Sciences Institute, Federal University of Mato Grosso, Sinop, MT, Brazil; ${ }^{9}$ Laboratory of Secretion Cell Biology, Department of Cell Biology and Genetics, State University of Maringá - (Maringál PR, Brazil); ${ }^{10}$ Laboratory of Secretion Cell Biology, Department of Cell Biology and Genetics, State University of Maringá - (Maringál PR, Brazil)/Fellowship of CAPES-Foundation, Ministry of Educat; ${ }^{11}$ Laboratory of Secretion Cell Biology, Department of Cell Biology and Genetics, State University of Maringá - (MaringálPR, Brazil)/ Fellowship of CNPq-Brazilian Research Agency

Research question: Antioxidant contained in food has showed effect against cardiometabolic disease. Isoflavones from soya beans are antioxidant and have estrogen-like (phytoestrogen) properties. Whether early chronic consume of isoflavones can attenuate adult obesity induced by milk-suckling excess in rat pups, was tested.

Methods: After birth, litters size was manipulated: normal litters, 9 pups (NL); and small litters, 3 pups (SL). Male rat offspring with 30 days old received by gavage $300 \mathrm{mg} / \mathrm{kg} /$ day of soya bean extract, containing $42.37 \%$ of mixture isoflavones (32.57\% daidzein; $0.14 \%$ genistein and $11.84 \%$ others) until 90-days-old. Adult animals were used to evaluate metabolism. Results: As expected SL adult offspring developed obesity. Body weight and food intake were not affected by the isoflavones treatment for both animal groups; however, fat tissue accretion was reduced in SL animals ( $p<0.05)$. Glucose intolerance and hyperinsulinemia of SL-obese rats were blocked by isoflavones exposure $(p<0.05)$. Isoflavones treatment did not changed testis weight, neither testosterone release by testis slices.

Conclusions: Early treatment with soya bean isoflavones programs metabolism of male adult rat offspring, without affecting testosterone production.

\section{2}

Maternal cafeteria-style diet intake during lactation programs to obesity with hepatic metabolism disruption in rat offspring at adulthood

Rodrigo Mello Gomes ${ }^{1}$, Flávio Andrade Francisco ${ }^{2}$, Lucas Paulo Jacinto Saavedra ${ }^{3}$, Rosiane Aparecida Miranda ${ }^{4}$, Solange Marta Franzoi de Moraes 5 , Vilma Aparecida Ferreira de Godoi ${ }^{6}$, Paulo Cesar de Freitas Mathias ${ }^{7}$, Claudinéia Conationi da Silva Franco ${ }^{8}$, Jean Carlos Fernando Besson ${ }^{9}$, Júlio Cezar de Oliveira $^{10}$, Maria Raquel Marçal Natali ${ }^{11}$

\footnotetext{
${ }^{1}$ Department of Physiological Sciences, State University of Maringa, Maringá, PR, Brazil; ${ }^{2}$ Laboratory of Secretion Cell Biology, State University of Maringa, Maringa, PR, Brazil; ${ }^{3}$ Laboratory of Secretion Cell Biology, Department of
} 
Biotechnology, Genetics and Cell Biology, State University of Maringá, 87020-900 Maringá, PR, Brazil; ${ }^{4}$ Laboratory of Secretion Cell Biology, Department of Biotechnology, Genetics and Cell Biology, State University of Maringá, 87020-900 Maringá, PR, Brazil; ${ }^{5}$ Department of Physiological Sciences, State University of Maringa, Maringá, PR, Brazil; ${ }^{6}$ Department of Physiological Sciences, State University of Maringa, Maringá, PR, Brazil; ${ }^{7}$ Laboratory of Secretion Cell Biology, Department of Biotechnology, Genetics and Cell Biology, State University of Maringá, 87020-900 Maringá, PR, Brazil; ${ }^{8}$ Laboratory of Secretion Cell Biology, Department of Biotechnology, Genetics and Cell Biology, State University of Maringá, 87020-900 Maringá, PR, Brazil; ${ }^{9}$ Department of Morphological Sciences, State University of Maringa, Maringá, PR, Brazil; ${ }^{10}$ Health Sciences Institute, Federal University of Mato Grosso, Sinop/MT, Brazil; ${ }^{11}$ Department of Morphological Sciences, State University of Maringa, Maringá, PR, Brazil

Background: Non-alcoholic fatty liver diseases (NAFLD) and diabetes are associated with obesity onset. During perinatal life, pups are vulnerable to malnourishment and others stressful factors, which increase risk to develop cardiometabolic diseases later in life, the $\mathrm{DOHaD}$ concept. Unbalanced milk composition can program offspring to worst metabolism. Using cafeteria-style diet during lactation, we aimed test whether offspring displays in adulthood obesity associated to hepatic malfunction.

Methods: Wistar dam rats, during lactation received cafeteriastyle diet, CAF group, and controls consumed standard rodent chow, $\mathrm{CO}$ group. All rats received $\mathrm{CO}$ diet from weaning until 90 days of age, whose were used to analyze hepatic metabolism.

Results: Milk from CAF dams showed increased cholesterol, triglycerides and protein $(\mathrm{p}<0.05)$. Adult offspring from CAF group presented: overweight, enhanced adiposity, dyslipdemia $(\mathrm{p}<0.05)$. Increased hepatic gluconeogenesis and blood alanine aminotransferase (ALT) and aspartate aminotransferase (AST) enzyme activities were also observed $(\mathrm{p}<0.05)$. Hepatic insulin resistance was recorded, with lower expression of insulin transduction signal proteins PI3K and Akt $(\mathrm{p}<0.05)$. Increased lipid inclusion was observed in hepatocytes from CAF animals $(\mathrm{p}<0.05)$.

Conclusions: Maternal CAF consumption might program to obesity late in life associated to hepatic dysfunctions. Unbalanced milk composition could be a target mechanism for this programming.

\section{2}

Maternal high fat diet promotes resistance to the hypolipidemic effect of fish oil in adolescent offspring rats

Lorraine Soares de Oliveira ${ }^{1}$, Aline Fonseca Pereira Souza ${ }^{2}$, Aline Cordeiro de Faria Fernandes ${ }^{3}$,

George Eduardo Gabriel Kluck ${ }^{4}$, Georgia Correa Atella ${ }^{5}$,
Isis Hara Trevenzoli ${ }^{6}$, Luana Lopes de Souza ${ }^{7}$, Carmen Cabanelas Pazos de Moura ${ }^{8}$

${ }^{1}$ Institute of Biophysics Carlos Chagas Filho; ${ }^{2}$ Institute of Biophysics Carlos Chagas Filho; ${ }^{3}$ Institute of Biophysics Carlos Chagas Filho; ${ }^{4}$ Institute of Medical Biochemistry; ${ }^{5}$ Institute of Medical Biochemistry; ${ }^{6}$ Institute of Biophysics Carlos Chagas Filho; ${ }^{7}$ Institute of Biophysics Carlos Chagas Filho; ${ }^{8}$ Institute of Biophysics Carlos Chagas Filho

Obesity prevalence has increased in adolescents, resulting in early comorbidities development. Maternal obesity (MO) promotes obesity and metabolic changes in offspring at weaning and adult life, but we do not know its effect on adolescent offspring. Fish oil (FO), an n-3 polyunsaturated fatty acid source, has hypolipidemic and anti-obesogenic effects in animal obese models. Thus, we evaluated MO impact on metabolic profile of adolescent offspring, with or without FO intervention. Female Wistar rats received standard ( $9 \%$ fat) or high fat diet $(29 \%$ fat $)$ prior mating, throughout pregnancy and lactation. After weaning, offspring received standard chow and, from 25th to 45th day, received oral administration of soybean oil (control) or FO $(0,4 \mathrm{ml} / 100 \mathrm{gBW})$. MO offspring showed higher body weight and glucose intolerance, however, FO attenuated these parameters. In control offspring, FO reduced serum triglyceride and cholesterol levels; nonetheless, this effect was abolished in $\mathrm{MO}$ group. MO offspring with $\mathrm{FO}$ intervention showed higher hepatic cholesterol content, higher hepatic SREBP-1 expression, and higher serum leptin and corticosterone levels. Hepatic triglycerides content was similar among groups. Therefore, FO intervention partially attenuated metabolic changes provoked by MO in adolescent offspring. However, even without serum lipid changes, MO group was resistant to FO hypolipidemic effect, suggesting signaling pathway impairment.

\section{2}

Maternal nutritional history impacts on the development of the neonatal enteric nervous system in a rat model

Kennedy Hao ${ }^{1}$, Rajka Borojevic ${ }^{2}$, Kaitlyn $\mathrm{Chan}^{3}$, Wajiha Gohir ${ }^{4}$, Elyanne Ratcliffe ${ }^{5}$, Deborah Sloboda ${ }^{6}$

${ }^{1}$ Biochemistry, McMaster University; ${ }^{2}$ Pediatrics, McMaster University; ${ }^{3}$ Biochemistry, McMaster University; ${ }^{4}$ Biochemistry, McMaster University; ${ }^{5}$ Pediatrics, McMaster University; ${ }^{6}$ Biochemistry, Pediatrics, Obstetrics and Gynecology, McMaster University

Research question: In utero nutritional adversity results in an increased risk of obesity in offspring, although the mechanisms are unclear. Normal gastrointestinal function is essential in regulating energy extraction and is regulated by the enteric nervous system (ENS). We investigated the effects of maternal diet on the development of the neonatal ENS. 
Methods: Pregnant rats were fed a control, undernourished (UN), or high fat (HF) diet during pregnancy. The ENS was studied in wholemount preparations of gut collected from day 4 neonates. The ENS was visualized using immunohistochemistry and analyzed for nerve density and neuronal number.

Results: Nutritionally-induced changes in offspring ENS development were dependant upon the in utero diet. Significant abnormalities in the myenteric plexi of HF and UN gut sections were revealed through immunolabeling. Loss of enteric neurons occurred in the myenteric plexi of the proximal and distal jejunum, in both UN and HF offspring, and the colon in HF offspring. Total number of neurons were reduced by $23-29 \%$.

Conclusion: Our results demonstrate that in utero exposure to nutritional adversity modifies the neonatal ENS. The decrease in enteric neurons may contribute to changes in energy extraction and nutrient absorption that could impact obesity risk later in life.

\section{2}

Fetal high fat programming reduces hepatic and skeletal muscle insulin receptor immunoreactivity in neonatal rats

Zibele Ndlovu ${ }^{1}$, Johan Louw ${ }^{2}$

${ }^{1}$ South African Herbal Science and Medicine Institute, University of the Western Cape; ${ }^{2}$ Diabetes Discovery Platform, South African Medical Research Council

Research question: Maintenance on a fetal high fat diet may programme hepatic and skeletal muscle insulin resistance. This study investigated the effects of maternal diets, varying in fat content, on hepatic and skeletal muscle expression of the proximal insulin signalling factors: insulin receptor (IR), insulin receptor substrate 2 (IRS2) and phosphoinositide 3-kinase (PI3K).

Methods: Pregnant rats were randomised into groups and maintained on diets with varying fat proportions: $10 \%$ (control), 20\% (20F), 30\% (30F) and 40\% (40F) fat as energy throughout gestation. The hepatic and skeletal muscle expression profiles of target insulin signalling factors were determined by qPCR and immunostaining followed by image analysis.

Results: Hepatic PI3K mRNA expression was elevated in 30F neonates compared to $20 \mathrm{~F}$ neonates with no changes in hepatic IR or IRS2 mRNA expression. In skeletal muscle, IR and PI3K mRNA expression were reduced in the $30 \mathrm{~F}$ and $40 \mathrm{~F}$ neonates compared to $20 \mathrm{~F}$ neonates. Hepatic IR $\alpha$ immunoreactivity was reduced in $40 \mathrm{~F}$ neonates compared to control and $20 \mathrm{~F}$ neonates. Further, skeletal muscle IR $\alpha$ immunoreactivity was reduced in $30 \mathrm{~F}$ and $40 \mathrm{~F}$ neonates compared to control neonates.

Conclusion: Fetal high fat programming reduced both hepatic and skeletal muscle IR $\alpha$ immunoreactivity which reflects impaired insulin signalling at the receptor level.
4433

High fat programming alters islet cell trajectories in neonate, weanling and adolescent rats

Charna Chapman ${ }^{1}$, Johan Louw ${ }^{2}$

${ }^{1}$ Diabetes Discovery Platform, South African Medical Research Council; ${ }^{2}$ Diabetes Discovery Platform, South African Medical Research Council

Research question: Islets are dynamic throughout life and influenced by metabolic states. We assessed beta and alpha cell trajectories in neonate, weanling and adolescent rats during development and after maintenance on a high fat diet.

Methods: Pancreata from neonatal (one-day-old), weanling (three-week-old) and adolescent (three-month-old) rats were double immunolabeled with insulin and glucagon in control progeny $(10 \%$ fat diet) and progeny maintained on a high fat diet ( $40 \%$ of mainly saturated fat as energy) during fetal, lactational and/or postnatal life (high fat programming). Beta and alpha cell number, size and volume were assessed.

Results: Postnatal high fat maintenance induced beta cell hyperplasia at weaning which persisted into adolescence concomitant with beta cell hypertrophy at adolescence. Physiologically and more markedly after postnatal high fat maintenance, alpha cell numbers increased with age, emerging at weaning and prevailing into adolescence. Alpha cell hyperplasia was maintained after fetal and postnatal high fat maintenance at weaning and adolescence. After postnatal high fat maintenance, alpha cell hypertrophy emerged earlier at weaning and persisted into adolescence. Alpha cell volume was consistently enhanced at weaning.

Conclusion: Beta and alpha cell populations vary in number, size and volume throughout development and are altered in response to high fat programming.

\section{9}

Methylglyoxal treatment during lactation induces metabolic programing and gluconeogenesis dysfunction in male offspring rats at weaning

Flávio Andrade Francisco ${ }^{1}$, Claudinéia Conationi da Silva Franco $^{2}$, Elaine Vieira ${ }^{3}$, Julio Cesar de Oliveira ${ }^{4}$, Kesia Palma Rigo $^{5}$, Latifa Najar ${ }^{6}$, Lucas Paulo Jacinto Saavedra ${ }^{7}$, Luiz Felipe Barella ${ }^{8}$, Rosana Torezan? , Rosiane Aparecida Miranda $^{10}$, Stéphane Firmin ${ }^{11}$, Vander Silva Alves ${ }^{12}$, Paulo Cezar de Freitas Mathias ${ }^{13}$, Rodrigo Mello Gomes ${ }^{13}$

${ }^{1}$ State University of Maringa, Maringa-PR, Brazil; ${ }^{2}$ State University of Maringa, Maringa-PR, Brazil; ${ }^{3}$ State University of Maringa, Maringa-PR, Brazil; ${ }^{4}$ Federal University of Mato Grosso, Sinop, MT, Brazil; ${ }^{5}$ State University of Maringa, Maringa-PR, Brazil; ${ }^{6}$ Institut Polytechnique LaSalle de Beauvais, Beauvais Cedex, France; ${ }^{7}$ State University of Maringa, MaringaPR, Brazil; ${ }^{8}$ National Institutes of Health, Bethesda, MD, USA; ${ }^{9}$ State University of Maringa, Maringa-PR, Brazil; ${ }^{10}$ State 
University of Maringa, Maringa-PR, Brazil; ${ }^{11}$ Institut

Polytechnique LaSalle de Beauvais, Beauvais Cedex, France;

${ }^{12}$ State University of Maringa, Maringa-PR, Brazil; ${ }^{13}$ State

University of Maringa, Maringa-PR, Brazil; ${ }^{14}$ State University of

Maringa, Maringa-PR, Brazil

Background/Aims: Increased levels of Advanced Glycation End products (AGEs) in the organism is associated with hyperglycemia, which is due to AGE-induced cell dysfunction. Therefore, our aim was to study the effects of chronic administration of an AGE precursor, Methylglyoxal, on the metabolism and pancreatic islet function of the offspring treated with MG during the two first weeks of lactation.

Methods: After birth, the puppies were divided into 2 groups: Control Group $(\mathrm{CO}, \mathrm{n}=10)$ treated with saline injection $(0.9 \% \mathrm{Kg}$ of BW/day) and the Methylglyoxal Group (MG, $\mathrm{n}=10)$, treated with Methylglyoxal $(6 \mathrm{mg} / \mathrm{Kg}$ of $\mathrm{BW} /$ day $)$ during the first 15 days of the lactation period. At 21 days-old, male pups were submitted to pyruvate tolerance test and euthanized for tissue collection.

Results: Treatment with MG decreased body weight, liver weight, periepididymal and mesenteric fat pads as compared with the CO group. MG group developed dyslipidemia with increased plasma levels of LDL, VLDL, total cholesterol and triglycerides $(p<0.05)$. Furthermore, pyruvate tolerance test showed an increase in gluconeogenesis in the MG group as compared to CO group $(\mathrm{p}<0.05)$.

Conclusion: Treatment with Methylglyoxal in the first two weeks of lactation period promoted metabolic programming inducing alterations in glucose and lipid metabolism.

\section{8}

\section{The Effect of Early Life Methyl Donor Supplementation on Obesity Development}

Teresa M. Reyes

\section{University of Cincinnati}

Excessive maternal weight gain during pregnancy contributes to an increased risk for obesity in the offspring. In a mouse model of excessive maternal weight gain, we find that offspring have increased preference for sucrose and fat, increased expression of genes that underlie reward-related behaviors, and both global and gene specific DNA hypomethylation. These changes in reward-related neural circuitry may contribute to the increased risk for the development of obesity in the offspring by altering the animal's response to highly palatable, energy dense foods. Methyl donor supplementation (MDS) during pregnancy can reverse some of these phenotypes, yet it is unknown whether postnatal MDS can reverse these phenotypes. To determine this, offspring from dams fed either a high fat diet (HFD) or control diet during gestation/lactation were fed a methyl donor supplemented diet during early life (age 3-6 weeks). We find that postnatal MDS significantly decreased body weight in both male and female adult HFD offspring, and does not alter body weights of control diet offspring. Further, postnatal MDS can normalize adult male fat preference and contributes to regional specific normalization of DNA hypomethylation.

\section{5}

Shunting diabetes: neonatal metformin treatment protects against obesity onset

Claudinéia Franco ${ }^{1}$, Carina Previate ${ }^{2}$, Isabela Martins ${ }^{3}$, Rosiane Miranda ${ }^{4}$, Flávio Francisco ${ }^{5}$, Audrei Pavanello ${ }^{6}$, Rodrigo Gomes ${ }^{7}$, Júlio de Oliveira ${ }^{8}$, Paulo Mathias ${ }^{9}$

${ }^{1}$ State University of Maringá - Laboratory of Secretion Cell Biology, Department of Biotechnology, Genetics and Cell Biology;

${ }^{2}$ State University of Maringá - Laboratory of Secretion Cell Biology, Department of Biotechnology, Genetics and Cell Biology; ${ }^{3}$ State University of Maringá - Laboratory of Secretion Cell Biology, Department of Biotechnology, Genetics and Cell Biology; ${ }^{4}$ State University of Maringá - Laboratory of Secretion Cell Biology, Department of Biotechnology, Genetics and Cell Biology; ${ }^{5}$ State University of Maringá - Laboratory of Secretion Cell Biology, Department of Biotechnology, Genetics and Cell Biology; ${ }^{6}$ State University of Maringá - Laboratory of Secretion Cell Biology, Department of Biotechnology, Genetics and Cell Biology; ${ }^{7}$ State University of Maringá - Department of Physiological Sciences; ${ }^{8}$ State University of Maringá - Laboratory of Secretion Cell Biology, Department of Biotechnology, Genetics and Cell Biology; ${ }^{9}$ State University of Maringá - Laboratory of Secretion Cell Biology, Department of Biotechnology, Genetics and Cell Biology

Background: Antidiabetic metformin has been used worldwide to treat metabolic diseases. Perinatal phases is known as window to program adulthood metabolism. The goal of this study was verified whether metformin treatment during lactation could attenuate early overnutrition, (small litter) induced obesity.

Methods: After birth normal litter size (NL) were adjusted to 9 pups or small litter (SL) with 3 pups per dam. From the first to the 12th day rat offspring received intraperitoneal injection of metformin, $100 \mathrm{mg} / \mathrm{kg}$ body weight (bw)/day, controls received saline. From weaning until 90-days-old, bw and food intake were taken. It was also evaluated fat pad stores, blood glucose and insulin levels during intravenous glucose tolerance test (ivGTT).

Results: As expected SL animals showed increased food consumption and bw compared to NL group. SL rats presented glucose intolerance and hyperinsulinemia $(\mathrm{p}<0.05)$. While, metformin did not alter food intake and bw of NL group, to SL rats these parameters were reduced $(\mathrm{p}<0.05)$. Fat tissue accretion was also decreased in SL rats by metformin early treatment $(\mathrm{p}<0.05)$. Glucose intolerance and hyperinsulinemia were normalized by metformin comparing to SL and NL rats untreated $(\mathrm{p}<0.05)$. 
4545

\section{Alterations in hippocampal mRNA expression of lambs born to ewes supplemented with fishmeal and challenged with endotoxin}

Rebecca Fisher-Heffernan ${ }^{1}$, Alison Lee ${ }^{2}$, Margaret Quinton ${ }^{3}$, Timothy Regnault ${ }^{4}$, Niel Karrow ${ }^{5}$

${ }^{1}$ University of Guelph; ${ }^{2}$ University of Guelph; ${ }^{3}$ University of Guelph; ${ }^{4}$ Western University; ${ }^{5}$ University of Guelph

Research question: Maternal stressors such as infection during pregnancy can alter the offspring's stress responsiveness throughout life. Research suggests that omega-3 fatty acids may help protect the fetus from these stressors. The purpose of this study was to assess if an endotoxin challenge, used to simulate a systemic bacterial infection during pregnancy, altered offspring hippocampal gene expression, and if supplementation with fishmeal (FM) affected these changes.

Methods: Ewes diets were supplemented with FM or soybean meal (SM) from 100 days of gestation (gd) throughout lactation. On gd135 half the ewes were challenged with lipopolysaccharide (LPS), while the other half received saline (CON). At 6-months-of-age, hippocampal tissues were collected from female offspring for gene expression analysis. The

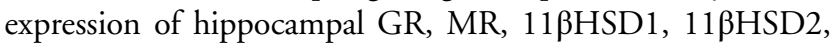
COX1, COX2, PPAR $\gamma$ and IL1 $\beta$ genes were assessed using RT-PCR.

Results and Conclusion: Results demonstrated that expression levels of GR and MR were greatest in FM + CON offspring compared to other treatment groups. FM + LPS offspring had greater GR expression compared to SM + LPS offspring while the reverse was true for MR and $11 \beta \mathrm{HSD} 1$ expression. Overall, simulated maternal bacterial infection caused alterations in hippocampal gene expression of 6-monthold offspring and these changes were affected by dietary supplementation.

\section{9}

Perinatal Over- or Undernutrition Programs Adipogenesis: A Common Wnt10b/PPAR $\gamma$ Pathway Mediated via Differential $\beta$-Catenin Signaling

Mina Desai ${ }^{1}$, Michael G Ross ${ }^{2}$, Guang $\mathrm{Han}^{3}$, Marie H Beall ${ }^{4}$, Robert H Lane ${ }^{5}$

${ }^{1}$ Geffen School of Medicine at UCLA; ${ }^{2}$ LABioMed at HarborUCLA Med. Ctr, Torrance, CA, United States; ${ }^{3}$ LABioMed at Harbor-UCLA Med. Ctr, Torrance, CA, United States; ${ }^{4} L A$ Perinatal Assoc, LA, CA, United States; ${ }^{5}$ Medical College of Wisconsin, Milwaukee, WI, United States

Introduction: Perinatal over- or under-nutrition results in obese adult offspring with programmed enhanced adipogenesis. We have shown that increased adiposity in both offspring groups is mediated via early upregulation of adipogenic transcription factor, PPAR $\gamma$, which promotes adipocyte differentiation. Induction of PPAR $\gamma$ and adipogenesis is regulated by factors including Wnt10b signal transduction. Activation of $\mathrm{Wnt} 10 \mathrm{~b}$ signalling inhibits adipogenesis through both canonical $\beta$-catenin dependent and non-canonical $\beta$ catenin independent pathways. We hypothesized that inhibition of Wnt $10 \mathrm{~b}$ contributes to increased PPAR $\gamma$ and enhanced adipogenesis in offspring exposed to maternal obesity and under-nutrition. We further determined whether these effects were mediated via $\beta$-catenin pathway.

\section{6}

\section{Detraining enhances high-fat-diet-induced obesity} in rats

Laize Peron Tófolo ${ }^{1}$, Kesia Palma Rigo ${ }^{2}$,

Adriane Barreto Gôngora ${ }^{3}$, Ananda Malta ${ }^{4}$, Lucas Eduardo Cardoso $^{5}$, Audrei Pavanello ${ }^{6}$, Wilson RInaldi ${ }^{7}$, Paulo Cezar de Freitas Mathias ${ }^{8}$

${ }^{1}$ State University of Maringá; ${ }^{2}$ State University of Maringá;

${ }^{3}$ State University of Maringá; ${ }^{4}$ State University of Maringá;

${ }^{5}$ State University of Maringá; ${ }^{6}$ State University of Maringá;

${ }^{7}$ State University of Maringá; ${ }^{8}$ State University of Maringá

Background: It has been suggested that continuous exercise can improve metabolism. High fat diet (HFD) impairs metabolism inducing to obesity onset. Whether or not detraining changes the magnitude of HFD-induced obesity was tested in the current work.

Methods: Wistar adult rats (90-days-old) were submitted to treadmill moderate exercise program $[55 \%$ from maximum oxygen uptake velocity (VO2max)], three times a week, during 30 days. Controls did not perform any training. At the end of training program, 120-days-old animals were exposed to HFD ( $30 \%$ of lard) during 30 days without any exercise training (detraining). Maximal effort tests were performed: before and after training program; and after detraining. After the rats were submitted to intravenous glucose tolerance test (ivGTT). Rats were sacrificed and retroperitoneal and periepididimal were removed and weighed. Data were submitted to two-way ANOVA and Tukey posttest.

Results: Just after training, animals showed to improve performance in effort test (50\%), comparing to sedentary $(\mathrm{p}<0.001)$. Detraining decreases the physical performance. HFD increased fat deposition in sedentary and detrained rats ( $\mathrm{pd}<0.001 ; \mathrm{d}=$ diet factor). As expected HFD induced glucose intolerance $(\mathrm{pd}<0.001)$ and hyperinsulinemia $(\mathrm{pd}<0.001)$. Detraining enhanced glucose and insulin blood concentration disturbs ( $\mathrm{pe}<0.001$; $\mathrm{e}=$ exercise factor).

Conclusion: Detraining might enhance metabolic disorders induced by HFD. 
4696

High-fat programming during lactation leads to vagal hyperactivity and impairs the pancreatic islet function in weanling rats

Luiz Felipe Barella ${ }^{1}$, Rosiane A. Miranda ${ }^{2}$, Claudinéia C. S. Franco $^{3}$, Flavio A. Francisco ${ }^{4}$, Vander S. Alves ${ }^{5}$, Laize P. Tófolo $^{6}$, Ananda Malta ${ }^{7}$, Clarice Gravena ${ }^{8}$, Rosana Torrezan ${ }^{9}$, Paulo C. F. Mathias ${ }^{10}$

${ }^{1}$ Molecular Signaling Section, Laboratory of Bioorganic Chemistry, National Institute of Diabetes and Digestive and Kidney Diseases, National Institutes of Health, Bethesda, MD, USA ${ }^{2}$ Laboratory of Secretion Cell Biology, Department of Biotechnology, Genetics and Cell Biology, State University of Maringá - Maringá, PR, Brazil;

${ }^{3}$ Laboratory of Secretion Cell Biology, Department of Biotechnology, Genetics and Cell Biology, State University of Maringá - Maringá, PR, Brazil; ${ }^{4}$ Laboratory of Secretion Cell Biology, Department of Biotechnology, Genetics and Cell Biology, State University of Maringá - Maringá, PR, Brazil; ${ }^{5}$ Laboratory of Secretion Cell Biology, Department of Biotechnology, Genetics and Cell Biology, State University of Maringá - Maringá, PR, Brazil; ${ }^{6}$ Laboratory of Secretion Cell Biology, Department of Biotechnology, Genetics and Cell Biology, State University of Maringá - Maringá, PR, Brazil; ${ }^{7}$ Laboratory of Secretion Cell Biology, Department of Biotechnology, Genetics and Cell Biology, State University of Maringá - Maringá, PR, Brazil; ${ }^{8}$ Laboratory of Secretion Cell Biology, Department of Biotechnology, Genetics and Cell Biology, State University of Maringá - Maringá, PR, Brazil; ${ }^{9}$ Department of Physiological Sciences, State University of Maringá - MaringálPR, Brazil; ${ }^{10}$ Laboratory of Secretion Cell Biology, Department of Biotechnology, Genetics and Cell Biology, State University of Maringá - Maringá, PR, Brazil

Background: We aimed to investigate the glucose metabolism, pancreatic beta cell function and parasympathetic activity in weanling rat offspring (at 22-day-old) from dams fed a normal (NF) or high-fat (HF) diet.

Methods: The HF diet (35\% of fat) was offered to dams from the delivery day until the end of offspring nursing (day 21). At 22-day-old the overnight fasted pups were sacrificed, the fat pads removed and weighed and the blood was collected. The pancreatic islets were isolated for functional and protein expression studies. Vagal nerve activity was measured directly using an electrode.

Results: HF pups became heavier and exhibited a great increase of the fat pads compared with the NF pups. Although no differences in the fasting glucose and corticosterone levels were found, the fasting insulinemia decreased $75 \%$ in HF rats. HF rats showed impaired islet function along with increased protein expression of the M3 muscarinic receptor. The vagal activity in the HF rats was greatly increased.

Conclusion: HF diet offered to dams during lactation induced an obese phenotype, beta cell dysfunction and vagus nerve hyperactivity in these weanling rats, which can result in the development of metabolic diseases when they become adults.
4874

Folic acid supplementation during the juvenile pubertal period in mice leads to persistent changes in the stem cell marker OCT- 4 and morphology differences in mammary gland structure

Karen Lillycrop ${ }^{1}$, Mark Burton ${ }^{2}$, Jinal Shah ${ }^{3}$, Graham Burdge ${ }^{4}$

${ }^{1}$ University of Southampton; ${ }^{2}$ University of Southampton;

${ }^{3}$ University of Southampton; ${ }^{4}$ University of Southampton

Research question: Folic acid intake has increased over the last decade; however, there are concerns that high levels of folic acid, particularly during early life, may increase breast cancer risk. Here we tested folic acid intake during specific periods of the life-course on the regulation of key cancer genes OCT- 4 and BRCA1 and mammary gland morphology.

Methods: Female C57BL/6 mice were fed a modified AIN93M semi-purified diet containing either $1 \mathrm{mg} / \mathrm{kg}$ (1x basal daily recommendation (BDR): $5 \mathrm{mg} / \mathrm{kg}$ ( $5 \mathrm{x}$ BDR) or $20 \mathrm{mg} / \mathrm{kg}$ (20x BDR) for 4 weeks during either juvenilepubertal (JP) or adult age.

Results: There was a persistent increase in the number of terminal end buds and a decrease in OCT- 4 and BRCA1 gene expression after folic acid supplementation at $20 \mathrm{mg} / \mathrm{kg}$ during the JP period. In contrast folic acid supplementation at $5 \mathrm{mg} / \mathrm{kg}$ during adulthood led to an increase in OCT-4 and BRCA1 gene expression as well as an increase in mammary gland epithelial density.

Conclusion: This data suggests that folic acid supplementation can induce persistent changes in mammary gland morphology and gene expression which are dose and time dependent.

\section{5}

Renal micro-RNAs expression and epithelial-to-mesenchymal transition in adult maternal low protein diet programmed male rats

LB Sene ${ }^{1}$, GJF Garcia ${ }^{2}$, R Carvalho ${ }^{3}$, J Gontijo ${ }^{4}$, PA Boer ${ }^{5}$

${ }^{1}$ Morphology Department, UNESP, Botucatu and Internal Medicine Department, UNICAMP, Campinas, São Paulo, Brazil; ${ }^{2}$ Morphology Department, UNESP, Botucatu and Internal Medicine Department, UNICAMP, Campinas, São Paulo, Brazil; ${ }^{3}$ Morphology Department, UNESP, Botucatu and Internal Medicine Department, UNICAMP, Campinas, São Paulo, Brazil; ${ }^{4}$ Morphology Department, UNESP, Botucatu and Internal Medicine Department, UNICAMP, Campinas, São Paulo, Brazil; ${ }^{5}$ Morphology Department, UNESP, Botucatu and Internal Medicine Department, UNICAMP, Campinas, São Paulo, Brazil

We have demonstrated that adult male rats from gestational protein-restricted mothers have reduced nephron number associated to structural and functional glomerular 
disorders. Additionally, using isolated glomeruli, we found downregulation of miR-200 family and enhanced TGF- $B 1$ and ZEB2 expression that are related to glomeruli epithelial-tomesenchymal transition (EMT) and fibrosis. Now, our objective was analyze the expression of miR-200 family and miR-192, mRNAs and proteins related to EMT in whole renal tissue of 16-wk-old male rats from protein-restricted mothers. During pregnancy Wistar rats were fed with normal (NP 17\% casein) or protein-restricted (LP 6\% casein) diet. The expression profile of miR-200 and miR-192 family and, mRNAs (ZEB1, ZEB2, desmin, fibronectin, ZO-1, E-cadherin, TGF- $\beta 1$, col $1 \mathrm{a} 1$ and col 1a2) were studied by RT-qPCR. Also, renal samples were processed to western blot and immunohistochemistry to mRNAs corresponding proteins. miR-192, miR-141, miR-200c and miR-429 expression were up regulated in LP. TGF- $ß 1$, ZEB1, fibronectin and collagen expression was enhanced whereas e-cadherin expression was reduced in LP. In conclusion, these results indicated the dissociation between glomerular and total kidney tissue analyses and led us to suggest that, in whole renal tissue, miR-200 family and miR-192 expression up-regulation induced TGF- $\beta 1$, ZEB1 and EMT in 16-wk-old male LP offspring.

\section{8}

Stem/progenitor cells and mitosis are reduced in the nephogenic cap of male rat fetus programmed by low protein diet in uteri

\author{
LB Sene ${ }^{1}$, JAR Gontijo ${ }^{2}$, WR Scarano ${ }^{3}$, PA Boer ${ }^{4}$ \\ ${ }^{1}$ Morphology Department, UNESP, Botucatu and Internal \\ Medicine Department, UNICAMP, Campinas, São Paulo, \\ Brazil; ${ }^{2}$ Morphology Department, UNESP, Botucatu and \\ Internal Medicine Department, UNICAMP, Campinas, São \\ Paulo, Brazil; ${ }^{3}$ Morphology Department, UNESP, Botucatu and \\ Internal Medicine Department, UNICAMP, Campinas, São \\ Paulo, Brazil; ${ }^{4}$ Morphology Department, UNESP, Botucatu and \\ Internal Medicine Department, UNICAMP, Campinas, São \\ Paulo, Brazil
}

We have demonstrated, in adult male offspring from dams submitted to gestational protein restriction, a significant reduction $(27 \%)$ of the nephron number. During nephrogenesis, the number of stem cells present in the mesenchymal cap $(\mathrm{CM})$, around of the ureteral bud tip, determines the number of nephrons to be formed and the transcriptional regulator Six2 is a regulator of progenitor self-renewal cap cells. Thus, in the present study we investigate the number of proliferating (Ki67+) and Six2+ cells in metanephros of fetus from mothers submitted or not to protein restriction. Wistar rats were fed during pregnancy with normal-protein (NP 17\% casein) or protein-restricted (LP $6 \%$ casein) diet. In the 17 th gestational day the metanephos from male embryos were processed for immunolocalization analyses. The LP nephrogenic zone was $17 \%$ reduced and Six $2+$ level was $70 \%$ reduced. In the CM from LP metanephros the number of Six $2+$ and mitotic cells was significantly reduced ( $27 \%$ and $34 \%$, respectively). For the first time, we demonstrate that gestational protein restriction causes reduced fetal kidney functional units probably, induced by loss of nephrogenic stem/progenitor cells in the same proportion found in adulthood.

\section{0}

Effects of protein restriction in utero on BNST and Amygdala of rats: study of 3D dendritic structure

Daniele Braz Torres ${ }^{1}$, Agnes da Silva Lopes ${ }^{2}$, Ana João Rodrigues $^{3}$, Ana Paula Silva-ventura ${ }^{4}$, Carina Soares-Cunha ${ }^{5}$, Barbara Coimbra ${ }^{6}$, José Antônio Rocha Gontijo ${ }^{7}$, Nuno Sousa ${ }^{8}$, Patrícia Aline Boer ${ }^{9}$

${ }^{1}$ Department of Internal Medicine School of Medicine, State University of Campinas; ${ }^{2}$ Department of Internal Medicine School of Medicine, State University of Campinas; ${ }^{3}$ Department of Internal Medicine School of Medicine, State University of Campinas; ${ }^{4}$ Department of Internal Medicine School of Medicine, State University of Campinas; ${ }^{5}$ Department of Internal Medicine School of Medicine, State University of Campinas; ${ }^{6}$ Department of Internal Medicine School of Medicine, State University of Campinas; ${ }^{7}$ Department of Internal Medicine School of Medicine, State University of Campinas; ${ }^{8}$ Department of Internal Medicine School of Medicine, State University of Campinas; ${ }^{9}$ Department of Internal Medicine School of Medicine, State University of Campinas

The bed nucleus of the stria terminalis (BNST) and the amygdala are involved in the stress response and are implicated in anxiety and fear response. We studied the possible effects of gestational protein restriction early in development. Male offspring rats subjected in utero protein restriction were compared with appropriated normoprotein intake controls in the general morphological structure of amygdala and BNST at PND7 and PND14. After pregnancy, female Wistar rats were divided into two groups and maintained on isocaloric standard rodent laboratory with normal protein content [NP17\% protein] or low protein content [LP6\% protein] diet ad libitum throughout the entire pregnancy. Both groups returned to the normal diet after the birth. We confirmed that maternal protein restriction caused a significant $10 \%$ reduction in the birth weight of the LP offspring when compared to NP group. This reduction was of $4 \%$ on PND7 and $14 \%$ on PND14. In 3D neuronal reconstruction analysis, we observed a significant reduction in the length of BNST neurons on PND14 p0.0082. In BNST stereology at PND14 we observed a significant anterodorsal volume reduction p0.0477. We conclude that gestational protein restriction leads to morphological changes in the BNST beyond 14th day of life and are perpetuated into adulthood. 
4434

\section{Post-natal low insulin might program lean adult phenotype}

Aline Amenencia de Souza ${ }^{1}$, Kelly Valerio Prates ${ }^{2}$, Julio Cezar de Oliveira ${ }^{3}$, Rosiane Aarecida Miranda ${ }^{4}$, Laize Tófoli Peron ${ }^{5}$, Kesia Palma-Rigo ${ }^{6}$, Ananda Malta ${ }^{7}$

${ }^{1}$ Universidade Estadual de Maringá, Brazil; ${ }^{2}$ Universidade Estadual de Maringá, Brazil; ${ }^{3}$ Federal University of Mato Grosso, Brazil; ${ }^{4}$ Universidade Estadual de Maringá, Brazil;

${ }^{5}$ Universidade Estadual de Maringá, Brazil; ${ }^{6}$ Universidade

Estadual de Maringá, Brazil; ${ }^{7}$ Universidade Estadual de

Maringá, Brazil

Background: In rodents, malnourishment during lactation programs lean adult animals. Knockout of cholinergic-muscarinic receptor subtype M3 showed low insulin secretion and lean phenotype in mice. Whether low insulin levels in lactation are implicated to lean phenotype in adulthood was tested.

Methods: After birth male rat pups, intraperitoneal, were injected anti-muscarinic drug buscopan (N-butyl scopolamine) $0.5 \mathrm{mg} / \mathrm{Kg}$ body weight (bw)/day during the first 12 days of lactation. Controls received saline. Food intake and growth were recorded until 90-days-old. Pancreatic islets were isolated from adult offspring to study insulin secretion response. Vagus nerve electrical activity was registered. M3 muscarinic receptor was evaluated by western-blotting technique. It was also measure leptin levels.

Results: Lean phenotype was observed in early offspring, as well as in adult ones $(\mathrm{p}<0.05)$. Vagus hypoactivity was registered in treated adult rats $(\mathrm{p}<0.05)$. Leptin, insulin levels and tissue fat were reduced in buscopan treated adult rats $(p<0.05)$. Glucose and acetylcholine stimulated low insulin secretion $(\mathrm{p}<0.05)$. Protein expression of M3 was reduced in treated adult rats. When treatment was halted pups with 10, 15 and 21 days of age showed 30\% decrease blood insulin concentration $(\mathrm{p}<0.05)$.

Conclusion: Low insulin during lactation is associated to programming lean adult phenotype.

4453

Is Prenatal Weight Gain associated with Delivery and Child BMI and Allergy Outcomes? Evidence from a US Birth Cohort (WHEALS)

Ganesa Wegienka ${ }^{1}$, Suzanne Havstad ${ }^{2}$, Christine Cole Johnson $^{3}$, Kimberley Woodcroft ${ }^{4}$, Andrea Cassidy-Bushrow ${ }^{5}$

${ }^{1}$ Department of Public Health Sciences, Henry Ford Hospital, Detroit, Michigan, USA; ${ }^{2}$ Department of Public Health Sciences, Henry Ford Hospital, Detroit, Michigan, USA; ${ }^{3}$ Department of Public Health Sciences, Henry Ford Hospital, Detroit, Michigan, USA; ${ }^{4}$ Department of Public Health Sciences, Henry Ford Hospital, Detroit, Michigan, USA; ${ }^{5}$ Department of Public Health Sciences, Henry Ford Hospital, Detroit, Michigan, USA
Research question: Is prenatal weight gain associated with delivery characteristics and the child's early-life BMI and allergic outcomes?

Methods: Height and antenatal weight measurements, delivery and neonatal characteristics were recorded from medical charts of pregnant women enrolled for study of their child in the racially diverse WHEALS birth cohort. Clinic visits at age 2 years assessed child eczema and allergic sensitization (skin prick tests [SPT] and allergen-specific $\operatorname{IgE}[\operatorname{sigE}]$ ). Parental report of asthma diagnosis at age 3-6 years was collected. Maternal weight gain over pregnancy was calculated and assessed for associations with outcomes.

Results: 785 women had at least one BMI measurement in pregnancy. Maternal weight gain was not associated with the following in the child: BMI at age 2 years, eczema, having an elevated $\operatorname{sgE}$, or doctor diagnosis of asthma. Women with a planned c-section had greater weight gain than women who delivered vaginally even after adjustment for baseline BMI $(\mathrm{p}=0.05)$. Prenatal weight gain was positively associated with the child's birthweight $z$-score $(p<0.05)$. Among women obese in the 1 st trimester, higher prenatal weight gain was associated with a lack of SPT positivity $(\mathrm{p}<0.05)$.

\section{0}

Offspring of High-Fat Diet Fed IL-6 Null Mice are Protected Against Early-Onset Overweight and Metabolic Programming of Pro-Asthmatic Phenotype

Katharina Dinger ${ }^{1}$, Philipp Kasper ${ }^{2}$, Christina Vohlen ${ }^{3}$, Eva Hucklenbruch-Rother ${ }^{4}$, Ruth Janoschek ${ }^{5}$, Inga Bae-Gartz ${ }^{6}$, Sarah Appel ${ }^{7}$, Jörg Dötsch ${ }^{8}$, Miguel Angel Alejandre Alcazar?

${ }^{1}$ Department of Pediatric and Adolescent Medicine, University of Cologne, Germany; ${ }^{2}$ Department of Pediatric and Adolescent Medicine, University of Cologne, Germany; ${ }^{3}$ Department of Pediatric and Adolescent Medicine, University of Cologne, Germany; ${ }^{4}$ Department of Pediatric and Adolescent Medicine, University of Cologne, Germany; ${ }^{5}$ Department of Pediatric and Adolescent Medicine, University of Cologne, Germany; ${ }^{6}$ Department of Pediatric and Adolescent Medicine, University of Cologne, Germany; ${ }^{7}$ Department of Pediatric and Adolescent Medicine, University of Cologne, Germany; ${ }^{8}$ Department of Pediatric and Adolescent Medicine, University of Cologne, Germany; ${ }^{9}$ Department of Pediatric and Adolescent Medicine, University of Cologne, Germany

Research question: Early-onset overweight induced by maternal obesity has been associated with bronchial asthma, but the mechanistic link remains unclear. As low-grade inflammation and interleukin-6 (IL-6), an adipocytokine, are important in lung diseases, we hypothesized that 1) IL-6 is linked to bronchial asthma following early-onset overweight, and 2) offspring of IL-6 null mice (IL-6-/-) are protected from this metabolic programming. 
Methods: Early-onset overweight in the offspring was induced in wildtype and IL-6-/- dams by high-fat diet (HFD), controls were fed standard chow. Intraperitoneal glucose tolerance was assessed at postnatal day (P) 21 and P70 and serum, lungs and white adipose tissue (WAT) were obtained. Some mice underwent whole-body-plethysmography at P70.

Results: The HFD group exhibited an accelerated body weight gain, increased WAT, impaired glucose tolerance and active intrinsic-pulmonary IL-6 signalling at P21, but not at P70 compared to Control. Moreover, assessment of lung morphometry showed a three-fold thicker bronchial smooth muscle layer and a two-fold higher airway resistance in the HFD than in the Control at P70. The offspring of IL-6-/- were protected from those changes.

Conclusion: Our study identified IL-6 as a novel mediator of metabolic programming of a pro-asthmatic phenotype and defines thereby a potential preventive strategy of obesityprogrammed Asthma.

\section{6}

Evidence of early life programming of the melancortin- 4 receptor in hypertension arising from maternal obesity

Amandine Mullier ${ }^{1}$, Nuria Macais Blasco ${ }^{2}$, Tatiana Novoselova ${ }^{3}$, Li Chan ${ }^{4}$, Joaquim M. Pombo ${ }^{5}$, Clive W. Coen ${ }^{6}$, Nina Balthasar ${ }^{7}$, Lucilla Poston ${ }^{8}$

${ }^{1}$ Division of Women's Health \& Women's Health Academic Centre, Faculty of Life Sciences \& Medicine, King's College London, London, UK; ${ }^{2}$ Division of Women's Health \& Women's Health Academic Centre, Faculty of Life Sciences \& Medicine, King's College London; ${ }^{3}$ Centre for Endocrinology, William Harvey Research Institute, St Mary Hospital, UK; ${ }^{4}$ Centre for Endocrinology, William Harvey Research Institute, St Mary Hospital, UK; ${ }^{5}$ Division of Women's Health \& Women's Health Academic Centre, Faculty of Life Sciences \& Medicine, King's College London; 'Division of Women's Health \& Women's Health Academic Centre, Faculty of Life Sciences \& Medicine, King's College London; ${ }^{7}$ School of Physiology and Pharmacology, University of Bristol, Bristol, UK; ${ }^{8}$ Division of Women's Health \& Women's Health Academic Centre, Faculty of Life Sciences \& Medicine, King's College London

Research question: Leptin acting on the hypothalamus (paraventricular nucleus, PVN) has been implicated in the pathogenesis of obesity-induced hypertension. The aim of this study was to determine if similar pathways are involved in early onset hypertension secondary to maternal obesity.

Methods: Transgenic mice re-expressing Mc4r in the PVN (Mc4rPVN) were compared with global Mc4r knockouts (Mc4rKO) in a model of maternal obesity. Female heterozygous (+/-) mice were rendered obese using an obesogenic diet [20\% LARD] supplemented with sweetened condensed milk and compared with control mothers. Offspring were weaned onto standard chow and ear tissue collected for genotyping. At 6 months of age, metabolic and cardiovascular parameters were analysed before and after leptin $(10 \mathrm{mg} / \mathrm{kg})$ and MTII $(80 \mu \mathrm{g}$ Mc4r agonist) challenges.

Results: Offspring of obese dams (OffOb) showed sympathetic mediated hypertension with a heightened pressure response to leptin in WT $(\mathrm{P}<0.05)$ and Mc4rPVN $(\mathrm{P}<0.001)$ mice, whereas the obese Mc4rKO remained normotensive and nonleptin responsive. MTII challenge in WT and MC4rPVN reduced food intake by $30-40 \%$ but remained unaltered in Mc4rKO. PVN Mc4r is thus sufficient to mediate MTII anorexic effect.

Conclusions: Mc4r in the PVN mediate the leptin-induced hyperphagia and hypertension arising from maternal obesity (British Heart Foundation, Tommy's).

\section{1}

Ethnic differences in the Cortisol Axis in early pregnancy The PRiDE-HPA study - Protocol and baseline characteristics

Hema Venkataraman ${ }^{1}$, Amitha Gopinath ${ }^{2}$, Gail Pounder ${ }^{3}$, Rebecca Reynolds ${ }^{4}$, Ponnusamy Saravanan ${ }^{5}$

${ }^{1}$ University of Warwick; ${ }^{2}$ George Eliot Hospital; ${ }^{3}$ George Eliot Hospital; ${ }^{4}$ University of Edinburgh; ${ }^{5}$ University of Warwick

Research question: Cortisol is closely linked to central adiposity, metabolic syndrome and low birth weight, all classical features in South Asians (SA).

1. Do South Asians (SA) have higher cortisol exposure compared to White Caucasians (WC)?

2. Does cortisol behaviour predict insulin resistance?

Methods: The PRiDE-HPA is a substudy of the PRiDE study, a multicentre high-risk pregnancy cohort.

Inclusion criteria: women of SA or WC from the PRiDE study. Exclusion criteria: Topical, oral, inhaled steroids $<3$ months prior to recruitment, multiple pregnancy.

Maternal data, anthropometry, blood are obtained with timed salivary collections and a 24 hour urine. Saliva is collected at waking, $30 \mathrm{~min}$ after, $4 \mathrm{pm}$ and bedtime twice in pregnancy. At oral glucose tolerance test (OGTT) blood samples are obtained half hourly to determine insulin-resistance.

Results: 102 women (52 SA, 52 WC) between Nov2014-Apr2015. 94 samples of 24 hour Urine and saliva collections were obtained. Mean(SD) of WC vs SA was Age:29.0(5.3) vs 31.2(5.2), BMI:29.6(6.8) vs 24.9(4.2), Subscapular skinfold thickness:28.2(9.1) vs 22.3(9.0) $(\mathrm{p}<0.02$ for all). Salivary and Urinary cortisol analysis is pending.

Conclusion: This is the first study assessing diurnal cortisol patterns in SA and in pregnancy. The study will be completed in July-2015 with results available in Sep-2015. 


\section{6}

Interaction between maternal weight in pregnancy and offspring birth weight on physical activity in late adulthood: the Helsinki birth cohort study

Niko Wasenius ${ }^{1}$, Minna Salonen ${ }^{2}$, Mikaela von Bonsdorff ${ }^{3}$, Eero Kajantie $^{4}$, Johan G. Eriksson ${ }^{5}$

${ }^{1}$ Department of General Practice and Primary Health Care, University of Helsinki, Finland; ${ }^{2}$ Folkhälsan Research Center, Helsinki, Finland; Department of Health, National Institute for Health and Welfare, Helsinki, Finland; ${ }^{3}$ Gerontology Research Center and Department of Health Sciences, University of Jyväskylä, Jyväskylä, Finland; ${ }^{4}$ Folkhälsan Research Center, Helsinki, Finland; Children's Hospital, Helsinki University Central Hospital, University of Helsinki, Finland; Department of Obstetrics and Gynaecology; ${ }^{5}$ Department of General Practice and Primary Health Care, University of Helsinki, Finland; Folkhälsan Research Center, Helsinki, Finland; Department of Health, National Institute for Health and Welfare, Helsinki, Finland

Research question: We investigated the interaction between maternal body mass index (BMI) and offspring birth weight on physical activity (PA) in late adulthood.

Methods: Objective total PA (SenseWear Armband $囚$ ) was measured in 2011-2013 in a total of 712 subjects born 1934-44. PA was expressed in metabolic equivalents of task (MET). Detailed maternal pregnancy BMI and birth weight information were collected from hospital birth records. Maternal BMI was dichotomized into normal BMI $\left(<25 \mathrm{~kg} / \mathrm{m}^{2}\right)$ and high BMI $\left(\geqslant 25 \mathrm{~kg} / \mathrm{m}^{2}\right)$.

Results: Age adjusted linear regression analysis revealed a significant interaction between maternal BMI and birth weight on volume of PA in late adulthood in men $(p=0.025)$ but not in women $(p=0.676)$. In analyses stratified with maternal BMI, a $1 \mathrm{~kg}$ increase in birth weight was associated with decrease of 1.3 MET-hours/day (95\% confidence interval -2.4, -0.2) in PA among males born to mother with high BMI. Adjustment for gestational age, childhood socioeconomic status, and adulthood body fat percentage did not attenuate the association. No significant association was found among males born to normal weight mothers.

Conclusion: High maternal BMI and birth weight are associated with decreased volume of total daily physical activity in late adulthood in men but not in women.

\section{6}

Fetal size and growth and the association with parental anthropometry in a multi-ethnic population

Line Sletner ${ }^{1}$, Anne Karen Jenum ${ }^{2}$, Svein Rasmussen ${ }^{3}$, Siri Vangen ${ }^{4}$

${ }^{1}$ Department of Pediatric and Adolescents Medicine, Akershus University Hospital, P.O. Box 1000, 1478 Lorenskog, Norway;

${ }^{2}$ Department of General Practice, Institute of Health and Society, University of Oslo, Oslo, Norway; ${ }^{3}$ Department of Clinical Science, University of Bergen, Bergen, Norway; ${ }^{4}$ Norwegian National
Advisory Unit on Women's Health, Department for Woman and Child Health, Oslo University Hospital, Oslo, Norway

Aims: To explore the associations between maternal and paternal height and BMI and fetal size from mid pregnancy until birth in a multi-ethnic population.

Material and methods: The sample is 509 parent/offspring triplets with South Asian $(n=111)$, Middle East/NorthAfrican $(n=86)$ or European $(n=301)$ ethnic origin, from a population-based, prospective cohort in Oslo, Norway. Associations between parental height and BMI, and z-scores of estimated fetal weight (EFW), head circumference, abdominal circumference and femur length (FL), in gestational week 24, 32 and 37 and at birth were analysed using separate General Linear Models, adjusting for other covariates.

Results: In week 24 paternal height was significantly associated with fetal EFW (for every $10 \mathrm{~cm}$ increase in paternal height fetal z-score increased by $0.19 \mathrm{SD}(0.05,0.33), \mathrm{p}=0.008)$, and all other fetal measures. Maternal height was only associated with fetal FL, and not with EFW (0.04 SD $(-0.13,0.20)$, $\mathrm{p}=0.6$ ). However, the impact of maternal height and BMI increased throughout pregnancy, but was stable for paternal anthropometry. The effect on ethnic differences of adjusting for parental anthropometry was most pronounced at birth, as both parents contributed.

Conclusions: Associations between fetal size and paternal height were present earlier in gestation than associations with maternal height and BMI.

\section{1}

Preconception maternal helminth infection influences offspring gut microbiome and immunity

Donald Nyangahu ${ }^{1}$, Heather Jaspan ${ }^{2}$, William Horsnell ${ }^{3}$, Matthew Darby ${ }^{4}$, Jerome Wendoh ${ }^{5}$, Enock Havyarimana ${ }^{6}$, Katie Viljoen ${ }^{7}$

${ }^{1}$ University of Cape Town; ${ }^{2}$ University of Cape Town, Seattle Children's Research Institute; ${ }^{3}$ University of Cape Town;

${ }^{4}$ University of Cape Town; ${ }^{5}$ University of Cape Town;

${ }^{6}$ University of Cape Town; ${ }^{7}$ University of Cape Town

Background and Research question: The early life intestinal microbiome is pivotal in determining the immunological status of the mammalian host. Helminths potentially interact with commensal bacteria in the intestine. We sought to determine how helminth-bacterial interactions prior to conception may alter offspring gut microbiome and immunity.

Methods: 6-week-old female BALB/c mice were infected with Nippostrongylus brasiliensis, treated with ivermectin after 7 days, and then mated three weeks post infection. Pups were killed two weeks postpartum and mucosal immunity analysed by Flow Cytometry. Fecal bacterial DNA was extracted and the $16 \mathrm{~S}$ gene, V6 region amplified. Deep sequencing was performed on the Illumina HiSeq platform. Sequence data was 
pre-processed in QIIME and UPARSE and imported into R for downstream analysis.

Results: Significant increases were observed in relative abundance of enterobacteriaceae and peptococcaceae families in offspring born to helminth-infected mothers compared to naïve controls (FDR adj $\mathrm{P}=0.039$ and 0.041 respectively). In addition, certain clostridia OTUs were significantly increased in pups born to infected dams. Further, we observed significant increases in total $\mathrm{T}$ and $\mathrm{B}$ lymphocytes, as well as specific subsets, in offspring born to infected mothers.

Conclusions: Our data shows for the first time that helminth driven alteration of maternal microbiome prior to pregnancy affects infant immunity.

\section{8}

Lower maternal concentrations of nicotinamide and related metabolites in late pregnancy are associated with offspring atopic eczema at age 12 months

Sarah El-Heis ${ }^{1}$, Sarah Crozier ${ }^{2}$, Sian Robinson ${ }^{3}$, Nick Harvey ${ }^{4}$, Cyrus Cooper ${ }^{5}$, Hazel Inskip ${ }^{6}$, Keith Godfrey ${ }^{7}$

${ }^{1} M R C$ Lifecourse Epidemiology Unit, University of Southampton; ${ }^{2} M R C$ Lifecourse Epidemiology Unit, University of Southampton; ${ }^{3} M R C$ Lifecourse Epidemiology Unit, University of Southampton; ${ }^{4} M R C$ Lifecourse Epidemiology Unit, University of Southampton; ${ }^{5} M R C$ Lifecourse Epidemiology Unit, University of Southampton; ${ }^{6} M R C$ Lifecourse Epidemiology Unit, University of Southampton; ${ }^{7}$ MRC Lifecourse Epidemiology Unit, University of Southampton

Research question: Topical nicotinamide has been reported to improve aspects of atopic eczema; we examined the relation of maternal serum concentrations of nicotinamide and related tryptophan metabolites to the risk of atopic eczema in the offspring.

Methods: Within the UK Southampton Women Survey, infantile atopic eczema at ages 6 and 12 months was ascertained (UK Working Party Criteria for the Definition of Atopic Dermatitis). Maternal serum levels of kynurenine, kynurenic acid, anthranilic acid, tryptophan, nicotinamide and N1methylnicotinamide were measured in late pregnancy (mass spectrometry, $\mathrm{n}=495$ ) and related to the odds ratio of infantile atopic eczema.

Results: Maternal nicotinamide and related metabolite concentrations were not associated with offspring atopic eczema age 6 months. Higher late pregnancy maternal concentrations of nicotinamide were, however, associated with a lower incidence of offspring eczema at age 12 months (odds ratio 0.69, 95\% CI 0.53-0.91 per SD change, $\mathrm{p}=0.007$ ); associations were similar for anthranilic acid $(\mathrm{p}=0.001)$ and were robust to adjustment for sex and potentially confounding maternal variables.

Conclusion: This is the first study linking maternal serum levels of nicotinamide and related metabolites to the risk of atopic eczema in the offspring. The findings point to potentially modifiable maternal influences on this complex, multifactorial condition.

\section{6}

The importance of the first $\mathbf{1 0 0 0}$ days - setting trajectories for future population wellbeing. Evidence from Growing Up in New Zealand

Sarah Berry ${ }^{1}$, Polly Atatoa Carr ${ }^{2}$

${ }^{1}$ University of Auckland; ${ }^{2}$ University of Auckland

Growing Up in New Zealand is the country's largest and most comprehensive longitudinal study, following the lives of nearly 7000 children born in 2009 and 2010 from before birth until they reach adulthood. This presentation will describe how these children, who represent the ethnic and socioeconomic diversity of the contemporary NZ preschool population, are developing throughout their critical first 1000 days of life when the foundations of so many later life health and wellbeing pathways are being established.

Patterns of early nutrition, growth and cognitive development will be detailed for the ethnically diverse cohort from maternal nutrition in pregnancy to children's dietary patterns and behaviours over their first 1000 days. The rich diversity of their families and the environments the children are growing up in will also be described as well as the relevance and effectiveness of current policies designed to enhance child wellbeing.

New Zealand's record in child wellbeing is very poor internationally, so this longitudinal study is explicitly designed to provide evidence to inform innovative, cross-sectoral solutions to improve wellbeing for all NZ children. While some toddlers have had a healthy start to life it is clear that we could do more for children who are most vulnerable.

\section{4}

Relationship of maternal hormones with glucose levels and Beta-cell function of the offspring in the neonatal and early postnatal periods

Marketa Toman ${ }^{1}$, Nigel John Crowther ${ }^{2}$, Franco Guidozzi ${ }^{3}$, Paul Rheeder ${ }^{4}$

${ }^{1}$ National Health Laboratory Service (NHLS) - Wits University, Johannesburg, South Africa; ${ }^{2}$ NHLS-Wits University,

Johannesburg, South Africa; ${ }^{3}$ Wits University, Johannesburg,

South Africa; ${ }^{4}$ University of Pretoria, South Africa

Research question: Do maternal glucose and hormone levels affect neonatal or early postnatal glucose tolerance and beta-cell function in the offspring of pregnant, healthy urban African women?

Methods: The study included 78 Black pregnant women from the Charlotte Maxeke Johannesburg Academic Hospital 
Antenatal Clinic. Maternal anthropometry and blood samples were collected at 20-24 (V1) and 30-36 (V2) gestational weeks whilst at 3-4 weeks (neonate) and 5-7 months (infant) data was collected from the offspring. Maternal samples were analysed for fasting glucose, placental lactogen (HPL), thyroid hormone (FT4), insulin, proinsulin, IGF-1 and cortisol. Offspring blood samples were analysed for fasting glucose, insulin and proinsulin. Results: Maternal HPL from both V1 and V2 correlated significantly with the infants' fasting glucose levels (beta = $0.40, \mathrm{p}=0.001$ and beta $=0.25, \mathrm{p}=0.034$ respectively). Maternal cortisol at V2 correlated positively with neonatal fasting glucose (beta $=0.37, p=0.001$ ), whilst maternal FT4 at the same time point correlated negatively (beta $=-0.33$, $p=0.003)$. A strong inverse relationship was found between maternal FT4 at V1 and neonatal fasting insulin level (beta = $0.37, \mathrm{p}=0.001$ ) and HOMA-IR index (beta $=-0.42$, $\mathrm{p}<0.001)$.

Conclusion: The outcomes of this study suggest an involvement of maternal hormones in neonatal and infant glucose tolerance and beta-cell function.

\section{7}

Relationship between maternal glucose and hormone levels and neonatal size in the offspring of healthy African urban pregnant women

Marketa Toman ${ }^{1}$, Franco Guidozzi ${ }^{2}$, Nigel John Crowther ${ }^{3}$

${ }^{1}$ National Health Laboratory Service - University of the

Witwatersrand, Johannesburg, South Africa; ${ }^{2}$ Wits University;

${ }^{3}$ NHLS-Wits University, Johannesburg, South Africa

Research question: We investigated whether neonatal size, expressed as weight/length (W/L) z-scores, was linked to maternal glucose or hormone levels.

Methods: Seventy eight healthy Black pregnant women attending the Charlotte Maxeke Johannesburg Academic Hospital Antenatal Clinic were investigated in their second (V1) and third (V2) trimester. Anthropometric measurements and a standard 75gOGTT were undertaken. Maternal glucose, insulin, proinsulin, C-peptide, IGF-1, human placental lactogen, thyroid hormones, cortisol and leptin were analysed. The neonatal weight and length were assessed at 3-4 weeks and the W/L z-scores were derived based on the WHO growth charts.

Results: Mothers of neonates with higher W/L ratio showed significantly lower fasting glucose $(\mathrm{p}=0.013$ at $\mathrm{V} 1 ; \mathrm{p}=0.004$ at V2), lower fasting $(p=0.0003, V 1 ; p=0.0002, V 2)$ and postprandial $(\mathrm{p}=0.0002, \mathrm{~V} 1 ; \mathrm{p}=0.0002, \mathrm{~V} 2)$ proinsulin levels, lower fasting $(\mathrm{p}=0.028, \mathrm{~V} 1 ; \mathrm{p}=0.024, \mathrm{~V} 2)$ and postprandial $(\mathrm{p}=0.002, \mathrm{~V} 1 ; \mathrm{p}=0.002, \mathrm{~V} 2) \mathrm{PI}$ to insulin ratio and lower insulin resistance at V1 $(p=0.003)$.

Conclusion: Disproportionate fetal growth is characterised by low glucose levels, more efficient proinsulin processing and greater insulin sensitivity in the mothers.
5012

Relationship of flow of uterine artery in the second trimester of pregnancy with neurodevelopment the second year of life

Ricardo Cavalli ${ }^{1}$, Marcos Okido ${ }^{2}$, Marco Barbieri $^{3}$, Heloisa Bettiol $^{4}$, Cristina Del Ben ${ }^{5}$, Viviane Cardoso ${ }^{6}$

${ }^{1}$ Department of Gynecology and Obstetric, Ribeirão Preto Medical School, University of São Paulo, Brazil; ${ }^{2}$ Department of Gynecology and Obstetric, Ribeirão Preto Medical School, University of São Paulo, Brazil; ${ }^{3}$ Department of Pediatrics, Ribeirão Preto Medical School, University of São Paulo, Brazil;

${ }^{4}$ Department of Pediatrics, Ribeirão Preto Medical School, University of São Paulo, Brazil; ${ }^{5}$ Department Neurosciences and Behavioral Sciences, Ribeirão Preto Medical School, University of São Paulo, Brazil; ' Department of Pediatrics, Ribeirāo Preto Medical School, University of São Paulo, Brazil

Research question: To evaluate the relationship of the pulsatility index of the uterine artery in the second trimester of pregnancy with the neurodevelopment of children in the second year of life.

Methods: A cohort study was performed involving 1370 women with singleton pregnancies. Pulsatility index of the uterine artery between 20 and 25 weeks was obtained. The psychomotor development of the children was evaluated in the second year of life, the Bayley Scales of Infant and Toddler Development Third Edition.

Results: The average pulsatility index of the uterine artery was 0.89 and 1.47 for the 95 th percentile. Evaluation of children, showed no increased risk associated with delay in pulsatility index of uterine artery greater than or equal to the 95 th percentile development (cognitive performance: RR 0.76, 95\% CI 0.3 to 1.96 , receptive communication: RR $1.17,95 \%$ CI 0.46 to 3.01, expressive communication: RR $0.66,95 \%$ CI 00.67 to 1,36 , fine motor: RR $1.26,95 \%$ CI 0.5 to 3.27 , gross motor: RR $1.1495 \%$ CI 0.44 to 2.94 ).

Conclusions: The high flow resistance of the uterine artery in the second quarter, without deleting other perinatal factors, cannot be considered as a risk factor for the delay in child development.

\section{4}

Adaptation of the Uterine Vasculature after Chronic Maternal Stress (CMS) During Late Pregnancy Protects the Fetus from Acute Stress Effects

Rakers Florian $^{1}$, Haase Michelle ${ }^{2}$, Sabine Bischoff ${ }^{3}$, Rene Schiffner ${ }^{4}$, Rupprecht Sven ${ }^{5}$, Schubert Harald ${ }^{6}$, Nathanielsz Peter $^{7}$, Matthias Schwab ${ }^{8}$

${ }^{1}$ Department of Neurology, Jena University Hospital, Jena, Germany; ${ }^{2}$ Department of Neurology, Jena University Hospital, Jena, Germany; ${ }^{3}$ Institute of Lab Animal Sciences, Jena University Hospital, Jena, Germany; ${ }^{4}$ Department of Neurology, Jena University Hospital, Jena, Germany; ${ }^{5}$ Department of Neurology, 
Jena University Hospital, Jena, Germany; 'Institute of Lab Animal Sciences, Jena University Hospital, Jena, Germany; ${ }^{7}$ Department of Animal Science, University of Wyoming, USA; ${ }^{8}$ Institute of Lab Animal Sciences, Jena University Hospital, Jena, Germany

Research question: Does CMS during late pregnancy aggravate fetal responses to acute maternal stress?

Methods: Six pregnant sheep (CMS group) underwent repeated isolation stress between 0.66 and 0.8 gestation for $3 \mathrm{~h}$ twice a week. Six pregnant ewes functioned as controls. Fetuses were chronically instrumented and an ultrasound flow probe was placed around the uterine artery. After recovery, ewes underwent acute isolation stress for $2 \mathrm{~h}$.

Results: Control ewes responded to isolation stress with an increase in maternal blood pressure (MBP), heart rate, cortisol and norepinephrine (NE) concentrations and a decrease in UBF $(\mathrm{P}<0.05)$. Following CMS, MBP increase was more prolonged (12 vs. $120 \mathrm{~min}$ ) but UBF decrease was shorter (6 vs. $32 \mathrm{~min})$ reaching the same nadir $(\mathrm{P}<0.05)$.

Fetal cortisol transiently increased twofold over a maximal of $60 \mathrm{~min}$ in both groups $(\mathrm{P}<0.05)$. Controls but not $\mathrm{CMS}$ fetuses showed a prolonged increase of NE from $4.91 .0 \mathrm{nmol}$. L-1 to 8.61.4 nmol.L-1 and a metabolic shift towards an anaerobic state over $2 \mathrm{~h}(\mathrm{P}<0.05)$.

Conclusion: CMS during late pregnancy decreases sensitivity of uterine vasculature to maternal catecholamines. This mechanism prevents prolonged metabolic effects of maternal stress in the fetus.

\section{4}

Fetal programming by maternal smoking exposure: kidney structure and function in adult offspring

DB Block ${ }^{1}$, FF Mesquita ${ }^{2}$, IP de Lima ${ }^{3}$, PA Boer $^{4}$, JA Gontijo ${ }^{5}$

${ }^{1}$ Department of Internal Medicine School of Medicine, State

University of Campinas; ${ }^{2}$ Department of Internal Medicine School of Medicine, State University of Campinas; ${ }^{3}$ Department of Internal Medicine School of Medicine, State University of Campinas; ${ }^{4}$ Department of Internal Medicine School of Medicine, State University of Campinas; ${ }^{5}$ Department of Internal Medicine School of Medicine, State University of Campinas

Maternal smoking leads to a decreased offspring's birth weight but the specific adverse mechanisms in association with renal morphology and function are unclear. In this study we investigate, in adult male rats, the effect of gestational smoking exposure (Sk) on kidney morphology and function in parallel to blood pressure evaluation. A group of pregnant rats was submitted to $\mathrm{Sk}$ and other not (Co). The 16-week old male rats were submitted to blood pressure (BP) measurement and lithium and creatinine clearance. The kidneys are collected and processed to stereological, histological and immunohistochemical techniques. Sk male offspring presented significant reduction in birth body mass. Beyond 13 weeks of age, the Sk offspring showed an increased systolic BP from (Sk: $130.5 \pm 2.43$ vs. $130.7 \pm 2.43 \mathrm{mmHg}$ in $\mathrm{Co}$ at 5 th week of age), to (Sk: 146.13 .5 vs. $134.23 .01 \mathrm{mmHg}$ in Co, at $13 \mathrm{nd}$ week of age; $p=0.0001)$. Sk rats also presented reduced urinary sodium excretion and higher TGF- $\beta 1$, collagen and fibronectin deposition intrinsically related to fibrotic process. Although the precise mechanism responsible for the renal response in Sk offspring is incompletely known, the current data suggest that the excess of renal sodium reabsorption and fibrotic process might potentiate the programming of adult hypertension.

\section{9}

Early protein restriction alters behavior and hippocampal cellular phenotype that are recovered by enriched environment exposure

Gabriel Boer Grigoletti ${ }^{1}$, Agnes da Silva Lopes ${ }^{2}$, Patrícia Aline Boer $^{3}$, José Antônio Rocha Gontijo ${ }^{4}$

${ }^{1}$ Department of Internal Medicine School of Medicine, State

University of Campinas; ${ }^{2}$ Department of Internal Medicine School of Medicine, State University of Campinas; ${ }^{3}$ Department of Internal Medicine School of Medicine, State University of Campinas; ${ }^{4}$ Department of Internal Medicine School of Medicine, State University of Campinas

\section{5}

Bromocriptine treatment to rats pups at the beginning of lactation programs for thyroid dysfunction at adulthood

Janaina Carvalho $^{1}$, Elaine Oliveira ${ }^{2}$, Patricia Lisboa ${ }^{3}$

${ }^{1}$ State University of Rio de Janeiro; ${ }^{2}$ State University of Rio de Janeiro; ${ }^{3}$ State University of Rio de Janeiro

Research question: Maternal treatment with bromocriptine (BRO, a dopaminergic agonist) at the end of lactation, used to decrease milk production, programmed for hypothyroidism in the adult offspring. Since BRO is transferred through the milk, we evaluated its direct effects on the pups.

Methods: Male offspring were treated with BRO $(0.1 \mathrm{mg} /$ once daily) or vehicle from first to tenth day of lactation. Body mass, food intake, thyroid function and prolactinemia were analyzed. Offspring were killed at 180 days (adults).

Results: At 180 days-old, BRO animals presented normal body weight and visceral adiposity but lower food intake $(-5 \%)$, hypoprolactinemia $(-66 \%)$, lower content of hypothalamic TRH $(-58 \%)$, pituitary TSH $(-32 \%)$, plasma T3 $(-30 \%)$, UCP-1 (-34\%) expression in BAT as well as lower mGPD $(-81 \%)$ activity and type 1 deiodinase (Dio1) expression $(-49 \%)$ in the liver. No changes were detected in the expressions of Dio2 or thyroid hormone receptors.

Conclusion: For the first time, we demonstrated that neonatal BRO treatment programs to hypothalamic hypothyroidism in the adult offspring, suggesting that imprinting factors that increase the dopaminergic tonus in this period can have 
deleterious consequences during development, maybe caused by a permanent alteration in dopaminergic pathways.

\section{5}

Pup Morphometry as a Pointer of Fructose-Induced Foetal Programming

Opeyemi Akindele ${ }^{1}$, Yinusa Raji ${ }^{2}$

${ }^{1}$ University of Ibadan; ${ }^{2}$ University of Ibadan

Research question: In developing countries, resources for analysing foetal and neonatal health are seldom accessible. We evaluated changes in pup and placental morphometry in term foetuses of fructose fed (FRUCTOSE) dams as a detection tool for foetal programming.

Methods: Twenty pregnant Wistar rats were assigned into two groups from gestation day (GD) 1-21; CONTROL and FRUCTOSE (received 10\% fructose solution as daily drinking water). Dams were sacrificed on GD21 and random blood glucose level was measured. Pups and placentas were extracted from the uterus and weighed. Head diameter, abdominal diameter, crown-to-rump length and anogenital distance of each pup; thickness and diameter of each placenta were measured using a digital Vernier Caliper. Data were expressed as mean \pm SEM and analysed using Student's t-test. $p \leqslant 0.05$ was considered statistically significant.

Results: FRUCTOSE offspring showed increased body weight; head circumference-to-abdominal circumference, waist-to-height, weight-to-height and foetal weight-toplacental weight ratios. Anogenital distance index was reduced in both male and female offspring.

Conclusion: Fructose consumption during pregnancy produces macrosomic offspring with shorter anogenital distances. Foetal morphometry can thus be used to detect offspring of fructose fed mothers who have been programmed to develop cardiometabolic and reproductive disorders and consequently promote early interventions to protect the health of future generations.

\section{0}

Impact of maternal sleep deprivation on reproductive functions in male offspring of Wistar rats

Opeyemi Akindele ${ }^{1}$, Olufadekemi Kunle-Alabi ${ }^{2}$, Yinusa Raji ${ }^{3}$

${ }^{1}$ Reproductive Physiology and Developmental Programming Unit, University of Ibadan; ${ }^{2}$ Reproductive Physiology and Developmental Programming Unit, University of Ibadan;

${ }^{3}$ Reproductive Physiology and Developmental Programming Unit, University of Ibadan

Research question: The impact of sleep deprivation at different stages of gestation on reproductive function in male offspring of Wistar rats was assessed.
Methods: Thirty pregnant rats were assigned into three groups $(\mathrm{n}=10)$ from GD 1-7, 8-14 and 15-21. Half of each group $(n=5)$ was sleep deprived (SD) using the modified multiple platform technique. The other half served as control (C). Dams littered naturally. Birth weight and anogenital distance were taken on postnatal day 1. Testes descent and preputial separation were monitored. At 6 months postnatal life, serum gonadotropin, testosterone, corticosterone and melatonin levels; sperm characteristics and gonadal histology were assessed. Data were summarized as mean \pm SEM and analyzed using Student's t-test. Differences in means were based on $95 \%$ confidence interval.

Results: Serum corticosterone was increased in GD1-7SD compared with GD1-7C. GD8-14SD had increased serum corticosterone and decreased serum melatonin levels compared with GD8-14C. GD15-21SD had increased corticosterone, reduced testosterone, birth weight, sperm motility, sperm count and delayed testes descent compared with GD15-21C. Testes and epididymes of GD15-21SD showed poor architecture.

Conclusion: These findings suggest that maternal sleep deprivation is detrimental to male reproductive system development particularly during gestation days 15-21 in Wistar rats.

\section{8}

Maternal Exposure to Dexamethasone during Pregnancy and Lactation Programmes Reproductive Functions in Male Offspring of Wistar Rats

Yinusa Raji ${ }^{1}$, Sikirullai O Jeje ${ }^{2}$

${ }^{1}$ University of Ibadan, Nigeria; ${ }^{2}$ University of Ibadan

Research question: Early exposure to glucocorticoids retards growth and alters serum corticosterone level. Excess glucocorticoids in males may inhibit gonadotropin secretion leading to subnormal testosterone secretion. The effects of maternal exposure to dexamethasone (DEX) on reproductive functions of male offspring in Wistar rats were therefore investigated.

Methods: Study 1 (gestation): Twenty-five pregnant rats divided into five groups $(\mathrm{n}=5)$ received $100 \mu \mathrm{g} / \mathrm{kg} /$ day DEX on gestation day (GD) 1-7, 8-14, 15-21 and 1-21 respectively. Study 2 (lactation): Twenty lactating rats divided into four groups $(\mathrm{n}=5)$ received $100 \mu \mathrm{g} / \mathrm{kg} /$ day DEX on postnatal day (PND) 1-7, 1-14 and 1-21 respectively. The control groups received $0.02 \mathrm{ml} / 100 \mathrm{~g} /$ day normal saline. DEX and saline were administered subcutaneously. Preputial separation was monitored in male offspring which were sacrificed on PND90 for serum testosterone, gonadotropins and corticosterone measurement. Sperm characteristics were analyzed. Data were expressed as mean \pm SEM and analyzed using ANOVA at $\mathrm{p}<0.05$.

Results: Onset of puberty was delayed and sperm characteristics were adversely altered in male offspring of DEX-treated dams. LH, FSH and testosterone levels were significantly 
reduced while GnRH and corticosterone levels were increased in these pups.

Conclusion: Maternal dexamethasone exposure delays puberty and alters sperm functions in male offspring. These programming effects may be mediated via the hypothalamic-pituitarygonadal axis.

\section{1}

Late pregnancy malnourishment programs rat adult offspring to high sensitivity caloric abundance

Júlio Cezar de Oliveira ${ }^{1}$, Audrei Pavanello ${ }^{2}$, Carina Previate ${ }^{3}$, Isabela Peixoto Martins ${ }^{4}$, Tatiane Aparecida da Silva Ribeiro ${ }^{5}$, Paulo Cezar de Freitas Mathias $^{6}$, Ananda Malta ${ }^{7}$

${ }^{1}$ Department of Physiology, Federal University of Mato Grosso(Sinop/MT, Brazil); ${ }^{2}$ Laboratory of Secretion Cell Biology, Department of Cell Biology and Genetics, State University of Maringá - (MaringálPR, Brazil); ${ }^{3}$ Laboratory of Secretion Cell Biology, Department of Cell Biology and Genetics, State University of Maringá-(MaringálPR, Brazil); ${ }^{4}$ Laboratory of Secretion Cell Biology, Department of Cell Biology and Genetics, State University of Maringá-(MaringálPR, Brazil); ${ }^{5}$ Laboratory of Secretion Cell Biology, Department of Cell Biology and Genetics, State University of Maringá-(MaringálPR, Brazil); 'Laboratory of Secretion Cell Biology, Department of Cell Biology and Genetics, State University of Maringá - (Maringá/PR, Brazil)/UPSP-EGEAL Institut Polytechnique LaSalle de Beauv; ${ }^{7}$ Laboratory of Secretion Cell Biology, Department of Cell Biology and Genetics, State University of Maringá - (MaringálPR, Brazil)

Background: Early protein restrictions can program different phenotypes depending on which phase rodents were submitted to undernourishment. Protein restriction, during lactation, programs to lean phenotype later in life; however, during adolescence programs to obesity. Pregnancy and lactation caloric restriction programs rats to obesity induced by an obesogenic diet after weaning. Whether or not protein restriction during later pregnancy can program to obesity exposing adult rats to a high fat diet (HFD) and which intensity, were tested.

Methods: In the final 1/3 of pregnancy rat dams were received low protein diet $(5 \%)$, LPP group, and controls received normoproteic diet (23\%), NPP group. After deliverance, dams and their offspring from both groups consumed normal protein and fat diet (NFD). Male offspring with 60-days-old were switched to HFD, containing $30 \%$ of fat. At 90 -days-old, adult offspring were used to analyze metabolism.

Results: Maternal low protein diet provokes low birth weight of pups, 30\% reduction $(\mathrm{p}<0.05)$. Adult animals from both groups submitted to HFD presented obesity associated to glucose intolerance, hyperinsulinemia and insulin resistance $(p<0.05)$; however, LPP group showed higher magnitude than NPP $(\mathrm{p}<0.05)$.
Conclusion: Later pregnancy protein restriction might program to metabolic syndrome with higher sensitivity to fat/ caloric abundance.

\section{4}

Exploring the thrifty gene theory: Calorie-restriction at begin of lactation programs rats to be lean and do not became obese by high-fat diet at adulthood

Audrei Pavanello ${ }^{1}$, Rosiane Aparecida Miranda ${ }^{2}$, Carina Previate $^{3}$, Ananda Malta ${ }^{4}$, Tatiane Aparecida Ribeiro 5 , Paulo Cezar de Freitas Mathias ${ }^{6}$, Júlio Cezar de Oliveira ${ }^{7}$

${ }^{1}$ Laboratory of Secretion Cell Biology, Department of Biotechnology, Cell Biology and Genetics, State University of Maringa, Maringá, PR, Brazil; ${ }^{2}$ Laboratory of Secretion Cell Biology, Department of Biotechnology, Cell Biology and Genetics, State University of Maringa, Maringá, PR, Brazil; ${ }^{3}$ Laboratory of Secretion Cell Biology, Department of Biotechnology, Cell Biology and Genetics, State University of Maringa, Maringá, PR, Brazil; ${ }^{4}$ Laboratory of Secretion Cell Biology, Department of Biotechnology, Cell Biology and Genetics, State University of Maringa, Maringá, PR, Brazil; ${ }^{5}$ Laboratory of Secretion Cell Biology, Department of Biotechnology, Cell Biology and Genetics, State University of Maringa, Maringá, PR, Brazil; ${ }^{6}$ Laboratory of Secretion Cell Biology, Department of Biotechnology, Cell Biology and Genetics, State University of Maringa, Maringá, PR, Brazil; ${ }^{7}$ Health Sciences Institute, Federal University of Mato Grosso, Sinop, MT, Brazil

Research question: Undernutrition early in life implicates metabolic disruption as long-term consequence. We aimed to study the effect of high-fat diet on metabolism of the adult rat malprogrammed during the suckling phase.

Methods: Lactating dams were fed a low-protein diet (4\%) during the first 2 weeks of lactation, control dams were fed a normal-protein diet (23\%). At weaning male rat offspring were fed a control diet until the age 60th when were fed a high-fat diet $(35 \%, \mathrm{NP} / \mathrm{HF}$ and $\mathrm{LP} / \mathrm{HF}$ groups) or normal-fat diet (7\%, NP/NF and LP/HF groups) until 90-day-old. Food consumption and body weight were evaluated every two days. At 90-day-old, rats were euthanized, fat pad stores were weighed, and blood samples collected from fasted rats to assess biochemical parameters.

Results: In relation to NP/NF, LP/NF rats were leaner; high-fat diet increased body weight around 1.2-folde in both groups $(p<0.01)$. Mesenteric and retroperitoneal fat stores were increased 2.5-folde in $\mathrm{NP} / \mathrm{HF}$ and 2-folde in $\mathrm{LP} / \mathrm{HF}$, respectively $(p<0.01)$. Even normoglycemic, LP/NF rats were hypoinsulinemic $(\mathrm{p}<0.01)$. High-fat diet induced hyperglycemia, hyperinsulinemia, hyperleptinemia and insulin resistance in both groups $(\mathrm{p}<0.001)$.

Conclusions: Adult rat malprogrammed by low-protein diet during lactation are less prominent to metabolic dysfunctions induced by a high-fat diet regimen. 
4556

Intrauterine growth restriction (IUGR) is associated with impairment of memory related to food and differential brain activation in response to palatable food images in adolescents

Amanda Brondani Mucellini ${ }^{1}$, Roberta Dalle Molle ${ }^{2}$, Danitsa Marcos Rodrigues ${ }^{3}$, Roberta Sena Reis ${ }^{4}$,

Tânia Diniz Machado ${ }^{5}$, Luciano Minuzzi ${ }^{6}$,

Alexandre Rosa Franco ${ }^{7}$, Augusto Buchweitz ${ }^{8}$, Andressa Bortoluzzi ${ }^{9}$, Rudineia Toazza ${ }^{10}$,

Giovanni Abrahão Salum Júnior ${ }^{11}$, Gisele Gus Manfro ${ }^{12}$, Patrícia Pelufo Silveira ${ }^{13}$

${ }^{1} P P G$ of Medical Sciences: Psychiatry of UFRGS, Porto Alegre, Brazil; ${ }^{2} P P G$ of Health of Child and Adolescent of UFRGS, Porto Alegre, Brazil; ${ }^{3} P P G$ of Neuroscience of UFRGS, Porto Alegre, Brazil; ${ }^{4} P P G$ of Health of Child and Adolescent of UFRGS, Porto Alegre, Brazil; ${ }^{5} P P G$ of Health of Child and Adolescent of UFRGS, Porto Alegre, Brazil; ${ }^{6}$ Department of Psychiatry and Behavioural Neurosciences, McMaster University, Hamilton, Canada; ${ }^{7}$ Ins Cer, PPG of Medicine and Health Sciences and PPG of Electrical Engineering of PUCRS, Porto Alegre, Brazil; ${ }^{8}$ InsCer, PPG of Medicine and Health Sciences and PPG of Letters of PUCRS, Porto Alegre, Brazil; ${ }^{9} P P G$ of Neuroscience of UFRGS, Porto Alegre, Brazil; ${ }^{10}$ PPG of Neuroscience of UFRGS, Porto Alegre, Brazil; ${ }^{11}$ PPG of Medical Sciences: Psychiatry of UFRGS, Porto Alegre, Brazil;

${ }^{12}$ PPG of Medical Sciences: Psychiatry and PPG of Neuroscience of UFRGS, Porto Alegre, Brazil, ${ }^{13}$ PPG of Health of Child and Adolescent and PPG of Neuroscience of UFRGS, Porto Alegre, Brazil

IUGR leads to typical food preferences and this can in part explain their increased risk for developing metabolic disorders. Could the impairment of memory related to food be implied? 69 adolescents performed an implicit memory test related to food responding which snack they would choose if they could eat it. They did not know but one of the snacks was what they had eaten months before. They performed a brain fMRI visualizing high, low palatable foods and neutral objects. External eating behavior was assessed using Dutch Eating Behavior Questionnaire. 24 of participants had IUGR and they recognized their own snack with less frequency than non-IUGR group $(P=0.01)$. For those who did not recognize the snack, the lower BWR, the more active is cuneus and less active is precuneus, middle and inferior frontal gyri when visualizing palatable foods (PFWE <0.05). For boys who did not recognize it, the lower BWR, the higher score of external eating $(B=-10,135$; $\mathrm{P}<0.01)$. Memory related to food may be associated with distinct feeding behavior in IUGR and brain activation changes in regions involved in visuospatial processing, memory, expectation of reward and consciousness. IUGR may interact with memory decline, leading to a higher external eating.
4638

Prenatal Synthetic Glucocorticoid Programs Juvenile Behavior and Stress Response: Transgenerational Paternal Transmission

Vasilis Moisiadis ${ }^{1}$, Andrea Constantinof ${ }^{2}$, Alisa Kostaki ${ }^{3}$, Stephen Matthews ${ }^{4}$

${ }^{1}$ University of Toronto, Department of Physiology; ${ }^{2}$ University of Toronto, Department of Physiology; ${ }^{3}$ University of Toronto, Department of Physiology; ${ }^{4}$ University of Toronto, Departments of Physiology, Ob-Gyn, Medicine; Fraser Mustard Institute for

Human Development

Glucocorticoids are powerful, necessary regulators of brain development. Fetal exposure to excess glucocorticoids programs aberrant stress response and behavior in young children. We investigated whether prenatal synthetic glucocorticoid exposure would transgenerationally program behavior and stress response in juvenile offspring following paternal transmission.

Pregnant guinea pigs received 3 courses of betamethasone (Beta; $1 \mathrm{mg} / \mathrm{kg}$ ) or saline (Veh) at $75 \%$ of gestation (term $\sim 69$ days). Adult male offspring were mated with naïve females to produce F2 and F3 offspring. Juvenile male and female offspring (F1, F2, F3) underwent behavioral and stress testing.

F1 and F3 Beta females were more active and traveled a greater distance in an aversive environment. 24h-home-cage activity profile was advanced one hour in F1 Beta males. In an aversive environment, F2 Beta females displayed increased, whereas F2 Beta males displayed reduced anxiety-like behavior. F1 and F2 Beta females produced a greater stress response. F2 Beta females displayed reduced attention. All results: Beta versus Veh, $\mathrm{P}<0.05$.

Fetal exposure to synthetic glucocorticoids during sensitive neurodevelopmental periods affected behavior and stress response across three generations of juvenile offspring. Female offspring appear to be more sensitive to programming by glucocorticoids. Transmission via the paternal lineage implicates germline epigenetic mechanisms.

\section{9}

The Effect of Prolonged Embryo Culture on Subsequent Development and Postnatal Health in a Mouse Model

Anan Aljahdali Aljahdali ${ }^{1}$, Ili Airina Raja ${ }^{2}$, Miguel Velazquez ${ }^{3}$, Bhav Sheth ${ }^{4}$, Neil Smyth ${ }^{5}$, Tom Fleming ${ }^{6}$

${ }^{1}$ Biological Science - University of Southampton;

${ }^{2}$ Biological Science - University of Southampton;

${ }^{3}$ Biological Science - University of Southampton; ${ }^{4}$ Biological

Science - University of Southampton; ${ }^{5}$ Biological Science -

University of Southampton; ${ }^{6}$ Biological Science - University of

Southampton

Several million babies have been born worldwide since in vitro fertilisation (IVF) and assisted reproductive technologies 
(ART) became available. However, reports link IVF techniques with adverse short and long-term health outcomes. Using a mouse model, we investigated the effect of IVF and length of culture on the total cell number in blastocyst stage embryos and the postnatal health of offspring.

Methods: Two-cell stage and blastocyst mouse embryos were collected from C57/BL6 superovulated females mated with CBA males (IVC). (IVF) conducted on isolated C57/BL6 oocytes from superovulated females using CBA sperm following an IVF protocol. Blastocyst trophectoderm (TE) and inner cell mass (ICM) cell numbers were determined by differential staining. IVF and IVC generated embryos at two-cell stage and blastocyst were transferred to MF1 pseudo pregnant recipients. Offspring were analysed for body weight, systolic blood pressure (SBP).

Results: IVC blastocysts had significantly more TE and ICM cells compared with IVF. Offspring from IVC and IVF embryos were significantly heavier than naturally mated(NM). IVF and IVC induced increased SBP in offspring compared to NM. We conclude IVF causes reduction in blastocyst cell numbers but superovulation and embryo transfer are sufficient to alter offspring growth and SBP. Further glucose tolerance analyses are underway.

\section{4}

Low glycaemic Index Diet and Physical Activity in Obese Pregnant Rats Does Not Improve Metabolic Profile of 3-Month Old Offspring

EA Domfeh, N Maicas Blasco, E Richardson, AM Samuelsson, J Pombo, PD Taylor, L Poston

Division of Women's Health, Faculty of Life Sciences \& Medicine, King's College London

Research question: We hypothesised that the adverse consequences of maternal obesity on offspring metabolic profile would be prevented by a maternal intervention combining a low glycemic index diet (LGI) and physical activity (phys).

Methods: At gestational day (GD) 7, control (Con) and diet-induced obese rats $(\mathrm{Ob})$ were assigned either to Con $(n=10), O b(n=9)$ or Ob-phys-LGI groups $(n=8)$. Physical activity was maintained until GD 17 and LGI diet until day 14 of lactation. Offspring (Off) were followed until 3 months. Glucose tolerance tests and blood pressure (BP) monitoring by radiotelemetry were performed at 3 months in offspring maintained on control chow.

Results: At the end of pregnancy, $\mathrm{Ob}$ dams had elevated fat pad mass $(p<0.001)$, insulin $(p<0.05)$ and serum leptin $(p<0.001)$ compared to Con. The intervention reduced weight gain during gestation $(p<0.001)$, lactation $(p<0.001)$ and fat pad mass $(p<0.001)$. Female OffOb had increased retroperitoneal fat mass $(p<0.05)$; impaired glucose tolerance $(p<0.05)$ and elevated systolic and mean arterial BP $(p<0.05)$ compared to OffCon. Maternal intervention had no apparent effect on offspring metabolic profile.

Conclusions: Low GI diet/exercise intervention improved maternal adiposity in obese pregnant rats but had no effect on metabolic and cardiovascular parameters in 3-month-old offspring of obese dams [EU-7-EarlyNutrition].

\section{8}

Varying dietary cholesterol exposure in the suckling period programs the intestinal cholesterol absorption during adulthood in mice

Lidiya G. Dimova $^{1}$, Jan Freark de Boer ${ }^{2}$, Henkjan J. Verkade ${ }^{3}$, Uwe J.F. Tietge ${ }^{4}$

${ }^{1}$ Department of Pediatrics, University of Groningen, University Medical Center Groningen, Hanzeplein 1, 9713GZ Groningen, the Netherlands; ${ }^{2}$ Department of Pediatrics, University of Groningen, University Medical Center Groningen, Hanzeplein 1, 9713GZ Groningen, the Netherlands; ${ }^{3}$ Department of Pediatrics, University of Groningen, University Medical Center Groningen, Hanzeplein 1, 9713GZ Groningen, the Netherlands;

${ }^{4}$ Department of Pediatrics, University of Groningen, University Medical Center Groningen, Hanzeplein 1, 9713GZ Groningen, the Netherlands

Research question: Breast milk has a high cholesterol content compared to infant formulas. Adults, who had been breastfed in infancy, have lower levels of proatherogenic LDL cholesterol than subjects fed formula. We aimed to assess whether reduced dietary cholesterol exposure during suckling alters cholesterol metabolism in adulthood.

Methods: Dietary cholesterol exposure in the first 3 weeks of life of hypercholesterolemic LDLR knockout mice offspring was either decreased by treatment with the cholesterol absorption inhibitor ezetimibe (eze) via the milk of the nursing dams, or left unaltered (control). The offspring was followed until 24 weeks when all relevant parameters of cholesterol metabolism were assessed including stable isotope kinetic studies.

Results: At 24 weeks, food intake, plasma cholesterol levels, biliary and fecal cholesterol excretion were not different between the experimental groups. Importantly, intestinal cholesterol absorption was decreased in post-eze offspring $(-30 \%$, $\mathrm{p}<0.001)$ coinciding with reduced mRNA expression of the main intestinal cholesterol uptake transporter, Npc1l1 $(-52 \%$, $\mathrm{p}<0.05)$.

Conclusion: In mice, reduced early life exposure to dietary cholesterol programs the intestine towards decreased cholesterol absorption in adulthood via altered expression of Npc1l1. These results might help delineate the mechanisms underlying the different long-term health outcomes of formula-fed and breast-fed individuals. 
4990

Maternal Obesity (MO) increases adult rat offspring liver oxidative stress (ROS) and programs mitochondrial electron transport chain (ETC) genes

Abulaitijiang Tuerxun ${ }^{1}$, Claudia Vega ${ }^{2}$, Claudia J Bautista ${ }^{3}$, Luis Reyes-Castro ${ }^{4}$, Guadelupe Rodriguez ${ }^{5}$, Carlos Ibáñez ${ }^{6}$, Consuelo Lomas ${ }^{7}$, Cun $\mathrm{Li}^{8}$, Laura A Cox ${ }^{9}$, Jeremy Glenn ${ }^{10}$, Elena Zambrano ${ }^{11}$

${ }^{1}$ Wyoming Pregnancy and Life Course Health Center, University of Wyoming, Laramie, WY, USA; ${ }^{2}$ Reproductive Biology,

Instituto Nacional de Ciencias Médicas y Nutrición Salvador Zubirán, Mexico City, Mexico; ${ }^{3}$ Reproductive Biology, Instituto

Nacional de Ciencias Médicas y Nutrición Salvador

Zubirán, Mexico City, Mexico; ${ }^{4}$ Reproductive Biology, Instituto

Nacional de Ciencias Médicas y Nutrición Salvador Zubirán,

Mexico City, Mexico; ${ }^{5}$ Reproductive Biology, Instituto

Nacional de Ciencias Médicas y Nutrición Salvador Zubirán,

Mexico City, Mexico; ${ }^{6}$ Reproductive Biology, Instituto Nacional de Ciencias Médicas y Nutrición Salvador Zubirán, Mexico City, Mexico; ${ }^{7}$ Reproductive Biology, Instituto Nacional de Ciencias Médicas y Nutrición Salvador Zubirán, Mexico City, Mexico;

${ }^{8}$ Wyoming Pregnancy and Life Course Health Center,

University of Wyoming, Laramie, WY; ${ }^{9}$ Department of Genetics and Southwest National Primate Research Center, Texas

Biomedical Research Institute, San Antonio, TX, USA;

${ }^{10}$ Department of Genetics and Southwest National Primate

Research Center, Texas Biomedical Research Institute, San

Antonio, TX, USA; ${ }^{11}$ Reproductive Biology, Instituto Nacional de Ciencias Médicas y Nutrición Salvador Zubirán, Mexico City,

Mexico

Research question: Offspring mitochondrial developmental programming is little explored. We evaluated effects of MO induced in female rats weaned onto a high fat diet, which was maintained through pregnancy and lactation on their110 day male offspring liver mitochondrial ETC, and ROS. We hypothesized that MO causes offspring liver ETC gene down regulation and increases ROS.

Methods: We conducted RNA Seq, ROS activity assays, oxidative stress marker nitrotyrosine immunohistochemistry, and blood and tissue ROS measures, malondialdehyde and anti-oxidant glutathione peroxidase activity on 110 day male offspring livers of control lean and MO mothers.

Results: All five ETC oxidative phosphorylation complexes showed gene down regulation e.g. Ndufb 2,3,6,8 and 11, Ndufa 9,10,11, Sdha, Qcr10, Co5b, and Ocsp genes. Serum malondialdehyde (control first - 305 \pm 22 vs. $523 \pm 39 \mathrm{nM} / 100 \mu \mathrm{l})$, liver malondialdehyde $(24.4 \pm 3.4$ vs. $36.1 \pm 3 \mathrm{nMol} / \mathrm{mg}$ protein), nitrotyrosine (12.4 \pm 1 vs. $25.13 \pm 2$ fraction immunostained \%) and glutathione peroxidase (33.9 \pm 1.6 vs. $58 \pm 5.9 \mathrm{mU} / \mathrm{mg}$ protein) all increased in MO offspring indicating increased ROS.

Conclusion: $\mathrm{MO}$ programs decreased offspring liver ETC gene expression, especially complex 1 the major site of ROS production, which was increased. These changes can lead to metabolic dysfunction and offspring obesity.

4441

Postnatal growth in preterm infants and later health outcomes: a systematic review

Ken K Ong ${ }^{1}$, Kathy Kennedy ${ }^{2}$, Euridice Castaneda Gutierrez ${ }^{3}$, Stewart Forsyth ${ }^{4}$, Keith Godfrey ${ }^{5}$, Berthold Koletzko ${ }^{6}$, Marie E Latulippe ${ }^{7}$, Susan E Ozanne ${ }^{8}$, Ricardo Rueda ${ }^{9}$, Marieke Schoemaker ${ }^{10}$, Eline $\mathrm{M}$ van der Beek ${ }^{11}$, Stef van Buuren ${ }^{12}$, Mary Fewtrell ${ }^{13}$

${ }^{1}$ MRC Epidemiology Unit, University of Cambridge, Cambridge, UK; ${ }^{2}$ Institute of Child Health, University College London, London, UK; ${ }^{3}$ Nestle Research Centre; ${ }^{4}$ DSM-Martek Division, Dundee, UK; ${ }^{5}$ MRC Lifecourse Epidemiology Unit + NIHR Southampton Biomedical Research Centre, University of Southampton and University Hospital Southampton NHS Foundation Trust, Southampton, UK; ${ }^{6}$ University of Munchen; ${ }^{7}$ Formely of: ILSI Europe, Brussels, Belgium; ${ }^{8}$ University of Cambridge Metabolic Research Laboratories and MRC Metabolic Disease Unit, Cambridge, UK; ${ }^{9}$ Abbott Nutrition, Granada, Spain; ${ }^{10}$ Mead Johnson Pediatric Nutrition Institute, Nijmegen, the Netherlands;

${ }^{11}$ Danone Nutricia Early Life Nutrition, Singapore; ${ }^{12}$ University of Utrecht, Utrecht, the Netherlands; ${ }^{13}$ Institute of Child Health, University College London, London, UK

Question: In preterm infants does growth during critical postnatal periods influence later neuro-development and/or metabolic risks?

Methods: We performed a systematic search of electronic databases for studies published before May 2013 reporting associations between postnatal growth and later health outcomes in preterm infants; 42 eligible studies were identified. In addition, seven reports published before May 2004 were extracted from an earlier review.

Results: Few intervention studies were identified; these provided little evidence for benefits or adverse effects of faster early growth. Observational studies showed generally consistent positive associations between postnatal weight gain (19 studies) or head growth (16 studies) and neuro-developmental outcomes from infancy onwards, with no obvious critical periods of growth. Two of the four observational studies reported positive associations between postnatal weight gain and later body fat; three of the four studies reported positive associations with insulin resistance; associations with cardiovascular disease were more variable.

Conclusion: The dissonance between findings of the limited intervention studies and more numerous observational studies raises the possibility of confounding by factors such as neonatal disease that may affect both growth and neuro-development. Future nutritional intervention studies should routinely report effects on weight gain and growth, as well as later body composition, metabolism and neuro-development. 
4456

Re-sampling Method for the Construction of Twin-Specific Birth Weight References: A New Concept

Xiu Qiu ${ }^{1}, \mathrm{Yu} \mathrm{Liu}{ }^{2}$, Guangjian $\mathrm{Liu}^{3}$, Jianrong $\mathrm{He}^{4}$, Xiaoyan $\mathrm{Xia}^{5}$, Ping Wang ${ }^{6}$, Weidong $\mathrm{Li}^{7}$, Hongwen Deng ${ }^{8}$, Huimin $\mathrm{Xia}^{9}$

${ }^{1}$ Guangzhou Women and Children's Medical Center;

${ }^{2}$ Guangzhou Women and Children's Medical Center;

${ }^{3}$ Guangzhou Women and Children's Medical Center;

${ }^{4}$ Guangzhou Women and Children's Medical Center;

${ }^{5}$ Guangzhou Women and Children's Medical Center;

${ }^{6}$ Guangzhou Women and Children's Medical Center;

${ }^{7}$ Guangzhou Women and Children's Medical Center; ${ }^{8}$ Tulane

University School of Public Health and Tropical Medicine;

${ }^{9}$ Guangzhou Women and Children's Medical Center

Research question: Currently twins-specific birthweight references do not differentiate their origins from like-sexed or unlike-sexed pairs. This introduces systematic errors due to the co-linearity within like-sexed twins, and underestimates the contribution of unlike-sexed twins. We aimed to find a method to overcome these deficiencies.

Methods: A total of 20,245 pairs of twins were included in the study. One twin was randomly selected from each like-sexed pair, and pooled with all same-sex neonates from unlike-sexed twins. The Generalized Additive Models for Location, Scale, and Shape was used to obtain birthweight percentiles by gestational age. The sampling and modeling procedures were repeated for 1000 times, and the final references were generated by smoothing the bootstrap percentiles using a cubic splines function. The references were compared with those generated using traditional method.

Results: Compared to traditional method, the birth weight percentile curves of RSM were slightly but systematically upshifted. Consequently, the estimated prevalence of SGA changed from $9.7 \%$ to $10.7 \%$. The differences between two methods were mainly determined by the mean birth weight differences between the same-sexed infants from unlike-sexed and like-sexed twins.

Conclusion: We proposed a useful method to eliminate the effect of sample dependence and correct the underestimation of unlike-sexed twins.

\section{7}

Adherence to 10 dietary principles and risk of preterm delivery - a study performed in the Norwegian Fit for Delivery study

Elling Bere ${ }^{1}$, Linda Reme Sagedal ${ }^{2}$, Ingvild Vistad ${ }^{3}$, Nina Cecilie Øverby ${ }^{4}$

${ }^{1}$ University of Agder, Kristiansand, Norway; ${ }^{2}$ Sorlandet hospital $H F$, Kristiansand, Norway; ${ }^{3}$ Sorlandet hospital HF, Kristiansand, Norway; ${ }^{4}$ University of Agder, Kristiansand, Norway
Research question: Is degree of adherence to 10 predefined dietary principles during pregnancy associated with risk of preterm delivery?

Methods: 591 healthy nulliparous women participating in the randomized controlled Norwegian Fit for Delivery (NFFD) study were included in the analysis. The dietary intervention targeted energy balance-related dietary behaviors. A baseline diet score was constructed from a 43-item food frequency questionnaire completed by participants before randomization. The score comprised 10 subscales addressing meal regularity, water vs. other beverages, fruit/vegetables, sweets/snacks, portion size, satiety, and awareness of food labeling. The score ranged from 0-10 points and was used to categorize participants into 'low', 'medium' or 'high' diet adherence. Risk of preterm delivery was assessed by multivariate logistic regression.

Results: A total of 34/591 (5.8\%) women delivered before 37 completed weeks. Adjusted for potential confounding, both high and medium vs. low diet adherence was associated with lower odds of preterm delivery (OR: 0.24; 95\% CI: 0.10-0.59; $\mathrm{p}=0.001$ and OR: 0.23; 95\% CI: 0.10-0.56; $\mathrm{p}=0.002$, respectively) (adjusted for maternal age, height, BMI, smoking status, education, cohabitant status, and randomization status). Conclusion: Higher adherence to 10 dietary principles in early pregnancy was associated with lower risk of preterm delivery in the NFFD study.

4469

Health-related quality of life among adults born preterm the Helsinki Study of Very Low Birth Weight Adults (HeSVA)

Petteri Hovi ${ }^{1}$, Anu-Katriina Pesonen ${ }^{2}$, Katri Räikkönen ${ }^{3}$, Anna-Liisa Järvenpää ${ }^{4}$, Johan G. Eriksson ${ }^{5}$, Sture Andersson ${ }^{6}$, Eero Kajantie $^{7}$

${ }^{1}$ National Institute for Health and Welfare, Children's Hospital, University of Helsinki and Helsinki University Hospital;

${ }^{2}$ Institute of Behavioral Sciences, University of Helsinki, Helsinki Finland; ${ }^{3}$ Institute of Behavioral Sciences, University of Helsinki, Helsinki Finland; ${ }^{4}$ University of Helsinki; ${ }^{5}$ University of Helsinki; ${ }^{6}$ Children's Hospital, University of Helsinki and Helsinki University Hospital; ${ }^{7}$ National Institute for Health and Welfare

Research question: Is the health-related quality of life (HRQoL) among very low birth weight (VLBW, < 1500g) adults comparable to that of adults born at term?

Methods: We used the 15D questionnaire to assess HRQoL of 164 VLBW and 172 control adults, born 1978-1985, now aged on average 22 years. The $15 \mathrm{D}$ questionnaire is a selfadministered, generic questionnaire that produces both a 15-dimensional profile and an aggregate $15 \mathrm{D}$ score.

Results: The VLBW group had comparable HRQoL to the control group in all 15 dimensions and the aggregate $15 \mathrm{D}$ score. However, further investigation of subgroups revealed 
significant differences; those born small for gestational age (birth weight $<2 \mathrm{SD}$ ), especially women, fared significantly worse than both controls and preterms born appropriate for gestational age

Conclusion: While preterm birth at VLBW per se is not a risk factor for poor adult HRQoL, VLBW accompanied by intrauterine growth restriction predicts poorer HRQoL, especially among women.

\section{6}

The effect of protein and energy intake in the first 28 days of life on the growth velocity of extremely low birth weight infants

Shilpee Raturi ${ }^{1}$, Charles Qi Shi Cheng ${ }^{2}$, Pratibha Agarwal ${ }^{3}$, Victor Samuel Rajadurai ${ }^{4}$, Lu Ming Shi ${ }^{5}$

${ }^{1}$ KK Women's and Children's Hospital; ${ }^{2}$ Singapore Clinical Research Institute; ${ }^{3} K K$ Women's and Children's Hospital; ${ }^{4} K K$ Women's and Children's Hospital; ${ }^{5}$ Singapore Clinical Research Institute

Aims: 1 . To describe nutritional practices among extremely low birth weight (ELBW) infants and their impact on growth.

2. To compare the differences in nutritional intervention and comorbidities between those with limited growth velocity (GV) $(<25$ th percentile) and those with GV $>25$ th percentile.

Methods: A prospective cohort study with analysis of demographics, anthropometry, co-morbidities, daily total protein and energy intake for week 1, days 14, 21 and 28 of life. Postnatal growth analysed by measuring GV using an exponential model.

Results: Median GV was $9.84 \mathrm{~g} / \mathrm{kg} /$ day $(1.70-28.12)$ on day 28 (GV28) and $11.87 \mathrm{~g} / \mathrm{kg} /$ day (0.31 to 15.40$)$ at discharge (GVdis). 27/105 infants had limited GV. Cut-off points for GV28 and GVdis were 8.06 and $10.60 \mathrm{~g} / \mathrm{kg} /$ day. Increased protein and energy intake was associated with higher GV at discharge. NEC and hypotension $>72$ hours were significantly associated with reduced GV at discharge $(\mathrm{p}=0.002$ and $<0.001)$. Small for gestational age babies increased from $22 \%$ at birth to $66.6 \%$ at discharge.

Conclusions: Although increasing protein and energy intake improved postnatal growth, comorbidities had a stronger effect on GV. Majority of ELBW infants had lower z scores at discharge than at birth.

\section{4}

Early growth after preterm birth and neurocognitive functioning in adulthood

Kati Heinonen ${ }^{1}$, Marius Lahti ${ }^{2}$, Jari Lahti ${ }^{3}$, Sture Andersson ${ }^{4}$, Aulikki Lano ${ }^{5}$, Timo Vartia ${ }^{6}$, Dieter Wolke ${ }^{7}$, Eero Kajantie ${ }^{8}$, Johan Eriksson ${ }^{9}$, Katri Räikkönen ${ }^{10}$

${ }^{1}$ Institute of Behavioural Sciences, University of Helsinki, Helsinki, Finland; ${ }^{2}$ Institute of Behavioural Sciences, University of Helsinki, Helsinki, Finland; ${ }^{3}$ Institute of Behavioural Sciences, University of Helsinki, Helsinki, Finland; Folkhälsan Research Centre, Helsinki, Finland; ${ }^{4}$ Children's Hospital, Helsinki University Central Hospital and University of Helsinki, Helsinki, Finland; ${ }^{5}$ Children's Hospital, Helsinki University Central Hospital and University of Helsinki, Helsinki, Finland; ${ }^{6}$ Children's and Adolescents' Clinic Pikkujätti, Helsinki, Finland; ${ }^{7}$ Department of Psychology and HSRI, University of Warwick, Coventry, UK; ${ }^{8}$ Children's Hospital, Helsinki University Central Hospital and University of Helsinki, Helsinki, Finland; National Institute for Health and Welfare, Helsinki, Finland; ${ }^{9}$ National Institute for Health and Welfare, Helsinki, Finland; Folkhälsan Research Centre, Helsinki, Finland; Department of General Practice and Primary Health Care, University of Helsinki; ${ }^{10}$ Institute of Behavioural Sciences, University of Helsinki, Helsinki, Finland

Research question: Faster growth after preterm birth may benefit childhood neurodevelopment. Yet, it remains unclear if this effect persists into young adulthood and extends across the range of prematurity. We examined whether faster growth in early childhood after different degrees of preterm birth predicts better neurocognitive functioning and school performance in young adulthood.

Methods: Weight, length, and head circumference of 150 preterm (39 very/moderate preterm: $<34+0$ gestational weeks +days; and 111 late preterm: $34+0$ to $36+6$ weeks + days) Arvo Ylppö Longitudinal Study participants were measured at birth and at 5, 20 and 56 months. At 25 years, the participants underwent psychological tests on neurocognitive functioning and self-reported the grade point average (GPA) on their comprehensive school diploma.

Results: Faster growth from birth to 5 months of corrected age, especially in weight and head circumference, was associated with better neurocognitive functioning, including general cognitive ability, memory, and executive functioning, as well as with a higher self-reported GPA. Growth thereafter was less consistently associated with adult neurocognitive outcomes. The result was largely similar in those born very/moderate vs. late preterm.

Conclusion: Faster growth in early infancy is associated with better neurocognitive functioning and school performance in young adults born prematurely.

\section{8}

Objectively measured physical activity in young adults born preterm - The ESTER study

Marjaana Tikanmäki ${ }^{1}$, Hanna-Maria Matinolli ${ }^{2}$,

Tuija Tammelin ${ }^{3}$, Nina Kaseva ${ }^{4}$, Marika Sipola-Leppänen ${ }^{5}$,

Harto Hakonen ${ }^{6}$, Ulf Ekelund ${ }^{7}$, Johan G Eriksson ${ }^{8}$, Marjo-Riitta Järvelin ${ }^{9}$, Marja Vääräsmäki ${ }^{10}$, Eero Kajantie ${ }^{11}$

${ }^{1}$ National Institute for Health and Welfare; ${ }^{2}$ National Institute for Health and Welfare; ${ }^{3}$ LIKES - Research Center for Sport and 
Health Sciences; ${ }^{4}$ National Institute for Health and Welfare; ${ }^{5}$ National Institute for Health and Welfare; ${ }^{6}$ LIKES Research Center for Sport and Health Sciences; ' Department of Sport Medicine, Norwegian School of Sport Sciences, Oslo,

Norway; ${ }^{8}$ Department of General Practice and Primary Health Care, University of Helsinki and Helsinki University Hospital, Helsinki, Finland; ${ }^{9}$ Department of Epidemiology and Biostatistics, MRC-PHE Centre for Environment \& Health, School of Public Health, Imperial College London, UK; ${ }^{10}$ Department of Obstetrics and Gynaecology, Medical Research Center Oulu, Oulu University Hospital and University of Oulu, Oulu, Finland; ${ }^{11} \mathrm{National}$ Institute for Health and Welfare

Adults born preterm report less physical activity (PA) and are less fit than their term-born peers. We explored whether objectively measured PA levels are lower among adults born preterm and whether the decrease in PA is associated with the degree of prematurity.

Methods: At the age of 23.1 (SD 1.2) years we measured PA of 286 participants of the ESTER study by using hip-worn accelerometer (ActiGraph $\AA$ ). Of the participants, 60 were born early preterm (EPT, <34 wk GA), 108 late preterm (LPT, 34-36 wk GA) and 178 were termborn controls. Group differences were examined by linear regression.

Results: The mean PA levels were similar among adults born either early $(\beta=-21 \mathrm{cpm}, 95 \%$ CI $-61,19 ; \mathrm{p}=0.31)$ or late $(\beta=5 \mathrm{cpm}, 95 \% \mathrm{CI}-27,38 ; \mathrm{p}=0.75)$ preterm, as compared with controls with mean PA of $303 \pm 129 \mathrm{cpm}$. Also there were no differences in time spent sedentary between preterm groups when compared to controls. Adjustments for early life confounders or current mediating characteristics did not change the results.

Conclusion: We found no difference in objectively measured PA or time spent sedentary between adults born preterm and term-born peers. This could indicate that the previously reported differences are limited to PA captured by self-report.

\section{6}

Aged female mice suffering from intrauterine growth restriction show increased plasma free fatty acids and endoplasmic reticulum stress in the adipose tissue

Violeta Stojanovska ${ }^{1}$, Neha Sharma ${ }^{2}$, Josee Plantinga ${ }^{3}$, SIcco Scherjon ${ }^{4}$, Hubert Schorle ${ }^{5}$, Torsten Plosch ${ }^{6}$

${ }^{1}$ Department of Obstetrics and Gynecology, University Medical Center Groningen, the Netherlands; ${ }^{2}$ Dept of Developmental Pathology, Institute of Pathology, University of Bonn, Medical School; ${ }^{3}$ Department of Obstetrics and Gynecology, University Medical Center Groningen, the Netherlands; ${ }^{4}$ Department of Obstetrics and Gynecology, University Medical Center Groningen, the Netherlands; ${ }^{5}$ Dept of Developmental Pathology, Institute of Pathology, University of Bonn, Medical School; ${ }^{6}$ Department of
Obstetrics and Gynecology, University Medical Center Groningen, The Netherlands

Research question: Does intrauterine growth restricted (IUGR) mice show metabolic deterioration and ER stress at adult age?

Methods: Biochemical plasma parameters were analyzed in fasted 12 months old IUGR mice and controls. Glucose clearance was assessed by intraperitoneal glucose tolerance test (IPGTT). Liver and white adipose tissues were used for gene expression analysis.

Results: Plasma free fatty acids (FFA) were increased ( $p<0.01)$ in the IUGR aged female mice in compare to controls. The total body glucose clearance did not show any significant differences between the groups. Gene expression analysis showed increased expression of genes involved in the free fatty acid synthesis in the adipose tissue, again only in the female IUGR group. This is consistent with the previously observed increased systemic FFA. In continuation, we found increased mRNA expression levels of endoplasmic reticulum (ER) stress markers in the adipose tissue.

Conclusion: Our data suggest that adult organisms, previously exposed to IUGR, have elevated plasma free fatty acids that can contribute to the adipose tissue ER stress in sex specific manner. Further research is needed in order to evaluate whether these IUGR and age-associated metabolic changes are causative to the growth restricted increased susceptibility to chronic non-communicable diseases.

\section{1}

Paternal grandparental mortality association with grandchildren's birth-weight: Mortality follow-up, Lifeways Cross-Generation Cohort Study, Ireland

Karien Viljoen ${ }^{1}$, Aakash Shrivastava ${ }^{2}$, Celine Murrin ${ }^{3}$, John O'Brien ${ }^{4}$, Cecily Kelleher ${ }^{5}$

${ }^{1}$ University College Dublin, School of Public Health, Physiotherapy and Population Science; ${ }^{2}$ University College Dublin, School of Public Health, Physiotherapy and Population Science; ${ }^{3}$ University College Dublin, School of Public Health, Physiotherapy and Population Science; ${ }^{4}$ University College Dublin, School of Public Health, Physiotherapy and Population Science; ${ }^{5}$ University College Dublin, School of Public Health, Physiotherapy and Population Science

Research question: There's increasing interest in crossgeneration transmission of risk for adult chronic disease, mediated through early childhood growth and development. We aim to differentiate associations of grandparental mortality patterns with infant birth-weight.

Methods: Grandparents were categorised as maternal grandmother (MGM), maternal grandfather (MGF), paternal grandmother (PGM) and paternal grandfather (PGF) and linked to grandchild's birth-weight (standardised for 
gestational age). Mean birth-weight was compared in families where only one index grandparent had died with those where none had died. Birth-weight was furthermore compared to lineage-specific survival relative to circulatory and noncirculatory deaths respectively.

Results: $\mathrm{n}=835$ deaths were identified, highest in PGFs (36.2\%), followed by MGFs (30.1\%), PGMs (19.6\%) and MGMs (13.6\%). All-cause mortality: after full adjustment, where the PGF was deceased the infants had a mean birthweight of \pm 300 grams greater to those with living grandparents $(p=0.01)$.Cause-specific mortality: after full adjustment the PGM had a significant decreased HR for death with each unit increase in infant birth-weight $(\mathrm{p}=0.04)$.

Conclusions: Results suggest a positive relationship between higher birth-weight infants and PGFs all-cause mortality, and a negative relationship for PGMs circulatory-cause mortality. Mechanisms for this merit exploration. Findings are important as few studies have paternal lineage data with capacity to explore these relationships.

\section{2}

TNF-2 gene -308 polymorphism among women with preterm delivery from two Brazilian cohorts with different socioeconomic profiles (BRISA Birth Cohort, Brazil)

Perla Lopes de Freitas ${ }^{1}$, Ricardo Carvalho Cavalli ${ }^{2}$, Antonio Augusto Moura da Silva ${ }^{3}$, Heloisa Bettiol ${ }^{4}$, Marco Antonio Barbieri ${ }^{5}$, Viviane Cunha Cardoso ${ }^{6}$, Rosangela Fernandes Lucena Batista ${ }^{7}$, Silma Regina Ferreira Pereira $^{8}$

${ }^{1}$ Laboratory of Genetics and Molecular Biology, Federal University of Maranhao, Brazil; ' Department of Obstetrics and Gynecology, Ribeirao Preto Medical School, University of Sao Paulo, Brazil;

${ }^{3}$ Department of Public Health, Federal University of Maranhao, Brazil; ${ }^{4}$ Department of Pediatrics, Ribeirao Preto Medical School, University of Sao Paulo, Brazil; ${ }^{5}$ Department of Pediatrics, Ribeirao Preto Medical School, University of Sao Paulo, Brazil; ${ }^{6}$ Department of Pediatrics, Ribeirao Preto Medical School, University of Sao Paulo, Brazil; ${ }^{7}$ Department of Public Health, Federal University of Maranhao, Brazil; ${ }^{8}$ Departmente of Biology, Laboratory of Genetics and Molecular Biology, Federal University of Maranhao, Brazil

Research question: Genes coding for pro- and antiinflammatory cytokines and their receptors have been considered to be possibly associated with preterm birth (PTB). In the present study we investigated the association between the presence of the TNF2 allele and the occurrence of PTB in two Brazilian cohorts.

Methods: Genotyping of DNA from peripheral blood of pregnant women was performed by real time PCR. Differences in allele and genotype distribution and in clinical and socioeconomic variables between pregnant women with PTB (cases) and controls were determined by the chi-square test. The association between polymorphism and PTB, and between polymorphism and a history of PTB was determined by the odds ratio.

Results: A total of 102 pregnant women with PTB (cases) and 204 with term delivery (controls) were genotyped in Sao Luis and 129 cases and 258 controls were genotyped in Ribeirao Preto. Among the sociodemographic variables, only a "history of PTB" showed a difference between groups $(p<0.001)$. There was no association between the presence of the TNF2 allele (homozygous and heterozygous genotypes) and PTB in either city: Sao Luis $(\mathrm{OR}=1.15$; 95\%CI 0.64-2.07, $\mathrm{p}=0.740)$ and Ribeirao Preto $(\mathrm{OR}=1.72 ; 0.98-3.00, \mathrm{p}=0.071)$.

Conclusion: The presence of the variant TNF2 allele, alone, does not influence PTB.

\section{0}

Cardiac Akt/mTOR-signaling is decreased in rat fetuses affected by uteroplacental insufficiency - survival at the cost of impaired heart development?

Felix Lechner ${ }^{1}$, Eva Nüsken ${ }^{2}$, Nava Mehdiani ${ }^{3}$, Gregor Lippach $^{4}$, Maria Wohlfarth ${ }^{5}$, Jörg Dötsch ${ }^{6}$

${ }^{1}$ University of Cologne, Department of Pediatrics; ${ }^{2}$ University of Cologne, Department of Pediatrics; ${ }^{3}$ University of Cologne,

Department of Pediatrics; ${ }^{4}$ University of Cologne, Department of Pediatrics; ${ }^{5}$ University of Cologne, Department of Pediatrics;

${ }^{6}$ University of Cologne, Department of Pediatrics

Introduction: Heart development is a tightly regulated process including a variety of signaling pathways. We performed a detailed analysis of Akt/mTOR signaling in fetuses affected by utero-placental insufficiency to identify dysregulations and to provide a time frame for interventional strategies.

Methods: Offspring of pregnant Wistar rats after bilateral uterine vessel ligation (LIG) or sham operation (SOP) at gestational day E19 (term is gestational day E22.5) were compared to offspring of un-operated controls (C). We obtained fetal hearts $4 \mathrm{~h}$ (E19), 24h (E20) and 72h (E22) after the intervention, and on postnatal days P1 and P7. Western blot analysis and mRNA-quantification were performed to determine changes in the regulation of Akt/mTOR-signaling.

Results: After LIG, a sequential, significant downregulation of p-Akt/Akt could be observed in the fetuses, normalizing after birth. IGF1 receptor expression, an upstream modulator of Akt, was decreased. Further downstream, p-4E-BP1, a mTORregulated protein capable of reducing protein synthesis, was similarly decreased.

Discussion: We show a temporary inhibition of $\mathrm{p}$-Akt/Akt and further downstream signaling after LIG. This may lead to decreased proliferation and protein synthesis, turning the heart into a "survival first"-state. Our study provides evidence that early interventional strategies activating mTor might improve cardiovascular outcome. 
4685

Supplementation of breast milk and development of preterm infants after hospital discharge: a randomized clinical trial

Fernando Lamy Filho ${ }^{1}$, Roxana Desterro e Silva da Cunha ${ }^{2}$, Eremita Val Rafael ${ }^{3}$, Zeni Carvalho Lamy ${ }^{4}$, André Luíz Guimarães Queiroz ${ }^{5}$

${ }^{1}$ Universidade Federal do Maranhão; ${ }^{2}$ Maranhão Federal University, Brazil; ${ }^{3}$ Maranhão Federal University, Brazil; ${ }^{4}$ Maranhão Federal University, Brazil; ${ }^{5}$ Maranhão Federal University, Brazil

Research question: Supplementation of breast milk after hospital discharge on preterm newborns affects their development at 12 months of corrected gestational age?

Methods: A randomized controled trial with 53 infants followed up from discharge from NICU until a corrected gestational age of twelve months was performed. Newborns in the intervention group were exclusively breast-fed and received two grams of multinutrient supplement (PRÉ-NAN ${ }^{\circledR}$, Nestlé) added to expressed breast milk twice a day until a corrected age from four to six months. The control group were breast-fed exclusively with maternal milk without supplementation. After monthly follow-up, developmental assessment was performed using the Bayley III Scale.

Results: There was no statistically significant difference between the mean values of Bayley III Scale in the two groups in any of the domains studied: Motor (intervention: 99,19 \pm 14,09; control: 95,55 $\pm 11.04 ; p=0.237$ ), cognitive

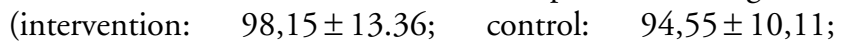
$\mathrm{p}=0.404$ ) and language (intervention: 99,19 $\pm 14,09$; control: $94,55 \pm 10,11 ; \mathrm{p}=0.356)$. However, scores in the three domains were always greater in the intervention group. There were a similar number of cases of developmental delay in both groups.

Conclusions: The results failed to show an association between multinutrient post-discharge supplementation and development of infants analyzed.

\section{0}

Excessive left venticular myocardial growth directly after preterm birth

Ulf Schubert

\section{Karolinska Institutet}

Research question: Early cardiac re-modelling and increased left ventricular mass (LVM) in young adults born preterm have been reported. We investigated LVM in infants for early adaptational myocardial changes during 6 months after preterm birth.

Method: Longitudinal echocardiographic study measuring LVM in 25 preterm infants (GA 26-30) directly after birth, at term and 3 months post-term, and comparison to 30 agematched term children after correction for body surface area $\left(\mathrm{m}^{2}\right)$.

Results: $\mathrm{LVM} / \mathrm{m} 2$ increased by $78 \%$ during the first three months after preterm birth $\left(37.43\right.$ to $66.73 \mathrm{~g} / \mathrm{m}^{2}$ ) compared to $13 \%$ in controls ( 49.39 to $55.70 \mathrm{~g} / \mathrm{m}^{2}$ ). At term, $\mathrm{LVM} / \mathrm{m} 2$ was significantly higher in the preterm group $\left(66.73\right.$ vs $49.39 \mathrm{~g} / \mathrm{m}^{2}$, $\mathrm{p}<0.001)$. Preterm infants developed even more absolute LVM (12.79 vs $10.79 \mathrm{~g}, \mathrm{p}=0.02)$ although they were slightly lighter $(3.18$ vs $3.45 \mathrm{~kg})$.

At three months of corrected age, no significant differences could be shown between the groups.

Conclusion: Preterm infants develop an immediate but transient increase in LVM. Premature myocardial maturation, increased afterload and a narrower vascular tree might be responsible for left ventricular hypertrophy. The impact on long-term left ventricular function is still unclear and has to be explored.

\section{1}

Neonatal antibiotics in preterm infants and risk of allergic diseases later in life

Annelies van $\mathrm{Zwol}^{1}$, Elisabeth Westerbeek ${ }^{2}$, Linda Carstens ${ }^{3}$

${ }^{1}$ Erasmus MC-Sophia Children's Hospital, Department of

Pediatrics, Rotterdam, The Netherlands; ${ }^{2} V U$ University Medical

Center, Department of Pediatrics, Amsterdam, The Netherlands;

${ }^{3} V U$ University Medical Center, Department of Pediatrics,

Amsterdam, The Netherlands

Research question: To determine the effect of antibiotics on the risk of developing allergic diseases later in life in preterm infants.

Methods: Preterm infants (gestational age (GA) $<32$ weeks and/or birth weight (BW) < 1500 grams) who participated in two large nutritional intervention studies were eligible. At six years of age, the prevalence of allergic diseases was assessed by a validated questionnaire. Antibiotic use was defined as the number of days of antibiotic use during the neonatal period (first 30days of life). Allergic diseases included atopic dermatitis, hay fever, recurrent wheeze and asthma. Data were analyzed by multivariable logistic regression.

Results: In total, 142/183 (78\%) children participated. Mean (SD) GA and BW were $29.4( \pm 1.9)$ weeks and $1259( \pm 349)$ grams, respectively. Prevalence of atopic dermatitis was $18 \%$, hay fever $8 \%$, recurrent wheeze $17 \%$ and asthma $13 \%$. Antibiotic use during $\geqslant 8$ days was significantly associated with the development of atopic dermatitis at six years of age (adjusted odds ratio $3.74 ; 95 \% \mathrm{CI}: 1.15-12.22 ; \mathrm{P}=0.03$ ). No association was found between antibiotic use and other allergic diseases.

Conclusion: Antibiotic use during neonatal period in preterm infants was associated with atopic dermatitis at six years of age. This indicates that neonatal antibiotics may have long term adverse consequences in preterm infants. 
4888

Gender differences in cord blood methylation profiles associated with birth weight and gestational age in a Brazilian cohort

Alexandra Brentani brentani ${ }^{1}$, Mariana Maschietto ${ }^{2}$, Laura Bastos ${ }^{3}$, Henrique Vieira ${ }^{4}$, Angelica Baumont ${ }^{5}$, Guilherme Polanczyk ${ }^{6}$, Sandra Grisi ${ }^{7}$, Euripedes Miguel ${ }^{8}$, Carlos Alberto Moreira ${ }^{9}$, Rossana Pulcinelli ${ }^{10}$, Ana maria Escobar ${ }^{11}$, Gunther Fink ${ }^{12}$

${ }^{1}$ Faculdade de medicina - USP; ${ }^{2}$ Laboratory of national Sciences CNPEM Campinas; ${ }^{3}$ Faculdade de medicina USP; ${ }^{4}$ Faculdade de Medicina USP, ${ }^{5}$ Faculdade de Medicina USP, ${ }^{6}$ Faculdade de Medicina USP; ${ }^{7}$ Faculdade de medicina USP, ${ }^{8}$ Faculdade de Medicina USP; ${ }^{9}$ Faculdade de medicina USP; ${ }^{10}$ Faculdade de medicina USP; ${ }^{11}$ Faculdade de medicina USP; ${ }^{12}$ Harvard School of Public health

The gender difference in psychiatric disorder prevalence is well established. A growing evidence suggests that exposure to toxic stress during gestation differentially impacts outcomes in males and females. This project aimed studing epigenetic alterations on psychiatric outcomes in boys and girls. We assessed the methylation and genomic profile using cord blood of 69 babies (39 females and 30 males). Mothers were interviewed about gestational exposures and toxic stress. Bisulphite-converted DNA was hybridized to Illumina HumanMethylation 450 BeadChips. Beta-values were generated from dye intensity tracking the methylation level of 487,557 sites. Probes located at $\mathrm{X}$ and $\mathrm{Y}$ chromosomes and those associated to SNPs population specific, were removed. We used Singular Value Decomposition Analysis to identify major drivers of variability of methylation. Gender $(p<10-10)$, tobacco and maternal health problems were identified as key drivers $(\mathrm{p}<0.05)$. Looking for $\mathrm{CpG}$ sites associated only with gender we found 3749 MVPs (p-value <0,05) representing 1620 unique genes. Considering CpG ( $\mathrm{p}$ value $<10-5$ we performed GO, KEGG, Disease enrichment analysis. Genes were differentially methylated according to gender (independent of stress exposure) and were enriched for neurodevelopment disorders, suggesting that gender influences methylation in the autosomal chromosomes shown to be predictive of psychiatric outcomes independent of stress exposure.

\section{6}

Secular trends of low birthweight and macrosomia and related maternal factors in Beijing, China: a longitudinal trend analysis

Fangfang Chen ${ }^{1}$, Wenpeng Wang ${ }^{2}$, Hongbo Dong ${ }^{3}$, Jie Mi ${ }^{4}$

${ }^{1}$ Capital Institute of Pediatrics; ${ }^{2}$ Capital Institute of Pediatrics;

${ }^{3}$ Peking Union Medical College; Capital Institute of Pediatrics;

${ }^{4}$ Capital Institute of Pediatrics
Objectives: To demonstrate tracking information of birthweight and related maternal risk factors in Beijing, hospital records from two major obstetrics and gynecology hospitals in urban districts in Beijing were analyzed.

Methods: Hospital records from 1996 to 2010 were retrieved. Information of prenatal examination and birth outcomes was entered into a structured database. Live births were used for trend analysis.

Results: A total of 63661 live births were delivered during 1996-2010 in the study hospitals. The percentage of low birthweight fluctuated around $4.0 \%$, while the percentage of preterm birth presented an upward trend. Both the percentages of macrosomia and excessive gestational weight gain (GWG) increased from 1996 to 2010. Proportion of suboptimal GWG was decreased significantly from $16.1 \%$ in 1996 to $11.7 \%$ in 2010 .

Conclusions: Continuously monitoring macrosomia is needed and intervention should focus on appropriate gestational weight gain and reduction of preterm birth.

\section{9}

Very preterm fetal responses to intraamniotic LPS are characterised by systemic IL-6 signalling and cellular proliferation in a sheep model of pregnancy

Matthew Kemp ${ }^{1}$, Timothy Molloy ${ }^{2}$, Haruo Usuda ${ }^{3}$, Eleanor Woodward ${ }^{4}$, Yuichiro Miura ${ }^{5}$, Matthew Payne ${ }^{6}$, Demelza Ireland $^{7}$, Alan Jobe ${ }^{8}$, Suhas Kallapur ${ }^{9}$, Sarah Stock ${ }^{10}$, Brad Spiller $^{11}$, John Newnham ${ }^{12}$, Masatoshi Saito ${ }^{13}$

${ }^{1}$ University of Western Australia; ${ }^{2}$ St Vincent's Centre for Applied Medical Research, Sydney, Australia; ${ }^{3}$ Tohoku University Hospital, Sendai, Japan; ${ }^{4}$ University of Western Australia; ${ }^{5}$ University of Western Australia; ${ }^{6}$ University of Western Australia; ${ }^{7}$ University of Western Australia; ${ }^{8}$ Cincinnati Children's Hospital Medical Centre; ${ }^{9}$ Cincinnati Children's Hospital Medical Centre; ${ }^{10}$ University of Edinburgh Queen's Medical Research Institute; ${ }^{11}$ Cardiff University, School of Medicine; ${ }^{12}$ University of Western Australia; ${ }^{13}$ Tohoku University Hospital, Sendai, Japan

Research question: Globally, preterm birth (delivery before 37 weeks' gestation) is a leading cause of death and life-long disability. Although intrauterine infection and inflammation play central role in preterm labour and neonatal injury, the responses of very preterm fetuses to microbial agonist are incompletely understood. We aimed to characterise the fetal responses to microbial agonist in very preterm fetuses using a chronically catheterised sheep model of pregnancy.

Methods: Fetal tracheal, jugular and amniotic fluid catheters were placed in 18 ewes carrying a singleton fetus at $92 \mathrm{~d}$ gestation (term $=150 \mathrm{~d}$ ). After recovery, animals were randomised to either: i) $10 \mathrm{mg}$ intraamniotic E.coli lipopolysaccharide (LPS; $\mathrm{n}=9)$; or ii) saline $(\mathrm{n}=9)$. Samples were collected for $2 \mathrm{~d}$ before 
euthanasia. Inflammatory signalling was measured with ovinespecific microarray, ELISA, and qPCR.

Results: LPS exposure generated robust tissue inflammation and significant $(\mathrm{p}<0.05)$ increases in amniotic fluid, tracheal and fetal plasma cytokine protein concentrations by $8 \mathrm{~h}$. Cytokine and chemokine mRNA expression was unchanged in the fetal blood at 48h; pathway analysis identified an interleukin-6 signalling response, characterised by cell cycle activation and proliferation.

Conclusion: The acute fetal systemic inflammation associated with preterm birth and fetal injury is driven by tissues exposed to the amniotic environment.

4932

\section{Consistency between different determinations of gestational age}

Kuruvilla Joseph Abraham ${ }^{1}$, Ricardo Carvalho Cavalli ${ }^{2}$, Viviane Cunha Cardoso ${ }^{3}$, Heloisa Bettiol ${ }^{4}$, Marco Antonio Barbieri $^{5}$, Manoel Romeu Gutierrez ${ }^{6}$, Antonio Augusto Moura da Silva ${ }^{7}$, Vanda Maria Ferreira Simōes ${ }^{8}$

${ }^{1}$ Estacio ICentro Universatio Uniseb, Ribeirao Preto, Brazil; ${ }^{2}$ Department of Gynaecology, University of Sao Paulo Ribeirao Preto, Brazil; ${ }^{3}$ Department of Pediatrics, University of Sao Paulo, Ribeirao Preto, Brazil; ${ }^{4}$ Department of Pediatrics, University of Sao Paulo, Ribeirao Preto, Brazil; ${ }^{5}$ Department of Pediatrics, University of Sao Paulo, Ribeirao Preto, Brazil; ${ }^{6}$ Department of Pediatrics, University of Sao Paulo, Ribeirao Preto, Brazil; ${ }^{7}$ Dept. of Public Health, Federal University of Maranhao, Brazil; ${ }^{8}$ Dept. of Public Health, Federal University of Maranhao, Brazil

Research Question: Low Gestational Age is an indicator of health problems in childhood and beyond. Gestational Age determination via the reported date of the last menstrual period (LMP) is somewhat unreliable, more reliable methods such as ultrasound are not always available. It is worth checking the consistency between these methods.

Methods: The data were obtained as part of a study of a birth cohort during the year 2010 in the region around Ribeirão Preto, Brazil. Gestational ages using both methods were available for 4319 of the 7702 children in the cohort. The study was approved by the local Committee for Ethics in Research. We discretize the 4319 gestational ages into three categories, $<37$ weeks, between 37 and 42 weeks and $>42$ weeks. The gestational ages were cross classified using both methods and the consistency between methods checked using the Maxwell test for homogeneity.

Results and Conclusion: The p-value is highly significant $(<10-10)$, indicating a lack of consistency, with the bulk of the discrepancy due to the relatively large number of infants classified with gestational age $>42$ weeks by LMP. This suggests gestational ages obtained by LMP may be overestimated.
4962

Continuing Low Rates of Obesity in young Australian Aboriginal adults born Growth Restricted

Gurmeet Singh $^{1}$, Belinda Davison ${ }^{2}$, Susan Sayers ${ }^{3}$

${ }^{1}$ Menzies School of Health Research; ${ }^{2}$ Menzies School of Health

Research; ${ }^{3}$ Menzies School of Health Research

Research question: To assess the development of overweight and obesity in young Australian Aborigines who were growth restricted at birth.

Methods: Findings from a prospective longitudinal birth cohort of 686 Australian Aboriginal people living in the Northern Territory at wave-3 (16-20 years) and wave-4 (23-28 years). Anthropometric measurements at birth and follow-up were collected directly from participants.

Results: Rates of FGR (less than 10th centile birth weight for gestational age) were high (25.9\%). Rates of obesity were $7.4 \%$ at wave- 3 and $14.5 \%$ at wave- 4 . Prevalence of obesity in nonFGR was higher than FGR: $10.3 \%$ versus $1.0 \%$ at wave-3 and increased to $15.5 \%$ versus $2.6 \%$ at wave- 4 . Overweight showed a similar trend; $20.3 \%$ versus $8.4 \%$ at wave- 3 and $24.6 \%$ versus $15.4 \%$ at wave- 4 . This pattern was also reflected in waist and fat percentage measures.

Conclusion: Rates of obesity were remained low over time, especially in FGR, in contrast to results from other longitudinal studies. The continuing low rates of obesity at this age offer a window of opportunity to target strategies to prevent obesity and possibly adult chronic disease in this population at very high risk of metabolic, renal and cardiovascular disease leading to premature mortality.

\section{5}

Cumulative pregnancy anxiety and preterm birth: the role of resiliency factors

Dr. Suzanne Tough ${ }^{1}$, Dr. Sheila McDonald ${ }^{2}$

${ }^{1}$ University of Calgary; ${ }^{2}$ University of Calgary

Research question: Investigate the relationship between cumulative pregnancy anxiety (CPA) and the risk of early preterm (EP;

\section{6}

Intrauterine growth restriction (IUGR) is associated with impairment of memory related to food and differential brain activation in response to palatable food images in adolescents

Amanda Brondani Mucellini ${ }^{1}$, Roberta Dalle Molle ${ }^{2}$, Danitsa Marcos Rodrigues ${ }^{3}$, Roberta Sena Reis ${ }^{4}$, Tânia Diniz Machado 5 , Luciano Minuzzi ${ }^{6}$, Alexandre Rosa Franco ${ }^{7}$, Augusto Buchweitz ${ }^{8}$, Andressa Bortoluzzi ${ }^{9}$, Rudineia Toazza ${ }^{10}$, 
Giovanni Abrahão Salum Júnior ${ }^{11}$, Gisele Gus Manfro ${ }^{12}$, Patrícia Pelufo Silveira ${ }^{13}$

${ }^{1} P P G$ of Medical Sciences: Psychiatry of UFRGS,

Porto Alegre, Brazil; ${ }^{2} P P G$ of Health of Child and Adolescent of

UFRGS, Porto Alegre, Brazil; ${ }^{3} P P G$ of Neuroscience of UFRGS,

Porto Alegre, Brazil; ${ }^{4} P P G$ of Health of Child and Adolescent of

UFRGS, Porto Alegre, Brazil; ${ }^{5} P P G$ of Health of Child and

Adolescent of UFRGS, Porto Alegre, Brazil; ${ }^{6}$ Department of

Psychiatry and Behavioural Neurosciences, McMaster

University, Hamilton, Canada ; ${ }^{7}$ Ins Cer, PPG of Medicine and

Health Sciences and PPG of Electrical Engineering of PUCRS, Porto Alegre, Brazil; ${ }^{8}$ InsCer, PPG of Medicine and Health Sciences and PPG of Letters of PUCRS, Porto Alegre, Brazil; ${ }^{9} P P G$ of

Neuroscience of UFRGS, Porto Alegre, Brazil;

${ }^{10} P P G$ of Neuroscience of UFRGS, Porto Alegre, Brazil; ${ }^{11} P P G$ of Medical Sciences: Psychiatry of UFRGS, Porto Alegre, Brazil; ${ }^{12} P P G$ of Medical Sciences: Psychiatry and PPG of Neuroscience of UFRGS, Porto Alegre, Brazil; ${ }^{13}$ PPG of Health of Child and Adolescent and PPG of Neuroscience of UFRGS, Porto Alegre, Brazil

IUGR leads to typical food preferences and this can in part explain their increased risk for developing metabolic disorders. Could the impairment of memory related to food be implied? 69 adolescents performed an implicit memory test related to food responding which snack they would choose if they could eat it. They did not know but one of the snacks was what they had eaten months before. They performed a brain fMRI visualizing high, low palatable foods and neutral objects. External eating behavior was assessed using Dutch Eating Behavior Questionnaire. 24 of participants had IUGR and they recognized their own snack with less frequency than non-IUGR group $(P=0.01)$. For those who did not recognize the snack, the lower BWR, the more active is cuneus and less active is precuneus, middle and inferior frontal gyri when visualizing palatable foods (PFWE <0.05). For boys who did not recognize it, the lower $B W R$, the higher score of external eating $(B=-10,135$; $\mathrm{P}<0.01)$. Memory related to food may be associated with distinct feeding behavior in IUGR and brain activation changes in regions involved in visuospatial processing, memory, expectation of reward and consciousness. IUGR may interact with memory decline, leading to a higher external eating.

\section{8}

Fetal growth interacts with multilocus genetic score reflecting dopamine signaling capacity and predicts spontaneous palatable food intake in children

Patrícia Pelufo Silveira ${ }^{1}$, Hélène Gaudreau ${ }^{2}$, Leslie Atkinson ${ }^{3}$, Alison S. Fleming ${ }^{4}$, Marla B. Sokolowski ${ }^{5}$, Meir Steiner ${ }^{6}$, James L. Kennedy ${ }^{7}$, Laurette Dubé ${ }^{8}$, Robert D. Levitan ${ }^{9}$, Michael J. Meaney ${ }^{10}$

${ }^{1}$ UFRGS, Porto Alegre/RS, Brazil; ${ }^{2}$ McGill University, Montreal, Quèbec, Canada; ${ }^{3}$ Ryerson University, Toronto, Canada;

${ }^{4}$ University of Toronto, Canada; ${ }^{5}$ University of Toronto, Canada;
${ }^{6}$ McMaster University, Hamilton, Canada; ${ }^{7}$ University of Toronto, Canada; ${ }^{8}$ McGill University, Montreal, Quèbec, Canada; ${ }^{9}$ University of Toronto, Canada; ${ }^{10}$ McGill University, Montreal, Quèbec, Canada

Research question: Intrauterine growth restriction(IUGR) leads to increased preference for palatable foods at different ages. In rodents, we demonstrated that poor striatal dopamine signaling may be involved in this behavior. Here we verified if a multilocus genetic score reflecting dopamine signaling capacity predicts spontaneous palatable food intake in IUGR children. Methods: 222 four-year old children from the MAVAN project were classified according to the birth weight ratio(observed $\mathrm{BW} /$ mean populational $\mathrm{BW}$ - sex and gestational age specific; IUGR if less than0.85). They were administered a snack test meal (milk, cheese, bread, milk, orange juice, apple, fibre cereal)containing palatable foods(chocolate muffin, croissant, sugared cereal) and genotyped for polymorphisms associated with low dopamine signaling capacity(TaqIA-A1 allele, DRD2-141CIns/Ins, DRD47-repeat, DAT1-10-repeat, Met/ Met-COMT); a composite score was calculated reflecting the total number of the five genotypes.

Results: GLM shows an interaction between IUGR status and the multilocus score on palatable food intake $(\mathrm{p}=0.03)$ and sugar in $\operatorname{grams}(\mathrm{p}=0.006)$ during the Snack Test.In the IUGR group only, poor dopamine signaling is associated with increased intake of palatable foods and sugar,but not total caloric intake or other macronutrients. There was no effect/ interactions with sex or BMI.

Conclusions: The genetic profile associated with poor dopamine signaling predicts palatable food intake in IUGR children. The results may have important implications for obesity prevention.

\section{2}

Comparable levels of eNOS in umbilical and systemic arteries of IUGR-derived endothelial cells of guinea pig fetuses at term

Bernado Krause ${ }^{1}$, Emilio A. Herrera ${ }^{2}$, Farah Díaz, ${ }^{3}$, Esteban Figueroa ${ }^{4}$, Cristián Villanueva ${ }^{5}$, Marcelo Farías ${ }^{6}$, Paola Casanello ${ }^{7}$

${ }^{1}$ Division of Obstetrics and Gynecology, School of Medicine, Pontificia Universidad Católica de Chile, Santiago, Chile;

${ }^{2}$ Division of Obstetrics and Gynecology, School of Medicine, Pontificia Universidad Católica de Chile, Santiago, Chile;

${ }^{3}$ Division of Obstetrics and Gynecology, School of Medicine, Pontificia Universidad Católica de Chile, Santiago, Chile;

${ }^{4}$ Division of Obstetrics and Gynecology, School of Medicine, Pontificia Universidad Católica de Chile, Santiago, Chile;

${ }^{5}$ Division of Obstetrics and Gynecology, School of Medicine, Pontificia Universidad Católica de Chile, Santiago, Chile;

${ }^{6}$ Division of Obstetrics and Gynecology, School of Medicine, Pontificia Universidad Católica de Chile, Santiago, Chile;

${ }^{7}$ Division of Obstetrics and Gynecology, School of Medicine, Pontificia Universidad Católica de Chile, Santiago, Chile 
IUGR relates with altered umbilico-placental vascular reactivity and increased postnatal cardiovascular risk. However, it is unknown whether these vascular changes reflect alterations in the endothelial function of systemic arteries in the IUGR fetus. We aimed to determine if IUGR-induced effects on NOSdependent relaxation and eNOS expression in umbilical and fetal systemic arteries.

IUGR was induced at mid gestation in guinea pig sows. At day 63 fetuses were euthanized, weighted and dissected. NOdependent vasoactive responses to SNP (NO donor), insulin or acetylcholine were assessed by wire myography in umbilical, aorta, carotid and femoral arteries. Expression of eNOS in endothelial cells was determined by qPCR.

Gradual uterine artery occlusion decreased fetal growth and increased placental vascular resistance. IUGR umbilical arteries showed a lower insulin-induced relaxation $(4.7 \pm 2.8 \% \mathrm{KCl}$ IUGR vs. $20.6 \pm 4.6 \% \mathrm{KCl} \mathrm{C)}$, and increased sensitivity to SNP. IUGR aortas showed a decreased sensitivity $(5.86 \pm 0.05$ IUGR vs. $6.89 \pm 0.13 \mathrm{C})$ to acetylcholine, and higher relaxation to SNP $(103.5 \pm 5.3 \% \mathrm{KCl}$ IUGR vs. $70.6 \pm 9.5 \% \mathrm{KCl} \mathrm{C})$ compared to controls. Similar results were observed in carotid and femoral arteries. These alterations in vasodilator function was associated with changes in eNOS.

In the IUGR endothelial function is heterogeneously affected at different vascular levels however eNOS expression seems be programmed in a uniform manner across vascular system.

\section{3}

Foetal macrosomia resulting from high pre-gestational weight in guinea pig dams associates with changes in vascular function

Bernardo Krause ${ }^{1}$, Herrera $\mathrm{EA}^{2}$, Díaz $\mathrm{F}^{3}$, Farías $\mathrm{M}^{4}$, Uauy $\mathrm{R}^{5}$, Casanello $\mathrm{P}^{6}$

${ }^{1}$ Division of Obstetrics \& Gynecology, 2Division of Pediatrics, Faculty of Medicine, Pontificia Universidad Católica de Chile, Santiago-Chile; ${ }^{2}$ Division of Obstetrics \& Gynecology, 2Division of Pediatrics, Faculty of Medicine, Pontificia Universidad Católica de Chile, Santiago-Chile; ${ }^{3}$ Division of Obstetrics \& Gynecology, 2Division of Pediatrics, Faculty of Medicine, Pontificia Universidad Católica de Chile, Santiago-Chile; ${ }^{4}$ Division of Obstetrics \& Gynecology, 2Division of Pediatrics, Faculty of Medicine, Pontificia Universidad Católica de Chile, SantiagoChile; ${ }^{5}$ Division of Obstetrics \& Gynecology, 2Division of Pediatrics, Faculty of Medicine, Pontificia Universidad Católica de Chile, Santiago-Chile; ' Division of Obstetrics \& Gynecology, 2Division of Pediatrics, Faculty of Medicine, Pontificia Universidad Católica de Chile, Santiago-Chile

Maternal obesity during pregnancy associates with long term cardiovascular risk in the offspring.

Aim: To determine whether increased pre-gestational weight in guinea pig sows results on altered foetal vascular reactivity in foetal systemic and umbilical arteries.
Methods: Sows were classified as control or high-weight (HW) according to their pre-mating weight, and their foetuses extracted at 0.9-gestation. Segments of foetal carotid, femoral and umbilical arteries were mounted in a wire-myograph, where the contractile response to $\mathrm{KCl}$ and the relaxation to NOS-dependent agents and NO were determined.

Results: Foetuses from HW sows were grouped according to their body weight as low (LFW, range 56-74.8g) or high (HFW, range $88.5-107.7 \mathrm{~g}$ ) foetal weight, based on the confidence interval $(76.5-84.9 \mathrm{~g})$ of the control group. No foetuses from HW sows were observed in the normal range. Umbilical arteries from HFW foetuses presented a lower response to $\mathrm{KCl}$ and insulin compared to controls, but a comparable response to SNP. Conversely carotid and femoral arteries from HFW foetuses showed an increased response to $\mathrm{KCl}$ and acetylcholine, altered eNOS-induced relaxation and sensitivity to NO donors.

Conclusion: Maternal obesity associates with increased risk of altered foetal growth and vascular function at term in guinea pigs.

\section{5}

Inflammatory markers mediating pregnant secondhand smoke exposure causing full-term low birth weight

Zhongzheng Niu ${ }^{1}$, Chuanbo Xie ${ }^{2}$, Xiaozhong Wen ${ }^{3}$, Fuying Tian ${ }^{4}$, Peng Ding 5 , Yanhui $\mathrm{He}^{6}$, Jianmiao Lin ${ }^{7}$, Shixin Yuan ${ }^{8}$, Xiaoling Guo ${ }^{9}$, Deqin Jia ${ }^{10}$, Weiqing Chen ${ }^{11}$

${ }^{1}$ Sun Yat-sen University; ${ }^{2}$ Sun Yat-sen University; ${ }^{3}$ State University of New York at Buffalo; ${ }^{4}$ Sun Yat-sen University; ${ }^{5}$ Sun Yat-sen University; ${ }^{6}$ Guangdong Provincial Center for Disease Control and Prevention; 'Shenzhen Women and Children's Hospital; ${ }^{8}$ Shenzhen Women and Children's Hospital; ${ }^{9}$ Foshan Women and Children's Hospital; ${ }^{10}$ Foshan Women and Children's Hospital; ${ }^{11}$ Sun Yat-sen University

Research question: Maternal secondhand smoke (SHS) during pregnancy increased maternal systemic inflammation and decreased placental weight, and both of them were associated with full-term low birth weight (LBW). This study aims to test mediation role of maternal inflammatory in maternal SHS leading to LBW.

Methods: 195 full-term LBW and 196 normal birth weight mother-newborn pairs were enquired about maternal SHS during pregnancy and socio-demographics in Shenzhen and Foshan Maternal and Child Hospital of China. Birth weight was measured. Maternal serum tumor necrosis factor- $\alpha$ (TNF$\alpha$ ), interleukin-1 $\beta$ (IL-1 $\beta$ ) and soluble vascular cell adhesion molecule-1 (VCAM-1) were measured by flow cytometry. Structural equation model was fitted to explore the mediation role.

Results: A good-fitted model was achieved (RMSEA =0.044, $\mathrm{GFI}=0.995$, IFI $=0.979$, and $\mathrm{CFI}=0.976)$. It indicated a plausible pathway that maternal gestational SHS exposure might first irritate the elevation of pro- inflammatory markers 
of TNF- $\alpha$, IL- $1 \beta$, which in turn affected the level of inflammatory mediators of VCAM-1 and CRP through IL-6; further, triggered inflammation lessened placental weight; at last, the lessened placenta along with aberrant levels of inflammatory markers lead to LBW.

Conclusion: Pregnant SHS may firstly lead to inflammation that subsequently spoil the placenta and ultimately causes LBW.

\section{0}

Changes in vegetables, fruits and berries consumption habits from pre-pregnancy to early pregnancy among Norwegian women

Elling Bere ${ }^{1}$, Linda Reme Sagedal ${ }^{2}$, Ingvild Vistad ${ }^{3}$, Nina Cecilie Øverby ${ }^{4}$

${ }^{1}$ Public Health, Sports and Nutrition; ${ }^{2}$ Sorlandet Hospital;

${ }^{3}$ Sorlandet Hospital; ${ }^{4}$ Public Health, Sports and Nutrition

Research question: Are there changes in consumption of vegetables, fruits and berries from pre-pregnancy to early pregnancy among Norwegian women?

Methods: Nulliparous women aged $\leqslant 18$ years with a singleton pregnancy and a BMI $\geqslant 19$, were consecutively recruited from April 2010 to January 2013 at primary health clinics in Southern Norway. At inclusion, in gestational week 16 (median, 9-20), 577 women reported how often they consumed various vegetables, fruits and berries at present, and in retrospect how often they consumed various vegetables, fruits and berries before they got pregnant. Changes in consumption were analysed with repeated measure analysis.

Results: The percentage of women reporting eating fruits and vegetables as in between meals (15\% v. 33\%), eating vegetables on their sandwich (15\% v. 19\%), eating apples, oranges, pears or bananas $(27 \%$ v. $46 \%)$ or eating other fruits and berries $(9 \%$ v. $15 \%)$ daily or more frequent all increased significantly from pre-pregnancy to early pregnancy ( $\mathrm{p}<0.001$ for all items), while the percentage of women who reported at least daily consumption of vegetables with dinner (24\% v. $24 \%)$ were stable.

Conclusion: There is a significant increase in consumption of vegetables, fruits and berries.

\section{2}

A comparative analysis of adolescent nutrition knowledge and socioeconomic status at an urban versus a peri-urban school in Hilton, South Africa

Frederick Veldman ${ }^{1}$, Susanna Kassier ${ }^{2}$

${ }^{1}$ University of KwaZulu Natal; ${ }^{2}$ University of KwaZulu Natal

Research Question: Does socioeconomic status, gender or ethnicity impact on the nutrition knowledge of South African adolescents?
Methods: A cross-sectional descriptive survey was conducted among 98 grade 9-11 learners from an urban high school and 111 grade 9-11 learners from a peri-urban high school in Hilton, KwaZulu Natal. A nutrition knowledge questionnaire was used to assess nutrition knowledge of learners. A sociodemographic questionnaire was used to collect information on parental education, employment and household data.

Results: The urban school had a higher mean nutrition knowledge scores than the peri-urban school. Significantly higher nutrition knowledge scores were observed in adolescents with educated mothers and full-time employed fathers $(\mathrm{p}<0.05)$, which was greater in the urban school than the peri-urban school. In the urban school, Black students had a significantly higher mean nutrition knowledge scores than White students $(\mathrm{p}<0.05)$. No significant differences in nutrition knowledge scores were found between age and gender in both schools.

Conclusion: A disparity in nutrition knowledge exists between adolescents from the urban and peri-urban school; but not according to age, gender or ethnicity. This indicates that socioeconomic status has an impact on nutrition knowledge. Adolescents from poorer backgrounds may benefit from nutrition education programs within the school setting.

\section{5}

Sitting time in pregnancy is associated with greater gestational weight gain, depressive symptoms and risk of gestational diabetes

Nithya Sukumar ${ }^{1}$, Jacqueline Farmer ${ }^{2}$, Hema Venkataraman ${ }^{3}$, Alexander Maraveyas ${ }^{4}$, Alex Shields ${ }^{5}$, Ponnusamy Saravanan ${ }^{6}$

${ }^{1}$ Warwick Medical School, University of Warwick, UK;

${ }^{2}$ George Eliot Hospital NHS Trust, Nuneaton, UK; ${ }^{3}$ Warwick Medical School, University of Warwick; ${ }^{4}$ Warwick Medical School, University of Warwick; ${ }^{5}$ Warwick Medical School, University of Warwick; ${ }^{6}$ Warwick Medical School, University of Warwick

Research Question: What is the level of physical activity in pregnancy and its relationship to glucose tolerance and depression?

Methods: A sub-study on physical activity during pregnancy was conducted within the longitudinal PRiDE study. Physical activity was assessed by the International Physical Activity Questionnaire (IPAQ) and depression by the PhQ-9 questionnaire.

Results: Completed questionnaires were obtained from women in the late first (mean gestation $12+3$ weeks, $\mathrm{n}=1263$ ) and second trimesters $(26+4$ weeks, $n=982)$. The frequency of doing any vigorous or moderate physical activity and walking for more than 10 mins/day were $18.6 \%, 38.9 \%$ and $83.7 \%$ in visit 1 and $23.8 \%, 50.9 \%$ and $96.9 \%$ in visit 2 respectively. Corresponding sitting times were 5.7 and 5.5 hours/day. In early pregnancy, sitting time was the only predictor of walking $(\beta=-$ $0.40, p=0.01)$ and longer sitting associated with onset of gestational diabetes later on ( 6.5 vs 5.7 hours, $\mathrm{p}=0.05)$. At visit 2 , duration of sitting per day was predicted by gestational weight 
gain $(\mathrm{GWG})(\beta=0.23, \mathrm{p}=0.01)$ and higher level of depression $(\beta=0.20, p<0.05)$ after correcting for age, BMI and socioeconomic status.

Conclusion: Self-reported sedentary behaviour is associated with depressive symptoms and GWG. If proven in objective assessments, reducing sitting time could improve metabolic risk in pregnancy.

\section{9}

Is smoking during pregnancy adversely affecting offspring bone development?

Sharon Brennan-Olsen ${ }^{1}$, John Wark ${ }^{2}$, David Moloney ${ }^{3}$, Kathy Bennett ${ }^{4}$, Julie Pasco ${ }^{5}$

${ }^{1}$ Deakin University \& University of Melbourne; ${ }^{2}$ University of Melbourne; ${ }^{3}$ Deakin University; ${ }^{4}$ Deakin University; ${ }^{5}$ Deakin University \& University of Melbourne

Research Question: Does smoking during pregnancy compromise offspring bone development in utero?

Methods: Pregnant women were recruited from the Geelong Hospital antenatal clinic before 16 weeks gestation (2002-04; $\mathrm{n}=475)$. Gestational maternal smoking status, anthropometric measures, dietary information and serum 25hydroxyvitamin D (25OHD) were collected. At birth, infant crown-heel length was measured using an Ellard newborn lengthboard $(n=393)$ and knee-heel length was measured using a handheld BK5 knemometer $(n=391)$.

Results: Infants of mothers who smoked had a lighter birthweight [median IQR: $3.39(3.11-3.77)$ vs $3.55(3.22-3.96) \mathrm{kg}$, $\mathrm{p}=0.02]$, shorter crown-heel length [49.5 (47.5-50.5) vs. 50.9 (49.0-52.0) cm, p < 0.0001], and shorter knee-heel length [84.41 (79.20-88.57) vs. $86.82(80.96-91.51) \mathrm{mm}, \mathrm{p}=0.01]$. Atrend was observed for shorter gestation [39 (38-40) vs. 40 (39-41) wk, $\mathrm{p}=0.09)$. When adjusted for maternal height, associations persisted for a shorter crown-heel length $(-1.11, \mathrm{SE} 0.32, \mathrm{p}=0.001)$ and a trend remained for lighter birth-weight $(\beta-0.130$, SE 0.07) and shorter knee-heel length (-2.1 SE 1.1) (both $p \leqslant 0.08)$. Furthermore, after adjustment for maternal height, dietary calcium and gestation length, associations with infant crown-heel length persisted (-0.86, SE 0.29, $\mathrm{p}=0.003)$; this was not confounded by maternal serum $25 \mathrm{OHD}$.

Conclusion: Maternal smoking during pregnancy may be detrimental to bone development in utero.

\section{1}

Alcohol consumption, smoking and physical exercise before and during pregnancy

Ana Maria Escobar ${ }^{1}$, Murilo Wadt ${ }^{2}$, André Covic ${ }^{3}$, Carolina Hofmeister Mansu ${ }^{4}$, Filumena Gomes ${ }^{5}$, Maria Helena Valente $^{6}$, Grisi Sandra ${ }^{7}$, Joana Abucham Ribeiro ${ }^{8}$

${ }^{1}$ University of Sao Paulo Medical School; ${ }^{2}$ University of São Paulo, School of Economics, Business Administration and
Accounting; ${ }^{3}$ State University of Campinas, Computer Science Institute and Institute of Philosophy and Human Sciences; ${ }^{4}$ Universtity of Sao Paulo School of Medicine; ${ }^{5}$ University of Sao Paulo Medical School; ${ }^{6}$ University of Sao Paulo Medical School;

${ }^{7}$ University of Sao Paulo Medical School; ${ }^{8}$ AJR Heath Education

Research question: Health information in a social network and pregnancy can change habits of pregnant women?

Methods: 649 pregnant women are followed since the first trimester of pregnancy, in a closed group within Facebook, an online social network. They accepted the terms and conditions, and filled out an online form. Participation is on a voluntary basis. Five structured questionnaires are used with the pregnant women's habits and health information. Every day we provide online behavior-changing health information, enabling women to go through a healthier and more stress-free pregnancy.

Results: 347 pregnant women completed the questionnaire. Physical exercises: From 154 women who did not exercise before pregnancy, 25 (16.2\%) of them started to work out after pregnancy. From 222 women who exercised before pregnancy, 116 (52.3\%) stopped exercising after pregnancy.

Smoking: From 25 people who smoked before pregnancy, 14 stopped smoking during pregnancy (56.0\%).

Alcohol consumption: From 182 people who drank alcohol before pregnancy, $163(89.6 \%)$ stopped drinking. In all three situations, we observed McNemar $\mathrm{p}<0.001$.

Conclusions: Pregnancy had an impact on the reduction of physical activity, smoking and alcohol consumption. The social network can be used as a tool to promote health.

\section{1}

Pregnancy planning status and its association with maternal characteristics and health during pregnancy

Tuck Seng Cheng ${ }^{1}$, See Ling Loy ${ }^{2}$, Yin Bun Cheung ${ }^{3}$, Jerry Kok Yen Chan, Keith Godfrey ${ }^{4}$, Peter Gluckman ${ }^{5}$, Kenneth Kwek ${ }^{6}$, Seang Mei Saw ${ }^{7}$, Yap-Seng Chong ${ }^{8}$, Yung Seng Lee ${ }^{9}$, Fabian Yap ${ }^{10}$, Ngee Lek ${ }^{11}$

${ }^{1}$ KK Women's and Children's Hospital, Singapore; ${ }^{2}$ KK Women's and Children's Hospital, Singapore; ${ }^{3}$ Duke-NUS Graduate Medical School, Singapore; ${ }^{4}$ University of Southampton, Southampton, UK; ${ }^{5}$ Agency for Science, Technology and Research, Singapore; 'KK Women's and Children's Hospital, Singapore;

${ }^{7}$ National University of Singapore, Singapore; ${ }^{8}$ National University of Singapore, Singapore; ${ }^{9}$ National University of Singapore, Singapore; ${ }^{10}$ KK Women's and Children's Hospital, Singapore; ${ }^{11} K K$ Women's and Children's Hospital, Singapore

Research question: Health behaviour may differ between mothers with and without pregnancy planning. This study aimed to describe differences in maternal characteristics and health across pregnancy planning status.

Methods: In a Singapore birth cohort study, maternal characteristics before and during pregnancy were captured at regular 
visits. At 26-28 weeks of gestations, mothers' responses about whether their pregnancy was planned and their level of enthusiasm towards current pregnancy were classified into planned, mistimed (unplanned but enthusiastic) and unintended (unplanned and unenthusiastic) pregnancies. Univariate analyses were performed to explore differences between groups.

Results: Of 1152 recruited mothers, 861 (481 planned, 334 mistimed and 46 unintended pregnancies) were included in this study. Compared to mothers of planned pregnancy, mothers of mistimed and unintended pregnancies attained a lower educational level, reported lower monthly household income and folic acid supplement intake, had higher exposure to secondhand smoke and were heavier. Moreover, more mothers of mistimed pregnancy were single, Malay and had pre-existing hypertension but not gestational diabetes, whereas more mothers of unintended pregnancy were older, anxious and had pre-existing Type 2 diabetes.

Conclusion: Differences in socio-demographic and lifestyles between planned, mistimed and unintended pregnancies exist and could affect the outcomes of their offspring.

\section{5}

Does timing of energy intake affect glucose intolerance during pregnancy?

See Ling Loy ${ }^{1}$, Tuck Seng Cheng ${ }^{2}$, Marjorelee T. Colega ${ }^{3}$, Yin Bun Cheung ${ }^{4}$, Keith Godfrey ${ }^{5}$, Peter Gluckman ${ }^{6}$, Kenneth $\mathrm{Kwek}^{7}$, Seang Mei Saw ${ }^{8}$, Yap-Seng Chong ${ }^{9}$, Ngee Lek ${ }^{10}$, Fabian Yap $^{11}$, Mary Foong-Fong Chong ${ }^{12}$, Jerry Kok Yen Chan ${ }^{13}$

${ }^{1}$ KK Women's and Children's Hospital, Singapore; ${ }^{2}$ KK Women's and Children's Hospital, Singapore; ${ }^{3}$ Agency for Science, Technology and Research, (A*STAR), Singapore; ${ }^{4}$ Duke-NUS Graduate Medical School, Singapore; ${ }^{5}$ University of Southampton, Southampton, UK; ${ }^{6}$ Agency for Science, Technology and Research, Singapore; 'KK Women's and Children's Hospital, Singapore; ${ }^{8}$ National University of Singapore, Singapore; ${ }^{9}$ National University of Singapore, Singapore; ${ }^{10} \mathrm{KK}$ Women's and Children's Hospital, Singapore; ${ }^{11} K K$ Women's and Children's Hospital, Singapore; ${ }^{12}$ Agency for Science, Technology and Research, (A $\left.{ }^{*} S T A R\right)$, Singapore; ${ }^{13}$ KK Women's and Children's Hospital, Singapore

Research question: Little is known about the effect of circadian rhythm on glucose regulation in pregnant women. We examined the role of maternal meal timing and weight status on glucose intolerance during pregnancy.

Methods: In a prospective cohort study of 985 pregnant women in Singapore, maternal fasting glucose, 2-hour glucose concentrations and 24-hour dietary recalls were collected at 2628 weeks' gestation. Women were categorised as day-time and night-time eaters according to consumption of higher proportion of calories during daylight (0700-1759h) or nightfall (1800-0659h) respectively. Women were defined as lean (BMI $\left.<23 \mathrm{~kg} / \mathrm{m}^{2}\right)$ or overweight $\left(\mathrm{BMI} \geqslant 23 \mathrm{~kg} / \mathrm{m}^{2}\right)$. Multivariate linear regression analyses were performed.
Results: There were $838 \quad(85.1 \%)$ day-time eaters and 147 (14.9\%) night-time eaters. Lean night-time eaters had higher fasting glucose $(\beta=0.12 \mathrm{mmol} / \mathrm{l} ; 95 \% \mathrm{CI}=0.03$, $0.20)$ than lean day-time eaters but no association was found for 2-hour glucose $(\beta=-0.11 \mathrm{mmol} / \mathrm{l} ; 95 \% \mathrm{CI}=-0.45$, 0.22 ). No difference in fasting and 2-hour glucose was observed between day-time and night-time eaters in overweight women.

Conclusion: Consuming higher proportion of calories at night is associated with higher fasting glucose in lean but not in overweight pregnant women.

\section{1}

\section{A lifestyle intervention including an exercise program helps women increase physical activity level in pregnancy}

Birgitte Sanda ${ }^{1}$, Linda Reme sagedal ${ }^{2}$, Ingvild Vistad ${ }^{3}$, Hilde Lohne Seiler ${ }^{4}$, Lene Anette Hagen Haakstad ${ }^{5}$, Monica Klungland Torstveit ${ }^{6}$

${ }^{1}$ Faculty of Health and Sport Sciences, University of Agder, Kristiansand, Norway; ${ }^{2}$ Department of Obstetrics and Gynecology, Sorlandet Hospital, Kristiansand, Norway; ${ }^{3}$ Department of Obstetrics and Gynecology, Sorlandet Hospital, Kristiansand, Norway; ${ }^{4}$ Faculty of Health and Sport Sciences, University of Agder, Kristiansand, Norway; ${ }^{5}$ Norwegian School of Sports Science, Oslo, Norway; ${ }^{6}$ Faculty of Health and Sport Sciences, University of Agder, Kristiansand, Norway

Research question: What is the effect of the Norwegian Fit for Delivery (NFFD) lifestyle intervention on physical activity (PA) level in pregnancy and postpartum?

Methods: The NFFD study is a randomized controlled trial in nulliparous healthy women, randomized to intervention (IG, $n=303$ ) or control group (CG, $n=303$ ). Participants in the IG were encouraged to attend two group-exercise sessions weekly and received dietary counseling twice by telephone. The CG received standard prenatal care. All participants answered the International Physical Activity Questionnaire (IPAQ) short form at gestational week 16, 36, as well as 6 and 12 months postpartum. PA level was computed in MET-min/week. Independent-sample t-tests were used to analyze data.

Results: There was no significant difference in PA level between the groups at inclusion (IG 1144.7 vs. CG 1137.0 MET-min/week, $\mathrm{p}=0.934)$. The IG increased their PA level significantly more than the CG at gestational week 36 (454.0 vs. 92.2 MET-min/week, $\mathrm{p}=0.005)$. No significant differences in PA level were observed between the groups at 6 months (931.9 vs. $769.2 \mathrm{MET}-\mathrm{min} /$ week, $\mathrm{p}=0.274$ ) or 12 months (689.0 vs. 466.5 MET-min/week, $\mathrm{p}=0.144$ ) postpartum compared to time at inclusion.

Conclusion: The NFFD lifestyle intervention increased PA level in pregnancy, but not in the postpartum period. 


\section{9}

Effect of regular exercise on blood pressure in normotensive pregnant women. A randomized controlled trial

Lene Haakstad $^{1}$, Elisabeth Edvardsen ${ }^{2}$, Kari Bø ${ }^{3}$

${ }^{1}$ Norwegian School of Sport Sciences; ${ }^{2}$ Oslo University Hospital;

${ }^{3}$ Norwegian School of Sport Sciences

Research question: The effect of regular exercise on maternal arterial blood pressure (BP) at rest and during uphill walking. Methods: Healthy, inactive nulliparous women were assigned to either exercise $(n=35)$ or control group $(n=26)$ at mean gestation week $17.6 \pm 4.2$ through a single blind, single center randomized controlled trial. The intervention included participation in two 60 minutes aerobic dance classes, plus $30 \mathrm{~min}$ utes of daily self-imposed physical activity performed for a minimum of 12 weeks. Primary outcome was mean difference in resting systolic and diastolic BP between the two groups. Secondary outcome was mean difference in systolic BP during uphill treadmill walking measured at critical power.

Results: Prior to intervention, resting systolic and diastolic BP was $115 / 66 \pm 12 / 7$ and $115 / 67 \pm 10 / 9 \mathrm{mmHg}$ in the exercise and control group, respectively. After intervention, resting systolic BP was $112 \pm 8$ in the exercise group and $119 \pm 14$ $\mathrm{mmHg}$ in the control group $(\mathrm{p}=0.03)$, whereas diastolic BP was $71 \pm 9$ and $76 \pm 8(\mathrm{p}=0.03)$, respectively. During treadmill walking at critical power, the exercise group had a lower increase in the systolic BP $(159 \pm 18$ vs. $165 \pm 21 \mathrm{mmHg})$ compared with the controls, but the difference did not reach statistical significance.

Conclusions: Aerobic exercise reduced resting blood pressure in healthy inactive pregnant women.

\section{8}

Sedentary behaviour with higher Socio-economic Status (SES) in South Asians in UK - A potential contributor of adverse metabolic risk? Evidence from the PRiDE study

Hema Venkataraman ${ }^{1}$, Selvin Selvamoni, Renjit Kurian ${ }^{2}$, Rajni Vekaria, Nithya Sukumar ${ }^{3}$

\section{${ }^{1}$ University of Warwick; ${ }^{2}$ University of Warwick; ${ }^{3}$ University of Warwick}

Research question: What are the ethnic differences in SES, anthropometry and physical activity(PA) among high-risk pregnant women in the UK?

Methods: Maternal anthropometry and demographic characteristics were obtained from the prospective, PRiDE study pregnancy cohort. Physical activity and socioeconomic status (SES) were collected via International-Physical-Activity-Questionnaire(IPAQ) and Socio-economic questionnaires.

Results: Of 1183 women, 16.7\% were South-Asians(SA) and 83.3\% White-Caucasians(WC).

SAs were older $[31.3 \pm 4.9$ vs $29.2 \pm 5.2(\mathrm{p}<0.001)]$ with lower adiposity: BMI $[25.7 \pm 5.1$ vs $32.2 \pm 6.9$ ( $\mathrm{p}<0.001)]$, waistcircumference $[86.8 \pm 11.7$ vs $102.3 \pm 16.5 \mathrm{~mm}(\mathrm{p}<0.001)]$, triceps skinfold [22.4 vs $25.7 \mathrm{~mm}(\mathrm{p}=0.02)$ ], and subscapular skinfold thickness [23.4 \pm 9.4 vs $27.2 \pm 8.1 \mathrm{~mm}(\mathrm{p}=0.02)]$.

SAs had a higher SES: Qualifications (degree and above) $[65.7 \%$ vs $39.3 \%(\mathrm{p}<0.001)]$, higher occupations $[84 \%$ vs $63.2 \%(\mathrm{p}<0.001)]$, household income $(>£ 20,800 \mathrm{pa})[70.9$ vs $59.4 \%(p=0.008)]$ and married [97.4 vs 44.4\% $(p<0.001)]$. SAs had lower PA: Walking (10 min in last 7 days) $[89.6 \%$ vs $96 \%(\mathrm{p}<0.001)]$, moderate activity [29\% vs $48.1 \%(\mathrm{p}<0.001)]$ and vigorous activity $[15.1 \%$ vs $23.4 \%(\mathrm{p}=0.014)]$.

FPG $[4.4 \pm 0.6$ vs $4.5 \pm 0.6(\mathrm{p}=0.2) \mathrm{mmol} / \mathrm{l}]$ and $2 \mathrm{hPG}$ $[5.8 \pm 1.4$ vs $5.8 \pm 1.4(\mathrm{p}=0.9) \mathrm{mmol} / \mathrm{l}]$ at $26-28$ weeks was similar in both.

Vigorous activity predicted FPG after adjustment for age, BMI, waist-circumference and height $(\mathrm{p}=0.008)$.

Conclusion: Despite higher SES, SAs exhibited more sedentary behaviour than WC. SA had similar glucose levels to obese WC's despite lower adiposity, which may, in part be due to lower levels of PA.

\section{9}

Soft drink consumption during pregnancy as a risk factor for large for gestational age birth: BRISA Birth Cohort, Brazil

Janaina Maiana Abreu Barbosa ${ }^{1}$, Antonio Augusto Moura da Silva $^{2}$, Maria Teresa Seabra S B Alves ${ }^{3}$, Vanda Maria Ferreira Simões ${ }^{4}$, Rosangela Fernandes Lucena Batista ${ }^{5}$, Marco Antonio Barbieri $^{6}$, Heloisa Bettiol ${ }^{7}$, Ricardo Carvalho Cavalli ${ }^{8}$, Viviane Cunha Cardoso ${ }^{9}$, Cecilia Claudia Costa Ribeiro ${ }^{10}$

${ }^{1}$ Public Health Post Graduate Program, Federal University of Maranhao, Brazil; ${ }^{2}$ Department of Public Health, Federal University of Maranhao, Brazil; ${ }^{3}$ Department of Public Health, Federal University of Maranhao, Brazil; ${ }^{4}$ Department of Medicine III, Federal University of Maranhao, Brazil; ${ }^{5}$ Department of Public Health, University of Maranhao, Brazil; ' Department of Pediatrics, Ribeirao Preto Medical School, University of Sao Paulo, Brazil; ${ }^{7}$ Department of Pediatrics, Ribeirao Preto Medical School, University of Sao Paulo, Brazil; ${ }^{8}$ Department of Gynecology and Obstetrics, Ribeirao Preto Medical School, University of Sao Paulo, Brazil; ${ }^{9}$ Department of Pediatrics, Ribeirao Preto Medical School, University of Sao Paulo, Brazil; ${ }^{10}$ Department of Dentistry II, Federal University of Maranhão-Brazil

Research question: This study analyzed the association between soft drink consumption during pregnancy and large for gestational age (LGA) birth.

Methods: Data from 1381 pregnant women and their newborns were analyzed in Sao Luis, Brazil. Theoretical assumptions of soft drink consumption (daily $\mathrm{x}$ weekly use) during pregnancy in association with LGA were analyzed by directed acyclic graphs. Two models were tested: 1) Total effect (TE) adjusted for confounders such as maternal age, family income, maternal education, marital status, parity, pre-pregnancy body 
mass index; 2) Direct effect (DE) adjusted for the above variables and for mediators such as junk food consumption, weight gain during pregnancy and gestational diabetes.

Results: The LGA rate was 5.5\%. High tertile of soft drink consumption was associated with LGA in TE (OR 2.80; 95\% CI 1.13-3.86) and DE (OR 2.15, 1.12-4.12). Overweight (OR 3.61, 2.09-6.22), obesity (OR 3.11, 1.33-7.23), weight gain (OR 1.90, 1.04-1.14) and diabetes (OR 3.76, 1.40-10.0) were also associated with LGA.

Conclusion: High soft drink consumption seems to be a risk factor for LGA birth, regardless of adjustment for confounders. Feeding practices during the fetal period need to be considered in order to prevent LGA births, which have shown adverse health effects along the

\section{4}

Labor physical activity during pregnancy and its association with adverse perinatal outcomes (BRISA birth cohort, Brazil)

Adriana Sousa Rego ${ }^{1}$, Maria Teresa Seabra S B Alves ${ }^{2}$, Rosangela Fernandes Lucena Batista ${ }^{3}$, Cecilia Claudia Costa Ribeiro $^{4}$, Heloisa Bettiol ${ }^{5}$, Viviane Cunha Cardoso ${ }^{6}$, Marco Antonio Barbieri ${ }^{7}$, Antonio Augusto Moura da Silva ${ }^{8}$

${ }^{1}$ Department of Public Health, Federal University of Maranhao, Brazil; ${ }^{2}$ Department of Public Health, Federal University of Maranhao, Brazil; ${ }^{3}$ Department of Public Health, Federal University of Maranhao, Brazil;; ${ }^{4}$ Department of Dentistry II, Federal University of Maranhao, Brazil; ${ }^{5}$ Department of Pediatrics, Ribeirao Preto Medical School, University of Sao Paulo, Brazil; ${ }^{6}$ Department of Pediatrics, Ribeirao Preto Medical School, University of Sao Paulo, Brazil; ${ }^{7}$ Department of Pediatrics, Ribeirao Preto Medical School, University of Sao Paulo, Brazil; ${ }^{8}$ Department of Public Health, Federal University of Maranhao, Brazil

Research question: Overloading, standing posture and long hours spent at work have been studied as risk factors for adverse perinatal outcomes. The aim of this study was to analyze the association between workload during pregnancy and adverse perinatal outcomes: low birth weight, preterm birth and intrauterine growth restriction in a sample of 5067 postpartum women from the BRISA cohort in Sao Luis, Brazil, who answered a birth questionnaire.

Methods: The workload during pregnancy was calculated based on three variables (working hours/week, work while standing up, and lifting weight at work). Three different Poisson regression models with robust adjustment of variance were used for each outcome variable, i.e., low birth weight, preterm birth and intrauterine growth restriction.

Results: The estimated workload was absent in $31.2 \%$ of cases, some load was present in $61 \%$ and all loads were present in 7.1 $\%$. There were no significant associations between workload and low birth weight (PR 0.82, 95\% CI 0.56-1.19), preterm birth (PR 0.89, 95\% CI 0.67-1.20) or intrauterine growth restriction (PR 1.13, 95\% CI 0.73-1.77).
Conclusion: The workload during pregnancy did not influence adverse perinatal outcomes such as low birth weight, preterm birth and intrauterine growth restriction.

\section{9}

The effects of maternal risk exposure during pregnancy and infant birth weight: A South African perspective

Wiedaad Slemming ${ }^{1}$, Braimoh Bello ${ }^{2}$, Linda Richter ${ }^{3}$, Haroon Saloojee ${ }^{4}$

${ }^{1}$ University of the Witwatersrand; ${ }^{2}$ School of Public Health, University of the Witwatersrand; ${ }^{3}$ Developmental Pathways to Health Research Unit, University of the Witwatersrand;

${ }^{4}$ Division of Community Paediatrics, University of the Witwatersrand

Research question: Birth weight is an important determinant of a newborn's immediate and future health. This study aimed to determine the associations between selected maternal risk factors and subsequent infant birth weight, utilising data from the Birth to Twenty Plus (Bt20+) longitudinal birth cohort study, Johannesburg, South Africa.

Methods: Exposure to nine maternal psychosocial and behavioural risk factors was assessed in 1228 women who completed an antenatal questionnaire and whose infants were delivered within a seven-week period in 1990 (the Bt20+ enrolment time).

Results: Three variables were significantly associated with infant birth weight $\mathrm{z}$-score $(\mathrm{p}<0.05)$ in the multivariate analysis. Tobacco use during pregnancy was associated with an approximate $97 \mathrm{~g}$ (95\% CI 23g, 173g); and unwanted pregnancy with an approximate $83 \mathrm{~g}(95 \%$ CI 30g, 136g) decrease in infant birth weight. Maternal parity was associated with an approximate increase in birth weight of 39g (95\% CI 19g,61g) for every additional child born to a woman. Exposure to more than one of the significant risks was associated with cumulative reductions in birth weight.

Conclusions: This study confirms that psychosocial risk exposures during pregnancy can significantly influence infant birth weight. Each of the identified risks is amenable to intervention through public health policies and programmes.

\section{1}

Nicotine exposure in early pregnancy: mid-gestational growth restriction correlates with differential mRNA and microRNA expression

Lisa Yamada ${ }^{1}$, Elizabeth Mason ${ }^{2}$, Christine Wells ${ }^{3}$, Suyinn Chong ${ }^{4}$

${ }^{1}$ Mater Research Institute - The University of Queensland; ${ }^{2}$ The University of Queensland; ${ }^{3}$ The University of Queensland; ${ }^{4}$ Mater Research Institute - The University of Queensland 
Research question: Exposure to nicotine for the entirety of gestation in animal models results in numerous phenotypic consequences in the offspring. The effect of nicotine exposure restricted to early gestation alone is poorly characterised. We aimed to determine what phenotypic and transcriptional effects moderate nicotine exposure during early gestation has on the $9.5 \mathrm{dpc}$ mouse embryo.

Methods: Pregnant C57BL/6J mice were provided with ad libitum access to $100 \mu \mathrm{g} / \mathrm{ml}$ nicotine from 0.5 to $8.5 \mathrm{dpc}$ (developmentally equivalent to the first 3-4 weeks of human gestation). Resultant embryos were assayed at $9.5 \mathrm{dpc}$ for crown-rump length and somite number, genome-wide gene expression, and microRNA expression.

Results: Crown-rump length was reduced in nicotineexposed embryos when compared to control embryos at $9.5 \mathrm{dpc}$; however there was no evidence of developmental delay. Of 105 differentially expressed genes, 21 correlated to embryonic size, of which many have known roles in growth and metabolism. 7 microRNAs differentially expressed and correlated to embryonic size also had predicted mRNA targets with roles in growth and metabolism.

Conclusion: Moderate nicotine exposure during early pregnancy, results in growth restriction at $9.5 \mathrm{dpc}$ with correlated changes in mRNAs and microRNAs predicted to be involved in growth and metabolism in the mouse.

\section{1}

Parental smoking, childhood household living conditions and adult respiratory health: findings from a multi-ethnic UK sample

Lauren Sherar ${ }^{1}$, Tom Norris ${ }^{2}$, Mark Orme ${ }^{3}$, Dale Esliger ${ }^{4}$, Andrew Kingsnorth ${ }^{5}$, Sally Singh ${ }^{6}$, Michael Steiner ${ }^{7}$, Mike Morgan ${ }^{8}$

${ }^{1}$ Loughborough University; ${ }^{2}$ University of Bristol; ${ }^{3}$ Loughborough University; ${ }^{4}$ Loughborough University; ${ }^{5}$ Loughborough University; ${ }^{6}$ University Hospitals of Leicester NHS Trust;

${ }^{7}$ University Hospitals of Leicester NHS Trust; ${ }^{8}$ University

Hospitals of Leicester NHS Trust

Scant literature has investigated the association between childhood environmental factors and adult respiratory function.

Aim: Investigate whether childhood household living conditions and parental smoking are associated with adult (40-76 years) respiratory function.

Methods: Respiratory function was measured using forced spirometry in a cross sectional sample of 404 adults $(29.2 \%$ South Asian, 45.5\% male, $24.8 \%$ diagnosed with COPD) from the UK Physical Activity and Respiratory Health (PhARaoH) Study. Childhood environmental factors were determined via the following indicators: presence/absence of bathroom, toilet, hot water, bath, shared bedroom, damp/ mould and parental smoking.
Results: After adjustment (adult SEP, current smoking status, childhood smoking exposure, COPD diagnosis and other indicators of childhood living conditions) those with access to a bath (312(77.2\%) of the sample) had a 5.8\% greater FEV1\%predicted and it was the only significant childhood household predictor. $126(31.5 \%)$ had a mother and $227(57.5 \%)$ had a father that smoked during their childhood. Maternal, but not paternal, smoking during childhood was associated with a $4 \%$ reduction in FEV1/FVC in adulthood with results persisting after adjustment.

Conclusion: Of the childhood environmental factors investigated only access to a bath in the home and maternal smoking was associated with adult lung function. Mechanisms underlying these associations need elucidating.

\section{2}

\section{A longitudinal analysis of maternal feeding practices and the diet quality of young children}

Megan Jarman ${ }^{1}$, Jane Ogden ${ }^{2}$, Rhonda Bell ${ }^{3}$, Hazel Inskip ${ }^{4}$, Wendy Lawrence ${ }^{5}$, Janis Baird ${ }^{6}$, Cyrus Cooper ${ }^{7}$, Sian Robinson $^{8}$, Mary Barker ${ }^{9}$

${ }^{1}$ MRC Lifecourse Edidemiology Unit; ${ }^{2}$ University of Surrey;

${ }^{3}$ University of Alberta; ${ }^{4} M R C$ Lifecourse Edidemiology Unit;

${ }^{5}$ MRC Lifecourse Edidemiology Unit; ${ }^{6} M R C$ Lifecourse

Edidemiology Unit; ${ }^{7}$ MRC Lifecourse Edidemiology Unit;

${ }^{8}$ MRC Lifecourse Edidemiology Unit; ${ }^{9}$ MRC Lifecourse

Edidemiology Unit

Research question: How do maternal feeding practices affect the establishment of healthy eating behaviours in early childhood?

Methods: Mothers with preschool children completed questionnaires about their use of covert and overt feeding practices, child food neophobia and demographics. The quality of children's diets was assessed using a validated food frequency questionnaire from which a standardised z-score was calculated. This questionnaire was repeated two years later. Complete data were available for 228 mother-child pairs.

Results: Mothers who used more covert practices had children with better quality diets $(\beta 0.05 \mathrm{SD}, \mathrm{P}=0.001$ at both time points). In addition, mothers who increased their use of covert practices had children whose diet quality improved ( $\beta$ 0.03 SD, $P=0.003$ ). Associations were independent of mothers' level of education. Mothers' use of overt practices was not associated with children's diet quality. However, mothers who increased their use of overt practices had children whose level of food neophobia increased $(\beta 0.12, \mathrm{P}=0.03)$ and furthermore, children with higher levels of neophobia had poorer quality diets $(\beta-0.07 \mathrm{SD}, \mathrm{P}=0.001$ at both time points).

Conclusion: Supporting mothers to adopt more covert techniques to control their children's eating habits may help to improve the quality of diets of young children. 
4970

Exercise only early in life in rats programs a healthier phenotype in adulthood

Filippe Falcao-Tebas ${ }^{1}$, Evelyn Marin ${ }^{2}$, Chelsea Arceri ${ }^{3}$, Jujiao Kuang ${ }^{4}$, David Bishop ${ }^{5}$, Glenn McConell ${ }^{6}$

${ }^{1}$ Institute of Sport, Exercise and Active Living (ISEAL), College of Sport and Exercise Science, Victoria University, Melbourne, Victoria, Australia; Institute of Sport, Exercise and Active Living (ISEAL), College of Sport and Exercise Science, Victoria University, Melbourne, Victoria, Australia; ${ }^{3}$ Institute of Sport, Exercise and Active Living (ISEAL), College of Sport and Exercise Science, Victoria University, Melbourne, Victoria, Australia; ${ }^{4}$ Institute of Sport, Exercise and Active Living (ISEAL), College of Sport and Exercise Science, Victoria University, Melbourne, Victoria, Australia; ${ }^{5}$ Institute of Sport, Exercise and Active Living (ISEAL), College of Sport and Exercise Science, Victoria University, Melbourne, Victoria, Australia; ${ }^{6}$ Institute of Sport, Exercise and Active Living (ISEAL), College of Sport and Exercise Science, Victoria University, Melbourne, Victoria, Australia; College of Health and Biomedici

Research question: Can exercise only early in life improve glucose tolerance and mitochondrial respiration in adult rats? Methods: Female Sprague-Dawley rats performed moderate intensity treadmill exercise (5 days/week, $60 \mathrm{~min} /$ day, $20 \mathrm{~m} /$ $\mathrm{min}$ ) from 5 to 9 weeks of age or remained sedentary. At 12 and 24 weeks of age ( $\mathrm{N}=9-10$ and 4 per group, respectively), an intraperitoneal glucose tolerance test was performed and plasma insulin, blood glucose and glucose area under the curve (AUC) were analyzed. At 25 weeks of age ( $\mathrm{N}=4$ per group), mitochondrial respiration (Oroboros Oxygraph-2k) was performed in plantaris muscles. Student's t test and repeatedmeasures ANOVA were performed.

Results: At 12 weeks of age exercised pups had lower (P-value).

\section{7}

The relationship between physical activity and glucose tolerance amongst pregnant women living in Soweto, South Africa

Tasneem Khan ${ }^{1}$, Estelle Watson ${ }^{2}$, Shelley Macaulay ${ }^{3}$

${ }^{1}$ University of the Witwatersrand; ${ }^{2}$ University of the

Witwatersrand; ${ }^{3}$ University of the Witwatersrand

Research Question: Physical activity levels of pregnant women and the influence on glucose tolerance.

Methods: Demographic and baseline measures collected at $<14$ weeks gestation. Time spent in moderate-vigorous physical activity (MVPA) was measured objectively using an ActiGraph accelerometer at 16-20 weeks gestation. A $75 \mathrm{~g}$ oral glucose tolerance test was administered at 24-28 weeks gestation, with samples taken at fasting, 60 and 120 minutes. The IADPSG (2010) diagnostic criterion was used to diagnose gestational diabetes mellitus (GDM). Participants were classified into active $(\geq 150 \mathrm{~min} / \mathrm{week}$ MVPA) and inactive groups ( $<150 \mathrm{~min} /$ week MVPA).

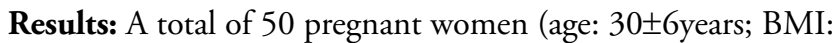
$27.2 \pm 5$; HIV+: $22 \%$ ) participated in the study. Thirty of the 50 women $(70 \%)$ were active and $30 \%$ were inactive. A normal glucose profile was observed in $86 \%$ of the women, while $14 \%$ were diagnosed with GDM. No differences were found between the MVPA, fasting glucose $(\mathrm{p}=0.418)$ and glucose tolerance at $60(\mathrm{p}=0.229)$ and $120(\mathrm{p}=0.81)$ minutes.

Conclusion: In contrast to previous studies, majority of the women were meeting the minimum activity guidelines. However, the prevalence of GDM in this small sample was quite high. In this small study physical activity levels did not provide a protective effect against GDM, or improve glucose metabolism.

\section{0}

Identifying opportunities to increase fruit and green leafy vegetable consumption in India using value chain analysis

Sarah Kehoe ${ }^{1}$, Kalyanaraman Kumaran ${ }^{2}$, Ramesh Potdar ${ }^{3}$, Aulo Gelli ${ }^{4}$, Harsha Chopra ${ }^{5}$, Caroline Fall

${ }^{1}$ University of Southampton; ${ }^{2}$ University of Southampton; ${ }^{3}$ Centre for Study of Social Change; ${ }^{4}$ IFPRI; ${ }^{5}$ Centre for Study of Social Change

Research question: A quarter of babies in India are born below $2.5 \mathrm{~kg}$ at term. Micronutrient-rich foods such as fruit and green leafy vegetables (GLV) have been associated with improved birth outcomes. Indian women of reproductive age have low intakes of these foods. We aim to identify modifiable determinants of diet and design interventions to increase intakes.

Methods: Agricultural scientists, economists and nutritionists are currently conducting a 'value chain analysis' of fruit and green leafy vegetables in an urban slum and rural villages in Maharashtra State. A 'value chain' refers to the addition of value along food supply chain for actors at different steps. This allows identification of opportunities whereby actors can benefit from marketing foods of higher nutritional value. We will collect qualitative data from policy makers, landowners, farmers, wholesalers, vendors and consumers. Primary data on determinants of fruit and GLV intake and secondary food price data will be used to calculate cost per nutrient metrics against which cost-efficiency of candidate interventions will be benchmarked.

Results and conclusion: recommendations for future interventions will be made and intervention studies initiated. These could range from policy change (eg subsidies) to introducing measures to increase consumer demand (eg reducing contamination of GLVs).

\section{2}

Dietary habits and eating practices and how they associate with obesity in black South African adolescents undergoing a health transition

Modiehi Sedibe ${ }^{1}$, PT Pisa ${ }^{2}$, A Feeley ${ }^{3}$, TM Pedro ${ }^{4}, K_{\text {Kahn }}^{5}$, SA Norris ${ }^{6}$

${ }^{1}$ Sefako Makgatho Health Sciences University; ${ }^{2}$ Sefako Makgatho Health Sciences University; ${ }^{3}$ Sefako Makgatho Health Sciences 
University; ${ }^{4}$ Sefako Makgatho Health Sciences University; ${ }^{5}$ Sefako Makgatho Health Sciences University; ${ }^{6}$ Sefako Makgatho Health Sciences University

Objectives: To compare dietary habits and eating practices between rural and urban adolescents and their associations with obesity risk.

Methods: Dietary habits, eating practices, and anthropometric measurements were collected in urban adolescents $(n=3098$, mean age $=14$ years $)$ and rural adolescents $(n=392$, mean age $=13$ years) from The Birth to Twenty (Bt20) cohort, and the Agincourt Health and Socio-demographic Surveillance System site respectively. Logistic regression was employed to examine the associations between dietary habits and eating practices, with obesity risk in both rural and urban adolescents. Results: Differences in dietary habits and eating practices by gender and between rural and urban adolescents within the home, community, and school were identified. Using the regression model that explained the most variance, among earlyadolescents (Year 13) the following dietary habits and eating practices in the home environment were associated with increased risk of being overweight and obese after adjusting for gender, site, and dietary habits and eating practices within the community and school environment: eating the main meal with family some days $(\mathrm{OR}=1.777,95 \% \mathrm{CI}=1.114-2.835 ; \mathrm{P} \leqslant 0.016)$, eating the main meal with family almost every day $(\mathrm{OR}=1.610$, $95 \% \mathrm{CI}=1.106-2.343 ; \mathrm{P} \leqslant 0.013)$, and irregular frequency of consuming breakfast on weekdays $(\mathrm{OR}=1.382,95 \% \mathrm{CI}=$ $1.007-1.896 ; \mathrm{P} \leqslant 0.045)$. For the same model, among midadolescent participants (Year 15) only the irregular frequency of consuming breakfast on weekends within the home environment $(\mathrm{OR}=1.530,95 \% \mathrm{CI}=1.099-2.129, \mathrm{P} \leqslant 0.012)$, was associated with increased risk of being overweight and obese. For both early- and mid-adolescents, being male and residing in a rural setting were associated with reduced risk of being overweight and obese.

Conclusion: Differences in dietary habits and eating practices by gender and site were observed. Only dietary habits and eating practices within the home environment were associated with increased risk of being obese and overweight.

\section{7}

The Analysis of Intake and Effects of Trans fats in relation to the risk of Cardiovascular Disease

Idowa Joseph Olaniyi ${ }^{1}$, Carole L. Marshall ${ }^{2}$

${ }^{1}$ Modibbo Adama University of Technology, Yola; ${ }^{2}$ Northumbria University, $U K$

A pilot study was conducted for the above named topic, to identify the association of trans fat intake with number of deaths from cardiovascular disease in the UK using dietary assessment. This is to understand the regional, socio-economic and ethnic variations in death numbers.

\section{Questions asked covered:}

- What was eaten?

- How much was eaten?

- How often is this?

Methods: Six participants (age range 19-64 years) from Northumbria University's School of Life Sciences (now Faculty of Health and Life Sciences) who are either Staff or Post-graduate Students took part in the study. Participants were recruited via mass email. The dietary assessments methods used are; (i) 3 Day Food Diary (ii) 24 Hour Recall and (iii) Weighed Food Intake. All participants were asked to complete all the three different dietary assessment methods.

Results: Trans fatty acid isomers (18:1, 18:2 and 18:3) were analysed in all assessments. No statistical significance (at 95\% CI) was obtained for all the three dietary assessment methods when compared with each other. P-values obtained were; 0.6337 , 0.8986 and 0.05464 for Weighed Food Intake v 24 Hour Recall, Weighed Food Intake v 3 Day Food Dairy and 24 Hours Recall v 3Day Food Dairy respectively. The comparison was deem necessary, in order to establish the appropriate dietary method.

Conclusion: 3 day food dairy was determined to be appropriate dietary method of analysing dietary trans-fat intake. The recorded intakes for 3 Day Food Dairy show the potential to provide quantitative information and are useful for dietary behaviour modification. Also, this study confirmed that it is essential to consolidate the use of dietary assessment by analysis via biomarkers.

\section{5}

How does the policy context affect the implementation of the national Healthy Start Workforce Project (HSWP)?

Anne Jaquiery ${ }^{1}$, Donna Congalton ${ }^{2}$, Mary Barker ${ }^{3}$, Wendy Lawrence ${ }^{4}$, Christine Vogel ${ }^{5}$

${ }^{1}$ Gravida, University of Auckland; ${ }^{2}$ Gravida, University of Auckland; ${ }^{3}$ MRC Lifecourse Epidemiology Unit, University of Southampton; ${ }^{4} M R C$ Lifecourse Epidemiology Unit, University of Southampton; ${ }^{5}$ MRC Lifecourse Epidemiology Unit, University of Southampton

Research question: How do policies impact implementation of a workforce education and behaviour change programme to improve the diets and lifestyles of pregnant women?

Methods: Local and national policies, resourcing structures and models of service delivery can impact health interventions. The HSWP provides an opportunity to assess how government and organisational policies affect delivery of a population-level intervention to support pregnant women adopt healthier diets and lifestyles. Drawing on MRC guidance for evaluating complex interventions, policy analysis and semi-structured interviews with the maternal and child workforce were completed to explore contextual factors facilitating or hindering intervention implementation. 
Results: National and local policy documents prioritising improving the diet and lifestyle of women and children and resources allocated to address this issue were identified. Findings from the document analysis and outputs from qualitative data analysis were used to describe organisations with optimal models of service delivery for implementing the HSWP intervention.

Conclusion: The need to address the developmental origins of health is increasingly recognised by governments around the world. The HSWP offers a sustainable workforce development solution that could be translated into action in other countries. The key contextual factors enabling or hindering implementation of the intervention provide insight for effective uptake in other communities.

\section{7}

Formative research for the development of an intervention to optimise body composition in early childhood

Catherine Draper ${ }^{1}$, Simone Tomaz ${ }^{2}$, Matthew Stone ${ }^{3}$, Shane Norris ${ }^{4}$, Trina Hinkley ${ }^{5}$, Rachel Jones ${ }^{6}$

${ }^{1}$ Division of Exercise Science and Sports Medicine, Department of Human Biology, University of Cape Town, Cape Town, South Africa; ${ }^{2}$ Division of Exercise Science and Sports Medicine, Department of Human Biology, University of Cape Town, Cape Town, South Africa; ${ }^{3}$ Department of Psychology, University of Cape Town Cape Town, South Africa; ${ }^{4} M R C /$ Wits Developmental Pathways for Health Research Unit, University of the Witwatersrand, Johannesburg, South Africa; ${ }^{5}$ Deakin University, Melbourne, Australia; ${ }^{6}$ Early Start Research Unit, University of Wollongong, Wollongong, Australia

Research question: This study collected formative data to be used for the development of an intervention to optimise body composition in urban and rural preschool children (4-6 years). Methods: Children from urban and rural settings were included in this study. Data includes physical activity (PA) measures (accelerometry, direct observation) environment observation, gross motor skill assessment, focus groups with preschool teachers and parents/caregivers, and questionnaires from parents/ caregivers.

Results: The formative findings have been used to develop the intervention, which involves teacher and parent/caregiver training components, with resources (including manuals, posters and pamphlets), and on-going support. The resources and training will provide guidance on activities to promote physical activity, decrease or break up sitting time in the school and home environments. In the focus groups, safety concerns for young children in the low-income urban and rural settings were raised. Therefore, a community intervention component will involve older children in the promotion of the safety of younger children in play spaces.

Conclusion: There is a need to promote PA and decrease sedentary behaviour in early childhood in order to optimise body composition in this age group - both in settings where overweight/obesity are a concern, and where the opportunity for primary prevention still exists.

4903

LifeLab: Exploring the link between socioeconomic status and alcohol awareness and consumption in secondary school students

Kathryn Woods-Townsend ${ }^{1}$, Emma Vaccari ${ }^{2}$, Ben Ainsworth ${ }^{3}$, Lisa Bagust ${ }^{4}$, Hannah Davey ${ }^{5}$, Donna Lovelock ${ }^{6}$, Marcus Grace ${ }^{7}$, Janice Griffiths ${ }^{8}$, Keith Godfrey $^{9}$, Mark Hanson ${ }^{10}$, Hazel Inskip ${ }^{11}$, Julia Sinclair ${ }^{12}$

${ }^{1}$ University of Southampton, NIHR Southampton Biomedical Research Centre in Nutrition, University Hospital Southampton NHS Foundation Trust; ${ }^{2}$ University of Southampton; ${ }^{3}$ University of Southampton; ${ }^{4}$ University of Southampton; ${ }^{5}$ University of Southampton; ${ }^{6}$ University of Southampton; ${ }^{7}$ University of Southampton; ${ }^{8}$ University of Southampton; ${ }^{9}$ University of Southampton, NIHR Southampton Biomedical Research Centre in Nutrition, University Hospital Southampton NHS Foundation Trust; ${ }^{10}$ University of Southampton, NIHR Southampton Biomedical Research Centre in Nutrition, University Hospital Southampton NHS Foundation Trust; ${ }^{11}$ MRC Lifecourse Epidemiology Unit, University of Southampton; ${ }^{12}$ University of Southampton

Research question: What is the relationship between socioeconomic status, alcohol awareness and alcohol consumption in adolescents?

Methods: 407 students aged 13-14 years completed questionnaires about exposure to alcohol (who they saw drinking and how often), alcohol unit awareness, knowledge about consequences of alcohol consumption and personal consumption. A subset completed a brand awareness app. Schools were classified as high, medium or low deprivation using standard deprivation indicators. Mixed-model ANCOVA examined effects of school-deprivation $\mathrm{x}$ brand (alcohol vs. non-alcohol) with age, gender, alcohol exposure and consumption as covariates.

Results: Students who had increased exposure to alcohol or reported alcohol consumption were significantly more accurate in recognising alcohol brands versus non-alcohol brands. Students from higher deprivation schools were significantly quicker at identifying alcohol brands than students from medium/low deprivation.

Conclusions: Recent evidence shows that adolescent alcohol exposure has potentially lasting consequences, acting through epigenetic processes. We showed associations between socioeconomic status, adolescent alcohol exposure and personal consumption. Research is needed to clarify the degree to which increased susceptibility to alcohol exposure and advertising might be moderated to reduce the higher burden of alcohol related disease in lower socioeconomic groups and to explore 
implications for the role of alcohol in determining health inequalities.

\section{1}

How are the IYCF knowledge and practices where the voluntary health workers (VHW) had received nutrition education 2 years ago?

Dwi Nastiti Iswarawanti ${ }^{1}$, Siti Muslimatun ${ }^{2}$, Jill A.

Dewayanti $^{3}$, Indriani A.A.S Oka ${ }^{4}$

${ }^{1}$ SEAMEO RECFON; ${ }^{2}$ Indonesia International Institute of Life Sciences, Jakarta, Indonesia; ${ }^{3}$ General Hospital DR. R. Sosodoro Djatikoesoemo, Bojonegoro, Indonesia; ${ }^{4}$ Save the Children

Indonesia, Jakarta, Indonesia

Research question: The objectives of the study were to assess knowledge and practices of caregivers on IYCF as well as adequacy of children nutrient intake in the area where in the previous two years a nutrition education on IYCF had been given to VHW with 2 different methods.

Methods: This cross sectional study interviewed caregivers (intervention 317 and comparison 319). Food intake of their 6-23 months children were measured by 2 days non-consecutive 24 hour recall. Two years prior to study, a nutrition education on IYCF had been given to $70 \mathrm{VHW}$ as a 3-day training (intervention area) and to $68 \mathrm{VHW}$ as a half day seminar (comparison area).

Results: The prevalence of caregivers who had good IYCF knowledge and practices in both areas were not significantly different. However the prevalence of nutrient intake inadequacy of protein, fat, Vitamin A, Iron, Calcium and Zinc amongst 6-23 months children in the intervention area were significantly lower than those in the comparison area.

Conclusion: The prevalence of nutrient inadequacies was significantly lower in areas nutrition education was provided in the form of 3-days training compared to in the form of half-day seminar in the previous 2 years.

\section{3}

A qualitative investigation of the life experiences of substance abusing women in Cape Town

Nwabisa Bikitsha ${ }^{1}$, Anita Padmanabhanunni ${ }^{2}$

${ }^{1}$ Human Sciences Research Council; ${ }^{2}$ University of the Western Cape

This aim of this study was to investigate the life experiences of substance abusing women participating in a substance abuse treatment programme in the Cape Town area. Much of the research that has been conducted on this topic has been quantitative and therefore removed from the context of lived experience. The current study thus utilized a qualitative methodology to explore substance abusers' own accounts of substance use, their trajectory towards substance abuse as well as their motivation for seeking treatment. A qualitative research design was employed, drawing on the interpretive framework which seeks to understand how people make sense of their experiences. Six participants were recruited from a substance abuse treatment center in the Cape Town area and data was collected using semi-structured interviews and analyzed using Interpretive Phenomenological Analysis (IPA). Partner substance use and psychological pain experienced by the women played a motivating role in their substance use. The reconstruction of one's own identity, perceptions of motherhood and mending relationships were found to be key elements in women's motivation for recovery. Therefore, in-depth analysis in women's intimate relationship dynamics, how these relationships influence health behaviours and the self-concept is required.

\section{3}

Training healthcare personnel for a client centred approach to healthy lifestyle change in Malaysia

Amanda Villiers-Tuthill ${ }^{1}$, Jutta Kloppenborg Heick Skau ${ }^{2}$, Awatef Binti Amer Nordin ${ }^{3}$, Siti Irma Fadhilah Binti Ismail ${ }^{4}$, Zubaidah Jamil Osman ${ }^{5}$, Mimi Karim ${ }^{6}$

${ }^{1}$ Perdana University; ${ }^{2}$ Faculty of Health Sciences, University of the Witwatersrand, Johannesburg, South Africa; ${ }^{3}$ Institute of Health System Research, Ministry of Health, Malaysia; ${ }^{4}$ Faculty of Medicine and Health Sciences, Universiti Putra Malaysia;

${ }^{5}$ Faculty of Medicine and Health Sciences, Universiti Putra Malaysia; ${ }^{6}$ Seremban District Health Department, Ministry of Health, Malaysia

Research question and background: Client centred approaches such as behaviour change counselling (BCC) are shown to be more effective in achieving client lifestyle change than traditional advice giving in multiple settings, yet are not routinely utilised in Malaysia.

Jom Mama is a pre-pregnancy lifestyle intervention incorporating community health promoter (CHP) consultations and e-health. We developed a training curriculum in conjunction with CHPs and local experts, and trained CHPs in using a 'BCC skills toolbox' to promote a client-centred approach within the context of Jom Mama.

Methods: Participants completed pre and post-assessments of nutrition, physical activity and BCC knowledge, and participated in focus group discussions after training.

Results: Knowledge and understanding of client centred care and BCC improved after training. Focus group discussions highlighted increased CHP understanding of pre-pregnancy health, higher engagement and role-satisfaction, and higher confidence to take a BCC approach. Ongoing support and practice are necessary to ensure continued CHP skill development in BCC.

Conclusion: Capacity building of healthcare personnel is critical in addressing the challenge of non-communicable 
disease management in Malaysia. Jom Mama aplies the WHO's Innovative Care for Chronic Conditions (ICCC) framework in promoting a shift from a reactive acute care model, towards a proactive, multidisciplinary, client centred approach focused on maintaining good health and reducing risks for the next generation.

\section{2}

\section{Promoting pre-conception health: An intervention} mapping approach

Julius Cheah $^{1}$, Shane A Norris ${ }^{2}$, Aswir Abd Rashed ${ }^{3}$, Vibeke Vinding ${ }^{4}$, Jutta KH Skau ${ }^{5}$, Regien Biesma ${ }^{6}$, Priya Matzen ${ }^{7}$, Jens Aagaard-Hansen ${ }^{8}$, Mark Hanson ${ }^{9}$

${ }^{1}$ School of Medicine and Health Sciences, Monash University Malaysia; ${ }^{2}$ University of the Witwatersrand, Johannesburg, South Africa; ${ }^{3}$ Nutrition Unit, Cardiovascular, Diabetes and Nutrition Research Center; ${ }^{4}$ IT University of Copenhagen; ${ }^{5}$ University of the Witwatersrand, Johannesburg, South Africa; ${ }^{6}$ Department of Epidemiology and Public Health Medicine, Royal College of Surgeons in Ireland; ${ }^{7}$ Institute of Developmental Sciences and NIHR Nutrition Biomedical Research Centre, University of Southampton, UK; ${ }^{8}$ Faculty of Health Sciences, University of the Witwatersrand, Johannesburg, South Africa; ${ }^{9}$ Institute of Developmental Sciences and NIHR Nutrition Biomedical Research Centre, University of Southampton, UK

Background: Malaysia is undergoing multiple transitions with an increasing burden of non-communicable diseases and high levels of obesity in women, which have serious implications not only for their health but also for the intergenerational transfer of metabolic risk. Pre-pregnancy health promotion may be a key to addressing intergenerational risk. Using Intervention Mapping (IM), this paper describes the development of the Jom Mama intervention, which is intended to improve the health of adult women and men in order to limit metabolic disease risk.

Methods/Design: This paper describes the application of the first four steps of IM. Evidence is provided to support the selection of key behavioural objectives for women that includes healthy dietary practices, increases physical activity, reduced sedentary behaviour, and improved social support around demanding and stressful lifestyles. An appropriate behaviour change technique is suggested (motivational interviewing) that draws upon the Theory of Triadic Influence. It is conceptualised that the Jom Mama intervention will be a health-system based intervention that includes health promoters to deliver a complex intervention to adult couples within the community.

Discussion: IM is an effective process to systematically frame evidence and assist in the development of a complex intervention. The Jom Mama intervention is novel, both in Malaysia and globally, as it is: (i) based on strong evidence, extensive formative work and best practice from evaluated interventions; (ii) combines theory with evidence to inform intervention components; (iii) includes multiple domains of influence; (iv) focuses on an at-risk target group; and (v) embeds within existing and planned health service priorities in Malaysia.

4472

Practical Approach towards reduction of low birth weight as a measure to achieve MDG goal 4

Ramesh Potdan

Self Employed

MDG 4 relates to Reduction of Child Mortality, major causes of which were pneumonia and preterm birth complications Achievement of MDG goals is globally SLOWER in the preterm birth complications.

Research question: What is the role of $\mathrm{DOHaD}$ concept with reference to Neonatal Morbidity and Mortality (using Low Birth Weight as an indirect indicator/predictor of preterm birth complications?

Methods: 1. Results of a food based intervention trial during prepregnancy and pregnancy phase amongst 6500 slum poor women to reduce Low Birth Weight between 2006 to 2012 and 2. Analysis of updated global literature with special focus on SEARO and INDIA were used for the study.

\section{Results:}

- The trial amongst 6500 women concluded that in women with normal BMI, food based micronutrient supplementation in preconception and prenatal period can significantly increase the Birth weight of the offspring $(\mathrm{p}=<0.05)$.

- Literature shows that low birth weight is a major contributor to neonatal mortality and morbidity.

- It appears that there is role of $\mathrm{DOHaD}$ concept with reference to Neonatal Morbidity and Mortality notwithstanding the regional differentiation.

Conclusions: With such an intervention, it may be possible envisage reduction in preterm birth complications and help to achieve MDG goal 4.

\section{8}

Perceptions, attitudes and knowledge of diabetes and preconception health in young couples in Malaysia

Awatef Binti Amer Nordin ${ }^{1}$, Ramli Zainal ${ }^{2}$, Khatijah Abdullah $^{3}$, Julius Cheah Chee Ho ${ }^{4}$, Regien Biesma ${ }^{5}$, Priya Matzen ${ }^{6}$, Jens Aagaard-Hansen ${ }^{7}$, Mark Hanson ${ }^{8}$, Shane A Norris ${ }^{9}$, Jutta KH Skau ${ }^{10}$

${ }^{1}$ Institute of Health System Research, Ministry of Health, Malaysia; ${ }^{2}$ Institute of Health System Research, Ministry of Health, Malaysia; ${ }^{3}$ Department of Nursing Science, Faculty of Medicine, University of Malaya; ${ }^{4}$ School of Medicine and Health Sciences, Monash University Malaysia; ${ }^{5}$ Department of Epidemiology and Public Health Medicine, Royal College of Surgeons in Ireland; ${ }^{6}$ Institute of Developmental Sciences and 
NIHR Nutrition Biomedical Research Centre, University of Southampton, UK; ${ }^{7}$ Faculty of Health Sciences, University of the Witwatersrand, Johannesburg, South Africa; ${ }^{8}$ Institute of Developmental Sciences and NIHR Nutrition Biomedical Research Centre, University of Southampton, UK; ${ }^{9}$ Faculty of Health Sciences, University of the Witwatersand, Johannesburg, South Africa; ${ }^{10}$ Faculty of Health Sciences, University of the Witwatersrand, Johannesburg, South Africa

Background: Malaysia is undergoing multiple transitions with an increasing burden of non-communicable diseases and high levels of obesity in women, which have serious implications not only for their health but also for the intergenerational transfer of metabolic risk. The purpose of the study was to explore the perceptions, attitudes and knowledge of diabetes and preconception health of young couples in Malaysia. This is done with the aim to understand barriers and facilitators of healthy lifestyles and preferred modes of delivery for potential preconception intervention programs.

Methods/Design: The research utilized an exploratory design using a qualitative research approach in the form of face-to-face semi-structured interviews to gain in-depth understanding of the challenges and barriers of young couples in leading a healthy lifestyle. The study was conducted in the district of Seremban, in the state of Negeri Sembilan, Malaysia. A total of 18 couples between the age of 18-35 years and 9 social influencers were interviewed.

Results and discussion: With the view to planning a lifestyle related health education intervention for young couples in Malaysia, key considerations highlighted by this study included attention to cultural values and norms and the influence of the working and social environment. Young couples face practical and perceived barriers to engaging in healthy behaviours, yet participants expressed important beliefs and values regarding family roles that motivate health behaviours. If perceptions regarding what behaviours are normative can be changed towards health promoting norms, this may increase the desire to comply with healthy behaviours, and facilitate a shift towards longer term engagement in healthy lifestyle choices

\section{6}

Mathematical model of pancreatic cell fate decisions, implication for the development of beta cells

Caïdi Aziza $^{1}$, Firmin Stéphane ${ }^{2}$, Bréant Bernadette ${ }^{3}$, Blondeau Bertrand $^{4}$, Abdennebi-Najar Latifa ${ }^{5}$, Bahi-Jaber Narges ${ }^{6}$

${ }^{1}$ UP 2012.10.101. EGEAL-Institut Polytechnique LaSalle Beauvais; ${ }^{2}$ UP 2012.10.101. EGEAL-Institut Polytechnique LaSalle Beauvais; ${ }^{3}$ INSERM UMRS 1138 - Université Pierre et Marie Curie; ${ }^{4}$ INSERM UMRS 1138 - Université Pierre et Marie Curie; ${ }^{5}$ UP 2012.10.101. EGEAL-Institut Polytechnique LaSalle Beauvais; ${ }^{6}$ UP 2012.10.101. EGEAL-Institut

Polytechnique LaSalle Beauvais

Research question: During pancreatic organogenesis, a complex and coordinated action of transcription factors (TF) ensures the differentiation of pancreatic precursors into several cell lineages. This gene regulatory network (GRN) is essential in determining pancreatic beta-cell development but complexity and non-linearity of gene interactions make difficult to evaluate the impact of TF expression rate modulation on beta cell density in early postnatal age.

Methods: We developed a time dependent stochastic model of the GNR involved in rat pancreatic development during the fetal period. By simulation of the model we investigated the temporal dynamics of gene expressions and analyzed the different trajectories of pancreatic precursors unto differentiated cells.

Results: Our model reproduced the time course of gene expressions in the different lineages in accordance with biological data. By varying gene expression rates we explored the impact of their regulation on cell fate decisions and beta cell density. Moreover, this impact depends on the time window at which gene regulation occurs.

Conclusion: this model might be used to predict the impact of deleterious expositions during fetal life, implicated in the regulation of TF expression, on the development of beta cell and by extension on the programming of type 2 diabetes at adulthood.

\section{1}

Anthropology, DOHaD, and Refurbished Retrospective Data Sources

Celeste Giordano ${ }^{1}$, Daniel Benyshek ${ }^{2}$

${ }^{1}$ University of Nevada Las Vegas; ${ }^{2}$ University of Nevada Las Vegas

Research question: As the diets of many 'populations in transition' undergo significant changes in less than a generation, longitudinal studies on early-life nutritional factors relevant to the development of disease in adulthood often remain out of reach. Nevertheless, these are the populations which are suffering the most from metabolic disorders known to have an early-life developmental component. Retrospective prenatal dietary histories, the value of which has received little attention for over 30 years, may be the only source of prenatal/early-life nutritional information in many communities. In other cases, "old" sources of information, like Guthrie cards, fingerprint ridge counts, ritually saved baby teeth and hair may offer new possibilities, despite having been originally collected for another purpose.

Methods: We investigated the contribution of market and local foods in the prenatal diets of Yup'ik Alaska Native women 20 to 40 years ago utilizing a remote recall food frequency questionnaire and conversational interviews.

Results and conclusion: Presenting our work with Yup'ik mothers as a case study, we argue that for many communities experiencing rapid dietary transitions, the investigation of nutrition-related chronic disorders suspected to have a developmental component will necessitate the retrospective collection of information on past diets or another reliable proxy. 


\section{5}

A Literature review translates current nutrition/physical activity research findings to support a maternal and infant health workforce education programme

Dr Caroline Gunn ${ }^{1}$, Jackie Gunn ${ }^{2}$, Dr Anne Jaquiery ${ }^{3}$

${ }^{1}$ Liggins Institute, University of Auckland; ${ }^{2}$ Gravida, University of Auckland; ${ }^{3}$ Gravida, University of Auckland

Research question: How does a literature review inform Gravida's Healthy Start Workforce Project (HSWP) education programme on nutrition and physical activity for pregnant women and young children?

Methods: A literature review provided a platform of evidence for HSWP e-learning programme on the importance for long term health of nutrition and physical activity during pregnancy and early life. Database searches included ScienceDirect, PubMed, Cochrane, DOAJ and Google Scholar with keywords: Pregnancy, nutrition, physical activity, breastfeeding, complementary feeding and $\mathrm{DOHaD}$. Gray literature included WHO and Ministry of Health (NZ) guidelines/reports. Evidence graded highest to lowest included Cochrane reports/ meta-analyses/ systematic reviews/ randomised controlled trials/ observational studies.

Results: The research literature demonstrates that during pregnancy, macro/micronutrient intake, weight gain, physical activity and gestational diabetes affect maternal health and the intrauterine environment. The fetal nutritional environment influences gene expression via epigenetic changes and is likely to have intergenerational effects. Breastfeeding and dietary patterns ( $0-2$ years) also may influence infants' long term health and BMI.

Conclusion: Maternal workforce education must prioritise and reflect current research evidence demonstrating the compelling need for healthy eating and physical activity during pregnancy and early life for long term individual and societal health and economic benefits.

\section{0}

Pre-service science teachers' understanding of epigenetics and their views on the implications of epigenetics for society, everyday life and education

Lisa Bagust ${ }^{1}$, Andri Christodoulou ${ }^{2}$, Kath Woods-Townsend ${ }^{3}$, Hannah Davey ${ }^{4}$, Donna Lovelock ${ }^{5}$, Karen Lillycrop ${ }^{6}$, Janice Griffiths $^{7}$, Keith Godfrey ${ }^{8}$, Mark Hanson ${ }^{9}$, Hazel Inskip ${ }^{10}$, Marcus Grace ${ }^{11}$

${ }^{1}$ Southampton University; ${ }^{2}$ Southampton University; ${ }^{3}$ Southampton University, NIHR Southampton Biomedical Research Centre in Nutrition; ${ }^{4}$ Southampton University; ${ }^{5}$ Southampton University; ${ }^{6}$ Southampton University; ${ }^{7}$ Southampton University; ${ }^{8}$ NIHR Southampton Biomedical Research Centre in Nutrition, Sothampton University; ${ }^{9}$ Southampton University, NIHR Southampton Biomedical
Research Centre in Nutrition; ${ }^{10}$ MRC Lifecourse Epidemiology Unit, University of Southampton; ${ }^{11}$ Southampton University

Research question: Do teachers and consequently pupils consider the aetiology of NCDs to be primarily a result of genetic predisposition coupled with unhealthy adult lifestyle?

Methods: Trainee science teachers $(n=56)$ completed a questionnaire to elicit their knowledge and understanding of epigenetics. This data was analysed using NVIVO.

Results: Respondents were asked about the contribution of fixed genetic make-up to risk of diabetes and coronary heart disease - none correctly identified the contribution as $<10 \%$, whilst $64 \%$ thought it was $>25 \%$. Only $52 \%$ had heard of epigenetics; of these, $68 \%$ attempted a definition which showed a partial understanding. Considering the implications for health, 37\% acknowledged the possibility of changing their own and pupils' health prospects. Only $8 \%$ appreciated the importance of learning about epigenetics in the future.

Conclusion: Education is key to tackling the problem of NCDs, reflected in the inclusion of NCDs in the UK national curriculum. Our findings suggest a major gap in science teachers' understanding of epigenetics. We propose a way to tackle this deficit through the LifeLab teaching programme which includes teacher professional development, coupled with a teaching activity designed to raise awareness of epigenetics amongst pupils and the impact on their health.

\section{7}

Understanding the Epigenetic Origins of Congenital Heart Disease May Lead to its Control

Rik De Decker ${ }^{1}$, Elmarie van der Merwe ${ }^{2}$

${ }^{1}$ Red Cross War Memorial Children's Hospital; ${ }^{2}$ Red Cross War Memorial Children's Hospital

Congenital heart disease (CHD), occurring in 1 per 100 births, remains the leading life-threatening abnormality present at birth. It is reasonable to assume that most, if not all, CHD stems from errors in the genetic control of heart development, and that knowing these controlling molecular mechanisms may allow us to understand the origins of CHD. With understanding comes the potential of prevention and possibly even early (fetal) repair.

Our operational research in the Western Cape has identified a severe epidemiological shortfall between the theoretical number of newborns that should present to us with severe CHD and those that actually do - we are referred only $50 \%$ of the children that (epidemiologically) should receive heart surgery. Even if we could identify all the missing children, our cardiac services would be unable to contend with the consequent demand. This stark dilemma plays itself out in all resource-constrained cardiac health services.

It is therefore of utmost importance that the origins of CHD that may be under environmental influence - the epigenetic causes of 
CHD - are prioritized in our quest to reduce heart defects. This paper will outline these influences and show how their understanding may begin to reduce an enormous health burden.

\section{4}

\section{The Intergenerational Effects on Birth Weight, São Paulo,} Brazil

Leide Irislayne Macena da Costa Silva ${ }^{1}$, Filumena Maria da Silva Gomes ${ }^{2}$, Maria Helena Valente ${ }^{3}$, Ana Maria de Ulhôa Escobar ${ }^{4}$, Alexandra Valéria Maria Brentani ${ }^{5}$, Sandra J. F. E. Grisi ${ }^{6}$

${ }^{1}$ Faculdade de Medicina da Universidade de São Paulo;

${ }^{2}$ Faculdade de Medicina da Universidade de São Paulo;

${ }^{3}$ Faculdade de Medicina da Universidade de São Paulo;

${ }^{4}$ Faculdade de Medicina da Universidade de São Paulo;

${ }^{5}$ Faculdade de Medicina da Universidade de São Paulo;

${ }^{6}$ Faculdade de Medicina da Universidade de São Paulo

The present research aims to study the correlation between the child's birth weight with the mother's birth weight, in hospital's medical records from the University of São Paulo. Identified 773 children born from 558 mothers. Group studies were constituted: birth weight $<2,500$ grams (g) and $\geqslant 3,500 \mathrm{~g}$. The length at birth was also studied in children $\leqslant 47.5 \mathrm{~cm}$ (lower quartile). Girls were heavier at birth than their mothers, with an average increase at birth weight between the generations of $79 \mathrm{~g}$. The child's weight at birth $\geqslant 3,500 \mathrm{~g}$ showed strong correlation with maternal weight at birth $\geqslant 3,500$ g (Chi2 sig 0; Spearman's Rho +0.142; OR 0.5 and OR upper 0.7). The higher the maternal pre-pregnancy BMI, the stronger the correlation with child's birth weight $\geqslant 3,500 \mathrm{~g}$ was (Chi2 sig 0.013; Spearman's Rho 0.09; OR 1.54 and OR upper 2.17 and Chi2 sig 0; Spearman's Rho 0.137; OR 2.58 and OR upper 4.26). The mother weight's birth $\geqslant 3,500 \mathrm{~g}$ and the prenatal overweight or obesity were correlated with the child's weight at birth $\geqslant 3,500 \mathrm{~g}$, coupled with the tendency of increasing birth weight between mothers's and girl's generations.

\section{6}

\section{MAGAs, Pregnancy Disorders \& miRNAs in DOHaD}

William Johnson ${ }^{1}$, Edward Stenroos ${ }^{2}$, Steven Buyske ${ }^{3}$, Zachary Cartun ${ }^{4}$

${ }^{1}$ Rutgers University; ${ }^{2}$ Rutgers University; ${ }^{3}$ Rutgers University;

${ }^{4}$ Rutgers University

$\mathrm{DOHaD}$ involves factors that affect fetal growth during gestation and are believed to be environmental in nature. Maternally acting gene alleles, MAGAs, are maternal genes that act in maternal tissues independently of any inheritance by the fetus. From the fetus' perspective, MAGAs are environmental factors since they act independently of any inheritance from mother-to-fetus and since their effects result from gene action in the mother not in the fetus. MAGAs produce gene products, e.g., proteins and miRNAs that may influence intrauterine environment. We previously analyzed reports of alleles of 38 MAGAs (DOHaD 2013), some implicated in PTB, SGA, IUGR/low birth weight/FGR), placental abruption, preeclampsia, utero-placental insufficiency or neurodevelopmental disorders. Now, we analyze 605 reports of 165 MAGAs. Distribution of gene categories was similar: genes related to 1 . folate $(12.7 \%), 2$. detoxification/ oxidative stress $(14.5 \%)$, and 3 . immune mechanisms $(23.0 \%)$, 4. Other genes $(50.0 \%)$ was now a category much larger than before. 36 of the 165 MAGAs contribute to IUGR/low birth weight/FGR $(21.8 \%)$ a significant fraction of the total. Since 2013, at least 6 additional reports delineate miRNAs in decidua. MAGAs and miRNAs acting in decidua may contribute to these disorders and to the "other" category of MAGAs.

\section{8}

\section{Exploring women's fear of childbirth in a high maternal mortality setting on the Arabian Peninsula}

Annica Kempe ${ }^{1}$, Töres Theorell ${ }^{2}$, Fatoom Noor-Aldin Alwazer $^{3}$, Samera Taher ${ }^{4}$, Kyllike Christensson ${ }^{5}$

${ }^{1}$ Karolinska Institutet; ${ }^{2}$ Karolinska Institutet and Stress Research Institute at Stockholm University; ${ }^{3}$ Ministry of Public

Health \& Population and the Yemeni Midwives Association;

${ }^{4}$ Ministry of Public Health \& Population and the Yemeni Midwives Association; ${ }^{5}$ Karolinska Institutet

Research question: Few studies from low-income countries have addressed women's fear of childbirth (FOC). The aim of this study was to explore FOC in a high maternal mortality setting, Yemen.

Methods: We interviewed 220 women with childbirth experience in rural/urban Yemen. Answers to the question "Were you afraid of giving birth?" were analyzed using qualitative content analysis.

Results: Women perceived childbirth as a place of danger. Fear of death and childbirth complications stemming from previous traumatic childbirth and traumatic experience in the community was rampant. Husbands' and in-laws' disappointment in a girl infant constituted a strong sociocultural component of FOC. Women's perception of living in tension "between worlds" of tradition and modernity reinforced fear of institutional childbirth.

Conclusion: The numerous maternal and infant deaths have a strong impact on women's fear. Antenatal care (ANC) has an important role in reducing fear including that of institutional childbirth and in strengthening a couple in welcoming a female infant. Staff should be sensitized to the fears of both husband and wife and women be allowed support 
during childbirth. Within the scope of the Millennium Development Goals (MDGs) and strengthening of reproductive mental health programs globally, FOC urgently needs to be addressed.

\section{7}

Socio-economic outcomes for adult men born with hypospadias; a register-based study

Anna Skarin Nordenvall ${ }^{1}$, Louise Frisén ${ }^{2}$, Anna Nordenström ${ }^{3}$, Catarina Almqvist Malmros ${ }^{4}$, Agneta Nordenskjöld ${ }^{5}$

${ }^{I}$ Department of Women's and Children's health, Karolinska Institute; ${ }^{2}$ Department of Clinical Neuroscience, Karolinska Institute; ${ }^{3}$ Department of Women's and Children's Health, Karolinska Institute; ${ }^{4}$ Department of Medical Epidemiology and Biostatistics, Karolinska Institute; ${ }^{5}$ Department of Women's and Children's Health, Karolinska Institute

Many long-term follow-up studies of men born with hypospadias focus on cosmetic results and sexual function. Some studies have suggested that men with hypospadias report lower health-related quality of life and mental health problems to a greater extent than healthy men. It is unknown to which extent this affects the general well-being in adulthood.

This register-based cohort-study includes men diagnosed with hypospadias, born in Sweden 1969-1993. Patients with hypospadias were matched with 100 non-affected males by birth year. Several prospectively collected socio-economic outcomes served as proxies for well-being in adulthood. Associations between hypospadias and outcomes were estimated with conditional logistic regression, expressed in OR (95\% CI).

4738 men with hypospadias were included. $53 \%$ were diagnosed with glandular or penile hypospadias, $4.6 \%$ were diagnosed with penoscrotal or perineal hypospadias. Men born with hypospadias were less likely to be eligible for upper secondary school, OR 0.85 (0.76-0.95), but achieved the same level of education and income as non-affected. No differences in probabilities of being married, OR 1.0 (0.891.12), or having children, OR 0.94 (0.86-1.04), were observed regardless the severity of hypospadias. An increased probability of obtaining disability pension was detected among all severities of hypospadias.

\section{9}

Broadspectrum Antibiotics Increases the Risk of Childhood Type 1 Diabetes in Offspring Delivered by Cesarean Section

Tine Dalsgaard Clausen ${ }^{1}$, Thomas Bergholt ${ }^{2}$, Olivier Bouaziz ${ }^{3}$, Rolf Magnus Arpi ${ }^{4}$, Steen Rasmussen ${ }^{5}$, Frank Eriksson ${ }^{6}$, Niels Keiding ${ }^{7}$, Ellen Løkkegaard ${ }^{8}$

${ }^{1}$ Department of Gynecology and Obstetrics, Nordsjaellands Hospital, University of Copenhagen, Denmark; ${ }^{2}$ Department of
Obstetrics, Rigshospitalet, University of Copenhagen, Denmark; ${ }^{3}$ Department of Public Health, Section of Biostatistics, University of Copenhagen, Denmark; ${ }^{4}$ Department of Clinical Microbiology, Herlev Hospital, University of Copenhagen, Denmark;

${ }^{5}$ Department of Microbiology, Hvidovre Hospital, University of Copenhagen, Denmark; ${ }^{6}$ Department of Public Health, Section of Biostatistics, University of Copenhagen, Denmark; ${ }^{7}$ Department of Public Health, Section of Biostatistics, University of Copenhagen, Denmark; ${ }^{8}$ Department of Gynecology and Obstetrics, Nordsjaellands Hospital, University of Copenhagen, Denmark

Environmental factors like antibiotic treatment and delivery mode influence the gut microbiota, which may in turn affect the risk of childhood type 1 diabetes.

Objective: To evaluate associations between children's use of antibiotics age 0-2 and subsequent childhood type 1 diabetes. Methods: Cox regression analyses provided multiple adjusted Hazard Ratios.

Results: A total of 858,201 singletons contributed 7,621,344 person-years, during which 1,503 were diagnosed with childhood type 1 diabetes. Respectively, $72.1 \%$ and $68.8 \%$ were treated with antibiotics in 1997 and 2010. The risk of childhood type 1 diabetes was associated with use of broad-spectrum (HR 1.13; 95\% CI 1.02- 1.25) but not with narrow-spectrum (HR 1.02; 95\% CI 0.92-1.13) antibiotics.

However, the effect of antibiotics was modified by mode of delivery. Compared to untreated children, risk of childhood type 1 diabetes was increased with broad-spectrum antibiotics in children delivered by elective (HR 1.63; 95\% CI 1.12-2.38) or emergency (HR 1.70; 95\% CI 1.15-2.51) cesarean section, but not in children born by vaginal delivery (HR 1.05; 95\% CI 0.94-1.18).

Conclusions: Treatment with broad-spectrum antibiotics during the first two years of life is associated with an increased risk of childhood type 1 diabetes in offspring delivered by caesarean section.

\section{3}

How and why do maternal cortisol profiles vary in a time dependent manner across the peri-conceptional period?

Katrina Salvante ${ }^{1}$, Leilei Zeng ${ }^{2}$, Chelsea Pyles ${ }^{3}$, Huiting MA ${ }^{4}$, Jason Blais ${ }^{5}$, Cindy Barha ${ }^{6}$

${ }^{1}$ Faculty of Health Sciences - Simon Fraser Unviersity; ${ }^{2}$ Statistics and Actuarial Science - University of Waterloo; ${ }^{3}$ Faculty of Health Sciences - Simon Fraser University; ${ }^{4}$ Department of Statistics and Actuarial Sciences, University of Waterloo; ${ }^{5}$ Department of Statistics and Actuarial Sciences, University of Waterloo; ${ }^{6}$ Faculty of Health Sciences - Simon Fraser University

Cortisol elevations are linked to suppression of reproductive function, increased risk of early miscarriage and fetal programming. Here we present the first longitudinal description of first morning urinary cortisol (FMUC) profiles across the peri- 
conceptional period (12-weeks centered on conception) $(n=22)$ and evaluate alternative functional explanations for said profiles. Reproductive hormones, to determine time of conception, and cortisol were quantified using immunoassays. Linear mixedeffects models with regression splines were used to evaluate the magnitude and significance of changes in cortisol trajectories. Overall, FMUC increased across the peri-conceptional period. The increasing cortisol trajectory $(+1.36 \% /$ day; $\mathrm{p}=0.007)$ was interrupted by a week-long decline immediately prior to conception $(-4.02 \% /$ day; $p=0.0013)$ and during the fifth week post-conception $(-6.60 \% /$ day; $p=0.0002)$. We propose that the overall increase in FMUC before conception may mediate a shift in energy allocation from investment in the last-born towards the production of a new offspring. Post-conception, energy would be allocated towards meeting the energetic demands of early gestation and storage for use later in pregnancy. Since high cortisol levels may elevate the risk of miscarriage and affect embryonic development, the observed cortisol trajectory may reflect a tradeoff between meeting pregnancy's energetic needs and minimizing overall cortisol exposure during specific windows of vulnerability.

\section{3}

Mother's marital status and offspring's health in adult life. The Helsinki Birth Cohort Study 1934-1944

Minna K. Salonen ${ }^{1}$, Johan G. Eriksson ${ }^{2}$, Eero Kajantie ${ }^{3}$

${ }^{1}$ Folkhälsan Research Centre, Helsinki, Finland; National Institute for Health and Welfare, Helsinki, Finland; ${ }^{2}$ Folkhälsan Research Centre, Helsinki, Finland; National Institute for Health and Welfare, Helsinki, Finland; University of Helsinki and Helsinki University Hospital, Helsinki, Finl; ${ }^{3}$ The National Institute for Health and Welfare; Children's Hospital, Helsinki University Central Hospital and University of Helsinki, Helsinki, Finland; Department of Obstetrics and

Research question: Growing up with single parent is a strong predictor of low socioeconomic position in adult life. However there is limited evidence of its lifetime consequences on health. We examined whether being born to an unmarried mother is associated with adulthood coronary heart disease (CHD) in adulthood.

Methods: We studied 6186 men and 5460 women born in Helsinki, Finland, in 1934-44. Using a unique personal identification number, we linked data from birth, child welfare clinic and school healthcare records with CHD diagnoses from national Hospital Discharge and Death Registers.

Results: Hazard ratios for CHD, adjusted for mother's age, birth order, birth weight and length of gestation, were 1.02 $(95 \%$ CI $0.75,1.37)$ for men and $1.68(1.10,2.58)$ for women born to an unmarried mother. They were little affected by adjustment for adult educational attainment but attenuated to non-significance when adjusted for childhood socio-economic position. No interaction between single parent background and sex was found $(p=0.10)$.
Conclusions: Despite its strong associations with low adult socioeconomic position, a single parent background does not stand out as an independent predictor of CHD.

\section{5}

An invitation to a fetal perspective - a new way of understanding fetal lifetime that leads to new concepts and new perspectives

Ann-Jeanett

Institut for fosterperspektiver (Institute for fetal perspective)

What would the world look like if we contemplated it through a theoretical and analytical fetal perspective? Could the fetal period be considered a lifetime? And in turn how would pregnancy and parenthood be conceived in such a view?

This is a research of whether and how it is possible to go beyond the current understandings of the fetal period, pregnancy and parenthood through the fetal perspective as a new concept. The fetal perspective is based on already existing research and on theoretical and meta-theoretical analyses through phenomenological and post-structuralistic thinking, thus providing a new construction of "reality" which challenges the current thinking about the fetal period, pregnancy and parenthood. With the concept of a fetal perspective, it is possible to question the existing understandings of life development.

I conclude that a fetal perspective as a new concept brings us the opportunity to view the fetus as a subject with a first-person perspective and the mother as an ontological world (both her inner world and our collective world) that frame the new human being from the very beginning. The way we speak, understand and act pregnancy as society and individuals defines the construct of which world the fetus has access to experience.

\section{0}

Intergenerational effect of high salt intake during pregnancy Maria Angélica Peres ${ }^{1}$, Luzia S Furukawa ${ }^{2}$, Ivone B Oliveira ${ }^{3}$, Joel Heimann ${ }^{4}$

${ }^{1}$ Universidade of Sao Paulo; ${ }^{2}$ Universidade de Sao Paulo;
${ }^{3}$ Universidade de Sao Paulo; ${ }^{4}$ Universidade de Sao Paulo

We hypothesized if phenotype of adult offspring of dams fed high salt diet during pregnancy can be transmitted further to next generations. Pregnant rats were fed with normal $(\mathrm{N})$ or high $(\mathrm{H})$ salt diet from the first day of gestation until delivery. Afterwards, all dams and offspring were fed with N. Body and organs weight (heart, adrenal glands, kidneys, testicles, epididymis, and retroperitoneal fat) were measured. Gene expression of the components of the renin-angiontensin system (RAS) and 11betahydroxysteroid dehydrogenase (11 $\beta$ HSD) 1 and 2 were evaluated in kidneys and testicles from males of the three generations as well as in the placenta and decidua from F1 and F3. Higher ratio of organ/body mass were observed in $\mathrm{H}$ in the three generations. Placenta/decidua mass was higher in $\mathrm{H}$ only in F3. RAS gene 
expression presented some isolated alterations in kidney and testis. In F3 male $\mathrm{H}$ fetus decidua $11 \beta \mathrm{HSD} 1$ and 2 were higher expressed. Higher organ mass from $\mathrm{F} 1$ to $\mathrm{F} 3$ in $\mathrm{H}$ group indicate that some developmental changes are in process.

\section{8}

Health can be programmed? Low Intensity exercise during pregnancy protects offspring from metabolic syndrome induced by early overnutrition

Audrei Pavanello ${ }^{1}$, Tatiane Aparecida Ribeiro ${ }^{2}$, Ananda Malta ${ }^{3}$, Laize Peron Tófolo ${ }^{4}$, Júlio Cezar de Oliveira ${ }^{5}$, Luiz Felipe Barella ${ }^{6}$, Rodrigo Mello Gomes ${ }^{7}$, Paulo Cezar de Freitas Mathias ${ }^{8}$

${ }^{1}$ Laboratory of Secretion Cell Biology, Department of Cell Biology and Genetics, State University of Maringá - (MaringálPR, Brazil); ${ }^{2}$ Laboratory of Secretion Cell Biology, Department of Cell Biology and Genetics, State University of Maringá - (MaringálPR, Brazil)/ Fellowship of CNPq-Brazilian Research Agency; ${ }^{3}$ Laboratory of Secretion Cell Biology, Department of Cell Biology and Genetics, State University of Maringá-(Maringá/PR, Brazil); ${ }^{4}$ Laboratory of Secretion Cell Biology, Department of Cell Biology and Genetics, State University of Maringá - (Maringá/PR, Brazil); ${ }^{5}$ Health Sciences Institute, Federal University of Mato Grosso, Sinop, MT, Brazil; ${ }^{6}$ Molecular Signaling Section, Laboratory of Bioorganic Chemistry, National Institute of Diabetes and Digestive and Kidney Diseases, National Institutes of Health, Bethesda, MD, USA; ${ }^{7}$ Laboratory of Secretion Cell Biology, Department of Cell Biology and Genetics, State University of Maringá - (MaringálPR, Brazil); ${ }^{8}$ Laboratory of Secretion Cell Biology, Department of Cell Biology and Genetics, State University of Maringá - (MaringálPR, Brazil)/ Fellowship of CNPq-Brazilian Research Agency

Research question: Perinatal life deviations can program the metabolism to develop metabolic diseases latter in life. Physical exercise is a key factor in controlling metabolic complications in obese people. To test whether low intensity exercise in pregnant and lactating female rats could program the metabolism of the offspring to be resistant to metabolic consequences was the aim of this work.

Methods: Rat dams were submitted to run in a treadmill during all pregnancy and lactation, 3-times/week with speed set to $30 \%$ of VO2max. Sedentary dams did not train. After birth litters were adjusted to 9 pups (normal-litter, NL) and 3 (small-litter, SL) to induce obesity. Body weight (bw) and food intake were registered from mothers and offspring. At 90-days-old adult offspring were used to evaluate metabolism. Results: Exercised mothers presented increased insulinemia; however, there were no changes in bw and food intake. As expected, litter reduction induced obesity, hyperinsulinemia, glucose intolerance and insulin resistance $(\mathrm{p}<0.05)$. Maternal exercise training drastically reduced obesity and improved metabolism overall of SL adult rats and blood pressure was improved $(\mathrm{p}<0.05)$.
Conclusion: Exercise training with low intensity and frequency prevents obesity onset in offspring later in life.

\section{5}

\section{The Effect of Maternal Seasonality on Pregnancy Weight Gain and Birthweights in a Canadian Cohort}

Robert Levitan ${ }^{1}$, Alissa Moody ${ }^{2}$, Stephen Matthews ${ }^{3}$, Stephen Lye $^{4}$, Alan Bocking ${ }^{5}$

${ }^{1}$ CAMH, University of Toronto; ${ }^{2}$ Mt. Sinai Hospital, Toronto;

${ }^{3}$ University of Toronto; ${ }^{4} \mathrm{Mt}$. Sinai Hospital, Lunenfeld Research Instutute, University of Toronto; ${ }^{5} \mathrm{Mt}$. Sinai Hospital, Lunenfeld Research Instutute, University of Toronto

At northern latitudes, highly seasonal women overeat and gain considerable weight over the fall/winter period. We examine whether highly seasonal women and/or spring births are associated with greater pregnancy weight gain and/or higher birthweights in the Ontario Birth Study in Toronto, Canada. Data are currently available for 88 pregnancies, including 22 (25\%) with high maternal seasonality. Controlling for maternal age and prepregnancy maternal BMI, we are finding an interesting pattern of pregnancy weight gain in mothers giving birth in the spring. For spring births only, highly seasonal mothers are gaining more weight in pregnancy than are low-seasonality mothers $(p=.04)$. This supports our general working hypothesis that spring births would differ significantly between mothers with high and low seasonality. A similar analysis using birth weight percentile as the dependent variable found a significant main effect of birth season independent of maternal seasonality scores. The mean birth weight percentile in newborns born in the spring was 58.5 compared to 38.2 in other children. These initial results suggest that seasonality may have relevance for pregnancy weight gain and/or fetal growth trajectories in Canadian children. The implications for childhood obesity risk based on birth season and maternal seasonality will be examined prospectively.

\section{2}

How has fetal health changed in the very long run? Western Europe and North America, 1700 to the present

Eric Schneider

London School of Economics and Political Science

The DOHaD research agenda has shown that conditions in utero are important. However, we currently have a very limited understanding of how fetal health has changed in the very long run. This paper analyses proxies of fetal health in Western Europe and North America since 1700. There were improvements in fetal health before 1860 as perinatal mortality rates (PNMR) declined. However, the evidence for improvements in fetal health since 1860 is mixed. PNMRs were fairly stagnant between 1860 and the late 1930s before they declined 
dramatically following the introduction of antibiotics. Antibiotics allowed doctors to conduct more invasive intrapartum interventions to save at risk births because they could effectively treat puerperal fever. Thus, declines in PNMR since the late 1930s may not reflect better conditions in utero but only improvements in intrapartum care. On top of this, birth weight means and distributions have remained relatively constant between the mid-nineteenth century and the present. This leads to two possible conclusions: (1) substantial improvements in postnatal health since 1860 have had a relatively small influence on fetal health or (2) these indicators of foetal health are problematic and researchers need to find new proxies of fetal health for the past and future.

\section{9}

\section{Teenage pregnancy outcomes in Colombia}

Julio Reina $^{1}$, Luis Bravo ${ }^{2}$

${ }^{1}$ Universidad del Valle; ${ }^{2}$ Universidad del Valle

Third world teenage mothers have a higher prevalence of premature and low birth weight outcomes compared to adult mothers. There is a nutritional competition between the growing adolescent mother and the developing gestation.

We studied 435 pregnant adolescents 13 to $17 y$. from a low socioeconomic community and poor living conditions in Cali, Colombia. We gathered socio-demographic, anthropometric, nutritional, gynecological and obstetrical health data, and electronic measurements of the knee height to estimate short term growth.

Results: Mean age $15 \mathrm{y}$. Mean weight gain during pregnancy: $9.2 \mathrm{~kg}$. Mean adolescent mother height: $154.1 \mathrm{~cm}$. Baseline low Body Mass Index (BMI) was associated with low birth weight ( $p<0.001)$. Mean newborn birth weight of the adolescent mother: $3115 \mathrm{~g}$ vs $3175 \mathrm{~g}$ mean newborn birth weight of the adult mothers. $81 \%$ of the pregnant adolescent mothers grew during the observation period, with no influence in the new born birth weight. $84 \%$ were born by normal vaginal delivery and $16 \%$ by cesarean section. $9.7 \%$ were premature and $7.7 \%$ low birth weight.

We did not find nutrient competition between the adolescent growing mother and the newborn birth weight. The adolescent mother age and the gynecological age did not influence the birth weight of the newborn.

\section{6}

Low levels of prenatal vitamin-d and increased risk of adult schizophrenia. Results from a societal experiment in Denmark. The D-tect study

\author{
Joachim Knop ${ }^{1}$, Kristina Ranc ${ }^{2}$ \\ ${ }^{1}$ Institute of Preventive Medicine Frederiksberg and Bispebjerg \\ Hospital, The Capital Region, Denmark; ${ }^{2}$ Institute of Preventive \\ Medicine Frederiksberg and Bispebjerg Hospital, The Capital \\ Region, Denmark
}

Research question: Animal experiments have demonstrated that prenatal vitamin-D deficiency may influence brain structure and function. Also, a few observational studies have suggested that prenatal vitamin-D-statusmay be related to later schizophrenia. However, results are few and inconsistent.

Methods: In Denmark, a mandatory fortification programme adding vitamin-D to margarine took place 1961-1985. Fortification supplied $13 \%$ of daily average intake. We used this "social experiment" to examine risk of schizophrenia among individuals from birth cohorts exposed/not-exposed to the extra vitamin-D during foetal life.

Individuals born two years before $(n=149.580)$ and two years after $(n=161.766)$ initiation of the fortification programme (1961) were selected from Danish registries via personal ID and followed for the subsequent 53 years for schitzophrenia in the Danish Psychiatric Register. Similarly, individual born before $(n=106.627)$ and after $(n=116.149)$ fortification termination (1985) were followed for 24 years.

Results: Compared to non-exposed individuals, exposed had a lower risk of schizophrenia both around the initiation $(\mathrm{HR}=$ $0.81 ; 95 \% \mathrm{CI}(0.70 ; 0.85))$ and the termination $(\mathrm{HR}=0.66$; $95 \%$ CI $(0.46 ; 0.87))$ of the fortification period.

Conclusion: Extra vitamin-D from fortification during foetal life seems to greatly reduce risk of adult schizophrenia. Food supplementation, which supplies small amounts of extra vitamin-D to pregnant woman and the growing foetus may be relevant for reducing risk of schizophrenia.

\section{4}

Prenatal Docosa-hexaenoic acid supplementation and offspring's health outcomes: a systematic review

Pallavi Nair ${ }^{1}$, Suganthi Jaganathan ${ }^{2}$, Garima Rautela ${ }^{3}$, Shivangi Sharma ${ }^{4}$, Poornima Prabhakaran ${ }^{5}$, Malvika Saxena ${ }^{6}$

${ }^{1}$ PHFI; ${ }^{2}$ CCDC; ${ }^{3}$ CCDC; ${ }^{4}$ PHFI; ${ }^{5}$ PHFI; ${ }^{6}$ PHFI

Research question: The objective of this review was to collate evidences from intervention studies of prenatal DHA supplementation on several parameters of offspring's growth and development.

Methods: A systematic review of prenatal DHA intervention studies was conducted using two freely available literature databases-PubMed and Google Scholar. Randomized clinical trials reporting DHA-specific results in children aged 0-2 years born to supplemented mothers were categorized under three primary outcomes of interest: anthropometry, immunity and neurodevelopment (sensory, cognition and others). The Critical Appraisal Skills Program (CASP) tool helped to assess the quality of the extracted studies.

Results: The preliminary search yielded 1355 unique citations; after stage-wise screening 19 studies pertaining to 12 unique trials were included for the final review. Among the total, six, four and nine studies reported about anthropometric, 
immunity and neurodevelopmental outcomes. Nine trials showed a significant improvement in anthropometric and immunity outcomes. However, only one study (of the nine) evaluating cognitive outcomes documented a significant beneficial impact of prenatal DHA on children.
Conclusion: Generic guidelines promoting DHA inclusion during pregnancy need to be re-examined. Well-designed interventions embedded in long term cohorts are required to assess the impact of different fatty acids on specific health outcomes. 